\title{
SUPPRESSION OF THE IMMUNE RESPONSE AGAINST TUBERCULOSIS BY NATURAL T REGULATORY CELLS
}

By

Kylie Margaret Quinn

\author{
A thesis \\ submitted to the Victoria University of Wellington \\ in fulfilment of the requirements for the degree of \\ Doctor of Philosophy \\ in Cell Biology
}

Victoria University of Wellington 


\section{Abstract}

The causative agents of Tuberculosis (Tb), Mycobacterium tuberculosis and Mycobacterium bovis, subvert a number of host immune mechanisms to promote persistence of infection in the human host. Infection with these immunoevasive pathogens is estimated to cause 1.8 million deaths per year. The only $\mathrm{Tb}$ vaccine currently available is an attenuated form of $M$. bovis, called bacille Calmette Guérin (BCG), and its efficacy is highly variable. Thus, development of more effective vaccine strategies for $\mathrm{Tb}$ is of significant importance.

Host-derived natural $\mathrm{CD} 4^{+} \mathrm{CD} 25^{+} \mathrm{T}$ regulatory cells (Tregs) suppress host immune responses and prevent autoimmunity. However, Tregs can also interfere with protective immune responses against chronic infections or with the protective efficacy of vaccines. There is speculation that mycobacteria exploit the suppressive activity of Tregs to permit persistence in the host and that Treg activity also diminishes the protective efficacy of the BCG vaccine.

Therefore, to explore the role of Tregs in anti-mycobacterial immunity, I first optimised a protocol to inactivate Tregs in vivo in a murine model, through administration of anti-CD25 monoclonal antibody. In addition, I developed a novel plasmid DNA replicon vaccine against $\mathrm{Tb}$ to aid with the assessment of the effect of Tregs on $\mathrm{Tb}$ vaccines.

I hypothesised that inactivating Tregs in vivo prior to mycobacterial infection would enhance acute immune responses that are thought to be protective under current dogma and thereby enhance bacterial clearance. Enhanced immune responses were observed but, contrary to the hypothesis, inactivation of Tregs did not alter bacterial numbers in the lung.

I also hypothesised that inactivating Tregs in vivo prior to live BCG or DNA vaccination would enhance primary and secondary immune responses that are thought to be protective against $\mathrm{Tb}$ infection and thereby enhance the protective efficacies of the vaccines. Enhanced vaccine-induced immune responses and accelerated secondary immune responses were observed for the BCG vaccine. However, Treg inactivation did not alter the protective efficacy of the BCG vaccine, and preliminary data suggests Treg 
inactivation may in fact decrease the protective efficacy of one plasmid DNA vaccine tested.

In summary, this thesis illustrates that inactivating natural Tregs does not enhance protection from mycobacterial infection. This implies that inactivation of natural Tregs cannot be used in a therapeutic setting to enhance treatment or vaccination approaches for $\mathrm{Tb}$. More importantly, Treg inactivation enhances immune responses that current dogma regard as protective against $\mathrm{Tb}$ infection, but does not correspondingly enhance protection. As a result of this disparity, this thesis questions the validity of measures that are commonly used as immune correlates of protection for $\mathrm{Tb}$ vaccine design. Ultimately, the development of more accurate immune correlates of protection for $\mathrm{Tb}$ will permit targeted design of more effective $\mathrm{Tb}$ vaccination strategies. 


\section{Acknowledgments}

I have been fortunate during the course of my studies to have the help of a supportive group of family and friends and a talented and enthusiastic group of colleagues.

I would like to thank Fenella Rich, Lisa Goldsack, Therése Petterssen, Prof. Brett Delahunt and Kasper Eckert for their assistance with experiments detailed in this thesis. In addition, the technical advice of Fenella Rich, Lisa Goldsack, Cyndy Watson, and Drs Rebecca McHugh, Thomas Bäckström, Tobias Ostler, Alex Khromykh and Ronan O'Toole, my co-supervisor at Victoria University, has enriched my studies immensely. I would also like to thank those who assisted with critical reading and editing this thesis.

A number of experiments were undertaken at AgResearch Wallaceville and I would like to sincerely thank the staff, in particular Gary Yates, Prof. Bryce Buddle and Dr Geoff De Lisle, for their assistance. Mice were housed at the Malaghan Institute for Medical Research Biomedical Research Unit and the AgResearch Wallaceville Biohazard Facility and I would like to acknowledge the excellent animal husbandry of staff at both facilities. I would like to acknowledge the advice and support of staff and students at both the Victoria University of Wellington School of Biological Sciences and the University of Otago. I have appreciated financial support provided by the Lottery Health Grants Board and Victoria University, which has permitted me to undertake these studies.

The Malaghan Institute has been an exciting, fun and supportive environment to study at during my PhD. I would like to sincerely thank all the people that contribute to that environment: my fellow PhD students, the scientific staff, the support staff, the senior scientists and (some great late night company from Apii!).

My friends and family have been extremely understanding and supportive. I want to thank in particular my mum Heather, my dad D'Arcy and my sister Shayna for their immeasurable support.

And finally I would like to thank my supervisor, Dr Joanna Kirman, who has been an outstanding mentor, teacher and support for me. I have admired and appreciated her consistent optimism and enthusiasm. 


\section{Table of Contents}

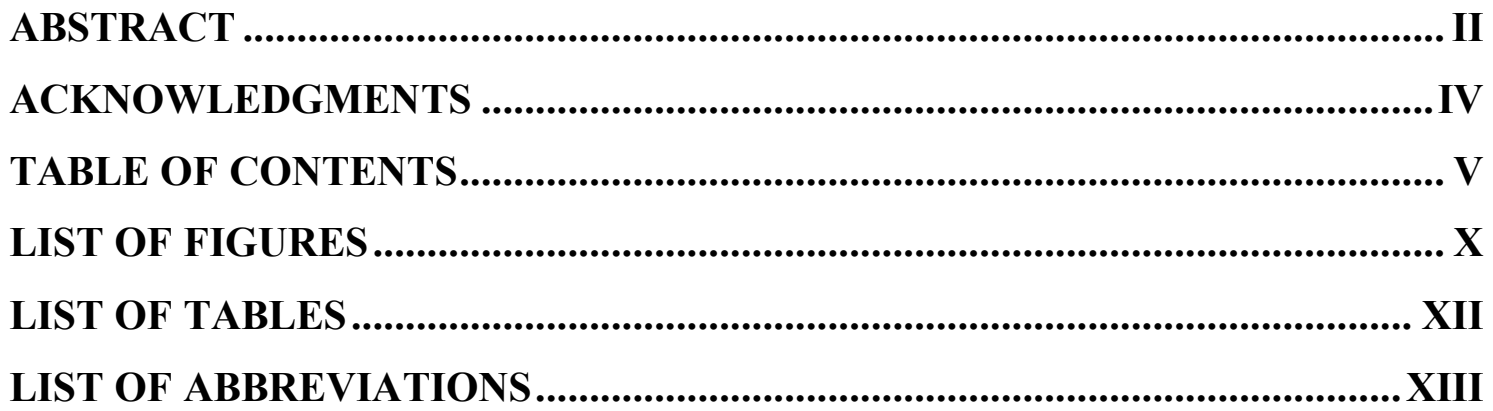

CHAPTER 1: GENERAL INTRODUCTION ................................................. 1

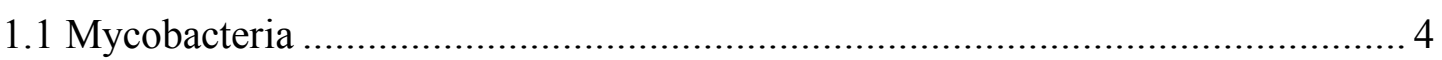

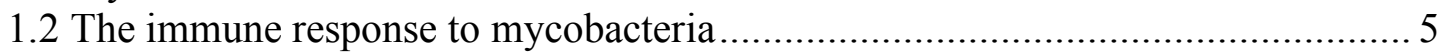

1.2.1 A general outline of the immune response ................................................ 5

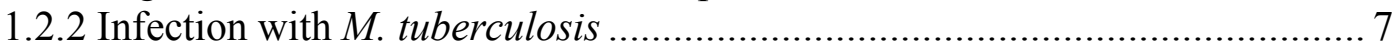

1.2.3 The primary immune response to $M$. tuberculosis ..................................... 9

1.2.3.1 Containing infection with the innate immune response..................... 9

1.2.3.2 The adaptive immune response .................................................. 11

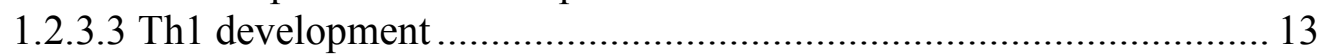

1.2.3.4 Immunomodulation of the immune response by cytokines .............. 15

1.2.3.5 Effector mechanisms of anti-mycobacterial immunity................... 17

1.2.4 Latent infection and the granuloma.................................................... 22

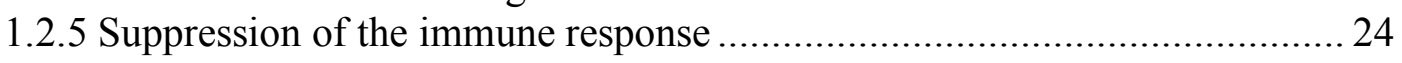

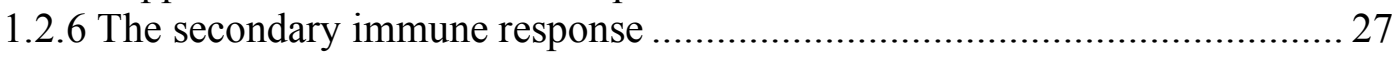

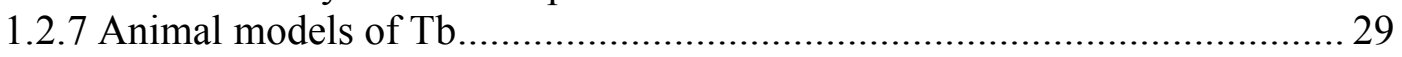

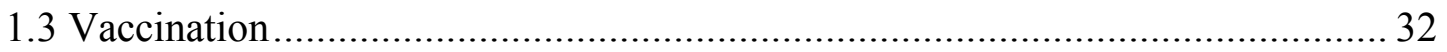

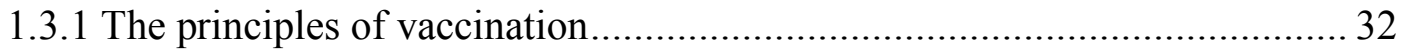

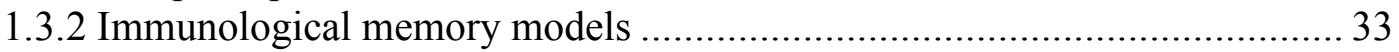

1.3.2.1 Humoral memory....................................................................... 34

1.3.2.2 Cell-mediated memory........................................................... 34

1.3.2.3 Factors mediating the development and maintenance of memory $\mathrm{T}$

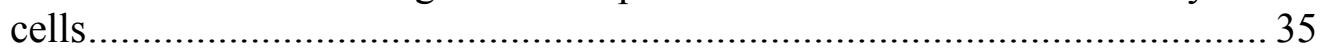

1.3.2.4 Models of memory $\mathrm{T}$ cell differentiation ........................................ 37

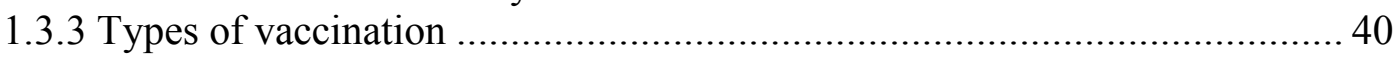

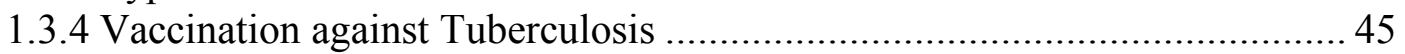

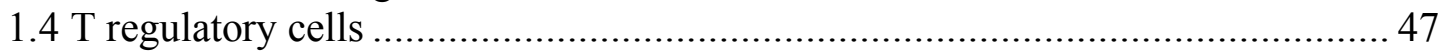

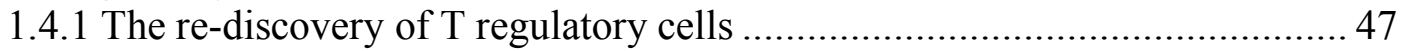

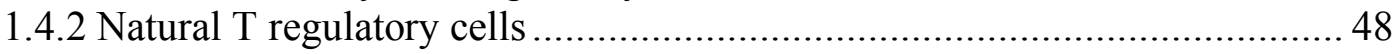

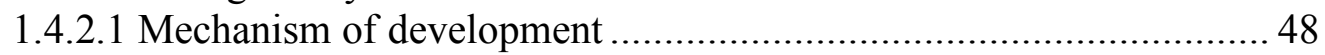

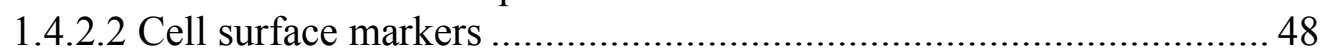

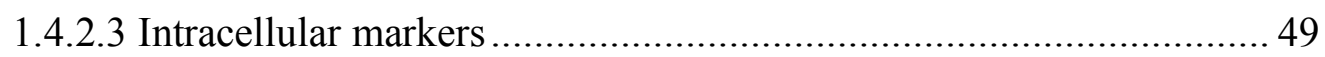

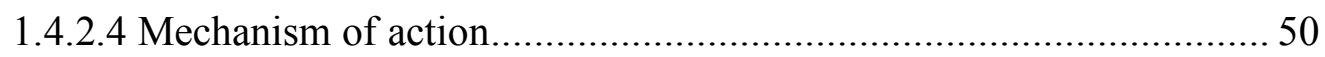

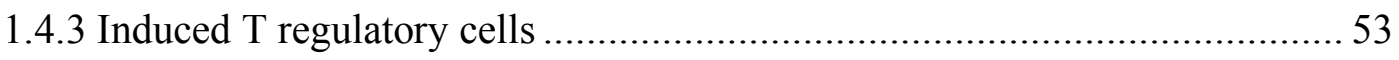

1.4.3.1 Low-dose antigen induced Tregs.............................................. 54

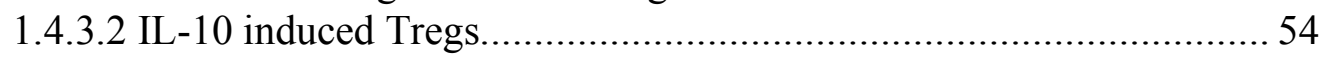

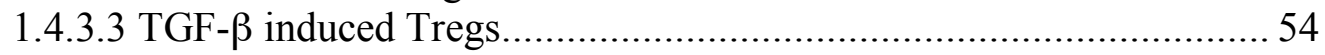

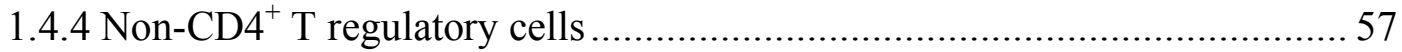

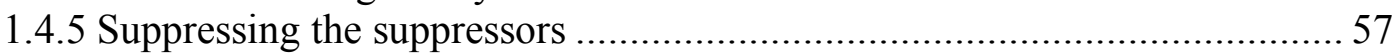


CHAPTER 2: MATERIALS AND METHODS ...................................................60

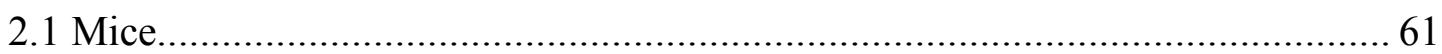

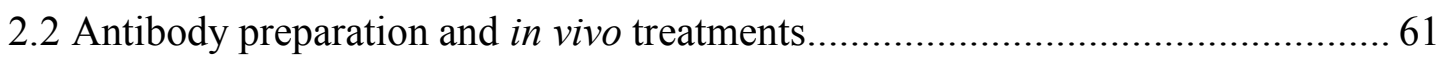

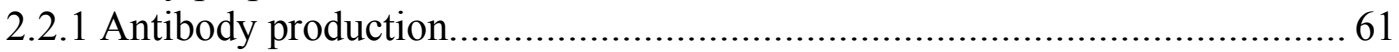

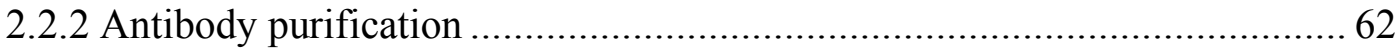

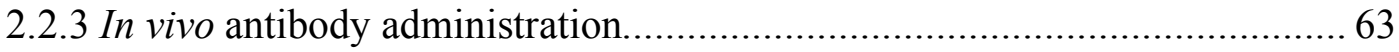

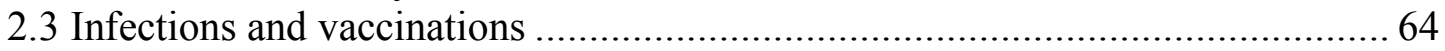

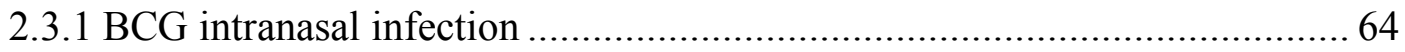

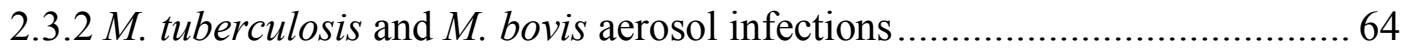

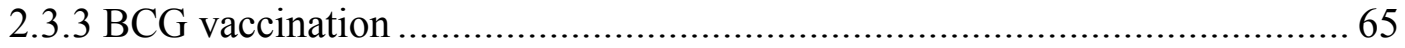

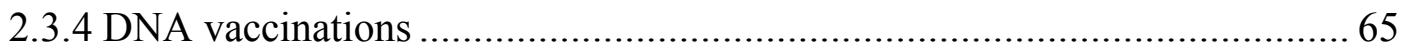

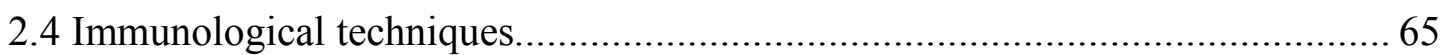

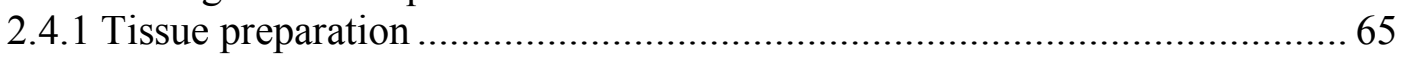

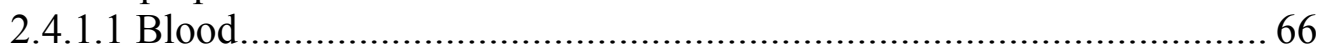

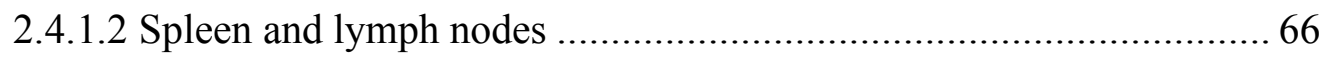

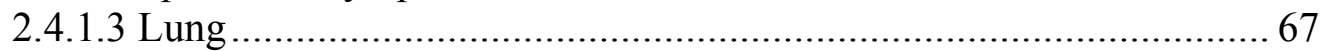

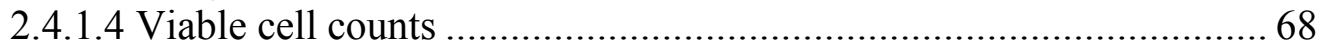

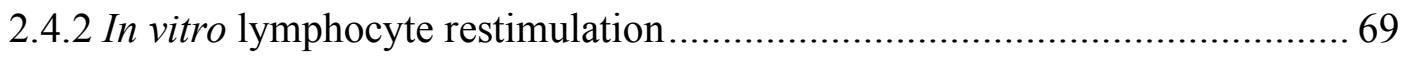

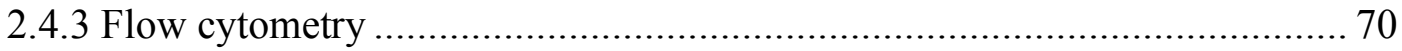

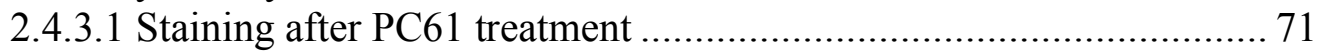

2.4.3.2 Cell surface staining for assessment of cytokine production ............ 72

2.4.3.3 Cell surface staining for assessment of Foxp3 expression............... 73

2.4.3.4 Intracellular cytokine staining .................................................. 73

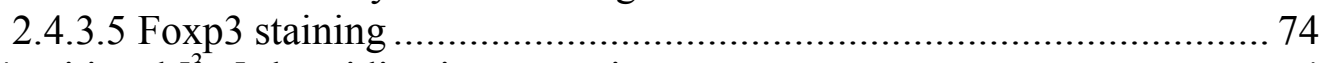

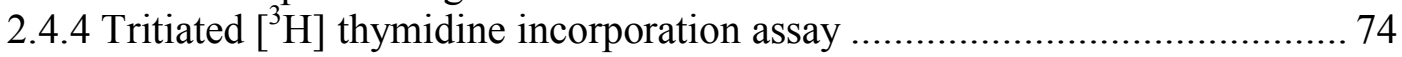

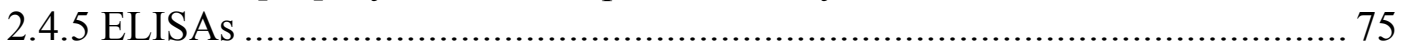

2.4.5.1 IFN- $\gamma$ ELISA of cell culture supernatants .................................. 75

2.4.5.2 Anti-CD25 (clone PC61) ELISA of murine serum ......................... 76

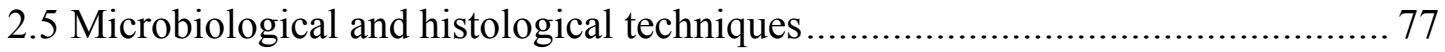

2.5.1 Determination of BCG bacterial load........................................................ 77

2.5.2 Determination of M. tuberculosis and M. bovis bacterial load .................... 77

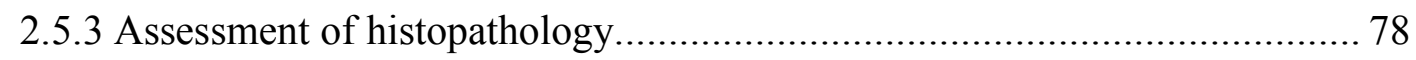

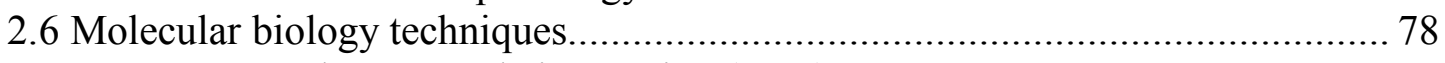

2.6.1 Ag85A polymerase chain reaction (PCR) ............................................ 78

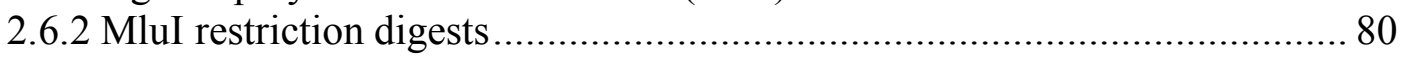

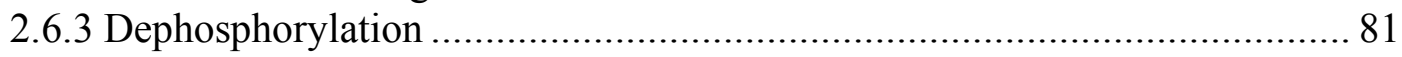

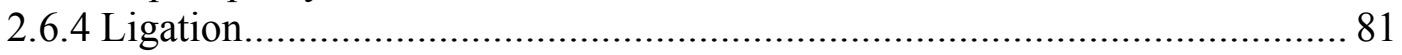

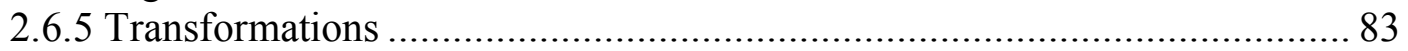

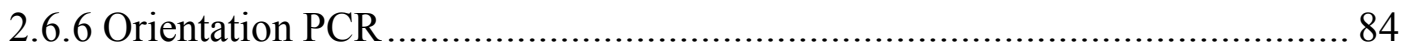

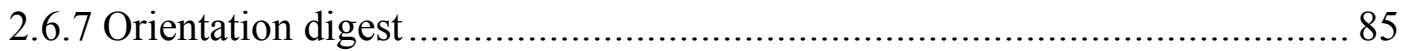

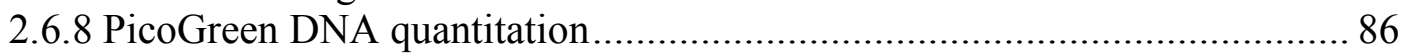

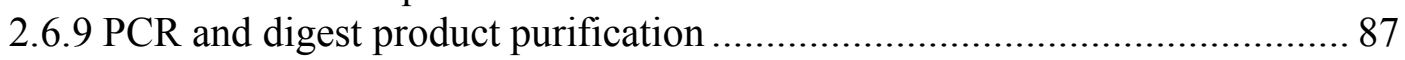

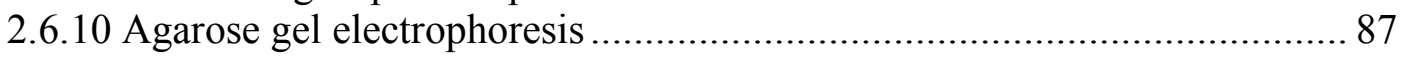

2.6.11 Qiagen Plasmid Mini-prep protocol ........................................................ 88

2.6.12 Qiagen Plasmid Endotoxin-Free Mega-prep protocol............................. 88

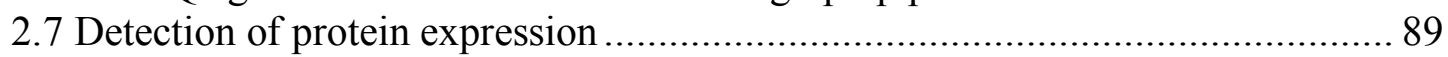

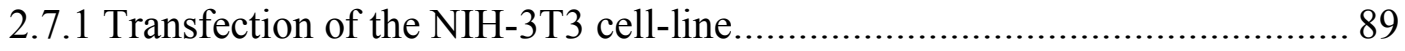

2.7.2 Lysis of NIH-3T3 cells and immunoprecipitation of target proteins ............ 90 
2.7.3 SDS-PAGE, Western transfer and immunoblotting................................ 91

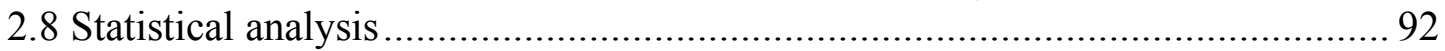

\section{CHAPTER 3: SUPPRESSION BY NATURAL TREGS DURING PRIMARY}

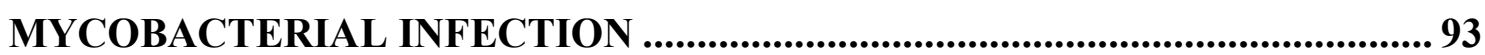

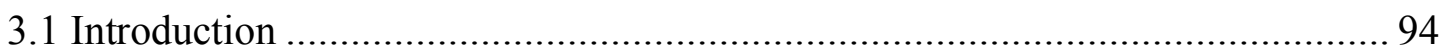

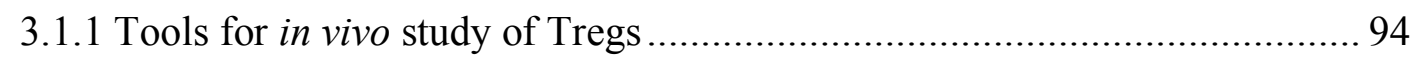

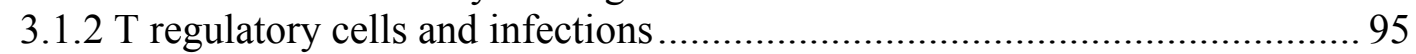

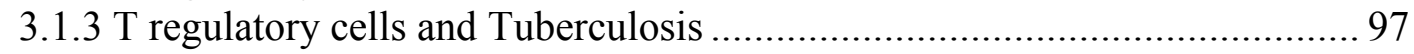

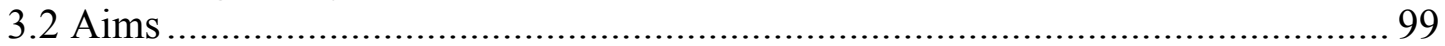

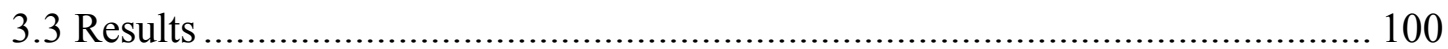

3.3.1 Treg inactivation in the lung and other tissues ....................................... 100

3.3.2 Repopulation by Tregs after PC61 administration................................... 104

3.3.3 Treg influx to the lung after mycobacterial infection.............................. 109

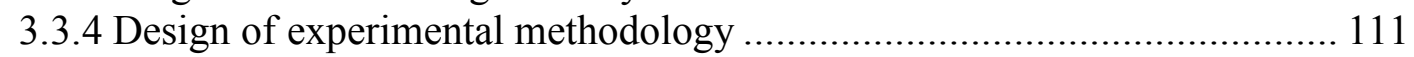

3.3.5 Natural Tregs and the immune response to primary mycobacterial infection

3.3.6 Lung bacterial numbers and inflammation during BCG or M. tuberculosis

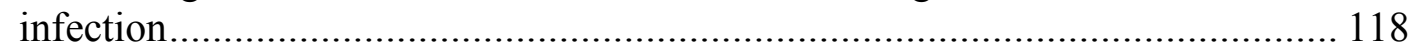

3.3.7 Lung bacterial numbers and inflammation during $M$. bovis infection ........ 122

3.3.8 Tregs during the chronic phase of mycobacterial infection..................... 126

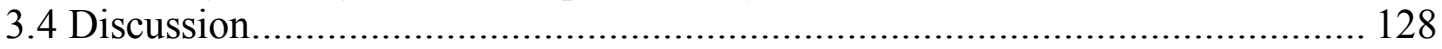

3.4.1 PC61 treatment and its limitations ....................................................... 129

3.4.2 Induction of Tregs during Tb infection ................................................ 131

3.4.3 Suppression by Tregs during chronic Tb infection ............................... 131

3.4.4 Immunopathology during M. bovis infection ...................................... 133

3.4.5 Subsequent published studies ........................................................... 134

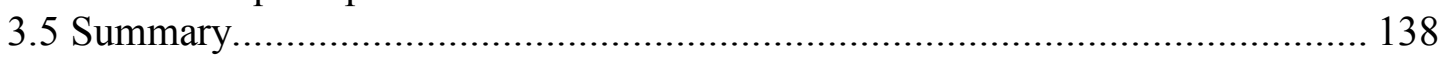

\section{CHAPTER 4: CONSTRUCTION OF A NOVEL PLASMID DNA REPLICON}

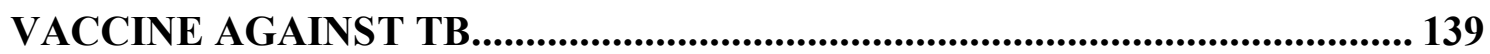

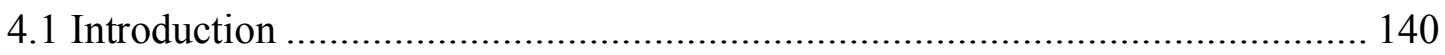

4.1.1 DNA plasmid vaccination for Tb: Pros and Cons.................................. 140

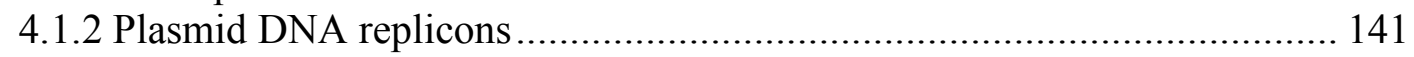

4.1.3 Apoptosis and DNA vaccine immunogenicity ..................................... 145

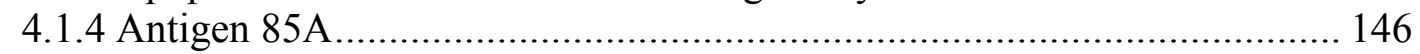

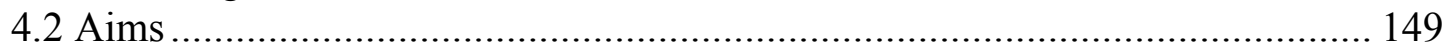

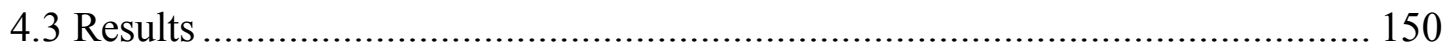

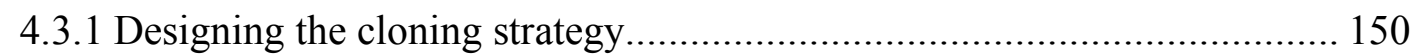

4.3.2 Preparation of Ag85A PCR product and pKUNrep6 vector DNA ............. 155

4.3.3 Cloning Ag85A into pKUNrep6 ............................................................ 157

4.3.4 Screening of transformants for pKUN85A.......................................... 157

4.3.5 Expression of Ag85A and NS-1 protein from pKUN85A ..................... 161

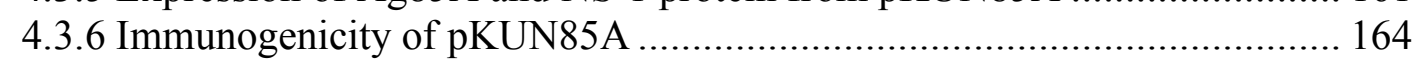

4.3.7 Short-term protection from infection afforded by pKUN85A................... 167

4.3.8 Long-term protection from infection afforded by pKUN85A ................... 170 
4.3.9 Pulmonary inflammation after pKUN85A vaccination followed by $\mathrm{Tb}$

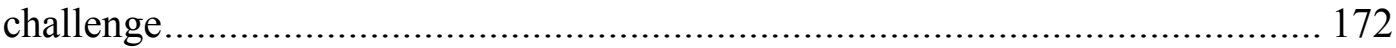

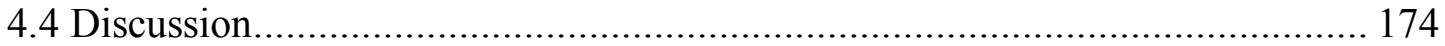

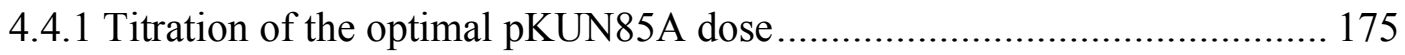

4.4.2 Longevity of protection after vaccination................................................ 176

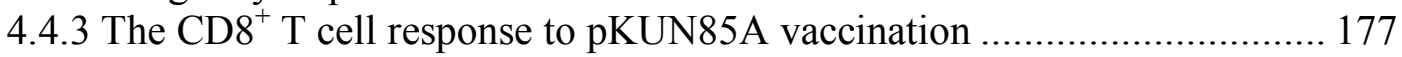

4.4.4 Future directions for pKUN85A vaccination....................................... 178

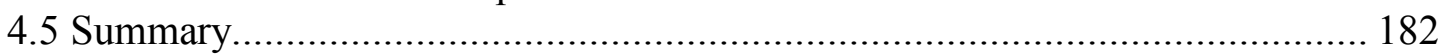

\section{CHAPTER 5: NATURAL TREG-MEDIATED SUPPRESSION OF}

PROTECTIVE IMMUNITY AGAINST TB ..................................................... 183

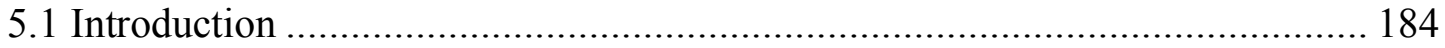

5.1.1 Tregs can suppress protective memory .............................................. 184

5.1.2 Tregs can facilitate protective memory ................................................ 186

5.1.3 Tregs and development of anti-mycobacterial immunity......................... 186

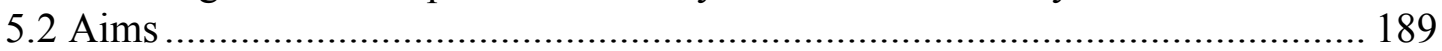

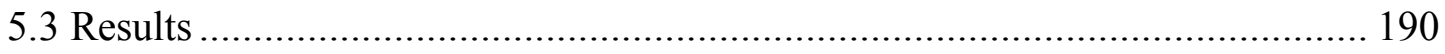

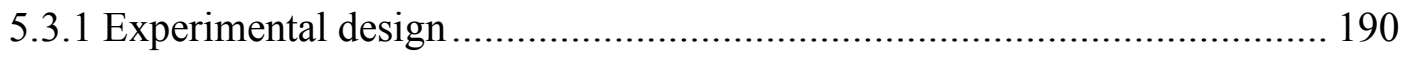

5.3.2 The vaccine-induced immune response to BCG vaccination ................... 193

5.3.3 Antigen-responsive lymphocytes after BCG vaccination ....................... 196

5.3.4 The secondary immune response for BCG vaccination ......................... 201

5.3.5 Short-term protection for BCG vaccination........................................ 204

5.3.6 Long-term protection for BCG vaccination........................................... 206

5.3.7 Lung pathology for BCG vaccination ................................................. 208

5.3.8 Vaccine-induced immune responses to Tb DNA vaccines....................... 210

5.3.9 Secondary immune responses for Tb DNA vaccines ............................ 213

5.3.10 Protection from infectious challenge for Tb DNA vaccines................... 218

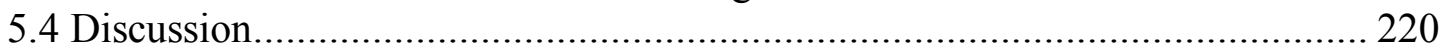

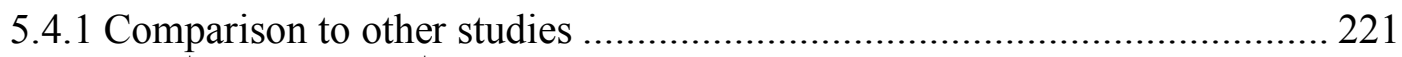

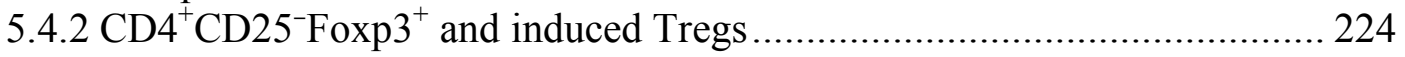

5.4.3 Implications for models of Th1 immunity......................................... 225

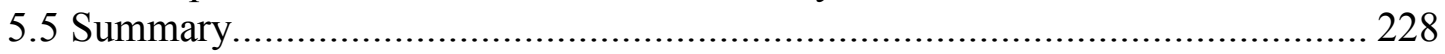

CHAPTER 6: GENERAL DISCUSSION.......................................................... 229

6.1 Summary of experiments...................................................................... 230

6.2 Optimisation of the pKUN85A DNA replicon vaccine ................................ 231

6.2.1 Techniques for optimising pKUN85A efficacy .................................. 231

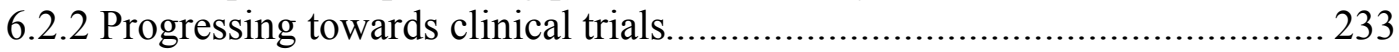

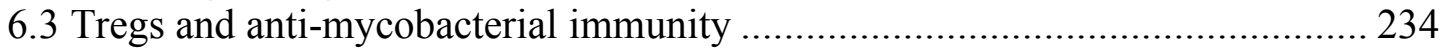

6.3.1 Induced Tregs and anti-mycobacterial immunity ................................. 236

6.3.2 Tregs during chronic mycobacterial infection ........................................ 238

6.3.3 Tregs during virulent mycobacterial disease ..................................... 240

6.3.4 Cross-reactivity or bystander Treg suppression...................................... 240

6.3.5 Treg memory................................................................................. 242

6.3.6 Recent progress and remaining questions............................................. 243

6.3.7 Summary: Tregs and anti-mycobacterial immunity ............................. 245

6.4 Lessons for the immunological basis of mycobacterial protection.................. 246

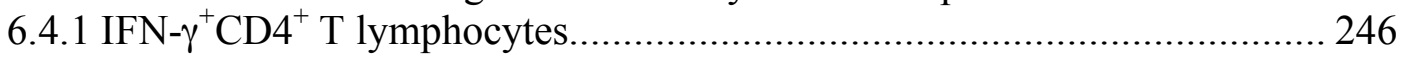




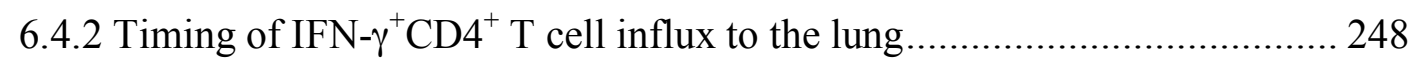

6.4.3 Multifunctional Th1 lymphocytes...................................................... 249

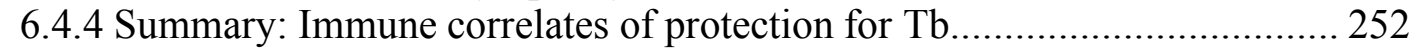

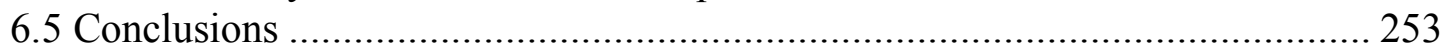

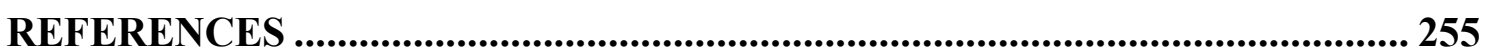

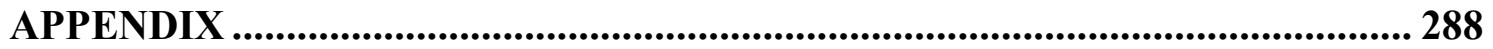

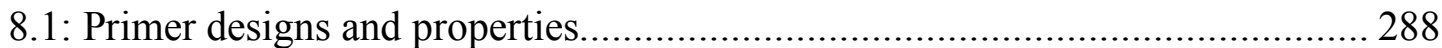

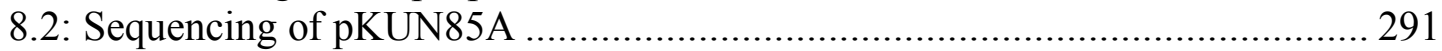

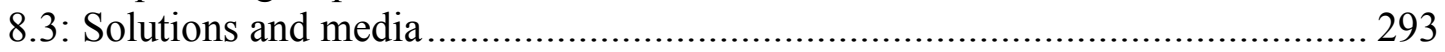

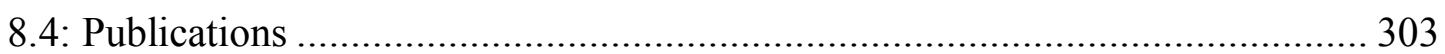




\section{List of Figures}

Figure 1.1: Possible outcomes after exposure to aerosolised $M$. tuberculosis ...........................8

Figure 1.2: Effector mechanisms of the immune response during Tb infection .....................21

Figure 1.3: The Linear and Branched Models of Memory T Cell Development....................38

Figure 1.4: The Process of DNA Vaccination ...........................................................................................43

Figure 1.5: Schematic outlining Treg subsets and mechanisms of action...............................56

Figure 2.1: Plot of spleen weight against viable lymphocyte count from spleen cell preparations.

Figure 3.1: Optimisation of the route of PC61 administration ................................................... 101

Figure 3.2: Treg inactivation in the blood, spleen and lung after PC61 treatment 103

Figure 3.3: Treg repopulation in peripheral blood of naïve or mycobacteria-infected mice

Figure 3.4: Treg repopulation in the lung after mycobacterial infection. 107

Figure 3.5: Persistence of PC61 mAb in circulation. 108

Figure 3.6: Comparison of Treg and total $\mathrm{CD4}^{+} \mathrm{T}$ cell influx to the lung tissue after mycobacterial infection 110

Figure 3.7: Experimental outline for testing whether natural Tregs suppress the acute phase of mycobacterial infection 112

Figure 3.8: ELISA analysis of IFN- $\gamma$ production during mycobacterial infection 114

Figure 3.9: IFN- $\gamma$ and IL-2 production by splenocytes after mycobacterial infection...... 116 Figure 3.10: IFN- $\gamma$ and IL-2 production by lung lymphocytes after mycobacterial infection

Figure 3.11: Lung bacterial numbers after BCG or M. tuberculosis infection 119

Figure 3.12: Pulmonary inflammation after BCG or M. tuberculosis infection 121

Figure 3.13: Tissue bacterial numbers after $M$. bovis infection 124

Figure 3.14: Pulmonary inflammation after $M$. bovis infection 125

Figure 3.15: Impact of Treg modulation on chronic M. tuberculosis infection 127

Figure 4.1: Proposed mechanism of action for plasmid DNA replicons 143

Figure 4.2: Schematic view of the composition of the mycobacterial cell wall 147

Figure 4.3: Plasmid diagrams for pcDNA3-85A, pKUNrep6, pKUN85AOF and pKUN85A

Figure 4.4: Schematic diagram of the translated product from pKUN85A

Figure 4.5: The cloning strategy for the creation of pKUN85A............................................ 153

Figure 4.6: Preparation of $\mathrm{Ag} 85 \mathrm{~A}$ PCR product and pKUNrep6 for cloning 156 
Figure 4.7: Orientation PCR of transformants ....................................................... 159

Figure 4.8: Sample orientation digest for transformants.............................................. 160

Figure 4.9: Protein expression from pKUN85A-transfected NIH-3T3 cells ........................ 163

Figure 4.10: Lymphocyte proliferation and IFN-g production after pKUN85A vaccination

Figure 4.11: Cytokine production by $\mathrm{CD4}^{+}$and $\mathrm{CD8}^{+} \mathrm{T}$ cells after pKUN85A vaccination 166

Figure 4.12: Short-term protection from $M$. tuberculosis infection after pKUN85A vaccination. 169

Figure 4.13: Long-term protection from $M$. tuberculosis infection after pKUN85A vaccination.

Figure 4.14: Lung pathology after pKUN85A vaccination.

Figure 5.1: Experimental outline for testing whether natural Tregs inhibit the efficacy of BCG and DNA vaccination

Figure 5.2: IFN- $\gamma$ and IL-2 production by CD4 ${ }^{+}$lymphocytes 2 weeks after BCG vaccination

Figure 5.3: Cytokine production by $\mathrm{CD4}^{+}$lymphocytes 2 weeks after BCG vaccination. 195

Figure 5.4: IFN- $\gamma$ production by $\mathrm{CD4}^{+}$and $\mathrm{CD8}^{+}$lymphocytes 6 weeks after BCG vaccination

Figure 5.5: IFN- $\gamma$ and IL-2 production by CD4 ${ }^{+}$lymphocytes 6 weeks after BCG vaccination

Figure 5.6: IFN- $\gamma$ and IL-2 co-production by CD4 ${ }^{+}$lymphocytes 6 weeks after BCG vaccination 200

Figure 5.7: The secondary immune response after i.n. BCG challenge.............................. 203

Figure 5.8: Tissue bacterial numbers after early virulent mycobacterial challenge .......... 205

Figure 5.9: Lung bacterial numbers after late $M$. tuberculosis or M. bovis challenge....... 207

Figure 5.10: Pulmonary inflammation after M. bovis infection ...................................... 209

Figure 5.11: Vaccine-induced immune responses after DNA vaccination ........................... 212

Figure 5.12: Secondary immune responses in the spleen after DNA vaccination............. 214

Figure 5.13: Secondary immune response in the lung after DNA vaccination.................. 216

Figure 5.14: IFN- $\gamma$ and IL-2 co-production by CD4 ${ }^{+}$lymphocytes after DNA vaccination

Figure 5.15: Protection afforded by DNA vaccination 219

Figure 5.16: Schematic for hypothesised effect of Treg inactivation on lymphocyte subsets and protection after $\mathrm{BCG}$ vaccination

Figure 8.1: Primer design for sequencing of transformant plasmids

Figure 8.2: Sequencing alignment for transformant 37 


\section{List of Tables}

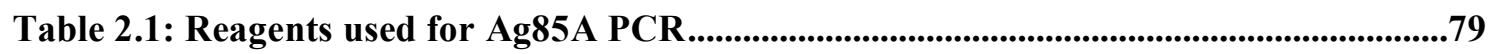

Table 2.2: PCR cycles used for Ag85A PCR ...........................................................................................79

Table 2.3: Reaction conditions for MluI digestion of Ag85A PCR product ............................80

Table 2.4: Reaction conditions for MluI digestion of pKUNrep6 .................................................81

Table 2.5: Ligation Reactions and Controls.....................................................................82

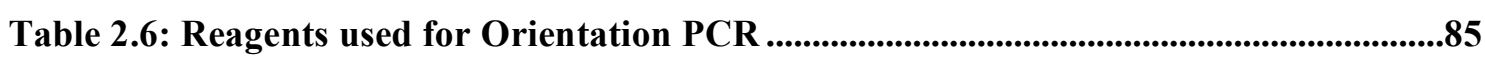

Table 2.7: PCR cycles used for Orientation PCR .............................................................85

Table 2.8: Reaction conditions for KspI and XbaI orientation digestion of pKUN85A .......86

Table 4.1: Ag85A PCR primer design........................................................................................................... 154

Table 8.1: Primers for amplification of the $\mathrm{Ag85A}$ insert......................................................... 288

Table 8.2: Primers for orientation PCR and sequencing of the $A g 85 A$ insert.................... 289 


\section{List of Abbreviations}

Tb

DNA

M. tuberculosis

APC

HIV

Treg

MTBC

M. bovis

BCG

PAMP

PRR

NK

DC

MHC

MHC I

MHC II

TCR

Th

IFN

TNF

IL

TLR

TNF

$-/-$

ER

mAb

WT

$\beta-2$ m

PIM

LAM

PBMC

TGF

ROI

M. microti

RNI
Tuberculosis

Deoxyribose nucleic acid

Mycobacterium tuberculosis

Antigen presenting cell

Human immunodeficiency virus

$T$ regulatory cells

Mycobacterium tuberculosis complex

Mycobacterium bovis

Mycobacterium bovis bacille Calmette Guérin

Pathogen associated molecular pattern

Pattern recognition receptor

Natural killer

Dendritic cell

Major-histocompatibility complex

Major-histocompatibility complex class I

Major-histocompatibility complex class II

$T$ cell receptor

T helper

Interferon

Tumour necrosis factor

Interleukin

Toll-like receptor

Tumour necrosis factor

Gene-disrupted

Endoplasmic reticulum

Monoclonal antibody

Wild-type

$\beta-2$ microglobulin

Phosphatidylinositol mannoside

Lipo-arabinomannan

Peripheral blood mononuclear cell

Transforming growth factor

Reactive oxygen intermediates

Mycobacterium microti

Reactive nitrogen intermediates 
NOS2

CTL

Acr

ESAT-6

TACO

STAT

CFU

s.c.

i.n.

PC

M. marinum

$\mathbf{T}_{\mathbf{E M}}$

$\mathbf{T}_{\mathrm{CM}}$

AICD

i.m.

MVA

MVA-85A

GITR

Foxp3

IPEX

LPS

GITRL

CIMDM

$\min$

d

PBS

PC61

i.p.

DTA-1

5F-1

sec

ELISA

CFP

Ag85A

CPM

HRP
Inducible nitric oxide synthase

Cytotoxic lymphocyte

$16 \mathrm{kDa}$ alpha-crystallin homologue

$6 \mathrm{kDa}$ early secreted antigenic target

Tryptophan aspartate-containing coat

Signal transducer and activator of transcription

Colony forming units

Subcutaneous

Intranasal

Physical containment

Mycobacterium marinum

Effector memory $T$ cell

Central memory $T$ cell

Activation induced cell death

Intramuscular

Modified vaccinia virus Ankara

Modified vaccinia virus Ankara viral vector containing

Antigen 85A from Mycobacterium tuberculosis

Glucocorticoid induced TNF receptor

Forkhead box P3 transcription factor

Immune dysregulation, polyendocrinopathy, enteropathy, $\mathrm{X}$ -

linked syndrome

Lipopolysaccharide

Glucocorticoid induced TNF receptor ligand

complete Iscove's Modified Dulbecco's Medium

Minute

Distilled

Phosphate buffered saline

Anti-CD25 mAb (clone PC61)

Intraperitoneal

Anti-GITR (clone DTA-1)

Anti-GITRL (clone 5F-1)

Seconds

Enzyme linked immuno-sorbent assay

Culture filtrate protein

Antigen 85A from Mycobacterium tuberculosis

Counts per minute

Horseradish peroxidase 
PCR

\section{TA}

CIAP

bp

rpm

LB Amp+ agar plates

iIMDM

SDS-PAGE

TBS

SEM

GEM

HSV

L. major

H. pylori

L. sigmodontis

GFP

Rag1

RNA

+ str

ds

- str

RIG-1

PKR

mRNA

NS

Ag85

ORF

CMV

FMDV

PolyA

aa

G, C, A and T

ELISpot

P. berghei

MFI
Polymerase chain reaction

Annealing temperature

Calf intestinal alkaline phosphotase

Base-pairs

Revolutions per minute

Luria Broth supplemented agar plates containing ampicillin Incomplete Iscove's Modified Dulbecco's Medium

Sodium dodecyl sulfate polyacrylamide gel electrophoresis

Tris-buffered Saline

Standard error of the mean

Standard error of the geometric mean

Herpes simplex virus

Leishmania major

Helicobacter pylori

Litomosoides sigmodontis

Green fluorescent protein

Recombinase activating gene 1

Ribonucleic acid

Positive-sense strand

Double-stranded

Negative-sense strand

Retinoic acid inducible gene-I

Protein kinase receptor

Messenger RNA

Non-structural

Antigen 85

Open reading frame

Cytomegalovirus

Foot and mouth disease virus

Polyadenylation

Amino acid

Guanine, cytosine, adenine and thymine

Enzyme-linked immunospot assay

Plasmodium berghei

Mean fluorescence intensity 
Chapter 1:

General Introduction 
Mycobacterium tuberculosis, the causative agent of Tuberculosis (Tb) in humans, has long been associated with disease in its human host. Mycobacterial deoxyribose nucleic acid (DNA) has been found in numerous mummified human remains, dating back several thousand years in Egypt and South America [1-4]. Co-evolution of $M$. tuberculosis with its human host has led to a complex disease process manifest in the immune response to $\mathrm{Tb}$.

The human immune system is a carefully orchestrated interplay of cells and hormones that detect and control or eliminate danger to the health of the host. The immune system has evolved to combine broad activation signals with highly specific recognition of antigens. Processing of these activation signals and presentation of specific epitopes by the innate arm of the immune system and antigen presenting cells (APCs) activates the adaptive arm, which has more targeted antigen-specific actions. It is vital to understand how this initial immune response unfolds after exposure to a specific pathogen in order to design effective immunotherapies or diagnostics for the resulting disease.

Importantly, if the immune system encounters a certain pathogen for a second time, it can exhibit immunological memory. This second encounter with a pathogen results in an immune response that is faster or larger and more effective at controlling or eradicating the pathogen than the initial encounter. Immunological memory forms the basis of vaccination, and vaccine design relies on understanding how an effective secondary response can be induced to a specific pathogen.

Unfortunately, the immune response to $\mathrm{Tb}$ is so complex that a satisfactory model of primary and secondary immune responses has not yet been developed. $M$. tuberculosis has evolved strategies to evade the immune response ranging from hiding or disguising itself to actually hijacking the cellular machinery of the infected host to actively shut the immune response down. This makes it challenging to formulate a general model of the primary immune response. Vaccine design for $\mathrm{Tb}$ has also been a challenge, as it is not understood which aspects of a primary immune response lead to a successful, protective secondary immune response.

The development of a new vaccine to combat $\mathrm{Tb}$ is of real importance as $\mathrm{Tb}$ is one of the top three infectious killers, alongside human immunodeficiency virus (HIV) and malaria. Tb killed an estimated 1.6 million people and newly infected 8.8 million people worldwide in 2005 [5]. While death and incidence rates seem to have stabilised 
worldwide, the total number of cases continue to grow in areas such as Africa, Eastern Mediterranean and South-East Asia.

The overall goal of my thesis was to evaluate the role of natural $\mathrm{T}$ regulatory cells (Tregs) in the primary immune response to mycobacterial infection and their impact on development of protective memory immunity induced by vaccination. Through this, I was able to suggest refinements of current models for protective primary and secondary immune responses against $\mathrm{Tb}$, and deeper understanding of this area will facilitate the generation of a more effective vaccine to combat $\mathrm{Tb}$.

Natural Tregs are a subset of lymphocytes that can suppress effector immune mechanisms, such as proliferation and cytokine production by other lymphocytes (section 1.4.2). Given the ability of natural Tregs to suppress immune mechanisms and the immunoevasive nature of mycobacteria (section 1.2.5), it has been widely hypothesised that Tregs are exploited by mycobacteria to suppress the host immune response. Therefore, I sought to evaluate whether natural Tregs suppress the immune response during mycobacterial infection in an in vivo murine model. Additionally, since natural Tregs can inhibit lymphocyte activation or cytokine production, and these factors are important for optimal development of immunological memory (section 1.3.2.3), activation of natural Tregs during vaccination could diminish Tb vaccine efficacy. Therefore, I used several Tb vaccines, including a novel DNA replicon vaccine developed during the course of this project, to investigate whether inactivating natural Tregs during vaccination would enhance the development of protective memory against Tb.

In this chapter, I review M. tuberculosis the organism (section 1.1), the primary and secondary immune responses to mycobacteria and mechanisms by which mycobacteria manipulate these responses (section 1.2). In addition, an overview of current models of immunological memory and strategies for vaccine design is included (section 1.3). Finally, as my thesis focuses on the role of Tregs, a review of the subtypes of these cells and their mechanisms of induction and action is presented (section 1.4). 


\subsection{Mycobacteria}

M. tuberculosis is a very resilient, niche-evolved pathogen. It is a $2-5 \mu \mathrm{m}$ long rodshaped Actinobacterium belonging to the genus Mycobacterium. The genus has traditionally been divided into rapid growers such a Mycobacterium smegmatis or Mycobacterium flavescens, which form colonies on solid media in less than 7 days, or slow growers such as Mycobacterium bovis, Mycobacterium avium and M. tuberculosis, which take longer than 7 days [6]. Within the slow-growing mycobacteria, $M$. tuberculosis is part of the M. tuberculosis complex (MTBC), a group of organisms so closely related that they are defined as subspecies of the same species. The MTBC consists of M. tuberculosis, Mycobacterium africanum, Mycobacterium canetti, $M$. bovis and Mycobacterium microti. It is noteworthy that while these organisms are highly related at a genetic level, they are remarkably different in terms of host range and pathogenicity. For example, M. bovis has a broad mammalian host range while $M$. microti is almost exclusively a rodent pathogen.

Mycobacteria possess a highly specialised cell wall, which allows them to survive within the host and remain viable for a period in the environment outside of a host. The cell wall contains some peptidoglycan, which is a feature of gram-positive bacteria, but mycobacteria are genetically more related to gram-negative bacteria [7]. However, their cell wall is so unique that they cannot be classified gram-positive or negative. The most striking feature of the cell wall is its high proportion of lipids. These lipids are largely made up of mycolic acids, which make up to $50-60 \%$ of the dry weight of the cell wall. The strong hydrophobicity of the lipid layer in the mycobacterial cell wall means that mycobacteria are classified as acid-fast, due to their ability to retain basic dyes upon acid or heat treatment. The classical stain for mycobacteria is the ZiehlNeelsen stain, whereby mycobacteria stain bright red/pink against surrounding tissue after a carbol-fuchsin stain followed by destain with acid-alcohol, although there are several variations on this technique [8].

As a slow-growing mycobacterial species, the replication time for $M$. tuberculosis is around 14-24 hours in vitro $[9,10]$ and 20-28 hours in vivo in murine models [11]. M. tuberculosis is a facultative intracellular pathogen as it can survive and replicate within host cells, most notably the alveolar macrophage, which is the resident phagocytic cell in the lung. While $M$. tuberculosis replicates optimally at the interface of the liquid media or the host tissue and air [10], it can also persist in anaerobic 
conditions. For example, as a pulmonary $\mathrm{Tb}$ infection progresses, mycobacteria are contained within fibrotic structures known as granulomas. The inside of a granuloma is believed to be highly hypoxic as the granuloma tissue is not supported by a blood supply [12]. During this period of anaerobic survival within the granuloma, $M$. tuberculosis enters a growth phase known as latency, which is addressed in section 1.2.4.

Currently genomes have been sequenced for a number of mycobacteria including laboratory strains and clinical isolates of $M$. tuberculosis [13, 14], an isolate of M. bovis [15] and the Pasteur strain of the attenuated vaccine, M. bovis bacille Calmette Guerin (BCG) [16]. Comparisons of the regions of difference between subspecies and strains has revealed how different clinical isolates of M. tuberculosis are and how similar sub-species of the MTBC are, considering marked differences in host tropism and pathogenicity (reviewed in [17]).

\subsection{The immune response to mycobacteria}

\subsubsection{A general outline of the immune response}

The human immune system has evolved to control pathogens such as M. tuberculosis. Pathogens possess two features essential to initiate and direct an immune response: pathogen associated molecular patterns (PAMPs) and antigens. PAMPs are molecules commonly found in viruses or bacteria, and allow the host to distinguish between cells that are host-derived and cells that are foreign [18]. Antigens are typically proteins that are pathogen-specific and enable the immune system to target one particular pathogen. As a result, PAMPs function to activate the immune response in a generic way, while antigens supply a target to direct the response against a specific pathogen.

An immune response to a pathogen progresses in two phases; the innate and adaptive phases. Upon infection, PAMPs from a pathogen initially interact with pattern recognition receptors (PRRs) on leukocytes and non-lymphoid cells in the infection site [18]. They induce the release of hormones known as chemokines and cytokines, which enhance recruitment of more leukocytes and activate specific functions of those leukocytes respectively. This initial inflammation at the infection site is known as the innate immune response. Essential cells recruited for the innate immune response 
include natural killer (NK) cells, eosinophils, basophils and mast cells, but also phagocytic cells, such as macrophages, neutrophils and dendritic cells (DCs). Phagocytosis is the process whereby extracellular components including large molecules can be taken up and contained within a vacuole called a phagosome. During an infection, entire microbes are phagocytosed and some phagocytic cells are equipped with anti-microbial functions to control growth or destroy the microbe within the phagosome.

A key activity of DCs is to initiate the adaptive phase of the immune response. When DCs engulf a pathogen two things occur. Firstly, PAMPs interact with PRRs and convey a danger signal to that DC, activating it and enabling it to migrate out of inflamed tissue to the local draining lymph node [18]. Secondly, DCs process antigens derived from the engulfed pathogen and load peptides from them onto molecules encoded by the major-histocompatibility complex (MHC) locus (reviewed in [19]). Once an activated DC is in the lymph node, it is a very effective APC, displaying antigen loaded onto MHC molecules to lymphocytes. There are three main types of MHC known as class I (MHC I), class II (MHC II) and the MHC I-like CD1 molecule [20], which interact with and activate lymphocytes, that are respectively $\mathrm{CD} 8^{+}, \mathrm{CD} 4^{+}$ and NK T cells, through their T cell receptors (TCRs). $\mathrm{CD} 8^{+}$and $\mathrm{CD} 4^{+} \mathrm{T}$ cells have what is known as variant TCR, since somatic recombination in the region of their genome that encodes their TCR during development results in individual lymphocytes that each express a unique TCR. Most NK T cells have a semi-invariant TCR, composed of an invariant $\alpha$-chain paired with a $\beta$-chain from a restricted reportiore. This semi-invariant TCR can recognise microbial glycolipid antigens loaded onto CD1.

When lymphocytes recognise their cognate antigen presented by an activated $\mathrm{DC}$, they begin to proliferate and activate their effector functions. $\mathrm{CD} 8^{+} \mathrm{T}$ cells secrete cytokines and can be cytotoxic upon encountering pathogen-infected cells. CD4 ${ }^{+} \mathrm{T}$ cells primarily exert their effects through cytokine secretion, and can be divided into subsets depending on their cytokine profile. The most well-known of these divisions is the $\mathrm{T}$ helper (Th) $1 /$ Th2 division [21]. Th1 cells produce interferon (IFN)- $\gamma$ and tumour necrosis factor (TNF)- $\alpha$ and promote macrophage anti-microbial activation, whereas Th2 cells produce interleukin (IL)-4, IL-5 and IL-13 and promote antibody production by B cells. After proliferation and activation in the lymph node, lymphocytes enter the circulation and are recruited into the infection site by chemokine signals produced by 
the innate immune response. These lymphocytes direct their effector functions against the pathogen and control the infection.

In this way the innate and adaptive arms of the immune response allow a rapid but generic innate response to control the pathogen until a more directed but delayed adaptive response can be initiated to control, and ideally eradicate, an infection.

\subsubsection{Infection with M. tuberculosis}

The most common form of disease caused by M. tuberculosis is pulmonary $\mathrm{Tb}$, since the host is infected primarily by inhalation of aerosolised droplets containing the bacterium. From the lung, the infection can disseminate to almost any other organ.

Tb infection induces local inflammation that contains the infection, which is known as a granuloma (section 1.2.4). Symptoms of Tb include night-sweats, tiredness and a persistent cough. The sputum that a patient produces from this cough eventually contains blood, as the integrity of the granuloma and lung degrade. The cough aerosolises bacilli present in the lung, enabling the transmission of infection to other individuals. As the inflammation consolidates lung tissue, the affected lung tissue is not able to function and perform efficient oxygen exchange. Death typically results when consolidated lung tissue can no longer support the oxygen requirements of the patient or infection has caused complications, such as pleural effusion.

Once a person is exposed to aerosolised M. tuberculosis, there are several different outcomes possible, reviewed in [22] (Fig. 1.1). 


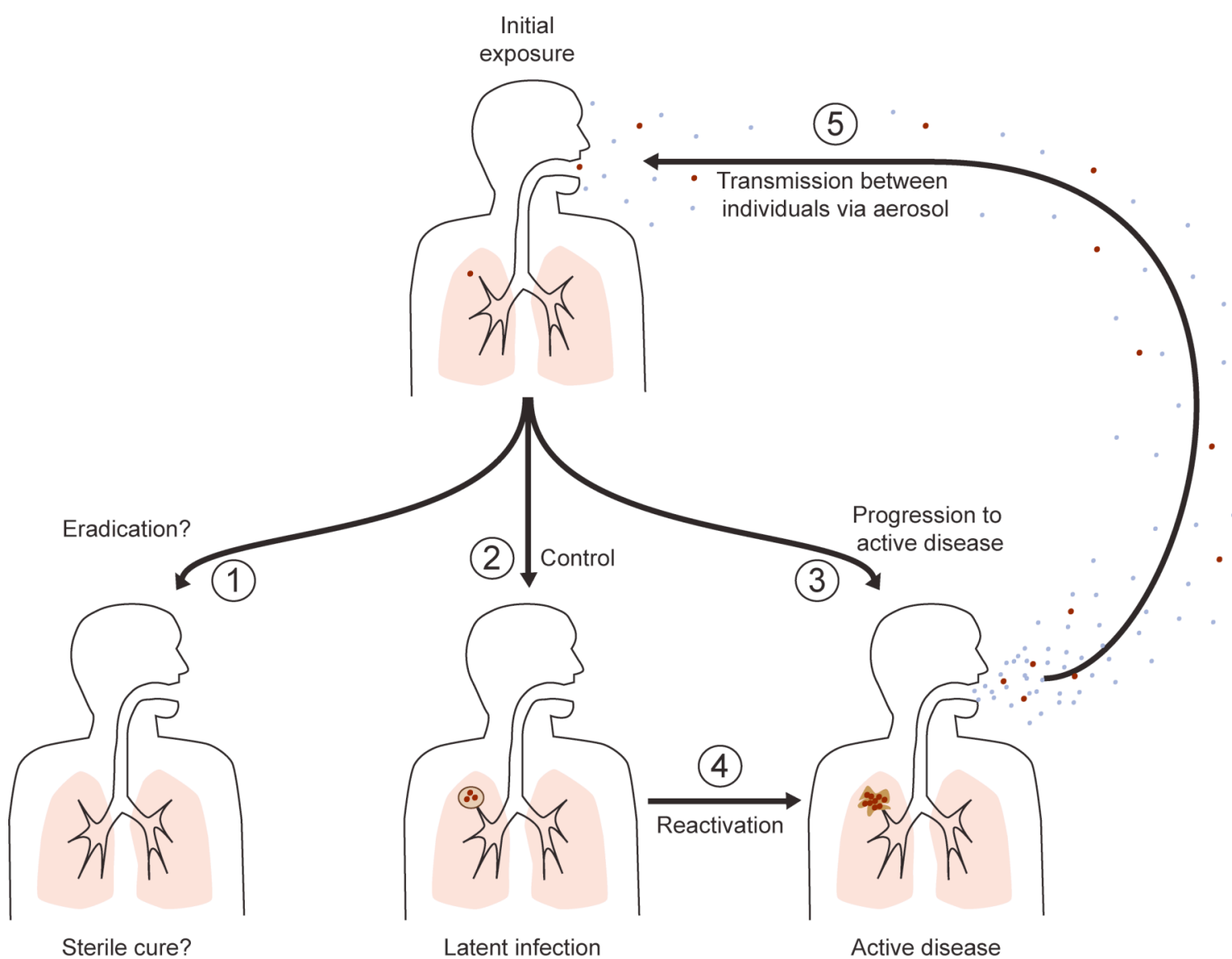

Figure 1.1: Possible outcomes after exposure to aerosolised M. tuberculosis

After exposure to aerosolised M. tuberculosis, several outcomes are possible. An exposed individual may: 1) eliminate the pathogen resulting in a sterile cure, 2) control the infection within a granuloma resulting in latent $\mathrm{Tb}, \mathbf{3}$ ) become infected and progress rapidly to exhibit symptoms of active $\mathrm{Tb}, 4)$ progress from latent to active $\mathrm{Tb}$ when the granuloma in the lung breaks down. 5) During active Tb infection, inflammation in the lung, loss of integrity of the granuloma and coughing enables M. tuberculosis to become aerosolised and facilitates transfer of infection between individuals. 
Some individuals exposed to $M$. tuberculosis do not develop reactivity to $M$.

tuberculosis antigens, as determined by the Mantoux skin test. The immune response in these individuals may have efficiently destroyed the bacterium, presumably using innate mechanisms of the immune response (addressed in section 1.2.3.4). Of the individuals that are exposed, $10-30 \%$ become infected and reactive in the Mantoux test. Of these infected individuals, $90 \%$ spend years without displaying clinical symptoms of active disease. This group is deemed to have a chronic or latent $\mathrm{Tb}$ infection, and it has been estimated that the lifetime risk of developing active $\mathrm{Tb}$ is approximately $12 \%$ in these individuals [23]. Latent infection can reactivate spontaneously in later life or in situations of lowered immunocompetence. The remaining $10 \%$ of infected individuals progress quickly from the initial exposure to display clinical symptoms of $\mathrm{Tb}$, termed as active $\mathrm{Tb}$. Individuals that progress directly to active $\mathrm{Tb}$ are unable to control the initial infection and are thought to possess characteristics that make them susceptible to $M$. tuberculosis infection. Therefore, within the human population, there is a spectrum of susceptibilities to M. tuberculosis infection and the susceptibility of an individual is a product of genetics, environment, health and stresses or a combination of these factors.

\subsubsection{The primary immune response to $M$. tuberculosis}

\subsubsection{Containing infection with the innate immune response}

Upon inhalation of M. tuberculosis, the bacteria are phagocytosed, predominantly by the alveolar macrophage. The alveolar macrophage is a lung resident phagocytic cell and is paradoxically both the preferred habitat of the mycobacteria and the first line of host defence against bacterial lung infections. Uptake of mycobacteria by alveolar macrophages is facilitated by a number of molecules including cholesterol [24] and mannose receptors that recognise the mannose-rich cell wall of the mycobacteria [25].

DCs and macrophages can both phagocytose mycobacteria, but each fulfils different functions after phagocytosis. DCs actively migrate to the draining lymph node and are particularly effective at producing IL-12 and presenting antigen, initiating the Th1 immune response and priming $\mathrm{CD}^{+}$and $\mathrm{CD} 8^{+} \mathrm{T}$ cells [26]. Macrophages once activated by IFN- $\gamma$, are very effective at eliminating mycobacteria, whereas DCs cannot kill the invading organisms even after IFN- $\gamma$ activation [27]. Therefore, while clearly 
necessary to initiate the adaptive immune response against $\mathrm{Tb}$, DCs may act as a reservoir for the mycobacterial growth and contribute to dissemination of infection though DC migration to the lymph node [27], in contrast to macrophages that possess the cellular machinery to control Tb infection.

PAMPs from invading mycobacteria interact with PRRs such as the toll-like receptors (TLRs) on host cells. Presumably early events such as signalling through TLRs are important for control of the infection, as mice deficient in MyD88, a central molecule involved in signal transduction from TLRs, succumb quickly to infection [28, 29]. A number of TLRs are considered vital for optimal control of mycobacterial infection, including TLR2, TLR4 and TLR9 [30]. Interaction with TLRs on phagocytic cells leads to activation of DCs and partial activation of anti-microbial activity in alveolar macrophages. TLR signalling also results in the release of pro-inflammatory cytokines such as IL-1, tumour necrosis factor (TNF)- $\alpha$ and most importantly IL-12 $[31,32]$ as well as chemokines, which initiate a site of inflammation to promote influx of monocytes, neutrophils and NK cells and later activated lymphocytes.

IL-12 produced by DCs is important for control of mycobacterial infection. IL12 is a heterodimeric cytokine composed of two subunits: p40 (shared with IL-23) and p35 (exclusive to IL-12), which together make active IL-12 p70. IL-12p40 and IL12 p35 gene-disrupted (-/-) mice exhibit defective immune responses and reduced protection to mycobacteria $[33,34]$. The importance of IL-12 in mice is mirrored by human studies in which patients with mutations in the IL-12 receptor gene are highly susceptible to mycobacterial infection [35]. In the local lung environment early during infection IL-12 acts on NK cells [36] and even back on the macrophage [37, 38] to induce IFN- $\gamma$ production. Thus, IL-12 production by DCs in the draining lymph node creates an environment that promotes Th1 development. 


\subsubsection{The adaptive immune response}

The adaptive immune response, initiated upon antigen presentation in the lymph node, is essential for the control of mycobacterial infection. Antigens presented on MHC I to $\mathrm{CD}^{+} \mathrm{T}$ cells are typically derived from cytosolic protein transported into the endoplasmic reticulum (ER) where they are loaded onto MHC I and exported to the cell surface for presentation (reviewed in [19]) (Fig. 1.2). Antigens presented on MHC II to $\mathrm{CD}^{+} \mathrm{T}$ cells are typically derived from phagocytosed protein processed through the endocytic pathway (reviewed in [19]) (Fig. 1.2). Since mycobacteria are phagocytosed, it is uncertain how mycobacterial antigen in the phagosome can access the MHC I pathway to activate $\mathrm{CD} 8^{+} \mathrm{T}$ cells but several mechanisms have been proposed. MHC I presentation in vitro [39] and activation of $\mathrm{CD}^{+} \mathrm{T}$ cells in vivo [40] can be efficiently induced when phagocytic cells engulf apoptotic vesicles containing mycobacterial antigen. It is thought these apoptotic bodies access the MHC I pathway through what has been called the detour pathway [41] and cross-presentation or cross-priming (reviewed in [42]). Alternatively, mycobacteria or their antigen may enter the cytoplasm of chronically infected cells and thus, following proteasomal degradation, be loaded onto MHC I in the ER and exported to the cell surface [43, 44]. Although the exact mechanisms that mediate presentation of mycobacterial antigens on MHC I remain uncertain, it is established that M. tuberculosis infection results in activation of $\mathrm{CD}^{+} \mathrm{T}$ cells.

Within the adaptive immune response, $\alpha \beta \mathrm{CD} 4^{+}$and $\mathrm{CD} 8^{+} \mathrm{T}$ cells are essential for control of mycobacterial infection. This was first demonstrated in depletion studies with monoclonal antibodies (mAbs) $[45,46]$ and was further validated by observing $M$. tuberculosis infection in gene-disrupted murine models. In wild-type (WT) mice, bacterial numbers increase steadily in the lung until around 3-4 weeks after infection, when they stabilise and remain constant for an extended period of time. Bacteriostasis in the lungs coincides with the influx of large numbers of $\mathrm{CD}^{+}$and $\mathrm{CD} 8^{+} \mathrm{T}$ cells to the lung [47]. In $\alpha \beta$ TCR -/- mice, which lack $C D 4^{+} \mathrm{T}$ cells, $\mathrm{CD} 8^{+} \mathrm{T}$ cells or NK T cells, and MHC class II -/- mice, which do not have $\mathrm{CD}^{+} \mathrm{T}$ cells, lung bacterial numbers increase rapidly after $\mathrm{Tb}$ infection and the infection is quickly lethal [48-50]. Therefore, it is clear that $\mathrm{CD} 4^{+} \mathrm{T}$ cells are vital for control of mycobacterial infection.

The contribution of $\mathrm{CD}^{+} \mathrm{T}$ cells to protection against $\mathrm{Tb}$ infection is less defined. In MHC class I -/- mice, which lack MHC Ia for presentation to $\mathrm{CD}^{+} \mathrm{T}$ cells 
and CD1 for NK T cells, lung bacterial numbers progress to $\sim 1 \log _{10}$ higher than wildtype mice by week 5 after infection but eventually stabilise [49]. In Transporterassociated-with-antigen-processing -/- mice, that lack the ability to load cytosolic antigen on MHC I, lung bacterial numbers also progress to $\sim 1 \log _{10}$ higher than wildtype mice and stabilise but this leads to earlier lethality [49, 51].

Other studies have tried to extricate the contribution by $\mathrm{CD} 8^{+} \mathrm{T}$ cells to protection against $\mathrm{Tb}$ infection from other MHC I-dependent cells by following $M$. tuberculosis infection in $\beta-2$ microglobulin $(\beta-2 \mathrm{~m})$-/-, CD8 -/- or MHC Ia -/- mice. $\beta$ $2 \mathrm{~m}$-/- mice cannot form MHC Ia, which presents antigen to $\mathrm{CD}^{+} \mathrm{T}$ cells. MHC Ia -/mice do not express MHC Ia and also cannot present antigen to $\mathrm{CD} 8^{+} \mathrm{T}$ cells. CD8 -/mice lack $\mathrm{CD}^{+} \mathrm{T}$ cells. All of these mice exhibit increased lung bacterial numbers and accelerated lethality compared to WT mice; however lung bacterial numbers and the rates of lethality are higher in $\beta-2 \mathrm{~m}-/-$ mice compared to MHC Ia -/- or CD8 -/- mice [52-54]. Increased disease severity in $\beta-2 \mathrm{~m}-/-$ mice may be due to a defect in iron metabolism caused by $\beta-2 \mathrm{~m}$ deficiency, which inhibits innate immune mechanisms [55]. In conclusion, $\mathrm{CD}^{+} \mathrm{T}$ cells clearly have a role in controlling the infectious burden but the degree to which they control bacterial growth has not yet been determined.

CD1-restricted T cells and NK T cells are also activated during the course of mycobacterial infection through presentation of glycolipids in the context of CD1. The mycobacterial cell wall is rich in glycolypids, such as phosphatidylinositol mannoside (PIM) or lipo-arabinomannan (LAM). PIM can be presented on CD1d to activate both human and murine NK T cells [56] and LAM can be presented on CD1b to activate human $\alpha \beta$ CD1-restricted T cells [57]. The importance of CD1-restricted T cells and NK T cells is unresolved. CD1d -/- mice, which do not have NK T cells, have a similar progression of infection to WT mice [51]. However, the murine model may not be appropriate to evaluate the impact of $\mathrm{CD} 1$-restricted responses on $\mathrm{Tb}$ infection (reviewed in [58]). Mice only express the Group 2 CD1 isoform CD1d that presents glycolipid to NK T cells, whereas humans also express the Group 1 CD1 isoforms CD1a, CD1b and CD1c that present glycolipid to CD1-restricted T cells. Therefore, humans can present a wider range of mycobacterial glycolipids to CD1-restricted T cells than mice, and murine models may underestimate control of bacterial numbers by $\mathrm{CD} 1-$ restricted responses during $\mathrm{Tb}$ infection. 
A further lymphocyte population, known as $\gamma \delta$ T cells, utilise a $\gamma \delta$-chain TCR and can recognise a diverse range of antigens (protein and non-protein), particularly with phosphate residues as recognition sites. $\gamma \delta$ T cells are activated by mycobacterial stimulation [59] but $\gamma \delta \mathrm{T}$ cell -/- mice do not differ from WT mice in terms of tissue bacterial numbers [49], although they do exhibit altered granuloma formation [60]. This altered granuloma formation may account for increased pathology and tissue bacterial numbers observed in $\gamma \delta \mathrm{T}$ cell -/- mice compared to WT mice after high dose intravenous infection [61]. However, $\gamma \delta \mathrm{T}$ cells make up only 2-3\% of peripheral blood mononuclear cells (PBMCs) in mice but make up 7\% of PBMCs in humans [62, 63]. Thus, the smaller proportion of $\gamma \delta \mathrm{T}$ cells in mice may lead to an underestimation of their role in anti-mycobacterial immunity in humans.

In summary, $\mathrm{CD}^{+} \mathrm{T}$ cells are integral for control of mycobacterial infection in the lung, although $\mathrm{CD}^{+} \mathrm{T}$ cells also contribute to this control. NK T cells and $\gamma \delta \mathrm{T}$ cells may contribute to control of $\mathrm{Tb}$ infection in humans but this will be difficult to accurately model in mice. While the effect of these lymphocytes on protection from primary mycobacterial infection has been explored using in vivo murine models, the effect of Tregs on protection from mycobacterial infection had not been assessed at the outset of this thesis. Tregs are capable of suppressing effector mechanisms of the lymphocyte subsets described above (section 1.4.2). A major question for this thesis was whether Tregs suppress effector mechanisms of $\mathrm{CD}^{+}$and $\mathrm{CD} 8^{+} \mathrm{T}$ cells during mycobacterial infection and thereby inhibited control of mycobacterial infection.

\subsubsection{Th1 development}

The main function of $\mathrm{CD}^{+} \mathrm{T}$ cells is to provide help for other lymphocytes, primarily by producing cytokines. When $\mathrm{CD} 4^{+} \mathrm{T}$ cells are activated by TCR triggering in the presence of IL-2 and IL-12, they proliferate and differentiate into CD4 ${ }^{+}$Th1 effector cells. The presence of certain cytokines such as IFN- $\gamma$ further augments this development while Th2 cytokines such as IL-4 can inhibit development [21]. Therefore, the cytokine millieu during intial TCR triggering is critical for predicting whether a Th1 response will develop. During early mycobacterial infection, activated DCs produce IL12 (section 1.2.3.1), and can produce IL-2 and IFN- $\gamma$. Production of IL-2 by DCs is even enhanced upon incubation with mycobacteria-infected neutrophils [496]. Other 
leukocytes such as NK cells [36], NK T cells [56, 57], macrophages [37, 38] and $\gamma \delta \mathrm{T}$ cells [59] can also produce IFN- $\gamma$ during early mycobacterial infection. Therefore, several cell types can contribute to the cytokine millieu during early mycobacterial infection and ensure preferential development of the Th1 immune response that is regarded as protective against $\mathrm{Tb}$.

CD4 ${ }^{+}$Th1 effector cells are characterised by activation of STAT1 and STAT4 and expression of the transcription factor, Tbet (reviewed in [497]). This programs the $\mathrm{CD} 4^{+}$Th1 effector cell to produce effector cytokines such as IFN- $\gamma$ and TNF- $\alpha$.

IFN- $\gamma$ is a key cytokine that mediates control of mycobacterial infection. IFN- $\gamma-$ /- mice are incapable of controlling Tb infection, and bacterial numbers in the lung climb exponentially until premature death of the mice between 30 and 60 days after infection [49, 64-66]. These results are mirrored in humans studies, since individuals known to be highly susceptible to mycobacterial infection were found to have mutations in the IFN- $\gamma$ receptor gene $[67,68]$. While these findings demonstrate that IFN- $\gamma$ production is important in mycobacterial infection, they do not indicate which cell must produce the cytokine.

IFN- $\gamma$ can be produced by several different leukocytes, such as infected macrophages [38], NK cells [69], NK T cells [70], $\mathrm{CD}^{+} \mathrm{T}^{\mathrm{T}}$ cells [71] and $\mathrm{CD} 4^{+} \mathrm{T}$ cells [72]. IFN- $\gamma$ production by cells of the innate immune system such as NK cells could initiate conditions conducive for later Th1 development. Deficiencies in IFN- $\gamma$ production by cells of the innate immune response could contribute to the observed high bacterial burdens in IFN- $\gamma-/-$ mice. Indeed NK cell activity during M. tuberculosis infection is affected by IFN- $\gamma$ gene disruption, along with the cytolytic activity of CD8 ${ }^{+}$ $\mathrm{T}$ cells and cytokine production by $\mathrm{CD} 4^{+} \mathrm{T}$ cells [66].

However IFN- $\gamma$ production by $\mathrm{CD}^{+} \mathrm{T}$ cells early during infection is widely regarded as necessary for optimal protection from $\mathrm{Tb}$ infection. Cellular infiltrate to the lung has been followed after infection in strains of mice that are deemed susceptible or resistant to M. tuberculosis infection [47]. The early appearance of IFN- $\gamma$ producing $\mathrm{CD}^{+}$and $\mathrm{CD}^{+} \mathrm{T}$ cells in the lungs of resistant mice correlated with lower tissue bacterial numbers and increased survival after infection compared to susceptible mice [47]. In addition, MHC II -/- mice or CD4 -/- mice that have no CD4 ${ }^{+} \mathrm{T}$ cells, have transiently decreased levels of IFN- $\gamma$ relative to WT mice two weeks after 
mycobacterial infection, but quickly reach similar levels of IFN- $\gamma$ production in the lung by the onset of the adaptive response at week 4 [50]. In the absence of $\mathrm{CD} 4^{+} \mathrm{T}$ cells, IFN- $\gamma$ is produced predominantly by $\mathrm{CD}^{+} \mathrm{T}$ cells during the adaptive response and, despite similar levels of IFN- $\gamma$ production by week 4 after infection, these mice succumb rapidly to infection. This study has been used to suggest that IFN- $\gamma$ produced by $\mathrm{CD}^{+} \mathrm{T}$ cells is key for control of mycobacterial infections. If $\mathrm{CD}^{+} \mathrm{T}$ cells recognise and interact with infected macrophages, it is hypothesised that $\mathrm{CD} 4^{+} \mathrm{T}$ cells would deliver IFN- $\gamma$ in a targeted, more effective fashion to the infected cell compared to other IFN- $\gamma$-producing leukocytes. However, there are alternative hypotheses. The transient deficiency in IFN- $\gamma$ at 2 weeks may affect the protective outcome of infection. Alternatively, $\mathrm{CD} 4^{+} \mathrm{T}$ cells may play a role in protection from $\mathrm{Tb}$ infection that is independent of IFN- $\gamma$ production. Therefore, it remains unresolved which cellular subset produces protective IFN- $\gamma$.

Another key cytokine for protection against mycobacterial infection is TNF- $\alpha$. TNF- $\alpha$ is produced by a number of different types of cell during infection, including macrophages, DCs, $\mathrm{CD} 4^{+}$and $\mathrm{CD} 8^{+} \mathrm{T}$ cells. Studies in both TNF- $\alpha$ receptor -/- mice or mice treated with TNF- $\alpha$ neutralising antibody demonstrated that a loss or deficiency in TNF- $\alpha$ signalling during the course of mycobacterial infection led to higher bacterial numbers in the lung, extensive necrosis of tissue due to lack of granuloma organisation and a rapidly lethal infection compared to WT mice [73]. Administration of TNF- $\alpha$ neutralising antibody after the onset of chronic infection led to granuloma instability and re-activation of infection [74]. Human cases again confirm the importance of TNF$\alpha$. Rheumatoid arthritis patients can be treated with anti-TNF- $\alpha$ antibodies, such as infliximab, to minimise arthritis-associated inflammation. Patients that were latently infected with $\mathrm{Tb}$, were found to develop active Tb after anti-TNF- $\alpha$ treatment [75]. Therefore, TNF- $\alpha$ is regarded as essential for formation and retention of the granuloma structure, enabling containment of mycobacterial infection.

\subsubsection{Immunomodulation of the immune response by cytokines}

Apart from the classical Th1 cytokines, IFN- $\gamma$ and TNF- $\alpha$, other cytokines can have immunomodulatory roles during mycobacterial infection. 
IL-17 is a recently characterised cytokine whose role in mycobacterial infection is not yet well defined. IL-17 is a pro-inflammatory cytokine that promotes the recruitment of neutrophils by inducing $\mathrm{CXC}$ chemokine production and cytokine production, such as IL-6, IL-8 and TNF- $\alpha$ by macrophages $[76,77]$. IL-17 is produced predominantly by Th17 differentiated $\mathrm{CD}^{+} \mathrm{T}$ cells, although $\gamma \delta \mathrm{T}$ cells also produce IL-17 during mycobacterial infection [78]. Th17 differentiation is promoted during $\mathrm{CD}^{+} \mathrm{T}$ cell activation by the presence of TGF- $\beta$ and IL- 6 and Th17 lymphocytes are maintained by IL-23 signalling [79-81]. IL-23 has been shown to be responsible for some protection and control of mycobacterial infection, using plasmid-mediated expression of IL-23 in the lung of murine models [82, 83]. Furthermore, mice deficient in the IL-12p40 subunit, shared by IL-12 and IL-23, have more severe disease compared to mice deficient in the IL-12p35 subunit, which is used solely by IL-12 [33]. While IL17 and IL-23 may contribute to protection in a primary response, it has also been suggested that $\mathrm{IL}-17^{+} \mathrm{CD} 4^{+} \mathrm{T}$ cells accelerate recruitment of IFN $-\gamma^{+} \mathrm{CD} 4^{+} \mathrm{T}$ cells to the lung upon secondary challenge and thereby mediate protective memory against $\mathrm{Tb}$ [84].

IL-6 has an important role early during the anti-mycobacterial immune response to promote inflammation. IL-6 -/- mice infected with M. tuberculosis by aerosol exhibit early increases in lung bacterial numbers and decreased IFN- $\gamma$ production from lung and spleen-derived leukocytes prior to the onset of adaptive immunity [85]. After the onset of the adaptive response, lung bacterial numbers stabilise in IL-6 -/- mice but remain $\sim 1 \log _{10}$ higher than in WT mice. This suggests that IL-6 production contributes to early control of M. tuberculosis infection, but does not impact on chronic infection.

IL-27 is a member of the IL-6/IL-12 family of cytokines and is released by APCs. Recently, it has emerged that IL-27 suppresses the development of Tregs and Th17 CD4 ${ }^{+}$T cells, favouring Th1 $\mathrm{CD}^{+}$effector T cell development [86]. $M$. tuberculosis infection of IL-27 receptor -/- mice leads to lower lung bacterial numbers compared to WT mice, despite both possessing similar numbers of IFN $-\gamma^{+} \mathrm{CD}^{+} \mathrm{T}$ cells in the lung [87]. However, the amount of IFN- $\gamma$ produced by an individual CD4 ${ }^{+} \mathrm{T}$ cell is decreased in IL-27 receptor -/- mice. The decrease in IFN- $\gamma$ production is hypothesised to promote survival of effector IFN $-\gamma^{+} \mathrm{CD} 4^{+} \mathrm{T}$ cells, as IFN- $\gamma$ production can enhance activation induced cell death (AICD) in $\mathrm{CD}^{+} \mathrm{T}$ cells [88] and lead to apoptosis of effector $\mathrm{CD}^{+}{ }^{+} \mathrm{T}$ cells after BCG infection $[89,90]$. 
There may also be roles for Th2 cytokines, such as IL-4, during M. tuberculosis infection. IL-4 -/- mice on a C57BL/6 background infected with M. tuberculosis by aerosol exhibited similar bacterial numbers in the lung, spleen and liver to WT C57BL/6 mice, during both the acute and chronic phases of infection $[85,91]$. However, IL-4 -/- mice display increased IFN- $\gamma$ production from splenocytes and increased IFN- $\gamma$ and TNF- $\alpha$ mRNA expression in the lung up to 4 weeks after infection [85]. An increase in IFN- $\gamma$ in the absence of IL-4 may be anticipated given that IL-4 negatively regulates IFN- $\gamma$ production, but it is important to note here that increased IFN- $\gamma$ production did not correlate with a decrease in tissue bacterial numbers. Finally, IL-10 and transforming growth factor (TGF)- $\beta$ are cytokines that are regarded as being anti-inflammatory. It is proposed that production of IL-10 or TGF- $\beta$ can initiate reactivation of $\mathrm{Tb}$ infection (section 1.2.4).

In conclusion, several cytokines contribute to protection at different stages of mycobacterial infection. IL-12 is vital early after infection, as may be IL-23. IFN- $\gamma$ is essential for bacteriostasis, TNF- $\alpha$ is necessary for granuloma formation and containment of infection and IL-17 may mediate protective memory. Other cytokines such as IL-6, IL-27 and IL-4 may modulate the immune response to infection. Several different cell types are capable of producing each of these cytokines but specific cell subsets may need to produce the cytokine for it to effectively reach the target, the $\mathrm{Tb}$ infected macrophage. Thus, once $\mathrm{CD} 4^{+}$and $\mathrm{CD} 8^{+} \mathrm{T}$ cells are activated and capable of producing cytokines, they can migrate into the inflamed tissue and interact with the infected macrophage, resulting in control of mycobacterial growth. Since Tregs can suppress cytokine production by other leukocyte subsets (section 1.4.2), I hypothesised that Tregs might suppress production of protective cytokines during mycobacterial infection. Inhibition of cytokine production that is necessary for control of primary mycobacterial infection could enhance survival of mycobacteria within the host.

\subsubsection{Effector mechanisms of anti-mycobacterial immunity}

There are several mechanisms by which infected macrophages control or eliminate mycobacterial infection (Fig. 1.2). Firstly, fusion of a phagosome containing microbes with a lysosome results in degradation of the microbes and facilitates further processing of the microbial components in the endocytic pathway and eventual presentation of 
antigen on MHC II. This process begins with phagocytosis and the creation of the phagosome in the cytosol. The phagosome can fuse directly with the lysosome or mature through interactions with endosomes that sequentially alter the protein contents of the phagosome before fusion with the lysosome $[92,93]$. The lysosome contains a host of hydrolytic enzymes, which function optimally at acidic $\mathrm{pH}$, and the lysosome is maintained at $\mathrm{pH}$ 4.5-5.0 by ATP-dependent proton pumps in the lysosomal membrane $[94,95]$. The hydrolytic enzymes are capable of degrading microbes contained within the phago-lysosome [96] through either enzymatic attack or the damaging effects of high $\mathrm{pH}$ and this constitutes a vital bacteriocidal function of macrophages.

If mycobacteria escape phago-lysosomal fusion (outlined in 1.2.5), IFN- $\gamma$ and TNF- $\alpha$ signalling to the infected macrophage activates alternative bacteriocidal mechanisms, including autophagy and reactive oxygen and nitrogen species production.

Autophagy is a process whereby a cell can degrade entire organelles in structures similar to lysosomes. The antigen contained in the organelle may enter the endocytic pathway and thereby the MHC II processing pathway. In mycobacteriainfected macrophages, phagosomes containing mycobacteria can enter the autophagic pathway $[97,98]$. Autophagy has been induced in mycobacteria-infected cells by starvation or IFN- $\gamma$ treatment and it decreases bacterial numbers in macrophage in vitro cultures [97]. Mycobacterial antigen can access MHC II on macrophages following autophagy of mycobacteria-infected phagosomes but whether this process also occurs in DCs in vivo is yet to be explored.

Reactive oxygen intermediates (ROI) are produced by macrophages in response to IFN- $\gamma$ and TNF- $\alpha$ activation [99]. In vitro evidence suggests ROI production does not inhibit M. tuberculosis growth [100-102]. However, ROI production in in vitro activated macrophages has been demonstrated to kill M. microti [103] and mice deficient in ROI production exhibit early transient defects in control of pulmonary $M$. tuberculosis infection [99, 104].

Reactive nitrogen intermediates (RNI) are also produced by macrophages in response to IFN- $\gamma$ signalling $[100,102,105,106]$ and this production is augmented by TNF- $\alpha$ signalling [107]. The cytokine signalling induces production of inducible nitric oxide synthase (NOS2), which uses L-arginine as the substrate for RNI production including nitric oxide. RNI production has been demonstrated to inhibit M. tuberculosis 
growth in vitro $[100,102,106]$ and in murine models [108]. Alveolar macrophages from human $\mathrm{Tb}$ patients have increased levels of NOS2 mRNA expression and increased staining with NOS2-reactive mAb $[109,110]$. Thus, RNI production is regarded as the most important mycobacteriocidal function of the cytokine-activated alveolar macrophage and the protective effects of IFN- $\gamma$ and TNF- $\alpha$ production by $\mathrm{CD}^{+} \mathrm{T}$ cells are thought to be due primarily to their ability to activate RNI production by the macrophage.

Infected macrophages can also be eliminated through cytotoxic lymphocyte (CTL) activity. Cytotoxicity can be mediated either by ligation of CD95 (Fas) on target cells to CD95 ligand (FasL) on the cytolytic cell or by exocytosis of cytolytic granules containing molecules, such as granzyme B, granulysin and perforin. Apoptosis or lysis of mycobacteria-infected cells can transfer mycobacteria from macrophages where phago-lysosomal fusion or RNI production has been inhibited to new phagocytic cells, which could be comparatively more proficient at eliminating the mycobacteria [111] or facilitating antigen presentation [40]. Molecules released by CTLs and the process of apoptosis may also have a direct effect on the viability of the mycobacteria.

Cytotoxic activity is regarded as the principal activity of the activated $\mathrm{CD} 8^{+} \mathrm{T}$ cells, although other leukocytes can also be cytotoxic against infected macrophages or mycobacteria, including: neutrophils [112], NK cells [113], NK T cells [114], $\gamma \delta$ T cells [115] and activated $\mathrm{CD} 4^{+} \mathrm{T}$ cells [116]. $\mathrm{CD}^{+} \mathrm{T}$ cells have two key effector functions: cytokine production and cytotoxicity. $\mathrm{CD}^{+} \mathrm{T}$ cells migrate into infected lung tissue and produce IFN- $\gamma$ and TNF- $\alpha[71,117]$, which can contribute to activation of macrophage bacteriocidal functions outlined above. However, the unique activity of CTLs and CD $8^{+}$ $\mathrm{T}$ cells is that they can directly lyse and kill target cells. This results in the death of the entire mycobacteria-infected macrophage and can also kill the mycobacteria themselves.

The expression of FasL, granulysin and perforin is upregulated by both $\mathrm{CD} 4^{+}$ and $\mathrm{CD}^{+} \mathrm{T}$ cells stimulated with M. tuberculosis [118]. While Fas-FasL signalling and granule exocytosis both induce apoptosis of mycobacteria-infected cells $[116,118]$, granule exocytosis has the more dramatic effect on mycobacterial viability. Granule exocytosis is utilised predominantly by $\mathrm{CD}^{+} \mathrm{T}$ cells as compared to other CTLs $[116$, $118,119]$. Perforin, granzyme B and granulysin are contained within these granules. The course of acute $\mathrm{Tb}$ infection is similar in perforin -/- and granzyme B -/- mice to 
WT mice $[120,121]$, although perforin -/- mice do not contain the chronic phase of $\mathrm{Tb}$ infection as well as WT mice, resulting in a slow but steady increase in lung bacterial numbers $[120,121]$. Granulysin released by cytotoxic lymphocytes has been shown to directly kill extracellular mycobacteria $[119,122]$. To kill intracellular mycobacteria, granulysin requires the presence of perforin, presumably to access intracellular compartments containing mycobacteria $[119,122]$.

The method by which granulysin kills mycobacteria is not certain. Mycobacteria that have been incubated with granulysin develop a distorted cell surface with numerous protrusions observed by electron scanning microscopy [122] and resulting changes in membrane permeability may impact on their viability [123]. The effects of granulysin on extracellular mycobacteria and infected macrophages in vitro are well documented but the role of granulysin in vivo in humans is unknown. Currently we lack tools to analyse the role of granulysin in vivo, as mice lack a granulysin homolog and granulysin -/- mice cannot be generated [124].

In summary, many effector mechanisms control growth or elimination of mycobacteria phagocytosed by the macrophage. These include: phago-lysosomal fusion in the macrophage, autophagy, cytokine signalling to macrophages to activate ROI or RNI production and cytotoxic mechanisms. While there are many host effector immune mechanisms that can control mycobacterial infection, mycobacteria have also developed many mechanisms to evade the host immune response (section 1.2.5). 


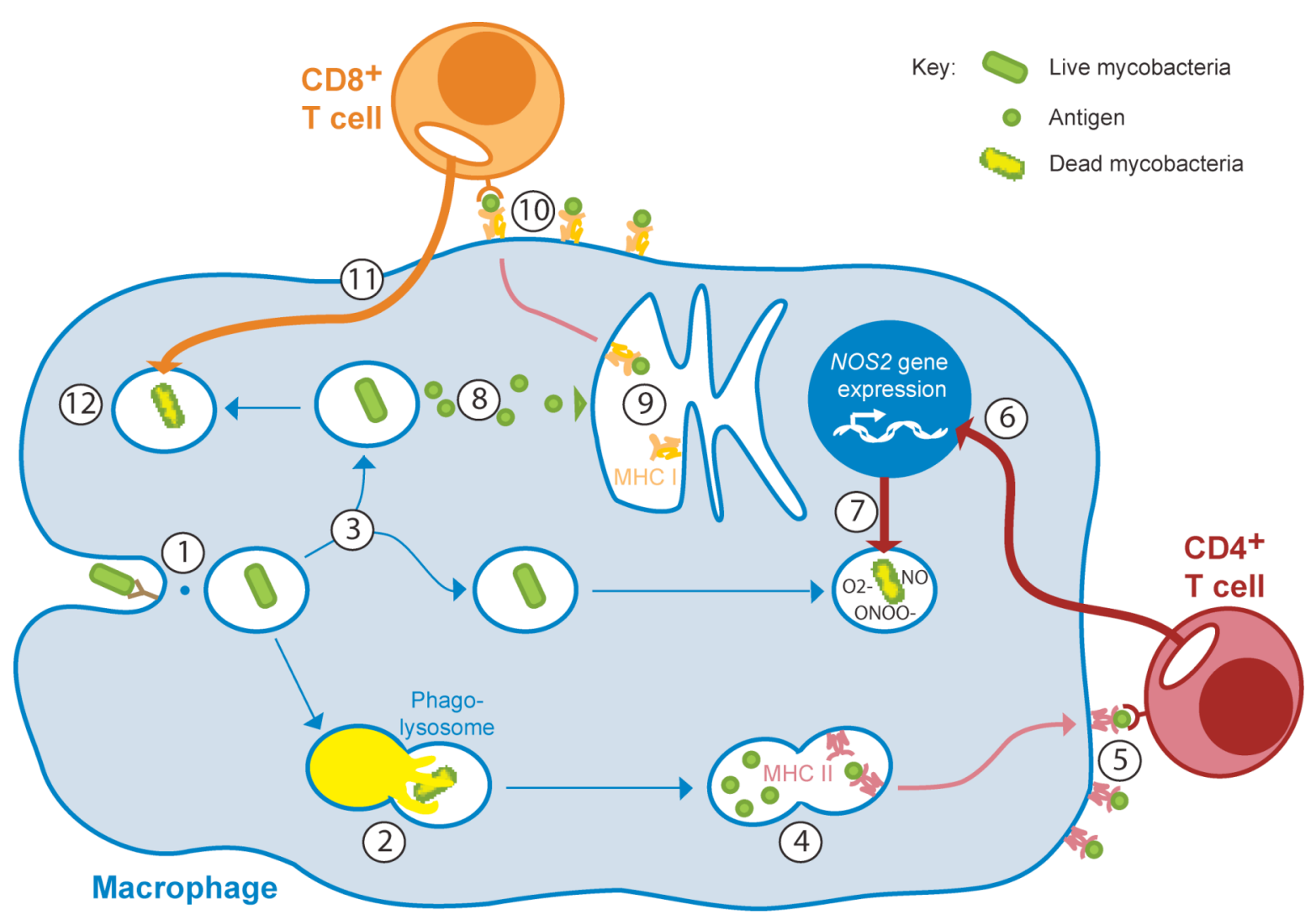

Figure 1.2: Effector mechanisms of the immune response during Tb infection

1) Mycobacteria are phagocytosed by the macrophage and contained within a phagosome. 2)

The phagosome can fuse with a lysosome degrading the mycobacteria contained or 3) the mycobacteria can evade phago-lysosomal fusion. 4) If the mycobacteria are degraded, the antigens are processed through the endocytic pathway and loaded onto MHC II, exported to the cell surface and 5) presented to activated $\mathrm{CD} 4^{+} \mathrm{T}$ cells. 6) Activated $\mathrm{CD} 4^{+} \mathrm{T}$ cells will release IFN- $\gamma$ and TNF- $\alpha$ that signal to the infected macrophage and 7) induce production of ROI and RNI to eliminate mycobacteria within stalled phagosomes. 8) Mycobacterial antigen could be cross-presented on MHC I if it escapes the phagosome and enters the cytosol. 9) This antigen can be processed and loaded onto MHC I in the ER 10) before being exported to the cell surface and presented to activated $\mathrm{CD} 8^{+} \mathrm{T}$ cells. 11) Activated $\mathrm{CD} 8^{+} \mathrm{T}$ cells release IFN- $\gamma$ and TNF- $\alpha$, as well as cytolytic granules containing perforin and granulysin. 12) The contents of the cytolytic granules can have direct cytotoxic effects on mycobacteria contained within the phagosome. 


\subsubsection{Latent infection and the granuloma}

In the murine model of $\mathrm{Tb}$, the acute phase of the infection is characterised by an exponential mycobacterial growth phase in the lung. This initial infectious insult is contained by the innate and adaptive immune responses and, from weeks 3-4 after infection, there is no net increase in bacterial numbers in the lung, resulting in a stable, chronic Tb infection [49]. This stabilisation in bacterial numbers is also thought to occur in humans exposed to $\mathrm{Tb}$, in situations where the initial immune response is sufficient to control the infection and contain it within infected macrophages but not eradicate it, resulting in latent $\mathrm{Tb}$. In the mouse, activated $\mathrm{CD} 4^{+}$and $\mathrm{CD} 8^{+} \mathrm{T}$ cells arrive in the lung at the same time as bacterial numbers stabilise, suggesting that these lymphocytes are capable of inducing bacteriostasis. It is interesting to note that although there is a slight reduction in bacterial numbers after the influx of $\mathrm{CD} 4^{+}$and $\mathrm{CD} 8^{+} \mathrm{T}$ cells, many bacteria persist, resulting in chronic infection [47]. When chronic or latent infection is established, an equilibrium is reached between the mycobacterial bacilli and the host. The vital structure for this equilibrium is the granuloma.

A granuloma is an immunological structure, comprising many leukocytes and non-haematopoietic cells, which forms to contain pathogen-infected cells. If the formation of the granuloma is prevented, as in ICAM-1 -/- mice that lack a key adhesion molecule, lung bacterial numbers stabilise but the mice suffer significant dissemination and pathology [125]. Therefore, granuloma formation is critical for physically containing the bacteria. However, once a granuloma has formed, it is in fact a dynamic structure. Studies in a murine model suggest that leukocytes migrate in and out of the granuloma, and structures similar to secondary lymphoid tissue and follicles form around its periphery [126]. Furthermore, following super-infection in a zebrafish model, mycobacteria-infected macrophages from outside the granuloma will home preferentially to an existing structure [127]. This suggests that mycobacterial infection of a macrophage induces phenotypic changes in that macrophage that facilitate its localisation to forming or pre-formed granulomas.

Granuloma formation is co-ordinated by chemokines and cytokine signalling. By comparing chemokine gene expression profiles in WT and TNF- $\alpha-/-$ mice, TNF- $\alpha$ has been shown to co-ordinate chemokine expression by $\mathrm{CD}_{1} 1 \mathrm{~b}^{+}$macrophages after $M$. tuberculosis infection, early during granuloma formation and later for maintenance of the granuloma during chronic infection [128]. In addition, administration of TNF- $\alpha$ 
neutralising antibody to chronically $M$. tuberculosis infected mice led to loss of granuloma structure and increased bacterial numbers in the lung [74].

The primary cell type believed to be important for production of TNF- $\alpha$ is the $\mathrm{CD} 4^{+} \mathrm{T}$ cell. Depletion of $\mathrm{CD} 4^{+} \mathrm{T}$ cells in chronically $\mathrm{Tb}$ infected mice leads to loss of granuloma integrity [129] and $\mathrm{CD}^{+} \mathrm{T}$ cells are the predominant lymphocyte subset within the murine granuloma [130]. $\mathrm{CD} 8^{+} \mathrm{T}$ cells are also important for granuloma maintenance. CD8 -/- mice or mice deficient in $\mathrm{CD}^{+} \mathrm{T}$ cell effector molecules, such as perforin and Fas/FasL, initially control bacterial numbers in the lung at similar levels to WT mice [131]. After the onset of adaptive immunity, the rate of increase of bacterial numbers in the lungs of CD8, perforin and Fas or FasL -/- mice is reduced, but unlike in WT mice, bacterial numbers do not stabilise. $\mathrm{CD} 8^{+} \mathrm{T}$ cells accumulate in the granuloma tissue more slowly than $\mathrm{CD}^{+} \mathrm{T}$ cells $[130]$ and $\mathrm{CD}^{+} \mathrm{T}$ cells switch from primarily producing IFN- $\gamma$ during the acute phase to displaying cytotoxic activity during the chronic phase of infection [132]. Therefore, the current model suggests $\mathrm{CD} 4^{+} \mathrm{T}$ cells maintain granuloma integrity, while $\mathrm{CD}^{+} \mathrm{T}$ cells function to control the number of mycobacteria within the granuloma and thereby contribute to containment of infection during latency.

Factors that are detrimental to granuloma integrity can lead to reactivation. A number of stresses that undermine the immune response can contribute to reactivation, such as malnutrition, physical and mental stress, aging, immunosuppressive treatments and HIV infection [133]. An imbalance in key immune system mediators, such as IL-10 and TGF- $\beta$, may also contribute. For example, transgenic mice on a C57BL/6 background were engineered to express IL-10 under the IL-2 promoter. When these mice were infected with M. tuberculosis, they over-expressed IL-10 [134]. This caused no difference in lung bacterial numbers between transgenic and WT mice during the acute phase of infection but differences became apparent during the chronic phase of the infection. Unlike WT mice, lung bacterial numbers did not stabilise in the transgenic mice but continued to increase. Since the murine model is not regarded as a good model of latent $\mathrm{Tb}$ (outlined in section 1.2.7), it is problematic to translate these results of increased bacterial numbers in the lung in the mouse during a chronic infection into an analogous human disease outcome. However, human patients with active Tb exhibit elevated serum IL-10 levels compared to subjects lacking symptoms that have been exposed to individuals with active $\mathrm{Tb}$ [135] and it is hypothesised that IL-10 promotes reactivation of $\mathrm{Tb}$ infection. 
TGF- $\beta$ may also inhibit T cell responses during Tb infection. TGF- $\beta$ production from human monocytes can be induced by stimulation with either M. tuberculosis [136] or mycobacterial components such as LAM [137]. In addition, in vitro culture of PBMCs from patients who have active $\mathrm{Tb}$, with neutralising antibody or natural inhibitors of TGF- $\beta$ enhances IFN- $\gamma$ production [138]. Thus, there is speculation that TGF- $\beta$ is produced and suppresses $\mathrm{T}$ cell effector mechanisms during active $\mathrm{Tb}$ infection; but this has not been assessed using an in vivo model.

The granuloma environment induces changes in the mycobacteria. Within the granuloma, there are lymphocytes producing cytokines, such as IFN- $\gamma$ and TNF- $\alpha$, as well as CTLs. As a result, macrophages containing mycobacteria become highly activated, anti-microbial and produce RNI. Furthermore, the conditions at the centre of the human $\mathrm{Tb}$ granuloma become hypoxic and toxic to both host cells and mycobacteria [12]. It is thought that due to these conditions, mycobacteria alter their metabolism and enter a dormancy phase by inducing expression of a number of mycobacterial genes. The best characterised of these are the genes controlled by the response regulator DosR (Rv3133c), comprising the DosR regulon. The 48 genes in the regulon are transcriptionally upregulated in vitro in response to hypoxia or RNI products [139]. Many of these genes are also upregulated in M. tuberculosis isolated from IFN- $\gamma$-treated macrophages [140] and chronically infected murine lung tissue [141] suggesting that the genes are involved in mycobacterial survival after the onset of adaptive immunity in vivo. The most well-known gene of the DosR regulon is $h s p X(\mathrm{Rv} 2031 \mathrm{c})$, which encodes the dominant protein in stationary phase cultures of $M$. tuberculosis, the $16 \mathrm{kDa}$ alpha-crystallin homologue (Acr) [142]. Inhibition of Acr production does not affect growth on media or infectivity but $h s p X$ deficient $M$. tuberculosis strains do not survive in murine or human macrophages in vitro [142]. Therefore, it is thought that genes of the DosR regulon, such as $h s p X$, are essential for survival in host macrophages after the onset of adaptive immunity.

\subsubsection{Suppression of the immune response}

During the long history of co-evolution between humans and mycobacteria, humans have evolved effector immune mechanisms to combat the intracellular infection; however, mycobacteria have evolved ways to evade or disrupt these mechanisms. 
Latency induces changes in mycobacteria that not only permit survival but also promote immunoevasion. The altered state of metabolism induced by latency changes the antigen expression profile [143]. Some immunogenic mycobacterial proteins such as the $6 \mathrm{kDa}$ early secreted antigenic target (ESAT-6) are highly expressed during active growth [144], but not during latency. Conversely, production of other proteins, such as Acr, are upregulated during latency [142]. Individuals with latent $\mathrm{Tb}$ infection mount $\mathrm{T}$ cell responses against Acr [145] but responses to Acr are lower than responses to antigens present during active growth in murine models [146]. Therefore, altering antigen expression during different metabolic stages can allow mycobacteria to evade the immune response.

Mycobacteria can also impair the immune response in several ways, including inhibition of: phago-lysosomal function, ROI and RNI production, macrophage activation and antigen presentation.

Phago-lysosomal function can be inhibited in macrophages infected with mycobacteria and this inhibition could be mediated in a number of ways. Fusion of the phagosome with the lysosome has been shown to be inhibited, since phagosomes containing live, viable mycobacteria are inhibited from fusing with lysosomes [147149]. However, phagosomes containing mycobacteria are capable of fusing with other endosomes, which suggests they are dynamic, fusion-competent structures that are selectively prevented from fusing with lysosomal compartments $[149,150]$. A host protein found in lymphoid and myeloid cells called tryptophan aspartate-containing coat (TACO) protein has been recently identified, and may inhibit fusion. TACO relocalises within cells to associate with phagosomal membranes containing live mycobacteria and is retained for prolonged periods [151, 152]. Phago-lysosomal function may also be inhibited by interference with the acidity of the compartments. The lysosome contains hydrolytic enzymes, which require a low, acidic $\mathrm{pH}$ for optimal function. Acidification is mediated by ATP-dependent proton pumps, but mycobacteria-infected phagosomes can exclude these proton pumps, preventing acidification [153-155]. Mycobacteria can also produce ammonia, which has been proposed to increase the phago-lysosomal $\mathrm{pH}$ [156].

Mycobacteria are also able to prevent damage caused by ROI and RNI produced by infected macrophages. Mycobacterial products such as LAMs and phenolicglycolipid-1 can act as free-radical scavengers and mitigate oxidative damage 
$[157,158]$. M. tuberculosis possesses the genes noxR 1 , noxR 3 and ahpC that can provide resistance to nitrosative and oxidative stress [159-161]. Furthermore, $M$. tuberculosis expresses a methionine sulfoxide reductase, which catalyses the reduction of methionine sulfoxide formed from the reaction of ONOO- and methionine residues in mycobacterial proteins and can repair damage caused by RNI [162].

For maximal anti-mycobacterial function, infected macrophages require IFN- $\gamma$ signalling and mycobacterial infection can also interfere with this process. Human macrophages infected with $M$. tuberculosis are refractory to the effects of IFN- $\gamma$ signalling and, since $M$. tuberculosis co-infection prevents the macrophage from killing Toxoplasma gondii, it is thought to be due to a generalised defect in macrophage function [163]. It is proposed that mycobacterial cell wall products such as the $19 \mathrm{kDa}$ lipoprotein [164] prevent interaction of signal transducer and activator of transcription (STAT)1, cyclic AMP response element-binding protein and p300, which are downstream mediators of IFN- $\gamma$ signalling [163]. This defect prevents effective IFN- $\gamma$ induced gene expression, demonstrating that mycobacteria can interfere with even the most integral host mechanisms for their elimination.

Finally, as intracellular pathogens, mycobacteria live inside macrophages or DCs and the immune system must detect mycobacterial antigens displayed on the surface of macrophages to effectively eliminate the mycobacteria that they contain. Ideally mycobacterial antigens would be displayed on MHC II to encourage IFN- $\gamma$ production by activated $\mathrm{CD} 4{ }^{+} \mathrm{T}$ cells and facilitate full activation of the macrophage. However macrophages infected with mycobacteria can down-regulate MHC II surface expression. Infected macrophages can sequester MHC II and prevent efficient antigen processing by preventing the arrival of MHC II into endosomes and phago-lysosomes containing mycobacterial antigen [165]. Furthermore, IFN- $\gamma$ signalling to macrophages leads to upregulation of MHC II expression, but mycobacterial infection suppresses expression of IFN- $\gamma$ induced genes, including MHC II [164]. Macrophages infected with mycobacteria have decreased expression of the locus encoding MHC II and thereby decreased protein production [166]. Thus, mycobacteria interfere with MHC II expression and loading in infected macrophages and can result in sub-optimal targeting by activated $\mathrm{CD} 4^{+} \mathrm{T}$ cells.

To conclude, mycobacteria have developed a number of different ways of evading the immune response: changing their antigen profile, preventing phago- 
lysosome fusion, reducing free radicals from ROI and RNI production, inhibiting IFN- $\gamma$ activation of macrophages and preventing optimal antigen presentation. Due to the wide array of evasive tactics largely directed at the infected macrophage, it is conceivable that mycobacteria may have mechanisms for manipulating or suppressing other cells of the immune response, such as activation of Tregs and their suppressive mechanisms.

\subsubsection{The secondary immune response}

A secondary immune response to mycobacteria occurs either in the situation where an individual is vaccinated with the BCG vaccine (section 1.3.4), or if they have had a $\mathrm{Tb}$ infection and have been treated with antibiotics, and are then exposed to a mycobacterial challenge. The traditional view of a secondary immune response to challenge is that the response is either accelerated, increased in magnitude or it possesses an enhanced quality essential for improved control of infection compared to a primary immune response. Thereby, the infectious challenge can be more effectively controlled or eliminated, resulting in less severe disease in the host. However, the mechanism by which a secondary immune response is more effective than a primary immune response for controlling $\mathrm{Tb}$ infection is undefined.

A strong Th1 cell-mediated immune response during primary challenge is vital for containment of primary $\mathrm{Tb}$ infection. Since IFN- $\gamma$ production by $\mathrm{CD} 4^{+} \mathrm{T}$ cells is important for a protective primary immune response, $\mathrm{Tb}$ vaccines have largely aimed to induce strong $\mathrm{CD} 4{ }^{+} \mathrm{IFN}-\gamma^{+} \mathrm{T}$ cell responses, in the belief that this would lead to a larger pool of $\mathrm{Tb}$-specific memory $\mathrm{CD} 4^{+} \mathrm{T}$ cells. However, this approach does not guarantee a more effective vaccine, as vaccinations that induce strong $\mathrm{CD} 4^{+} \mathrm{IFN}-\gamma^{+} \mathrm{T}$ cell responses do not consistently result in enhanced protection from $\mathrm{Tb}$ infection [167]. This may be indicative of the fact that inducing strong $\mathrm{CD} 4^{+} \mathrm{IFN}-\gamma^{+} \mathrm{T}$ cell responses contradicts the current model of $\mathrm{CD}^{+}$Th1 memory induction, outlined in section 1.3.2.4.

While it remains difficult to define aspects of the primary immune response that correlate with enhanced secondary immune responses to $\mathrm{Tb}$, some components of the immune system have been shown to be vital for protective memory. Transfer of spleenderived $\mathrm{T}$ cells from mice vaccinated intravenously with $\mathrm{BCG}$ into naïve animals protected recipients against $\mathrm{Tb}$ or BCG infection to a similar degree as BCG vaccination [168], illustrating that $\mathrm{T}$ cells mediate protection against $\mathrm{Tb}$ after BCG vaccination. 
$\mathrm{CD}^{+} \mathrm{T}$ cell depleted splenocytes transferred from BCG infected animals into naïve recipients do not protect as well against $M$. tuberculosis infection when compared to total or even $\mathrm{CD}^{+} \mathrm{T}$ cell depleted splenocytes [169]. In addition, adoptive transfer of purified $\mathrm{CD}^{+}$or $\mathrm{CD} 8^{+} \mathrm{T}$ cells from $\mathrm{BCG}$ vaccinated mice into naïve recipients demonstrated that $\mathrm{CD} 4^{+} \mathrm{T}$ cell transfer resulted in reduced bacterial burden in the lung and spleen after BCG challenge, while $\mathrm{CD} 8^{+} \mathrm{T}$ cell transfer reduced burden only in the spleen [170]. These results indicate that the $\mathrm{CD}^{+} \mathrm{T}$ cell population is vital for the reduced bacterial numbers found in the lung of vaccinated compared to naïve animals, whereas $\mathrm{CD}^{+} \mathrm{T}$ cells can inhibit dissemination of the secondary infection to other tissues.

It has not been widely explored how $\mathrm{CD} 4^{+}$and $\mathrm{CD} 8^{+} \mathrm{T}$ cells mediate their effects during a secondary immune response and their mechanisms might not parallel the behaviour of these populations during a primary immune response. As previously mentioned, a critical cytokine for the anti-mycobacterial primary immune response is IFN- $\gamma$ but the amount of IFN- $\gamma$ induced following vaccination does not correlate with protection against secondary mycobacterial challenge [167]. However, the appearance of IFN $-\gamma^{+} \mathrm{CD}^{+} \mathrm{T}$ cells in the lung correlates with the onset of bacteriostasis during a primary mycobacterial infection [47], and the earlier appearance of IFN- $\gamma^{+} \mathrm{CD} 4^{+} \mathrm{T}$ cells correlates with the earlier onset of bacteriostasis in vaccinated compared to naïve mice after mycobacterial challenge [171]. Therefore, it has been suggested that the earlier appearance of IFN $-\gamma^{+} \mathrm{CD} 4^{+} \mathrm{T}$ cells in the lung is responsible for the reduced bacterial numbers in the lung observed in BCG vaccinated compared to naïve mice. Building on this model of mycobacterial immunity using peptide vaccinated mice, the arrival of IL$17^{+} \mathrm{CD} 4^{+} \mathrm{T}$ cells in the lung during the secondary immune response precedes the appearance of IFN- $\gamma^{+} \mathrm{CD} 4^{+} \mathrm{T}$ cells [84]. The arrival of $\mathrm{IL}-17^{+} \mathrm{CD} 4^{+} \mathrm{T}$ cells correlates with the upregulation of chemokine transcription in the lung and depletion of IL-17 reduced the accumulation of IFN $-\gamma^{+} \mathrm{CD} 4^{+} \mathrm{T}$ cells. Thus, the current model for the mechanism of protection during a secondary immune response suggests that an accelerated appearance in the lung of $\mathrm{IL}-17^{+} \mathrm{CD} 4^{+} \mathrm{T}$ cells induces chemokine production, providing an environment that encourages the earlier influx of IFN- $\gamma^{+} \mathrm{CD} 4^{+}$ T cells.

While there exists an obvious role for IFN $-\gamma^{+} \mathrm{CD} 4^{+} \mathrm{T}$ cells in induction of bacteriostasis in the lung, the question that remains is whether this is the only 
mechanism that facilitates vaccine-mediated protection. Naive and BCG vaccinated IFN- $\gamma-/-$ mice both succumb to M. tuberculosis infection quickly and display higher bacterial burdens compared to WT naïve and BCG vaccinated mice, demonstrating the importance of IFN- $\gamma$ in both primary and secondary immune responses [172]. However, when IFN- $\gamma-/-$ mice were vaccinated with BCG, the vaccinated mice had a significantly longer survival time compared to naïve IFN- $\gamma-/-$ mice [172]. IFN- $\gamma-/-$ BCG vaccinated mice also displayed lung bacterial numbers $\sim 1 \log _{10}$ lower than naïve IFN- $\gamma-/-$ mice after the first 20 days of infection and exhibited diminished lung pathology [172]. Interestingly, when the $\mathrm{CD}^{+} \mathrm{T}$ cell population was depleted in IFN- $\gamma-/-\mathrm{BCG}$ vaccinated mice, their survival time reverts to that of IFN- $\gamma-/-$ naive mice, as does their lung pathology [172]. The collective observations from this study indicates that BCG vaccination can confer a measure of protection to vaccinated mice that is independent of IFN- $\gamma$ mediated mechanisms but is mediated by $\mathrm{CD} 4^{+} \mathrm{T}$ cells. As a result, alternative mechanisms of protection for $\mathrm{Tb}$ immunity are being proposed (reviewed in [173]) and the concordance of several of these mechanisms with my findings will be discussed in chapter 6.

Although the roles of IFN- $\gamma, \mathrm{CD} 4^{+}$and $\mathrm{CD}^{+} \mathrm{T}$ cells during secondary protective immune responses are have been investigated, the impact of Tregs on the development of protective memory against $\mathrm{Tb}$ had not been assessed prior to this thesis. Tregs can suppress proliferation and cytokine production by $\mathrm{CD} 4^{+}$and $\mathrm{CD} 8^{+} \mathrm{T}$ cells (section 1.4.2). Proliferation and production of certain cytokines are essential for optimal $\mathrm{T}$ cell memory development (sections 1.3.2.2 and 1.3.2.3). Thus, Tregs could suppress the development of protective memory against $\mathrm{Tb}$ during vaccination and this possibility is explored in my thesis.

\subsubsection{Animal models of Tb}

Animal models have contributed significantly to our understanding of pathogenesis, immunology and host-bacilli interactions during Tb infection. Several different animals can be used as models of Tb due to their susceptibility to M. tuberculosis or related mycobacterial infections, such as M. bovis and M. marinum. Each model has advantages and disadvantages that must be considered. $\mathrm{Tb}$ is a complex disease in humans, with resistant and susceptible individuals and multiple stages of disease that can affect a 
variety of tissues. Some animal models are better suited to model specific aspects of disease than others.

The mouse is the most widely used model of $\mathrm{Tb}$ infection. Mice are easily housed and their genome is well characterised. There are also a wide variety of immunological tools, such as murine-directed antibodies and gene-deficient strains of mice, which can be utilised when working with murine models. Mice can be infected in a number of ways but a low-dose infection of $\sim 50$ colony forming units (CFU) of $M$. tuberculosis by aerosol is regarded as the most physiologically relevant to human $\mathrm{Tb}$ infection (reviewed in $[22,174]$ ). Using this approach, lung bacterial numbers increase slowly but in a log linear fashion until 3-4 weeks after infection, when the lung bacterial numbers stabilise, coinciding with the influx of activated $\mathrm{T}$ cells into the lung. Thereafter lung bacterial numbers remain high at $\sim 10^{6}$ CFU but relatively stable until mice succumb to infection. Although bacterial numbers in the lung stabilise, lung pathology does progress. Discrete lung lesions that correspond to the implantation sites of the aerosolised M. tuberculosis accumulate and consist of infected macrophage and lymphocytic infiltrate with some fibrosis evident at later stages. The survival times of mice differ according to strain. $\mathrm{C} 57 \mathrm{BL} / 6$ and $\mathrm{BALB} / \mathrm{c}$ mice survive on average to 250 days post infection and are termed resistant to infection, while $\mathrm{DBA} / 2, \mathrm{C} 3 \mathrm{H}, \mathrm{CBA}$ and 129Sv mice survive on average to 100 days post infection and are termed susceptible [175].

Dissemination of mycobacteria from the lung of infected mice occurs through the lymph and blood to the lymph node and other tissues such as the liver and spleen. This occurs prior to the onset of adaptive immunity, typically around 1-2 weeks after infection [176] and bacterial numbers in these tissues increase until the influx of activated T cells at 3-4 weeks after infection when bacterial numbers stabilise. After stabilisation of bacterial numbers, the chronic phase of $\mathrm{Tb}$ infection ensues.

The chronic phase of $\mathrm{Tb}$ infection in the murine model is not regarded as an accurate representation of human latent infection, since murine lung lesion structure does not replicate that of the human granuloma. Human granulomas are highly structured with infected macrophages surrounded by activated lymphocytes and fibroblasts enclosing the structure, and progress to become necrotic in the centre (reviewed in [177]. Murine lung lesions do not exhibit the same organised structure, possibly as lung architecture of a mouse is much smaller than that of humans [178]. 
Murine lung lesions also do not exhibit the same degree of central necrosis, possibly as the centres of murine lung lesions do not reach the extreme oxidative stress levels observed in human granuloma tissue due to the smaller size of murine lesions [12]. In addition, bacterial numbers in the murine lung after the onset of stationary phase is a lot higher than bacterial numbers observed in human tissue (reviewed in [179]).

Despite these differences, mice are a useful model for $\mathrm{Tb}$ vaccination. Subcutaneous (s.c.) BCG vaccination of mice results in Th1 immune responses against mycobacterial antigens. After an aerosol M. tuberculosis challenge, a $\sim 1 \log _{10}$ decrease in lung bacterial numbers is observed in BCG vaccinated mice compared to naïve controls [22]. This $\sim 1 \log _{10}$ decrease is regarded in pre-clinical vaccine trials to be the gold standard level of protection, against which novel vaccines are easily compared $[180,181]$.

BCG can also be used to model Tb infection. When administered to mice intranasally (i.n.), BCG initiates a pulmonary mycobacterial infection with recruitment to the lung of cellular subsets similar to those observed during pulmonary $M$. tuberculosis infection, including IFN- $\gamma$ producing CD4 ${ }^{+} \mathrm{T}$ cells $[182,183]$. Since BCG does not cause significant disease in immunocompetent humans, this model permits analysis of pulmonary mycobacterial infection in physical containment level (PC)-2 conditions and parallels may be drawn with virulent $M$. tuberculosis infection. Similar to $M$. tuberculosis infection, early growth of BCG is observed in the lung, followed by dissemination to other tissues, and upon the onset of adaptive immunity bacterial numbers in tissues stabilise $[182,183]$. However, unlike M. tuberculosis infection, the tissue bacteria numbers then slowly decline $[182,183]$.

Other small animal models of $\mathrm{Tb}$ infection include the guinea pig and rabbit infection models. Both models exhibit similar kinetics of bacterial growth to the murine model after low-dose aerosol infection [184, 185]. By contrast to the murine model, both models exhibit lung pathology more representative of human $\mathrm{Tb}$ with tissue necrosis $[185,186]$. Guinea pig and rabbit models share the limitations that they are larger and more difficult to house than mice and reagents available for immunological analysis are limited.

More recently, other animal models of mycobacterial infection have been developed, such as the non-human primate model and fish or amphibian models. The strength of the non-human primate model is its ability to model the onset and 
progression of latent infection [187], which cannot be as faithfully represented with other animal models, but there are significant costs associated with housing the nonhuman primate model. M. marinum is a natural pathogen of fish and amphibians that induces granuloma formation, and infection of zebrafish embryos with fluorescently labelled M. marinum can be used to visualise mycobacteria within a living host [127]. While the M. marinum zebrafish model enables visualisation of mycobacteria in vivo, there are vast differences between immune systems of zebrafish embryos and adult humans, and data should be interpreted with this in mind.

As outlined above, there are a number of different animal models for $\mathrm{Tb}$ infection available and one should consider their advantages and disadvantages when designing experiments. This thesis focuses on characterising the acute phase of the immune response to infection and vaccination rather than the latent phase of infection, which makes the well defined and well resourced murine model of Tb ideal. Furthermore, the BCG i.n. infection model was used to enable immunological analyses within a PC-2 facility, but some immunological findings and protection data were also obtained using virulent $M$. tuberculosis or $M$. bovis infections.

\subsection{Vaccination}

\subsubsection{The principles of vaccination}

The idea of using small doses of a whole or part of a pathogen to prevent full infection with the virulent organism is an old one (reviewed in [188]); evidence exists in ancient Chinese and Indian literature for the use of variolation to prevent smallpox, and more recently in history, Edward Jenner observed that cowpox infection prevented smallpox infection. However, all utilise the same basic principle of immunology: immunological memory, which will be discussed below.

There are several broad concerns to consider when designing vaccines to prevent infection. Firstly, the vaccine must be safe to administer. Vaccines are given to a range of different people, from healthy adults through to the immunocompromised, the elderly or the very young, whose immune systems may be underdeveloped or impaired. A vaccine must induce protective immunity without resulting in immunopathology or infection. Secondly the vaccine must induce the correct type of immune response, 
targeted for the specific pathogen. Just as there are different types of primary immune response, there are different types of secondary immune responses and targeting an aberrant immune response for that pathogen could lead to more severe disease in vaccinated individuals [189]. Designing a vaccine to induce the optimal immune response to prevent disease caused by a specific pathogen is known as rational design.

The rational design approach is at the interface of vaccinology and immunology, where researchers endeavour to use the principles of immunology to enhance vaccine design. Traditionally, vaccine design has used an empirical approach, in which a vaccine was created and tested for protection against infection and detailed prior knowledge of the protective immune response to the pathogen was not essential. The empirical approach has worked for many pathogens but as the understanding of different types of immune responses advanced, it emerged that classical vaccine designs typically induce strong antibody responses. Antibody responses can control extracellular bacterial infections and some viral and protozoal infections.

By contrast, classical vaccine designs have not resulted in successful vaccines for infections including HIV, malaria and Tb (reviewed in [190]). It has emerged that cell-mediated responses are needed to control or eradicate these infections. Thus, it is unsurprising that classical vaccine designs are ineffective for these infections and there is a push to understand more about the induction of cell-mediated immunity to enable rational vaccine design. However, large gaps remain between vaccinology and immunology, as the most desirable primary and secondary immune responses for protective immunity against these infections are not well defined. Therefore, while rational design is desirable for infections like HIV malaria and $\mathrm{Tb}$, it is not wholly attainable until the protective immune response for these infections is better understood.

\subsubsection{Immunological memory models}

Immunological memory denotes the enhancement or acceleration of secondary immune responses compared to that induced by the initial pathogen encounter and the response is antigen specific rather than a generalised heightened responsiveness of the immune system. Cells that mediate memory immune responses are termed memory lymphocytes, which are long-lived, antigen-specific B or T cells. Memory lymphocytes develop from naïve lymphocytes after an initial encounter with their specific antigen 
during the primary immune response. They become memory lymphocytes that can persist long-term and, upon secondary stimulation with their cognate antigen, their response is typically quantitatively and/or qualitatively enhanced compared to the primary response. The process of memory development differs between B and T cells and there are also memory subsets within these two populations. For vaccinology, it is key to determine how the optimal memory B or T cell is generated to prevent disease caused by a target pathogen.

\subsubsection{Humoral memory}

B cell memory is also referred to as humoral immunity, which encompasses the memory B lymphocytes and some $\mathrm{CD}^{+} \mathrm{Th} 2$ cells. Memory B lymphocytes include both quiescent memory B cells located in secondary lymphoid tissue and long-lived plasma cells located in the bone marrow. Primary antigenic stimulation is delivered through the $\mathrm{B}$ cell receptor and, with $\mathrm{CD}^{+} \mathrm{T}$ cell help in the form of cytokine production and co-stimulatory signals, promotes B cell survival and immunoglobulin (Ig) class switching and the establishment of plasma and memory B cell populations early during a primary immune response (reviewed in [191]). Plasma cells continue to produce high-affinity antibody that is released into circulation, and quiescent memory $\mathrm{B}$ cells produce high-affinity antibody after secondary antigenic stimulation, (reviewed in [192]). Therefore the key mediator of humoral memory is high-affinity serum antibody, first from the plasma cell and then from the activated memory B cell. Antibodymediated immunity is particularly effective at controlling extracellular bacterial infections and viral infections before they enter their target host cell or after lysis.

\subsubsection{Cell-mediated memory}

Cell-mediated memory encompasses $\mathrm{CD} 4^{+}$and $\mathrm{CD} 8^{+} \mathrm{T}$ cells and their effector mechanisms, including cytokine production and cytotoxicity. T cell memory is more complex than B cell memory to define, as there are several $\mathrm{T}$ cell subsets with different proposed models of memory development. Even within these defined subsets, there is significant heterogeneity in terms of surface and functional marker expression, cellular location and survival requirements. 
Memory $\mathrm{T}$ cell subpopulations have been proposed on the basis of proliferation [193] or by division into fast and slow responder subsets based on their capacity to initiate effector mechanisms after antigenic stimulation [194]. These subpopulations have recently been refined by studies relating memory $\mathrm{T}$ cell subsets to their location. A study by Sallusto, et al. of human memory cells, defined as CD45RA ${ }^{\text {lo }}$ and CD45RO ${ }^{\text {hi }}$, demonstrated differential cell surface expression of CCR7 within the memory population in both $\mathrm{CD}^{+}$and $\mathrm{CD} 8^{+} \mathrm{T}$ cells [195]. CCR7 is a chemokine receptor that promotes lymphocyte homing to and retention within the lymph node. $\mathrm{CCR} 7^{-}$memory $\mathrm{T}$ cells were predominantly found in peripheral tissues and circulation, whereas CCR $7^{+}$ memory $\mathrm{T}$ cells were predominantly found in lymphoid tissue. $\mathrm{CCR}^{-}$memory $\mathrm{T}$ cells were termed effector memory $\mathrm{T}$ cells $\left(\mathrm{T}_{\mathrm{EM}}\right)$, as this subset was able to rapidly upregulate effector mechanisms such as IFN- $\gamma$ production upon stimulation. CCR7 $7^{+}$ memory $T$ cells were termed central memory $T$ cells $\left(\mathrm{T}_{\mathrm{CM}}\right)$ due to their location in lymphoid tissue. $\mathrm{T}_{\mathrm{CM}}$ were less effective at rapid cytokine production but proliferated more readily than $\mathrm{T}_{\mathrm{EM}}$ and could differentiate into $\mathrm{T}_{\mathrm{EM}}$ upon polyclonal stimulation. Memory T cells were also demonstrated to differentially express CD62L [195]. CD62L is also a lymph node homing marker and $\mathrm{T}_{\mathrm{CM}}$ were found to be predominantly $\mathrm{CD}_{2} \mathrm{~L}^{\mathrm{hi}}$, while $\mathrm{T}_{\mathrm{EM}}$ were CD62 $\mathrm{L}^{\text {lo }}$.

Subsequent to this study, it has emerged that the $\mathrm{T}_{\mathrm{CM}} / \mathrm{T}_{\mathrm{EM}}$ distinction may not hold true in all situations. Memory T cell populations defined by CCR7 expression, as suggested by Sallusto, et al., have been shown to exhibit the distinct proliferative capacities and to localise to distinct tissue locations in other studies. However, a distinction between the populations in terms of effector responses has not always been apparent [196-198]. Differentiation of memory T cell subsets based on CD62L expression has also not translated into differences in effector responses in other studies $[63,199]$. Therefore while the $\mathrm{T}_{\mathrm{CM}} / \mathrm{T}_{\mathrm{EM}}$ distinction based on CCR7 or CD62L expression may assist in delineating probable location and proliferative capacity of memory $\mathrm{T}$ cells, replicable markers of memory $\mathrm{T}$ cell effector capacity remain to be identified.

\subsubsection{Factors mediating the development and maintenance of memory $T$ cells}

There are several basic factors that mediate development of a memory $\mathrm{T}$ cell population, although requirements for these can differ between $\mathrm{CD} 8^{+}$and $\mathrm{CD} 4^{+} \mathrm{T}$ cell 
populations. Key factors for the development or maintenance of memory $\mathrm{T}$ cells include signalling from the $\gamma c$ chain family of cytokines and TCR signalling at various stages of memory development. The $\gamma \mathrm{c}$ chain family of cytokines all use the common $\gamma$ chain as part of their receptor for cell signalling. Cytokines from this family that have been shown to enhance memory T cell development include IL-2, IL-7 and IL-15.

For efficient memory induction, there is an established role for early IL-2 signalling. IL-2 is predominantly secreted by CD4 $4^{+} \mathrm{T}$ cells and IL-2 signalling during priming has been shown to be important for development of both $\mathrm{CD}^{+}$and $\mathrm{CD} 8^{+}$ memory T cells. Effective IL-2 signalling to naïve $\mathrm{CD}^{+} \mathrm{T}$ cells undergoing initial expansion promotes their long-term survival, presumably inducing upregulation of the IL-7 receptor $\alpha$ chain $[200,201]$. IL-2 signalling to $\mathrm{CD}^{+} \mathrm{T}$ cells during priming may only slightly affect the number of effector or memory $\mathrm{CD}^{+} \mathrm{T}$ cells but is essential to develop memory cells capable of expanding upon secondary stimulation [202].

It has also been shown that $\mathrm{CD} 4^{+} \mathrm{T}$ cells promote the development of $\mathrm{CD} 8^{+}$ memory T cells [203]. It was suggested that this was due to the capacity of CD4 ${ }^{+} \mathrm{T}$ cells to secrete IL-2 during priming, but it appears that $\mathrm{CD}^{+} \mathrm{T}$ cells also enhance maintenance of $\mathrm{CD}^{+}$memory T cells [204].

IL-7 and IL-15 also have established roles in the maintenance of memory T cells in vivo. IL-7 is secreted constantly by fibroblasts and its availability is thought to be controlled by its consumption rather than production. Thereby, the constant amount of IL-7 maintains a fixed population size for memory T cells. For $\mathrm{CD}^{+}$effector T cells, upregulation of the IL-7 receptor $\alpha$ chain correlates with their transition into a memory cell population [201] and $\mathrm{CD}^{+}$memory T cells do not persist in IL-7 -/- mice [205]. Thus, IL-7 is important for optimal transition and maintenance of $\mathrm{CD}^{+}$memory $\mathrm{T}$ cells.

TCR signalling also promotes survival of $\mathrm{CD}^{+}$memory T cells [205] and IL-7 and TCR signalling can synergise for the optimal survival conditions of CD $4^{+}$memory $\mathrm{T}$ cells. For $\mathrm{CD}^{+}$memory $\mathrm{T}$ cells, TCR signalling does not appear to be as important, as transfer of $\mathrm{CD}^{+}$memory $\mathrm{T}$ cells into $\mathrm{MHCI}-/-$ mice does not affect the ability of the cells to persist [206]. Instead, maintenance of $\mathrm{CD}^{+}$memory $\mathrm{T}$ cells is dependent on IL7 and IL-15 signalling [207-209]. 
Therefore, the $\gamma \mathrm{c}$ chain family of cytokines and TCR signalling play roles in $\mathrm{CD}^{+}$and $\mathrm{CD}^{+}$memory $\mathrm{T}$ cell development and maintenance. $\mathrm{CD} 4^{+}$memory $\mathrm{T}$ cells require IL-7 and TCR signalling and CD ${ }^{+}$memory T cells require IL-15 but TCR signalling is not as essential. Both $\mathrm{CD}^{+}$and $\mathrm{CD} 8^{+}$memory $\mathrm{T}$ cells require early IL-2 signalling for optimal memory development, but this leads to subtly different outcomes for each population. Tregs can affect proliferation and cytokine production, particularly IL-2 production by effector T cells (section 1.4.2). Therefore, it is possible that Tregmediated suppression could impede the efficient development of immunological memory.

\subsubsection{Models of memory $T$ cell differentiation}

Two models of memory differentiation have been used to explain memory $\mathrm{T}$ cell development: the sequential or linear differentiation model and the branched differentiation model, as described in Fig. 1.3 [210].

Some evidence has been used to argue in favour of the sequential model for memory development. It was found that effector $\mathrm{T}$ cells generated in vitro or in vivo and transferred into a naïve host can confer memory and naïve $\mathrm{T}$ cells must divide a number of times before they can form a memory population [211-214]. These studies have been used to suggest that memory differentiation progresses from a naïve $\mathrm{T}$ cell, to an effector $\mathrm{T}$ cell defined as a cell that has proliferated, to a memory $\mathrm{T}$ cell. However, the definition of an effector $\mathrm{T}$ cell as a $\mathrm{T}$ cell that has proliferated is too simplistic. 


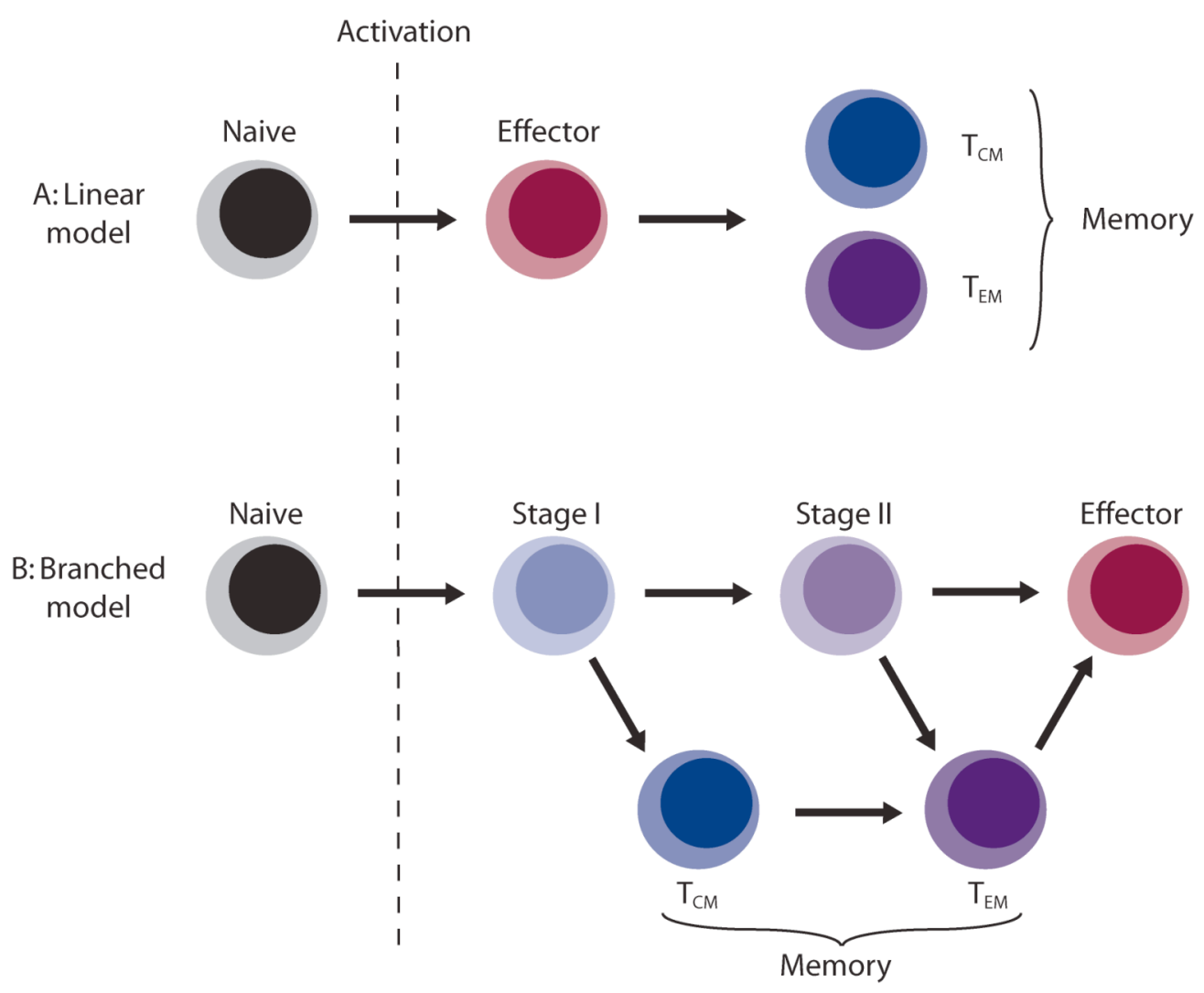

\section{Figure 1.3: The Linear and Branched Models of Memory T Cell Development.}

A) The linear model suggests progression from an individual naïve $T$ cell, which upon antigenic stimulation, proliferates to become an effector $\mathrm{T}$ cell, then finally to a memory $\mathrm{T}$ cell. Within the memory $\mathrm{T}$ cell population, progression from $\mathrm{T}_{\mathrm{CM}}$ to $\mathrm{T}_{\mathrm{EM}}$ cells and vice versa has been suggested. B) The branched differentiation model suggests progression from an individual naïve $\mathrm{T}$ cell to an effector $\mathrm{T}$ cell, with several differentiation stages. During effector T cell differentiation, activated T cells may branch off and establish memory cell populations. It is possible that $\mathrm{T}_{\mathrm{CM}}$ and $\mathrm{T}_{\mathrm{EM}}$ cell populations are established from discrete stages along this course. However, in the branched differentiation model, the effector $\mathrm{T}$ cell is a state of terminal differentiation and the effector $\mathrm{T}$ cell population is destined to die and not contribute to protective memory. This figure is based on figures in Farber, et al. [217]. 
Proliferated T cells do not necessarily acquire full effector functions including cytokine production and cytotoxicity. After a primary antigenic stimulation, cells proliferate and can acquire effector capabilities but a spectrum of cells displaying these different capabilities is induced. For example, if naïve $\mathrm{CD}^{+} \mathrm{T}$ cells are activated in vitro with their cognate antigen and high-level IL-2, they proliferate and develop into full effectors that demonstrate cytokine production and cytotoxicity [215]. In contrast, if naïve $\mathrm{CD}^{+} \mathrm{T}$ cells are activated in vitro with their cognate antigen and IL-15 or lowlevel IL-2, they proliferate and produce cytokines but do not acquire cytotoxicity and are thereby not full $\mathrm{CD} 8^{+}$effector $\mathrm{T}$ cells [215]. T cells that are not full effectors can also be generated in vivo. Administration of heat-killed Listeria monocytogenes leads to expansion of antigen-specific $\mathrm{CD}^{+} \mathrm{T}$ cells but cells do not acquire cytokine production or cytotoxic [216]. The point along this differentiation pathway at which cells also acquire the capability to become memory cells is not clear, although some stimulation and proliferation is clearly necessary.

Broad models of $\mathrm{T}$ cell memory such as the sequential or branched models of memory cell development can be helpful, but there is no clearly defined path for T cell memory induction on an individual cell basis. After TCR stimulation there is heterogeneity within the effector $\mathrm{T}$ cell population in terms of division cycles undergone and effector function. It is not known which cells within the heterogeneous population persist and contribute to protective immunity. Furthermore, the applicability of basic models of memory differentiation differs between $\mathrm{CD} 8^{+}, \mathrm{CD} 4^{+} \mathrm{Th} 1$ and $\mathrm{Th} 2$ cells and possibly other $\mathrm{T}$ cell subsets. For $\mathrm{CD}^{+} \mathrm{T}$ cells, the initial burst size, or number of activated antigen-specific $\mathrm{CD} 8^{+} \mathrm{T}$ cells during a primary immune response, corresponds to the magnitude of the memory responses [218]. It is therefore conceivable that within a larger total effector cell population, there are more cells destined to become memory cells, leading to a bigger memory response. These results have been used to suggest the linear model of memory differentiation is more suited for $\mathrm{CD}^{+} \mathrm{T}$ cells.

By contrast, the ability of $\mathrm{CD}^{+}$effector $\mathrm{T}$ cells to become memory cells depends on the cytokines they produce. A study using sorted TCR transgenic CD $4^{+} \mathrm{T}$ cells specific for a model antigen has demonstrated that IFN- $\gamma^{+} \mathrm{CD} 4^{+} \mathrm{T}$ cells do not contribute to memory development [219]. IFN- $\gamma^{+} \mathrm{CD} 4^{+}$and IFN $-\gamma^{-} \mathrm{CD} 4^{+}$populations sorted from short-term Th1-promoting in vitro cultures of TCR transgenic T cells were transferred into naïve congenic mice and tracked in vivo long-term. IFN- $\gamma^{+} \mathrm{CD} 4^{+} \mathrm{T}$ cells 
quickly disappeared but IFN $-\gamma^{-} \mathrm{CD} 4^{+} \mathrm{T}$ cells persisted and produced IFN- $\gamma$ upon secondary restimulation. It is therefore believed that IFN $-\gamma^{+} \mathrm{CD} 4^{+} \mathrm{T}$ cells do not persist long-term after primary challenge. IFN- $\gamma$ expression confers an enhanced susceptibility to AICD, as has been demonstrated for Th1 cells compared to Th2 cells [88, 220]. In addition, IFN- $\gamma$ production has been shown to lead to apoptosis of effector $\mathrm{CD}^{+} \mathrm{T}$ cells after BCG infection $[89,90]$. This has led to a branched model of memory CD4 ${ }^{+}$Th1 cell differentiation [221]. In this model, antigenic stimulation leads to proliferation and activation of naïve $\mathrm{CD} 4^{+} \mathrm{T}$ cells. If $\mathrm{T}$ cells differentiate to the point that they express IFN- $\gamma$, they die during contraction of the primary immune response and do not contribute to memory populations. However, activated IFN $-\gamma^{-} \mathrm{CD}^{+} \mathrm{T}$ cells can establish long-lived memory populations, producing IFN- $\gamma$ upon subsequent challenge and it is proposed that these cells mediate immunological memory and protection from subsequent infections.

This model of $\mathrm{CD}^{+} \mathrm{Th} 1$ memory is particularly relevant for $\mathrm{Tb}$ vaccine development, since it is thought that natural immunity for Tb relies on $\mathrm{CD} 4^{+} \mathrm{Th} 1$ memory. Previously, many $\mathrm{Tb}$ vaccine approaches have sought to increase the number of IFN- $\gamma^{+} \mathrm{CD}^{+} \mathrm{T}$ cells after vaccination but it has been demonstrated that increased IFN- $\gamma$ production during a primary immune response does not correlate with protection induced by Tb vaccination [167]. An essential gap in our current knowledge is an immunological marker from the primary immune response that correlates with protection from $\mathrm{Tb}$ infection upon secondary challenge. The branched model of Th1 memory differentiation suggests that a correlate of protection for $\mathrm{Tb}$ may be found within the IFN $-\gamma^{-} \mathrm{CD} 4^{+} \mathrm{T}$ cell population and that, if either the induction or subsequent function of the IFN $-\gamma^{-} \mathrm{CD} 4^{+} \mathrm{T}$ cell population is enhanced, $\mathrm{Tb}$ vaccine strategies can be improved.

\subsubsection{Types of vaccination}

When designing a vaccine, one must consider the key components required to successfully initiate an immune response. An adjuvant must be included, to provide danger signals to activate APCs, such as PAMPs. An antigen must also be included, to direct an adaptive immune response against the target pathogen. A vaccine can contain a single antigen from a pathogen that has immunodominant antigens. When a broader 
response is necessary, a mixture of several antigens or the entire killed or weakened pathogen can be used. There are several different types of vaccines: live, inactivated or subunit vaccines (either protein or DNA).

With live vaccines, recipients are vaccinated with a live organism that does not cause disease, as it has been altered to remove virulence but retain immunogenicity. Some of the first available vaccines were live, attenuated vaccines and attenuation was initially achieved by culturing virulent strains for long periods (reviewed in [188]), during which the organisms lost or mutated genomic material not essential for in vitro survival, including virulence factors. Attenuation of virulent organisms through in vitro culture, or more recently targeted mutation of candidate virulence genes, has been widely used to develop bacterial and viral vaccines. The advantage of live, attenuated vaccines is that they simultaneously deliver many different antigens for the pathogen with a spectrum of PAMPs and can induce something close to natural protective immunity. Furthermore, some live vaccines persist for a period of time and, if maintenance of natural immunity requires antigen persistence, immunity can be maintained for a prolonged period of time. However, there are safety concerns with live vaccines. Attenuated strains could revert to a virulent phenotype by point mutation reversion in virulence genes or acquisition of genetic material from co-infections with other related organisms. Furthermore, in susceptible or immunocompromised individuals, even attenuated pathogens can result in a productive infection, such as BCG lymphadenitis, which is the most common complication of BCG vaccination [222].

Due to these safety issues, inactivated vaccines were developed, whereby pathogens were inactivated or killed before being used to vaccinate an individual. Common approaches for pathogen inactivation include heat treatment or treatment with chemicals such as formaldehyde, acetone or phenol [223]. The inactivated pathogens again deliver antigen and PAMPs in one physically associated unit but are often less effective than live vaccination [224]. This may because the treatments alter the integrity and conformation of pathogen antigens or, since inactivation kills the pathogen, it may prevent secretion or production of an antigen or PAMP essential for immunogenicity. Safety of inactivated vaccines is not guaranteed as inconsistent validation of pathogen inactivation has lead to scenarios where vaccine batches have been released and used when not fully inactivated [225]. 
To further refine vaccines, subunit approaches have been used. Subunit vaccines are purified immunogenic components from the pathogen administered in conjunction with a formulated adjuvant. The antigen is usually an immunodominant protein from the pathogen, which may be purified from cultures of that pathogen [226] or produced by recombinant bacteria [227]. Adjuvants can be formulated to ensure localised, gradual delivery of the antigen and PAMPs, called the depot effect. Subunit vaccines are regarded as safer than live or inactivated vaccines but are typically less immunogenic, require repeated administration and careful choice of the antigen and adjuvant. The adjuvant must ensure effective activation of DCs and the selected antigen must be immunogenic, if not immunodominant. For some pathogens, there are no immunodominant antigens or they may produce different antigens or antigenic variants at different periods of their life-cycle [228] or infection [142, 144], so the breadth of antigens delivered by subunit vaccination may be limiting compared to a live vaccine.

A key characteristic of protein-based subunit vaccines is that they induce strong antibody responses; however, many intracellular infections require cell-mediated immune mechanisms for their control. Thus, researchers have endeavoured to develop subunit vaccines for intracellular infections that target the MHC I processing pathway and induce CTLs. In 1990, it was shown that intramuscular (i.m.) injection of plasmid DNA in saline resulted in transfection of the surrounding myocytes [229]. This was a significant finding for gene therapy but it was subsequently found that this process resulted in an immune response, as antibodies directed against protein from transfected genes were induced [230]. The definitive step came when it was shown that transfection also induced CTLs directed against protein expressed by the plasmid DNA, and this conferred protective immunity against a target pathogen [231]. As a result the process of DNA vaccination, as outlined in Fig. 1.4, was born. 


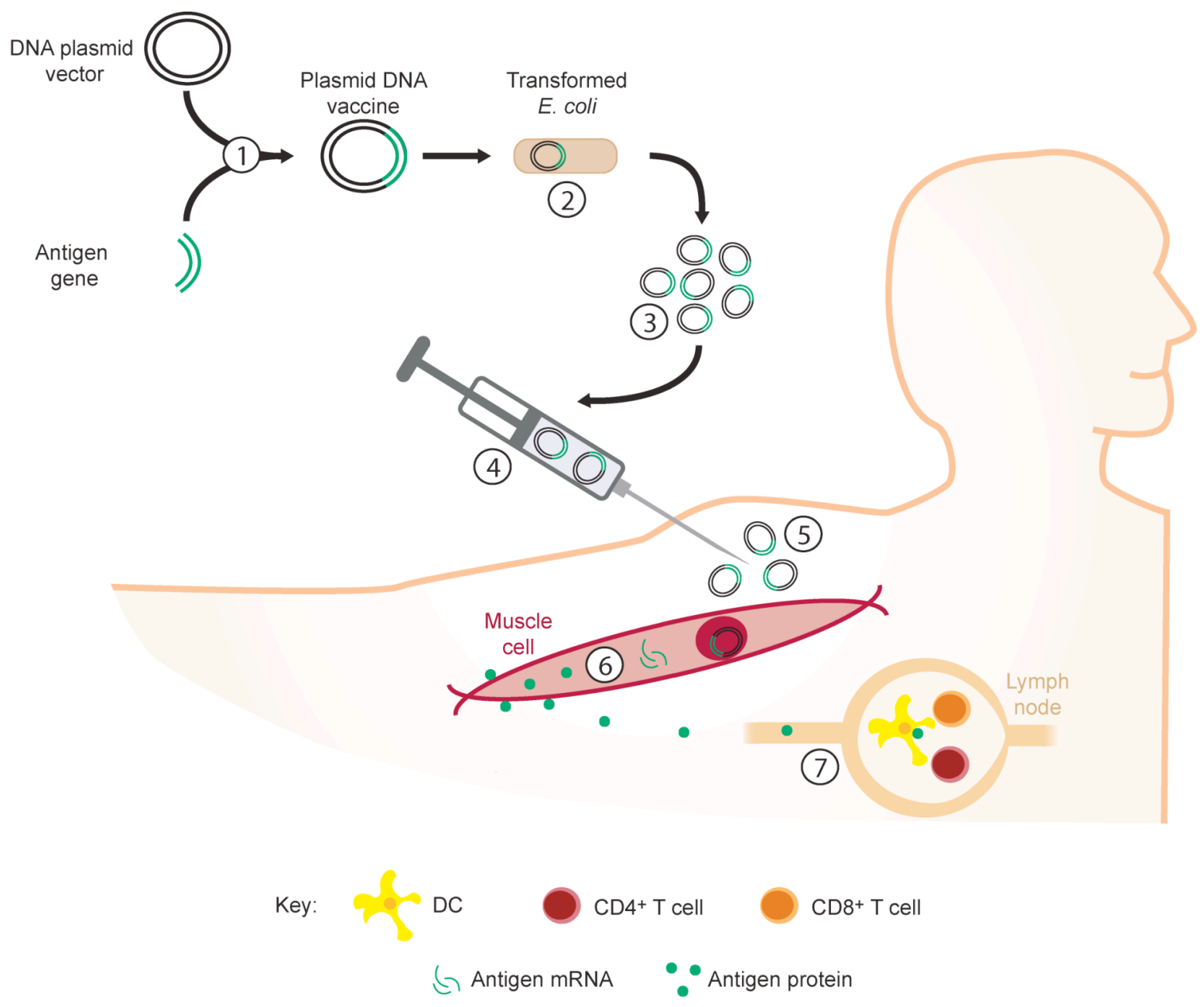

\section{Figure 1.4: The Process of DNA Vaccination}

1) Genomic material encoding an antigen from a pathogen (green) is cloned into a DNA vector (black). 2) This construct is transformed into bacteria 3) before being propagated and purified. 4) Purified DNA plasmids are injected into an individual. 5) The DNA plasmid transfects cells surrounding the injection site by endocytosis or receptor-mediated uptake of nucleic acid and translocates to the nucleus of the target cell by undefined mechanisms. 6) The antigen is expressed using the molecular machinery of the transfected cell 7) and the antigen is presented to specific $\mathrm{T}$ cells and initiates an immune response in the vaccinated individual. It is unknown currently which cell must be transfected to facilitate antigen presentation. Non-lymphoid cells surrounding the injection site (such as myocytes shown above) are transfected and may transfer antigen to APCs [241] or present antigen themselves [239]. Alternatively, APCs resident in the tissue may be directly transfected and thereby mediate antigen presentation [242]. 
DNA vaccination has several advantages compared to other approaches (reviewed in $[232,233])$. While direct injection of naked DNA encoding an antigen fails to induce antibody titres comparable to protein antigen and adjuvant co-administration, DNA vaccination can induce robust cell-mediated responses. In addition, specialised delivery methods such as gene-gun administration can be used to enhance antibody titres. Purified DNA plasmids can be efficiently manufactured and do not require refrigerated storage in contrast to many live or subunit vaccines, which improves the prospects of using these vaccines in the field. Antigens chosen can be well defined and manipulated to remove any toxic regions, making DNA vaccines very safe, even for immunocompromised patients. However, the mechanisms by which DNA vaccines access MHC I and II processing pathways and induce cell-mediated and humoral immunity remain to be defined [234-240].

For $\mathrm{Tb}$ vaccines, researchers have pursued vaccine designs that induce $\mathrm{CD} 4^{+}$ Th1 immunity, since IFN- $\gamma$ production and $\mathrm{CD}^{+} \mathrm{T}$ cells are important for protection from primary $\mathrm{Tb}$ infection (section 1.2.3) and are assumed to be important for protection from secondary $\mathrm{Tb}$ challenge (section 1.2.6). Live BCG vaccination has had some success for combating $\mathrm{Tb}$ (section 1.3.4). However, the focus of $\mathrm{Tb}$ vaccine research is beginning to shift from inducing IFN- $\gamma$ producing $\mathrm{CD}^{+} \mathrm{T}$ cells, as it becomes increasingly clear that it can not be assumed that IFN- $\gamma$ producing $\mathrm{CD}^{+} \mathrm{T}$ cells are the sole cellular subset responsible for protective memory against $\mathrm{Tb}$. Other live vaccines have been developed to target $\mathrm{Tb}$ and are currently in pre-clinical trials (section 1.3.4). Several of these enhance $\mathrm{CD}^{+} \mathrm{T}$ cell activation compared to $\mathrm{BCG}$ vaccination and have shown comparable or increased protective efficacy [180, 181]. The induction of $\mathrm{CD}^{+} \mathrm{T}$ cell memory is a promising target for $\mathrm{Tb}$ vaccine design and DNA vaccines provide a method to induce $\mathrm{CD} 8^{+} \mathrm{T}$ cell responses. Therefore, during the course of this thesis, a novel DNA replicon vaccine was created to induce $\mathrm{CD} 8^{+} \mathrm{T}$ cell memory and tested for protective efficacy.

It must be remembered that many factors need to be considered for vaccine design to induce the optimal type of immunity for a pathogen. Further ways of optimising vaccines will be addressed in chapter 4 but include: optimisation of antigen, adjuvant, immunogenicity, route and method of administration, and promotion of longterm immunity. Vaccine approaches have largely been developed empirically so the exact mechanisms governing their interactions with the immune system are yet to be fully defined. A deeper understanding of how different factors enhance or impede 
memory development will enable vaccinology to advance and develop truly rational vaccine design approaches.

\subsubsection{Vaccination against Tuberculosis}

The only commercially available vaccine for Tb is BCG, a live attenuated strain of $M$. bovis. BCG was first developed by Calmette and Guérin by passaging a virulent $M$. bovis strain isolated from a bovine mastitis sample 230 times through glycerinated bile potato medium, resulting in gene deletions and duplication and attenuation. BCG was first given to a human in 1921 (reviewed in [243]) and BCG has become since then the most widely administered vaccine globally.

Unfortunately, the effectiveness of BCG is inconsistent. BCG can effectively prevent forms of childhood $\mathrm{Tb}$ involving dissemination [244], probably due to its ability to control blood-borne bacilli [245]. However, a meta-analysis of literature estimates the effectiveness of the BCG vaccine in adults to be $50 \%$ [246]. The effectiveness varies substantially between studies included in the meta-analysis, ranging from $80 \%$ to no protection. Additionally, in some studies the effectiveness of BCG wanes over time [247], whereas in others similar levels of protection have been observed up to 60 years after vaccination [248].

There are several possible explanations for the variation in effectiveness between different BCG vaccinated populations. There are multiple substrains of BCG in use, due to passaging in different laboratories, which have diverged genetically and immunogenically from one another (reviewed in [243]). Different BCG substrains can confer different levels of protection against $\mathrm{Tb}$ [249]. Variation may also be a result of host genetics, with genetic elements prevalent in different populations contributing to the variable protection afforded by BCG. Moreover, the virulence of different strains of M. tuberculosis can vary and the virulence of the strain of $M$. tuberculosis endemic to a population could affect the effectiveness of the BCG vaccine.

Finally, there may be environmental factors that affect the effectiveness of the BCG vaccine. Populations that exhibit low BCG effectiveness are commonly in developing countries, which have greater exposure to helminth infections and environmental mycobacteria than populations in developed countries. Helminth infection at the time of BCG vaccination is hypothesised to result in either immunosuppression of the BCG immune response or skew the response to an 
unprotective Th2 profile [250]. Similar hypotheses have been suggested for the interaction between environmental mycobacteria and BCG vaccination [251]. BCG may be subject to immunosuppression if environmental mycobacteria have tolerised or skewed the immune response for antigens that are cross-reactive with BCG. Suboptimal APC priming after exposure to environmental mycobacteria [252] could result in Treg induction (section 1.4.3), or could prime a Th2 instead of Th1 immune response to conserved mycobacterial antigens, resulting in an unprotective immune response to $\mathrm{Tb}$ [252]. Alternatively, exposure to environmental mycobacteria may prime an immune response that reacts robustly to the BCG but clears the bacillus before it can establish protective immunity against $\mathrm{Tb}[253]$.

Since BCG fails to consistently protect against $\mathrm{Tb}$, significant effort has been directed towards developing new vaccine strategies against $\mathrm{Tb}$. At least twelve vaccines are moving towards or currently in clinical trials (reviewed in [254]). These include:

- A SecA2 deletional mutant of M. tuberculosis. SecA2 encodes a component of a virulence-associated secretion system that secretes superoxide dismutase A. Deletion attenuates the pathogen but promotes apoptosis of infected macrophages in vitro, enhancing $\mathrm{CD} 8^{+} \mathrm{T}$ cells priming in vivo and making it more protective than BCG in mouse and guinea pig models [181].

- Recombinant BCG that expresses the listeriolysin O protein from Listeria monocytogenes. The listeriolysin $\mathrm{O}$ protein forms pores in the phagosomal membrane, giving mycobacterial protein access to the MHC I processing pathway, permitting strong $\mathrm{CD}^{+} \mathrm{T}$ cell priming [180]. This vaccine exhibited better protection against virulent $M$. tuberculosis than the BCG vaccine in a murine model [180].

- A modified vaccinia virus Ankara (MVA) viral vector containing Antigen 85A, a mycobacterial antigen (MVA-85A) [255].

Administration of this construct can boost mycobacterial antigen-specific immune responses in subjects primed with the BCG vaccine, and boosting is not affected by the time elapsed between priming and boosting [256].

Therefore, a variety of new vaccine strategies are being developed for Tb. While all possess a rational immunological basis, truly rational vaccine design for $\mathrm{Tb}$ is not 
feasible due to gaps in knowledge between Tb immunology, protective immunity and vaccinology.

A final consideration is that neither BCG vaccination nor prior M. tuberculosis infection treated with antibiotics enables the host to eliminate the infection, however they enable earlier control of the infection [257]. Since natural immunity against $\mathrm{Tb}$ infection does not eliminate infection, we must question whether natural immunity is what we want to replicate through $\mathrm{Tb}$ vaccination.

\section{$1.4 T$ regulatory cells}

\subsubsection{The re-discovery of $\mathrm{T}$ regulatory cells}

The concept of a lymphocyte that suppresses other lymphocytes was first proposed in the early 1970s [258, 259]. These cells were known as suppressor T cells but an inability to identify factors that mediated their mechanism of action or clone the cells themselves led to the demise of this area of research [260]. However, in the mid-1990s this concept was revived [261] and has led to a plethora of research on suppressive T cells, now known as Tregs.

The key observation that implied the existence of a suppressive $T$ cell subset was that murine neonates thymectomised before day 3 post-birth developed symptoms of autoimmunity, while neonates thymectomised after this time point did not develop these complications $[262,263]$. It was hypothesised that a distinct $\mathrm{T}$ cell subset leaving the thymus around day 3 post-birth was responsible for suppressing the immune response and preventing autoimmunity. Subsequently, cell surface markers for this $\mathrm{T}$ cell subset were identified, initially relying on CD4 [262], CD25 [261] and CD45RA [264]. Now many more markers, both on the cell surface and inside the cell are used to define the different subsets of Tregs. The first type of Treg defined in 1995 [261] is now known as the natural or intrinsic Treg, described in section 1.4.2, and the natural Treg subset is a major focus of this thesis. In addition, there are other suppressive $\mathrm{T}$ cells in the periphery that are induced in certain conditions and are collectively known as induced or adaptive Tregs, outlined in section 1.4.3. 


\subsubsection{Natural T regulatory cells}

\subsubsection{Mechanism of development}

Two models have been suggested for selection of natural Tregs in the thymus. Developing thymocytes undergo both positive and negative selection (reviewed in [265]). Positive selection ensures that the specific TCR on a thymocyte can engage MHC/self-peptide complex and deliver a survival signal. If this signal is not received, the thymocyte dies of neglect. Negative selection ensures that the affinity of the interaction with $\mathrm{MHC} /$ self-peptide complex is not too high. If thymocytes with a very high affinity TCR for MHC/self-peptide complex escaped the thymus, they could be auto-reactive and initiate autoimmunity in the periphery. Thymocytes with very high affinity TCRs are deleted due to negative selection. Therefore, thymocytes with low affinity TCRs for MHC/self-peptide complex survive to emerge as single positive CD4 or CD8 T cells.

It was initially hypothesised that natural Tregs are selected using MHC and TCR signals similar to other T cells, but using different signalling thresholds [266, 267]. It was thought that natural Tregs develop from thymocytes with TCRs of intermediate affinity for the MHC II/self-peptide complex. A higher TCR signal compared to conventional T cells was thought to trigger the development of the Treg suppressive phenotype.

However, a more recent model suggests that thymocytes require a specific signal to commit to the Treg lineage, either from an unidentified invariant signal or selection on an unconventional TCR ligand [268]. The precise mechanism of natural Treg selection in the thymus is undefined, as are the antigen-presenting cell subsets required for their selection, but it is important to note that while natural Tregs may use selfpeptide for selection [269], Tregs in the periphery possess a diverse TCR repertoire [266].

\subsubsection{Cell surface markers}

Natural Tregs emerge from the thymus as $\mathrm{CD}^{+} \mathrm{T}$ cells (Fig. 1.5) of which the majority express high levels of CD25 [261], CD152 [270] and glucocorticoid induced TNF 
receptor (GITR) [271] but low CD127 [272, 273] on the cell surface. Natural Tregs acquire CD25 expression at the CD4 single positive stage of thymocyte development [274]. After TCR engagement, Tregs can either act in the lymphoid tissue to inhibit effector $\mathrm{T}$ cell development or in the periphery at the site of inflammation. A subpopulation of Tregs express CCR7 and these inhibit proliferation in the lymph nodes draining the site of inflammation [275]. Another sub-population of Tregs express high levels of adhesion molecules, such as CD103, and chemokine receptors, such as CCR5, and these promote extravasation and homing of Tregs to inflammation sites [276]. By virtue of the fact that natural Tregs are likely to be selected for a higher affinity for a self-peptide/MHC complex in the thymus, they should again encounter this selfpeptide/MHC complex in the periphery, and are thought to undergo continual low-level TCR stimulation. This may explain why many cell surface markers for Tregs are also markers of $\mathrm{T}$ cell activation, giving Tregs a semi-activated phenotype, even in naïve mice.

\subsubsection{Intracellular markers}

A significant hindrance for the study of natural Tregs was the lack of a marker specific for Tregs. While the majority of Tregs express CD25, GITR or CD152 in a naïve mouse, expression of these markers does not correlate totally with suppressive activity. Furthermore, identifying Tregs during an active immune response was exceedingly problematic, as naïve $\mathrm{T}$ cells upregulate activation markers once activated, leading to confusion between activated $\mathrm{T}$ cells and Tregs. Therefore, the recent identification of forkhead box P3 transcription factor (Foxp3), otherwise known as scurfin, as a highly specific marker for the suppressive Treg phenotype was a significant advance. Foxp3 is selectively produced in $\mathrm{CD} 4{ }^{+} \mathrm{CD} 25^{+} \mathrm{T}$ cells $[277,278]$, and its effects in vivo are illustrated in both a natural mouse Foxp 3 mutant, the scurfy mouse, and a human condition affecting Foxp 3 called immune dysregulation, polyendocrinopathy, enteropathy, X-linked syndrome (IPEX). Both the scurfy mouse and IPEX patients exhibit autoimmunity, immune dysregulation and a deficiency in Tregs [279, 280].

Foxp3 expression correlates very specifically with the Treg suppressive phenotype, and it has been postulated to be the "master regulator" of the suppressive phenotype [281, 282]. There is considerable homology of the Foxp3 gene across species, which suggests conserved function [281]. The gene is located on the $\mathrm{X}$ 
chromosome, which leads to disease in the event of Foxp 3 deficiency in hemizygous males and in homozygous, but not heterozygous, females [283]. It has been demonstrated with chromatin immunoprecipitation and mouse genome array tiling that there are binding regions for Foxp3 in approximately 700 genes [284]. When compared to mRNA microarray data [285], only $10 \%$ of the genes whose expression is altered in Tregs bind directly to Foxp3, suggesting Foxp3 initiates a genetic program in Tregs, which may be carried out by other transcription factors. There is evidence that Foxp3 can act both as a repressor and as an activator and this may depend on histone modifications around its binding site $[284,286]$. Furthermore, it has become apparent that Foxp3 interacts with other transcription factors such as NFAT and NF-кB [287] and can override the Th17-promoting effect of ROR $\gamma \mathrm{t}$ [288]. An internal balance between the amounts of these transcription factors is thought to regulate the programming of an individual cell and whether it becomes an effector T cell or a Treg.

\subsubsection{Mechanism of action}

Several mechanisms of action have been suggested for natural Tregs (Fig. 1.5), but it is known that natural Tregs need TCR stimulation [289, 290] and IL-2 signalling [291] to activate their suppressive mechanisms. Once activated, $\mathrm{CD} 4{ }^{+} \mathrm{CD} 25^{+} \mathrm{T}$ cells can suppress proliferation of $\mathrm{CD}^{+}$[289] and $\mathrm{CD}^{+}$[292] responder T cells in vitro and, once stimulated with their specific antigen, they can suppress in an antigen-non-specific manner. In vitro, the failure of the responder population to proliferate correlates with a decrease in IL-2 transcription and thereafter production by responder T cells [289, 291]. IL-2 feeds an autocrine loop necessary for maximal proliferation and full activation of responder $\mathrm{T}$ cell effector mechanisms, such as cytokine production and CTL activity. Furthermore, cell populations dependent on full activation of responder T cells are also affected by Treg suppression, such as B cells [293, 294], macrophages and NK T cells $[295,296]$.

The mechanism by which natural Tregs inhibit IL-2 production in responder cells, and whether IL-2 inhibition acts alone or in concert with other mechanisms to result in suppression, is not understood. A number of Treg suppressive mechanisms have been characterised, and each predominate in different in vitro and in vivo scenarios. It is known that responder T cells and Tregs need to be in close proximity to each other for suppression to occur in vitro [290]. However, whether this extends to in 
vivo conditions and whether Tregs act through APCs interacting with responders $\mathrm{T}$ cells or directly on responder $\mathrm{T}$ cells, is uncertain. Thus, proposed mechanisms of Treg suppression are divided into indirect or direct mechanisms.

Models of indirect Treg suppression suggest the APC mediates suppression by Tregs of the responder T cells. One indirect model suggests that a Treg will direct an APC presenting antigen to downregulate co-stimulatory signals [297]. Subsequent antigen presentation by that APC to responder T cells would result in inefficient responder T cell activation and failure to induce IL-2 production and proliferation. However, it is possible that Tregs simply use APCs as a platform to bring them in close proximity to responder T cells undergoing TCR stimulation. As a result, Treg suppressive mechanisms would be enhanced by the presence of APC but Tregs would act on responder $\mathrm{T}$ cells through direct mechanisms.

Models of direct Treg suppression mechanisms refer to a direct interaction between the Treg and the responder T cell. In an APC-free system, using peptide loaded onto MHC I tetramers to initiate $\mathrm{CD} 8^{+} \mathrm{T}$ cell activation, the addition of $\mathrm{CD} 4^{+} \mathrm{CD} 25^{+}$ Tregs still suppressed $\mathrm{CD}^{+} \mathrm{T}$ cell proliferation and cytokine production [292]. Therefore, in in vitro cultures, Tregs can signal to and suppress responder T cells directly.

Many effector molecules have been postulated to mediate the indirect and direct suppressive mechanisms of Tregs.

CD25 is the alpha chain of the high affinity IL-2 receptor and is expressed at high levels on natural Tregs. Tregs need IL-2 from responder T cells early after TCR signalling to activate the Treg suppressive function and, in the periphery, IL-2 is required for the homeostasis of Tregs [298, 299]. High CD25 expression may give Tregs a competitive advantage over responder T cells for IL-2, which could be responsible for the suppressive phenotype of Tregs. It has recently been demonstrated in vitro that uptake of IL-2 by Tregs can suppress $\mathrm{CD} 4^{+}$responder $\mathrm{T}$ cells by depriving them of IL-2 early during proliferation, resulting in apoptosis of the responder cells [300].

CD152 is also expressed at high levels on natural Tregs and has also been implicated in the suppressive activity of Treg. CD152 is expressed late after activation on T cells and binds to CD80 and CD86 on APCs. Binding of CD152 and CD80 or 
CD86 initiates reverse signalling, delivering negative signals to the APC and the T cell. This negative signalling ordinarily suppresses proliferation and as CD152 is upregulated on $\mathrm{T}$ cells late after activation, it is thought to prevent over-activation of the immune response (reviewed in [301]). Administration of anti-CD152 mAb or its Fab fragment inhibits Treg-mediated suppression in vitro [302] and administration of anti-CD152 $\mathrm{mAb}$ contributes to autoimmunity in vivo [270]. However, it is contentious as to how anti-CD152 mAbs act in vivo (reviewed in [303]). They may cross-link CD152 on the Treg and deliver a negative signal to inhibit suppressive mechanisms of Tregs. AntiCD152 mAbs could also bind to and block CD152 on Tregs, preventing it from binding to CD80 and CD86 on APCs and thereby preventing Tregs from delivering a suppressive signal to the APCs.

Tregs also express and secrete cytokines that can mediate suppression, such as IL-10 and TGF- $\beta$. In vitro studies using neutralising antibodies against IL-10 or TGF- $\beta$ or Tregs from IL-10-deficient mice, demonstrate that Tregs can still efficiently suppress $\mathrm{CD}^{+} \mathrm{CD} 25^{-} \mathrm{T}$ cell proliferation in the absence of these soluble factors [289]. However, while soluble cytokine may not be vital for Treg suppression in vitro, membrane-bound cytokine could mediate the effects of Tregs. Tregs stain extracellularly with anti-TGF- $\beta$ polyclonal antibody after in vitro activation and high concentrations of anti-TGF- $\beta$ neutralising antibody can inhibit suppression of $\mathrm{CD} 4^{+} \mathrm{CD} 25^{-}$responder T cells [304]. These observations suggest that Tregs express membrane-bound TGF- $\beta$ in its inactive form and, upon encounter with the target T cell, membrane-bound TGF- $\beta$ is converted to active TGF- $\beta$ at the immunological synapse. Only high amounts of neutralising antibody could penetrate the synapse between the Treg and the target cell, explaining the dose-dependent affects of TGF- $\beta$ neutralising antibody.

There is confusion about the importance of cytokines for suppression by Tregs in vitro, but many in vivo models indicate that IL-10 and TGF- $\beta$ are critical. In the wellcharacterised murine Leishmania major infection model, administration of IL-10 neutralising antibody abrogates Treg suppression [305]. In a murine model of type I diabetes, expression of a dominant negative TGF- $\beta$ receptor on responder $\mathrm{CD} 8^{+} \mathrm{T}$ cells meant these lymphocytes could no longer be suppressed by Tregs expressing TGF- $\beta$, leading to disease progression $[286,306]$.

Finally, Tregs can develop cytolytic activity in some situations and inhibit immune responses by directly killing responder lymphocytes or APCs. Human CD4 
CD25 $5^{+}$Tregs activated with anti-CD3 and anti-CD46 mAbs, expressed high levels of granzyme A and human induced Tregs expressed high levels of granzyme B [307]. Both types of Treg were effective at killing a range of leukocytes in vitro through a perforindependent mechanism. Supporting the human studies, murine natural Tregs upregulate granzyme B after activation and Tregs from granzyme-B-deficient mice are not suppressive [308], although, in contrast to human studies, Tregs from perforin-deficient mice retain their suppressive activity [308]. Thus, Tregs can mediate suppression through cytolytic activity, but it remains uncertain what the target cells of cytolysis are (the APC or the responder T cells), and which molecules mediate these effects in vivo [309].

Overall, there are several proposed mechanisms of suppression for Tregs. There is variation between in vitro and in vivo models of suppression but different mechanisms may be important in different states of disease or infection. Therefore, there may be a combination of several suppressive mechanisms that result in the local or systemic environment of suppression in vivo. Of particular interest for my study is whether natural Treg mechanisms are activated and if natural Tregs exert their suppressive effects during mycobacterial infection or during vaccination against $\mathrm{Tb}$.

\subsubsection{Induced T regulatory cells}

$\mathrm{T}$ cells with suppressive abilities can also arise in the periphery from conventional $\mathrm{T}$ cells and these induced or adaptive Tregs are important for immune control during certain infections. There are several types of induced Treg and there are also several mechanisms of induction proposed that are outlined below, but all need an initial TCR signal. If TCR triggering is optimal and co-stimulatory signals are effectively induced on APCs this results in normal effector T cell development. However, if TCR triggering is sub-optimal or co-stimulatory signals are lacking then induced Tregs can develop. It is interesting to note that natural Tregs can promote the induction of Tregs. Natural Tregs can affect the co-stimulatory capacity of APCs and so can cause sub-optimal responder T cell activation [297]. Furthermore, natural Tregs produce cytokines, such as IL-10 and TGF- $\beta$, that can induce Foxp 3 expression and/or a suppressive Treg phenotype, as discussed below. 


\subsubsection{Low-dose antigen induced Tregs}

Low amounts of antigen can induce Foxp 3 expression in $\mathrm{CD}^{+} \mathrm{T}$ cells and these cells become functional suppressors (Fig. 1.5). Delivery of low amounts of antigen to DCs by coupling antigen to an anti-DEC205 mAb led to development of $\mathrm{CD}^{+}{ }^{+} \mathrm{Foxp} 3^{+} \mathrm{T}$ cells, whose suppressive function was dependent on TGF- $\beta$ [310]. Low-dose antigen induction of Tregs is also relevant to in vivo autoimmunity models, where tolerance is induced by low-dose antigen exposure [311]. Therefore, a subset of inducible Tregs can arise in situations of low-dose antigen.

\subsubsection{IL-10 induced Tregs}

Tregs that develop in the presence of IL-10 are termed Tr1 cells (Fig. 1.5). These cells were initially generated by a combination of vitamin D, antigen and immunosuppressants [312]. It was subsequently demonstrated that, if IL-10 is present during TCR triggering of $\mathrm{CD}^{+}{ }^{+} \mathrm{T}$ cells, then Tregs develop that produce large amounts of IL-10, as well as other cytokines such as TGF- $\beta$, IFN- $\gamma$ and IL-5 [313]. Some suppression by $\operatorname{Tr} 1$ cells is likely to be mediated by IL-10 production but they may also utilise IL-10-independent mechanisms [314]. Unlike other induced Treg populations, homogeneous populations of $\operatorname{Tr} 1$ cells generated in vitro and in vivo do not produce Foxp3 [314]. IL-10-induced Tregs have been shown to develop during the course of some infections. For example, in Bordetella pertussis infection, the filamentous haemagglutinin antigen induces IL-10 production by DCs and macrophages but inhibits IL-12 production, leading to development of IL-10 producing antigen-specific Tr1 cells [315]. Therefore, IL-10-induced Tr1 cells may arise under specific conditions during infection.

\subsubsection{TGF- $\beta$ induced Tregs}

Another induced Treg subtype develops in the presence of TGF- $\beta$ and is termed Th3 (Fig. 1.5). If TGF- $\beta$ is present during TCR signalling, CD25 and Foxp3 production can be induced in $\mathrm{CD} 4^{+} \mathrm{CD} 25^{-} \mathrm{T}$ cells [316]. These cells are highly suppressive and have increased cell-surface expression of CD25 and CD152. Studies of human T cells differentiated in these conditions indicate that suppression is contact dependent [317]. 
However, the presence of TGF- $\beta$ during $\mathrm{CD}^{+}{ }^{+} \mathrm{T}$ cell TCR triggering does not guarantee induction of a Treg. In vitro studies have demonstrated that the suppressive Treg phenotype is induced in the presence of TGF- $\beta$ and IL-2, which results in Foxp3 expression [79, 318, 319]. However, the inflammatory Th17 phenotype can develop in the presence of TGF- $\beta$ and IL- 6 , and is stabilised by IL-23 signalling [79, 80]. Within the same cell there can be induction of Foxp3 and ROR $\gamma$ t, the transcription factor responsible for the Th17 phenotype [288]. Therefore, in vivo where IL-2, TGF- $\beta$ and IL-6 are all present during TCR signalling, for example in the mucosa, a diverse spectrum of cellular phenotypes can develop, ranging from the induced Treg through to the Th17 phenotype. 


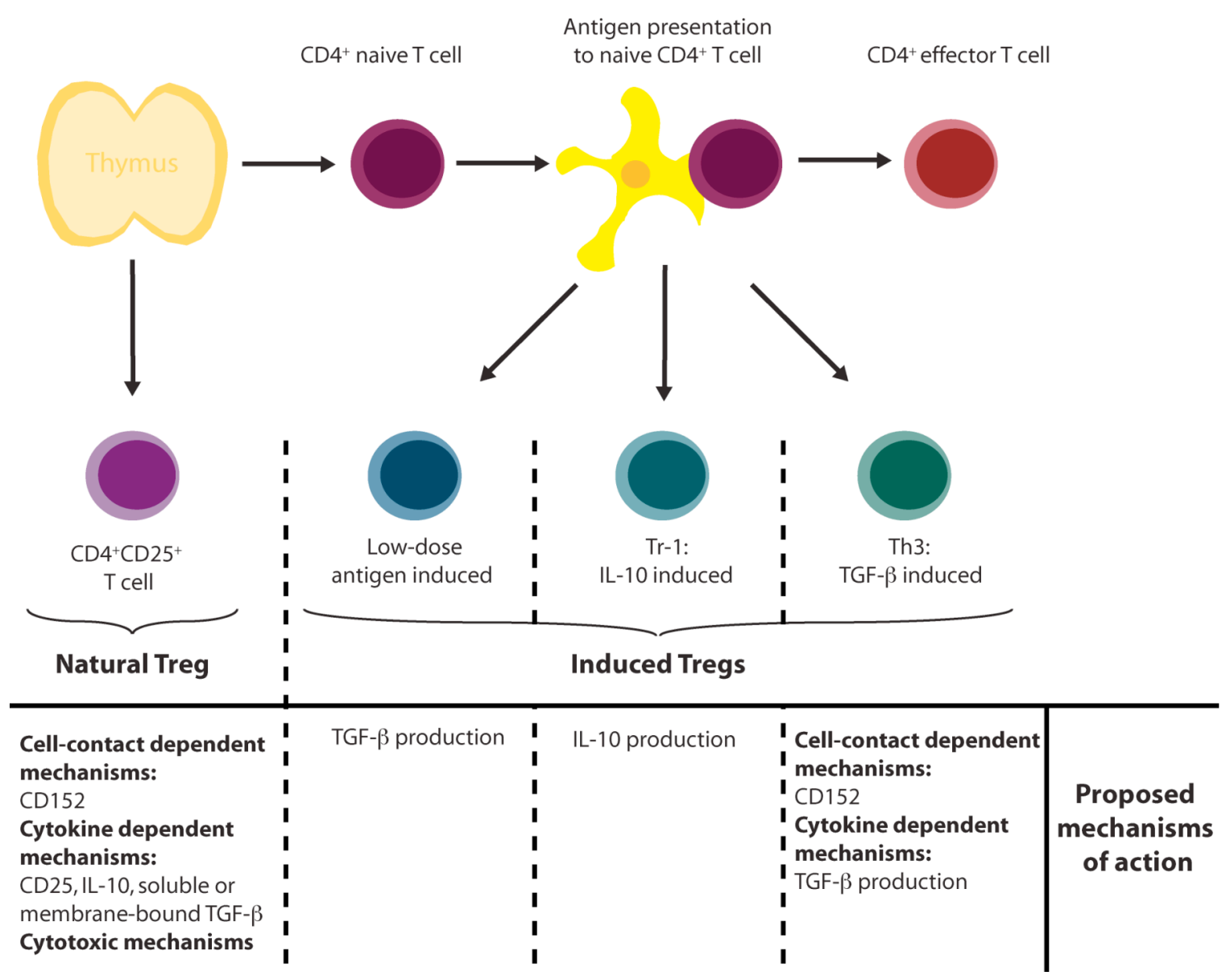

Figure 1.5: Schematic outlining Treg subsets and mechanisms of action.

Natural $\mathrm{CD} 4{ }^{+} \mathrm{CD} 25^{+}$Tregs are derived directly from the thymus and suppress by cell-contact dependent, cytokine dependent or cytotoxic mechanisms. Induced Tregs arise in the periphery after antigen presentation to naïve $\mathrm{CD} 4^{+} \mathrm{T}$ cells in sub-optimal conditions. The subtypes of induced Tregs are: low-dose antigen induced Tregs that typically suppress through TGF- $\beta$; Tr-1 Tregs that are induced by the presence of IL-10 during antigen presentation and typically suppress through IL-10; and Th3 Tregs that are induced by the presence of TGF- $\beta$ during antigen presentation and typically suppress by cell-contact or cytokine dependent mechanisms. If antigen presentation is optimal, activated effector T cells develop. 


\subsubsection{Non-CD4 ${ }^{+} \mathrm{T}$ regulatory cells}

In addition to $\mathrm{CD} 4^{+}$Tregs there are other subsets of lymphocytes that exhibit suppressive phenotypes. These have similar mechanisms of induction and suppression to $\mathrm{CD}^{+}$Tregs.

Suppressive $\mathrm{CD}^{+} \mathrm{T}$ cells have been found in several models. In a murine model of autoimmunity, transfer of $\mathrm{CD} 8^{+} \mathrm{T}$ cells from recovered mice protects recipient mice from autoimmunity [320]. Protection was mediated by $\mathrm{CD}^{+} \mathrm{T}$ cells that recognised self-peptide presented on auto-reactive $\mathrm{CD} 4^{+} \mathrm{T}$ cells and either directly killed autoreactive cells or suppress their activation by cytokine production [320]. Furthermore, antigen-presentation in vitro by IL-10-secreting plasmacytoid DCs induced a suppressive phenotype in $\mathrm{CD}^{+} \mathrm{T}$ cells, similar to the $\mathrm{Tr} 1$ subtype of $\mathrm{CD} 4^{+}$Tregs [321].

$\mathrm{CD}^{-} \mathrm{CD}^{-} \mathrm{T}$ cells can also exhibit suppressive qualities. Human $\mathrm{CD}^{-} \mathrm{CD}^{-} \mathrm{T}$ cells can be highly suppressive and cytotoxic to syngeneic $\mathrm{T}$ cells bearing the same TCR [322]. The origin of $\mathrm{CD}^{-} \mathrm{CD}^{-} \mathrm{T}$ cells is uncertain but it has been recently demonstrated that $\mathrm{CD} 4^{+} \mathrm{T}$ cells can convert to highly suppressive, cytotoxic $\mathrm{CD} 4^{-} \mathrm{CD} 8^{-}$ $\mathrm{T}$ cells during the course of $\mathrm{CD}^{+} \mathrm{T}$ cell activation and differentiation [323].

\subsubsection{Suppressing the suppressors}

Tregs mediate a feedback loop on the immune response. Once activated, they prevent immune responses against self-antigen from accelerating into autoimmunity and responses against foreign antigen from becoming excessive and causing immunopathology. Sometimes Tregs need to be controlled to enable an appropriate immune response to develop, so immune mechanisms exist that suppress the suppressive functions of Tregs.

Signalling through TLRs on APCs or Tregs can inhibit activation of Tregs or their suppressive functions. Ligation of TLRs on APCs induces secretion of cytokines $[324,325]$ that render responder T cells resistant to the suppressive effects of Tregs. TLR ligation on Tregs themselves can abrogate their suppressive functions. Addition of TLR2 ligand to Treg cultures promoted Treg expansion but abrogated their suppressive function [326] and knock-down of TLR 8 expression on human $\mathrm{CD} 4{ }^{+} \mathrm{CD} 25^{+}$Tregs 
resulted in increased suppressive function [327]. However, TLR ligation on Tregs can also promote suppressive functions. Addition of the TLR4 ligand, lipopolysaccharide (LPS), enhanced Treg activation and suppression function [328]. TLR signalling and activation of the APC can also upregulate co-stimulatory signal molecules such as GITR ligand (GITRL) and signalling through the GITR/GITL pathway can inhibit Treg activity (outlined in section 3.1). Therefore TLR signalling to Tregs modulates their function, either enhancing or inhibiting Treg activity.

In summary, there are several types of Tregs with different but overlapping phenotypes and mechanisms of action (Fig. 1.5). The role of a particular subset or subsets of Tregs varies depending on the environment during the induction and maintenance of an immune response [329]. Therefore, a number of factors contribute to either activating or suppressing Tregs subsets at various points during an immune response.

\subsection{Aims}

The broad aim of this thesis is to examine mechanisms of immune control exerted by $M$. tuberculosis. This thesis focuses on the role of Tregs, specifically natural $\mathrm{CD} 4{ }^{+} \mathrm{CD} 25^{+}$ Tregs, and whether these cells have been exploited by M. tuberculosis as an immunoevasive tactic.

As will be discussed in Chapter 3, Tregs have been shown in other chronic infections to suppress immune responses and immunopathology but promote pathogen survival $[305,315,330-335]$. There has been much speculation that Tregs similarly enable pathogen persistence during $\mathrm{Tb}$ infection.

Furthermore, Tregs have also been shown to inhibit vaccine-induced immune responses and reduce protective efficacy of vaccines [336-339]. Since there is no consistent vaccine against $\mathrm{Tb}$, it is also speculated that Tregs reduce $\mathrm{Tb}$ vaccine efficacy and improved vaccine strategies against $\mathrm{Tb}$ are of the upmost importance, as will be outlined in Chapters 4 and 5. Thus, the aims of this thesis can be summarised as follows:

1. To develop a protocol to target and inactivate Tregs in vivo in a murine model of mycobacterial infection or vaccination against $\mathrm{Tb}$. 
2. To evaluate whether Treg inactivation prior to mycobacterial infection, using the protocol defined in Aim 1:

- Enhances the acute immune response

- Decreases tissue bacterial numbers

- Increases tissue pathology

3. To develop a novel DNA vaccine for Tb called pKUN85A, and perform preliminary testing to evaluate vaccine efficacy.

4. To evaluate whether Treg inactivation prior to vaccination against $\mathrm{Tb}$, using several vaccines including $\mathrm{pKUN85A}$ :

- Enhances the primary immune response to vaccination

- Enhances the secondary immune response to infectious challenge

- Enhances vaccine protective efficacy against infectious challenge

5. To create a model to examine the role of Tregs during vaccination against $\mathrm{Tb}$.

When these studies were commenced, the role of Tregs during mycobacterial immunity had not been explored using an in vivo model. It was widely speculated that modulation of Treg activity during mycobacterial infection or vaccination could be used to enhance the efficacy of therapies and vaccines directed against $\mathrm{Tb}$. Therefore, these studies formally examine an issue that has invited much interest; does Treg-mediated immunosuppression aid immunoevasion by $\mathrm{Tb}$ or inhibit the efficacy of vaccines directed against $\mathrm{Tb}$ ? 


\subsection{Mice}

Inbred C57BL/6 mice were bred and housed in a conventional animal facility at the Malaghan Institute of Medical Research Biomedical Research Unit, Wellington, New Zealand. Mice infected with M. tuberculosis or M. bovis were housed at the Animal Containment Facility, AgResearch Wallaceville, Upper Hutt, New Zealand. All animal experimental protocols used in this thesis were approved by the Victoria University of Wellington Animal Ethics Committee and the AgResearch Wallaceville Animal Ethics Committee. At the commencement of each experiment, mice were aged between 6 and 12 weeks and mice within each experiment were age- and sex-matched.

\subsection{Antibody preparation and in vivo treatments}

B cell hybridoma clones were cultured to produce mAbs in-house. The clones PC61, DTA-1 and 5F-1 were cultured to produce mAbs for Treg modulation and the clones $2.4 \mathrm{G} 2,2 \mathrm{C} 11$ and 37.51 were cultured to produce mAbs for lymphocyte restimulation or staining for flow cytometry.

One $\mathrm{mL}$ aliquots of the cell-lines at $2 \times 10^{6}$ cells $/ \mathrm{mL}$ were stored in Freezing Media (Appendix 8.3) in liquid nitrogen before being rapidly defrosted with $10 \mathrm{~mL}$ of $37{ }^{\circ} \mathrm{C} 10 \%$ complete Iscove's Modified Dulbecco’s Medium (cIMDM) (Appendix 8.3) and grown in a Falcon ${ }^{\mathrm{TM}}$ T25 tissue culture flask (BD Biosciences Labware, Bedford, MA, USA) until confluent. Cells were re-plated with $5 \%$ cIMDM (Appendix 8.3) in several Falcon ${ }^{\mathrm{TM}}$ T75 tissue culture flasks (BD Biosciences Labware) and cells were expanded to acquire at least $3 \times 10^{7}$ cells.

The $3 \times 10^{7}$ cells were used to seed a CELLine CL1000 flask (INTEGRA Biosciences AG, Chur, Switzerland). The CL1000 flask consists of an upper compartment and a lower compartment separated by a $10 \mathrm{kDa}$ semi-permeable membrane, which allows exchange of metabolic waste while retaining the mAb within the lower compartment. A silicon membrane on the bottom of the lower compartment permits gas exchange. Cells were suspended in $15 \mathrm{~mL}$ of $20 \%$ cIMDM (Appendix 8.3) in the lower compartment of the flask. The upper compartment of the flask was filled 
with $500 \mathrm{~mL}$ of $1 \%$ cIMDM (Appendix 8.3). Every 2-4 days, the cells were harvested from the lower compartment and viable cells were counted by trypan blue exclusion (section 2.4.1). After viable cell counts were determined, $5 \times 10^{7}$ cells were removed, volume was adjusted to $15 \mathrm{~mL}$ with $20 \%$ cIMDM and these cells were used to re-seed the lower compartment of the flask. The $1 \%$ cIMDM in the upper compartment was also replaced and the flask was incubated at $37{ }^{\circ} \mathrm{C}$ with $5 \% \mathrm{CO}_{2}$.

The remaining suspension of cells in media from the lower compartment was centrifuged at $800 \mathrm{x}$ g for $15 \mathrm{~min}$ to pellet cells and debris. The supernatant, containing $\mathrm{mAb}$ secreted by the hybridoma, was harvested and kept either at $4{ }^{\circ} \mathrm{C}$ for short-term storage or at $-20{ }^{\circ} \mathrm{C}$ for long-term storage, until sufficient supernatant had been harvested for purification of antibodies.

Once approximately $100 \mathrm{~mL}$ or more of supernatant had been harvested, the supernatant was subjected to low-pressure chromatography for mAb purification. Supernatant was prepared for purification by centrifuging again at $4000 \mathrm{x}$ g at $4{ }^{\circ} \mathrm{C}$ for $30 \mathrm{~min}$ followed by filtration through a Serum $0.2 \mu \mathrm{m}$ Acrodisc filter (Pall Life Sciences, Pall Corp., Cornwall, UK) to remove residual cell debris. A $5 \mathrm{~mL} \mathrm{Hi}$-Trap $^{\mathrm{TM}}$ Protein G HP sepharose column (GE Healthcare Bio-Sciences AB, Uppsala, Sweden) was used to bind antibodies and these were stored in $20 \%$ ethanol (BDH, Poole, UK) when not in use. BioLogic low-pressure chromatography apparatus with a BioFrac Fraction Collector and LP Data View Software (all from BioRad, Hercules, CA, USA) were used for purification. The flow rate was set at $1.5 \mathrm{~mL} / \mathrm{min}$ and the column was initially washed with $20 \%$ ethanol for 10 minutes (min), followed by degassed, distilled (d) $\mathrm{H}_{2} 0$ for $10 \mathrm{~min}$. The UV spectrophotometer was zeroed and degassed $\mathrm{dH}_{2} \mathrm{O}$ was run for a further 5 min to establish a baseline zero reading. The column was washed with Purification Wash Buffer (Appendix 8.3) for $20 \mathrm{~min}$ to equilibrate the $\mathrm{pH}$ of the column to 8.0. After equilibration, supernatant was run through the column to bind the $\mathrm{mAb}$ to the column. The column was washed with purification wash buffer to remove other proteins present in the supernatant until absorbance readings returned to baseline. The antibody was eluted into $1.5 \mathrm{~mL}$ fractions by passing Purification Elution Buffer (Appendix 8.3) at $\mathrm{pH} 2.7$ through the column. The $1.5 \mathrm{~mL}$ fractions were treated with 
$37.5 \mu \mathrm{L}$ of Neutralisation/Stabilisation Buffer (Appendix 8.3) at $\mathrm{pH} 9.0$ to neutralise the $\mathrm{pH}$ of the solution containing the mAb.

Fractions containing mAb were identified by UV spectrophotometry at $280 \mathrm{~nm}$ and pooled fractions were dialysed in $1 \mathrm{~L}$ of phosphate buffered saline (PBS) (Appendix 8.3) using a Slide-A-Lyzer $10 \mathrm{kDa}$ dialysis cassette (Pierce, Rockford, IL, USA). PBS was changed once and the dialysed solution containing $\mathrm{mAb}$ was filtered using a Serum $0.2 \mu \mathrm{m}$ Acrodisc filter to sterilise. One $\mathrm{mL}$ aliquots of $\mathrm{mAb}$ were prepared and stored at $-80{ }^{\circ} \mathrm{C}$ until use. Final mAb concentration was determined using 1:10 dilutions of dialysed, filtered solution with UV spectrophotometry at $280 \mathrm{~nm}$ and the equation [340]:

Protein $(\mathrm{mg} / \mathrm{mL})=\mathrm{A}_{280} \times 0.74$ (for Rat $\left.\operatorname{IgG}\right) \times$ dilution factor $(10)$

\section{In vivo}

To assess the effect of Treg inactivation on acute mycobacterial infection or vaccination, mice received $100 \mu \mathrm{g}$ of anti-CD25 mAb (clone PC61) in $200 \mu 1$ of PBS by intraperitoneal (i.p.) injection to inactivate Tregs. Control mice received $100 \mu \mathrm{g}$ of rat IgG (Sigma-Aldrich, St Louis, MO, USA) in $200 \mu$ of PBS by i.p. injection. Inactivation was confirmed by assessing the proportion of $\mathrm{CD} 4{ }^{+} \mathrm{CD} 25^{+}$lymphocytes in peripheral blood samples by flow cytometry two days after antibody administration (see sections 2.4.1 and 2.4.3), unless otherwise stated.

To assess the effect of Treg inactivation or modulation of GITR/GITRL signaling on chronic mycobacterial infection, mice were administered mAbs in $200 \mu 1$ of PBS by i.p. injection at weeks 6 and 8 after infection. Mice received doses of either $500 \mu \mathrm{g}$ of rat IgG (Sigma-Aldrich), $100 \mu \mathrm{g}$ of anti-CD25 mAb (clone PC61), $500 \mu \mathrm{g}$ of anti-GITR (clone DTA-1), $500 \mu \mathrm{g}$ of anti-GITRL (clone 5F-1) or both $100 \mu \mathrm{g}$ of antiCD25 mAb (clone PC61) and $500 \mu \mathrm{g}$ of anti-GITR (clone DTA-1). 


\subsection{Infections and vaccinations}

M. bovis BCG Pasteur strain 1173P2 stocks were prepared by growing BCG in Tween Albumin Broth (Appendix 8.3). Logarithmically growing BCG cultures were harvested and pooled. Cultures were centrifuged at $5000 \mathrm{x}$ g for $30 \mathrm{~min}$ at $4{ }^{\circ} \mathrm{C}$ and washed twice in PBS/0.05\% Tween 80 (Appendix 8.3) before being resuspended in PBS/0.05\% Tween 80 . Aliquots were prepared and frozen at $-80{ }^{\circ} \mathrm{C}$ for future use. One aliquot was defrosted and ten-fold serial dilutions were plated onto Middlebrook 7H11 agar (Appendix 8.3) to determine CFU/mL. Colonies were counted after 2-3 weeks of incubation at $37^{\circ} \mathrm{C}$. Undiluted aliquots were also plated on blood agar and incubated for 5 days at $37^{\circ} \mathrm{C}$ to confirm that the batch of BCG was free from other bacterial contaminants.

To prepare BCG for infections, an aliquot of M. bovis BCG Pasteur strain 1173P2 was thawed at $4{ }^{\circ} \mathrm{C}$. BCG was sonicated 3 times for 30 seconds (sec) using an Ultrasonic Sonicator (Unisonics Pty. Ltd., Sydney, Australia) with 1 min rests between sonications on ice, before dilution in PBS. To facilitate i.n. BCG infection, mice were anaesthetised with an i.p. injection of $1 \mathrm{x}$ anaesthetic (Appendix 8.3) using a dose of 2.6 $\mathrm{mg}$ of xylazine and $87 \mathrm{mg}$ of ketamine per $\mathrm{kg}$ of body weight. Anaesthetised mice were placed on their backs and challenged with a $50 \mu$ inoculum containing $1 \times 10^{4} \mathrm{CFU}$ of BCG, which was slowly pipetted onto their noses and inhaled.

\section{M. tuberculosis M. bovis}

M. tuberculosis Erdman strain (originally from Prof Ian M. Orme, Colorado State University, CO, USA) and the M. bovis WAg201 strain [341] were grown from a lowpassage seed lot in Tween Albumin Broth [342] to early mid-log phase. Cultures were divided into aliquots and frozen at $-70^{\circ} \mathrm{C}$, until use. To infect mice by low-dose aerosol exposure, diluted thawed stock was administered using a Madison chamber aerosol generation device (University of Wisconsin, Madison, WI, USA) calibrated to deliver approximately 50 bacteria into the lungs. Aerosol infections, maintenance and 
manipulation of infected mice were performed under strict isolation conditions in the AgResearch Wallaceville Biohazard Facility.

To vaccinate mice with BCG, an aliquot of $M$. bovis BCG Pasteur strain 1173P2 was thawed at $4{ }^{\circ} \mathrm{C}$. BCG was sonicated 3 times for $30 \mathrm{sec}$ using an Ultrasonic Sonicator with 1 min rests between sonications on ice before dilution in PBS. Mice were vaccinated s.c. on the left flank with $200 \mu$ PBS containing 1 x $10^{6} \mathrm{CFU}$ of BCG.

To vaccinate mice with pcDNA-85A, purified plasmid (section 2.6.12) was prepared in PBS to deliver a $100 \mu \mathrm{g}$ dose of plasmid in $100 \mu \mathrm{L}$. To vaccinate mice with pKUN85A, purified plasmid from section 2.6.12 was prepared in PBS to deliver the indicated dose of plasmid in $100 \mu \mathrm{L}$. For experiments in chapter 4, 100, 25, 10 or $5 \mu \mathrm{g}$ doses of pKUN85A were used but, for experiments in chapter 5, just a $25 \mu \mathrm{g}$ dose of pKUN85A was used. Control mice were vaccinated with $100 \mu \mathrm{g}$ of pKUNrep6 prepared as indicated in section 2.6.12, resuspended in $100 \mu \mathrm{L}$ of PBS. DNA vaccines were delivered i.m. to the gluteal muscles of the hind limb, with approximately $50 \mu \mathrm{L}$ of the total dose administered to each hind limb of the mouse.

\subsection{Immunological techniques}

Tissues were harvested from animals at indicated times. If mice were euthanised for tissue harvest, this was performed primarily by cervical dislocation. If mediastinal lymph nodes were to be harvested, mice were euthanised by lethal anaesthetic so as to preserve the architecture of the pleural cavity and facilitate recovery of these lymph nodes. Mice were euthanised through lethal anaesthesia by i.p. administration of $1 \mathrm{x}$ 
anaesthetic using a dose of $10.4 \mathrm{mg}$ of xylazine and $348 \mathrm{mg}$ of ketamine per $\mathrm{kg}$ of body weight.

\subsubsection{Blood}

Blood was harvested from ear marked mice by warming them for approximately $5 \mathrm{~min}$ under a heat lamp before restraining mice and gently cutting across the tail vein on the dorsal side of the tail with a sterile scalpel blade. Approximately 10 drops of blood were collected directly into a $1 \mathrm{~mL}$ aliquot of chilled Alsevers Solution (Appendix 8.3). Pressure was applied to the cut site and, once blood-flow was stemmed, mice were returned to their cages.

The blood samples were stored on ice until samples could be processed. Samples were centrifuged at $400 \mathrm{x}$ g for $10 \mathrm{~min}$ at $4{ }^{\circ} \mathrm{C}$, supernatants removed and pellets were washed with $3 \mathrm{~mL}$ of $5 \%$ cIMDM. Red blood cells were then lysed by resuspending pellets in $3 \mathrm{~mL}$ of room temperature ACT Lysis Buffer (Appendix 8.3) and incubating for $8 \mathrm{~min}$. Samples were topped up with $10 \mathrm{~mL}$ of $5 \%$ cIMDM and centrifuged at $400 \mathrm{x}$ $\mathrm{g}$ for $10 \mathrm{~min}$ at $4{ }^{\circ} \mathrm{C}$. Pellets were resuspended in $200 \mu \mathrm{L}$ of Flow Cytometry Buffer (Appendix 8.3) and transferred into 96-well plate format for antibody staining described in section 2.4.3 in preparation for flow cytometric analysis.

\subsubsection{Spleen and lymph nodes}

Spleens and inguinal, mediastinal or popliteal lymph nodes were harvested from euthanised mice with minimal attached adipose tissue, placed in chilled 5\% cIMDM and stored on ice until samples could be processed.

Spleens and lymph nodes were placed on Falcon ${ }^{\mathrm{TM}} 70 \mu \mathrm{m}$ Cell Strainers (BD Biosciences Labware) moistened with 5\% cIMDM and cells were released by gently disrupting the tissues with the sterile plunger from a $2 \mathrm{~mL}$ syringe (BD Biosciences Labware). Lymph nodes from the same treatment groups were pooled at this stage as noted to allow sufficient cell numbers for further immunological analyses. Filters were washed with $15 \mathrm{~mL}$ of $5 \%$ cIMDM to wash cells through into $50 \mathrm{~mL}$ Falcon $^{\mathrm{TM}}$ tubes (BD Biosciences Labware) and tubes were centrifuged to pellet cells. 
Lymph node cells were resuspended in $1 \mathrm{~mL}$ of chilled 5\% cIMDM before being counted as described below and used in immunological analyses. Spleen cells were resuspended in $3 \mathrm{~mL}$ of room temperature ACT Lysis Buffer and incubated for $8 \mathrm{~min}$ to lyse red blood cells. Spleen samples were then topped up with $10 \mathrm{~mL}$ of $5 \%$ cIMDM to inhibit further lysis and centrifuged at $400 \mathrm{x}$ g for $10 \mathrm{~min}$ at $4{ }^{\circ} \mathrm{C}$. Spleen pellets were resuspended in $5 \mathrm{~mL}$ of chilled 5\% cIMDM and filtered through Falcon ${ }^{\mathrm{TM}} 70 \mu \mathrm{m}$ Cell Strainers into $50 \mathrm{~mL}$ Falcon ${ }^{\mathrm{TM}}$ tubes before being counted as described below and used in immunological analyses.

\subsubsection{Lung}

Lungs were harvested from euthanised mice, placed in chilled 5\% cIMDM and stored on ice until samples could be processed.

Lung samples were digested with collagenase to release cells. Lung tissue was cut into small pieces with sterile dissection scissors, placed in one well of a sterile 6 well plate (BD Biosciences Labware) and incubated with $5 \mathrm{~mL}$ of freshly made Lung Digestion Media (Appendix 8.3) at $37^{\circ} \mathrm{C}$ for $60 \mathrm{~min}$. After incubation, cells were dissociated by drawing the solution up and down a sterile transfer pipette (SAMCO, San Fernando, CA, USA) several times. The solution was filtered through Falcon ${ }^{\mathrm{TM}} 70 \mu \mathrm{m}$ Cell Strainers into $50 \mathrm{~mL}$ Falcon ${ }^{\mathrm{TM}}$ tubes. Lung tissue that remained in the filter was gently disrupted with a sterile plunger from a $2 \mathrm{~mL}$ syringe before the filter was washed with $15 \mathrm{~mL}$ of $5 \%$ cIMDM.

Cells were pelleted by centrifugation at $400 \mathrm{x}$ g for $10 \mathrm{~min}$ at $4{ }^{\circ} \mathrm{C}$ and resuspended in $15 \mathrm{~mL}$ of 5\% cIMDM. After resuspension, $10 \mathrm{~mL}$ of $70 \%$ Percoll (Appendix 8.3) was carefully layered beneath the cell suspension to create a clear interface. Samples were centrifuged at $836 \mathrm{x}$ g for 20 min at $4{ }^{\circ} \mathrm{C}$ with minimal deceleration and the lymphocyte fraction accumulated at the interface. Approximately $7-10 \mathrm{~mL}$ containing the lymphocyte fraction was harvested from this interface and washed with $20 \mathrm{~mL}$ of $5 \%$ cIMDM. The pelleted cells were resuspended in $10 \mathrm{~mL}$ of $5 \%$ cIMDM and filtered through Falcon ${ }^{\mathrm{TM}} 70 \mu \mathrm{m}$ Cell Strainers into $50 \mathrm{~mL}$ Falcon $^{\mathrm{TM}}$ tubes, before being counted as described below and used in immunological analyses. 


\subsubsection{Viable cell counts}

Viable lymphocyte counts were determined for tissues from naïve, BCG vaccinated and BCG infected mice by trypan blue exclusion. Aliquots of lymphocyte preparations from tissues were diluted initially at 1:10 in 0.4 \% Trypan Blue stain (Gibco BRL) and $10 \mu \mathrm{L}$ of the dilutions was applied to the side of a cell on an AC1000 Improved Neubauer counting chamber (Hawksley, Lancing, UK). Counting chamber cells were analysed by light microscopy and lymphocytes that excluded the dye were counted as viable. The number of lymphocytes per $\mathrm{mL}$ was determined by the equation:

Lymphocytes per $\mathrm{mL}=\left(\right.$ Viable count per cell / 4) $\mathrm{x}$ dilution factor $\times 10^{4}$

Since trypan blue exclusion requires analysis of slides outside of a Class II biological safety cabinet, spleen lymphocyte counts were determined for M. tuberculosis infected mice through approximation based on spleen weight. As the basis for approximation, naïve mice were culled their spleens were weighed, processed as described above and viable lymphocyte counts were performed by trypan blue exclusion. These cell counts were plotted against spleen weights and the equation for the resulting function was used to estimate viable lymphocyte count by spleen weight in $M$. tuberculosis infected mice (Fig. 2.1). 


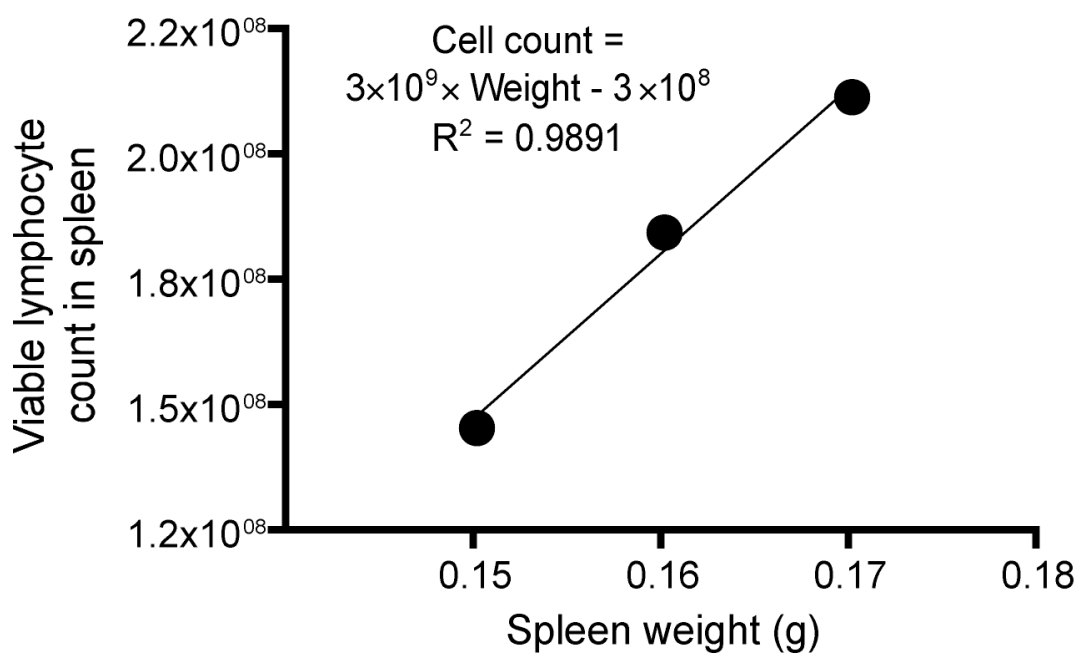

Figure 2.1: Plot of spleen weight against viable lymphocyte count from spleen cell preparations.

Spleens were harvested from naïve mice and weight was determined. Spleens were subsequently processed and viable lymphocyte counts were determined by trypan blue exclusion. Viable lymphocyte counts were plotted against spleen weight and the linear regression analysis was performed. The equation of the resulting line of best fit was used to estimate viable lymphocyte counts from spleen weight during processing of $M$. tuberculosis infected spleens.

After counting, lymphocyte preparations were restimulated for further immunological analyses, such as flow cytometry, $\left[{ }^{3} \mathrm{H}\right]$ thymidine proliferation assays or IFN- $\gamma$ enzymelinked immuno-sorbent assays (ELISAs).

For flow cytometric analysis of cytokine production, cells were restimulated with anti-CD28 mAb (to provide co-stimulation) and mycobacterial antigen as indicated, or anti-CD28 mAb and anti-CD3 mAb (as a polyclonal stimulus) for a positive control. Anti-CD28 mAb (clone 37.51) was prepared in-house and used at 2 $\mu \mathrm{g} / \mathrm{ml}$. Anti-CD3 mAb (clone 2C11) was also prepared in-house and used at $2 \mu \mathrm{g} / \mathrm{ml}$. Mycobacterial antigens used included M. bovis culture filtrate protein (CFP, gifted by AgResearch Wallaceville, Upper Hutt, New Zealand) used at $10 \mu \mathrm{g} / \mathrm{ml}$ or purified antigen 85A (Ag85A, provided by Assoc Prof John T. Belilse and Dr Karen M. Dobos, Colorado State University, CO, USA) used at $10 \mu \mathrm{g} / \mathrm{ml}$. Cells were plated at $1 \times 10^{6}$ 
cells per well in $150 \mu \mathrm{L}$ in round-bottomed 96-well plates (BD Biosciences Labware) with $5 \%$ cIMDM containing either anti-CD28 mAb/mycobacterial antigen or anti$\mathrm{CD} 28 \mathrm{mAb} / \mathrm{anti}-\mathrm{CD} 3 \mathrm{mAb}$. Cells were incubated for approximately 16 hours before the addition of monensin at $20 \mathrm{nM}$ for 4 hours to inhibit export of cytokine. Cells were then stained for flow cytometry to assess cell surface molecule expression (section 2.4.3.2) and cytokine production (section 2.4.3.3).

For $\left[{ }^{3} \mathrm{H}\right]$ thymidine proliferation assays and IFN- $\gamma$ ELISAs, cells were restimulated with either mycobacterial antigen (CFP or Ag85A, $10 \mu \mathrm{g} / \mathrm{ml}$ ), anti-CD3 $\mathrm{mAb}$ (clone $2 \mathrm{C} 11,2 \mu \mathrm{g} / \mathrm{ml}$ ) as a positive control, or left unstimulated as a negative control. Cells were plated at $1 \times 10^{6}$ cells per well in $150 \mu \mathrm{L}$ in flat-bottomed 96-well plates (BD Biosciences Labware) with $5 \%$ cIMDM containing either mycobacterial antigen, anti-CD3 mAb or nothing. Samples were plated in triplicate and incubated as indicated in section 2.4.4 for proliferation or incubated for 72 hours before being prepared for storage for the IFN- $\gamma$ ELISA. The 96-well plates were frozen at $-20{ }^{\circ} \mathrm{C}$ until use of the supernatants in the IFN- $\gamma$ ELISA (section 2.4.5.1).

Cells harvested after M. tuberculosis infection were plated at $2 \times 10^{6}$ cells per well in $2 \mathrm{~mL}$ in flat-bottomed 24-well plates (BD Biosciences Labware) with $5 \%$ cIMDM containing either CFP $(10 \mu \mathrm{g} / \mathrm{ml})$, anti-CD3 mAb (clone 2C11, $2 \mu \mathrm{g} / \mathrm{ml}$ ) or nothing. Cells were incubated for 72 hours before supernatants were harvested. Supernatants were filtered using low-protein binding $0.2 \mu \mathrm{m}$ Acrodisc filters (Pall Life Sciences) to sterilise samples and aliquots were frozen at $-20{ }^{\circ} \mathrm{C}$ until use of the supernatants in the IFN- $\gamma$ ELISA (section 2.4.5.1). Supernatant samples pre- and postfiltration were also plated on Middlebrooks 7H11 agar and incubated for up to 5 weeks to confirm supernatant sterility.

Samples were prepared in round-bottom 96-well plates (BD Biosciences Labware) and centrifugations were performed at $400 \mathrm{x}$ g for $10 \mathrm{~min}$ at $4{ }^{\circ} \mathrm{C}$. Samples were kept on ice and protected from light throughout staining.

After staining, described below, cells were prepared for flow cytometric analysis by washing them washed twice and then resuspending them in $200 \mu \mathrm{L}$ of chilled Flow 
Cytometry Buffer (Appendix 8.3). Samples were filtered through gauze into Titertube ${ }^{\circledR}$ Microtubes (BioRad) and stored on ice until analysis on a BD FACSort ${ }^{\mathrm{TM}}$ or BD FACSCalibur $^{\mathrm{TM}}$ flow cytometer using BD CellQuest ${ }^{\mathrm{TM}}$ Pro software (all from BD Biosciences, San Jose, CA, USA). The antibodies used were all pre-titrated to an optimal concentration for staining, to maximise separation between populations and minimise background staining.

For each restimulation condition and each tissue, non-stained and single-stained samples were also prepared. Single-stained samples were prepared with anti-CD4-FITC (clone GK1.5), anti-CD4-PE (clone GK1.5) anti-CD4-PerCP (clone RM4-5) and antiCD4-APC (clone RM4-5), (all from BD Biosciences Pharmingen, San Diego, CA, USA), which were all used $0.05 \mu \mathrm{g}$ per $100 \mu \mathrm{L}$ stain volume and prepared in the same way as the samples of interest. Non-stained samples were used to adjust the voltage settings and single-stained samples were used to adjust compensation settings.

Three types of flow cytometric analysis were performed in this thesis:

1. Screening of peripheral blood samples (section 2.4.1.1) for $\mathrm{CD} 4{ }^{+} \mathrm{CD} 25^{+}$ lymphocytes after PC61 treatment prior to mycobacterial infection or vaccination (section 2.4.3.1).

2. Analysis of in vitro restimulated samples from the spleen, lung or lymph nodes (sections 2.4.1 and 2.4.2) for IL-2 and IFN- $\gamma$ production by $\mathrm{CD} 4^{+}$ and $\mathrm{CD}^{+}$lymphocytes (sections 2.4.3.2 and 2.4.3.4).

3. Analysis of unstimulated samples from the spleen, lung or blood (section 2.4.1) for Foxp3 and CD25 expression by $\mathrm{CD}^{+}$lymphocytes (sections 2.4.3.3 and 2.4.3.5).

\subsubsection{Staining after PC61 treatment}

To evaluate the efficacy of $\mathrm{CD} 25^{+}$Treg inactivation following PC61 treatment, lymphocytes from peripheral blood samples were stained with anti-CD25-FITC (clone 7D4) and anti-CD4-PerCP (clone RM4-5) (both from BD Biosciences Pharmingen). The 7D4 clone of anti-CD25 mAb recognises a different epitope within the CD25 protein from the epitope recognized by the PC61 clone [343, 344], and therefore should detect any CD25-expressing cells in mice that had been treated with PC61. 
Prepared blood samples were plated in $200 \mu \mathrm{L}$ into round-bottom 96-well plates, centrifuged and then washed with $200 \mu \mathrm{L}$ of Flow Cytometry Buffer. Cells were then resuspended with $50 \mu \mathrm{L}$ of Flow Cytometry Buffer containing $1 \mu \mathrm{g}$ of purified antiCD16/CD32 (clone 2.4G2) and incubated for $15 \mathrm{~min}$. Anti-CD16/CD32 binds to and blocks the Fc $\gamma$ III/II receptors to prevent antibody binding to these receptors and thus non-specific staining. After incubation $1 \mu \mathrm{g}$ of anti-CD25-FITC and $0.05 \mu \mathrm{g}$ of antiCD4-PerCP in $50 \mu \mathrm{L}$ of Flow Cytometry Buffer was added and samples were incubated for $30 \mathrm{~min}$.

Cells were prepared for flow cytometry as described above and a minimum of 40,000 events were collected in the lymphocyte gate. FlowJo Version 4.5 was used for data analysis.

\subsubsection{Cell surface staining for assessment of cytokine production}

In vitro restimulated lymphocytes from the spleen, the lymph node or lung, were first stained for cell surface molecules with anti-CD8-FITC (clone 53-6.7) and anti-CD4PerCP (clone RM4-5) (both from BD Biosciences Pharmingen) before being fixed for intracellular cytokine staining.

Restimulated samples were centrifuged and then washed with $200 \mu \mathrm{L}$ of Flow Cytometry Buffer. Cells were then resuspended with $50 \mu \mathrm{L}$ of Flow Cytometry Buffer containing $1 \mu \mathrm{g}$ of purified anti-CD16/CD32 (clone 2.4G2) and incubated for $15 \mathrm{~min}$. After incubation $0.125 \mu \mathrm{g}$ of anti-CD8-FITC and $0.05 \mu \mathrm{g}$ of anti-CD4-PerCP in $50 \mu \mathrm{L}$ of Flow Cytometry Buffer was added and samples were incubated for $30 \mathrm{~min}$ in the dark.

Cells were pelleted and then washed in $200 \mu \mathrm{L}$ of PBS before being fixed with $100 \mu \mathrm{L}$ of $4 \%$ paraformaldehyde (Appendix 8.3) for $10 \mathrm{~min}$ at room temperature. After incubation, $100 \mu \mathrm{L}$ of PBS was added and cells were pelleted, washed in $200 \mu \mathrm{L}$ of PBS and then used for intracellular cytokine staining (section 2.4.3.4). 


\subsubsection{Cell surface staining for assessment of Foxp3 expression}

Unstimulated lymphocytes isolated from the spleen, the lymph node or blood, were first stained for cell surface molecules with anti-CD25-FITC (clone 7D4) and anti-CD4PerCP (clone RM4-5) (both from BD Biosciences Pharmingen) before being fixed for Foxp3 staining.

Samples were centrifuged and then washed with $200 \mu \mathrm{L}$ of Flow Cytometry Buffer. Cells were then resuspended with $50 \mu \mathrm{L}$ of Flow Cytometry Buffer containing 1 $\mu \mathrm{g}$ of purified anti-CD16/CD32 (clone 2.4G2) and incubated for $15 \mathrm{~min}$. After incubation, $1 \mu \mathrm{g}$ of anti-CD25-FITC and $0.05 \mu \mathrm{g}$ of anti-CD4-PerCP in $50 \mu \mathrm{L}$ of Flow Cytometry Buffer was added and samples were incubated for $30 \mathrm{~min}$ in the dark.

Cells were fixed prior to Foxp3 staining (section 2.4.3.5) using Fix/Perm Buffer composed of 1 part Fix/Perm Concentrate and 3 parts Fix/Perm Diluent (both from eBioscience Inc., San Diego, Ca, USA). Cells were pelleted and then washed twice in $200 \mu \mathrm{L}$ of PBS before being resuspended in $200 \mu \mathrm{L}$ of Fix/Perm Buffer and incubated overnight at $4{ }^{\circ} \mathrm{C}$. Cells were pelleted, and washed twice with $200 \mu \mathrm{L}$ of PBS, then used in the Foxp3 staining protocol (section 2.4.3.5).

\subsubsection{Intracellular cytokine staining}

Fixed cells were permeabilised for intracellular staining by incubation with $200 \mu \mathrm{L}$ of Saponin Buffer (Appendix 8.3) for 30 minutes. Cells were pelleted and resuspended in $100 \mu \mathrm{L}$ of Saponin Buffer containing either $0.1 \mu \mathrm{g}$ each of anti-IL-2-PE (clone JES65H4) and anti-IFN- $\gamma$-APC (clone XMG1.2), or $0.1 \mu \mathrm{g}$ each of rat IgG2b-PE and rat IgG1-APC as isotype controls (all from BD Biosciences Pharmingen). Cells were incubated with antibodies for 30 min before being washed twice with $200 \mu \mathrm{L}$ of Saponin Buffer and prepared for flow cytometric analysis as described above. A minimum of 100000 events were collected from the lymphocyte gate for each sample. Background staining (isotype control) was subtracted from cytokine (IL-2 or IFN- $\gamma$ ) staining. FlowJo Version 4.5 was used for data analysis. 


\subsubsection{Foxp3 staining}

Fixed cells were permeabilised for Foxp3 staining by washing cells twice with $200 \mu \mathrm{L}$ of 1x Permeabilisation Buffer (eBioscience Inc.). Cells were pelleted and resuspended in $100 \mu \mathrm{L}$ of $1 \mathrm{x}$ Permeabilisation Buffer containing either $0.1 \mu \mathrm{g}$ of anti-Foxp3-PE (clone FJK-16s) (eBioscience Inc.) or $0.1 \mu \mathrm{g}$ of rat IgG2a-PE (BD Biosciences Pharmingen) as an isotype control. Cells were incubated with antibodies for $45 \mathrm{~min}$ before being washed twice with $200 \mu \mathrm{L}$ of 1x Permeabilisation Buffer and being prepared for flow cytometric analysis as described above. A minimum of 100000 events were collected from the lymphocyte gate for each sample. Background staining (isotype control) was subtracted from Foxp3 staining. FlowJo Version 4.5 was used for data analysis.

After plating (section 2.4.2), cells were incubated at $37^{\circ} \mathrm{C}$ for 60 hours for antigen stimulated or unstimulated cells, or 24 hours for anti-CD3 stimulated cells. Cells were observed under the inverted microscope to ensure lymphocytes were undergoing activation and proliferation at the times indicated above. To each well, $10 \mu \mathrm{L}$ of 0.5 $\mathrm{mCi}\left[{ }^{3} \mathrm{H}\right]$ thymidine (GE Healthcare) diluted in 5\% cIMDM was added at either 60 or 24 hours as indicated above. Cells were incubated for a further 12 hours and frozen at $20{ }^{\circ} \mathrm{C}$ until they were harvested.

Cells from defrosted plates were harvested onto printed glass fiber filtermats (Wallac, Turku, Finland) using the Harvester $96^{\circledR}$ (Tomtec Inc., Hamden, CT, USA). Each filtermat was thoroughly dried in a microwave oven and sealed in a sample bag with $5 \mathrm{~mL}$ of Betaplate Scint Fluid (both from Wallac). Scintillation from the content of each well was assessed using a 1450 MicroBeta Plus liquid scintillation counter and MicroBeta Windows software (both from Wallac). The triplicate cultures for each sample were averaged to give a measurement of counts per minute (CPM) for each sample. 


\subsubsection{IFN- $\gamma$ ELISA of cell culture supernatants}

Culture plates (section 2.4.2) were thawed, cell debris was pelleted by centrifugation at $600 \mathrm{x} g$ for $15 \mathrm{~min}$ and supernatants were used immediately in the BD OptEIA Mouse IFN- $\gamma$ assay kit (BD Biosciences Pharmingen).

Nunc-Immuno $^{\mathrm{TM}} 96$ Microwell $^{\mathrm{TM}}$ plates (Nunc A/S, Roskilde, Denmark) were incubated overnight at $4{ }^{\circ} \mathrm{C}$ with $100 \mu \mathrm{L}$ of anti-mouse IFN- $\gamma$ capture antibody (BD Biosciences Pharmingen) diluted 1:250 in ELISA Coating Buffer (Appendix 8.3). Wells were washed 4 times with ELISA Wash Buffer (Appendix 8.3) and incubated with 200 $\mu \mathrm{L}$ of ELISA Assay Diluent (Appendix 8.3) for 60 min to block non-specific protein binding.

While the plates were blocking, the supernatant samples were prepared. Unstimulated cultures were prepared at neat and 1:10 dilutions, antigen (CFP or Ag85A) stimulated cultures were prepared at neat and 1:20 dilutions and anti-CD3 stimulated cultures were prepared at 1:40 and 1:200 dilutions. After blocking, plates were washed 4 times with ELISA Wash Buffer, $100 \mu \mathrm{L}$ of the appropriate samples or a two-fold serial dilution of the recombinant IFN- $\gamma$ standard (BD Biosciences

Pharmingen) were added to wells. Plates were sealed and incubated for 2 hours at room temperature then washed 4 times with ELISA Wash Buffer. Plates were incubated for $60 \mathrm{~min}$ at room temperature with $100 \mu \mathrm{L}$ of ELISA Assay Diluent containing biotinylated anti-mouse IFN- $\gamma$ detection antibody diluted 1:250 and avidin-horseradish peroxidase (HRP) conjugate enzyme reagent diluted 1:250 (both BD Biosciences Pharmingen). Plates were washed 6 times with ELISA Wash Buffer and $100 \mu \mathrm{L}$ of freshly prepared, room temperature TMB Substrate Reagent (BD Biosciences Pharmingen) was added.

Plates were incubated until a clear gradient of colour was observed across the recombinant IFN- $\gamma$ standard dilutions, at which point $50 \mu \mathrm{L}$ of ELISA Stop Solution (Appendix 8.3) was added. After stopping the reactions, the absorbance of each well was read at $450 \mathrm{~nm}$ and converted to absorbance values to amount of IFN- $\gamma$ in $\mathrm{pg} / \mathrm{mL}$ using the standard curve plotted from the recombinant IFN- $\gamma$ standard dilutions. The 
limit of detection was $32.5 \mathrm{pg} / \mathrm{mL}$. The triplicate cultures for each sample were averaged to give a mean measurement of IFN- $\gamma$ production for each sample.

\subsubsection{Anti-CD25 (clone PC61) ELISA of murine serum}

To detect PC61 mAb in murine serum after i.p. administration, a sandwich ELISA to detect rat IgG in murine sera was attempted based on a protocol outlined in Loughry, et al. [345]. This assay ineffective as the detection limit was higher than the concentration of PC61 in the serum of a mouse that had just received $100 \mu \mathrm{g}$ of the antibody.

Therefore, a more sensitive ELISA was used to evaluate PC61 presence in murine sera based on a method described in Cao, et al. [346].

Mice were administered $100 \mu \mathrm{g}$ of PC61 i.p. and bled at days 3, 10, 14 and 21 after treatment. Mice were also bled immediately prior to PC61 treatment to establish a baseline measurement. Sera were harvested and stored at $4{ }^{\circ} \mathrm{C}$ until use.

Nunc-Immuno $^{\mathrm{TM}} 96$ Microwell $^{\mathrm{TM}}$ plates (Nunc A/S) were incubated with 12.5 ng per well of recombinant mouse IL-2R $\alpha$ (CD25) (R\&D Systems Inc., Minneapolis, $\mathrm{MN}, \mathrm{USA}$ ) in $50 \mu \mathrm{L}$ ELISA Coating Buffer. Wells were washed 5 times with ELISA Wash Buffer and incubated with $200 \mu \mathrm{L}$ of ELISA Assay Diluent for $60 \mathrm{~min}$ at room temperature to block non-specific protein binding. After incubation, sera samples were prepared as a five-fold serial dilution from neat to 1:31250 and the PC61 standard was prepared as a 1:2 serial dilution from $100 \mathrm{ng} / \mathrm{mL}$ to $0.05 \mathrm{ng} / \mathrm{mL}$. Wells were washed 5 times with ELISA Wash Buffer and $50 \mu \mathrm{L}$ of sera sample dilutions or PC61 standard dilutions were added to wells and incubated at room temperature for 2 hours. Sera samples were plated in duplicate at each dilution. Wells were washed 5 times with ELISA Wash Buffer and $50 \mu \mathrm{L}$ of biotinylated anti-Rat IgG1 Fc domain mAb (clone RG11/39.4) (BD Biosciences Pharmingen) at $4 \mu \mathrm{g} / \mathrm{mL}$ diluted in ELISA Assay Diluent was added to wells and plates were incubated at room temperature for $60 \mathrm{~min}$. Wells were washed 5 times with ELISA Wash Buffer and $50 \mu \mathrm{L}$ of avidin-HRP conjugate enzyme reagent diluted 1:250 (BD Biosciences Pharmingen) was added to wells and plates were incubated at room temperature for $60 \mathrm{~min}$. Plates were washed 10 times with ELISA Wash Buffer and $100 \mu \mathrm{L}$ of freshly prepared, room temperature TMB Substrate Reagent (BD Biosciences Pharmingen) was added. 
Plates were incubated in the dark at room temperature until a clear gradient of colour was observed across PC61 standard dilutions, at which point $50 \mu \mathrm{L}$ of ELISA Stop Solution was added. After stopping the reactions, the absorbance of each well was read at $450 \mathrm{~nm}$. Absorbance values were converted to the amount of PC61 in ng/mL serum using the standard curve plotted from the standard dilutions. The concentration was then used to estimate the amount of PC61 in circulation per mouse, assuming a total serum volume in a $30 \mathrm{~g} 8$ week old mouse of approximately $0.945 \mathrm{~mL}$, given an estimated total plasma volume of $3.15 \mathrm{~mL}$ per $100 \mathrm{~g}$ body weight [347]. The limit of detection was $0.014 \mu \mathrm{g} /$ mouse. The mean of duplicate dilutions for each sample was used to determine the average amount of circulating PC61 per mouse at each time point assessed.

\subsection{Microbiological and histological techniques}

Lung or spleen samples were mechanically homogenised in $3 \mathrm{ml}$ of PBS/Tween $800.5 \%$ (Appendix 8.3) using an Ultra Turrax T25 Disperser (IKA Works, Selangor, Malaysia) and a freshly autoclaved S25N-8G dispersing tool (IKA Works). The dispersing tool was cleaned with PBS/Tween 80 between samples, and sterilised with $70 \%$ ethanol and rinsed with PBS/Tween 80 between groups. Homogenised samples were used to make five 10 -fold dilutions and $3 \times 10 \mu \mathrm{L}$ of each dilution was plated on Middlebrooks $7 \mathrm{H} 11$ agar supplemented for BCG. Plates were incubated at $37^{\circ} \mathrm{C}$ for $2-3$ weeks before colonies were counted. The limit of detection for this assay was $100 \mathrm{CFU}$ per organ. Spleen samples or lung samples early after infection that did not result in colonies were assigned a value of $90 \mathrm{CFU}$ (just below the detection limit), rather than zero, to prevent exaggerating statistical differences between treatment groups.

\section{M. tuberculosis M. bovis}

Lung or spleen samples were mechanically homogenised in $3 \mathrm{ml}$ of PBS/ Tween80 0.5\% using a Seward Stomacher ${ }^{\circledR} 80$ (Seward, Norfolk, UK). Homogenised samples were used to make five or two 10-fold dilutions for lungs or spleens respectively. Three 
volumes of $10 \mu \mathrm{L}$ for each dilution and the neat homogenate were plated on Middlebrooks 7H11 agar supplemented for either M. bovis or M. tuberculosis. Plates were incubated at $37^{\circ} \mathrm{C}$ for $2-3$ weeks before colonies were counted. The limit of detection for this assay was 100 CFU per organ. Spleen samples or lung samples early after infection that did not result in colonies were assigned a value of 90 CFU (just below the detection limit), rather than zero, to prevent exaggerating statistical differences between treatment groups.

The lower left lobe of the lung was inflated and fixed with $10 \%$ phosphate-buffered formalin (Sigma-Aldrich). After fixation, samples were embedded in paraffin, sectioned and stained with haematoxylin and eosin through the Pathology Department, Wellington School of Medicine, University of Otago. Samples were analysed by light microscopy by pathologist Professor Brett Delahunt, Department of Pathology, Wellington School of Medicine. Individual lung sections were graded for degree and extent of inflammation, and composite scores for inflammation or numbers of lung lesions per sample were used to evaluate the statistical significance of differences between treatment groups.

\subsection{Molecular biology techniques}

PCR primers 5'Ag85A and 3'Ag85A (Sigma-Genosys, Castle Hill, Australia) (Appendix 8.1) were designed to amplify the $A g 85 A$ gene, using the pcDNA3-85A plasmid (Fig. 4.3.A) as template DNA.

PCR amplification was performed using the AccuPrime Pfx PCR system (Invitrogen). PCR reactions were prepared on ice in PCR Strip Tubes (Axygen Scientific, Union City, CA, USA) and run in an iCycler Thermal Cycler (BioRad). The optimal annealing temperature $\left(\mathrm{T}_{\mathrm{A}}\right)$ was determined to be $68{ }^{\circ} \mathrm{C}$ by a temperature gradient PCR ranging from $66-70{ }^{\circ} \mathrm{C}$. A suitable $\mathrm{Mg}^{2+}$ concentration was determined to 
be $1 \mathrm{mM}$ after performing PCRs ranging from 1-5 $\mathrm{mM} \mathrm{Mg}^{2+}$. Individual $25 \mu \mathrm{L}$ reactions were composed of reagents detailed in Table 2.1 and run using the PCR conditions detailed in Table 2.2. PCR products were visualised by agarose gel electrophoresis (section 2.6.10).

To prepare enough Ag85A PCR product for cloning, fifteen $25 \mu \mathrm{L}$ reactions were run and pooled before PCR product was purified with the UltraClean ${ }^{\mathrm{TM}} \mathrm{PCR}$ Clean-Up Kit (MO BIO, Carlsbad, CA, USA) (section 2.6.9) and quantified (section 2.6.8).

Table 2.1: Reagents used for Ag85A PCR

\begin{tabular}{llll}
\hline Reagent & $\begin{array}{l}\text { Volume Added } \\
\text { per Reaction }\end{array}$ & $\begin{array}{l}\text { Stock } \\
\text { Concentration }\end{array}$ & $\begin{array}{l}\text { Final } \\
\text { Concentration }\end{array}$ \\
\hline 10x AccuPrime $P f x$ Reaction Mix & $2.5 \mu \mathrm{L}$ & $10 \mathrm{x}$ & $1 \mathrm{x}$ \\
5' Ag85A primer & $0.5 \mu \mathrm{L}$ & $20 \mu \mathrm{M}$ & $0.4 \mu \mathrm{M}$ \\
3' Ag85A primer & $0.5 \mu \mathrm{L}$ & $20 \mu \mathrm{M}$ & $0.4 \mu \mathrm{M}$ \\
AccuPrime $P f x$ DNA polymerase & $0.2 \mu \mathrm{L}$ & $2.5 \mathrm{U} / \mu \mathrm{L}$ & $0.1 \mathrm{U} / \mu \mathrm{L}$ \\
pcDNA3-85A template DNA & $1 \mu \mathrm{L}$ & $10 \mathrm{pg} / \mu \mathrm{L}$ & $0.4 \mathrm{pg} / \mu \mathrm{L}$ \\
DNAase/RNAase free $\mathrm{H}_{2} 0$ & $20.3 \mu \mathrm{L}$ & &
\end{tabular}

10x AccuPrime Pfx Reaction Mix contains $\mathrm{MgSO}_{4}$ and dNTPs that, when the mix is diluted, reach final concentrations of $1 \mathrm{mM}$ and $0.3 \mathrm{mM}$ respectively.

Table 2.2: PCR cycles used for Ag85A PCR

\begin{tabular}{llll}
\hline Type of cycle & Temperature & Time & Number of cycles \\
\hline Initial denaturation & $95^{\circ} \mathrm{C}$ & $2 \mathrm{~min}$ & 1 \\
\hline Denaturation & $95^{\circ} \mathrm{C}$ & $15 \mathrm{sec}$ & $30 \mathrm{x}$ \\
Annealing & $68{ }^{\circ} \mathrm{C}$ & $30 \mathrm{sec}$ & \\
Extension & $72{ }^{\circ} \mathrm{C}$ & $60 \mathrm{sec}$ & 1 \\
\hline Final Extension & $72{ }^{\circ} \mathrm{C}$ & $7 \mathrm{~min}$ & \\
\hline
\end{tabular}


To create digested $A g 85 A$ stock for ligation, twelve $20 \mu \mathrm{L}$ restriction digest reactions as detailed in Table 2.3 were performed. MluI restriction enzyme and $\mathrm{H}$ buffer (Roche Diagnostics GmbH, Mannheim, Germany) were used in the reactions. Digests were incubated at $37{ }^{\circ} \mathrm{C}$ for 3 hours before being purified using the UltraClean ${ }^{\mathrm{TM}}$ PCR CleanUp Kit (section 2.6.9).

Table 2.3: Reaction conditions for MluI digestion of Ag85A PCR product

\begin{tabular}{llll}
\hline Reagent & $\begin{array}{l}\text { Volume } \\
\text { Added per } \\
\text { Reaction }\end{array}$ & $\begin{array}{l}\text { Stock } \\
\text { Concentration }\end{array}$ & $\begin{array}{l}\text { Final } \\
\text { Concentration }\end{array}$ \\
\hline $\begin{array}{l}\text { Purified } A g 85 A \text { PCR } \\
\text { product }\end{array}$ & $17 \mu \mathrm{L}$ & $87.93 \mathrm{ng} / \mu \mathrm{L}$ & $74.74 \mathrm{ng} / \mu \mathrm{L}$ \\
$10 \mathrm{x}$ SuRE/Cut H buffer & $2 \mu \mathrm{L}$ & $10 \mathrm{x}$ & $1 \mathrm{x}$ \\
$M l u I$ & $1 \mu \mathrm{L}$ & $10 \mathrm{U} / \mu \mathrm{L}$ & $0.5 \mathrm{U} / \mu \mathrm{L}$ \\
\hline Total reaction volume & $\mathbf{2 0} \mu \mathrm{L}$ & & \\
\hline
\end{tabular}

To create the digested pKUNrep6 (Fig. 4.3.B) stock for ligation, ten $20 \mu \mathrm{L}$ restriction digest reactions as detailed in Table 2.4 were performed. MluI restriction enzyme and $\mathrm{H}$ buffer were used in the reactions. One reaction was also run with $1 \mu \mathrm{L}$ of DNAase/RNAase free $\mathrm{H}_{2} 0$ substituted for the $1 \mu \mathrm{L}$ of $M l u I$ to provide the undigested pKUNrep6 ligation control. Digest reactions and controls were incubated at $37^{\circ} \mathrm{C}$ for 3 hours before undergoing dephosphorylation (section 2.6.3). 
Table 2.4: Reaction conditions for MluI digestion of pKUNrep6

\begin{tabular}{llll}
\hline Reagent & $\begin{array}{l}\text { Volume } \\
\text { Added per } \\
\text { Reaction }\end{array}$ & $\begin{array}{l}\text { Stock } \\
\text { Concentration }\end{array}$ & $\begin{array}{l}\text { Final } \\
\text { Concentration }\end{array}$ \\
\hline pKUNrep6 DNA & $15 \mu \mathrm{L}$ & $167.01 \mathrm{ng} / \mu \mathrm{L}$ & $125.25 \mathrm{ng} / \mu \mathrm{L}$ \\
$10 \mathrm{x}$ SuRE/Cut H buffer & $2 \mu \mathrm{L}$ & $10 \mathrm{x}$ & $1 \mathrm{x}$ \\
MluI & $1 \mu \mathrm{L}$ & $10 \mathrm{U} / \mu \mathrm{L}$ & $0.5 \mathrm{U} / \mu \mathrm{L}$ \\
$\begin{array}{l}\text { DNAase/RNAase free } \\
\mathrm{H}_{2} \mathrm{O}\end{array}$ & $2 \mu \mathrm{L}$ & & \\
\hline Total reaction volume & $\mathbf{2 0} \mu \mathrm{L}$ & & \\
\hline
\end{tabular}

After a 3 hour incubation for MluI digestion of pKUNrep6, $17.5 \mathrm{U}$ of calf intestinal alkaline phosphotase (CIAP) (Stratgene, La Jolla, CA, USA) in $1 \mu \mathrm{L}$ was added to 9 of the digest reactions. One tube was not treated with CIAP to provide a phosphorylated, digested pKUNrep6 ligation control. Dephosphorylation reactions and controls were incubated at $37{ }^{\circ} \mathrm{C}$ for 60 min before being purified using the UltraClean ${ }^{\mathrm{TM}}$ PCR CleanUp Kit (section 2.6.9).

After the pKUNrep6 vector and $A g 85 A$ insert were prepared (sections 2.6.1-2.6.3), they were quantified (section 2.6.8) and ligated together using T4 DNA ligase (Invitrogen). To ensure a suitable ratio of pKUNrep6 vector to $\mathrm{Ag} 85 \mathrm{~A}$ insert in each reaction, pmol ends of both pKUNrep6 and $A g 85 A$ were determined using the following equation [340], where the number of base pairs (bp) for pKUNrep6 was $13481 \mathrm{bp}$ and for $\mathrm{Ag} 85 \mathrm{~A}$ was 989 bp:

pmol ends of dsDNA $=$ $2 \times 10^{6} \times$ (amount in $\mu \mathrm{g}$ of dsDNA) 
Ligation reactions were prepared on ice in PCR Strip Tubes and run at $14{ }^{\circ} \mathrm{C}$ overnight in an iCycler Thermal Cycler. Ligation reactions are detailed in Table 2.5.

Table 2.5: Ligation Reactions and Controls

\begin{tabular}{|c|c|c|c|c|c|}
\hline $\begin{array}{l}\text { Ratio of Insert } \\
\text { to Vector }\end{array}$ & $\begin{array}{c}\text { Insert } \\
(A g 85 A)\end{array}$ & $\begin{array}{c}\text { Vector } \\
\text { (pKUNrep6) }\end{array}$ & $\begin{array}{c}\text { T4 DNA } \\
\text { Ligase }\end{array}$ & $\begin{array}{c}5 \times \text { T4 DNA } \\
\text { Ligase } \\
\text { Buffer }\end{array}$ & $\begin{array}{c}\text { DNAase/ } \\
\text { RNAase free } \\
\mathrm{H}_{2} \mathbf{0}\end{array}$ \\
\hline \multicolumn{6}{|c|}{ Experimental ligations } \\
\hline $1: 2$ & $60 \mathrm{fmol}$ & $120 \mathrm{fmol}$ & $1 \mu \mathrm{L}$ & $4 \mu \mathrm{L}$ & Up to $20 \mu \mathrm{L}$ \\
\hline $1: 1$ & $60 \mathrm{fmol}$ & $60 \mathrm{fmol}$ & $1 \mu \mathrm{L}$ & $4 \mu \mathrm{L}$ & Up to $20 \mu \mathrm{L}$ \\
\hline $3: 1$ & $180 \mathrm{fmol}$ & $60 \mathrm{fmol}$ & $1 \mu \mathrm{L}$ & $4 \mu \mathrm{L}$ & Up to $20 \mu \mathrm{L}$ \\
\hline $5: 1$ & $300 \mathrm{fmol}$ & $60 \mathrm{fmol}$ & $1 \mu \mathrm{L}$ & $4 \mu \mathrm{L}$ & Up to $20 \mu \mathrm{L}$ \\
\hline $10: 1$ & $600 \mathrm{fmol}$ & $60 \mathrm{fmol}$ & $1 \mu \mathrm{L}$ & $4 \mu \mathrm{L}$ & $\mathrm{Up}$ to $20 \mu \mathrm{L}$ \\
\hline
\end{tabular}

\section{Ligation controls}

\begin{tabular}{|c|c|c|c|c|c|}
\hline $0: 1$ - no ligase & $0 \mathrm{fmol}$ & $60 \mathrm{fmol}$ & $0 \mu \mathrm{L}$ & $4 \mu \mathrm{L}$ & Up to $20 \mu \mathrm{L}$ \\
\hline $\begin{array}{l}0: 1-\text { using } \\
\text { phosphorylated } \\
\text { pKUNrep6 }\end{array}$ & $0 \mathrm{fmol}$ & $60 \mathrm{fmol}$ & $1 \mu \mathrm{L}$ & $4 \mu \mathrm{L}$ & Up to $20 \mu \mathrm{L}$ \\
\hline $0: 1$ - ligase & $0 \mathrm{fmol}$ & $60 \mathrm{fmol}$ & $1 \mu \mathrm{L}$ & $4 \mu \mathrm{L}$ & Up to $20 \mu \mathrm{L}$ \\
\hline $1: 1$ - no ligase & $60 \mathrm{fmol}$ & $60 \mathrm{fmol}$ & $0 \mu \mathrm{L}$ & $4 \mu \mathrm{L}$ & Up to $20 \mu \mathrm{L}$ \\
\hline $\begin{array}{l}0: 1 \text { - using } \\
\text { undigested } \\
\text { pKUNrep6 }\end{array}$ & $0 \mathrm{fmol}$ & $60 \mathrm{fmol}$ & $0 \mu \mathrm{L}$ & $4 \mu \mathrm{L}$ & Up to $20 \mu \mathrm{L}$ \\
\hline
\end{tabular}

Ligation controls were also included to validate the experimental ligation reactions (Table 2.5). The ligation control with 0:1 ratio and no ligase could indicate either if digestion has not been complete or if dephosphorylation has not been successful and the plasmid has religated, although a sample of digested, dephosphorylated pKUNrep6 was visualised on an agarose gel prior to ligation to confirm linear conformation. The $0: 1$ ratio with ligase using the phosphorylated vector would validate that the digested pKUNrep6 vector can be religated and enzymatic digestion has not damaged the vector ends. The 0:1 ratio with ligase would validate that the digested and dephosphorylated 
pKUNrep6 vector can be religated and dephosphorylation has not damaged vector ends. The 1:1 ratio without ligase would give a baseline for comparison to experimental ligations, in case there was low-level religation of pKUNrep6 or undigested pKUNrep6 in the vector DNA. The inclusion of undigested pKUNrep6 could indicate if the ligation reaction itself affects the integrity of the vector DNA but also provides a positive control to confirm the vector DNA confers antibiotic resistance to $E$ coli after transformation.

Transformations were performed using chemically competent MAX Efficiency ${ }^{\circledR}$ DH5a ${ }^{\mathrm{TM}}$ Escherichia coli (Invitrogen). Fifty $\mu \mathrm{L}$ of $E$. coli was thawed on wet ice for each transformation reaction and dispensed into pre-chilled eppendorf tubes. For transformations with intact pKUNrep6 DNA, $1 \mu \mathrm{L}$ of DNA at $200 \mathrm{ng} / \mu \mathrm{L}$ was added to the thawed E. coli. For transformations using ligation reactions from section 2.6.4, 7.5 $\mu \mathrm{L}$ of the ligation reaction was added to the thawed $E$ coli. For transformation controls, one transformation reaction was left without the addition of plasmid as a negative control and one transformation reaction received $2.5 \mu 1$ of $\mathrm{pUC} 19$ at $10 \mathrm{pg} / \mu \mathrm{L}$ as a positive control. After addition of DNA, transformation reactions were gently tapped, left on ice for $30 \mathrm{~min}$ and then heat-shocked by placing in a $42{ }^{\circ} \mathrm{C}$ waterbath for $45 \mathrm{sec}$. Reactions were placed on ice for $2 \mathrm{~min}$ before the addition of $0.45 \mathrm{~mL}$ of SOC media (Invitrogen), then incubated, shaking at 225 revolutions per minute (rpm) at $37{ }^{\circ} \mathrm{C}$, for $60 \mathrm{~min}$ in an Innova ${ }^{\mathrm{TM}} 4200$ Incubator (New Brunswick Scientific, Edison, NJ, USA).

After incubation, $50 \mu \mathrm{L}$ of transformation reaction was plated onto Luria Broth supplemented agar plates containing ampicillin (LB Amp ${ }^{+}$agar plates) (Appendix 8.3) and incubated overnight at $37^{\circ} \mathrm{C}$. Both pKUNrep6 and pUC19 contain ampicillin resistance genes and colonies that grow contain the desired plasmids. Individual colonies were selected from transformant plates, streaked out onto a second plate and incubated overnight at $37{ }^{\circ} \mathrm{C}$. An individual colony from the second plate was chosen, streaked out onto a master plate and incubated overnight at $37{ }^{\circ} \mathrm{C}$. A colony from this transformant master plate was inoculated into $6 \mathrm{~mL}$ of LB Amp ${ }^{+}$liquid culture (Appendix 8.3) and incubated overnight at $37^{\circ} \mathrm{C}$. One $\mathrm{mL}$ of this liquid culture was used to make Glycerol Stocks (Appendix 8.3) and the other $5 \mathrm{~mL}$ was used in the 
Qiagen Miniprep Plasmid protocol (section 2.6.11) to provide a plasmid DNA sample. The transformants were then screened for the insertion of the $A g 85 \mathrm{~A}$ gene into their plasmids by orientation PCR (section 2.6.6), orientation restriction digest (section 2.6.7) and sequencing.

PCR primers 5'CS and 3' Mid (Sigma-Genosys)(Appendix 8.1) were designed to amplify the sequence spanning the pKUNrep6 vector DNA into the 5' region of the Ag85A gene (Fig. 4.7).

Template DNA for each transformant was acquired by inoculating a colony from the transformant master plate into a $20 \mu \mathrm{L}$ aliquot of DNAase/RNAase free $\mathrm{H}_{2} 0$. These aliquots were heated to $95{ }^{\circ} \mathrm{C}$ for 2-5 min, cell debris was pelleted by centrifuging at $16000 \mathrm{x} g$ for $2 \mathrm{~min}$. Supernatants containing the plasmid released by cell lysis were transferred to fresh tubes. PCR amplification was performed using the Expand High Fidelity ${ }^{\text {PLUS }}$ PCR system (Roche Diagnostics GmbH). PCR reactions were prepared on ice in PCR Strip Tubes and run in an iCycler Thermal Cycler. Reactions were run with pKUNrep6 as template DNA or $\mathrm{H}_{2} 0$ instead of template DNA for negative controls, and pKUN85AOF (Fig. $4.3 \mathrm{C}$ ) as template DNA for a positive control. Reagents for individual $25 \mu \mathrm{L}$ reactions are detailed in Table 2.6. Reactions were run using the conditions detailed in Table 2.7 before being visualised by agarose gel electrophoresis (section 2.6.10). 
Table 2.6: Reagents used for Orientation PCR

\begin{tabular}{llll}
\hline Reagent & $\begin{array}{l}\text { Volume Added } \\
\text { per Reaction }\end{array}$ & $\begin{array}{l}\text { Stock } \\
\text { Concentration }\end{array}$ & $\begin{array}{l}\text { Final } \\
\text { Concentration }\end{array}$ \\
\hline $\begin{array}{l}5 \text { x Expand High Fidelity }{ }^{\text {PLUS }} \\
\text { Reaction Buffer }\end{array}$ & $5 \mu \mathrm{L}$ & $5 \mathrm{x}$ & $1 \mathrm{x}$ \\
$\mathrm{MgCl}_{2}$ solution & $2.5 \mu \mathrm{L}$ & $25 \mathrm{mM}$ & $2.5 \mathrm{mM}$ \\
dNTPs & $1 \mu \mathrm{L}$ & $10 \mathrm{mM}$ & $400 \mu \mathrm{M}$ \\
5' CS primer & $3.125 \mu \mathrm{L}$ & $3.2 \mu \mathrm{M}$ & $0.4 \mu \mathrm{M}$ \\
3' Mid primer & $3.125 \mu \mathrm{L}$ & $3.2 \mu \mathrm{M}$ & $0.4 \mu \mathrm{M}$ \\
$\begin{array}{l}\text { Expand High Fidelity }{ }^{\text {PLus }} \text { DNA } \\
\text { polymerase }\end{array}$ & $0.25 \mu \mathrm{L}$ & $10 \mathrm{U} / \mu \mathrm{L}$ & $0.1 \mathrm{U} / \mu \mathrm{L}$ \\
Boiled E. coli template DNA or & $2 \mu \mathrm{L}$ & & Variable or \\
Control template DNA & & $10 \mathrm{ng} / \mu \mathrm{L}$ & $0.8 \mathrm{pg} / \mu \mathrm{L}$ \\
DNAase/RNAase free $\mathrm{H}_{2} 0$ & $8 \mu \mathrm{L}$ & & \\
\hline Total reaction volume & $\mathbf{2 5} \mu \mathrm{L}$ & & \\
\hline
\end{tabular}

Table 2.7: PCR cycles used for Orientation PCR

\begin{tabular}{llll}
\hline Type of cycle & Temperature & Time & Number of cycles \\
\hline Initial denaturation & $95{ }^{\circ} \mathrm{C}$ & $2 \mathrm{~min}$ & 1 \\
\hline Denaturation & $95^{\circ} \mathrm{C}$ & $15 \mathrm{sec}$ & \\
Annealing & $59{ }^{\circ} \mathrm{C}$ & $30 \mathrm{sec}$ & $30 \mathrm{x}$ \\
Extension & $72{ }^{\circ} \mathrm{C}$ & $60 \mathrm{sec}$ & 1 \\
\hline Final Extension & $72{ }^{\circ} \mathrm{C}$ & $7 \mathrm{~min}$ & \\
\hline
\end{tabular}

Orientation restriction digest reactions were performed as detailed in Table 2.8, using plasmid DNA prepared from transformants (section 2.6.11) and KspI and XbaI restriction enzymes and L buffer (Roche Diagnostics GmbH). pKUNrep6 was used as a negative control, and pKUN85AOF as a positive control. Digest reactions and controls 
were incubated at $37{ }^{\circ} \mathrm{C}$ for $60 \mathrm{~min}$ before being visualised by agarose gel electrophoresis (section 2.6.10).

Table 2.8: Reaction conditions for KspI and $\mathrm{XbaI}$ orientation digestion of pKUN85A

\begin{tabular}{llll}
\hline Reagent & $\begin{array}{l}\text { Volume } \\
\text { Added per } \\
\text { Reaction }\end{array}$ & $\begin{array}{l}\text { Stock } \\
\text { Concentration }\end{array}$ & $\begin{array}{l}\text { Final } \\
\text { Concentration }\end{array}$ \\
\hline Template DNA & $5 \mu \mathrm{L}$ & $40-400 \mathrm{ng} / \mu \mathrm{L}$ & $2-20 \mathrm{ng} / \mu \mathrm{L}$ \\
$10 \mathrm{x}$ SuRE/Cut L buffer & $2 \mu \mathrm{L}$ & $10 \mathrm{x}$ & $1 \mathrm{x}$ \\
KspI & $0.2 \mu \mathrm{L}$ & $10 \mathrm{U} / \mu \mathrm{L}$ & $0.1 \mathrm{U} / \mu \mathrm{L}$ \\
XbaI & $0.2 \mu \mathrm{L}$ & $10 \mathrm{U} / \mu \mathrm{L}$ & $0.1 \mathrm{U} / \mu \mathrm{L}$ \\
DNAase/RNAase free & $12.6 \mu \mathrm{L}$ & & \\
$\mathrm{H}_{2} 0$ & & & \\
\hline Total reaction volume & $\mathbf{2 0} \mu \mathrm{L}$ & & \\
\end{tabular}

Purified DNA samples, including PCR products, restriction digest products and E. coli plasmid extractions, were quantified using the Quant-iT ${ }^{\mathrm{TM}}$ PicoGreen $^{\circledR}$ dsDNA Assay Kit (Invitrogen Molecular Probes, Eugene, Oregon, USA). To each well of a 96-well FluoroNunc $^{\text {TM }}$ plate (NUNC A/S), $1 \mu \mathrm{L}$ of DNA to be quantified was added to $99 \mu 1$ of DNAase/RNAase free $\mathrm{H}_{2} 0$ and $100 \mu \mathrm{L}$ of a 1:200 dilution of PicoGreen reagent.

Samples were incubated for 5 minutes in the dark and then quantified by measuring fluorescence intensity, with excitation at $480 \mathrm{~nm}$ and emission at $520 \mathrm{~nm}$ in the FLUOstar Optima microplate multi-detection reader (BMG Labtech GmbH, Offenburg, Germany). Fluorescence was measure in triplicate and amount of DNA was calculated by comparison with a standard curve ranging from $0.5-200 \mathrm{ng} / \mu \mathrm{L}$ created using bacteriophage lambda DNA supplied with the Quant-iT ${ }^{\mathrm{TM}}$ PicoGreen ${ }^{\circledR}$ dsDNA Assay Kit (Invitrogen Molecular Probes). 
PCR and digests products were purified using the UltraClean ${ }^{\mathrm{TM}}$ PCR Clean-Up Kit (MO BIO). Five volumes of SpinBind buffer, containing guanidine hydrochloride and isopropanol, were added to the pooled PCR or digest product, mixed and transferred to a filter unit. Filter units were centrifuged at $12000 \mathrm{x}$ g for $30 \mathrm{sec}$ to bind DNA in the solution to the filter and filtered solution was discarded. The bound DNA was washed twice with $300 \mu \mathrm{L}$ of SpinClean buffer, containing ethanol, and filtered solution was discarded. Empty filter units were centrifuged at $16000 \mathrm{x}$ g for $60 \mathrm{sec}$ to remove any traces of SpinClean buffer. Fifty $\mu \mathrm{L}$ of DNAase/RNAase free $\mathrm{H}_{2} \mathrm{O}$ was added to the filter, left for $1 \mathrm{~min}$ and centrifuged at $16000 \mathrm{x} \mathrm{g}$ for $60 \mathrm{sec}$ into a fresh eppendorf tube to elute the DNA.

Agarose gel electrophoresis was used to analyse the size and integrity of PCR and digest products. Typically $1-10 \mu \mathrm{L}$ of product was mixed at a 5:1 ratio of product : Loading Buffer (Appendix 8.3) and loaded onto the agarose gel. The gels used were either $2 \%$ or $1.5 \%$ for the $A g 85 A$ PCR product and orientation PCR products or $0.8 \%$ for pKUNrep6 and pKUN85A digest products (Appendix 8.3). Typically $4 \mu \mathrm{L}$ of a DNA ladder (High Mass DNA Ladder, Low Mass DNA Ladder or 100 bp Ladder as appropriate for the anticipated fragment size, all from Invitrogen) was loaded to calculate the size of PCR or digest products. Samples were run at 80-100 V for 1-2 hours to ensure bands of interest were separated. Gels were stained with ethidium bromide in $\mathrm{dH}_{2} \mathrm{O}(500 \mathrm{ng} / \mathrm{mL})$ for $30 \mathrm{~min}$ and destained in $\mathrm{dH}_{2} 0$ for $10-15 \mathrm{~min}$. Gels were visualised using a TFX-35M UV transilluminator (Gibco BRL, Gaithersburg, MD, USA) and the Kodak Digital Science Electrophoresis Documentation and Analysis System 120 (Eastman Kodak Co., Windsor, CO, USA) and fragment size was estimated using the Kodak Digital Science 1D program (Eastman Kodak Co.) 
Plasmid was amplified and purified for cloning, orientation digests and sequencing using the Qiagen Plasmid Mini-prep kit (Qiagen $\mathrm{GmbH}$ ). E. coli containing the target plasmids were inoculated into $5 \mathrm{~mL}$ of LB Amp ${ }^{+}$liquid cultures (Appendix 8.3) and grown for 16 hours, shaking at $225 \mathrm{rpm}$ at $37^{\circ} \mathrm{C}$ in an Innova ${ }^{\mathrm{TM}} 4200$ Incubator. The liquid cultures were centrifuged at $600 \mathrm{x}$ g for $8 \mathrm{~min}$ to pellet the $E$. coli and pellets were frozen overnight.

Pellets were thawed and resuspended in $0.5 \mathrm{~mL}$ of Buffer P1 (50 mM Tris- $\mathrm{HCl}$, pH 8.0; 10mM EDTA; $100 \mu \mathrm{g} / \mathrm{mL})$. A further $0.5 \mathrm{~mL}$ of Buffer P2 $(200 \mathrm{M} \mathrm{NaOH} ; 1 \%$ SDS) was added and samples were inverted 6 times to lyse the cells. After incubation at room-temperature for $5 \mathrm{~min}, 0.5 \mathrm{~mL}$ of Buffer P3 was added, samples were inverted 6 times and incubated on ice for $5 \mathrm{~min}$ to precipitate protein. Samples were centrifuged at $4600 \mathrm{x}$ g for $10 \mathrm{~min}$ to pellet protein precipitate and genomic DNA, and supernatants containing the plasmid DNA were applied to an equilibrated Qiagen-tip 20. The Qiagen-tip 20 was washed four times with $1 \mathrm{~mL}$ of Buffer QC before DNA was eluted from the Qiagen-tip 20 into a fresh $15 \mathrm{~mL}$ tube with $0.8 \mathrm{~mL}$ of Buffer QF. DNA was precipitated with $0.56 \mathrm{~mL}$ of room-temperature isopropanol (BDH) before being pelleted by centrifugation at $4600 \mathrm{x}$ g for $60 \mathrm{~min}$. The supernatant was decanted and the DNA pellet was washed with $1 \mathrm{~mL}$ of $70 \%$ ethanol (BDH) to remove excess salts and isopropanol. The DNA pellet was dried overnight and redissolved in $50 \mu \mathrm{L}$ of DNAase/RNAase free $\mathrm{H}_{2} 0$, typically yielding from 2-20 $\mu \mathrm{g}$ of plasmid DNA per reaction.

Plasmid was amplified and purified for protein expression and vaccination studies using the Qiagen Plasmid Mega-prep Endotoxin-free kit (Qiagen GmbH). E. coli containing the pKUN85A plasmid were inoculated into a $10 \mathrm{~mL}$ starter $\mathrm{LB} \mathrm{Amp}{ }^{+}$liquid culture (Appendix 8.3) and grown for 8 hours, shaking at $225 \mathrm{rpm}$ at $37^{\circ} \mathrm{C}$ in an Innova ${ }^{\mathrm{TM}} 4200$ Incubator. This starter culture was inoculated into $2 \mathrm{~L}_{\text {of }} \mathrm{LB} \mathrm{Amp}{ }^{+}$liquid culture and grown for 16 hours, shaking at $225 \mathrm{rpm}$ at $37^{\circ} \mathrm{C}$ in an Innova ${ }^{\mathrm{TM}} 4200$ Incubator. The liquid culture was pelleted by centrifugation at $600 \mathrm{x}$ g for $15 \mathrm{~min}$ and pellets were frozen overnight. 
The pellet was thawed and resuspended in $55 \mathrm{~mL}$ of Buffer P1 (50 mM Tris$\mathrm{HCl}, \mathrm{pH} 8.0 ; 10 \mathrm{mM}$ EDTA; $100 \mu \mathrm{g} / \mathrm{mL}$ ) in a $1 \mathrm{~L}$ Schott bottle. A further $55 \mathrm{~mL}$ of Buffer P2 (200 M NaOH, $1 \%$ SDS) was added and the suspension was inverted 10 times to lyse the cells. After incubation at room temperature for $5 \mathrm{~min}, 55 \mathrm{~mL}$ of $4^{\circ} \mathrm{C}$ Buffer P3 (3.0 M potassium acetate, $\mathrm{pH}$ 5.5) was added to precipitate protein. The suspension was poured into a QIAfilter Mega Cartridge and left to incubate for $10 \mathrm{~min}$. The suspension was filtered to remove protein precipitate and genomic DNA and the filtrate was treated with $3.75 \mathrm{~mL}$ of ER buffer (isopropanol; polyethylene glycol octyl phenyl ether) to remove endotoxin. The suspension was applied to an equilibrated Qiagen-tip 2500. The Qiagen-tip 2500 was washed with $200 \mathrm{~mL}$ of Buffer QC (1.0 M $\mathrm{NaCl} ; 50 \mathrm{mM}$ MOPS, pH 7.0; 15\% isopropanol). DNA was eluted from the Qiagen-tip 2500 with $35 \mathrm{~mL}$ of Buffer QF (1.6 M NaCl; $50 \mathrm{mM}$ MOPS, pH 7.0; 15\% isopropanol). DNA was precipitated with $24.5 \mathrm{~mL}$ of room-temperature isopropanol before being pelleted by centrifugation at $4600 \mathrm{x}$ g for $60 \mathrm{~min}$. The supernatant was decanted and the DNA pellet was washed with $5 \mathrm{~mL}$ of $70 \%$ ethanol to remove excess salts and isopropanol. The DNA pellet was dried overnight and redissolved in $500 \mu \mathrm{L}$ of DNAase/RNAase free $\mathrm{H}_{2} 0$, typically yielding from $0.7-1.5 \mathrm{mg}$ of plasmid DNA per reaction.

\subsection{Detection of protein expression}

The NIH-3T3 cells are a continuous adherent murine fibroblast-derived cell-line [348]. They were selected to be target cells for transfection with pKUN85A to model protein expression from the construct in mammalian cells. NIH-3T3 cells were plated at $5 \times 10^{4}$ cells $/ \mathrm{mL}$ in 5\% cIMDM with $2 \mathrm{~mL}$ per well in 6-well flat-bottom plates (BD Biosciences Labware) and incubated for 24 hours at $37{ }^{\circ} \mathrm{C}$ with $5 \% \mathrm{CO}_{2}$. After 24 hours, supernatant was aspirated and $1 \mathrm{~mL}$ of incomplete Iscove's Modified Dulbecco's Medium (iIMDM) (Appendix 8.3) was added per well.

Transfection mixtures were made up for each plate. The mixture contained 564 $\mu \mathrm{L}$ of iIMDM, $36 \mu \mathrm{l}$ of room temperature FuGene 6 Transfection Reagent (Roche Diagnostics $\mathrm{GmbH}$ ) and $15 \mu \mathrm{g}$ of a target DNA in a 5-50 $\mu \mathrm{L}$ volume. The FuGene 
Transfection Reagent was added directly to the iIMDM followed by the target DNA. Control mixtures containing FuGene or DNA only were included to test for toxic effects of the FuGene reagent or the target DNA respectively. A control mixture with only iIMDM was also included. The DNA plasmids used in transfection mixtures were pKUNrep6, pKUN85AOF, pcDNA3-85A and pKUN85A. One 6-well plate was used for each transfection mixture and, for each well in the 6 -well plate, $100 \mu \mathrm{L}$ of transfection mixture was gently pipetted in a dropwise circular pattern onto cells before the plates were returned to the incubator for 5 hours. After 5 hours, $1 \mathrm{~mL}$ of $10 \%$ cIMDM was added per well and cells were returned to the incubator for 72 hours. Transfected cells were then lysed and cell lysates were used for immunoprecipitation of the target proteins, NS-1 and Ag85A (section 2.7.2).

After 72 hours of incubation, cell culture supernatants were harvested and individual wells in the 6-well plate were pooled. Cells were also harvested by treatment of each well with $300 \mu \mathrm{L}$ of Gibco Trypsin-EDTA (Invitrogen) and wells were washed out with $2 \mathrm{~mL}$ of 5\% cIMDM then pooled for each 6-well plate. Cells were pelleted by centrifugation at $400 \mathrm{x}$ g for $8 \mathrm{~min}$ and washed three times with $2 \mathrm{~mL}$ of PBS. The cell pellets were resuspended in $300 \mu \mathrm{L}$ of Lysis Buffer (Appendix 8.3) and incubated at $4^{\circ} \mathrm{C}$ for $15 \mathrm{~min}$. Insoluble material was pelleted by centrifugation at $12000 \mathrm{xg}$ for 10 min at $4{ }^{\circ} \mathrm{C}$ and solubilised material in $250 \mu \mathrm{L}$ of the supernatants was transferred to fresh tubes for immunoprecipitation.

To the $250 \mu \mathrm{L}$ of cell lysates, $50 \mu \mathrm{l}$ of anti-mouse IgG beads (eBiosciences Inc.) was added, incubated at $4{ }^{\circ} \mathrm{C}$ for $30 \mathrm{~min}$ and centrifuged at $12000 \mathrm{x}$ g for 3 min at $4{ }^{\circ} \mathrm{C}$ to pre-clear the cell lysate. Supernatants were transferred to fresh tubes and primary antibodies were added that corresponded to primary antibodies to be used for the immunodetection in section 2.15. For NS-1 blots, $25 \mu \mathrm{L}$ of anti-NS-1 antibody was added and for Ag85A blots $50 \mu \mathrm{L}$ of anti-Ag85A antibody was added. Cell lysates were incubated at $4{ }^{\circ} \mathrm{C}$ for $60 \mathrm{~min}$ before $50 \mu \mathrm{l}$ of anti-mouse IgG beads (eBiosciences Inc.) was added. The cell lysates and beads were incubated at $4{ }^{\circ} \mathrm{C}$ for 60 min while gently rotating and centrifuged at $12000 \mathrm{xg}$ for $1 \mathrm{~min}$ at $4{ }^{\circ} \mathrm{C}$ to immunoprecipitate the target 
protein with the pelleted beads. Pellets were washed twice with $300 \mu \mathrm{L}$ of Lysis Buffer and frozen until bead pellets were used for sodium dodecyl sulfate polyacrylamide gel electrophoresis (SDS-PAGE), Western transfer and immunodetection of NS-1 or Ag85A (section 2.7.3).

Bead pellets were thawed and $4 \mu 1$ of Sample Reducing Dye, $10 \mu 1$ of LDS Loading Buffer and $26 \mu \mathrm{L}$ of 1x MOPS Buffer (all from Invitrogen) were added to prepare the samples for electrophoresis. Samples were heated to $95{ }^{\circ} \mathrm{C}$ for $10 \mathrm{~min}$, to denature antimouse IgG antibodies and release the immunoprecipitated protein into solution, and centrifuged at $12000 \mathrm{x}$ g for $3 \mathrm{~min}$ to pellet the beads. Twenty $\mu \mathrm{L}$ of the sample was loaded per well onto a 4-12\% Bis-Tris gel (Invitrogen). Seven $\mu \mathrm{L}$ of the MultiMark multi-coloured standard 1x (Invitrogen) was also loaded onto the gel to estimate protein molecular weight. The gel was run using the X-Cell Sure-Lock ${ }^{\mathrm{TM}}$ Electrophoresis Cell System (Invitrogen), with 1x MOPS Running Buffer (Invitrogen) in the outer reservoir and 1x MOPS Running Buffer and Antioxidant (Invitrogen) in the inner reservoir. The gel was run at $200 \mathrm{~V}$ for $50 \mathrm{~min}$, equilibrated in 1x Western Transfer Buffer (Appendix 8.3).

The gel was then assembled into the X-Cell II ${ }^{\mathrm{TM}}$ Blot Module (Invitrogen) and transferred onto a nitrocellulose membrane (BioRad). The Western transfer was run using 1x Western Transfer Buffer at 100V for 75 min. After transfer, protein was visualised on the nitrocellulose membrane by brief incubation with $0.5 \%$ Ponceau-S solution (Sigma-Aldrich) followed by destain in water, to ensure effective transfer of protein and even loading of samples.

The membrane was blocked by incubation in $15 \mathrm{~mL}$ of Western Blocking Solution (Appendix 8.3) for $60 \mathrm{~min}$ at room temperature while gently rocking. Blocking solution was rinsed off the membrane with Tris Buffered Saline (TBS) (Appendix 8.3) and $5 \mathrm{~mL}$ of primary antibody diluted in TBS was added. For NS-1 blots, the supernatant containing anti-NS-1 antibody was used at a 1:50 dilution. For Ag85A blots, the supernatant containing anti-Ag85A antibody was used neat. The membrane was incubated in primary antibody overnight at $4{ }^{\circ} \mathrm{C}$ on a rocker, then washed five times with $15 \mathrm{~mL}$ of TBS for $5 \mathrm{~min}$. Five $\mathrm{mL}$ of secondary anti-native mouse IgG-HRP 
antibody (eBiosciences Inc.) diluted in TBS was added. For NS-1 blots and Ag85A blots, the secondary anti-native mouse IgG-HRP antibody was used at a 1:1000 dilution. Proteins bands were detected with addition of $2 \mathrm{~mL}$ of SuperSignal ${ }^{\circledR}$ West Pico ChemiLuminescent substrate (Pierce) and exposure of the membrane to Kodak EKTASCAN B/RA film (Eastman Kodak Co.). Film was exposed from 1 min to 8 hours depending of the sensitivity of the assay and developed in a Kodak X-OMAT 1000 processor (Eastman Kodak Co.).

\subsection{Statistical analysis}

Normal distribution of variation within in vivo experimental groups was not assumed and therefore statistical significance between treatment groups or between experimental time points was determined by the non-parametric two-tailed Mann-Whitney test. Differences that gave $\mathrm{p}$ values $\leq 0.05$ were considered statistically significant. For flow cytometric, $[3 \mathrm{H}]$ thymidine proliferation, ELISA and pathology scoring data, graphs represent mean \pm the standard error of the mean (SEM). For data assessing bacterial numbers in the tissues, graphs represent geometric mean \pm the standard error of the geometric mean (GEM). 
Chapter 3: Suppression by natural Tregs during primary mycobacterial infection 


\subsection{Introduction}

M. tuberculosis has evolved numerous ways to subvert the immune response and promote its survival in the infected host (section 1.2.5). A further way to escape host immune responses, exploited by many infectious organisms, is to enhance of Treg function in the infected host $[305,334,335,349]$. Therefore, I chose to investigate whether M. tuberculosis also exploits the host natural Treg population to suppress the immune response and thereby enhance survival of $M$. tuberculosis in the infected host.

\subsubsection{Tools for in vivo study of Tregs}

Before it could be examined whether natural Tregs suppressed the host immune response to $M$. tuberculosis infection, a protocol for manipulating natural Treg activity in vivo had to be selected and optimised. Natural Tregs, conventionally defined as $\mathrm{CD} 4{ }^{+} \mathrm{CD} 25^{+} \mathrm{T}$ cells in a naïve mouse [261], can be functionally inactivated in vivo by the administration of anti-CD25 mAbs [350]. Two anti-CD25 mAbs from different clones have been used in vivo: PC61 and 7D4. PC61 is a rat anti-mouse CD25 IgG1 antibody and 7D4 is a rat anti-mouse CD25 IgM antibody. Each mAb targets distinct epitopes on CD25 [343, 344]. Different doses of both antibodies have been used in different studies, ranging from $125 \mu \mathrm{g}$ to $1 \mathrm{mg}$ per mouse [333, 351, 352]. In vivo administration of either antibody has been shown to decrease the number of $\mathrm{CD} 4{ }^{+} \mathrm{CD} 25^{+} \mathrm{T}$ cells in peripheral blood and tissues as detected by flow cytometry, and promote autoimmune disease [350].

Both PC61 and 7D4 were initially regarded as depleting antibodies, but recent studies suggest that 7D4 predominantly inactivates rather than depletes Tregs [353]. The mechanism of action of PC61 seems to be a combination of depleting and inactivating Tregs [352] and the implications of these studies on the results presented in this chapter will be addressed in the discussion. CD25 is also transiently upregulated on activated T cells [354] and it has been suggested that anti-CD25 mAbs administered during an active immune response could also target recently activated $\mathrm{CD} 4^{+} \mathrm{CD} 25^{+}$ effector $\mathrm{T}$ cells for depletion or inactivation.

GITR is another cell surface marker upregulated on both Tregs and activated effector T cells and GITR, with its ligand GITRL, are thought to play a key role in 
modulating Treg activity [355]. The current model of GITR/GITRL signalling suggests that when GITRL on an APC interacts with GITR on a responder T cell, it delivers a costimulatory signal to the responder $\mathrm{T}$ cell that promotes proliferation and makes it initially refractory to the suppressive effects of Tregs [356].

Two antibodies are used to modulate the GITR/GITRL signalling pathway: DTA-1 and 5F-1. DTA-1 is a rat anti-mouse GITR antibody and when used in vitro, it abrogates the ability of $\mathrm{CD} 4{ }^{+} \mathrm{CD} 25^{+}$Tregs to suppress the proliferation of naïve $\mathrm{CD} 4^{+} \mathrm{CD} 25^{-} \mathrm{T}$ cells upon TCR stimulation [357]. Therefore, DTA-1 is thought to be an agonistic antibody that signals through GITR to make responder T cells refractory to the suppressive effects of Tregs [355]. 5F-1 is an anti-GITRL antibody and when used in vitro, it enhances suppression of $\mathrm{CD} 4^{+} \mathrm{CD} 25^{-} \mathrm{T}$ cells by $\mathrm{CD} 4^{+} \mathrm{CD} 25^{+}$Tregs [356]. Thus, $5 \mathrm{~F}-1$ is thought to be an antagonistic antibody that binds to GITRL on APCs and prevents it from signalling through GITR on responder T cells [355].

Therefore, tools like anti-CD25, -GITR and -GITRL mAbs can be used to identify and in some cases manipulate Tregs in vivo. However, there are some limitations inherent in their use and these limitations will be outlined and discussed during the course of this chapter.

\subsection{2 $\mathrm{T}$ regulatory cells and infections}

The survival of many infectious organisms in the host can improve as a result of natural Treg action [305, 330, 333, 334]. Antibody-mediated inactivation of Tregs, or interference with their proposed mechanisms of action, can impact greatly on the degree of tissue damage, pathogen load and the development of immunological memory. Infection of mice with L. major was the first well-defined example of Treg involvement in the maintenance of a chronic infection [305].

L. major is a protozoal organism that establishes an intracellular infection of macrophages in the skin. While the infection in C57BL/6 mice is controlled by a Th1 immune response, the protozoa are not entirely eliminated. $\mathrm{CD} 4{ }^{+} \mathrm{CD} 25^{+}$Tregs are present in these chronic skin lesions and are essential to suppress the local immune response and permit pathogen persistence [305]. In addition, a proposed Treg suppressive mechanism is the secretion of IL-10, and blockade of the IL-10 receptor 
enhanced the immune response, resulting in complete clearance of L. major from the site of infection $[305,358]$. However, while it has been shown that $\mathrm{CD} 4^{+} \mathrm{CD} 25^{+} \mathrm{Foxp}^{+}$ Tregs are found in L. major lesions and can produce IL-10 [305, 359], the bulk of IL-10 is produced by $\mathrm{CD} 4^{+} \mathrm{CD} 25^{-} \mathrm{Foxp} 3^{-}$effector T cells that may co-produce cytokines such as IFN- $\gamma[360,361]$. Therefore, the current model for Treg involvement in L. major infection is that $\mathrm{CD} 4^{+} \mathrm{CD} 25^{+} \mathrm{Foxp}^{+}$Tregs located in the lesion suppress the effector immune response by directing $\mathrm{CD} 4^{+} \mathrm{CD} 25^{-} \mathrm{Foxp}^{-}$effector T cells to produce IL-10.

Prolonged or increased disease as a result of Treg activity has also been demonstrated for other infectious agents including Plasmodium berghei [333], Plasmodium yoelii [334], Litomosoides sigmodontis [330], and hepatitis C virus [349]. Treatment with anti-CD25 mAbs prior to infection can result in increased immunopathology in some models $[331,362]$ but can also result in increased pathogen clearance $[330,333,334] . \mathrm{CD}^{+} \mathrm{CD} 25^{+} \mathrm{Foxp}^{+}$Treg activity is often associated with chronic infections and it has been hypothesised that chronic infectious agents in particular can promote Treg activation, thereby hijacking this host immune mechanism to suppress the immune response and permit long-term immunoevasion.

Immunosuppression mediated by Tregs can increase pathogen load during infection, but it can also benefit the host by preventing over-activation of effector immune mechanisms and immunopathology $[331,332]$. Helicobacter pylori infection can cause chronic gastritis and natural Tregs have been shown to prevent the development of this pathology in a murine model [332]. Athymic nude mice were reconstituted with total lymphocytes from C57BL/6 or lymphocytes depleted of $\mathrm{CD} 25^{+}$ cells, and then infected with $H$. pylori. Mice that received lymphocytes depleted of natural $\mathrm{CD} 4{ }^{+} \mathrm{CD} 25^{+}$Tregs had increased IFN- $\gamma$ responses to $H$. pylori antigens and reduced $H$. pylori colonisation of the gut compared to mice that received total lymphocytes. However, the mice that received lymphocytes depleted of natural $\mathrm{CD} 4^{+} \mathrm{CD} 25^{+}$Tregs also had earlier onset and enhanced severity of gastritis compared to mice that received total lymphocytes. Enhanced immunopathology with depletion of Tregs has been observed with other infections such as ocular lesion formation in herpes simplex virus (HSV)-1 infection [331].

Therefore, suppression by Tregs during an infection can be detrimental to the host by increasing pathogen load, and beneficial to the pathogen by permitting its persistence. However, suppression by Tregs during an infection can also benefit the host 
by preventing either over-activation of the immune response or suppressing a persistent immune response, both of which could cause host tissue immunopathology.

\subsubsection{T regulatory cells and Tuberculosis}

During early studies of suppressor T cells, it was observed that PBMCs from human subjects that were incubated with BCG in vitro acquired a suppressive phenotype [363]. These cells were $\mathrm{CD}^{+}$, sensitive to irradiation and their suppressive phenotype enabled them to inhibit proliferation of fresh PBMCs from human subjects corresponding with a decrease in IL-2 production [363, 364]. Subsequently, the concept of suppressor T cells became unfavoured; as did the idea that a suppressive lymphocyte subset could regulate immune responses to mycobacteria.

However, more recent studies of human $\mathrm{Tb}$ patients suggest that a suppressive lymphocyte subset could inhibit the immune response during M. tuberculosis infection. A Cambodian study revealed a subset of chronically infected patients who were unresponsive to the Mantoux skin test [365]. Lymphocytes from these patients proliferated poorly in response to in vitro stimulation with mycobacterial antigen, and had decreased levels of IL-2 and enhanced IL-10 secretion. Since Tregs can secrete IL10 and inhibit lymphocyte proliferation, these observations are consistent with the proposed mechanism and effects of Tregs. Furthermore, studies have demonstrated that patients with active $M$. tuberculosis infections have increased proportions of $\mathrm{CD} 4{ }^{+} \mathrm{CD} 25^{+}[366,367]$ and $\mathrm{CD} 4{ }^{+} \mathrm{CD} 25^{+}$Foxp $^{+}$Tregs [368-371] in their PBMCs and disease sites compared to uninfected and, in some studies, latently infected individuals. Depletion of CD25 $5^{+}$lymphocytes from PBMC samples prior to in vitro restimulation with mycobacterial antigen led to increased IFN- $\gamma$ production $[366,367,372]$. However, the addition of IL-10 or TGF- $\beta$ neutralising antibodies to in vitro restimulations did not affect IFN- $\gamma$ production [372]. This suggests that a CD25 leukocyte population is responsible for suppression of IFN- $\gamma$ production during active Tb infection via IL-10- and soluble TGF- $\beta$-independent mechanisms.

If Tregs suppressed the immune response during active $\mathrm{Tb}$ infection, it had been speculated that depletion of Tregs in vivo would relieve this suppression and result in better control or resolution of the infection [373]. Human Tb studies demonstrated increased IFN- $\gamma$ production in vitro by PBMCs depleted of CD $25^{+}$leukocytes, but the 
effect of Tregs in vivo on bacterial numbers in tissue had not been explored.

Additionally, excess IFN- $\gamma$ production can be damaging and enhanced IFN- $\gamma$ production in the absence of Tregs could enhance lung pathology. Therefore, it was unresolved as to whether the increased numbers of Tregs observed in PBMCs of Tb patients affected outcomes of $\mathrm{Tb}$ infection, such as bacterial numbers in the lung or tissue pathology. In order to address these questions, I employed in vivo PC61 mAb treatment to inactivate Tregs in a murine model prior to mycobacterial infection and evaluated the ensuing immune responses and protection from infection. 


\subsection{Aims}

The results presented in this chapter aimed to assess whether natural Tregs inhibit the acute response to mycobacterial infection and facilitate mycobacterial survival.

Given the impact of Tregs during other infections, I hypothesised that inactivation of $\mathrm{CD} 4{ }^{+} \mathrm{CD} 25^{+}$Tregs prior to mycobacterial infection would increase the acute immune response and decrease bacterial numbers in the tissues, but could cause increased immunopathology in the lung.

To test this hypothesis, $\mathrm{CD} 4^{+} \mathrm{CD} 25^{+}$Tregs were inactivated in the $\mathrm{C} 57 \mathrm{BL} / 6$ murine model of $\mathrm{Tb}$ infection through administration of the anti-CD25 mAb (PC61) prior to infection with mycobacteria. Thereafter, the immune response and outcomes of infection, such as bacterial numbers in tissues and lung pathology, were assessed. My main objectives were to:

- Optimise and characterise in vivo inactivation of $\mathrm{CD} 4^{+} \mathrm{CD} 25^{+}$Tregs by administration of PC61.

- Characterise the acute immune response to mycobacterial infection after inactivation of $\mathrm{CD} 4^{+} \mathrm{CD} 25^{+}$Tregs.

- Characterise the outcomes of mycobacterial infection after inactivation of $\mathrm{CD} 4^{+} \mathrm{CD} 25^{+}$Tregs, such as tissue bacterial numbers and lung pathology.

These outcomes were assessed using several mycobacterial infections: BCG, M. bovis and M. tuberculosis. Modulation of Treg activity during the chronic phase of infection was also explored, as Tregs are particularly relevant in other chronic infections for promoting pathogen persistence.

Results from this chapter were published as the first assessment of immunosuppression by natural Tregs in an in vivo model of $\mathrm{Tb}$ [374]. 


\subsection{Results}

\subsubsection{Treg inactivation in the lung and other tissues}

In order to set up an in vivo model assessing natural Treg-mediated suppression during mycobacterial infection, an appropriate dose of the anti-CD25 mAb, PC61, was chosen. Published studies have used $1 \mathrm{mg}$ [351], $200 \mu \mathrm{g}$ [352] and $125 \mu \mathrm{g}$ [333] doses of PC61 per mouse. A lower $100 \mu \mathrm{g}$ dose of PC61 per mouse had been used to inactivate Tregs in vivo (personal communication, R.S. McHugh) and was found to be sufficient to inactivate Tregs (Figs. 3.1-3.4). This lower dose of PC61 was selected with the aim of minimising persistence of the antibody in vivo, to minimise any effect of PC61 on activated effector T cells that could transiently express CD25 [354] after mycobacterial infection.

Before it could be assessed whether natural Tregs suppress immune responses and bacterial clearance during mycobacterial infection, the process of inactivating Tregs prior to infection had to be validated. The route of administration of PC61 needed to be optimised and Treg inactivation had to be assessed in lymphoid and non-lymphoid tissues.

Two routes of administration for PC61 antibody were compared to determine how to most effectively inactivate $\mathrm{CD} 4{ }^{+} \mathrm{CD} 25^{+}$Tregs in the lung tissue, since the lung is the primary site of mycobacterial infection. Administration of PC61 i.p. is routinely used because the antibody enters the lymphatics and venous drainage, enabling broad distribution through multiple tissues. Administration of PC61 i.n. would deliver the antibody directly to the lung tissue, which is the primary site of pulmonary mycobacterial infection.

To determine the most effective route of administration, PC61 was administered i.p., i.n. or both i.p. and i.n. $\mathrm{CD} 4{ }^{+} \mathrm{CD} 25^{+} \mathrm{Foxp} 3^{+}$Treg presence in the lung tissue was evaluated by flow cytometry two days later (Fig. 3.1 A). When lymphocytes were stained for CD25 expression in preparation for flow cytometry, the anti-CD25 mAb clone 7D4 was used. The 7D4 clone recognises a different epitope within the CD25 protein to the epitope that PC61 recognises $[343,344]$ and thereby should minimise epitope masking when labeling cells from mice treated with PC61. 
A.

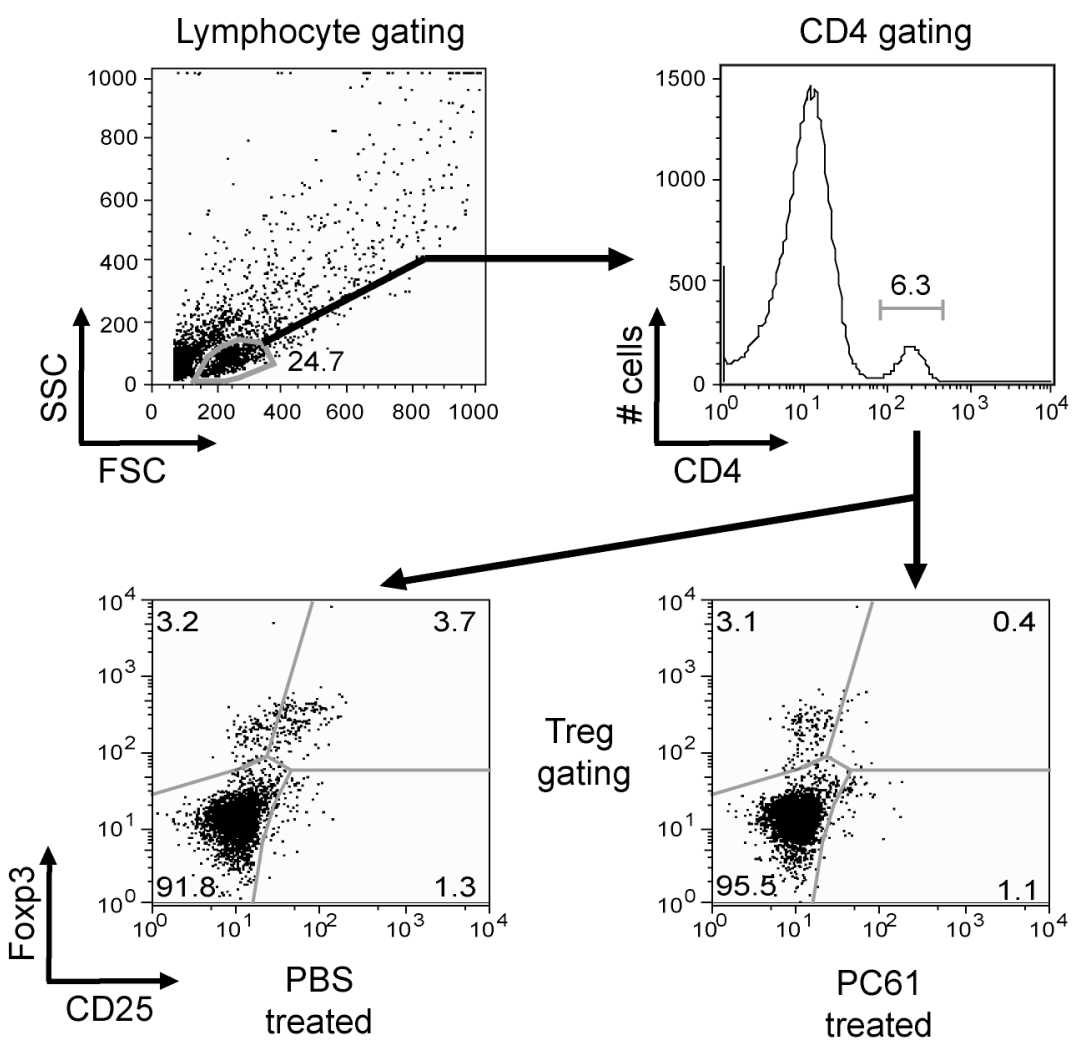

B.

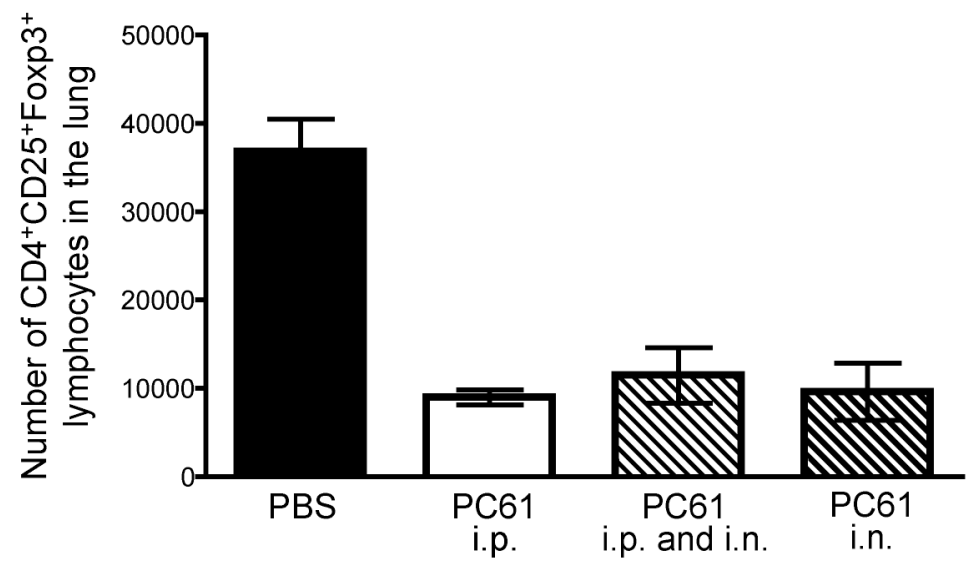

Treatment groups

Figure 3.1: Optimisation of the route of PC61 administration

Mice were treated with either PBS, $100 \mu \mathrm{g}$ of PC61 in $200 \mu \mathrm{L}$ of PBS i.p., $100 \mu \mathrm{g}$ of PC61 in $50 \mu \mathrm{L}$ of PBS i.n., or $50 \mu \mathrm{g}$ of PC61 in $200 \mu \mathrm{L}$ of PBS i.p. as well as $50 \mu \mathrm{g}$ of PC61 in $50 \mu \mathrm{L}$ of PBS i.n. Two days later, lung lymphocytes were isolated and $\mathrm{CD} 4^{+} \mathrm{CD} 25^{+} \mathrm{Foxp} 3^{+} \mathrm{T}$ cells were identified by flow cytometry. A) Sample flow cytometry plots showing lymphocyte gating, CD4 gating and CD25 (using anti-CD25 mAb clone 7D4) and Foxp3 staining of $\mathrm{CD}^{+}$gated lymphocytes from lungs of mice treated with PBS or PC61 i.p. B) Number of $\mathrm{CD} 4^{+} \mathrm{CD} 25^{+} \mathrm{Foxp}^{+}{ }^{+} \mathrm{T}$ cells in the lung after the different PC61 administration routes. Bars represent mean values \pm SEM. Five animals were analysed per treatment group. Results represent one experiment. 
All routes of administration resulted in significant but similar reductions in the average number of $\mathrm{CD}^{+} \mathrm{CD} 25^{+} \mathrm{Foxp}^{+}$Tregs in the lung (Fig 3.1 B). However, i.n. administration of PC61 increased the variation in $\mathrm{CD}^{+} \mathrm{CD} 25^{+} \mathrm{Foxp}^{+}$Treg numbers in the lung as detectable by flow cytometry (Fig 3.1 B). The cause of variation was not assessed but could be due to loss of antibody during i.n. administration, as some antibody solution could enter the oesophagus rather than the trachea and the lung. Regardless of the cause of variation associated with i.n. PC61 administration, i.p. administration was chosen as the most consistent way to inactivate natural Tregs in vivo in the lung.

While i.p. administration of PC61 was found to effectively reduce numbers of $\mathrm{CD} 4{ }^{+} \mathrm{CD} 25^{+} \mathrm{Foxp}^{+}$Tregs in the lung, the ability of PC61 to inactivate CD ${ }^{+} \mathrm{CD} 25^{+}$ Tregs across different tissues remained to be assessed. Mice were treated with either PC61 or control rat IgG antibody and percentages of Tregs were evaluated in several tissues by flow cytometry 3 days later. The percentages of Tregs were compared in the blood, the spleen to represent lymphoid tissue and the lung to represent a peripheral tissue and the primary site of mycobacterial infection (Fig. 3.2).

The percentage of $\mathrm{CD} 4^{+}$lymphocytes that were $\mathrm{CD} 25^{+}$was significantly reduced in all tissues in PC61 treated mice compared to control treated mice: a 74\% reduction in the blood, a $70 \%$ reduction in the spleen and a $61 \%$ reduction in the lung (Fig. 3.2 A). The presence of Foxp3 $3^{+}$lymphocytes was also evaluated by flow cytometry. The percentage of $\mathrm{CD} 4^{+}$lymphocytes that were $\mathrm{CD} 25^{+}$and Foxp $3^{+}$was also significantly reduced in all tissues in PC61 treated mice compared to control treated mice: an $89 \%$ reduction in the blood, a $91 \%$ reduction in the spleen and a $93 \%$ reduction in the lung. The reduction in $\mathrm{CD} 4^{+} \mathrm{CD} 25^{+} \mathrm{Foxp}^{+}$lymphocytes was more pronounced than for total $\mathrm{CD} 4{ }^{+} \mathrm{CD} 25^{+}$lymphocytes, suggesting that PC61 targets the Foxp ${ }^{+}$Treg subset more effectively within the $\mathrm{CD} 4^{+} \mathrm{CD} 25^{+}$population (Fig. 3.2 B). Unsurprisingly, since PC61 targets the CD25 surface molecule, there was no significant difference in the percentage of $\mathrm{CD} 4^{+}$lymphocytes that were $\mathrm{CD} 25^{-}$and $\mathrm{Foxp}^{+}$in any tissue analysed (Fig. 3.2 C). Of note, $\mathrm{CD} 4^{+} \mathrm{CD} 25^{-}$Foxp $3^{+}$lymphocytes made up a higher proportion of the $\mathrm{CD} 4^{+}$lymphocyte population in tissues such as the spleen or lung than in the blood (Fig. 3.2 C) and it is important to note that these cells, which possess Treg function [282], were still present after PC61 treatment. 
A.

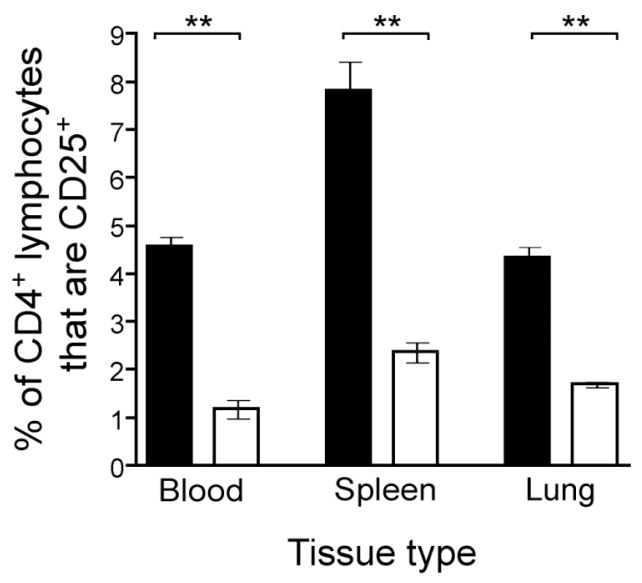

B.

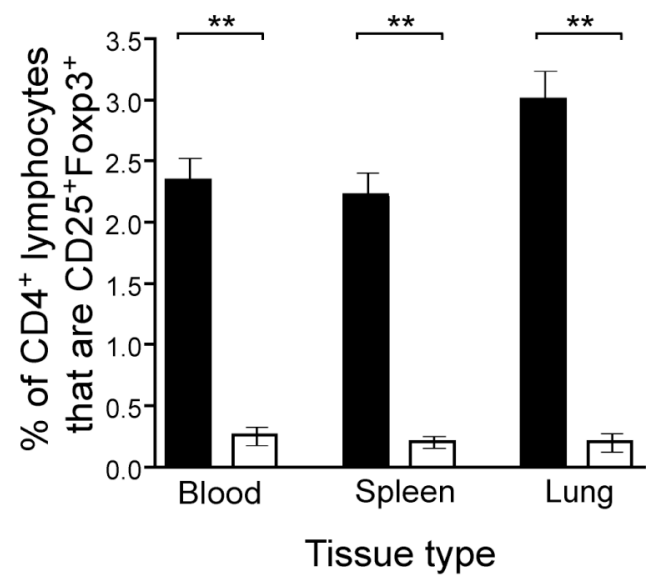

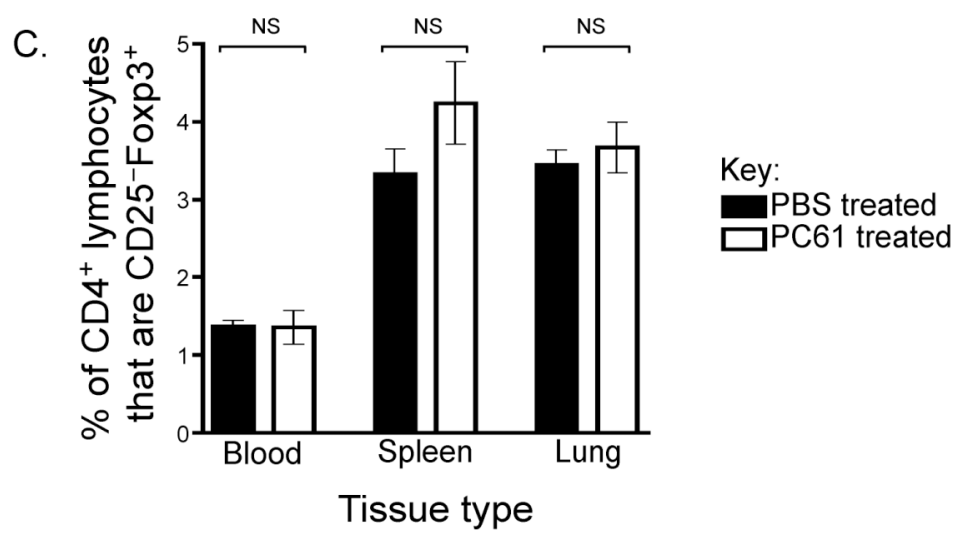

Figure 3.2: Treg inactivation in the blood, spleen and lung after PC61 treatment

Mice were treated with PBS (filled bars) or $100 \mu \mathrm{g}$ of PC61 (open bars) i.p. Three days later, lymphocytes were isolated from the peripheral blood, spleen and lung, counted and stained for CD4, CD25 (using anti-CD25 mAb clone 7D4) and Foxp3. Samples were analysed by flow cytometry for the percentage of $\mathrm{CD} 4^{+}$lymphocytes that were A) $\mathrm{CD} 25^{+}$, B) $\mathrm{CD} 25^{+} \mathrm{Foxp} 3^{+}$and C) $\mathrm{CD} 25^{-} \mathrm{Foxp}^{+}$. Bars represent mean values \pm SEM. Five animals were analysed per treatment group. Results are representative of two replicate experiments. Treatment groups were compared using the two-tailed Mann Whitney test; NS indicates $\mathrm{p}>0.05, * *$ indicates $\mathrm{p} \leq 0.01$. Results are representative of two replicate experiments. 


\subsubsection{Repopulation by Tregs after PC61 administration}

After PC61 treatment, $\mathrm{CD} 4^{+} \mathrm{CD} 25^{+} \mathrm{Foxp}^{+}$lymphocytes as detected by flow cytometry are dramatically reduced. However, $\mathrm{CD} 4^{+} \mathrm{CD} 25^{+} \mathrm{Foxp}^{+}$Tregs eventually repopulate the periphery, either after homeostatic proliferation, expression of CD25 by $\mathrm{CD}^{+} \mathrm{CD} 25^{-} \mathrm{Foxp}^{+}$Tregs or after reconstitution from the thymus. To assess the effect of Tregs on mycobacterial infection, $\mathrm{CD} 4^{+} \mathrm{CD} 25^{+}$Tregs had to remain inactivated after PC61 treatment during the course of the infection. Therefore, Treg repopulation in the periphery after PC61 treatment was followed.

Mice were treated with either PC61 or control rat IgG antibody and peripheral blood samples were analysed for the presence of $\mathrm{CD} 4{ }^{+} \mathrm{CD} 25^{+}$Tregs by flow cytometry up to day 56 after initial antibody treatment. Foxp3 was not assessed, as an anti-Foxp3 $\mathrm{mAb}$ for flow cytometry was not commercially available at the time these experiments were conducted. The mean percentage of $\mathrm{CD} 4^{+}$lymphocytes that were $\mathrm{CD} 25^{+}$was significantly reduced in peripheral blood samples for up to 29 days after PC61 treatment (Fig. 3.3 A). From day 23 after PC61 treatment, some mice within the PC61 treated group exhibited higher percentages of Tregs compared to earlier time points, such as day 5 after PC61 treatment (Fig. 3.3 B/C). This led to increased variation in percentages of Tregs within the PC61 treated group from day 23 (Fig. 3.3 A) and indicated that mice began repopulation of the Treg lymphocyte compartment from around day 23 after PC61 treatment.

Since Treg repopulation of the periphery may be altered by homeostatic cues, such as cytokine release during an active immune response, Treg repopulation in peripheral blood during mycobacterial infection was also followed.

Mice were treated with PC61 or control rat IgG antibody and infected with BCG i.n. 3 days later. Peripheral blood samples were taken up to 56 days after treatment and samples were analysed for the presence of $\mathrm{CD} 4^{+} \mathrm{CD} 25^{+}$lymphocytes by flow cytometry. The mean percentage of $\mathrm{CD}^{+}$lymphocytes that were $\mathrm{CD} 25^{+}$was significantly reduced in peripheral blood samples for up to 29 days after PC61 treatment compared to control treated mice (Fig. 3.3 A). Similar to Treg repopulation observed in the naïve mouse, mice within the PC61 treated group infected with BCG began to exhibit increased variation from day 23 after treatment (Fig. $3.3 \mathrm{~A} / \mathrm{B} / \mathrm{C}$ ), indicating that Treg repopulation also began in these mice around day 23. 
A.

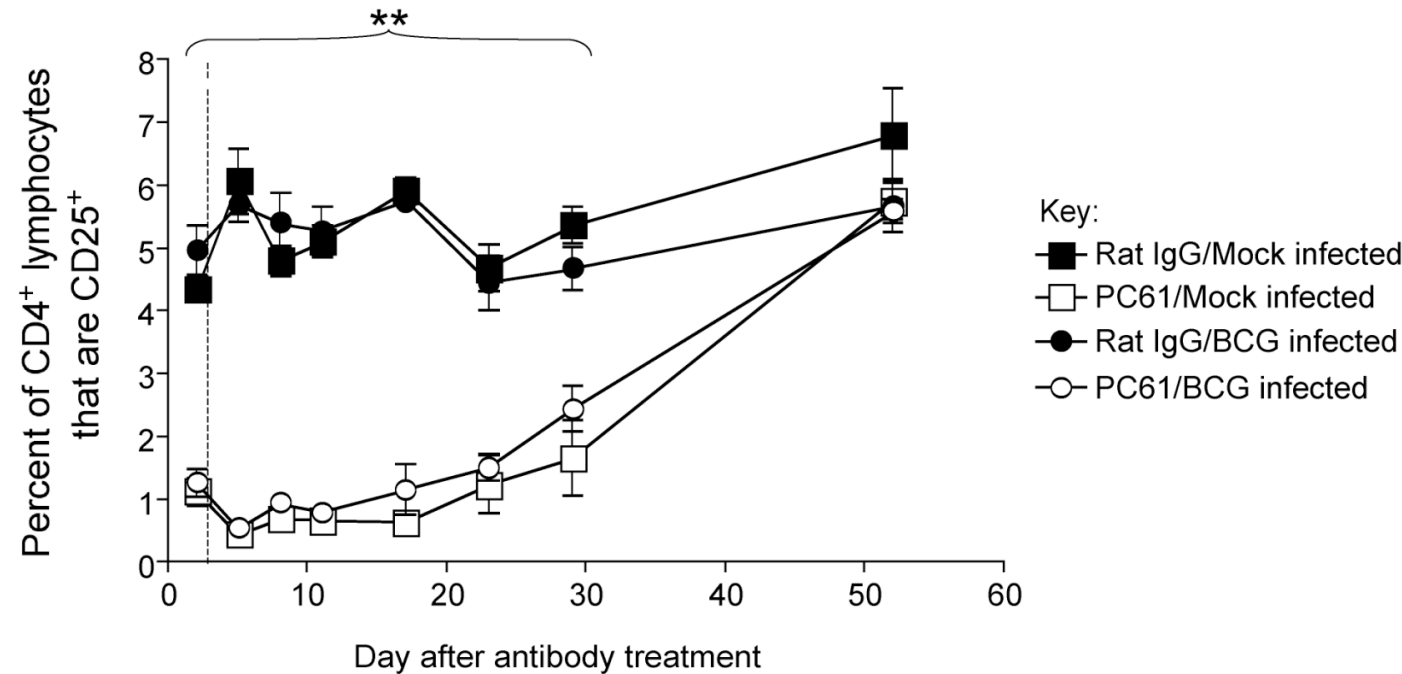

B.

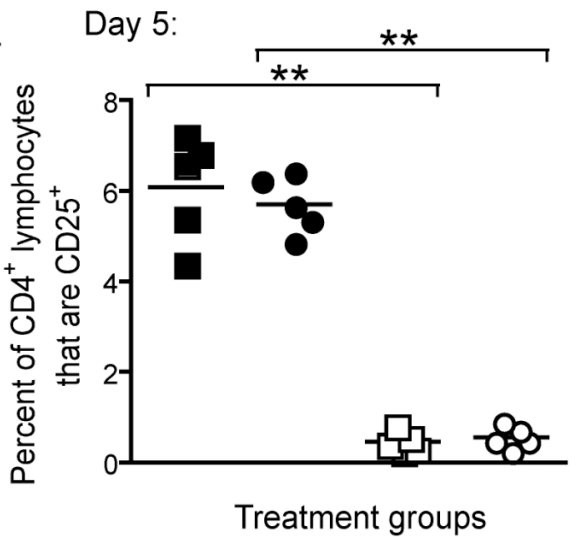

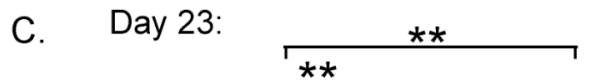

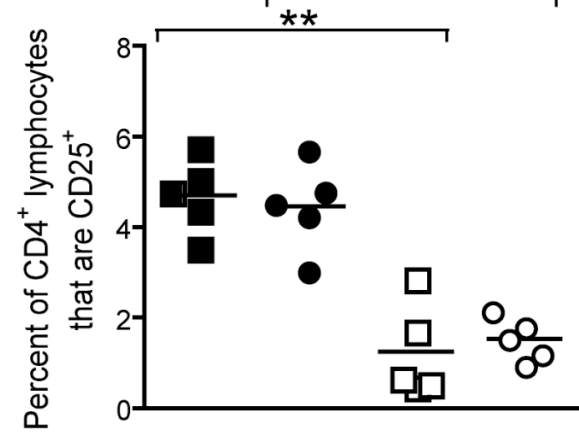

Treatment groups

Figure 3.3: Treg repopulation in peripheral blood of naïve or mycobacteria-infected mice

Groups of mice were administered either $100 \mu \mathrm{g}$ of rat IgG (filled symbols) or $100 \mu \mathrm{g}$ of PC61 (empty symbols) i.p. In addition, groups of mice were either mock infected with PBS (squares) or infected with BCG (circles) i.n. three days (dashed line) after antibody treatment. Treg repopulation of peripheral blood was followed up to day 56. Peripheral blood samples were analysed for the presence of $\mathrm{CD} 4{ }^{+} \mathrm{CD} 25^{+}$lymphocytes by flow cytometry. For surface staining of $\mathrm{CD} 25$, the anti-CD25 mAb clone 7D4 was used. A) $\mathrm{CD} 4{ }^{+} \mathrm{CD} 25^{+}$repopulation in peripheral blood. Symbols represent mean values \pm SEM. $\mathrm{CD} 4^{+} \mathrm{CD} 25^{+}$lymphocytes were significantly reduced in PC61 treated mice compared to control treated mice until day 29 after PC61 treatment in both infected and uninfected mice. Scatter plots of the percent of CD4 ${ }^{+}$ lymphocytes that were $\mathrm{CD} 25^{+}$at B) day 5 and C) day 23 after PC61 treatment. Symbols represent percentages from individual mice and lines represent mean values for each treatment group. Five animals were analysed per treatment group at each time point. Treatment groups were compared using the two-tailed Mann Whitney test; ** indicates $\mathrm{p} \leq 0.01$. Results represent one experiment. 
In the previous experiments, Treg repopulation was monitored in the peripheral blood but Treg repopulation may be different in the periphery, as Tregs could be recruited into sites of active inflammation. In order to address this issue, mice were treated with PC61 or control rat IgG antibody and infected 3 days later with BCG i.n. (Fig. 3.4 A) or $M$. tuberculosis by aerosol (Fig. 3.4 B). Lung lymphocytes were analysed for the presence of $\mathrm{CD} 4^{+} \mathrm{CD} 25^{+} \mathrm{Foxp}^{+}$lymphocytes during early infection by flow cytometry. Numbers of $\mathrm{CD} 4^{+} \mathrm{CD} 25^{+} \mathrm{Foxp}^{+}$lymphocytes were significantly reduced in the lung in PC61 treated mice compared to control treated mice up to day 24 after antibody treatment for BCG infection (Fig. 3.4 A) and day 17 after antibody treatment for M. tuberculosis infection (Fig. 3.4 B).

At the outset of these experiments, a low dose of PC61 mAb was chosen in order to effectively inactivate Tregs but minimise antibody persistence in vivo. There was speculation that, if anti-CD25 mAbs persist in vivo, they could interfere with effector $\mathrm{T}$ cell responses, as recently activated effector $\mathrm{T}$ cells transiently upregulate CD25 [354]. The persistence of PC61 in vivo after a $100 \mu \mathrm{g}$ dose had not been documented.

To assess the persistence of the PC61 mAb in circulation in vivo, mice were treated with PC61 and serum was harvested immediately prior to PC61 treatment and at days $3,10,14$, and 21 after treatment. Serum was tested by ELISA to assess the serum concentration of PC61 and this was used to estimate the total amount of PC61 per mouse in circulation at each time point. Mice were treated with a $100 \mu \mathrm{g}$ dose of PC61 $\mathrm{mAb}$ and the limit of detection for the ELISA was equivalent to $0.014 \mu \mathrm{g}$ of PC61 per mouse. At day 3 after PC61 treatment, the mean amount of PC61 detected per mouse was $13.4 \mu \mathrm{g}$ (Fig. 3.5). By day 10, this had dropped to $5 \mu \mathrm{g}$ per mouse and by days 14 and 21 the amount of PC61 per mouse was very low and usually below the limit of detection for the ELISA (Fig. 3.5). Therefore, after a $100 \mu \mathrm{g}$ dose of PC61, the antibody was detectable in circulation up to day 10 after administration but was generally undetectable by day 14 after administration. PC61 could potentially affect activation of effector T cells up to 14 days after PC61 administration and the implications of this for the results presented in this thesis are outlined in section 3.4.3. 
A.

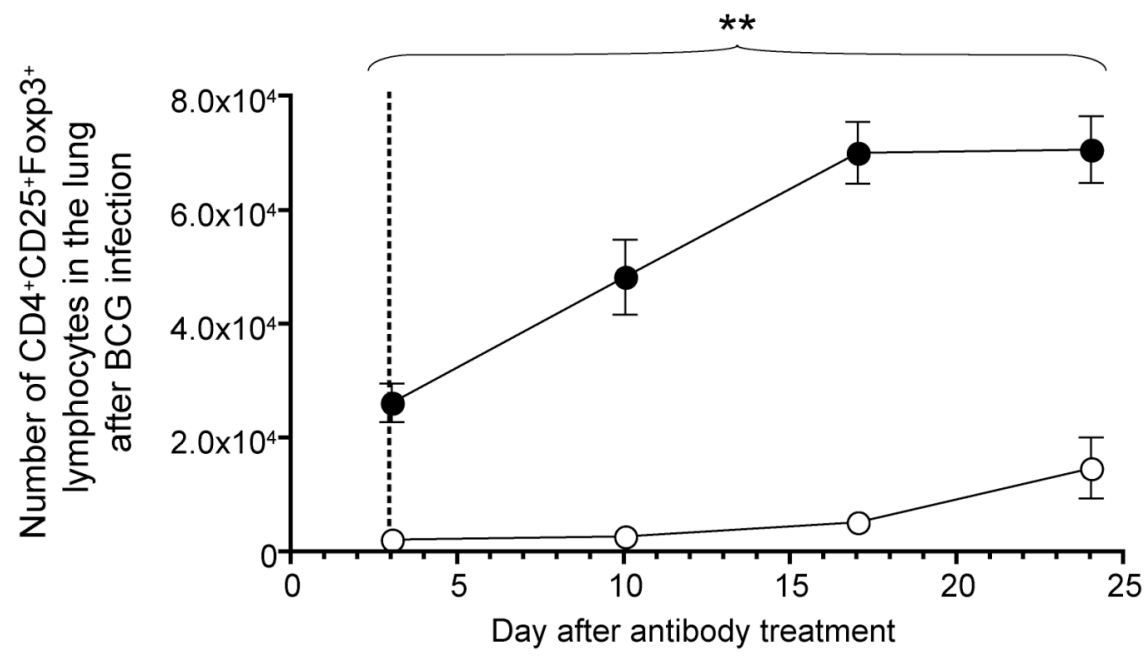

B.

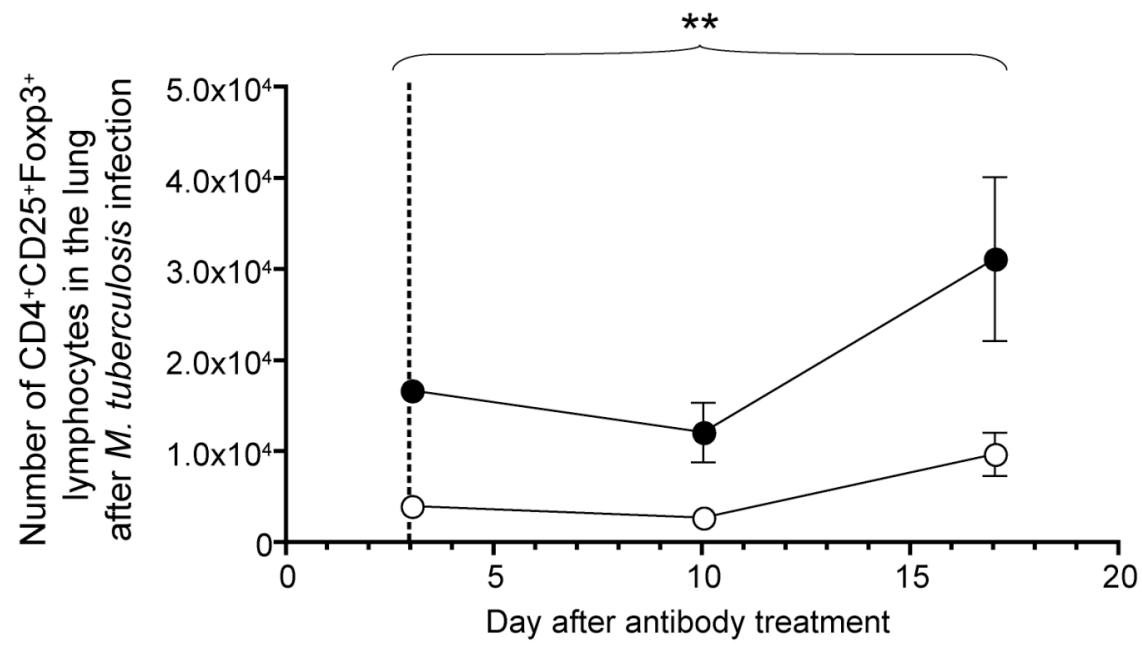

Key:

- Rat IgG treated

- PC61 treated

Figure 3.4: Treg repopulation in the lung after mycobacterial infection

Mice were administered $100 \mu \mathrm{g}$ of rat $\operatorname{IgG}$ (filled circles) or $100 \mu \mathrm{g}$ of PC61 (empty circles) i.p. and infected i.n. with BCG or infected with M. tuberculosis by aerosol three days (dashed line) after antibody treatment. Lung lymphocytes were isolated and analysed for the presence of $\mathrm{CD}^{+} \mathrm{CD} 25^{+} \mathrm{Foxp}^{+}$lymphocytes by flow cytometry and Treg influx to the lungs was followed. Surface staining for CD25 used the anti-CD25 mAb clone 7D4. The number of $\mathrm{CD} 4{ }^{+} \mathrm{CD} 25^{+} \mathrm{Foxp}^{+}{ }^{+}$lymphocytes in the lung during A) BCG infection or B) M. tuberculosis infection. Symbols represent mean values \pm SEM, and some symbols obscure error bars. Four to five animals were analysed per treatment group at each time point. Treatment groups were compared using the two-tailed Mann Whitney test and there were significant differences at all time points; $* *$ indicates $\mathrm{p} \leq 0.01$. Results represent single experiements. 


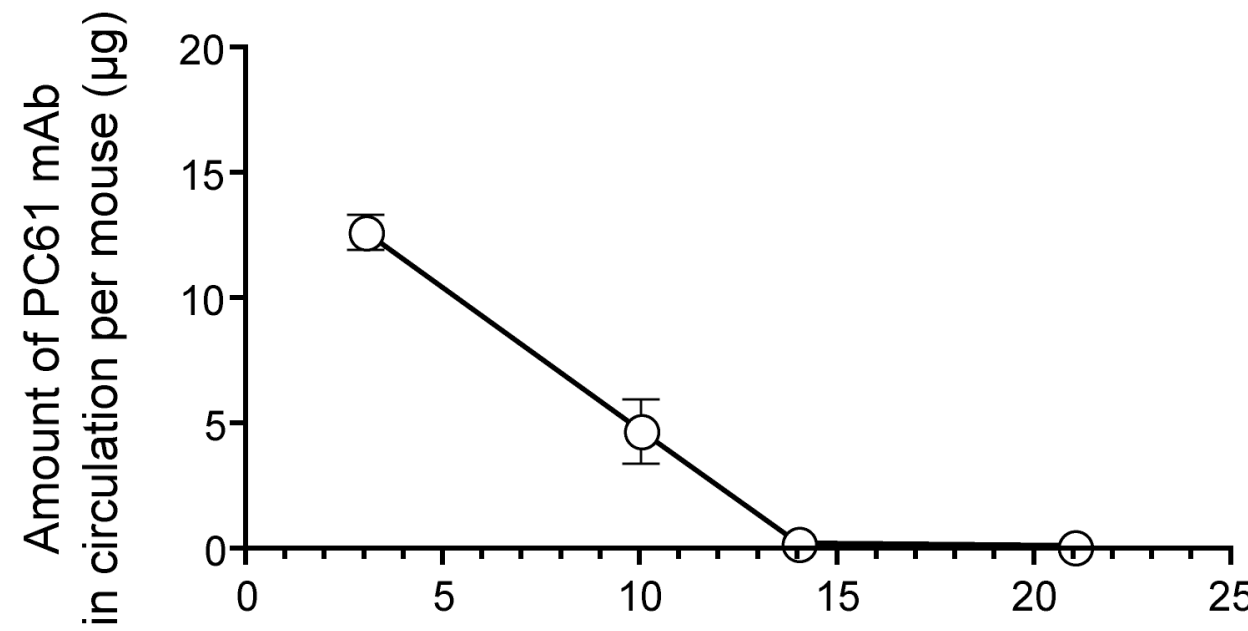

Day after antibody treatment

\section{Figure 3.5: Persistence of PC61 mAb in circulation}

Mice were administered $100 \mu \mathrm{g}$ of PC61 i.p. and serum was harvested immediately prior to treatment, and at days 3, 10, 14 and 21 after PC61 administration. Serum was then used in an ELISA to assess the amount of PC61 per mouse after antibody treatment. The limit of detection for this assay was $0.014 \mu \mathrm{g}$ per mouse. Sera collected from mice prior to PC61 treatment were below this limit of detection. Symbols represent mean values \pm SEM. Five to ten animals were analysed at each time point. Results are representative of one experiment.

In conclusion, Tregs repopulated the periphery from day 23 after PC61 treatment, regardless of whether or not there was a concurrent mycobacterial infection. The acute phase of the immune response to mycobacterial infection in the C57BL/6 murine model extends up to 28 days after infection (section 1.2.7). $\mathrm{CD} 4^{+} \mathrm{CD} 25^{+}$Tregs were reduced as a result of PC61 treatment for this period of time and reduced in both the peripheral blood and the lung. Therefore, this model can be used to successfully reduce $\mathrm{CD} 4{ }^{+} \mathrm{CD} 25^{+}$Treg numbers during acute mycobacterial infection, when initial cellular proliferation occurs in the draining lymphoid tissue and cytokine producing lymphocytes initially migrate to affected tissues. However, it is important to note that after PC61 treatment, antibody can persist in circulation for up to 14 days and the effect of PC61 on an active immune response is not well defined. 


\subsubsection{Treg influx to the lung after mycobacterial infection}

Tregs can mediate suppression of other leukocytes in the draining lymph node, by suppressing proliferation and lymphocyte activation, or in peripheral tissues, by suppressing cytokine production and other effector cell functions. Therefore, lymphocyte populations in the lung during mycobacterial infection were evaluated to monitor Treg numbers and determine their proportion relative to total $\mathrm{CD} 4^{+} \mathrm{T}$ cells.

Mice were infected with BCG i.n. or M. tuberculosis by aerosol and lung lymphocytes were analysed for the presence of $\mathrm{CD} 4{ }^{+} \mathrm{CD} 25^{+} \mathrm{Foxp} 3^{+}$Tregs and total $\mathrm{CD} 4{ }^{+}$lymphocytes over the early course of infection. During both BCG and $M$. tuberculosis pulmonary infections, the number of $\mathrm{CD} 4{ }^{+} \mathrm{CD} 25^{+} \mathrm{Foxp} 3^{+}$Tregs increased significantly in the lung over the early course of mycobacterial infection compared to immediately prior to infection (Fig. 3.6). This increase occurred in parallel with an accumulation of total $\mathrm{CD}^{+}$lymphocytes during both BCG infection (Fig. $3.6 \mathrm{~A}$ ) and $M$. tuberculosis infection (Fig. 3.6 B).

This illustrated that $\mathrm{CD} 4^{+} \mathrm{CD} 25^{+} \mathrm{Foxp}^{+}$Tregs accumulated in mycobacteriainfected lungs but suggests Tregs accumulated with similar kinetics to total CD4 ${ }^{+} \mathrm{T}$ cells and were present in the lung at the same time as other $\mathrm{CD}^{+} \mathrm{T}$ cells. 
A.
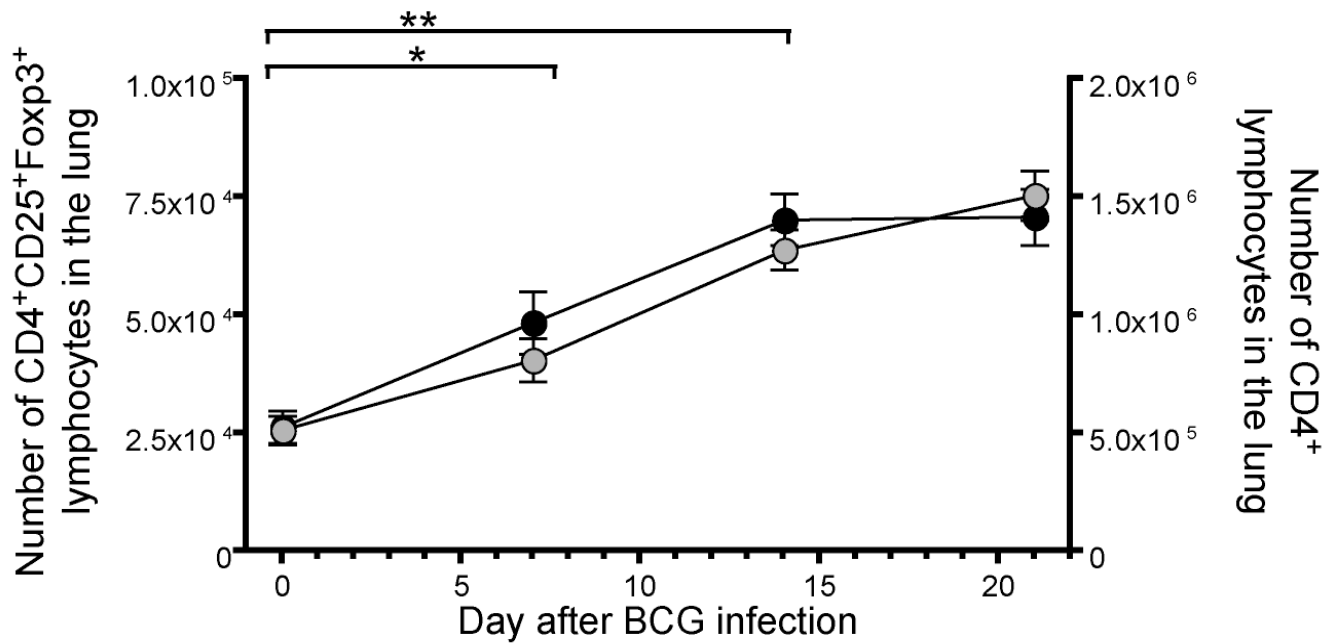

B.
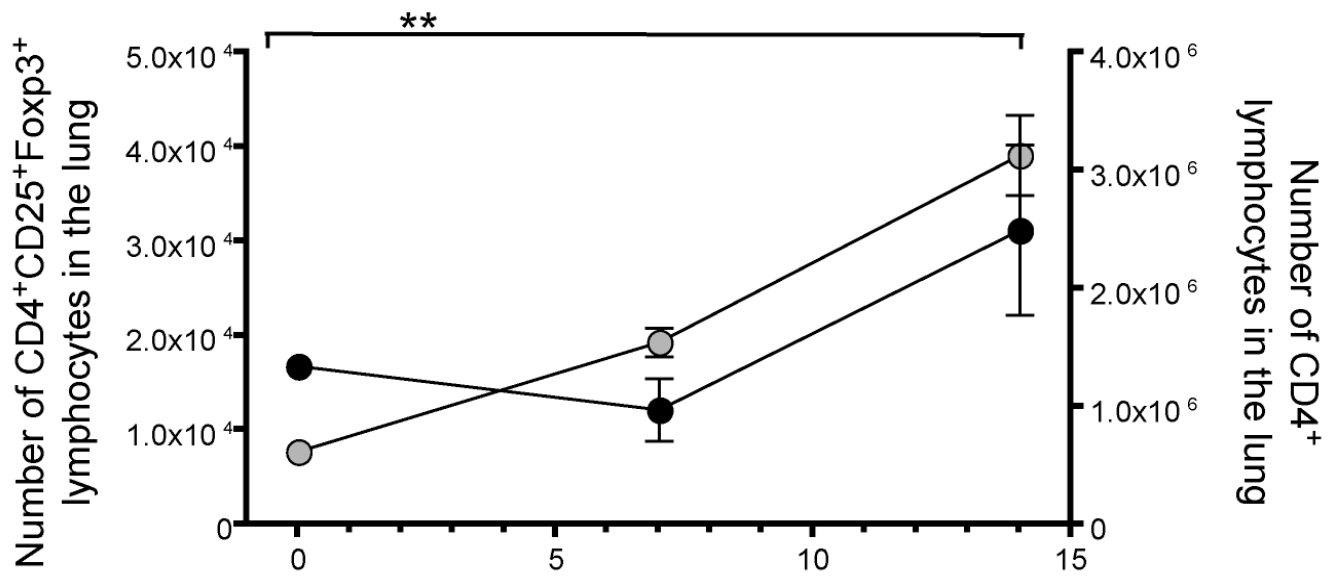

Day after $M$. tuberculosis infection

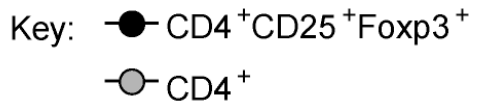

Figure 3.6: Comparison of Treg and total $\mathrm{CD4}^{+} \mathrm{T}$ cell influx to the lung tissue after mycobacterial infection

Mice were infected A) i.n. with BCG or B) by aerosol with M. tuberculosis three days after treatment with rat IgG. Lung lymphocytes were isolated and analysed for the presence of $\mathrm{CD} 4^{+} \mathrm{CD} 25^{+} \mathrm{Foxp}^{+}{ }^{+}$lymphocytes (black circles) and total $\mathrm{CD} 4^{+}$lymphocytes (grey circles) by flow cytometry. Symbols represent mean values \pm SEM, and some symbols obscure error bars. Four to five animals were analysed per treatment group at each time point. Numbers of $\mathrm{CD} 4{ }^{+} \mathrm{CD} 25^{+} \mathrm{Foxp}^{+}$lymphocytes at time points indicated were compared to day 0 , immediately prior to infection, using the two-tailed Mann Whitney test; * indicates $\mathrm{p} \leq 0.05, * *$ indicates $\mathrm{p} \leq$ 0.01 . Results represent single experiments. 


\subsubsection{Design of experimental methodology}

The results outlined above demonstrate that PC61 treatment effectively inactivates natural Tregs in the peripheral blood, lymphoid tissue and peripheral tissue, and that repopulation in the blood and peripheral tissues does not begin until around day 23 after PC61 treatment, even in the presence of an active infection. Based on the results outlined above, an experimental protocol was designed to evaluate whether natural $\mathrm{CD} 4{ }^{+} \mathrm{CD} 25^{+}$Tregs suppress the acute phase of the immune response to mycobacterial infection, resulting in increased tissue bacterial numbers but diminished tissue pathology (Fig. 3.7).

Mice were administered PC61 i.p. and, two days later, peripheral blood samples were taken to confirm inactivation of Tregs (Fig. 3.7 A). Surface staining for CD25 expression in these samples used the anti-CD25 mAb clone 7D4. Treg inactivation, as assessed by flow cytometry, was consistent in all mice that were administered PC61 across all experiments (data not shown). The following day, mice were infected with mycobacteria to initiate a pulmonary infection and samples were taken during the course of the infection to assess immune responses, tissue bacterial numbers and pulmonary inflammation (Fig. 3.7 B). 
A.

$$
\text { Day -3: }
$$

Antibody treatment

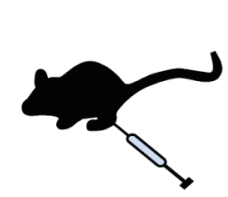

Day -1:

Assess CD25

Tregs

Day 0:

Mycobacterial infection

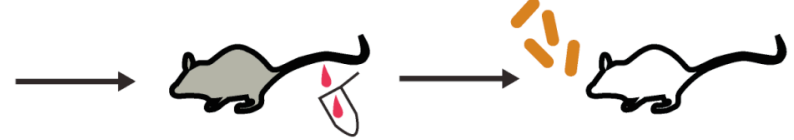

B.

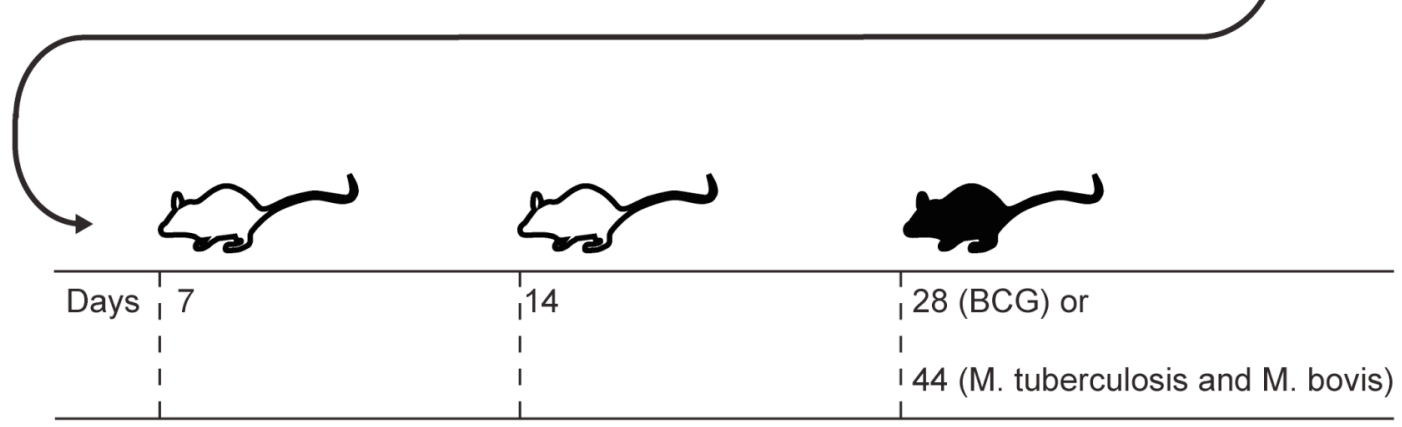

- Evaluate immune response: ELISA and flow cytometry

- Evaluate lung bacterial numbers

- Evaluate lung pathology

Key:
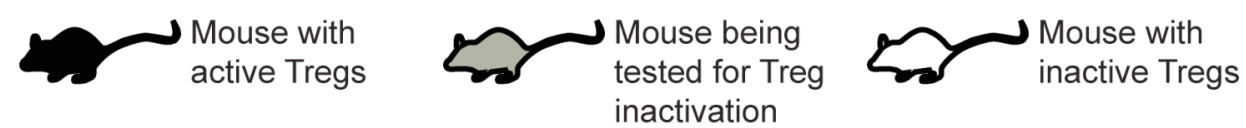
tested for Tractivation

Figure 3.7: Experimental outline for testing whether natural Tregs suppress the acute phase of mycobacterial infection

A) Mice were treated with $100 \mu \mathrm{g}$ of rat IgG or $100 \mu \mathrm{g}$ of PC61 i.p. and two days later a peripheral blood sample was taken and assessed by flow cytometry for the presence of $\mathrm{CD} 4{ }^{+} \mathrm{CD} 25^{+}$lymphocytes. The following day, mice were infected either with BCG i.n. or by aerosol with M. bovis or M. tuberculosis. B) At 7, 14 and 28 days after BCG infection or 44 days after M. bovis or M. tuberculosis infection, the immune response was evaluated by flow cytometry and/or ELISA, and lung bacterial numbers and pulmonary inflammation were evaluated. Black mice represent when Tregs were present, grey mice represent when mice were being tested for the presence of Tregs and white mice represent when Tregs were significantly reduced compared to control mice. 


\subsubsection{Natural Tregs and the immune response to primary mycobacterial infection}

Inactivation of Tregs prior to other infections has resulted in increases in effector lymphocyte proliferation and cytokine production in response to antigen stimulus [330, $333,375]$. Therefore, it was hypothesised that inactivation of Tregs prior to a mycobacterial infection in vivo, using the approach outlined in Fig. 3.7, would enhance the ensuing immune response. Since IFN- $\gamma$ is important for protection against primary mycobacterial infection $[64,65,376]$, IFN- $\gamma$ production was assessed by ELISA during the course of BCG infection.

At day 14 after infection, PC61 treated mice displayed a trend for increased antigen-driven IFN- $\gamma$ production compared to control mice, although this difference was not statistically significant (Fig 3.8 A/B). IFN- $\gamma$ production was comparable between PC61 treated and control mice by day 28 after infection (Fig 3.8 A), by which time Tregs had begun to repopulate the periphery (Fig 3.3 A). IFN- $\gamma$ production was also evaluated at day 14 after M. tuberculosis infection (Fig 3.8 C), although modified restimulation conditions were used. Fewer cells were plated per volume of media, resulting in a lower overall cytokine production compared to samples assessed in Fig. $3.8 \mathrm{~A} / \mathrm{B}$. However, PC61 treated mice again displayed a trend for increased antigendriven IFN- $\gamma$ production after $M$. tuberculosis infection compared to control mice, similar to BCG infection but again not statistically significant (Fig 3.8 C). 
A.
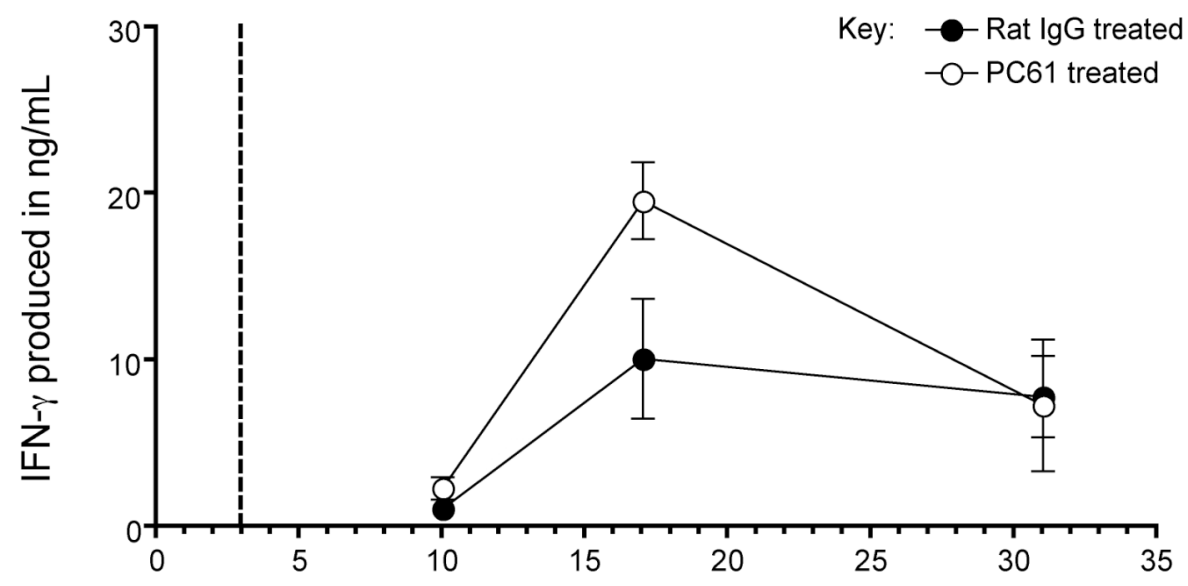

Day after antibody treatment

B.

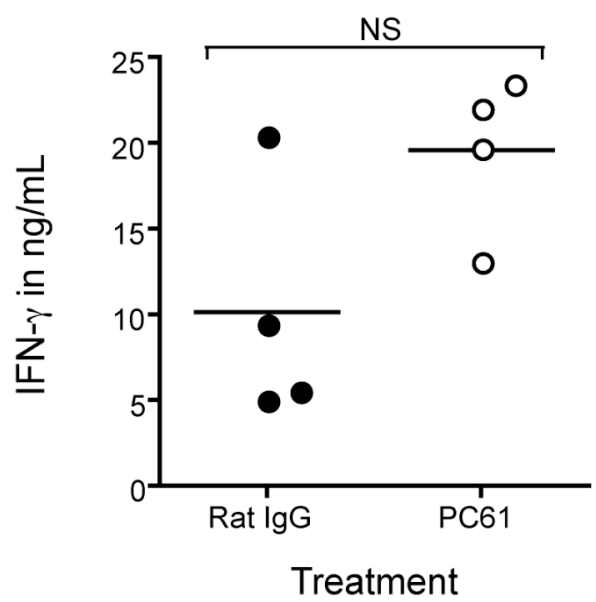

C.

M. tuberculosis infection:

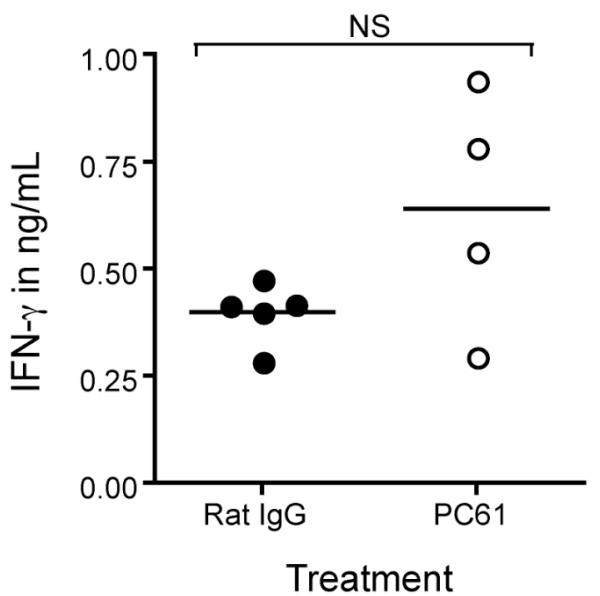

Figure 3.8: ELISA analysis of IFN- $\gamma$ production during mycobacterial infection

Mice were administered rat IgG (filled circles) or PC61 antibody (empty circles) i.p., 3 days before mycobacterial infection (dashed line) with either BCG or M. tuberculosis. At day 7, 14 or 28 after infection, splenocytes were harvested and restimulated with CFP before supernatants were used in ELISAs to assess IFN- $\gamma$ production. A) Time course of IFN- $\gamma$ production after BCG infection. B) The amount of IFN- $\gamma$ in supernatants from splenocytes of rat IgG treated and PC61 treated mice at day 14 after BCG infection. C) The amount of IFN- $\gamma$ in supernatants from splenocytes of rat IgG treated and PC61 treated mice at day 14 after M. tuberculosis infection. Symbols in A) represent mean values \pm SEM and symbols in $\mathbf{B}$ ) and $\mathbf{C}$ ) represent individual values and black lines represent mean values. The limit of detection for this assay was 32.5 $\mathrm{pg} / \mathrm{mL}$. Four to five animals were analysed per treatment group at each time point. Treatment groups were compared using the two-tailed Mann Whitney test; NS indicates $p>0.05$. Results for BCG infection are representative of two replicate experiments, while results for $M$. tuberculosis infection represent a single experiment. 
Total IFN- $\gamma$ production was increased at day 14 after infection, but to determine which lymphocyte population contributed to the increase, cytokine production was assessed by flow cytometry. Lymphocytes from the spleen (Fig. 3.9) and lung (Fig. 3.10) were harvested at day 14 after BCG infection, restimulated with CFP in vitro overnight and analysed by flow cytometry for IFN- $\gamma$ or IL-2 production. In the spleen, the percentage of $\mathrm{CD}^{+}$lymphocytes producing IFN- $\gamma$ and the percentage of total lymphocytes producing IL-2 were significantly increased in mice treated with PC61 compared to control mice (Fig. 3.9 B/C). A significant increase in IFN- $\gamma$ was not observed in the lung (data not shown), although, the percentage of $\mathrm{CD}^{+}$lymphocytes from the lung producing IL-2 was significantly higher in PC61 treated mice than in control mice (Fig. $3.9 \mathrm{~B})$.

$\mathrm{CD} 8^{+}$lymphocytes also produce cytokines in the lung following mycobacterial lung infection [71]. Although cytokine production by $\mathrm{CD} 8^{+}$lung-resident lymphocytes was detected, there was no statistically significant difference in the percentages producing IFN- $\gamma$ or IL-2 from mice treated with PC61, compared to control mice (data not shown). Together, these data illustrate that antibody-mediated Treg inactivation induced a modest increase in the proportion of $\mathrm{CD} 4^{+}$lymphocytes producing cytokines known to be protective in a mycobacterial infection [64, 65, 376, 377]. 
A.
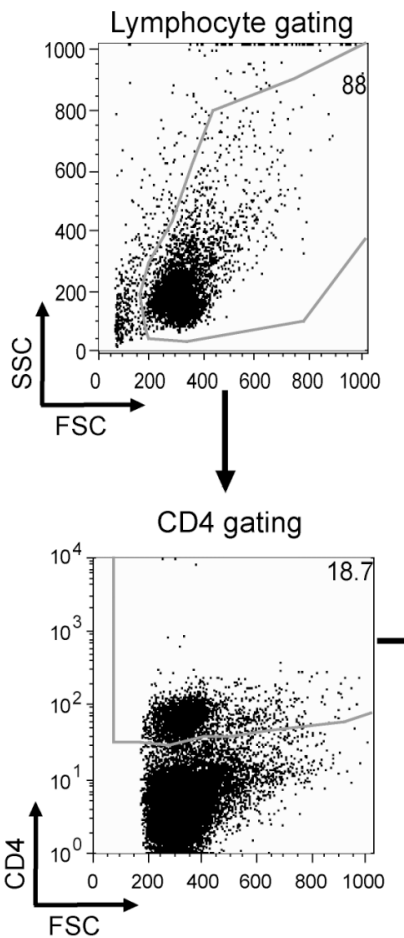

B.

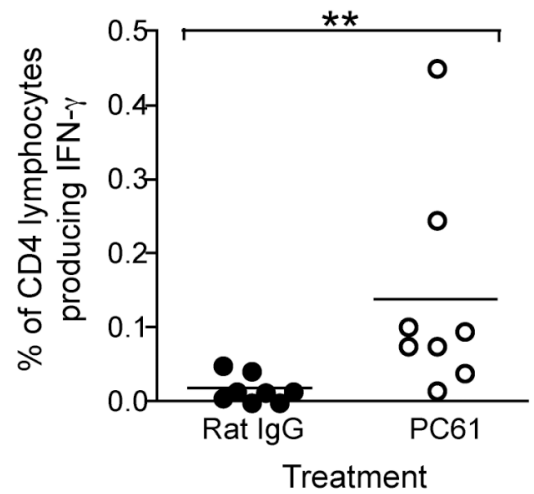

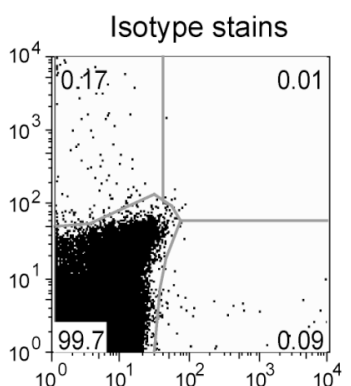

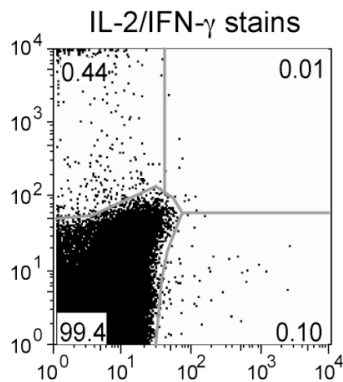

Rat $\lg G$

treated
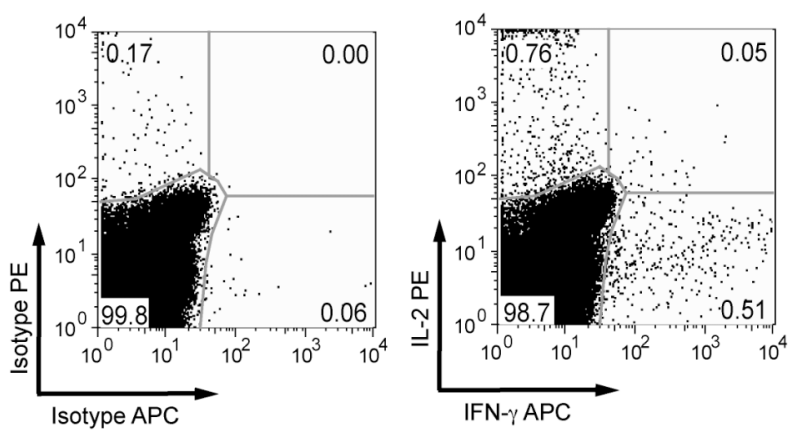

PC61

treated

Figure 3.9: IFN- $\gamma$ and IL-2 production by splenocytes after mycobacterial infection

Mice were treated with rat IgG or PC61 and infected with BCG 3 days later. At 14 days after infection, spleens were harvested and isolated splenocytes were stimulated with CFP overnight before being assessed for cytokine production by flow cytometry. A) Representative flow cytometry plots of lymphocyte gating, CD4 gating and isotype or IFN- $\gamma / \mathrm{IL}-2$ staining for restimulated splenocytes from rat IgG treated and PC61 treated mice. B) Percentage of CD4 ${ }^{+}$ lymphocytes producing IFN- $\gamma$. C) Percentage of total lymphocytes producing IL-2. Symbols represent values for individual mice and black lines indicate mean values; 8 animals were analysed per treatment group. Results are representative of two replicate experiments.

Treatment groups were compared using the two-tailed Mann Whitney test; ** indicates $\mathrm{p} \leq$ $0.01, * * *$ indicates $\mathrm{p} \leq 0.001$. Results are representative of two replicate experiments. 
A.

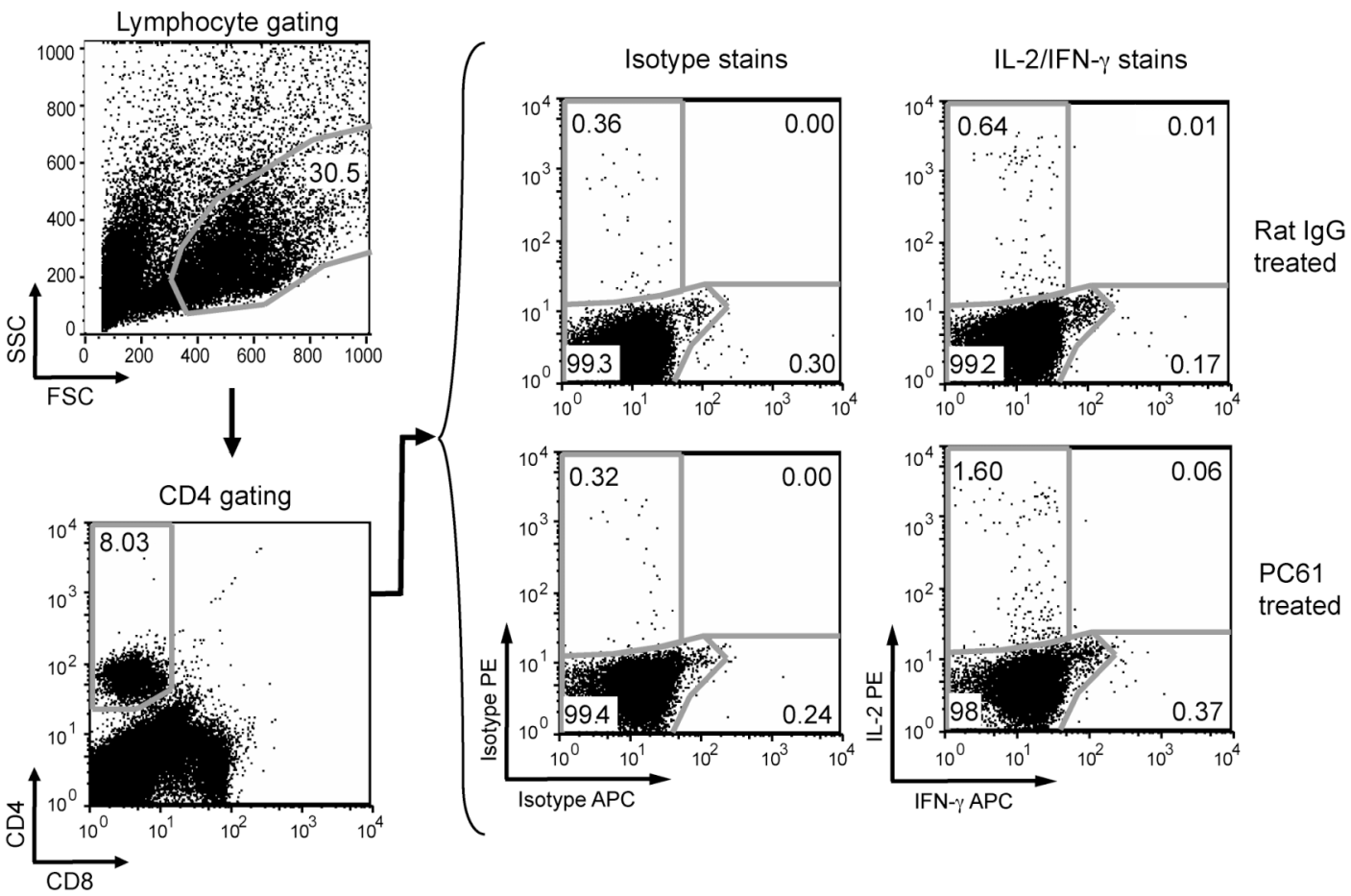

B.

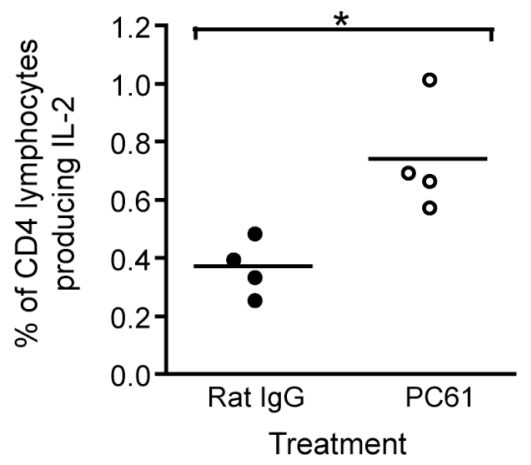

Figure 3.10: IFN- $\gamma$ and IL-2 production by lung lymphocytes after mycobacterial infection Mice were treated with rat IgG or PC61 and infected with BCG 3 days later. At 14 days after infection, lungs were harvested and isolated lymphocytes were stimulated with CFP overnight before being assessed for cytokine production by flow cytometry. A) Representative flow cytometry plots of lymphocyte gating, CD4 gating and isotype or IFN- $\gamma / \mathrm{IL}-2$ staining for restimulated lung lymphocytes from rat IgG treated and PC61 treated mice. B) Percentage of $\mathrm{CD} 4^{+}$lymphocytes producing IL-2. Symbols represent values for individual mice and black lines indicate mean values; 4 animals were analysed per treatment group. Results are representative of two replicate experiments. Treatment groups were compared using the twotailed Mann Whitney test; * indicates $\mathrm{p} \leq 0.05$. Results are representative of two replicate experiments. 


\subsubsection{Lung bacterial numbers and inflammation during BCG or $\boldsymbol{M}$. tuberculosis infection}

Inactivation of $\mathrm{CD} 4{ }^{+} \mathrm{CD} 25^{+}$Tregs modestly enhanced antigen-driven cytokine production during the acute immune response to mycobacterial infection. Studies in murine models of other infections have shown that pre-treatment with anti-CD25 mAbs can enhance the immune response and reduce pathogen burden on the host $[330,333$, 334]. Therefore, it was hypothesised that inactivation of Tregs during mycobacterial infection would reduce the number of bacteria in infected tissues.

To assess this, mice were treated with either PC61 or control rat IgG followed by infection with either BCG or M. tuberculosis (Fig. 3.7). Since BCG bacterial numbers in the lung stabilise earlier than virulent $M$. tuberculosis, lung bacterial numbers in BCG infected mice were assessed at days 7, 14 and 28 (Fig. 3.11 A), while lung bacterial numbers were assessed in M. tuberculosis infected mice at days 7, 14 and 44 (Fig. 3.11 B). In contrast to other infection models [330, 333, 334], bacterial numbers in the lungs were comparable between PC61 treated and rat IgG treated mice after both BCG and M. tuberculosis infections (Fig. $3.11 \mathrm{~A} / \mathrm{B}$ ). This suggests that $\mathrm{CD} 4{ }^{+} \mathrm{CD} 25^{+}$Treg inactivation did not affect bacterial growth or clearance after BCG or M. tuberculosis infection. 
A.

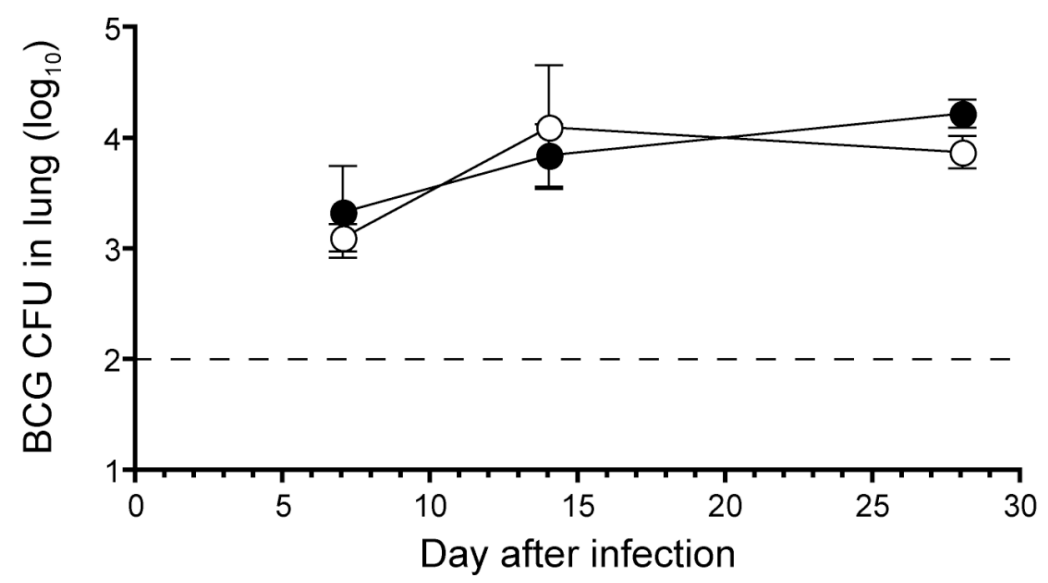

B.

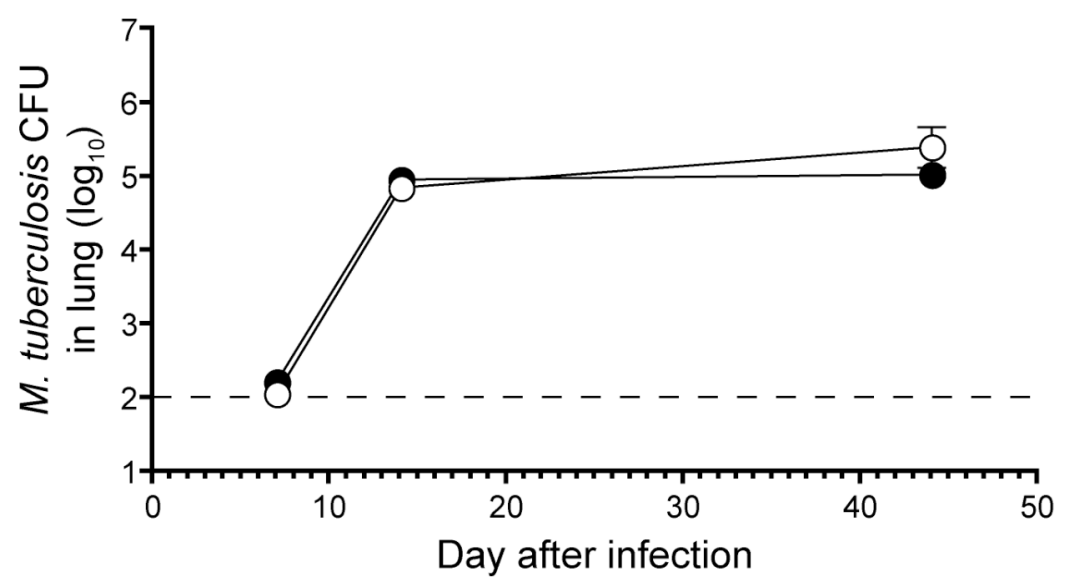

Key:

- Rat IgG treated -O- PC61 treated

\section{Figure 3.11: Lung bacterial numbers after BCG or M. tuberculosis infection}

Mice were administered rat IgG (filled circles) or PC61 (empty circles) i.p., and 3 days later were infected A) with BCG i.n. or B) by aerosol with M. tuberculosis. Bacterial numbers in the lung tissue homogenates were determined during the course of the infections. Symbols represent geometric mean values \pm GEM, and some symbols obscure error bars. The limit of detection for this assay at $2 \log _{10} \mathrm{CFU}$ is indicated by the dotted lines. Five animals were analysed per treatment group at each time point. Treatment groups were compared using the two-tailed Mann Whitney test; no significant differences were observed. Results for BCG infection are representative of two replicate experiments, while results for M. tuberculosis infection represent a single experiment. 
In other infection models, $\mathrm{CD} 4{ }^{+} \mathrm{CD} 25^{+}$Treg inactivation has enhanced infectioninduced pathology $[331,332]$. Although the modestly enhanced immune response observed after Treg inactivation did not enhance clearance of the mycobacteria, it was possible that it could exacerbate inflammation in the lung and pathology associated with $\mathrm{Tb}$.

In order to address this, mice were treated with either PC61 or control rat IgG followed by infection with either BCG or M. tuberculosis. Lung samples were collected over the course of the infections and the tissue was prepared for histological analysis. A similar degree of moderate granulomatous inflammation, with predominantly interstitial infiltrating lymphoid cells and epithelial histocytes, was observed in both PC61 treated and rat IgG treated mice at day 28 after BCG infection and day 44 after $M$. tuberculosis infection (Fig. 3.12 A). When inflammation was graded, composite pathology scores (with scores ranging from 0 (least severe) to 5 (most severe)) for both BCG and $M$. tuberculosis infections were low and averaged below 2 for PC61 and rat IgG treatment groups at all times assessed.

Thus, distinct from studies using $\mathrm{CD} 4^{+} \mathrm{CD} 25^{+}$Treg inactivation prior to other infections, $\mathrm{CD} 4{ }^{+} \mathrm{CD} 25^{+}$Treg inactivation prior to infection with either BCG or $M$. tuberculosis did not affect either the bacterial numbers in the lung or the degree of disease-associated lung pathology that developed. 


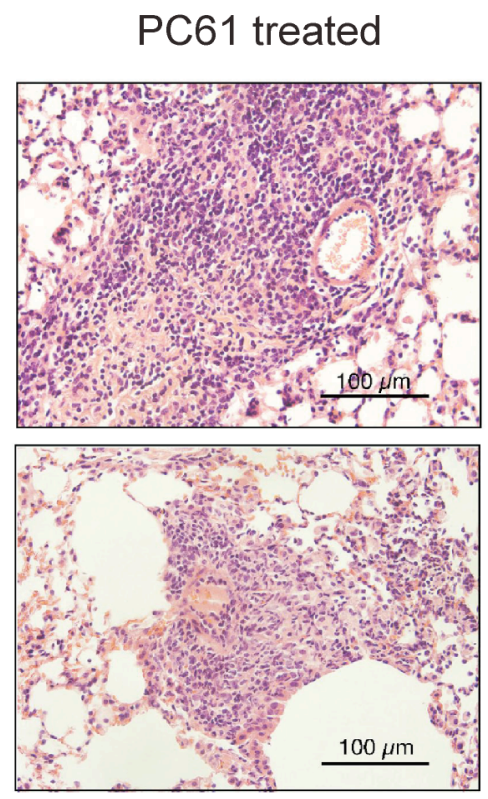

Rat IgG treated
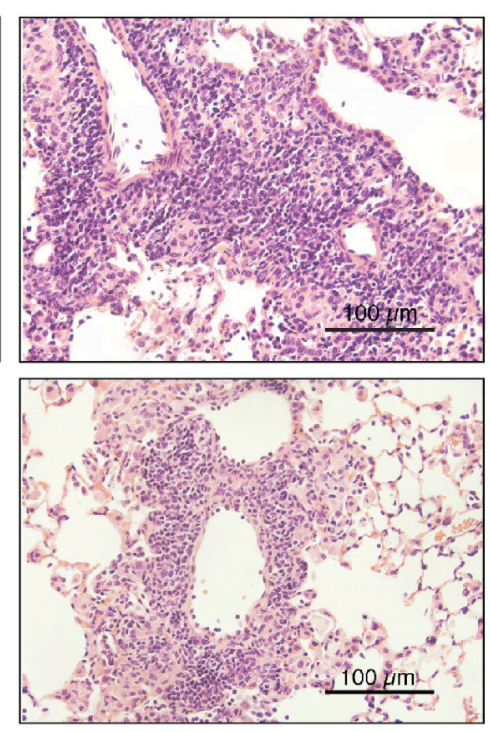

BCG

infected lung

Day 28

\section{M. tuberculosis infected lung \\ Day 44}

\section{Figure 3.12: Pulmonary inflammation after BCG or M. tuberculosis infection}

Mice were administered either control rat IgG antibody (filled circles) or PC61 (empty circles) i.p., and three days later were infected i.n. with BCG or by aerosol with M. tuberculosis. Lung tissue samples were taken in parallel with lung samples taken for Fig. 3.11, fixed, sectioned and stained with haematoxylin and eosin before being graded for pulmonary inflammation. Sections from lungs taken on day 28 after BCG infection or on day 44 after M. tuberculosis infection are shown. One micrograph, representative of sections from five animals, is shown for each treatment group. All micrographs were taken at 40x magnification; scale bars, $100 \mu \mathrm{m}$. Results for BCG infection are representative of two replicate experiments, while results for $M$. tuberculosis infection represent a single experiment. 


\subsubsection{Lung bacterial numbers and inflammation during $M$. bovis infection}

M. bovis is another member of the MTBC and it is the causative agent of bovine $\mathrm{Tb}$. New Zealand has relatively high rates of $M$. bovis infection in cattle and livestock, which compromise products such as dairy and beef (reviewed in [378]). Improved understanding of the regulation of the immune response to $M$. bovis infection could facilitate the development of improved therapies and vaccines to manage bovine $\mathrm{Tb}$. It is possible that suppression by Tregs could promote pathogen persistence during $M$. bovis infection and this point can also be explored using the murine model (Fig. 3.7), since $M$. bovis infection also causes disease in mice. It was anticipated that, similar to results obtained with $M$. tuberculosis and BCG infection, $\mathrm{CD} 4{ }^{+} \mathrm{CD} 25^{+}$Treg inactivation prior to $M$. bovis infection would not affect either the bacterial numbers in the lung or the degree of disease-associated lung pathology.

To validate this, bacterial numbers were assessed in the lung after either PC61 or control rat IgG treatment followed M. bovis infection, and lung samples were collected in parallel for histological analysis. Lung bacterial numbers were evaluated at days 7, 14 and 44 after infection and were comparable between PC61 treated and control treated mice up to day 44 after infection (Fig. 3.13 A). There were no obvious differences in pulmonary inflammation early after $M$. bovis infection but there was a dramatic difference between PC61 treated and control mice at day 44 after infection. Lungs of control mice exhibited moderate to severe granulomatous lesions, which were multifocal and coalescing, with involvement of airways and evidence of some necrosis (Fig. 3.14 A). In comparison, lungs of PC61 treated mice exhibited diminished inflammation, with mild granulomatous lesions that were multifocal but not coalescing, with no airway involvement and no necrosis (Fig. 3.14 A). Median composite lung pathology scores revealed a significantly diminished level of inflammation in the lung at day 44 after infection in PC61 treated compared to control mice (Fig. 3.14 B).

To further define when differences in inflammation due to PC61 treatment arose and whether they affected dissemination of the infection, bacterial load and pulmonary inflammation around day 44 was examined more closely. Bacterial numbers in the lungs were comparable between PC61 treated and control mice at days 38, 48 and 60 after $M$. bovis infection (Fig. $3.13 \mathrm{~B}$ ), demonstrating that alterations in pulmonary inflammation were not preceded or followed by alterations in lung bacterial numbers. Bacterial 
numbers in the spleens were also comparable between PC61 treated and control mice (Fig. $3.13 \mathrm{C}$ ), demonstrating that the altered inflammation in the lung did not affect dissemination of infection to other tissues. PC61 treated mice had a slightly lower median composite lung pathology score than control mice at day 38 , but this difference was not significant and lung pathology scores were comparable between PC61 treated and control mice at days 48 and 60 (Fig. 3.14 C).

In summary, inactivation of $\mathrm{CD} 4{ }^{+} \mathrm{CD} 25^{+}$Tregs prior to M. bovis infection may result in a different outcome to those observed with BCG and M. tuberculosis infections. Although Treg inactivation prior to infection did not affect bacterial numbers in the lung for any of the mycobacterial infections tested in this thesis, it significantly diminished pulmonary inflammation induced by $M$. bovis infection in the murine model at day 44 after infection. During subsequent experiments, slightly diminished pulmonary inflammation was also observed in PC61 treated compared to control mice at day 38 after M. bovis infection. Therefore, further analysis of the effect of Treg inactivation on pulmonary inflammation after $M$. bovis infection is required to determine if this is a real effect and the implications of this are discussed in section 3.4.4. 

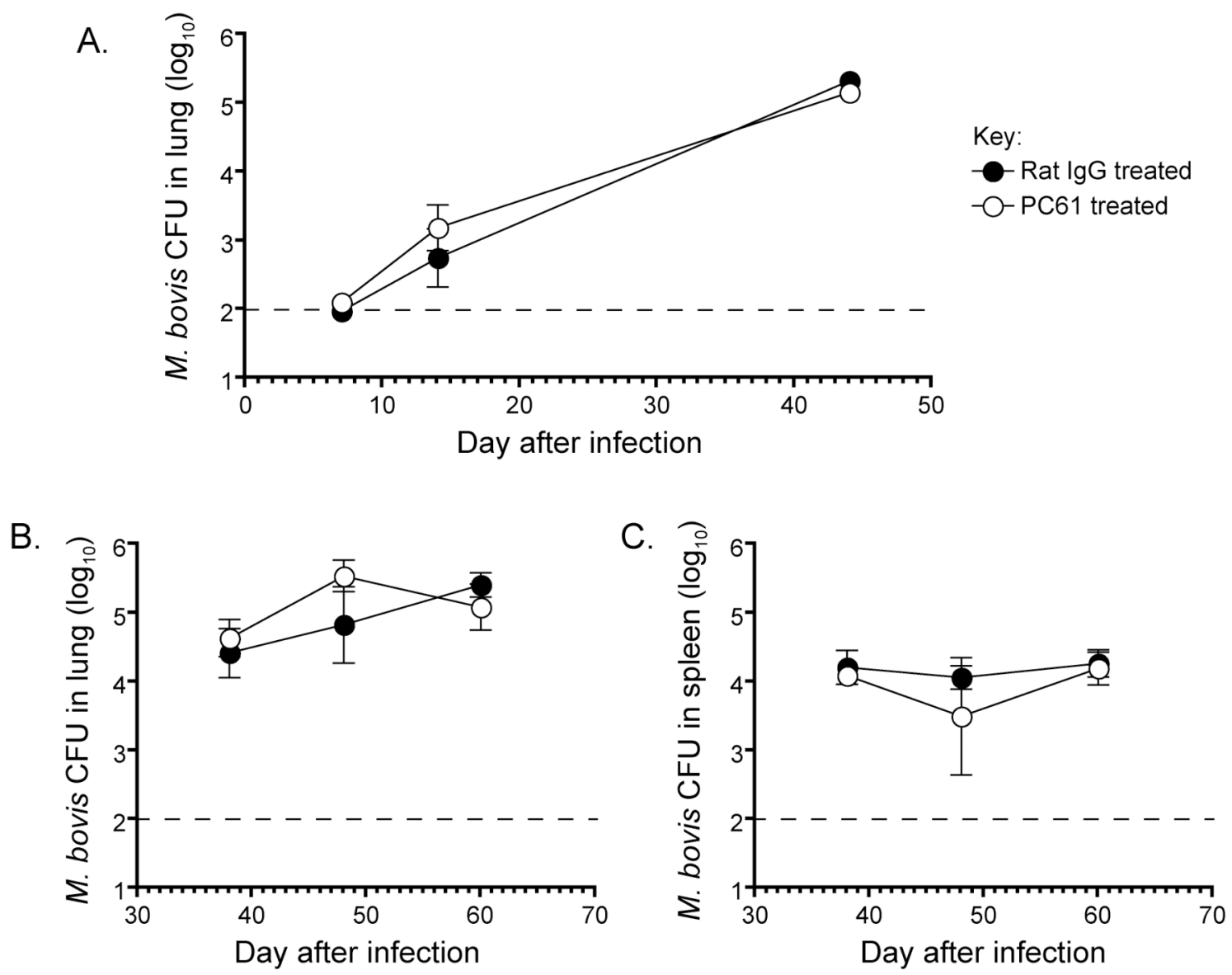

\section{Figure 3.13: Tissue bacterial numbers after $M$. bovis infection}

Mice were administered either control rat IgG antibody (filled circles) or PC61 (empty circles) i.p., and 3 days later were infected by aerosol with M. bovis. Bacterial numbers in the lung and spleen homogenates were determined during the course of the infection. Bacterial numbers were determined from days 7-44 and, in a separate experiment, from days 38-60. A) Bacterial numbers in the lung for days 7-44 after infection. B) Bacterial numbers in the lung for days 3860 after infection. C) Bacterial numbers in the spleen for days 38-60 after infection. Symbols represent geometric mean values \pm GEM, and some symbols obscure error bars. The limit of detection for this assay at $2 \log _{10} \mathrm{CFU}$ is indicated by the dotted lines. Four to five animals were analysed per time point. Treatment groups were compared using the two-tailed Mann Whitney test; no significant differences were observed. Results represent single experiments. 
A.

PC61 treated/M. bovis infected Day 44

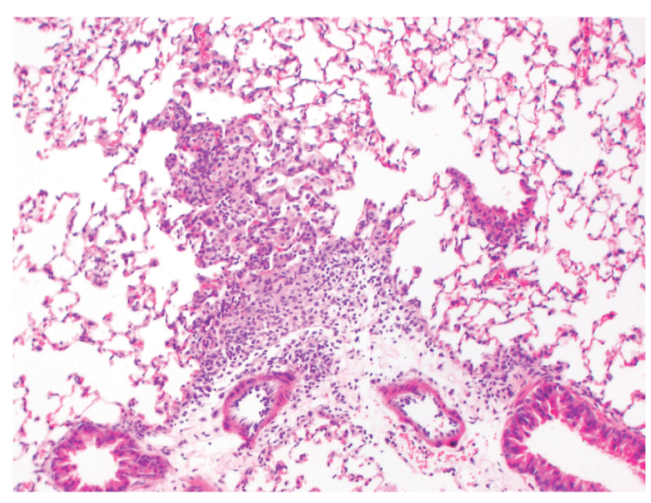

Rat IgG treated/M. bovis infected Day 44

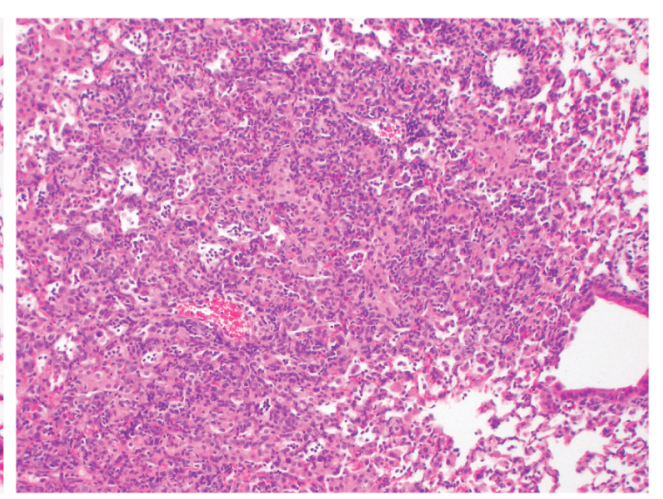

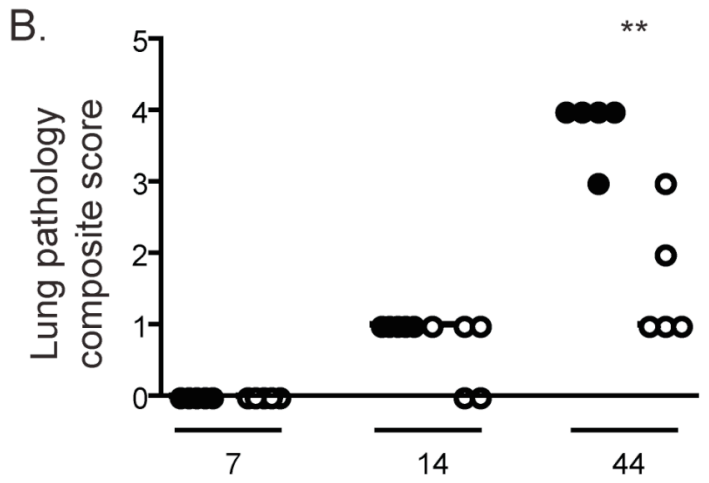

Days after infection
C.

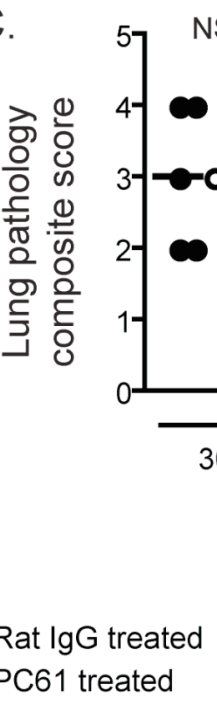

Figure 3.14: Pulmonary inflammation after $M$. bovis infection

Mice were administered either rat IgG (filled circles) or PC61 (empty circles) i.p., and 3 days later were infected by aerosol with M. bovis. Lung tissue samples were taken in parallel with samples taken for Fig. 3.12, fixed, sectioned and stained with haematoxylin and eosin before being graded for pulmonary inflammation. Inflammation was assessed from days 7-44 and, in a separate experiment, days 38-60. A) Sections from lungs taken on day 44 after M. bovis infection. One micrograph, representative of sections from five animals, is shown for each treatment group. Both micrographs were originally taken at 10x magnification. B) Composite pathology scores for lung samples taken at days 7-44 after M. bovis infection. C) Composite pathology scores for lung samples taken at days 38-60 after M. bovis infection. Scores ranged from 0 (least severe) to 5 (most severe). Symbols represent individual values and median values are indicated by the horizontal black line (although some symbols obscure this line). Four to five animals were analysed per treatment group at each time point. Treatment groups were compared using the two-tailed Mann Whitney test; NS indicates $\mathrm{p}>0.05, * *$ indicates $\mathrm{p} \leq 0.01$. Results represent single experiments. 


\subsubsection{Tregs during the chronic phase of mycobacterial infection}

Tregs are hypothesised to promote pathogen persistence in chronic infections, and modulation of Treg activity during a chronic infection can impact on disease outcomes [330]. Although inactivation of Tregs prior to mycobacterial infection did not affect bacterial numbers in the lung during the acute phase of infection, it was hypothesised that natural Tregs suppress bacterial clearance during chronic Tb infection. If that were the case, abrogation of Treg-mediated suppression in chronically infected mice by either DTA-1 or PC61 treatment could decrease bacterial numbers in the lung. Conversely, it was hypothesised that enhancement of Treg-mediated suppression by $5 \mathrm{~F}-1$ treatment could increase bacterial numbers in the lung.

Mice were infected with M. tuberculosis for 6 weeks to permit lung bacterial numbers to stabilise and lung lesions containing $\mathrm{CD}^{+}$effector $\mathrm{T}$ cells and Tregs to develop. At weeks 6 and 8 after infection, groups of mice received either PBS or rat IgG as negative controls, or PC61 alone, DTA-1 alone, 5F-1 alone or a combination of PC61 and DTA-1. At week 10, lung samples were taken for assessment of pathology and evaluation of bacterial numbers. There were no significant differences between treatment groups for either pulmonary inflammation (data not shown) or bacterial numbers (Fig. 3.15) in the lung. Therefore, modulation of Treg activity with PC61, DTA-1 or 5F-1 mAbs during the chronic phase of $M$. tuberculosis infection did not result in significant changes in lung bacterial numbers or disease-associated pathology in the C57BL/6 model. 


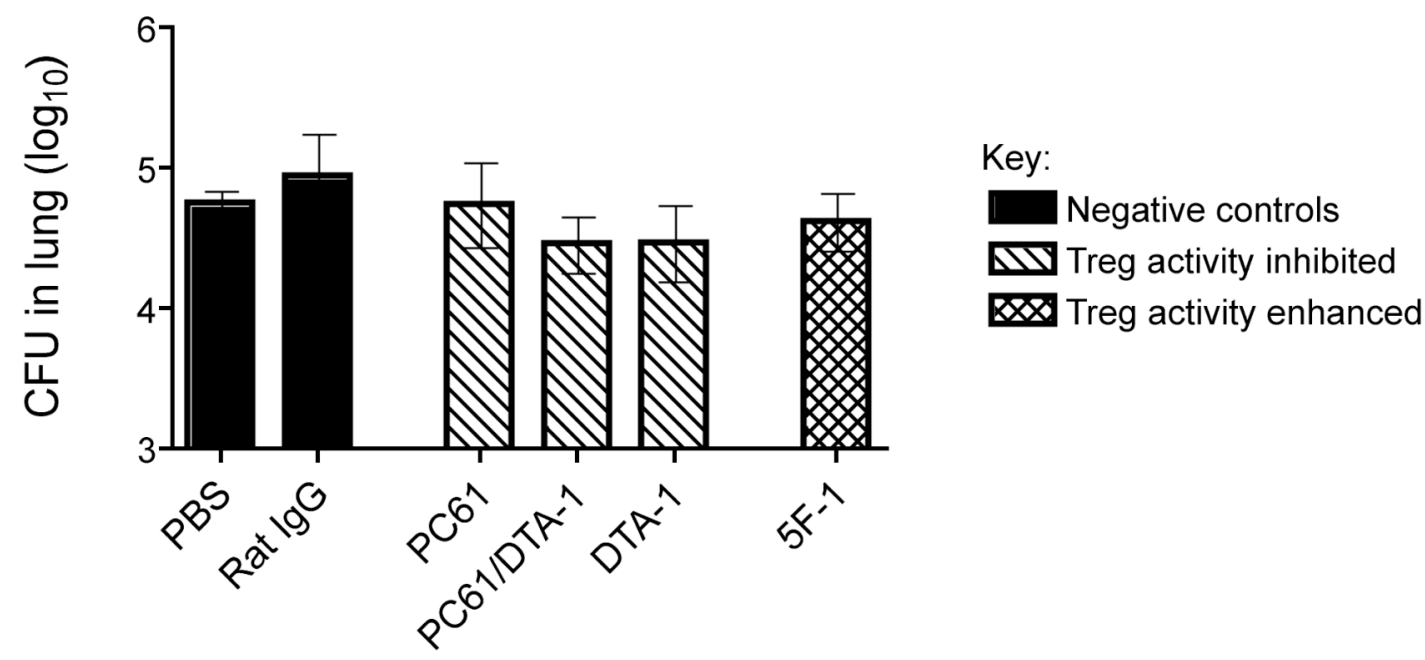

Antibody treatment

\section{Figure 3.15: Impact of Treg modulation on chronic M. tuberculosis infection}

Mice were infected with M. tuberculosis for 6 weeks and, at weeks 6 and 8, mice were administered Treg-modulating mAbs i.p. Groups received PBS or rat IgG control antibody as negative controls (black bars), PC61 (anti-CD25) alone, DTA-1 (anti-GITR) alone, or a combination of PC61 and DTA-1 as mAbs hypothesised to inhibit Treg activity (striped bars), or 5F-1 (anti-GITRL) alone as an mAb hypothesised to enhance Treg activity (hatched bar). At week 10 after infection, lung samples were harvested for determination of lung bacterial numbers. Bars represent geometric mean values \pm GEM. Three to five animals were analysed per treatment group. Treatment groups were compared to the PBS treated group using the twotailed Mann Whitney test; no significant differences were observed. Results represent one experiment. 


\subsection{Discussion}

In this chapter, I aimed to assess whether natural $\mathrm{CD} 4{ }^{+} \mathrm{CD} 25^{+}$Tregs suppressed the acute response to mycobacterial infection. To achieve this, methodology using PC61 treatment to target $\mathrm{CD} 4^{+} \mathrm{CD} 25^{+}$Tregs, outlined in Fig. 3.7, was developed to inactivate Tregs in vivo and limitations of that approach were identified.

I hypothesised that PC61 treatment prior to mycobacterial infection would enhance the immune response to infection and correspondingly decrease bacterial numbers in the tissues, but also lead to enhanced immunopathology in the lung. When these parameters were evaluated, PC61 treatment prior to infection did correlate with modest increase in IFN- $\gamma$ and IL-2 production after infection. For BCG and $M$. tuberculosis infections, lung bacterial numbers and pulmonary inflammation during the acute phase of the infection was unaffected by PC61 treatment. For M. bovis infection, PC61 treatment may correlate with diminished pulmonary inflammation around day 44 after infection, although PC61 treatment did not alter lung bacterial numbers. Therefore, although natural $\mathrm{CD} 4{ }^{+} \mathrm{CD} 25^{+}$Tregs suppressed the immune response during mycobacterial infection, they did not impact on measures of protection, such as pathogen clearance or lung pathology after BCG or M. tuberculosis infection, but may enhance lung pathology after M. bovis infection.

The lack of effect on lung bacterial numbers in this study contrasts with the increased pathogen clearance observed after treatments that interfere with Treg function prior to, and during other infections [305, 330, 333, 334]. Although many pathogens exploit natural Tregs to suppress the immune response to their advantage, it is interesting that a similar role for natural Tregs in mycobacterial infection is not apparent from these results. This study focussed solely on the role of natural Tregs, as defined by $\mathrm{CD} 25$ expression, during early mycobacterial infection. It remains undetermined whether other subgroups of Tregs mediate immunosuppression during early mycobacterial infection and whether natural or induced Tregs suppress immune responses and enable pathogen persistence during later stages of M. tuberculosis infection. 


\subsubsection{PC61 treatment and its limitations}

While PC61 has been widely used to inactivate Tregs in vivo, this chapter illustrates an important limitation of this approach. A sub-population of $\mathrm{CD} 4^{+} \mathrm{CD} 25^{-} \mathrm{Foxp} 3^{+}$Tregs were not affected by PC61 treatment and, although many studies define $\mathrm{CD} 4^{+} \mathrm{CD} 25^{+}$ lymphocytes in a naïve mouse as natural Tregs, the marker that correlates best with the suppressive phenotype of Tregs is Foxp3. A CD $4{ }^{+} \mathrm{CD} 25^{\text {lo }}$ Foxp $3^{+}$population has been observed in naïve transgenic mice that express Foxp3 as a fusion protein with green fluorescent protein (GFP) [282]. In these mice, the $\mathrm{CD} 25^{\mathrm{lo}} \mathrm{Foxp} 3^{+}$population has increased expression of genes that encode proteins characteristically produced by Tregs, including GITR, IL-10 and CD152 [282], and possess the Treg suppressive phenotype [282], similar to CD $25^{\mathrm{hi}} \mathrm{Foxp}^{+}$Tregs.

However, CD $25^{\text {hi }} \mathrm{Foxp}^{+}$Tregs differ from $\mathrm{CD} 25^{\text {lo }} \mathrm{Foxp} 3^{+}$Tregs, as the latter have increased expression of inducible T-cell co-stimulator precursor, chemokine receptors and $\beta 1$-integrin, which are associated with the ability to enter tissue [282]. $\mathrm{CD} 25^{\text {lo }}$ Foxp $3^{+}$Tregs are found at low levels in the peripheral blood but make up a larger proportion of the total Foxp $3^{+}$lymphocyte population in tissues such as spleen and lung [282]. Thus, it is suggested that the CD25 ${ }^{\text {lo }}$ Foxp $3^{+}$population may have a tissue-homing/effector phenotype [282].

In this chapter, the percentage of $\mathrm{CD}^{+}$lymphocytes that were $\mathrm{CD} 25^{-}$and Foxp $3^{+}$was unaffected by PC61 treatment in all tissues analysed (Fig 3.2 C). The lung and spleen also had a higher percentage of resident $\mathrm{CD} 4^{+} \mathrm{CD} 25^{-}$Foxp $3^{+}$lymphocytes than the blood (Fig 3.2 C). Mice were infected with mycobacteria by aerosol or i.n. infection, since aerosol exposure is the most common way to contract an $M$. tuberculosis infection. Therefore, the $\mathrm{CD} 4^{+} \mathrm{CD} 25^{-} \mathrm{Foxp} 3^{+}$population, a significant Treg subset in the lung that persists despite PC61 treatment, could suppress local immune responses during a pulmonary mycobacterial infection. This possibility remains to be evaluated as novel techniques are developed for exploring the role of $\mathrm{CD} 4^{+} \mathrm{CD} 25^{-}$ Foxp $3^{+}$Tregs.

Recently, there has been considerable controversy about the mechanism of action of PC61 when it is administered in vivo. The anti-CD25 mAbs, 7D4 and PC61, had been thought to deplete Tregs in vivo. However, a recent report suggested that administration of 7D4 caused functional inactivation, rather than depletion of the 
$\mathrm{CD} 4{ }^{+} \mathrm{CD} 25^{+}$Treg population, by downregulating or inducing shedding of CD25 from the surface of Tregs [353]. It was suggested that PC61 acts similarly in vivo to 7D4, but this was indirectly implied through analysis of Foxp3 mRNA transcript rather than expression of Foxp3 protein. A recent report followed the kinetics of CD25 and Foxp3 protein expression in vivo after PC61 treatment in $\mathrm{CD}^{+} \mathrm{T}$ cells [352]. This showed that, while $\mathrm{CD} 4{ }^{+} \mathrm{CD} 25^{+}$Foxp $^{+}$Tregs can shed CD25 early after PC61 treatment and become $\mathrm{CD} 4^{+} \mathrm{CD} 25^{-} \mathrm{Foxp}^{+} \mathrm{T}$ cells, cells with a naïve $\mathrm{CD} 69^{\text {lo }}$ phenotype are subsequently depleted [352]. The authors suggested that depletion could be due to lack of IL-2 signalling as a homeostatic survival signal [352]. Therefore, in vivo administration of PC61 depletes a subset of naïve Tregs and converts Tregs possessing a more activated phenotype into a $\mathrm{CD} 25^{-} \mathrm{Foxp}^{+}$population that is functionally inactivated, possibly due to the lack of IL-2 receptor alpha chain. Notably, a significant increase in the percentage of $\mathrm{CD}^{+} \mathrm{CD}^{2} 5^{-}$Foxp $^{+}{ }^{+}$cells was not observed at day 3 after PC61 treatment in the blood, spleen or lungs (Fig. 5.2 C), supporting the suggestion that at least a substantial population of Tregs are depleted by day 3 after PC61 treatment. Notwithstanding current debate over the mechanism of action for PC61, the ability of PC61 to inhibit Treg activity in vivo is well-documented [333, 350-352] and PC61 is referred to as inactivating, rather than depleting, Tregs in this thesis.

A further point of contention is whether Tregs repopulate the periphery after PC61 treatment more rapidly during active immune responses due to altered homeostatic signals compared to naïve animals. In a study by Couper, et al., Tregs began to repopulate the periphery from 14 days after PC61 treatment but malarial infection subsequent to PC61 treatment slightly accelerated CD $4^{+} \mathrm{CD} 25^{+}$Treg repopulation of the periphery compared to a naïve mouse [379]. In this thesis, repopulation of the periphery by $\mathrm{CD} 4{ }^{+} \mathrm{CD} 25^{+}$Tregs was not evident until 23 days after PC61 treatment for both naïve and mycobacteria infected animals (Fig. 3.3). The PC61 $\mathrm{mAb}$ used in this study was prepared in-house and, there may be differences in the production, stability and function in vivo of the antibody between the two studies. The study by Couper, et al. also used a higher dose of PC61 than was used in this thesis, but it is interesting that the two models display different effects of active infection on Treg repopulation. The difference could imply that malarial infection induces stronger Treg homeostatic or expansion signals compared to a mycobacterial infection. 


\subsubsection{Induction of Tregs during Tb infection}

According to current dogma, conditions during mycobacterial infection are favourable for the development of induced Tregs. Antigen presentation to naïve $\mathrm{CD} 4{ }^{+} \mathrm{CD} 25^{-} \mathrm{T}$ cells in conditions of low-dose antigen, high IL-10 or high TGF- $\beta$ is sufficient for conversion of naive $\mathrm{T}$ cells to a Treg suppressive phenotype, as outlined in section 1.4.3. IL-10 not only promotes the induction of Tregs but some induced Treg subsets also produce IL-10 as a mechanism of immunosuppression. Human Tb patients unresponsive to the purified protein derivative skin test, exhibit increased IL-10 production from their PBMCs when cultured in vitro with mycobacterial antigens [365]. Murine studies also point to the importance of IL-10 during infection with $M$. tuberculosis, as IL-10 mRNA synthesis is induced in the lung in WT mice infected by aerosol [380]. Although IL-10 -/- mice do not display increased resistance to BCG infection [381] or M. tuberculosis infection [91], mice expressing increased levels of IL-10 have a higher rate of disease reactivation during later stages of infection compared to control mice [134]. These previous studies in humans and murine models have shown that IL-10 is produced during periods of antigen presentation in a $M$. tuberculosis infection and increased amounts of IL-10 correspond to increased reactivation of disease. IL-10 production during the course of mycobacterial infection was not assessed in this thesis, but, given evidence that induced Tregs may be involved in M. tuberculosis infection and contribute to reactivation of latent disease, this warrants further investigation.

\subsubsection{Suppression by Tregs during chronic Tb infection}

Immunosuppression mediated by Tregs during chronic infection has been previously reported. During the chronic stages of filarial infection with $L$. sigmodontis, combined treatment with PC61 and DTA-1 caused a significant decrease in filarial load [330]. This study suggests that during a chronic infection there is an equilibrium between effector lymphocytes and natural Tregs and interference with this to enhance effector lymphocyte function can improve pathogen clearance. However, it is difficult to analyse and manipulate natural Tregs during an active infection. 
Dissecting the role of Tregs during active infection is limited by the tools available. The use of in vivo administration of PC61 as a technique to target Tregs during an active immune response has been controversial. PC61 could theoretically also target recently activated $\mathrm{CD} 4^{+}$effector $\mathrm{T}$ cells, which transiently upregulate CD25 after TCR stimulation [354]. Despite this, PC61 has been administered during active immune responses and was found to enhance these responses [330, 339], suggesting it does not inhibit effector T cells but targets Tregs preferentially. Results presented in this thesis also suggest that the presence of PC61 does not significantly inhibit effector immune responses. PC61 was shown to persist in circulation up to 14 days after administration (Fig. 3.5). In the protocol outlined in Fig. 3.7, PC61 would persist in circulation during the first week of mycobacterial infection. However, this does not inhibit the immune response, since IFN- $\gamma$ production is the same after the first week of mycobacterial infection and is enhanced after the second week in PC61 treated compared to control treated mice (Fig. 3.8).

Despite potential complications inherent in using CD25 and GITR to target Tregs, PC61, DTA-1 and 5F-1 mAbs were used in this chapter to modulate the activity of Tregs during the chronic phase of a mycobacterial infection. In contrast to $L$. sigmodontis infection, combined treatment with PC61 and DTA-1 did not affect bacterial numbers in the lung (Fig. 3.15), implying that inhibiting Treg activity during the chronic phase of mycobacterial infection might not promote pathogen clearance. Treatment with 5F-1 also did not affect bacterial numbers in the lung (Fig. 3.15), implying that enhancing Treg activity during the chronic phase might not promote pathogen persistence.

The DTA-1 and 5F-1 mAbs target the GITR/GITRL signalling pathway. $L$. sigmodontis lesion sites accumulate large numbers of $\mathrm{GITR}^{+}$effector $\mathrm{T}$ cells and Tregs [330], implying that the GITR/GITRL signalling pathway is important in L. sigmodontis infection. GITR expression has been observed on $\mathrm{CD} 4^{+} \mathrm{CD} 25^{+}$Foxp $3^{+}$Tregs in the PBMCs of patients with active Tb infection [371] and Tregs of this phenotype can be expanded from patients with latent $\mathrm{Tb}$ infection [370]. However, the accumulation of $\mathrm{GITR}^{+}$effector $\mathrm{T}$ cells and Tregs in lung lesions has not been assessed in a murine $\mathrm{Tb}$ model. It is unknown if the GITR/GITRL signalling pathway plays a significant role in regulating Treg activity during mycobacterial infection, but these preliminary results indicate that the pathway does not. However, Treg subsets or mechanisms unaffected by 
the GITR/GITRL signalling pathway may promote mycobacterial persistence during the chronic phase of M. tuberculosis infection.

\subsubsection{Immunopathology during $M$. bovis infection}

PC61 treatment prior to $M$. bovis infection caused a significant reduction in pulmonary inflammation around day 44 after infection (Fig. 3.14 A/B), which was not observed with PC61 treatment prior to BCG or M. tuberculosis infections (Fig. 3.12). Subsequent experiments exhibited a similar trend at day 38 after $M$. bovis infection, but this was not statistically significant and further analysis is required (Fig. 3.14 C). M. bovis infection causes more extensive inflammation in mice than BCG or M. tuberculosis infections [382], thus any reduction in lung pathology that may be mediated by PC61 treatment could be restricted to infections with more extensive inflammation.

A decrease in lung pathology with inactivation of Tregs was not anticipated; it was hypothesised that inactivation of Tregs would increase IFN- $\gamma$ production and thereby increase lung pathology. The reduction in pathology observed with M. bovis infection appears to be transient and could reflect a pro-inflammatory mechanism present during $M$. bovis infection that may be amplified by Tregs as they repopulate the periphery. Natural Tregs can produce TGF- $\beta$ and induction of the highly inflammatory Th-17 cell is dependent on the presence of TGF- $\beta$ and IL- 6 during TCR stimulation [79]. Therefore, removal of natural Tregs may remove a source of TGF- $\beta$ that Th-17 cells use to differentiate during the initial stages of a M. bovis infection, thus preventing Th-17 cell-mediated immunopathology. Future studies aim to evaluate the composition of the M. bovis granuloma. Samples of M. bovis infected lung with or without PC61 treatment over the course of infection have been prepared and stored. These will be used in immunohistochemical analysis to evaluate granuloma composition and the presence of $\mathrm{T}$ cells producing cytokines, including IFN- $\gamma$, IL-10, TGF- $\beta$ and IL-17. 


\subsubsection{Subsequent published studies}

Recently, two studies were published that also investigated the role of Tregs in a murine model of $\mathrm{Tb}$ infection and used approaches that did not inactivate Tregs by administration of anti-CD25 mAbs.

The first study by Kursar, et al. reconstituted recombinase activating gene 1 (Rag1) -/- mice with sorted $\mathrm{CD} 4^{+} \mathrm{CD} 25^{-} \mathrm{T}$ cells alone, sorted $\mathrm{CD} 4^{+} \mathrm{CD} 25^{+} \mathrm{T}$ cells alone or a mix of sorted $\mathrm{CD} 4{ }^{+} \mathrm{CD} 25^{+}$and $\mathrm{CD} 4^{+} \mathrm{CD} 25^{-} \mathrm{T}$ cells [383]. The reconstituted mice and WT C57BL/6 mice were then infected with M. tuberculosis by aerosol and bacterial numbers in tissues were evaluated at day 30 post-infection. Mice that had received $\mathrm{CD} 4^{+} \mathrm{CD} 25^{-} \mathrm{T}$ cells alone had significantly lower bacterial numbers in the lung and spleen than mice that had received $\mathrm{CD} 4{ }^{+} \mathrm{CD} 25^{+} \mathrm{T}$ cells. The enhanced protection observed in $\mathrm{CD} 4^{+} \mathrm{CD} 25^{-} \mathrm{T}$ cell reconstituted mice did not correlate with increased numbers of IFN $-\gamma^{+} \mathrm{CD} 4^{+} \mathrm{T}$ cells or changes in other mediators of mycobacterial immunity. Therefore, the mechanism for reduction of bacterial numbers in the absence of $\mathrm{CD} 4^{+} \mathrm{CD} 25^{+}$Tregs in this model is undefined.

However, this study has limitations inherent in its design. Firstly, a control group of Rag 1 -/- mice reconstituted with total $\mathrm{CD}^{+} \mathrm{T}$ cells was not included. Total $\mathrm{CD}^{+} \mathrm{T}$ cells would have contained a more physiologically relevant proportion of Tregs than the mixture of $\mathrm{CD} 4^{+} \mathrm{CD} 25^{+}$and $\mathrm{CD} 4^{+} \mathrm{CD} 25^{-} \mathrm{T}$ cells that was used. Normally, $\mathrm{CD} 4{ }^{+} \mathrm{CD} 25^{+}$Tregs are present in the periphery at a $1: 10$ to $1: 20$ ratio to $\mathrm{CD} 4{ }^{+} \mathrm{CD} 25^{-} \mathrm{T}$ cells. In the study of Kursar, et al. Tregs were transferred at a 1:2 ratio and were present at a 1:1 ratio by day 30 after infection. Thus, Tregs comprised an unphysiologically high proportion of $\mathrm{CD} 4^{+} \mathrm{T}$ cells in mice that received the mixture of $\mathrm{CD} 4{ }^{+} \mathrm{CD} 25^{+}$and $\mathrm{CD} 4^{+} \mathrm{CD} 25^{-} \mathrm{T}$ cells. Mice that received this mixture were used as a baseline to approximate the WT phenotype but, due to the high proportion of Tregs, may have actually approximated a phenotype when Tregs are unphysiologically and perhaps pathologically high.

Similar to the experimental approach used in this chapter, the methodology used by Kursar, et al. did not eliminate $\mathrm{CD} 4^{+} \mathrm{CD} 25^{-} \mathrm{Foxp} 3^{+}$Tregs. Additionally, by day 30 after infection, all groups of reconstituted mice had comparable numbers of $\mathrm{CD} 4^{+} \mathrm{Foxp}^{+}{ }^{+}$Tregs, suggesting there is a homeostatic mechanism in reconstituted mice that promotes Treg expansion in mice that received $\mathrm{CD} 4^{+} \mathrm{CD} 25^{-} \mathrm{T}$ cells. 
Finally, it appears that values for tissues with undetectable CFUs were recorded as zero rather than a value at or just below the detection limit. If these CFU values were recorded as zero, rather than at the detection limit, this would have amplified the statistical significance of the decrease in bacterial numbers between mice that received $\mathrm{CD} 4^{+} \mathrm{CD} 25^{-} \mathrm{T}$ cells alone and mice that received $\mathrm{CD} 4^{+} \mathrm{CD} 25^{+}$and $\mathrm{CD} 4^{+} \mathrm{CD} 25^{-} \mathrm{T}$ cells.

The second study by Scott-Browne, et al., used mixed bone marrow chimeras in combination with antibody depletion to eliminate Foxp ${ }^{+}$Tregs in vivo [384]. Chimeras were created containing Thy $1.1^{+}$Foxp3+/+ and Thy $1.2^{+}$Foxp3+/+ bone marrow, or Thy $1.1^{+}$Foxp3+/+ and Thy $1.2^{+}$Foxp3-/- bone marrow. Chimeric mice were treated with anti-Thy1.1 depleting mAb, infected with M. tuberculosis and treated with antiThy $1.1 \mathrm{mAb}$ weekly to maintain depletion of Thy $1.1^{+}$leukocytes during the infection. Thereby, Thy $1.1^{+}$Foxp3+/+:Thy $1.2^{+}$Foxp3+/+ chimeric mice possessed Tregs while Thy $1.1^{+}$Foxp3+/+:Thy1.2 $2^{+}$Foxp3-/- chimeric mice were depleted of total Foxp3 ${ }^{+}$Tregs during mycobacterial infection.

This elegant methodology enabled assessment of suppression by both $\mathrm{CD} 4^{+} \mathrm{CD} 25^{+} \mathrm{Foxp}^{+}$and $\mathrm{CD} 4^{+} \mathrm{CD} 25^{-} \mathrm{Foxp}^{+}$Treg populations during mycobacterial infection. A significant decrease in lung bacterial numbers was observed in Treg depleted chimeric mice compared to chimeric mice that possessed Tregs. In addition, this study demonstrated that IFN- $\gamma$ production in response to polyclonal restimulation was amplified in Treg depleted chimeric mice, although there was no significant increase in the antigen-specific response to a peptide from ESAT-6. In agreement with our study (Fig. 3.6), Scott-Browne, et al. also demonstrated parallel accumulation of $\mathrm{CD} 4^{+} \mathrm{Foxp}^{+}$Tregs and $\mathrm{CD} 4^{+}$effector $\mathrm{T}$ cells in the lungs after mycobacterial infection [384].

However, methodology employed by Scott-Browne, et al. was complicated by several factors that were acknowledged and explored by the authors. The efficacy of Thy1.1 depletion waned over time and in Thy $1.1^{+}$Foxp3+/+:Thy $1.2^{+}$Foxp3-/- chimeric mice there was significant expansion of Tregs. As a result, all groups of chimeric mice possessed comparable numbers of $\mathrm{CD} 4^{+} \mathrm{Foxp}^{+}$Tregs by day 30 after infection [384].

Furthermore, the chimeric Treg deficient mice displayed signs of autoimmunity, characterised by robust and generalised immune activation [384]. A complete deficiency of Tregs can provoke autoimmunity as evidenced by the scurfy mouse model and by athymic mice reconstituted with $\mathrm{CD} 4^{+} \mathrm{CD} 25^{-} \mathrm{T}$ cells $[261,280]$. As a result, it is 
not surprising that the chimeric Treg deficient animals exhibited a degree of autoimmunity [384]. From these data, it is not possible to separate the impact of autoimmunity on mycobacterial growth from the effect of heightened mycobacteriaspecific responses on bacterial growth. The generalised immune activity induced in the chimeric Treg deficient mice could contribute to a decrease in bacterial numbers in tissues during mycobacterial infection.

Finally, the study evaluated antigen-specific responses by restimulating with one peptide from ESAT- 6 that contained a known $\mathrm{CD}^{+} \mathrm{T}$ cell epitope [384]. While this effectively highlights antigen-specific immune responses to that epitope, it limits the ability of the study to detect the overall effect of Treg depletion on antigen-driven immune responses. Infection with $M$. tuberculosis does not result in an immune response to a single immunodominant epitope and there is no single immunodominant protein [385]. Responses to various mycobacterial peptides and proteins can be very different. Furthermore, the degree of overlap between the TCR repertoires of effector $\mathrm{CD} 4^{+} \mathrm{T}$ cells and Tregs directed against non-self peptides has not been resolved, although some overlap is apparent with self-peptides [386]. The selected $\mathrm{CD}^{+} \mathrm{T}$ cell epitope from ESAT-6 may restimulate CD4 effector T cells, but it may not engage a TCR within the Treg repertoire. Therefore, it would have been valuable to evaluate immune responses not only to the ESAT-6 peptide but also to other peptides, if not entire proteins or mixed mycobacterial protein preparations.

By contrast to these two studies, the results presented in this chapter correlate inactivation of Tregs with an increase in mycobacteria-specific cytokine production (Figs. 3.8-3.10). Other studies have used polyclonal stimulation and not observed any differences in cytokine production [383] or observed an enhanced polyclonal response with no enhancement of mycobacterial peptide-specific cytokine production [384]. However, the methodology used in this chapter, which targeted $\mathrm{CD} 4{ }^{+} \mathrm{CD} 25^{+}$but not $\mathrm{CD} 4^{+} \mathrm{CD} 25^{-} \mathrm{Foxp}^{+}$Tregs, did not result in reduced bacterial numbers in the tissues following mycobacterial infection (Figs. 3.11/3.13), while the other studies exhibited significant reductions [383, 384]. The methodology used by Kursar, et al. and ScottBrowne, et al. resulted in more profound differences in proportions of $\mathrm{CD} 4{ }^{+} \mathrm{CD} 25^{+}$ Tregs and $\mathrm{CD} 4{ }^{+} \mathrm{Foxp}^{+}$Tregs respectively, relative to the total $\mathrm{CD} 4^{+} \mathrm{T}$ cell population. This could account for the differences in bacterial numbers observed. The more profound depletion of Tregs, and in particular the ability to deplete total $\mathrm{CD} 4{ }^{+} \mathrm{Foxp}^{+}$ 
Tregs in the study by Scott-Browne, et al., is a significant advantage over the limitations of in vivo PC61 administration used to inactivate CD25 $5^{+}$Tregs.

However, interpretation of the studies by Kursar, et al. and Scott-Browne, et al. is complicated by the fact that they induced more profound differences in proportions or numbers of Tregs than in vivo PC61 administration. The profound differences in Treg numbers and ratios might not reflect fluctuations in Tregs that occur during natural mycobacterial infection, and the physiological relevance of these studies is unknown. Furthermore, a complete lack of Tregs can induce generalised immune activation and autoimmunity, as was pointed out by Scott-Browne, et al. As a result, it is difficult to control for the effect of generalised immune activation and the resulting cytokine and chemokine milieu on bacterial numbers. Either mycobacteria-specific responses or generalised responses could result in the observed decreases in bacterial numbers.

In summary, several studies, including mine, have explored the role of Tregs in M. tuberculosis infection using different murine models that possess different advantages and disadvantages. To date, it is still unclear whether natural Tregs allow mycobacterial infections to take hold and persist within the murine models, let alone during human $\mathrm{Tb}$, under normal physiological conditions. As more tools are developed for manipulation of Treg subsets in vivo (section 6.2.6), more extensive assessment of Treg suppression during mycobacterial infection will be possible. 


\subsection{Summary}

The work presented in this chapter demonstrates that inactivation of $\mathrm{CD} 4{ }^{+} \mathrm{CD} 25^{+}$Tregs with PC61 treatment prior to mycobacterial infection resulted in modest increases in cytokine production but did not affect bacterial numbers in the lungs after BCG, $M$. tuberculosis or M. bovis infection. This suggests that $\mathrm{CD} 4^{+} \mathrm{CD} 25^{+}$Tregs mildly suppress immune responses induced by mycobacterial infection, but do not suppress immune responses to the point where pathogen clearance is inhibited. This outcome contrasts with other published studies where inactivation of Tregs in infection models can enhance both immune responses and pathogen clearance. This implies that mycobacteria are not as sensitive to the effects of Treg inactivation as other infectious agents that have been tested.

Preliminary studies suggest that PC61 treatment prior to M. bovis infection may result in a transient reduction in pulmonary inflammation around day 44 after infection. This could indicate that Tregs contribute to the increased severity of pathology that is observed during M. bovis infection compared to other mycobacterial infections in the murine model. $\mathrm{CD} 4{ }^{+} \mathrm{CD} 25^{+}$Treg activity was also experimentally modulated during the chronic phase of infection but neither enhancement nor inhibition of Treg activity significantly affected lung bacterial numbers or pathology.

This study illustrates that, despite modestly enhancing acute immune responses to mycobacterial infection, inactivation of $\mathrm{CD} 4{ }^{+} \mathrm{CD} 25^{+}$Tregs did not affect bacterial numbers during the acute phase of a virulent mycobacterial infection. It must be noted that this does not entirely preclude a role for Tregs during mycobacterial infection. Treg suppression during chronic infection and the effect of $\mathrm{CD} 4^{+} \mathrm{CD} 25^{-} \mathrm{Foxp} 3^{+}$and induced Treg subsets during mycobacterial infection remain exciting possibilities that can be explored, as novel tools for in vivo Treg manipulation are developed. 
Chapter 4: Construction of a novel plasmid DNA replicon vaccine against $\mathrm{Tb}$ 


\subsection{Introduction}

The efficacy of the BCG vaccine is highly variable (section 1.3.4) and as a result there is a considerable drive to develop new vaccines against $\mathrm{Tb}$. Although many candidate $\mathrm{Tb}$ vaccines induce cell-mediated immunity with both $\mathrm{CD} 4^{+}$and $\mathrm{CD} 8^{+} \mathrm{T}$ cell activation, many researchers have focussed primarily on enhancing $\mathrm{CD}^{+} \mathrm{Th} 1$ immunity (section 1.2.6). However, the contribution of $\mathrm{CD} 8^{+} \mathrm{T}$ cells to vaccination against $\mathrm{Tb}$ is just beginning to be explored (section 1.2.6) and DNA vaccination offers a promising way to induce $\mathrm{CD}^{+} \mathrm{T}$ cell-mediated immunity.

\subsubsection{DNA plasmid vaccination for Tb: Pros and Cons}

DNA plasmid vaccination can establish effective humoral and cell-mediated immunity [230, 231], making it an attractive approach for preventing infections such as $\mathrm{Tb}$.

Plasmid constructs encoding mycobacterial antigens have been used as DNA plasmid vaccines against $\mathrm{Tb}$ predominantly in murine models, inducing both humoral responses and cell-mediated responses and resulting in a level of protection comparable to BCG $[387,388]$. For protection against Tb, DNA plasmid vaccines should be administered i.m. rather than by gene-gun bombardment, as gene-gun administration favours humoral rather than cellular immunity [389]. DNA plasmid vaccination can also broaden the epitopic repertoire against the target antigen as compared to the repertoire induced for the same antigen by BCG vaccination [390].

A significant problem facing DNA plasmid vaccination in general is that DNA plasmid vaccination of humans and non-human primates has not resulted in the levels of protection seen in murine models (reviewed in [232]). Immunogenicity can be enhanced by modulation of one or more factors, such as codon optimisation, optimising $\mathrm{CpG}$ motifs, using immunological adjuvants or incorporating DNA vaccines into prime-boost approaches. These strategies will be further described in section 4.4.4. Even so, the immunogenicity of DNA vaccines is poor when translated into humans. This may be due to the fact that this technique has been optimised for murine models, but not humans with respect to dose amount, dose volume, site of administration and method of delivery. In addition to the observed variation in immunogenicity across species, direct 
scaling of DNA plasmid vaccine dosage from a $25 \mathrm{~g}$ mouse into a $75 \mathrm{~kg}$ human means a 3000 -fold increase in the amount of DNA required. This is challenging for vaccine manufacture and administration, and may complicate the transition of DNA plasmid vaccines into human trials [391]. Reducing the required dose of DNA, as well as optimising immunogenicity, will be vital for the successful translation of DNA plasmid vaccination from murine models into humans.

\subsubsection{Plasmid DNA replicons}

To minimise some of the limitations of conventional DNA plasmid vaccines, plasmid DNA replicons have been developed. Plasmid DNA replicons encode the target antigen as well as a ribonucleic acid (RNA) replicase gene, which enhances vaccine immunogenicity, often at lower doses than conventional DNA plasmids [392]. The RNA replicases used in DNA replicons are derived from single-stranded, positive-sense strand (+str) RNA viruses that require an RNA replicase to replicate their genome in infected cells. Most DNA replicon vectors used for vaccination have been derived from two families of viruses: alphaviruses, such as the Semliki Forest virus [393] and Sindbis virus [394], and flaviviruses, such as the Yellow Fever virus [395] and Kunjin virus [396].

Plasmid DNA replicons exhibit increased immunogenicity at lower doses than conventional plasmids. It was originally hypothesised that, since RNA replicase can amplify the amount of mRNA encoding the target antigen, the amount of target antigen produced is also amplified. It is difficult to compare antigen expression between conventional DNA plasmids and plasmid DNA replicons in vivo but it is unlikely that increased antigen expression alone accounts for the pronounced increase in immunogenicity observed with plasmid DNA replicons [392, 397].

The formation of double-stranded (ds) RNA has also been proposed to enhance the immunogenicity of plasmid DNA replicons [397]. The amplification of +str RNA by an RNA replicase requires that + str RNA serves as a template for negative-sense strand (-str) RNA synthesis, and during this process dsRNA is formed (Fig. 4.1). In a eukaryotic cell, dsRNA is typically only present during viral infections and thereby dsRNA acts as a PAMP. The interaction of dsRNA with several PRRs initiates signalling through several pathways, outlined in Fig. 4.1. The PRRs include TLR3, 
retinoic acid inducible gene-I (RIG-1) and protein kinase receptor (PKR) (Fig. 4.1). These signalling pathways are responsible for inhibition of protein synthesis in virally infected cells, as well as initiation of inflammation through type I IFN release, promoting the development of a robust immune response (Fig. 4.1).

Type I interferon production also has the effect of activating the RNase L enzyme. Once activated, RNase L can degrade RNA within the cell but it can also initiate caspase-dependent apoptosis. The initiation of the RNase L pathway has been shown to be vital for cell-mediated immunity after vaccination with plasmid DNA replicons, as a deficiency in RNase L [397, 398] or type I IFNs [399] can abrogate vaccine efficacy in murine models. Collectively, the current model is that plasmid DNA replicons cause type I interferon production, which activates the immune response, but also results in inhibition of protein synthesis, degradation of RNA and apoptosis of the transfected cell (Fig. 4.1). 


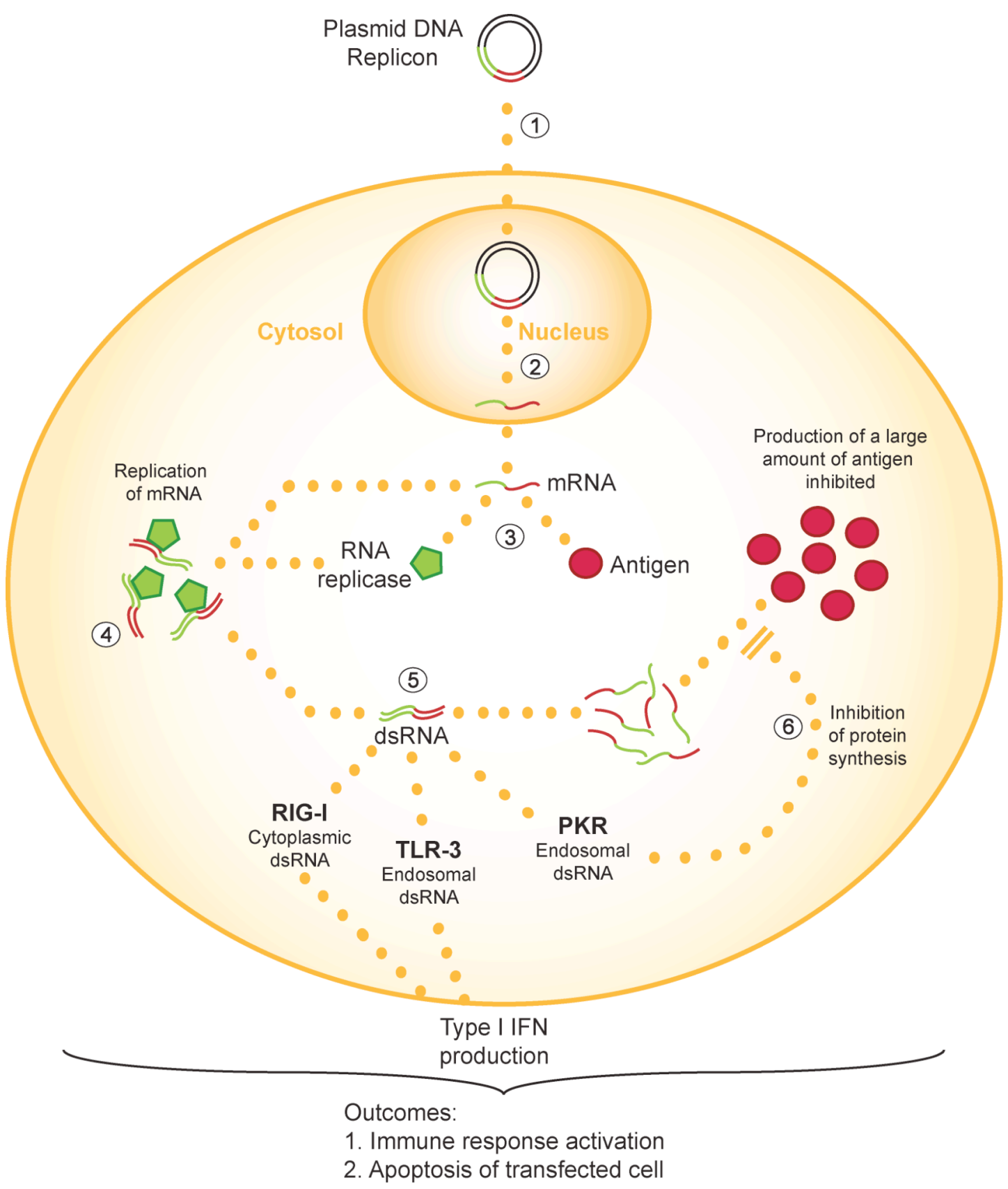

\section{Figure 4.1: Proposed mechanism of action for plasmid DNA replicons}

1) Like conventional DNA plasmids, plasmid DNA replicons are taken up by the host cell and presumably translocate to the nucleus. 2) In the nucleus, polycistronic + str messenger RNA (mRNA) is transcribed and transported to the cytosol. 3) In the cytosol, the +str mRNA is translated into both the target antigen and the RNA replicase. 4) The RNA replicase can bind the + str mRNA and use it as a template to replicate -str mRNA and subsequently more + str mRNA [400]. 5) The newly formed dsRNA can interact with a number of different PRRs. Extracellular and endosomal dsRNA binds to TLR3, which is present on cell membranes, and initiates a danger signal cascade that leads to type I IFN production and, in APCs, this also leads to upregulation of activation markers [401]. Cytoplasmic dsRNA binds RIG-I present in the cytosol, which activates interferon regulatory factor-3 and NF- $\kappa \mathrm{B}$, resulting in type I interferon 
production [402]. Cytoplasmic dsRNA can also bind to cytoplasmic PKR [403, 404]. Binding of dsRNA or even IFN signalling activates PKR and leads to the phosphorylation and inhibition of eukaryotic translation initiation factor 2 (reviewed in [405]). 6) PKR activation inhibits protein expression, which may explain why vastly increased antigen expression is not observed after DNA replicon administration despite amplification of the mRNA encoding the target antigen. Overall, transfection of a cell with a plasmid DNA replicon results in type I IFN production, which acts as danger signal to initiate the immune response, but also eventual apoptosis of the host cell. 


\subsubsection{Apoptosis and DNA vaccine immunogenicity}

Minimising apoptosis of DNA-transfected cells can enhance the efficacy of DNA vaccines. Co-administration of the Bcl-xL anti-apoptotic gene on a conventional plasmid DNA vaccine resulted in enhanced anti-tumour responses [406], showing that inhibiting apoptosis can enhance DNA vaccination. Apoptosis is thought to diminish DNA vaccine efficacy by reducing the amount of antigen expressed, reducing the window of time during which antigen is expressed or by removing transfected APCs that would otherwise present antigen.

On the other hand, minimising apoptosis of DNA-transfected cells can decrease DNA vaccine immunogenicity. Plasmid DNA replicons are typically cytopathic and interference with apoptosis of transfected cells can diminish the immunogenicity of plasmid DNA replicon vaccines. Co-administration of the Bcl-xL anti-apoptotic gene on a cytopathic plasmid DNA replicon reduced protection by the vaccine against a tumour challenge [407]. The current rationale for this is that large number of apoptotic transfected cells expressing the target antigen and releasing type I IFNs result in a localised inflammatory environment at the vaccination site [408]. APCs attracted into this environment would phagocytose large amounts of apoptotic debris containing target antigen, resulting in efficient distribution of antigen to APCs and increased antigen presentation [408]. Therefore, apoptosis is regarded as a mechanism that enhances efficacy of cytopathic plasmid DNA replicon vaccines.

Non-cytopathic plasmid DNA replicons are also available, such as the Kunjinbased plasmid DNA replicons. Kunjin-based plasmid DNA replicons can replicate mRNA transcripts and express protein encoded on the replicon in vitro and in vivo for prolonged periods of time, as the Kunjin RNA replicase possesses intrinsic antiapoptotic activity [396, 409]. The non-structural (NS) proteins expressed by Kunjinbased plasmid DNA replicons inhibit both IFN- $\alpha / \beta$ and IFN- $\gamma$ signalling, presumably by preventing phosphorylation of STAT1 and STAT2, which mediate IFN signalling in the transfected cell [410]. Decreased IFN signalling means the transfected cell is less likely to undergo apoptosis.

Vaccination with Kunjin-based plasmid DNA replicons has been shown to confer protective immunity in tumour and infectious challenge murine models at lower doses compared with conventional plasmids [411-413]. Kunjin-based plasmid DNA 
replicons have been shown to induce strong $\mathrm{CD}^{+} \mathrm{T}$ cell responses with IFN- $\gamma$ production and cytolytic activity $[411,414]$, presumably due to production of antigen in the cytosol that would effectively access the MHC I presentation pathway. Therefore, Kunjin-based plasmid DNA replicons are immunogenic, particularly for $\mathrm{CD}^{+} \mathrm{T}$ cells, and require lower doses than conventional plasmid DNA vaccines to induce equivalent responses. Due to their non-cytopathic nature, Kunjin-based plasmid DNA replicons are thought to produce antigen for longer periods in vivo than cytopathic plasmid DNA replicons.

\subsubsection{Antigen 85A}

The Antigen 85 (Ag85) complex comprises 3 highly related proteins [415, 416] Ag85A, $\mathrm{B}$ and $\mathrm{C}$, which are encoded by separate genes, $f b p A, f b p B$ and $f b p C$, in the genome of M. tuberculosis [417-419]. Ag85 complex gene homologs are present in other mycobacteria including M. bovis BCG, M. bovis and M. leprae [417-420], suggesting a fundamental role for this complex in mycobacterial physiology. Ag85 complex proteins are the major secreted proteins in mycobacterial liquid cultures but they are also thought to associate transiently with the cell wall of mycobacteria (Fig. 4.2). The proteins share a fibronectin-binding capacity, which suggests they may be necessary for complement receptor phagocytosis of $M$. tuberculosis [421, 422]. Their major function appears to be as mycolyl-transferases, to mediate the production of the glycolipids $\alpha, \alpha^{\prime}$-trehalose dimycolate and $\alpha, \alpha^{\prime}$-trehalose monomycolate [423]. Blocking mycolyl-transferase activity inhibited growth of Mycobacterium aurum $\mathrm{A}^{+}$, a surrogate organism used for M. tuberculosis drug testing, but did not inhibit growth of E. coli on solid media [423]. Therefore, Ag85A, B and C are attractive anti-mycobacterial drug targets [423]. These glycolipids form a pseudo-lipid bilayer with mycolic acids (Fig. 4.2) and regulate mycobacterial cell wall permeability. Thus, Ag85 complex proteins are critical for the final stages of mycobacterial cell wall biogenesis and can be exposed on outer surface of the mycobacterial cell wall. 


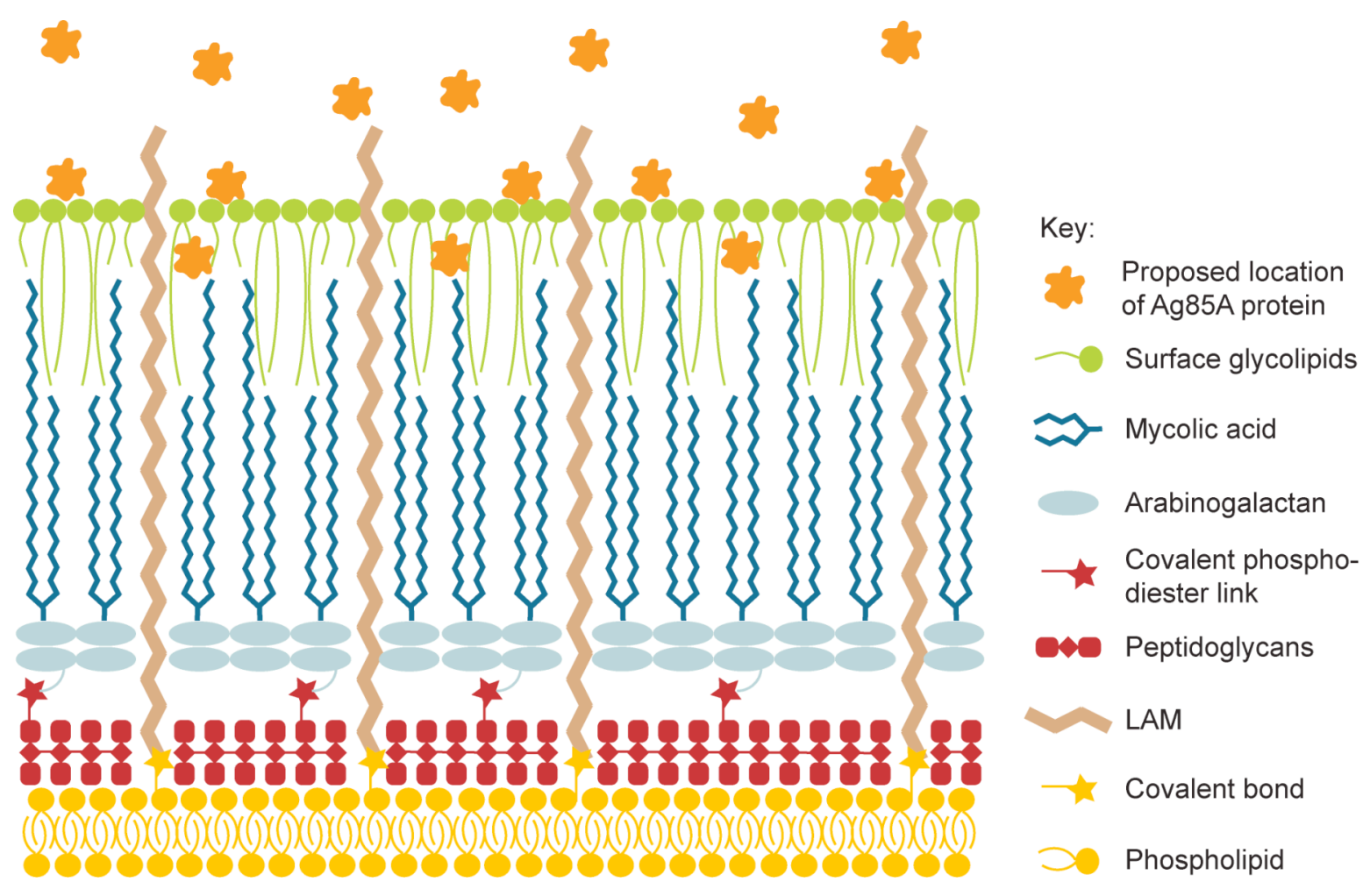

Figure 4.2: Schematic view of the composition of the mycobacterial cell wall

This schematic, based on a figure in McNeil, et al. [424], demonstrates several key features of the mycobacterial cell wall from slow-growing mycobacteria; the presence of a thin layer of peptidoglycan that is covalently bound to a layer of arabinogalactan via a phosphodiester linker, LAM covalently bound to the cell membrane, the presence of large amounts of mycolic acid bound to arabinogalactan and glycolipids intercalating with the mycolic acid on the surface to form a "pseudo-lipid bilayer" (reviewed in [425]). 
Since Ag85 complex proteins are present on the cell wall and secreted from mycobacteria in large amounts, they are also prime antigenic targets for the immune response and have been reported to be highly immunogenic. Initial studies showed that Ag85 complex proteins could be used for in vitro restimulation to induce proliferation and IFN- $\gamma$ secretion in splenocytes from BCG-vaccinated mice [426] and in PBMCs from BCG-vaccinated or M. tuberculosis-infected human subjects [427, 428].

Subsequently, components of the Ag85 complex have been further characterised for use in vaccination.

The Th1 epitopes in Ag85A, B and C have been mapped in seven strains of BCG-infected mice [429], M. tuberculosis-infected BALB/c and C57BL/6 mice [430] as well as in Mantoux test positive and M. tuberculosis-infected human subjects [431]. Despite significant homology between the proteins, there appears to be distinct regions within each of these proteins that induce strong $\mathrm{T}$ cell responses [430]. Ag85A and B proteins both generate strong Th1 responses and CTL responses when they are incorporated into conventional DNA plasmids and used in murine vaccination models [387, 432]. In contrast, Ag85C incorporated into a DNA plasmid vaccine does not generate effective Th1 and CTL responses [432] and, as a result, most vaccine approaches have utilised Ag85A and Ag85B as candidate Tb antigens.

Proteins from the Ag85 complex have also been incorporated into cytopathic plasmid DNA replicon vaccines. The $A g 85 A$ gene was incorporated into a cytopathic plasmid DNA replicon encoding a Sindbis-derived RNA replicase [433]. Vaccination of $\mathrm{BALB} / \mathrm{c}$ mice with this construct conferred a $0.77 \log _{10}$ reduction in lung bacterial numbers after virulent $M$. tuberculosis challenge and this protection was maintained long-term. The $A g 85 B$ gene has also been incorporated into a Sindbis-derived plasmid DNA replicon and conferred significant protection against $M$. tuberculosis infection [398]. However, a protein from the Ag85 complex has not yet been used in a noncytopathic DNA replicon system. Thus, the aim of this chapter was to develop a novel non-cytopathic plasmid DNA replicon, encoding Ag85A, which I hypothesised would result in long-term expression of antigen in a highly immunogenic construct and strong induction of $\mathrm{CD} 8^{+} \mathrm{T}$ cell responses. 


\subsection{Aims}

In this chapter, I aimed to develop and test a novel DNA replicon vaccine against $\mathrm{Tb}$ infection. This novel construct would express Ag85A as the target antigen and also express RNA replicase from the Kunjin virus, resulting in the first non-cytopathic plasmid DNA replicon vaccine directed against $\mathrm{Tb}$.

I hypothesised that pKUN85A would induce cell-mediated immunity with strong $\mathrm{CD} 8^{+} \mathrm{T}$ cell responses, which would control infection in the lung and mitigate dissemination to other organs. I anticipated that protection from $\mathrm{Tb}$ infection would be conferred by pKUN85A at lower doses than conventional DNA vaccines. My main objectives were to:

- Clone Ag85A into a Kunjin-based vector called pKUNrep6, resulting in a novel plasmid DNA replicon vaccine called pKUN85A.

- Test pKUN85A for Ag85A protein expression in a mammalian cell line.

- Evaluate pKUN85A for immunogenicity in the C57BL/6 murine model.

- Evaluate protection afforded by pKUN85A against Tb infection, dissemination of infection and infection-induced pathology in the C57BL/6 murine model.

Furthermore, as Kunjin-based vectors are non-cytopathic, pKUN85A should express protein in vivo for a longer period of time compared to cytopathic plasmid DNA replicons. If antigen persistence is required for maintenance of mycobacterial immunity, then protection conferred by pKUN85A will be relatively long-lived. 


\subsection{Results}

\subsubsection{Designing the cloning strategy}

The first step towards developing the novel DNA replicon vaccine, pKUN85A, was to design a strategy for cloning the $A g 85 A$ gene into the cloning site of the pKUNrep6 vector. The $A g 85 A$ coding sequence was to be amplified from a construct encoding the Ag85A gene called pcDNA3-85A (Fig. $4.3 \mathrm{~A}$ ), which has been used as a conventional plasmid DNA vaccine against $\mathrm{Tb}$ [433]. The $\mathrm{Ag} 85 \mathrm{~A}$ gene was then cloned into the MluI cloning site of the pKUNrep6 DNA vector (Fig. 4.3 B). The pKUNrep6 DNA vector was supplied by Dr Alex Khromykh, University of Queensland, Brisbane, Australia.

The sequence cloned into the MluI cloning site of pKUNrep6 must be in-frame with the open reading frame (ORF) surrounding the cloning site. This ORF stretches from just after the cytomegalovirus (CMV) EI promoter to the end of NS-5, resulting in a polycistronic mRNA. In transfected cells, this polycistronic mRNA is translated into a polypeptide containing foot and mouth disease virus (FMDV) autoprotease domains either side of the cloning site (Fig. 4.4). The autoproteolytic action of the FMDV domains releases the peptide sequence encoded between them, releasing target antigens cloned into this site. Downstream of the cloning site on the same polypeptide are regions for the Kunjin NS proteins: NS-1, NS-2A, NS-2B, NS-3, NS-4A, NS-4B and NS-5 (Fig. 4.4). The Kunjin NS proteins form a complex that functions as the RNA replicase of the Kunjin virus [434, 435]. The cloning cassette in pKUNrep6 contains only the MluI restriction site. Therefore, the Ag85A gene must be cloned into the MluI cloning site of pKUNrep6, in-frame with the surrounding ORF, to ensure effective transcription and translation both of Ag85A and the NS proteins of the Kunjin RNA replicase.

A cloning strategy (Fig. 4.5) was developed to clone the $A g 85 A$ gene into the MluI site of pKUNrep6, creating the new plasmid pKUN85A (Fig. 4.3 D). The $A g 85 A$ coding sequence was amplified by PCR using primers with 5'ends that incorporated MluI restriction sites at either end of the PCR product (Table 4.1). 
A.

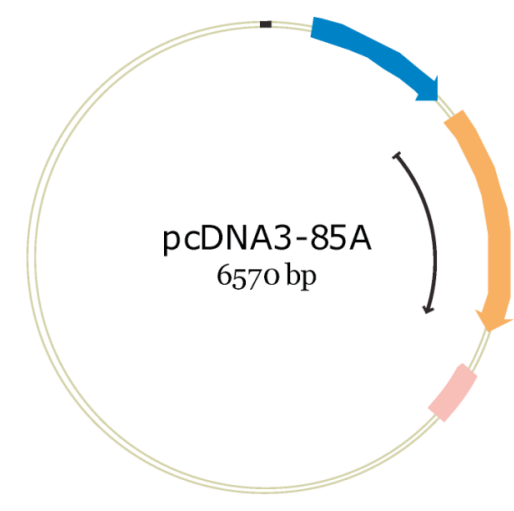

C.

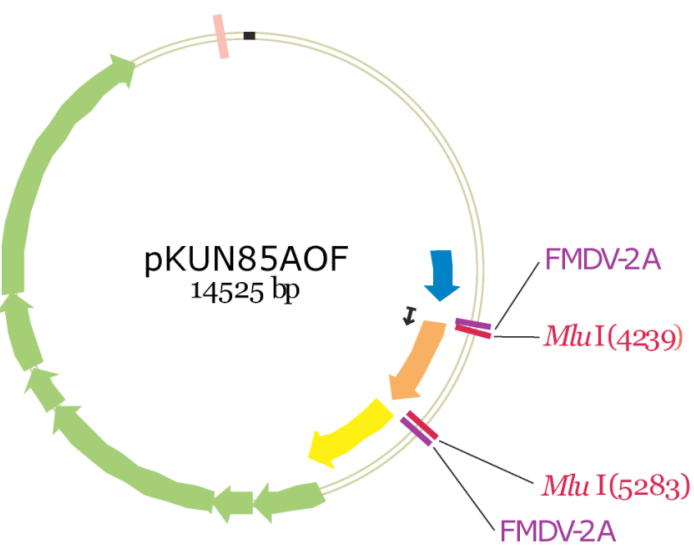

$\begin{aligned} \text { Key: } & \Rightarrow \text { CMV El promoter } & & \Rightarrow \text { NS-1 } \\ & \Rightarrow \text { Ag85A } & \Rightarrow & \text { Other NS genes }\end{aligned}$
B.

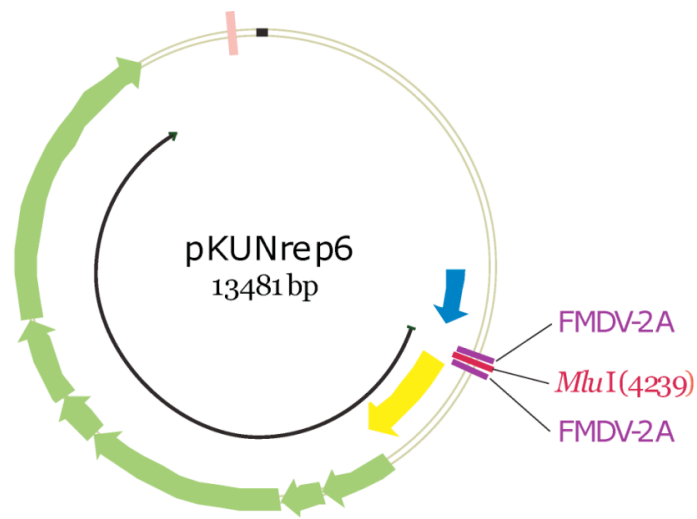

D.

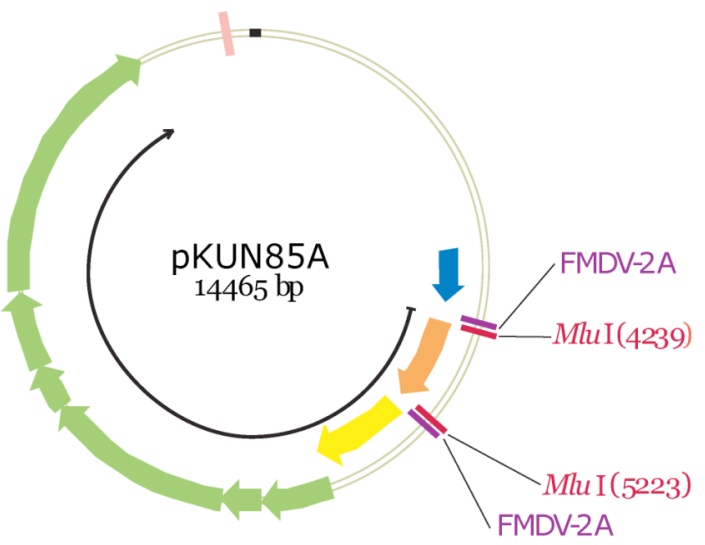

$\mapsto \mathrm{ORF}$
- PolyA site

- Mlul restriction site

- FMDV-2A autoprotease domain

\section{Figure 4.3: Plasmid diagrams for pcDNA3-85A, pKUNrep6, pKUN85AOF and pKUN85A}

Plasmid features include CMV EI promoter regions (dark blue), Ag85A (orange), $N S-1$ (yellow), the other Kunjin NS genes $N S-2 A, N S-2 B, N S-3, N S-4 A, N S-4 B$ and $N S-5$ in sequence (green), MluI restriction sites (red), foot and mouth disease virus autoprotease domains (purple), polyadenylation (PolyA) sites (light pink) and the ORFs running from start to termination codons (black arrows). Total bp size of the plasmids is indicated and the first bp is designated by the black notch at the top of the plasmid. A) The pcDNA3-85A plasmid containing Ag85A in an ORF running from 932 to $1975 \mathrm{bp}$. B) The pKUNrep6 vector with the MluI cloning site at $4239 \mathrm{bp}$ and the ORF spanning the MluI site, NS-1 and the other NS genes from 4090 to 12330 bp. C) The pKUN85AOF construct with $A g 85 A$ inserted into the $M l u I$ site out of frame. $A g 85 A$ insertion introduces a premature stop codon at $4242 \mathrm{bp}$, which truncates the ORF so it only spans from 4090 to $4242 \mathrm{bp}$ and prevents translation of NS-1 and the other NS genes. D) The pKUN85A construct with $A g 85 A$ inserted into the MluI site in the correct orientation and inframe with the surrounding ORF. This creates a large ORF spanning from 4090 to $13314 \mathrm{bp}$ that includes $A g 85 A, N S-1$ and the other NS genes. 


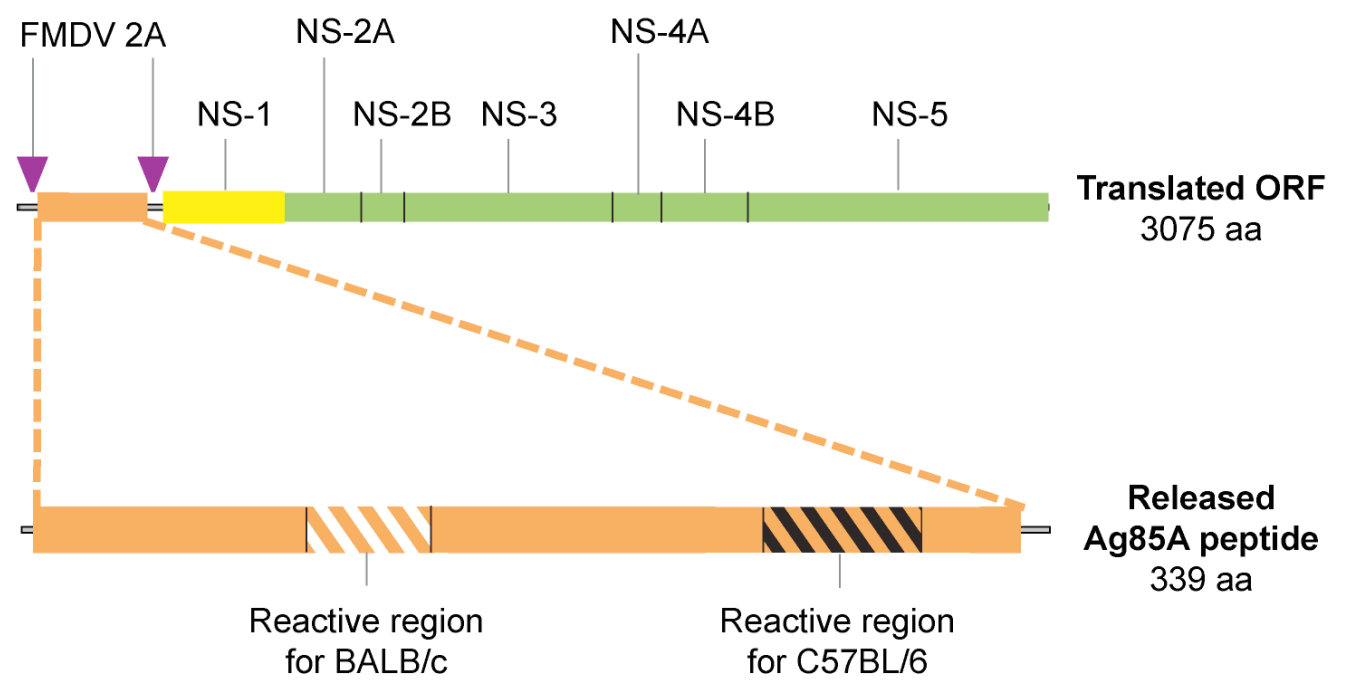

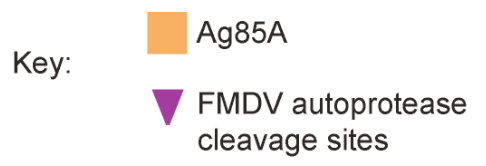
cleavage sites
NS-1

Reactive region of Ag85A for $\mathrm{BALB} / \mathrm{c}$
Other NS peptides

Reactive region of Ag85A for C57BL/6

\section{Figure 4.4: Schematic diagram of the translated product from pKUN85A}

The pKUN85A ORF (outlined in Fig. 4.3 D) encodes the peptides outlined above. The translated ORF is a 3075 amino acid (aa) polypeptide containing the Ag85A peptide and the Kunjin virus NS peptides NS-1, NS-2A, NS-2B, NS-3, NS-4A, NS-4B and NS-5. The Ag85A peptide is bordered by FMDV 2A auto-protease domains, which mediate the release of the Ag85A peptide from the polypeptide. Other proteolytic domains between the NS peptides mediate the release of these peptides and permit formation of the Kunjin RNA replicase. The released 339 aa peptide encoding Ag85A does not contain the full-length Ag85A protein but it contains the regions of Ag85A that encode epitopes, which induce strong cytokine production in BCG vaccinated or M. tuberculosis infected BALB/c and C57BL/6 mice. 


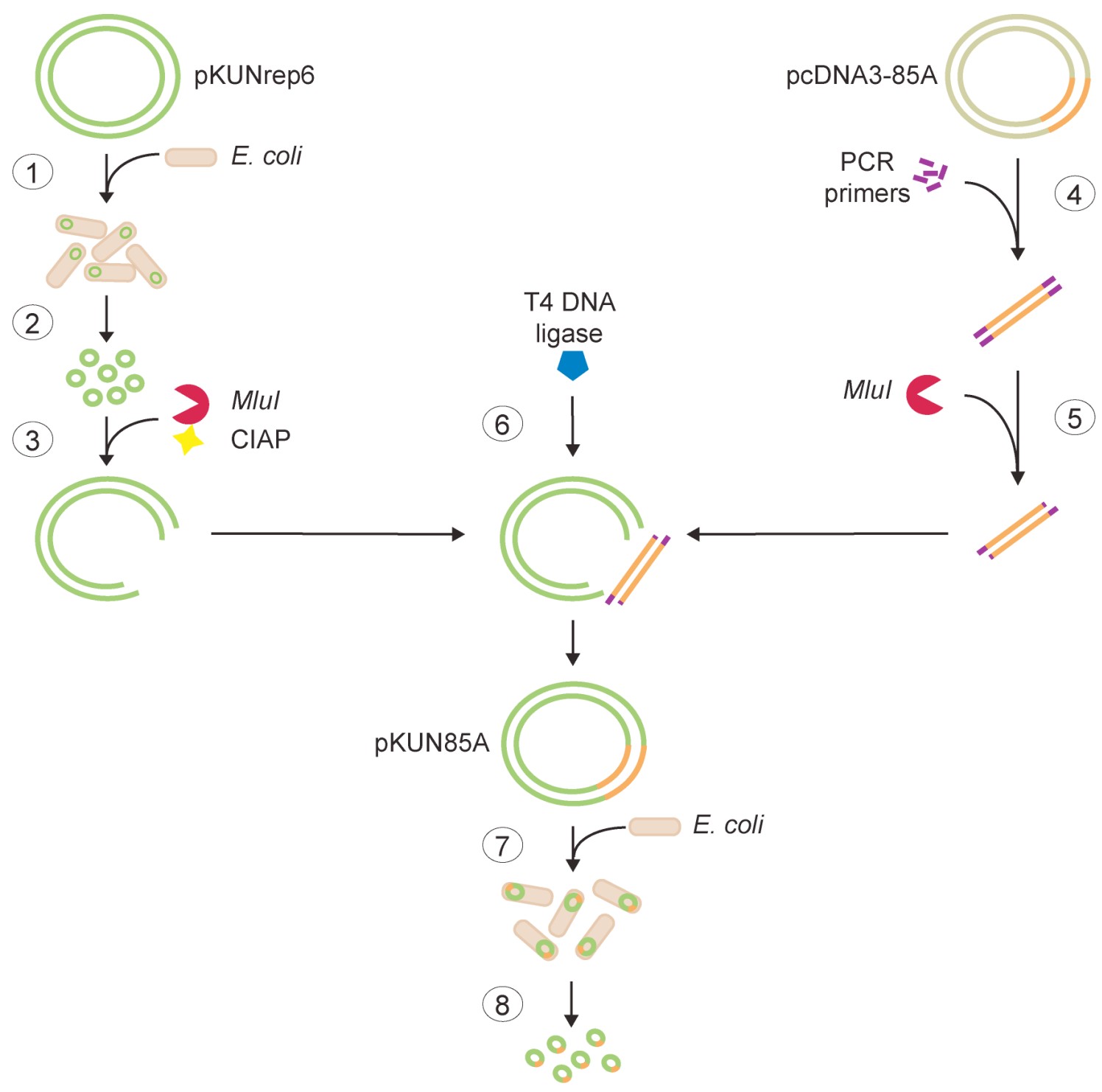

\section{Figure 4.5: The cloning strategy for the creation of pKUN85A}

1) pKUNrep6 is amplified by transformation into E. coli. 2) E. coli are propagated and pKUNrep6 plasmid stock is purified by plasmid mini-prep. 3) pKUNrep6 is digested with MluI, dephosphorylated with CIAP, purified and quantified in preparation for ligation. 4) The $A g 85 A$ gene is PCR amplified from pcDNA3-85A, which introduces MluI restriction sites to either end of the product, and the product is purified. 5) The $A g 85 A$ PCR product is digested with MluI, purified and quantified in preparation for ligation. 6) The $A g 85 A$ PCR product and pKUNrep6 are ligated using T4 DNA ligase, creating pKUN85A. 7) E. coli are transformed with ligation reactions and transformants are screened for the presence of intact pKUN85A with orientation PCRs, orientation digests and DNA sequencing. 8) A transformant containing pKUN85A is selected, propagated and used for vaccine production. 
The positioning of the primers, called 5' Ag85A and 3' Ag85A, and MluI restriction sites relative to the $A g 85 A$ gene (Table 4.1) was chosen to ensure that after cloning, the $A g 85 A$ coding sequence would be in-frame with the ORF that extends over the cloning site (Fig. 4.4). The sequence was also selected to avoid incorporating start or stop codons. One complication that arose during primer design was that the 3 ' end of the $A g 85 A$ gene is rich in guanine $(\mathrm{G})$ and cytosine $(\mathrm{C})$ bases rather than adenine (A) and thymine $(\mathrm{T})$ bases, making design of primers to target this region difficult. High GC content in a PCR primer increases the melting temperature of the primer and prolonged periods at higher heat decreases the efficacy of the DNA polymerase used in the PCR reaction. Therefore, while the $5^{\prime}$ primer includes the first codon after the $\mathrm{Ag} 85 \mathrm{~A}$ start codon, the 3' primer truncates the end of $A g 85 A$, as it was designed to bind to a less GC-rich sequence. However, the sequence amplified by the primers retains regions of the $\mathrm{Ag} 85 \mathrm{~A}$ protein that have been reported to be immunogenic in BALB/c and $\mathrm{C} 57 \mathrm{BL} / 6$ mice (Fig. 4.4) [430].

Table 4.1: Ag85A PCR primer design

5' Ag85A primer: $\quad$ GCGACGCGTTGCAGCTTGTTGACAGGGTTCG

\begin{tabular}{l|l}
\hline GC content (\%) & 61.29 \\
Projected secondary structure & Weak \\
Projected primer dimer & None
\end{tabular}

3' Ag85A primer: $\quad$ GATACGCGTGACCCAGTGCCCGTTGCAGG

\begin{tabular}{l|l}
\hline GC content (\%) & 65.52 \\
Projected secondary structure & Weak \\
Projected primer dimer & None
\end{tabular}

Bases underlined indicate MluI restriction enzyme sites and bases in bold indicated sequence coding for Ag85A. Three filler bases were added 5' of the MluI restriction site to promote efficient enzyme scanning and digestion. 
A second primer pair was designed to clone the $A g 85 A$ gene into pKUNrep6 out of frame, creating the second plasmid pKUN85AOF (Fig. $4.3 \mathrm{C}$ ). The primers, called 5' Ag85A2 and 3' Ag85A2 (Appendix 8.1), were designed to incorporate MluI restriction sites at either end of the $\mathrm{Ag} 85 \mathrm{~A}$ gene and facilitate insertion of the gene into the MluI site using the cloning strategy described above. However, in this construct, the $\mathrm{Ag} 85 \mathrm{~A}$ coding sequence is out of frame with the ORF spanning the cloning site and the cloned section incorporates a stop codon to prematurely terminate transcription (Fig. 4.3 C).

Thus, the pKUN85AOF plasmid possesses the $A g 85 A$ coding sequence in the correct site and orientation but does not express either Ag85A or the Kunjin RNA replicase. The pKUN85AOF plasmid was used as a positive control for orientation PCRs and digests and a negative control for protein expression studies. The pKUNrep6 plasmid expresses only Kunjin RNA replicase and was used as a negative control for orientation PCR and digests and a positive control for Kunjin RNA replicase protein expression studies.

\subsubsection{Preparation of Ag85A PCR product and pKUNrep6 vector DNA}

Using pcDNA3-85A as template DNA, a PCR reaction to amplify the $A g 85 A$ gene was optimised and conditions are outlined in section 2.6.1. To amplify the $A g 85 A$ gene, PCR reactions used the high-fidelity AccuPrime Pfx PCR system, which has several features that ensure purity of the $A g 85 A$ PCR product. AccuPrime $P f x$ PCR system uses $P f x$ DNA polymerase to amplify the target sequence, which has intrinsic proofreading activity to minimise the risk of incorporating mutations into the amplified sequence. Anti- $P f x$ antibodies are also included, to prevent non-specific activity of $P f x$ at low temperatures, as are accessory proteins, to prevent mispriming during PCR amplification. Following PCR amplification of the $\mathrm{Ag} 85 \mathrm{~A}$ gene, a $\sim 990 \mathrm{bp}$ PCR product was observed (Fig. 4.6 A), which was purified and quantified in preparation for cloning (sections 2.6.8 and 2.6.9).

The pKUNrep6 vector was also prepared for cloning. DH5 $\alpha$ E. coli were transformed with pKUNrep6 plasmid (section 2.6.5), and a transformant containing the pKUNrep6 plasmid was selected and used to propagate the plasmid. The pKUNrep6 DNA was purified and quantified (sections 2.6.8 and 2.6.9) and MluI digestion and 
dephosphorylation of the pKUNrep6 vector were optimised in preparation for cloning (Fig. 4.6 B). Conditions are outlined in sections 2.6.2 and 2.6.3.
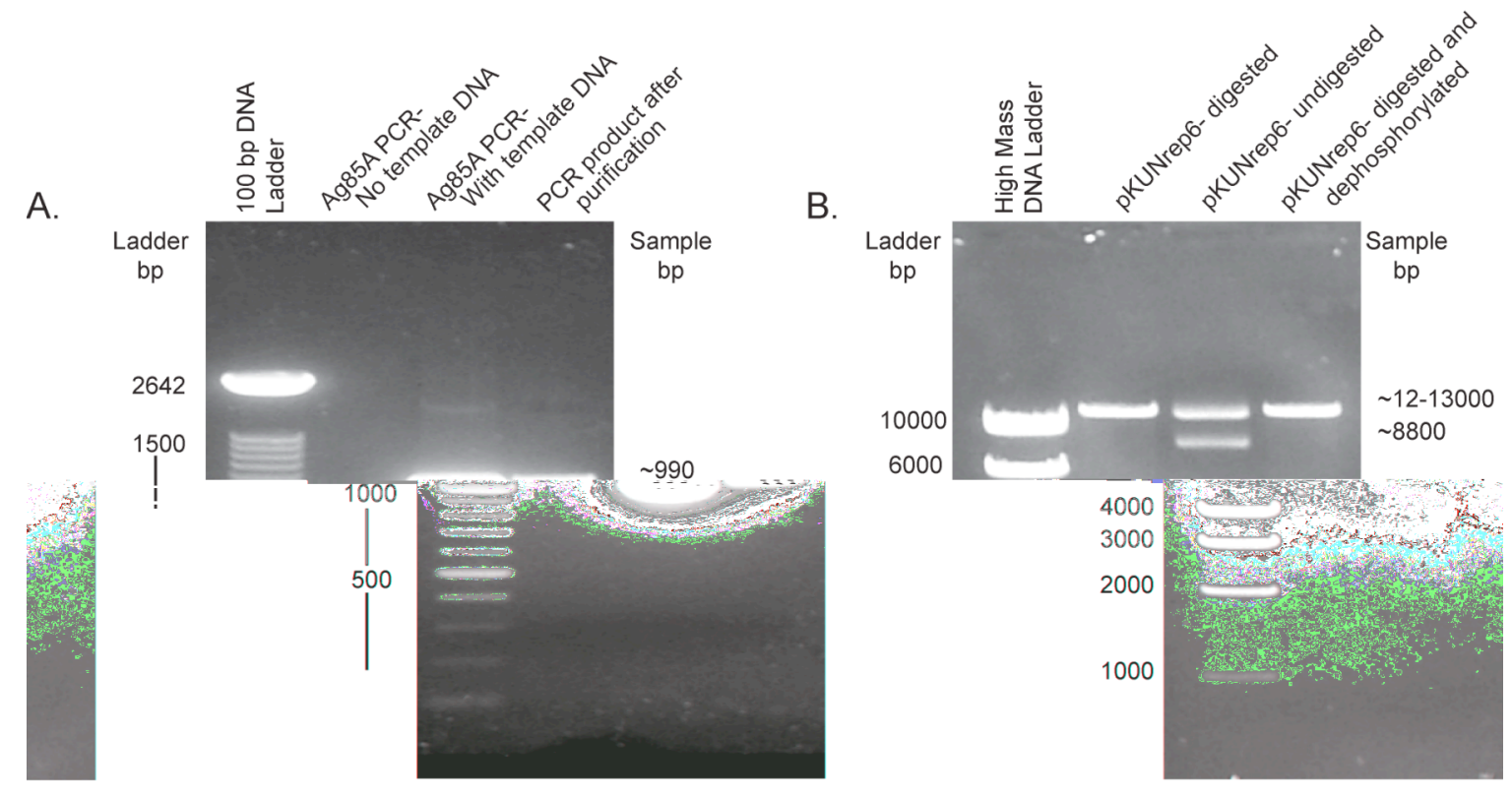

\section{Figure 4.6: Preparation of $\boldsymbol{A g} 85 \mathrm{~A}$ PCR product and pKUNrep6 for cloning}

A) Ten $\mu \mathrm{L}$ of $A g 85 A$ PCR products and $1 \mu 1$ of the purified $A g 85 A$ PCR product were run on a $1.5 \%$ agarose gel at $80 \mathrm{~V}$ for 1 hour. Four $\mu \mathrm{L}$ of the $100 \mathrm{bp}$ DNA ladder (Invitrogen) was run to estimate PCR product size. PCR product size was estimated at approximately $990 \mathrm{bp}$, which corresponds with the expected size of $996 \mathrm{bp}$. B) Three $\mu \mathrm{L}$ of pKUNrep6 digest products were run on a $0.8 \%$ agarose gel at $100 \mathrm{~V}$ for 1 hour. Four $\mu \mathrm{L}$ of the High Mass DNA ladder (Invitrogen) was run to estimate digest product size. Undigested pKUNrep6 ran as two bands at approximately 12000-13000 bp, which represents plasmid DNA in the open circle format, and a faster band at approximately $8800 \mathrm{bp}$, which represents more compact supercoiled plasmid DNA. Both digested and digested/dephosphorylated pKUNrep6 products ran as one band that was estimated at 12000-13000 bp, which corresponds with the expected size of linear pKUNrep6 at 13481 bp. Figures are representative of two replicate experiments. 


\subsubsection{Cloning Ag85A into pKUNrep6}

After the $A g 85 A$ PCR product and the pKUNrep6 vector had been prepared, they were used in the cloning strategy outlined in Fig. 4.5 (described in sections 2.6.2 to 2.6.5). The Ag85A PCR product and the pKUNrep6 vector were digested with MluI. Digested, linearised pKUNrep6 was also treated with CIAP. This removes the 5' phosphate group from the DNA backbone of the linearised vector, and prevents the digested ends of the vector from religating prior to ligase treatment.

The digested stocks of the $A g 85 A$ gene and pKUNrep6 were purified, quantified and ligated. Ligation reactions were incubated overnight and then transformed into $E$. coli. Transformation reactions were plated onto LB Amp ${ }^{+}$agar plates, as the pKUNrep6 vector contains an ampicillin resistance gene, to screen for transformants and resulting colonies were counted (data not shown). Positive controls were included, such as E. coli transformed with undigested pKUNrep6 DNA or pUC19 plasmid DNA, which also contains an ampicillin resistance gene, and these resulted in a large number of colonies. A negative control for transformation was also included, which was $E$. coli that were left untransformed, and this did not yield any colonies. E. coli transformed with target ligation reactions yielded colonies and these were screened for the insertion of the Ag85A gene into pKUNrep6 and orientation of the gene.

\subsubsection{Screening of transformants for pKUN85A}

Transformants were screened to check for the presence and orientation of the $\mathrm{Ag} 85 \mathrm{~A}$ insert in three ways: orientation PCR, orientation restriction digest and sequencing.

Colonies on transformant master plates were used for the orientation PCR (section 2.6.6), using the primer pair 5' CS and 3' Mid (Appendix 8.1). This primer pair is designed to amplify the sequence extending from within the pKUNrep6 vector upstream of the cloning site into the middle of $A g 85 A$, resulting in an $852 \mathrm{bp} \mathrm{PCR}$ product if the gene has inserted correctly (Fig. $4.7 \mathrm{~A}$ ). Three transformants containing the vector with the $A g 85 A$ gene inserted in the correct orientation were identified using the orientation PCR: numbers 33, 37 and 42 (Fig. 4.7 B). 
The orientation PCR is a rapid way to assess the orientation of the $A g 85 A$ insert but a restriction digest was performed to confirm these results. DNA samples were prepared from transformants 33, 37 and 42 (section 2.6.11) and used in an orientation restriction digest (section 2.6.7). The orientation restriction digest used the restriction enzymes $\mathrm{XbaI}$ and $\mathrm{KspI}$ (Fig. 4.8 A). Digestion of the projected plasmids with these enzymes results in fragments of 8802, 5196 and 467 bp if $A g 85 A$ is inserted in the incorrect orientation but fragments of 8064, 5196 and $1205 \mathrm{bp}$ if $\mathrm{Ag} 85 \mathrm{~A}$ is inserted in the correct orientation (Fig. $4.8 \mathrm{~B}$ ). All three transformants identified in the orientation PCR had bands at 8064, 5196 and 1205 bp after restriction digest, confirming that the $\mathrm{Ag} 85 \mathrm{~A}$ gene had inserted in the correct orientation.

Transformants 33, 37 and 42 contained the vector with the $A g 85 A$ insert in the correct orientation, as indicated by orientation PCR and orientation digest. These transformants were then sequenced using the Alan Wilson Centre Sequencing Service, Massey University, Palmerston North, New Zealand. Four primer sites were chosen to sequence the region spanning the cloning site in both the forward and reverse directions (Appendix 8.1 and 8.2). The projected construct sequence was constructed using Vector NTI Software (Invitrogen). A compiled sequence acquired for each transformant sample was compared to the projected construct sequence (Appendix 8.2) using the bl2seq Basic Local Alignment Search Tool, which is available on the National Center for Biotechnology Information website

(http://www.ncbi.nlm.nih.gov/BLAST/bl2seq/wblast2.cgi). Sequence comparison confirmed that transformants 33 (data not shown), 37 (Appendix 8.2) and 42 (data not shown) all contained the $A g 85 A$ gene, without mutations, and that insertion was inframe with the ORF spanning the cloning site. Therefore, transformants 33, 37 and 42 would all have been suitable for vaccine propagation, but transformant number 37 was chosen and used as the master transformant for pKUN85A (Appendix 8.2). Large liquid cultures of transformant number 37 were prepared and the pKUN85A plasmid was purified using endotoxin removal solution and endotoxin free buffers (section 2.6.12). Purified pKUN85A plasmid was used in protein expression, immunogenicity and protection assays. 
A.
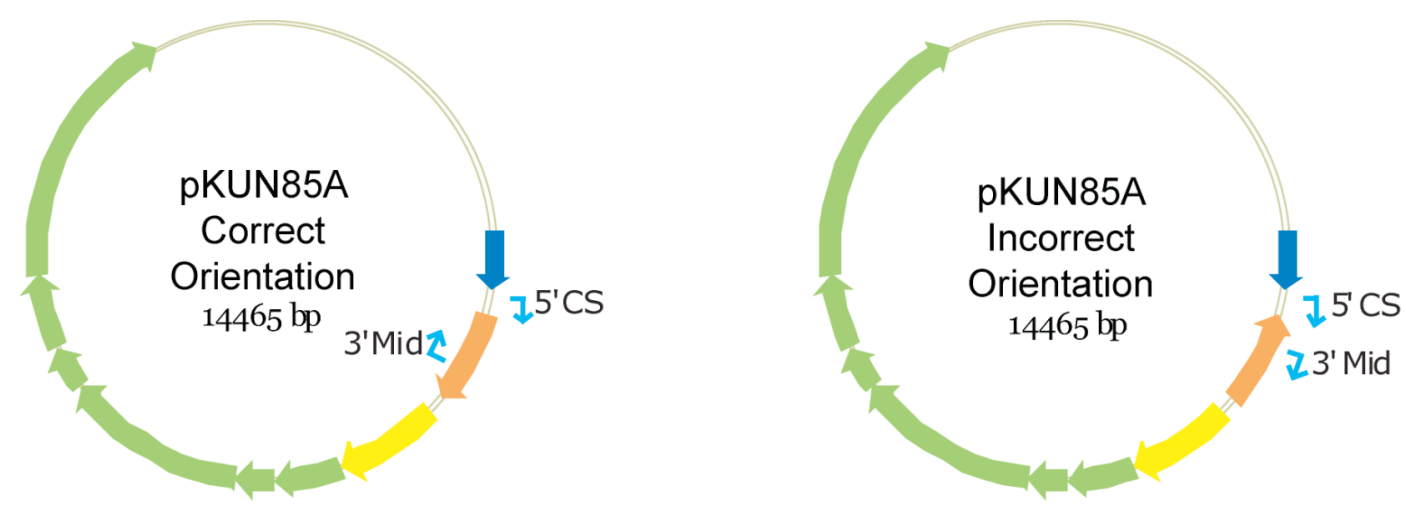

$$
\begin{aligned}
\text { Key: } & \Rightarrow \text { CMV El promoter } & \Rightarrow N S-1 & \mapsto \text { PCR primer sites } \\
& \Rightarrow \text { Ag85A } & \Rightarrow \text { Other NS genes } &
\end{aligned}
$$

B.

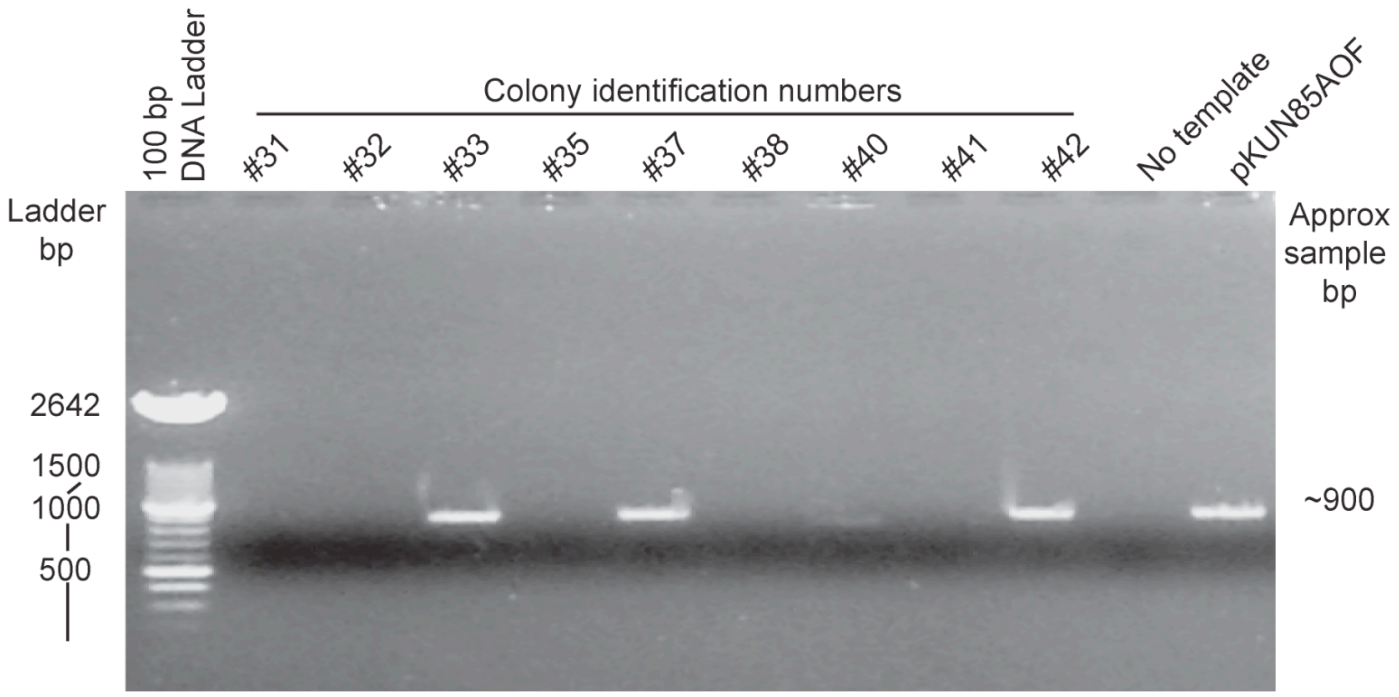

\section{Figure 4.7: Orientation PCR of transformants}

A) Transformants were screened for the presence and orientation of the $A g 85 A$ insert initially by orientation PCR, using the PCR primers 5' CS and 3' Mid. If the $A g 85 A$ gene had inserted in the correct orientation, an 852 bp PCR product was observed. If the $\mathrm{Ag} 85 \mathrm{~A}$ gene had not inserted or had inserted in the wrong orientation, there was no product. B) Ten $\mu$ l of orientation PCR products were run on an agarose gel (1.5\%) at $100 \mathrm{~V}$ for 1 hour. Four $\mu \mathrm{L}$ of the $100 \mathrm{bp}$ DNA ladder (Invitrogen) was run to estimate PCR product size. Orientation PCR product size was estimated at approximately $900 \mathrm{bp}$, which corresponds with the expected size of $852 \mathrm{bp}$. PCR products were observed in the pKUN85AOF positive control and three transformants; colony numbers 33, 37 and 42. Orientation PCRs were performed once to screen transformant colonies and results were confirmed using orientation digests and sequencing. 


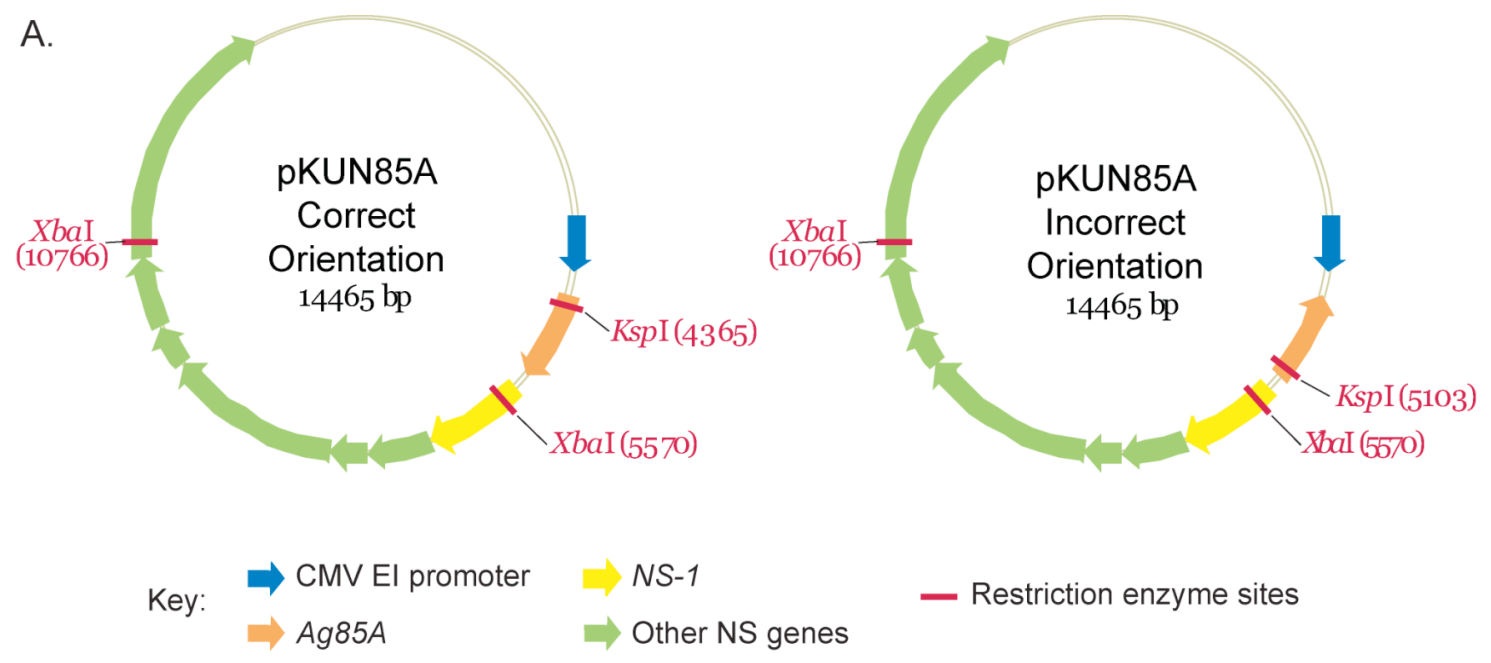

B.

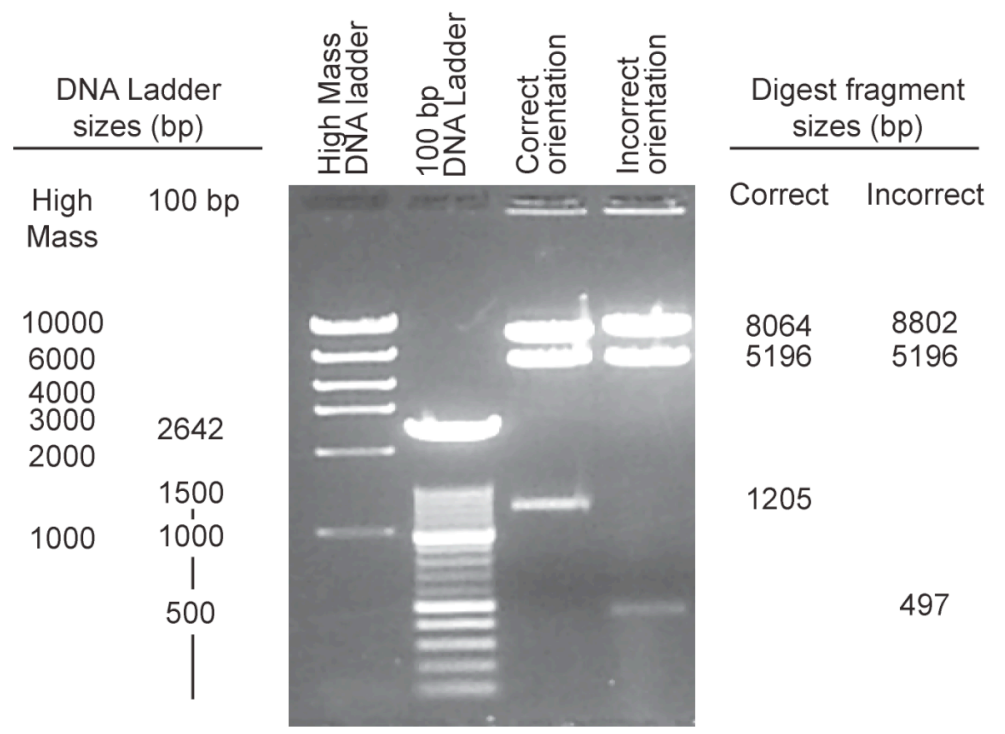

\section{Figure 4.8: Sample orientation digest for transformants}

A) Transformants were also screened for the presence and orientation of the $\mathrm{Ag} 85 \mathrm{~A}$ insert by an orientation digest using the restriction enzymes XbaI and KspI. B) Three $\mu 1$ of sample orientation digest products from pKUN85A transformants were run on a $1 \%$ agarose gel at 100 $\mathrm{V}$ for 2 hours to ensure optimal separation of larger fragments. Four $\mu \mathrm{L}$ of the 100 bp DNA ladder (Invitrogen) and four $\mu \mathrm{L}$ of the High Mass DNA ladder (Invitrogen) were run to estimate digest fragment size. If the $\mathrm{Ag} 85 \mathrm{~A}$ gene inserted in the correct orientation, 8064, 5196 and 1205 bp fragments were observed. If the $\mathrm{Ag} 85 \mathrm{~A}$ gene inserted in the wrong orientation, 8802, 5196 and $497 \mathrm{bp}$ fragments were observed. Orientation digests were performed on miniprep DNA from transformants previously identified by orientation PCRs and results were confirmed using sequencing. 


\subsubsection{Expression of Ag85A and NS-1 protein from pKUN85A}

The $A g 85 A$ gene had inserted into pKUNrep6, as predicted; however protein expression from the pKUN85A plasmid had to be tested in transfected mammalian cells to confirm that pKUN85A expressed the Ag85A protein and the Kunjin RNA replicase NS proteins. To analyse Ag85A and NS-1 protein expression, pKUN85A was transfected into the murine fibroblast cell-line NIH-3T3 (section 2.7.1). Seventy-two hours after transfection, supernatants and cells were harvested and lysed, and cell lysates were immunoprecipitated to enrich for either the Ag85A protein or the NS-1 protein (section 2.7.2). These immunoprecipitated cell lysates were then used in SDS-PAGE gel electrophoresis, Western transfer and immunodetection of protein expression (section 2.7.3). Antibody pairs used for immunodetection targeted either the Ag85A or the NS-1 protein and detection was optimised using dot blots.

The mouse anti-Ag85A primary antibody (clone 32.15 ) is not commercially available, and cell culture supernatant containing the antibody was sourced from Dr Kris Huygen, Institut Pasteur, Brussels, Belgium [429]. Supernatant containing the mouse anti-Ag85A primary antibody was used undiluted and anti-native mouse IgGHRP secondary antibody was used at a 1:1000 dilution for detection of Ag85A. A specific band for Ag85A should resolve on a Western blot at $\sim 32 \mathrm{kDa}$, but all cell lysates displayed a non-specific band at $\sim 12 \mathrm{kDa}$, including negative controls and the positive control containing purified Ag85A protein (data not shown). This indicated that the anti-Ag85A antibody (clone 32.15) was not specific for Ag85A or was not sufficient to detect Ag85A in our assay.

The mouse anti-NS-1 primary antibody is also not commercially available, and cell culture supernatant containing the antibody was sourced from Dr Alex Khromykh and Dr Rob Hall, University of Queensland, Brisbane, Australia. Supernatant containing the mouse anti-NS-1 primary antibody was used at a 1:50 dilution and anti-native mouse IgG-HRP secondary antibody was used at a 1:1000 dilution for detection of NS1 , and these concentrations resulted in a specific band on a Western blot. Ponceau-S staining of the blots indicated that loading of the immunoprecipitated cell lysates was consistent in terms of the total amount of protein applied (Fig. 4.9 A).

The predicted size of NS-1 is 39-41 kDa. After immunodetection, there was a clear band in this region for the positive control, which was cell lysate from pKUNrep6 
transfected cells (Fig. 4.9 B). The pKUN85AOF construct has the $A g 85 A$ gene inserted out of frame with the ORF spanning the cloning site and introduces a premature stop codon (Fig. 4.3 C). This construct should not produce Ag85A protein and should also terminate the polypeptide prematurely, preventing expression of NS-1. As predicted, there was no band for NS-1 in the cell lysate sample from pKUN85AOF transfected cells (Fig. 4.9 B). Notably, there was a clear 39-41 kDa band in the cell lysate sample from pKUN85A transfected cells (Fig. $4.9 \mathrm{~B}$ ). This demonstrates that ORF sequence downstream of the $\mathrm{Ag} 85 \mathrm{~A}$ insertion site was translated in pKUN85A transfected cells. Translation of the NS genes is essential for expression of the RNA replicase, which is essential for dsRNA production and the enhanced immunogenicity of the DNA replicon.

Translation from $N S-1$ implies that $A g 85 A$ inserted upstream of $N S-1$ is also translated, as sequencing has shown $\mathrm{Ag} 85 \mathrm{~A}$ is in-frame with the same ORF that contains $N S-1$. Therefore, while the anti-Ag85A primary antibody proved unsuitable for Western transfer and immunodetection, expression of Ag85A protein by the pKUN85A construct was implied by expression of the NS-1 protein. 
A.

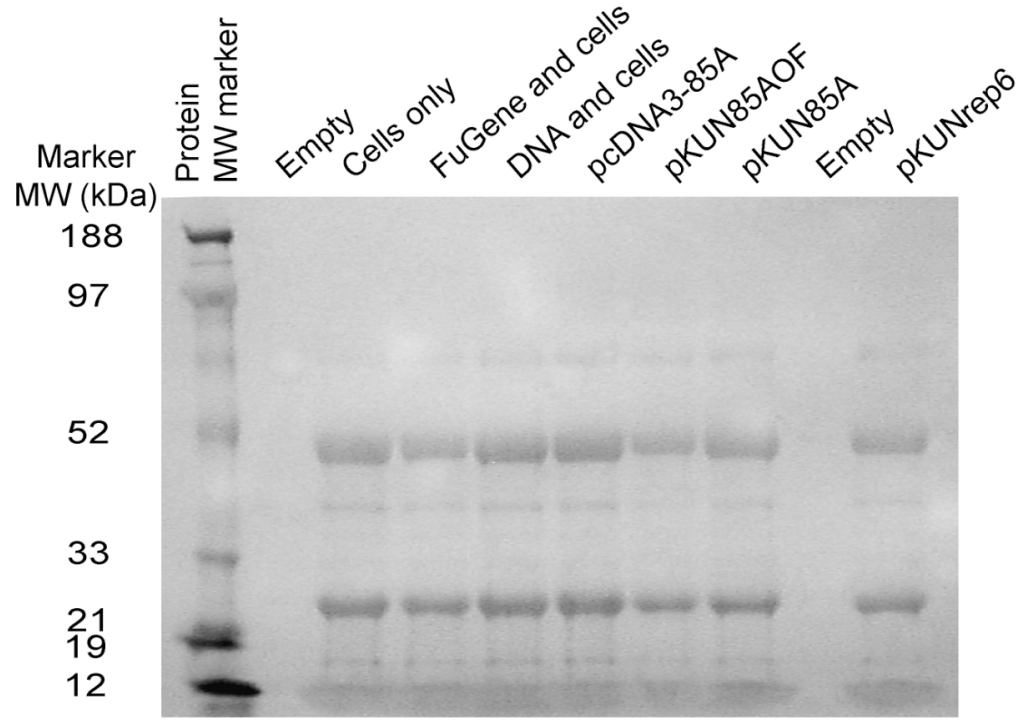

B.

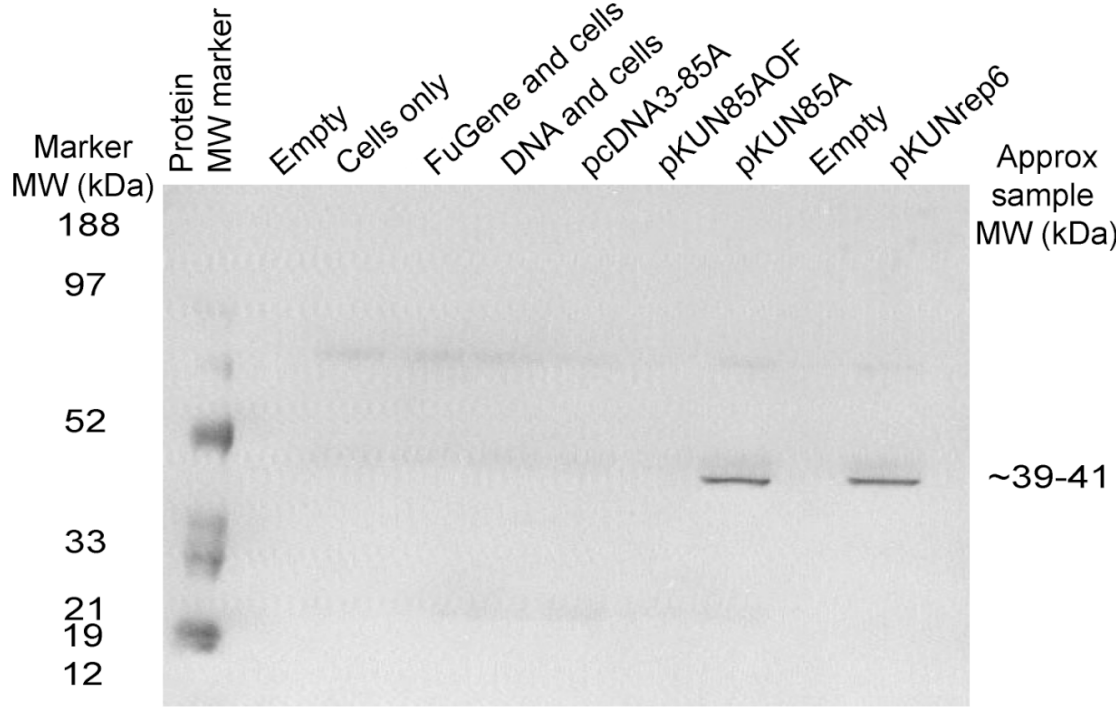

\section{Figure 4.9: Protein expression from pKUN85A-transfected NIH-3T3 cells}

DNA plasmids including pKUN85A were assessed for their ability to express NS-1 in mammalian cells. Plasmids were transfected into NIH-3T3 cells and left in culture for 72 hours to allow protein expression. Cell lysates were prepared and immunoprecipitated for NS-1, before being used in SDS-PAGE, Western transfer and immunodetection with antibodies specific for NS-1. A) Ponceau-S stained blot for NS-1. The Ponceau-S stained membrane demonstrates even loading of cell lysates. B) Western blot for NS-1. The membrane from A) was immunoblotted with mouse anti-NS-1 primary antibody and anti-native mouse IgG HRPconjugated secondary antibody. A 39-41 kDa band, corresponding to the expected size for NS1, was observed in cell lysate from pKUNrep6 transfected cells and from pKUN85A transfected cells. No bands were observed in negative controls. Results represent one experiment. 


\subsubsection{Immunogenicity of pKUN85A}

Protein from the pKUN85A construct was expressed by transfected mammalian cells in in vitro culture. However, the mechanism mediating in vivo transfection with DNA plasmids is not well understood and whether cells could be transfected by pKUN85A and express protein to induce an immune response in vivo remained to be tested. Furthermore, the dose of plasmid DNA replicons required for vaccination is typically lower than the dose for conventional DNA plasmids. Therefore, a dose titration was performed to assess the immunogenicity of pKUN85A.

Different groups of mice were vaccinated i.m. with doses of pKUN85A ranging from $5 \mu \mathrm{g}$ to $100 \mu \mathrm{g}$. Mice were primed, then boosted two weeks later, with the same vaccine dose. Two weeks after boosting, tissues were harvested and processed for in vitro restimulation. Lymphocyte proliferation was assessed by a $\left[{ }^{3} \mathrm{H}\right]$ thymidine incorporation assay, total IFN- $\gamma$ production was assessed by ELISA and cytokine production by $\mathrm{CD} 4^{+}$and $\mathrm{CD} 8^{+} \mathrm{T}$ cells was assessed by flow cytometry.

Proliferation in response to purified Ag85A protein was highest in mice vaccinated with $25 \mu \mathrm{g}$ of pKUN85A and was significantly higher compared to pKUNrep6 vaccinated control mice (Fig. 4.10 A). The average total IFN- $\gamma$ production in supernatants from restimulated cultures was highest, although not significantly different, in mice vaccinated with $25 \mu \mathrm{g}$ of pKUN85A (Fig. 4.10 B). Total IFN- $\gamma$ production was significantly higher in mice vaccinated with $100 \mu \mathrm{g}$ of pKUN85A compared to PBS treated and pKUNrep6 vaccinated control mice (Fig. 4.10 B). The average numbers of IFN- $\gamma$-producing $\mathrm{CD}^{+}{ }^{+}$lymphocytes and IFN- $\gamma$ - or IL-2-producing $\mathrm{CD}^{+} \mathrm{T}$ lymphocytes were highest in mice vaccinated with $25 \mu \mathrm{g}$ of pKUN85A (Fig. 4.11) and were significantly higher than PBS treated and pKUNrep6 vaccinated mice (Fig. 4.11). Notably, cytokine production and proliferation appeared to decrease with a $100 \mu \mathrm{g}$ dose of pKUN85A compared to a $25 \mu \mathrm{g}$ dose. With plasmid DNA replicons, higher doses often decrease the immunogenicity of the vaccine [433]. Therefore, i.m. vaccination with pKUN85A induced an Ag85A-specific immune response, with cytokine production and proliferation. As a result of these experiments, a $25 \mu \mathrm{g}$ dose was chosen for experiments using pKUN85A in chapter 5. 


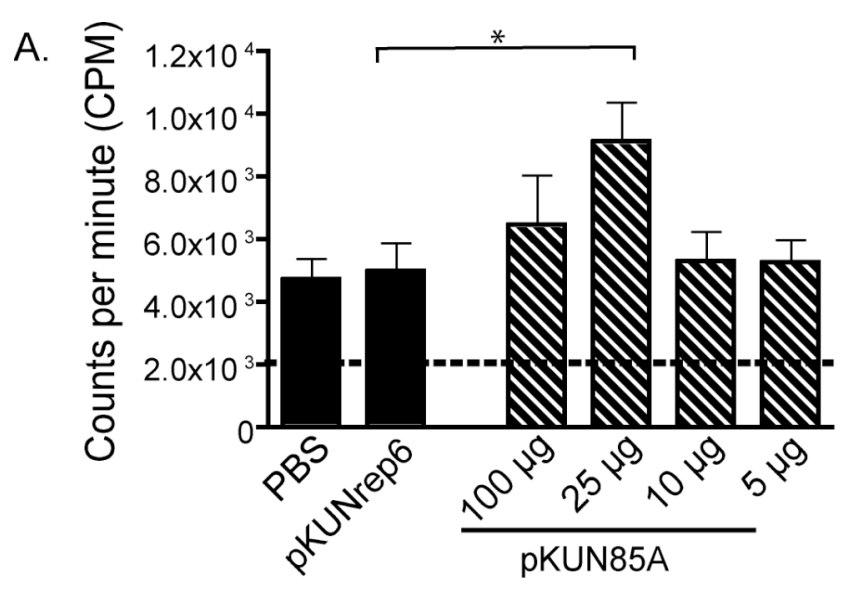

Vaccination and dose

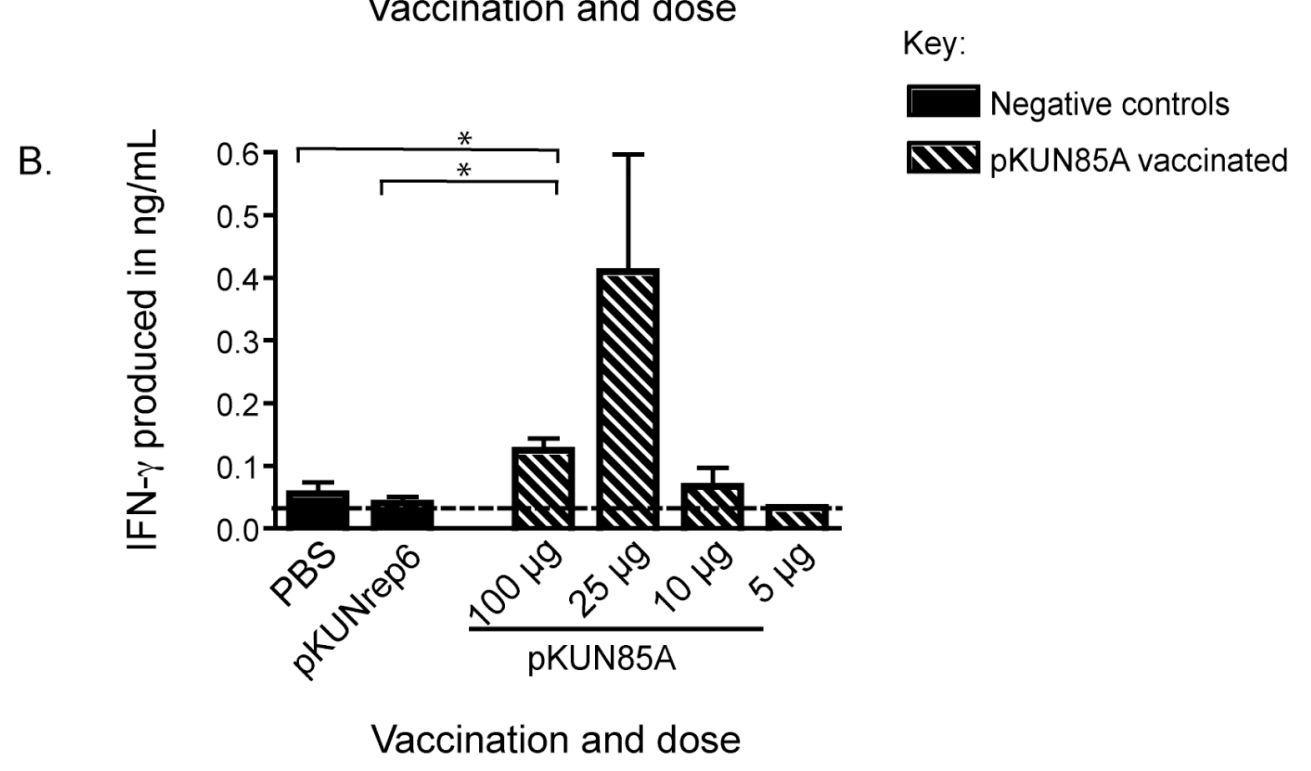

Figure 4.10: Lymphocyte proliferation and IFN- $\gamma$ production after $p K U N 85 A$ vaccination

Groups of mice were primed and then boosted two weeks later i.m. with the indicated dose of pKUN85A, $100 \mu \mathrm{g}$ of pKUNrep6 or PBS alone. Mice were left two weeks before spleens were harvested and processed. Lymphocytes were plated and restimulated with Ag85A and anti$\mathrm{CD} 28 \mathrm{mAb}$ to assess either proliferation by $\left[{ }^{3} \mathrm{H}\right]$ thymidine incorporation or IFN- $\gamma$ production by ELISA. A) Antigen-driven proliferation of lymphocytes from vaccinated animals. Dotted line indicates maximal background proliferation. B) IFN- $\gamma$ production by lymphocytes from vaccinated animals measured by ELISA. The dotted line indicates limit of detection of ELISA at $0.0315 \mathrm{ng} / \mathrm{mL}$. Bars represent mean values \pm SEM. Five mice were analysed per treatment group. Treatment groups were compared using the two-tailed Mann Whitney test; * indicates $p$ $\leq 0.05$. Results represent one experiment, although proliferation data for the $25 \mu \mathrm{g}$ dose of pKUN85A has been replicated. 
A.

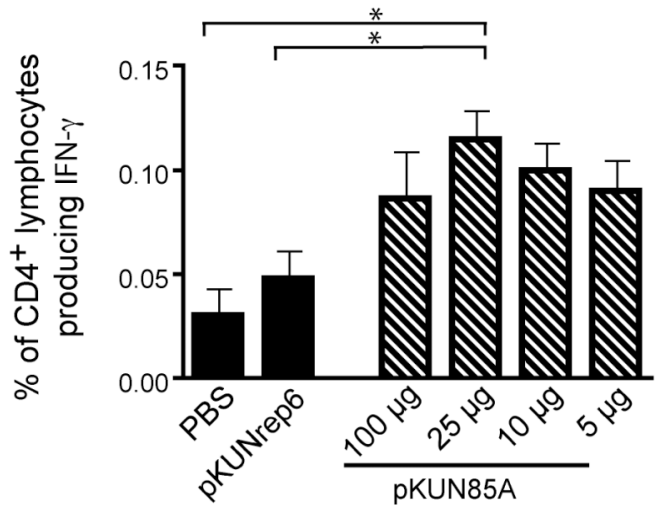

Vaccination and dose

C.

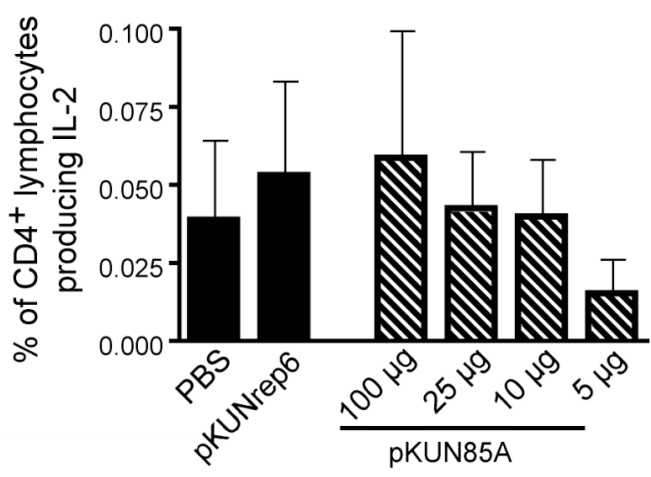

Vaccination and dose
B.

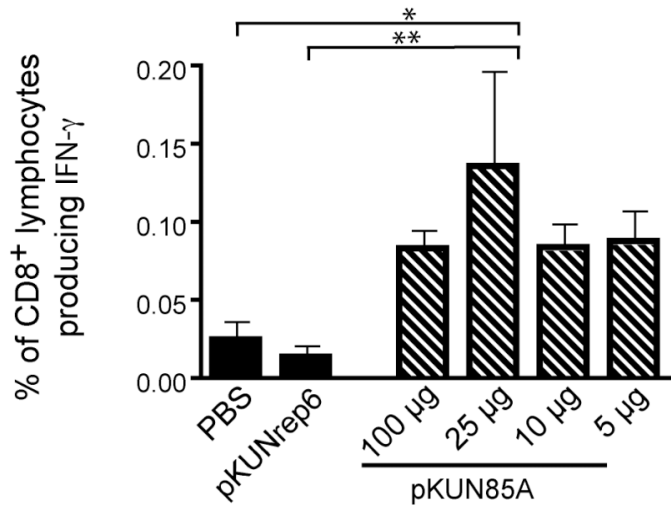

Vaccination and dose

D.

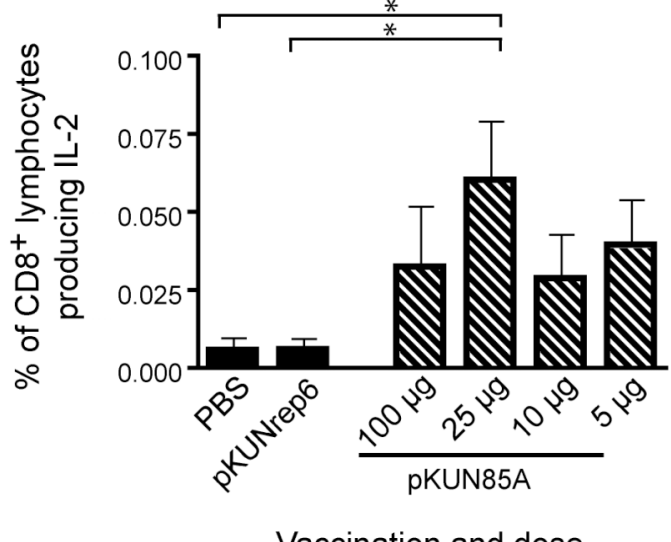

Key:

Negative controls

MI pKUN85A vaccinated

Figure 4.11: Cytokine production by $\mathrm{CD4}^{+}$and $\mathrm{CD8}^{+} \mathrm{T}$ cells after $\mathrm{pKUN85A}$ vaccination Groups of mice were primed and then boosted two weeks later i.m. with the indicated dose of pKUN85A, $100 \mu \mathrm{g}$ of pKUNrep6 or PBS alone. Mice were left two weeks before spleens were harvested and processed. Lymphocytes were plated and restimulated with Ag85 A and anti$\mathrm{CD} 28 \mathrm{mAb}$ to assess cytokine production via intracellular cytokine staining. A) Percentage of $\mathrm{CD} 4^{+} \mathrm{T}$ cells producing IFN- $\gamma$. B) Percentage of $\mathrm{CD} 8^{+} \mathrm{T}$ cells producing IFN- $\gamma$. C) Percentage of $\mathrm{CD}^{+} \mathrm{T}$ cells producing IL-2. D) Percentage of $\mathrm{CD} 8^{+} \mathrm{T}$ cells producing IL-2. Bars represent mean values \pm SEM. Five mice were analysed per treatment group. Treatment groups were compared using the two-tailed Mann Whitney test; * indicates $\mathrm{p} \leq 0.05, * *$ indicates $\mathrm{p} \leq 0.01$. Results represent one experiment. 


\subsubsection{Short-term protection from infection afforded by pKUN85A}

Vaccination with pKUN85A induced an immune response directed against mycobacterial antigens, with antigen-driven IFN- $\gamma$ production by $\mathrm{CD} 4{ }^{+} \mathrm{T}$ cells. This is often used as an indicator of immunity against $\mathrm{Tb}$. However, it is becoming increasingly obvious that IFN- $\gamma$ production by $\mathrm{CD}^{+} \mathrm{T}$ cells is not a good correlate of $\mathrm{Tb}$ vaccine efficacy [167]. Therefore, protective efficacy of pKUN85A against a virulent challenge with M. tuberculosis at a range of doses needed to be assessed.

To evaluate protection afforded by pKUN85A, different groups of mice were vaccinated i.m. with doses of pKUN85A ranging from $5 \mu \mathrm{g}$ to $100 \mu \mathrm{g}$. Mice were primed, then boosted two weeks later with the same vaccine dose. As a positive control, one group of mice was vaccinated with BCG s.c. at the same time as DNA boosting. Six weeks after boosting, all groups of mice were challenged by aerosol with $M$.

tuberculosis. Five weeks after infection, lung and spleen tissue was harvested; a lung sample was taken for histology and the spleen and remainder of the lung tissue were processed to evaluate tissue bacterial numbers.

Upon collection of tissue bacterial numbers, some samples were observed that had undetectable CFUs for both plated lung and spleen tissue homogenates. The murine model of $M$. tuberculosis infection used in this study aims to mimic natural human infection by using a low infection dose of approximately $50 \mathrm{CFU}$ of $M$. tuberculosis per mouse. Due to this low infection dose, there is the risk that infections can be too low, resulting in variation and uninfected mice. In this study, six mice in five of the eight treatment groups were uninfected and within groups for mice that were infected, there was more variation in tissue bacterial numbers than is usually seen. In subsequent experiments, the infection dose was increased but, for analysis of this study, data from uninfected mice were excluded from analyses of lung and spleen bacterial numbers.

Lung bacterial numbers were evaluated to assess pathogen control at the primary site of infection, the lung. BCG vaccinated mice displayed a $0.88 \log _{10}$ reduction in total lung bacterial numbers compared to the naïve PBS treated mice (Fig. 4.12 A), which was statistically significant but slightly less than the $\sim 1 \log _{10}$ reduction typically seen in the $\mathrm{C} 57 \mathrm{BL} / 6$ murine model of $\mathrm{Tb}$ [171]. The pcDNA3-85A vaccination was included as another positive control and this resulted in a $0.48 \log _{10}$ reduction in total lung bacterial numbers compared to naïve PBS treated mice (Fig. 4.12 A), which was also statistically 
significant but less than the $0.70 \log _{10}$ reduction seen in previous vaccination studies [433]. Mice vaccinated with the empty pKUNrep6 vector displayed a $0.30 \log _{10}$ reduction in lung bacterial numbers compared to the naïve PBS treated mice, which was not statistically significant (Fig 3.10 A). Non-coding DNA can enhance protection against $\mathrm{Tb}$ in a heterologous prime-boost setting, presumably due the inherent adjuvancy of the bacteria-derived DNA [436], but this decrease in bacterial numbers was not seen in other short- or long-term protection studies (data not shown) so is unlikely to be a real effect. Mice vaccinated with 100,25 or $5 \mu \mathrm{g}$ of pKUN85A did not display significant reductions in lung bacterial numbers. Mice that received $10 \mu \mathrm{g}$ of pKUN85A displayed a $0.39 \log _{10}$ reduction in lung bacterial numbers compared to naïve PBS treated mice (Fig. 4.12 A), but this was not statistically significant $(p=0.0635)$.

Bacterial numbers in the spleen were evaluated to assess dissemination of $\mathrm{Tb}$ infection to tissues beyond the primary site of infection in the lung. BCG vaccinated mice displayed a $1.74 \log _{10}$ reduction in average spleen bacterial numbers compared to naïve PBS treated mice, but this difference was not significant $(\mathrm{p}=0.0571)$ due to large variation within treatment groups and small sample size (Fig. 4.12 B). Mice vaccinated with all other constructs did not display significant reductions in total spleen bacterial numbers compared to naïve PBS treated mice (Fig. 4.12 B).

Overall in this study, the infection dose was relatively low and there was more variation than usual in bacterial numbers in the tissues for each treatment group. The low infection dose resulted in mice with undetectable lung bacterial numbers and these mice were not included in data analysis. In spite of this, significant reductions in bacterial numbers in the lung were observed for mice vaccinated with BCG or pcDNA3-85A compared to naïve PBS control mice. Mice vaccinated with pKUN85A did not display significant reductions in bacterial numbers in the lung or spleen compared to naïve PBS control mice in this study, but short-term protection against an M. tuberculosis challenge will be re-assessed in the future with a higher infection dose. 


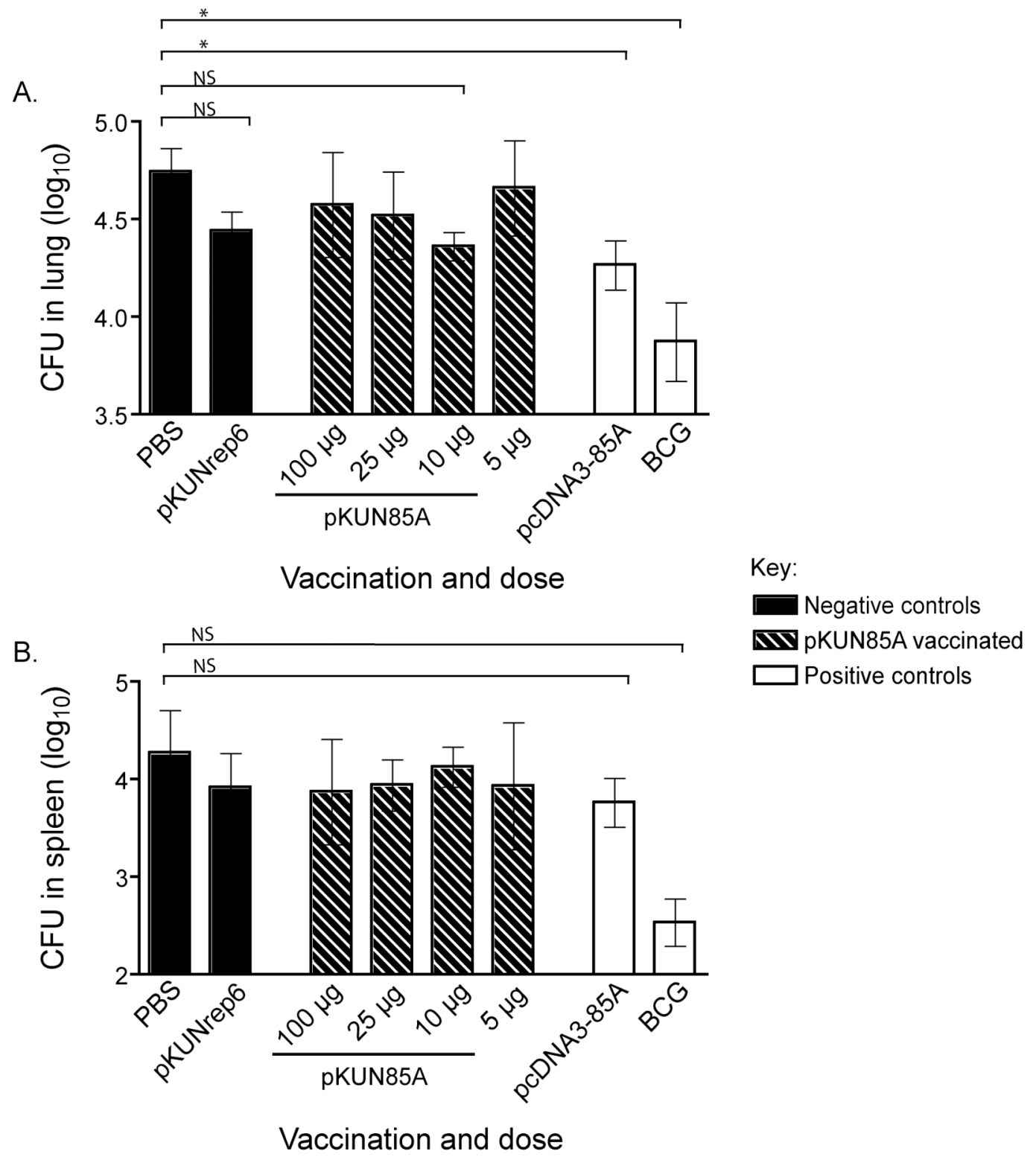

Figure 4.12: Short-term protection from M. tuberculosis infection after pKUN85A vaccination

Mice were primed and then boosted two weeks later i.m. with the indicated dose of pKUN85A, $100 \mu \mathrm{g}$ of pKUNrep6 or $100 \mu \mathrm{g}$ of pcDNA3-85A. Control groups of mice were also vaccinated with PBS alone or $10^{6} \mathrm{CFU}$ BCG s.c. at the time of DNA boosting in experimental groups of mice. Mice were left 6 weeks before being challenged by aerosol with M. tuberculosis. Five weeks after infection, lungs and spleens were harvested, homogenised and plated to evaluate tissue bacterial numbers. A) Bacterial numbers in the lung after M. tuberculosis challenge. B) Bacterial numbers in the spleen after M. tuberculosis challenge. Bars represent geometric mean values \pm GEM. Three to five mice were analysed per treatment group. Treatment groups were compared using the two-tailed Mann Whitney test; NS indicates $\mathrm{p}>0.05$, * indicates $\mathrm{p} \leq 0.05$. Results represent one experiment, although data for the $25 \mu \mathrm{g}$ dose of pKUN85A has been replicated. 


\subsubsection{Long-term protection from infection afforded by pKUN85A}

A candidate $\mathrm{Tb}$ vaccine should confer protection that is long-lived; otherwise subjects may need to be periodically revaccinated. Therefore, a long-term protection study was undertaken using the same experimental design as the short-term protection study, except that mice were left for sixteen weeks after boosting before challenge with $M$. tuberculosis.

The infection dose for this study was higher and all mice had detectable lung bacterial numbers. Therefore, differences in vaccine efficacy could be accurately evaluated. BCG vaccinated mice displayed a statistically significant $0.86 \log _{10}$ reduction in lung bacterial numbers compared to naïve PBS treated mice (Fig. 4.13). The pcDNA3-85A vaccinated mice did not display a significant reduction in lung bacterial numbers compared to naïve PBS treated mice (Fig. 4.13), as expected since protection has been seen to wane over time in other studies with pcDNA3-85A [433]. Finally, significant reductions in lung bacterial numbers were not observed for mice vaccinated with either $100 \mu \mathrm{g}$ or $10 \mu \mathrm{g}$ of pKUN85A (Fig. 4.13).

Overall, the long-term protection afforded by pKUN85A was difficult to compare to short-term protection due to the low infection dose and large amount of variation of bacterial numbers in the short-term study. However, only BCG vaccination, and not pKUN85A or pcDNA3-85A vaccination, resulted in a statistically significant reduction in lung bacterial numbers compared to naïve PBS treated mice after a longterm challenge with $M$. tuberculosis. 


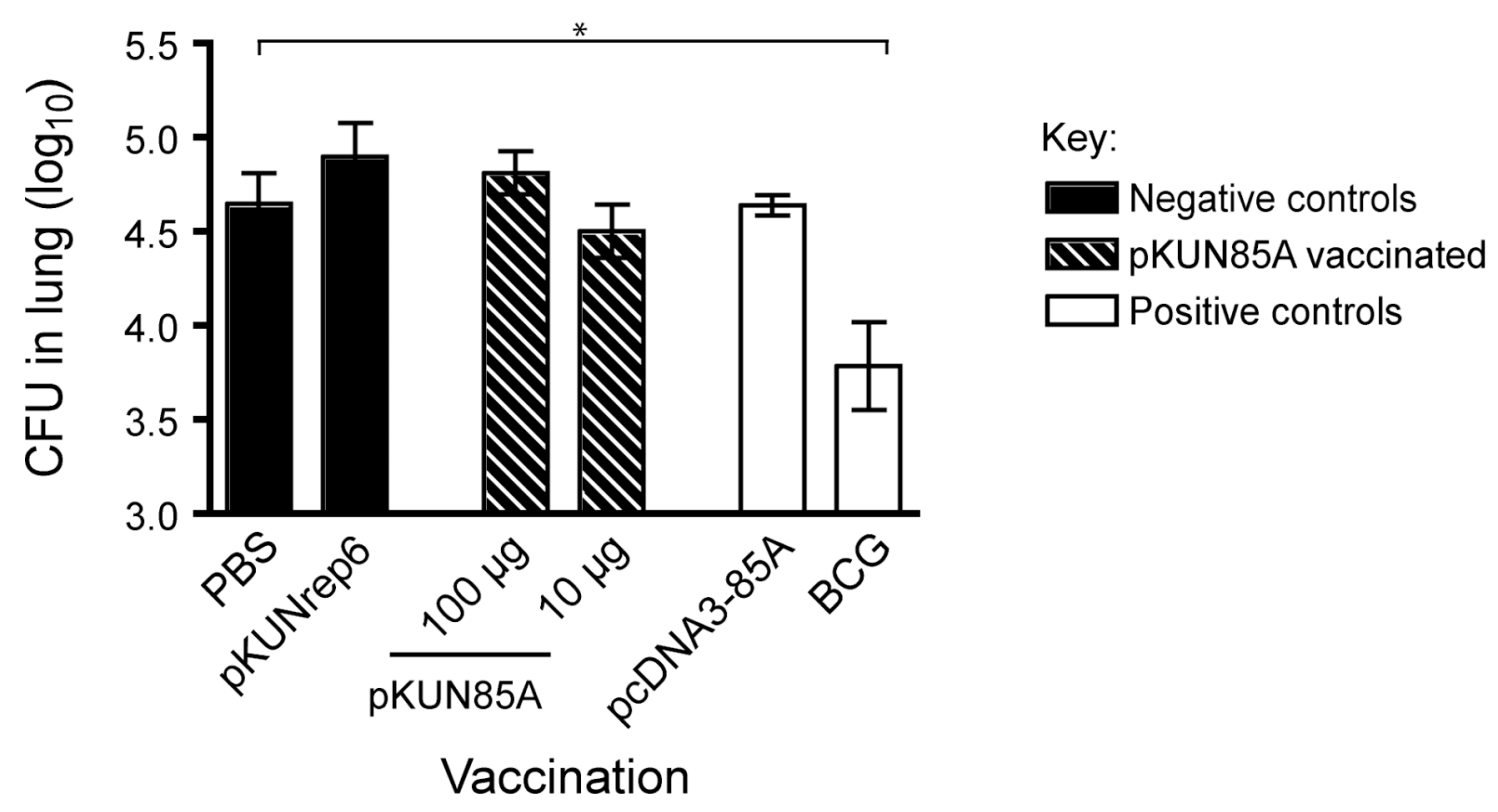

Figure 4.13: Long-term protection from M. tuberculosis infection after pKUN85A vaccination

Mice were primed and then boosted two weeks later i.m. with the indicated dose of pKUN85A, $100 \mu \mathrm{g}$ of pKUNrep6 or $100 \mu \mathrm{g}$ of pcDNA3-85A. Control groups of mice were also vaccinated with PBS alone or $10^{6} \mathrm{CFU}$ BCG s.c. at the time of DNA boosting in experimental groups of mice. Mice were left 15 weeks before being challenged by aerosol with M. tuberculosis. Five weeks after infection, lungs were harvested, homogenised and plated to evaluate tissue bacterial numbers. Bars represent geometric mean values \pm GEM. Five mice were analysed per treatment group. Treatment groups were compared using the two-tailed Mann Whitney test; * indicates $\mathrm{p}$ $\leq 0.05$. Results represent one experiment. 


\subsubsection{Pulmonary inflammation after pKUN85A vaccination followed by Tb challenge}

Inflammation in the lung tissue causes the pathology associated with $\mathrm{Tb}$ disease resulting in morbidity and death (outlined in chapter 1). A candidate $\mathrm{Tb}$ vaccine must control the infection in the tissues, as well as minimise immunopathology in the lung, to reduce morbidity associated with $\mathrm{Tb}$ disease. Therefore, pulmonary inflammation after virulent challenge is another important measure of the protective efficacy of a vaccine.

Lung samples were taken in parallel to the studies assessing short-term and long-term protection in Fig. 4.12 and 4.13. Samples were prepared for histological analysis and graded for the degree of pulmonary inflammation. Samples were harvested 5 weeks after infection with $M$. tuberculosis and the degree of inflammation overall was low. As a result, differences in inflammation between treatment groups were statistically insignificant (Fig. 4.14 A/B) and, for better assessment of pathological changes in the lung tissue, future experiments should harvest tissue later after infection.

Mild, localised granulomatous inflammation, with epithelioid histiocytes and lymphocytes present within the pulmonary interstitium and alveolar spaces, was observed in all groups after M. tuberculosis challenges either short- or long-term after vaccination (Fig. $4.14 \mathrm{~A} / \mathrm{B})$. The degree of inflammation appeared reduced in BCG vaccinated mice, although the median score did not differ significantly from naïve PBS treated mice (Fig. $4.14 \mathrm{~A} / \mathrm{B}$ ). In mice challenged long-term after vaccination, inflammation also appeared reduced to a level comparable to BCG vaccinated groups in groups vaccinated with $100 \mu \mathrm{g}$ of pKUN85A or $100 \mu \mathrm{g}$ of pcDNA3-85A, although scores did not differ significantly from PBS treated control mice (Fig. 4.14 B).

Therefore, pKUN85A vaccination does not significantly decrease pulmonary inflammation induced 5 weeks after $M$. tuberculosis challenge compared to PBS treated controls, although differences between treatment groups may become more apparent later after infection. 
A.

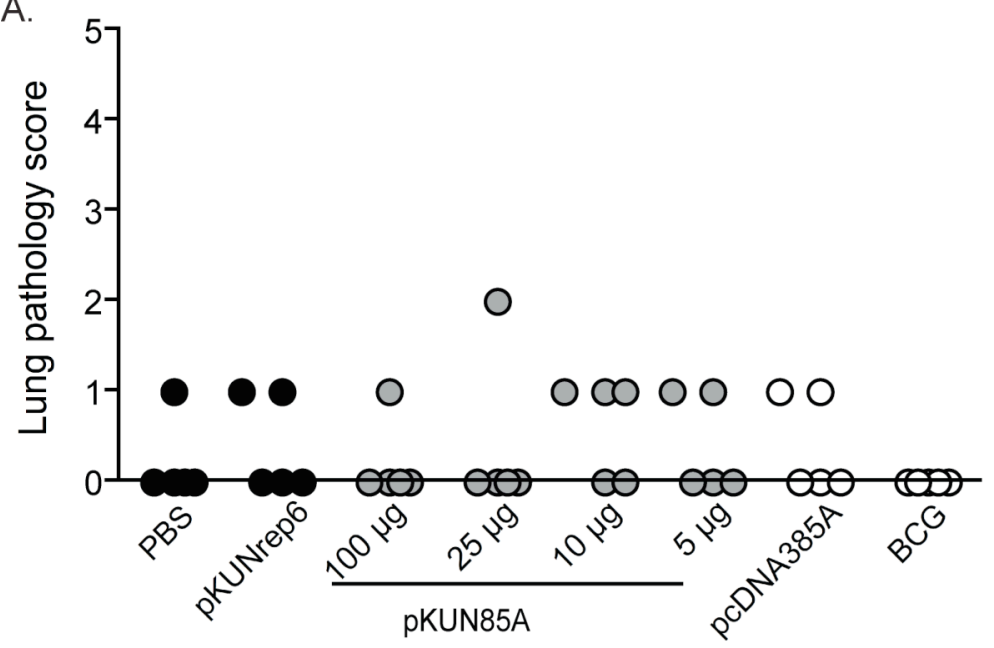

Short-term protection: 6 weeks

Vaccination and dose

Key:

Negative controls

pKUN85A vaccinated

B

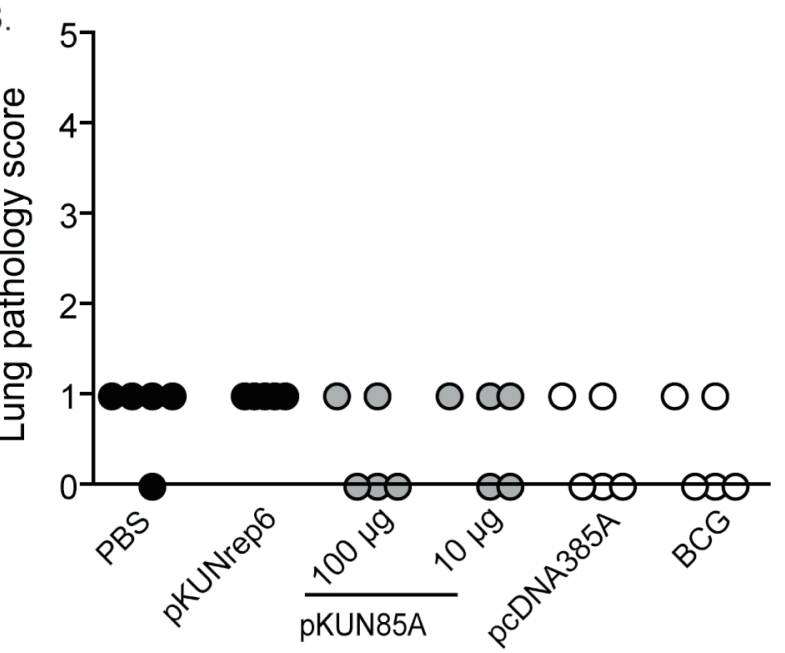

Positive controls

Long-term protection:

15 weeks

Vaccination and dose

\section{Figure 4.14: Lung pathology after pKUN85A vaccination}

Mice were primed and then boosted two weeks later i.m. with the indicated dose of pKUN85A, $100 \mu \mathrm{g}$ of pKUNrep6 or $100 \mu \mathrm{g}$ of pcDNA3-85A. Control groups of mice were also vaccinated with PBS alone or $10^{6} \mathrm{CFU}$ BCG s.c. at the time of DNA boosting in experimental groups of mice. Mice were left 6 or 15 weeks before being challenged by aerosol with M. tuberculosis. Five weeks after infection, lung samples were harvested, inflated and fixed. Samples were paraffin-embedded, sectioned and stained with haematoxylin and eosin. Sections were analysed by light microscopy and graded for inflammation. A) Composite lung pathology scores for $M$. tuberculosis challenge 6 weeks after vaccination. B) Composite lung pathology scores for $M$. tuberculosis challenge 15 weeks after vaccination. Pathology scores ranged from 0 (least severe) to 5 (most severe). Symbols represent individual values. Lung samples from five mice were analysed for each treatment group. Results in A represent one experiment, although data for the $25 \mu \mathrm{g}$ dose of pKUN85A has been replicated. Results in B represent one experiment. 


\subsection{Discussion}

This chapter outlines the creation of pKUN85A, a novel plasmid DNA replicon vaccine for protection against $\mathrm{Tb}$ infection, and initial testing of vaccine efficacy. The $\mathrm{Ag} 85 \mathrm{~A}$ gene was cloned into the pKUNrep6 vector and a suitable transformant was selected for propagation and preparation of the pKUN85A vaccine (Figs. 4.3-4.8). The pKUN85A construct expressed protein from the ORF containing the $A g 85 A$ and the Kunjin RNA replicase genes in a mammalian cell-line (Fig. 4.9). Vaccination with pKUN85A induced an immune response directed against Ag85A, as assessed by antigen-driven proliferation and IFN- $\gamma$ and IL-2 production by $\mathrm{CD} 4^{+}$and $\mathrm{CD} 8^{+}$lymphocytes (Figs. 4.10/4.11). Studies assessing short-term and long-term protection (Figs. 4.12/4.13) remain to be repeated before firm conclusions can be drawn regarding vaccine efficacy. Vaccination with pKUN85A did not induce protection comparable to the BCG vaccine in either study (Figs. 4.12/4.13), indicating that homologous prime-boost vaccination with pKUN85A is not as effective as BCG vaccination in the murine model of $\mathrm{Tb}$. However, refinements are can be made in order to improve the protective efficacy of the pKUN85A plasmid DNA replicon against $\mathrm{Tb}$, as addressed in section 4.4.4.

Tb vaccine efficacy studies conducted in PC-3 facilities can be complicated and must be carefully designed. Studies are lengthy and expensive in terms of reagents and space, as large numbers of mice must be vaccinated, boosted and challenged with virulent mycobacteria in specialised PC-3 facilities over several months and physical space in facilities is limited. For the short-term protection study, the infection dose was low to mimic low-dose natural human infection, but this resulted in some mice being uninfected (Fig. 4.12). Those that were infected had variable bacterial numbers in the tissues within treatment groups and the degree of inflammation in the lung was low (Fig. 4.14). This meant that while trends over treatment groups were apparent, differences between treatment groups were not statistically significant. There is variation inherent in the process of aerosol infection and the kinetics of mycobacterial infection. Factors such as vaccine batch and infection inoculum batch can also lead to variation in bacterial numbers. Therefore, for more accurate assessment of the protection afforded by pKUN85A against $M$. tuberculosis infection, these results will be repeated with a larger number of mice and higher infection dose, and lung pathology will be assessed later after virulent infection. 


\subsubsection{Titration of the optimal pKUN85A dose}

This study demonstrates a current issue for development of Tb vaccines; there is no reliable immunological correlate of protection that can be used to assess vaccine efficacy after vaccination or before infection [167]. The optimal dose of pKUN85A for immunogenicity at two weeks after boost vaccination was determined to be $25 \mu \mathrm{g}$. The $25 \mu \mathrm{g}$ dose of pKUN85A consistently induced increased markers of immunogenicity compared to the lower 10 and $5 \mu \mathrm{g}$ doses of pKUN85A, such as proliferation and cytokine production in response to Ag85A stimulation (Fig. 4.10/4.11). However, the $25 \mu \mathrm{g}$ dose of pKUN85A did not confer measurable protection in a short-term challenge protocol (Fig. 4.12). Bacterial numbers for the short-term protection study were variable within treatment groups and some mice were uninfected, but the $25 \mu \mathrm{g}$ dose of pKUN85A also did not provide a measurable degree of protection when used in experiments in chapter 5 (Fig. 5.15). Furthermore, proliferation and cytokine production was analysed only at 2 weeks after vaccination. While the $25 \mu \mathrm{g}$ dose induced the highest average proliferative and cytokine responses at two weeks after vaccination, the other doses of pKUN85A may have had different kinetics and maximal responses for antigen-driven proliferation or cytokine production may have occurred at different times after vaccination. These results illustrate that while it is important to demonstrate an immune response against the target mycobacterial antigen has been elicited after vaccination, that response does not predict whether the $\mathrm{Tb}$ vaccine will confer protection against infectious challenge.

This study also illustrates the importance of dose for plasmid DNA replicon vaccines. It has been shown that plasmid DNA replicons have a defined window for the optimal dose, rather than increased doses of vaccine resulting in better protection [433]. As the exact mechanism and target cells for plasmid DNA replicons is unknown, the basis for the optimal dose effect is yet to be defined. However, it is clear that the dose of plasmid DNA replicons must be titrated with respect to protection, as a given dose of a plasmid DNA replicon can induce a strong immune response but slight increases or decreases in dose may affect vaccine immunogenicity dramatically.

As there is no reliable immune correlate for protection against Tb, plasmid DNA replicon dose may need to be titrated with respect to protection from $\mathrm{Tb}$ for clinical implementation of a $\mathrm{Tb}$ vaccine. While dose titrations can easily be performed for agricultural vaccines, this could be an inherent problem for translating plasmid DNA 
replicon $\mathrm{Tb}$ vaccines into humans. $\mathrm{Tb}$ protection studies in humans are conducted over long periods of time, as $\mathrm{Tb}$ infection rarely progresses directly to an active infection but usually persists for years in a latent form, which is difficult to diagnose. The combination of the length of human protection studies and the complexity of characterising a dosing window for plasmid DNA replicons may prove prohibitive for the implementation of plasmid DNA replicons as $\mathrm{Tb}$ vaccines in humans.

\subsubsection{Longevity of protection after vaccination}

After vaccination, protection afforded by a vaccine can wane over time. Protection afforded by vaccination with the conventional pcDNA3-85A plasmid wanes over time (Figs. 4.12/4.13) [433], and protection afforded by BCG vaccination can also eventually wane in murine models and human studies [247, 257]. As pKUN85A uses a noncytopathic construct, it is conjectured to result in long-lived antigen expression in vivo relative to cytopathic plasmid DNA replicon systems. However, antigen persistence was not assessed in this study and it is not known how long-lived antigen expression would affect longevity of protection.

It is difficult to evaluate antigen expression in vivo unless a reporter gene, such as $\beta$-galactosidase or GFP, is included in the pKUN85A construct [397, 409]. While persistence of protein expression is difficult to define accurately, persistence of the DNA plasmid or mRNA transcription from the plasmid is more easily assessed. Persistence of the pKUN85A DNA plasmid can be evaluated by PCR amplification of plasmid from total DNA preparations of tissue from, around or draining the vaccination site [437]. Persistence of mRNA transcription from the pKUN85A DNA plasmid can be evaluated by reverse transcriptase PCR of genes encoded by the plasmid from preparations of complimentary DNA from vaccinated tissue [437].

Once persistence of either DNA plasmid or mRNA is assessed, this could be related to longevity of protection after pKUN85A vaccination. It is contentious whether persistent antigen is needed to maintain either $\mathrm{CD}^{+}$[438-440] or $\mathrm{CD}^{+}[441,442]$ memory $\mathrm{T}$ cells. Currently, long-lived protection against chronic intracellular pathogens like L. major and M. tuberculosis is mediated by live vaccines that persist in the host $[305,443]$. 
The relationship between persistence of the vaccine and the longevity of protection afforded by the vaccine would be interesting to evaluate, particularly comparing non-cytopathic and cytopathic plasmid DNA replicons. $\mathrm{CD} 8^{+} \mathrm{T}$ cell responses, antibody responses and protection have been compared between a noncytopathic Kunjin virus-based plasmid DNA replicon and a cytopathic Semliki forest virus-based plasmid DNA replicon, both expressing a $\beta$-galactosidase reporter gene [444]. $\mathrm{CD}^{+} \mathrm{T}$ cell responses and protection from infection with vaccinia virus producing $\beta$-galactosidase were similar for both plasmid DNA replicon platforms, but protection was only evaluated 14 days after vaccination and the longevity of protection was not compared. The pSINCP-85A vaccine is a cytopathic DNA replicon, which uses the Sindbis RNA replicase and contains the $\mathrm{Ag} 85 \mathrm{~A}$ gene [433]. Comparison of the persistence of protection mediated by pKUN85A and pSINCP-85A could have implications for the whether prolonged or persistent antigen expression enhances the longevity of protection against $\mathrm{Tb}$.

\subsubsection{The $\mathrm{CD}^{+} \mathrm{T}$ cell response to pKUN85A vaccination}

While the pKUN85A construct has been shown to induce a Th1 immune response in this study, the particular advantage of Kunjin-based plasmid DNA replicons is that they are very effective at inducing $\mathrm{CD} 8^{+} \mathrm{T}$ cell activation $[411,414] . \mathrm{CD}^{+} \mathrm{T}$ cells are important in the control of primary $\mathrm{Tb}$ infection, most likely for their cytotoxic function that can control bacterial numbers and contain infection within the granuloma (sections 1.2.3.4 and 1.2.4). $\mathrm{CD}^{+} \mathrm{T}$ cells also provide a measure of protection during secondary responses in BCG vaccinated animals, particularly for prevention of infection dissemination to tissues outside the lung (section 1.2.6).

Despite Kunjin-based plasmid DNA replicons being effective at inducing CD8 ${ }^{+}$ $\mathrm{T}$ cell activation, dissemination to other tissues was not reduced as bacterial numbers in the spleen were not significantly reduced in pKUN85A vaccinated mice compared to PBS treated control mice, although there was large variation in bacterial numbers (Fig. 4.12). However, $\mathrm{Ag} 85 \mathrm{~A}$-specific $\mathrm{CD}^{+} \mathrm{T}$ cell activation in response to $\mathrm{pKUN85 \textrm {A }}$ vaccination is yet to be assessed and the dose for maximal $\mathrm{CD}^{+} \mathrm{T}$ cell activation remains to be determined. 
The number of IFN- $\gamma$ - or IL-2-producing $\mathrm{CD}^{+} \mathrm{T}$ cells from $\mathrm{pKUN85 \textrm {A }}$ vaccinated mice was increased upon restimulation with Ag85A protein (Fig. $4.11 \mathrm{~B} / \mathrm{D}$ ) but cytokine production by $\mathrm{CD} 8^{+} \mathrm{T}$ cells after 20 hours of restimulation with whole protein is unlikely to represent antigen-specific cytokine production. The Ag85A protein would have to be processed and cross-presented on MHC I to induce true antigen-specific cytokine production. Cytokine production by $\mathrm{CD} 8^{+} \mathrm{T}$ cells after overnight restimulation with whole protein and anti-CD28 mAb is more likely to represent bystander activation of previously activated $\mathrm{CD}^{+} \mathrm{T}$ cells in response to $\mathrm{CD} 28$ signalling [445] or cytokine production by $\mathrm{CD}^{+} \mathrm{T}$ cells. Therefore, the restimulation conditions used in the experiments described above are likely to underestimate the total $\mathrm{CD}^{+} \mathrm{T}$ cell response and not account for the antigen-specific $\mathrm{CD}^{+} \mathrm{T}$ cell response.

The antigen-specific $\mathrm{CD}^{+} \mathrm{T}$ cell response has not been assessed for pKUN85A due to the complexity of assessing the $\mathrm{CD}^{+} \mathrm{T}$ cell responses for $\mathrm{Ag} 85 \mathrm{~A}$ epitopes. Using the C57BL/6 murine model of Tb, peptide library screening has not identified an immunogenic MHC I restricted epitope for $\operatorname{Ag} 85 \mathrm{~A}[430,446]$, although antigenspecific in vitro cytotoxic activity against Ag85A whole protein-loaded target cells is observed after DNA plasmid vaccination using Ag85A [432]. In contrast to C57BL/6 mice, there are several immunogenic $\mathrm{CD}^{+} \mathrm{T}$ cell MHC I epitopes defined for BALB/c mice [430, 447, 448], which can potentially be used as peptides loaded onto target cells for in vitro and in vivo cytotoxicity assays. Therefore, future experiments to assess the

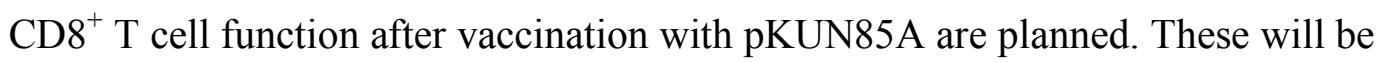
performed in a BALB/c model of $\mathrm{Tb}$ infection and correlated with protection studies also performed in $\mathrm{BALB} / \mathrm{c}$ mice.

\subsubsection{Future directions for pKUN85A vaccination}

While the mechanism of action of pKUN85A and its ability to activate antigen-specific $\mathrm{CD} 8{ }^{+} \mathrm{T}$ cells will be explored in the future, the pKUN85A vaccine and its administration regimen could also be further improved. Vaccination with pKUN85A could be optimised on several levels, which are outlined below: 
1. Cloning into the pKUNrep6 vector an antigen containing a $\mathrm{CD} 8^{+} \mathrm{T}$ cell epitope that is stronger than the epitopes found in Ag85A could enhance $\mathrm{CD}^{+} \mathrm{T}$ cell responses. The mycobacterial antigen, PstS-3, has been shown in murine models to induce strong $\mathrm{CD}^{+} \mathrm{T}$ cell responses, has a welldefined $\mathrm{CD}^{+} \mathrm{T}$ cell epitope and is a strong vaccine candidate [449].

2. Expression of antigens encoded in the pKUNrep6 vector could also be enhanced. Mycobacterial genes are optimised for the prokaryotic translational machinery with slightly different codon usage to the eukaryotic host. Therefore, optimising codons in $A g 85 A$ or $P s t S$-3 could promote efficient translation of the antigen using host cellular machinery. Optimal expression of antigen genes also relies on efficient transfection of target cells. A basic anti-viral mechanism is the secretion of nucleases and rapid degradation of extracellular DNA [450]. The challenge for DNA vaccination is to protect the integrity of the DNA and transfect it efficiently into target cells. Pathogen-like particles (reviewed in [451]), chemical transfection agents $[452,453][454]$ or electroporation $[455,456]$ can all be used to enhance transfection in vivo, and may also enhance vaccine immunogenicity. Electroporation in particular is becoming an increasingly viable option for DNA vaccination in humans as technology is developed to permit electroporation in the clinic and field. Eletroporation runs an electric current through tissue immediately after immunisation with naked DNA plasmid $[455,456]$. This can be used to force DNA into the cells but it also causes local tissue damage and inflammation, which may enhance recruitment of APCs and enhance vaccine immunogenicity.

3. The immunogenicity of pKUN85A could be optimised using a number of techniques. Cytokines, chemokines and co-stimulatory molecules can be included, either as a gene encoded by the same or co-administered plasmids or the protein can be administered to the vaccination site, to create a local inflammatory milieu essential for maximal priming [457-459]. Furthermore, unmethylated $\mathrm{CpG}$ motifs found in genetic material from bacteria interact with TLR 9 in the early endosome and activate type I interferon production 
[460], contributing to local inflammatory signals. The pKUN85A plasmid sequence could be optimised for the murine model to include more of these motifs.

4. The route of administration for pKUN85A could also be optimised. Administration of DNA vaccines to different tissues could lead to transfection of different cell types, altering mechanisms of APC activation and antigen presentation. Gene-gun bombardment, which delivers plasmidcoated microbeads to the epidermis, and i.m. injection of DNA plasmids are the most common routes of administration used for DNA vaccination. For Tb DNA vaccines, i.m. injection has been shown to induce better protection, presumably as gene-gun bombardment favours the development of robust humoral immunity rather than cell-mediated immunity, which is important for $\mathrm{Tb}$ protective memory [389]. Other routes of administration have also been used for DNA vaccines. Mucosal administration of DNA vaccines can result in tolergenic responses to the antigen encoded in the construct, so may diminish rather than enhance immunogenicity [461]. Subsutaneous vaccination has also been used for DNA vaccines and s.c. vaccination with the pcDNA3-85A vaccine confers short-term protection against $M$. tuberculosis challenge [433]. Other promising routes of administration include epicutaneous vaccination, which involves tape-stripping the upper layers of the skin and painting the stripped area with a vaccine [462], and intradermal vaccination [463]. These methods of administration are thought to deliver antigen to the potent APCs that are resident in the skin, such as Langerhans cells in the epidermis and dermal DCs in the dermis, as well as activating these cells and triggering their migration. Targeting DNA vaccines to these potent APCs could enhance their efficacy.

5. While the choice of antigen, its expression and immunogenicity can be optimised for the pKUN85A construct, it is difficult to generate long-lived immunity, particularly cell-mediated immunity. Boosting has been used to prolong immunity but repeated administration of the same vaccine, or homologous boosting, is not always beneficial due to interactions with pre- 
existing immunity to the vaccine. The other option is to use a heterologous prime-boost, whereby the immune response is primed against a target antigen using one vector and boosted against the target antigen using another vector (reviewed in [464]). The heterologous prime-boost approach can use a combination of live vaccines, adjuvanted protein, viral vectors or DNA vaccines and promotes cell-mediated immunity.

The immunological principle that heterologous prime-boost approaches exploit is the concept of "original antigenic sin". This states that repeated administration of a dominant antigen amplifies a response directed against that antigen during subsequent challenge, even using a challenge vector that shares only partial homology with the priming vector. In this way the size of the target antigen-specific $\mathrm{T}$ cell pool is expanded in preference to other antigens that may be included in either vector, and the avidity of the expanded antigen-specific $\mathrm{T}$ cell population is increased [465]. These factors combined can increase the number and functionality of the antigen-specific $\mathrm{T}$ cells over the thresholds required for protection against infection and result in not just additive but synergistic increases in measures of protection [466]. The effect of vaccinating with BCG or adenoviral or MVA vectors encoding Ag85A, which would enhance CD8 ${ }^{+}$ $\mathrm{T}$ cell activation, in combination with pKUN85A in a prime-boost approach could be explored in the future in an effort to provide robust and long-lived immunity against $\mathrm{Tb}$. 


\subsection{Summary}

In summary, a novel plasmid DNA replicon vaccine against Tb, pKUN85A, was created. The Ag85A gene from M. tuberculosis was cloned into the ORF of the noncytopathic pKUNrep6 construct and in-frame with NS genes derived from the Kunjin virus. The pKUN85A construct was administered in vivo in a C57BL/6 murine model, and the $25 \mu \mathrm{g}$ dose was immunogenic two weeks after boosting; however preliminary protection studies did not indicate that homologous prime-boost vaccination with pKUN85A provides protection against $M$. tuberculosis challenge equivalent to the BCG vaccine.

As with all plasmid DNA replicons, the dose of pKUN85A must be optimised for maximal protection against $\mathrm{Tb}$ and, as can occur with $\mathrm{Tb}$ vaccines, protection may wane over time. Further development of the pKUN84A vaccine will be undertaken to enhance immunogenicity and protection mediated by pKUN85A, particularly the longevity of protection. In the future, the $\mathrm{CD}^{+} \mathrm{T}$ cell responses induced by the pKUN85A vaccine will be assessed and correlated with protection data to explore the role of $\mathrm{CD}^{+} \mathrm{T}$ cells during vaccination and this could provide a more useful correlate of protection from $\mathrm{Tb}$ infection. 
Chapter 5: Natural Treg-Mediated Suppression of Protective Immunity Against Tb 


\subsection{Introduction}

The current $\mathrm{Tb}$ vaccine, $\mathrm{BCG}$, is highly variable in its effectiveness (section 1.3.4). This variability gives rise to two key questions:

- What can diminish the efficacy of the BCG vaccine?

- How can the efficacy of BCG and other Tb vaccines be enhanced?

The primary immune response to mycobacterial infection has been well characterised (section 1.2.3); however, less is known about the development of protective memory against $\mathrm{Tb}$ and which aspects of the BCG vaccine-induced immune response result in protective memory (section 1.2.6). To develop a more effective $\mathrm{Tb}$ vaccine, the memory cell subsets that mediate protection from $\mathrm{Tb}$ infection and how they are regulated need to be better understood.

A cellular subset that may play a role in suppressing the BCG vaccine-induced immune response is the Treg population. Results presented in chapter 3 illustrate that Treg inactivation prior to mycobacterial infection increases the number of antigen responsive cells producing cytokines such as IL-2. Proliferation and signalling by the $\gamma \mathrm{c}$ chain family of cytokines, including IL-2, are vital for optimal T cell memory development (sections 1.3.2.2 and 1.3.2.3). Thus, it has been suggested that immunosuppression mediated by Tregs during a BCG vaccine-induced immune response would diminish the protective immunity that BCG vaccination confers against $\mathrm{Tb}$ [339]. Treg inactivation prior to vaccination against several other diseases can enhance protective immunity, but it can also diminish resulting protective immunity, as discussed below.

\subsubsection{Tregs can suppress protective memory}

Antibody-mediated inactivation of natural CD25 $5^{+}$Tregs prior to or concurrently with vaccination has been shown to increase vaccine-induced immune responses and enhance the protective efficacy of vaccines [336, 338, 339].

A recent study by Moore, et al. [339] assessed the effect of antibody-mediated inactivation of $\mathrm{CD} 4{ }^{+} \mathrm{CD} 25^{+}$natural Tregs prior to or concurrently with vaccine 
administration. The types of vaccine that were assessed included viral vector and protein subunit vaccines, using a variety of bacterial, protozoal and tumour antigens.

Treg inactivation enhanced vaccine-induced immune responses, as assessed by numbers of IFN- $\gamma$-producing lymphocytes measured by enzyme-linked immunospot assay (ELISpot) 10-14 days after vaccination. The effect of Treg inactivation prior to or concurrently with priming was also assessed for both homologous and heterologous prime/boost vaccines. Treg inactivation enhanced vaccine-induced immune responses after boosting for both prime/boost approaches. Therefore, it is well established that Treg inactivation can enhance vaccine-induced immune responses to a variety of formulations.

Moore, et al. then focussed on a malarial vaccine in greater depth, assessing whether the primary immune response as well as the secondary immune response and protection against infectious challenge were enhanced by Treg inactivation during priming [339]. They used a fowlpox vector prime followed by a MVA vector boost and both vectors encoded the malarial Plasmodium berghei circumsporozoite protein. Moore, et al. demonstrated that, if Tregs were inactivated concurrently with priming, the primary immune response persisted longer and the response to sub-dominant epitopes within the target antigen was significantly enhanced as measured by IFN- $\gamma$ ELISpot. Furthermore, when Tregs were inactivated, $100 \%$ of vaccinated mice were protected upon challenge with malarial sporozoites compared to $40-60 \%$ protection in vaccinated control mice.

Collectively, the study by Moore, et al. illustrates that inactivating Tregs prior to or concurrently with a number of different vaccines enhances the vaccine-induced immune responses and, at least for the malarial vaccine that was studied, prolongs the vaccine-induced immune response and enhances protection from pathogen challenge.

In support of these results, studies in which Tregs were inactivated prior to HSV-1 vaccination or infection, or prior to hepatitis B virus vaccination, have shown amplified vaccine-induced primary immune responses and increased numbers of antigen-specific T lymphocytes after resolution of the primary response [336, 338, 375]. In the HSV-1 studies, when Tregs were inactivated prior to infection or vaccination, an earlier decline in viral plaque forming units in the tissues was observed following infectious challenge [338, 375]. Enhanced protection was observed after infectious challenge when Tregs were inactivated prior to priming with a live HSV-1 infection, an 
HSV-derived peptide vaccine or a plasmid DNA vaccine encoding an HSV-1 immunodominant protein $[338,375]$.

These studies illustrate that inactivation of Tregs prior to or concurrently with vaccination can result in enhanced primary immune responses, increased pools of antigen responsive lymphocytes after vaccination and, importantly, can enhance the efficacy of different types of vaccines, including live, subunit and DNA plasmid vaccines.

\subsubsection{Tregs can facilitate protective memory}

In contrast to the studies described above, while Tregs can suppress primary immune responses and negatively impact on the resulting protective immunity, in some circumstances Tregs are required to facilitate the development of protective immunity.

In the L. major C57BL/6 murine model, maintenance of protective immunity in a mouse that has been previously exposed or vaccinated with live L. major is dependent on the persistence of low numbers of the protozoa in skin lesions [305]. If L. major infected or vaccinated animals lose these persistent protozoa, protective immunity is lost and mice are vulnerable to re-infection and disease. Protective immunity can also be lost when natural Tregs are depleted [305] or IL-10 signalling is blocked [358] during infection. Both of these scenarios result in enhanced infection-induced primary immune responses and enhanced clearance of the primary infection. It is proposed that the lack of persisting organisms then leads to a loss of memory maintenance and a lack of protection from subsequent infectious challenge [305]. Therefore, suppression of the primary immune response, mediated by Tregs, is essential for development of protective immunity in situations where antigen persistence is essential for ongoing maintenance of immunological memory.

\subsubsection{Tregs and development of anti-mycobacterial immunity}

As described above, the inactivation of $\mathrm{CD} 25^{+}$Tregs in several infection and vaccination models can enhance the primary immune response and thereby modulate the development and maintenance of protective immunity. The effect of Treg activity in 
the models described above has implications for the effect of Tregs on BCG, other vaccines against $\mathrm{Tb}$ and overall protective immunity against $\mathrm{Tb}$.

The effect of Treg inactivation or depletion on antigen persistence after primary mycobacterial infection has been addressed. Following publication of my results (outlined in chapter 3) [374], two other groups have published their investigations, using alternative approaches, evaluating the role of Tregs during primary mycobacterial infection [383, 384] (discussed in section 3.4.5). The results presented in chapter 3 illustrate that antibody-mediated Treg inactivation prior to BCG, M. tuberculosis and $M$. bovis infections did not result in clearance or even reductions in lung bacterial numbers (Figs. 3.11/3.13). Kursar, et al. [383] used an adoptive transfer model to show that Rag1 -/- mice that received $\mathrm{CD} 4^{+} \mathrm{CD} 25^{-}$donor $\mathrm{T}$ cells had reduced bacterial numbers in the lung and spleen after $M$. tuberculosis challenge compared to mice that received a mixture of $\mathrm{CD} 4{ }^{+} \mathrm{CD} 25^{-}$and $\mathrm{CD} 4{ }^{+} \mathrm{CD} 25^{+}$donor T cells. Scott-Browne, et al. [384] used a mixed chimera model where irradiated recipient mice were reconstituted with Foxp3 /- and +/+ bone marrow. Foxp3 -/- bone marrow was derived from Thy $1.2^{+}$donor mice and Foxp $3+/+$ bone marrow from Thy $1.1^{+}$donor mice, and administration of Thy 1.1 depleting $\mathrm{mAb}$ to recipient mice resulted in depletion of all Foxp $3^{+}$Tregs [384]. When these mice were infected with $M$. tuberculosis, bacterial numbers in the lung were reduced compared to chimeras reconstituted with Foxp3 +/+ Thy $1.1^{+}$and Foxp3 +/+ Thy $1.2^{+}$bone marrow, which retained Foxp $3^{+}$Tregs [384]. From these studies the effect of Tregs in WT mice on tissue bacterial numbers in vivo is not clear; however, it is important to note that mice were not completely cured of mycobacterial infection in any study. Therefore, if antigen persistence is required for maintenance of antimycobacterial immunity, antibody-mediated inactivation of Tregs is unlikely to affect BCG persistence. As a result, and in contrast to L. major infection and live vaccination, I hypothesised that Treg inactivation prior to BCG vaccination would be unlikely to inhibit the development and maintenance of protective immunity.

As described in chapter 3, inactivation of Tregs during primary mycobacterial infection increased numbers of cytokine-producing lymphocytes at two weeks after infection (Fig. 3.7/3.8). In support of this data, the study by Moore, et al. inactivated Tregs concurrently with priming using a BCG / MVA-85A prime-boost vaccine against Tb. This resulted in increased numbers of IFN- $\gamma^{+}$lymphocytes measured by ELISpot in response to stimulation with a MHC II-restricted epitope two weeks after boosting [339]. Therefore, inactivation of Tregs during primary exposure to mycobacteria can 
enhance the immune response. Given the impact of Tregs in these other vaccination settings, I hypothesised along with others [339] that Treg inactivation during a primary immune response to mycobacterial antigen could enhance both the secondary immune response and development of protective immunity against $\mathrm{Tb}$.

Prior to this study, it had not been assessed whether Treg activity during priming with BCG or other Tb vaccines suppressed secondary immune responses or the protective efficacy of vaccines against a virulent mycobacterial challenge. If inactivation of $\mathrm{CD} 25^{+}$Tregs did enhance the protective efficacy of the BCG vaccine or other $\mathrm{Tb}$ vaccines, vaccination strategies could be developed to modulate Treg activity at the time vaccination and thereby optimise vaccine efficacy. Therefore, in this chapter, I evaluated the effect of Treg inactivation on the development of protective $\mathrm{Tb}$ immunity in a murine model. This was achieved by antibody-mediated inactivation of $\mathrm{CD} 25^{+}$Tregs prior to vaccination with $\mathrm{BCG}$ or plasmid DNA vaccines encoding $\mathrm{Tb}$ antigen, and assessment of the primary and secondary immune responses. Most importantly, functional protection from virulent $M$. tuberculosis or M. bovis aerosol challenge was evaluated. 


\subsection{Aims}

This chapter builds upon findings from chapter 3 and utilises the plasmid DNA replicon vaccine developed in chapter 4 , to examine whether Treg inactivation prior to $\mathrm{Tb}$ vaccination enhances primary and secondary immune responses and enhances the development of protective $\mathrm{Tb}$ immunity.

Inactivation of Tregs prior to vaccination can enhance vaccine efficacy [338, $339,375]$, if it does not interfere with mechanisms of memory maintenance, such as antigen persistence [305]. As inactivation of Tregs prior to BCG infection increases the primary immune response but does not decrease antigen persistence (Figs. 3.8-3.11), it was hypothesised that inactivation of Tregs prior to BCG vaccination would amplify primary and secondary immune responses and enhance protective efficacy. In addition, inactivation of Tregs prior to DNA vaccination can enhance vaccine efficacy [338]. Therefore, it was hypothesised that inactivation of Tregs prior to Tb DNA vaccination would enhance primary and secondary immune responses, as well as protective efficacy.

To explore these hypotheses, $\mathrm{CD} 4^{+} \mathrm{CD} 25^{+}$Tregs were inactivated in the $\mathrm{C} 57 \mathrm{BL} / 6$ murine model prior to vaccination with one of three different $\mathrm{Tb}$ vaccines and subsequently challenged with $M$. tuberculosis or M. bovis by aerosol. The vaccines used were $\mathrm{BCG}$ as a live vaccine, pcDNA3-85A as a conventional plasmid DNA vaccine or pKUN85A, which was developed in chapter 4 and is a non-cytopathic plasmid DNA replicon vaccine. My main objectives were to:

- Assess the effect of Treg inactivation on primary immune responses to BCG, pKUN85A or pcDNA3-85A vaccination.

- Assess the effect of Treg inactivation on secondary immune responses to the vaccines listed above.

- Characterise the protective efficacy of the vaccines listed above after inactivation of $\mathrm{CD} 4^{+} \mathrm{CD} 25^{+}$Tregs by evaluating tissue bacterial numbers and lung pathology. 


\subsection{Results}

\subsubsection{Experimental design}

In order to evaluate whether natural $\mathrm{CD} 4^{+} \mathrm{CD} 25^{+}$Tregs act during the primary immune response to inhibit $\mathrm{Tb}$ vaccine efficacy, the experimental protocol used in chapter 3 was modified to inactivate Tregs prior to either BCG or DNA vaccination (Fig. 5.1).

Mice were administered anti-CD25 mAb (PC61) or control rat IgG i.p. and two days later peripheral blood samples were taken to confirm inactivation of Tregs. Surface staining for CD25 expression in these samples used the anti-CD25 mAb clone 7D4 to avoid epitope masking after PC61 treatment. Treg inactivation as assessed by flow cytometry was consistent in all mice that received PC61 across all experiments (data not shown). Three days after antibody treatment, mice were vaccinated with one of three vaccines: BCG, pcDNA3-85A or pKUN85A. Groups vaccinated with pcDNA3-85A or pKUN85A received a homologous boost vaccination two weeks later. Results from chapter 3 demonstrate that PC61 treatment inactivates $\mathrm{CD} 4{ }^{+} \mathrm{CD} 25^{+}$Tregs up to day 23 after treatment and therefore Tregs were inactivated during the acute vaccine-induced immune response.

After vaccination, mice were rested for either 6 or 15 weeks, to assess protection against an infectious challenge early or late after vaccination respectively, before receiving either an aerosol challenge with M. tuberculosis or M. bovis (Fig. $5.1 \mathrm{D})$, or an i.n. challenge with BCG (Fig. 5.1 C). Aerosol M. tuberculosis or M. bovis challenges were used to evaluate tissue bacterial load and lung pathology as measures of the protective efficacy of BCG or DNA vaccination. BCG i.n. challenge was used as a PC2 model of mycobacterial infection to permit analysis of infected tissues in a PC2 flow cytometry facility and assessment of the secondary immune response after vaccination. Results from chapter 3 illustrate that, in terms of the acute immune response (Fig. 3.8) and tissue bacterial numbers (Fig. 3.11), primary BCG infection is a suitable PC-2 model for virulent mycobacterial infections, such as M. tuberculosis and M. bovis. In addition, BCG infection has been used as an infectious challenge to assess secondary immune responses in other $\mathrm{Tb}$ vaccine pre-clinical studies [467]. Therefore, BCG infection is a relevant PC2 model of virulent mycobacterial infection and can be used for assessment of immune responses to vaccination. 
A.

$\begin{array}{cc}\text { Day }-3: & \text { Day }-1: \\ \text { Antibody } & \text { Assess CD25+ } \\ \text { treatment } & \text { Tregs }\end{array}$

BCG vaccination
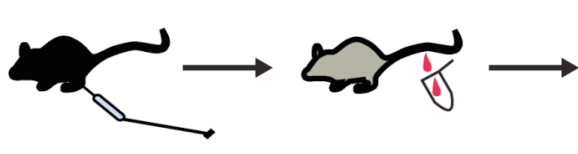

Tregs accination
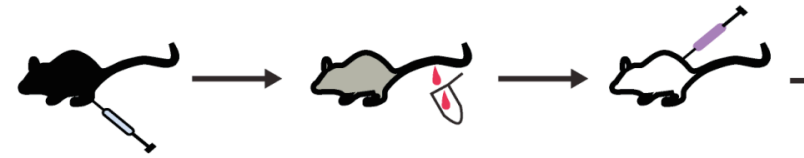

Day -15 :

Day -17:

treatment Assess CD25+ Tregs

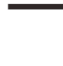

Day -14 :
DNA prime

\section{2 weeks between prime and boost}

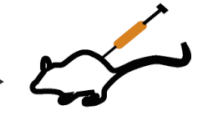
BCG
Week 0: vaccination
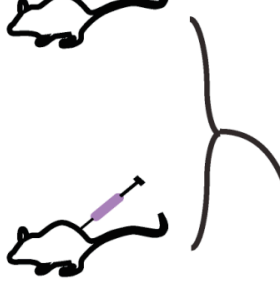

Week 0:

DNA

boost

B.

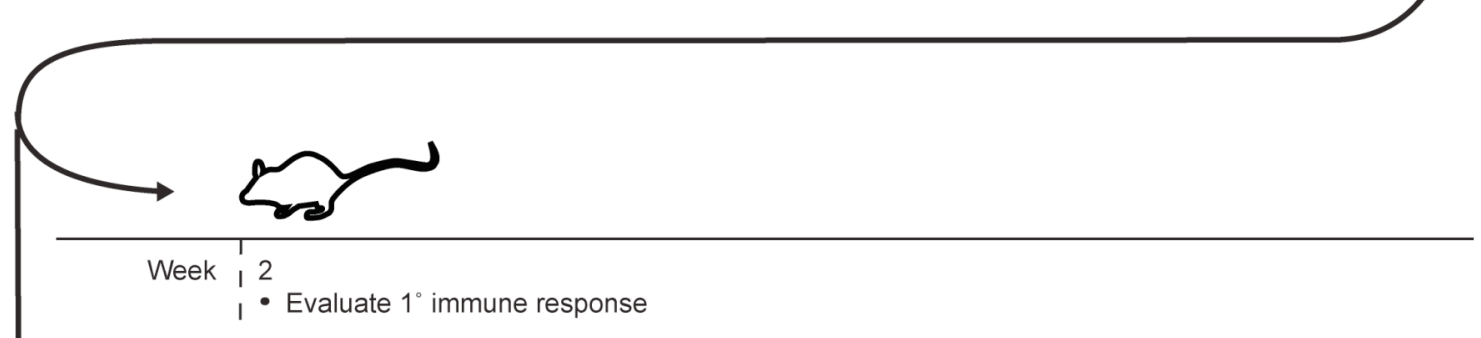

C.

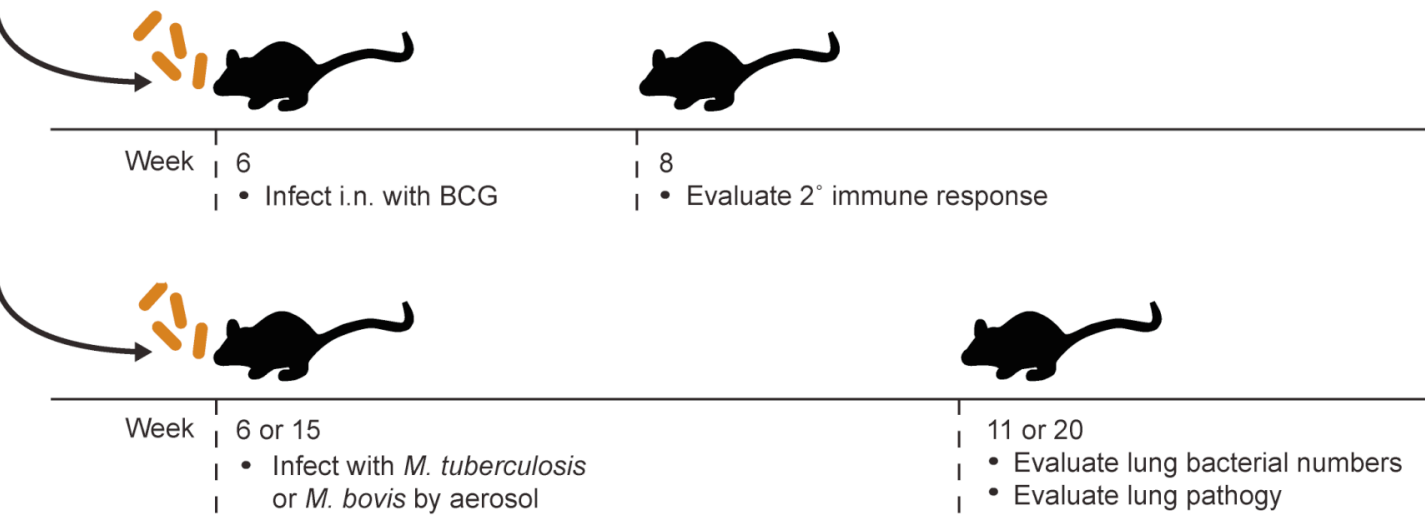

Key:
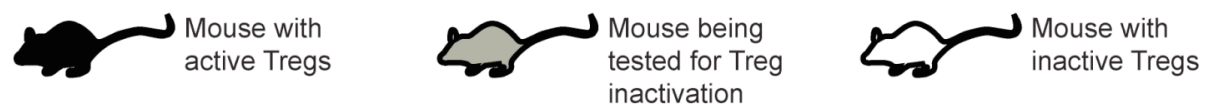

Figure 5.1: Experimental outline for testing whether natural Tregs inhibit the efficacy of

\section{BCG and DNA vaccination}

A) Mice were treated with $100 \mu \mathrm{g}$ of rat $\operatorname{IgG}$ or $100 \mu \mathrm{g}$ of PC61 i.p. and two days later a peripheral blood sample was taken and assessed by flow cytometry for the presence of $\mathrm{CD} 4{ }^{+} \mathrm{CD} 25^{+}$lymphocytes. The following day, mice were vaccinated with $\mathrm{BCG}$ or vaccinated with one of 2 DNA vaccines: pKUN85A or pcDNA3-85A. Mice receiving DNA vaccines were boosted with the same vaccine two weeks later. B) Two weeks after BCG vaccination or the boost DNA vaccinations, one group of mice was culled and the primary immune response assessed. C) Six weeks after BCG vaccination or the boost DNA vaccination, a second group of 
mice were infected i.n. with BCG and the secondary immune response was assessed immediately prior to infection and at days 3, 8 and 15 after the infection. D) Six or fifteen weeks after BCG vaccination or the boost DNA vaccination, the final groups of mice were infected by aerosol with M. bovis or M. tuberculosis and lung bacterial numbers and pathology were evaluated five weeks later. Black mice indicate when Tregs were present, grey mice indicate when mice were being tested for the presence of Tregs and white mice indicate when Tregs were significantly reduced compared to control mice. 
Therefore, the protocol outlined in Fig. 5.1 was used to test the hypothesis that inactivation of natural $\mathrm{CD} 25^{+}$Tregs during vaccination enhances primary and secondary immune responses and protective efficacies of $\mathrm{Tb}$ vaccines, initially focussing on BCG vaccination and later on pcDNA3-85A and pKUN85A vaccination.

\subsubsection{The vaccine-induced immune response to BCG vaccination}

To test the hypothesis that Treg inactivation prior to BCG vaccination would enhance the primary immune response, the vaccine-induced immune response was assessed two weeks after BCG vaccination. Mice were treated with either PC61 or control rat IgG, followed 3 days later by vaccination with BCG or a PBS vehicle control. Two weeks later, lymphocytes were isolated from the spleen and from the inguinal lymph nodes draining the site of vaccination (Fig. 5.1 B). Isolated lymphocytes were restimulated with either CFP or Ag85A and analysed by flow cytometry for IFN- $\gamma$ or IL-2 production. Of note, at two weeks after BCG vaccination, IL-2 and IFN- $\gamma$ producing antigen-specific $\mathrm{CD}^{+}$lymphocytes fell into distinct populations after either Ag85A restimulation (Fig. 5.2) or CFP restimulation (data not shown), with no significant coproduction of the cytokines.

After CFP restimulation, pooled lymphocytes from the draining inguinal lymph nodes of PC61 treated/BCG vaccinated mice had a higher percentage (data not shown) and average number (Fig. 5.3 A) of IFN- $\gamma^{+} \mathrm{CD} 4^{+}$lymphocytes compared to rat IgG treated/BCG vaccinated mice. A similar trend was observed in CFP-restimulated splenocytes, although differences were not statistically significant (data not shown and Fig. 5.3 B).

After Ag85A restimulation, the average number of $\mathrm{IL}-2^{+} \mathrm{CD} 4^{+} \mathrm{T}$ lymphocytes in the spleen was significantly increased in PC61 treated/BCG vaccinated compared to rat IgG-treated/BCG vaccinated mice (Fig. $5.3 \mathrm{C}$ ), although the average number of IFN- $\gamma$ producing $\mathrm{CD}^{+} \mathrm{T}$ lymphocytes remained unchanged (data not shown).

Overall, in agreement with previous studies, inactivation of Tregs during BCG vaccination led to enhanced cytokine production and a modest increase in the primary immune response. 


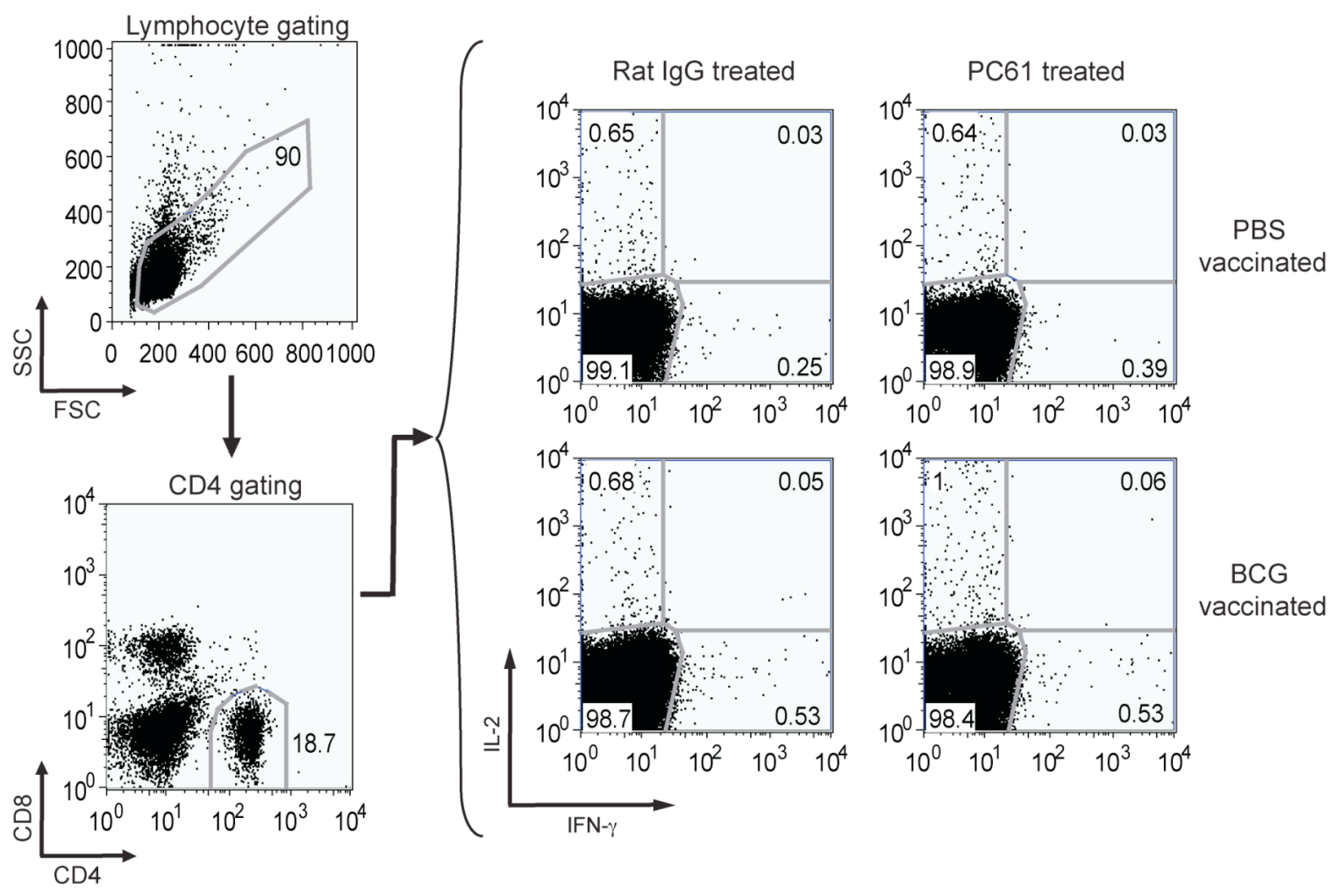

Figure 5.2: IFN- $\gamma$ and IL-2 production by $\mathrm{CD4}^{+}$lymphocytes 2 weeks after BCG vaccination

Mice were administered either rat IgG or PC61 antibody i.p and vaccinated 3 days later with BCG or mock vaccinated with PBS. At two weeks after vaccination, spleens were harvested and isolated splenocytes were stimulated with Ag85A overnight before being assessed for cytokine production. Displayed are representative flow cytometry plots of lymphocyte gating, CD4 gating and IFN- $\gamma / \mathrm{IL}-2$ staining for restimulated splenocytes from rat IgG treated and PC61 treated mice treated with PBS or vaccinated with BCG. Numbers in the gates on flow cytometry plots represent the percent of lymphocytes within that gate from the total parent population. Four to five animals were analysed per treatment group. Results represent two replicate experiments. 
A.

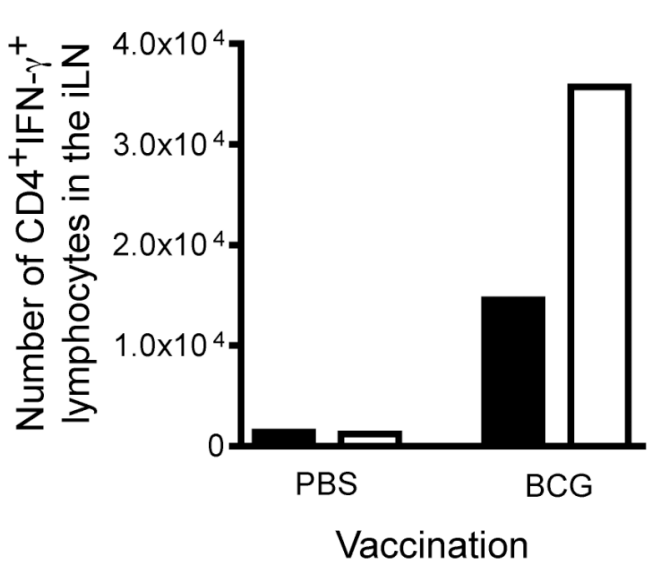

B.

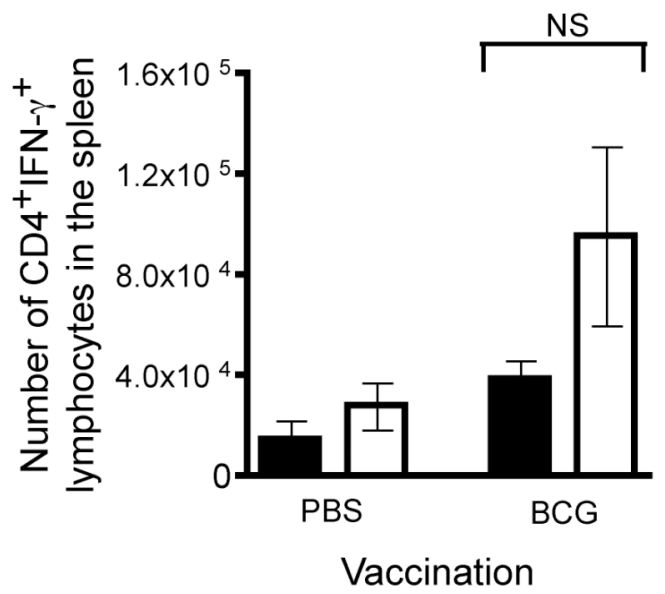

C.

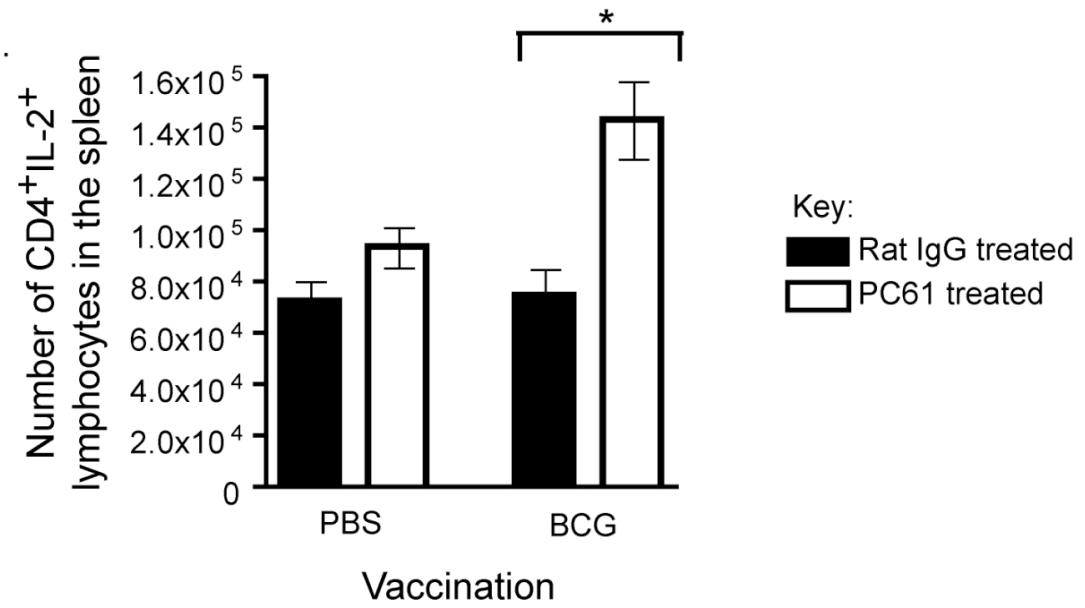

Figure 5.3: Cytokine production by $\mathrm{CD4}^{+}$lymphocytes 2 weeks after BCG vaccination

Mice were administered either rat IgG or PC61 antibody i.p and vaccinated 3 days later with BCG or mock vaccinated with PBS. At two weeks after vaccination, lymphocytes were isolated from tissues and restimulated with $\mathbf{A} / \mathbf{B}$ ) CFP or $\mathbf{C}$ ) Ag85A overnight before being assessed for cytokine production. A) Average number of $\mathrm{CD}^{+}{ }^{+} \mathrm{IFN}-\gamma^{+}$lymphocytes in the inguinal lymph node after CFP restimulation. Lymph nodes were pooled to enable sufficient numbers of cells for restimulation and flow cytometric analysis. B) Average number of $\mathrm{CD} 4^{+} \mathrm{IFN}-\gamma^{+}$ lymphocytes in the spleen after CFP restimulation. C) Average number of CD4 $4^{+} \mathrm{IL}-2^{+}$ lymphocytes in the spleen after $\mathrm{Ag} 85 \mathrm{~A}$ restimulation. Results in $\mathbf{A})$ and $\mathbf{B}$ ) are representative of two replicate experiments. Bars represent mean values \pm SEM. Four to five animals were analysed per treatment group. Treatment groups were compared using the two-tailed Mann Whitney test; NS indicates $p>0.05, *$ indicates $p \leq 0.05$. Results represent two replicate experiments. 


\subsubsection{Antigen-responsive lymphocytes after BCG vaccination}

PC61 treatment prior to BCG vaccination increased the numbers of antigen-responsive $\mathrm{CD}^{+} \mathrm{T}$ lymphocytes 2 weeks after BCG vaccination. However, the effector lymphocyte population goes through a contraction phase after activation $[182,183]$ and factors regulating this contraction phase in the context of mycobacterial infection and vaccination are not understood. Therefore, while there are more antigen-responsive lymphocytes 2 weeks after BCG vaccination in PC61 treated mice, it does not necessarily mean that more cells will be retained at later times to respond to an infectious challenge. In other vaccination models, primary immune responses have been shown to be prolonged and larger pools of antigen-responsive cells were present after contraction if Tregs were inactivated prior to or concurrently with vaccination [336, $338,339]$. After primary exposure to BCG, the T cell mediated immune response peaks around weeks 3-4 and begins to subside by about week $6[182,183]$. Therefore, numbers of cytokine-producing lymphocytes were quantified at 6 weeks after BCG vaccination to assess whether Treg inactivation resulted in a larger pool of antigenresponsive lymphocytes after contraction of the BCG vaccine-induced immune response.

Lymphocytes from the spleen and pooled inguinal lymph nodes were isolated from mice 6 weeks after vaccination with either BCG, or PBS as a negative control. Lymphocytes were restimulated with CFP, processed for intracellular cytokine staining and analysed by flow cytometry for the production of IFN- $\gamma$ and IL-2. In the spleen, the average number of CD4 ${ }^{+}$IFN $-\gamma^{+}$lymphocytes was increased significantly in PC61 treated/BCG vaccinated mice compared to rat IgG treated/BCG vaccinated mice (Fig. 5.4 A). This trend was also observed in the lung, although differences were not statistically significant (Fig. 5.4 B). The average number of CD $4^{+}$IFN $-\gamma^{+}$lymphocytes in BCG vaccinated mice 6 weeks after vaccination (Fig. 5.4 A) was similar to that observed 2 weeks after vaccination (Fig. 5.3 B). This is consistent with further expansion and then subsequent contraction occurring between 2 and 6 weeks after primary exposure to BCG $[182,183]$. The average number of $\mathrm{CD}^{+} \mathrm{IFN}-\gamma^{+}$lymphocytes was also significantly increased in lung, but not the spleen, of PC61 treated/BCG vaccinated mice compared to rat $\mathrm{IgG}$ treated/BCG vaccinated mice (Fig. 5.4 C/D). 


\section{Spleen}

A.

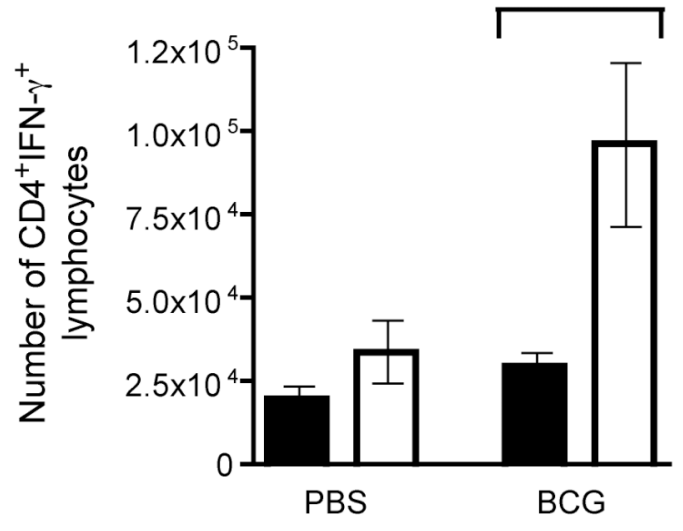

Vaccination

C.

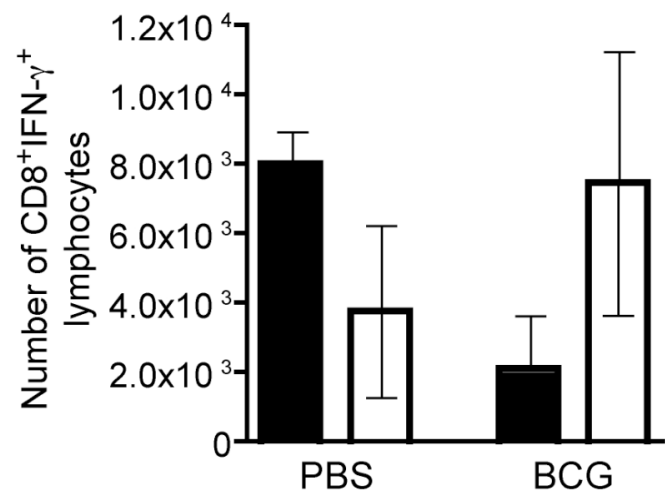

Vaccination
Lung

B.

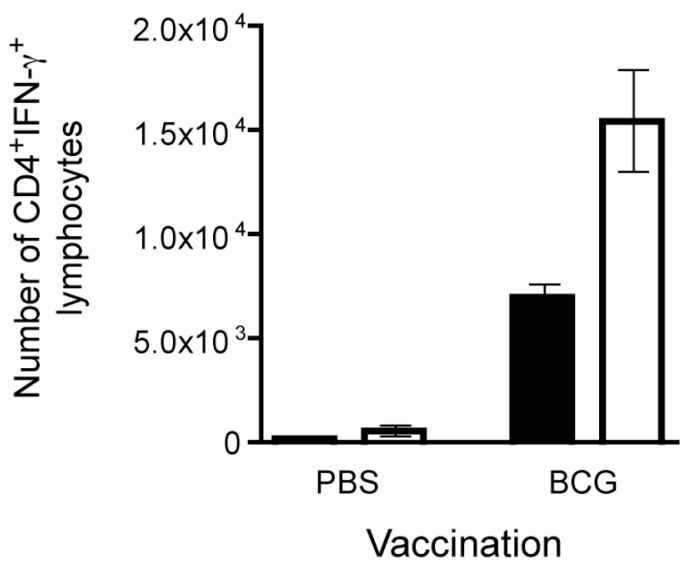

D.

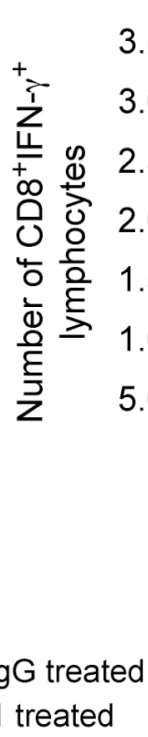

Figure 5.4: IFN- $\gamma$ production by $\mathrm{CD}^{+}$and $\mathrm{CD8}^{+}$lymphocytes 6 weeks after BCG vaccination

Mice were administered either rat IgG or PC61 antibody i.p and vaccinated 3 days later with BCG or mock vaccinated with PBS. At six weeks after vaccination, lymphocytes were isolated from spleens and lungs and restimulated with CFP overnight before being assessed for cytokine production by flow cytometry. A) Average number of $\mathrm{CD} 4^{+} \mathrm{IFN}-\gamma^{+}$lymphocytes in the spleen. B) Average number of $\mathrm{CD}^{+}$IFN- $\gamma^{+}$lymphocytes in the lung. C) Average number of CD8 ${ }^{+} \mathrm{IFN}-$ $\gamma^{+}$lymphocytes in the spleen. D) Average number of $\mathrm{CD}^{+} \mathrm{IFN}^{-}{ }^{+}$lymphocytes in the lung. Bars represent mean values \pm SEM. Five animals were analysed per treatment group. Treatment groups were compared using the two-tailed Mann Whitney test; * indicates $p \leq 0.05$. Results represent one experiment. 
Interestingly, when $\mathrm{CD}^{+}$lymphocytes from $\mathrm{BCG}$ vaccinated animals were assessed for cytokine production 6 weeks after vaccination, a small population of lymphocytes was observed that produced both IFN- $\gamma$ and IL-2 (Fig. 5.5). In both the spleen and lung, the numbers of IFN- $\gamma^{+} \mathrm{IL}-2^{+} \mathrm{CD} 4^{+}$lymphocytes were significantly increased in PC61 treated compared to rat IgG treated BCG vaccinated mice (Fig. 5.6 A/B). However, when the proportion of IFN- $\gamma^{+} \mathrm{IL}-2^{+} \mathrm{CD} 4^{+}$lymphocytes within the total IFN- $\gamma$ or IL-2 producing $\mathrm{CD}^{+}$lymphocyte population was assessed in $\mathrm{BCG}$ vaccinated mice, this proportion was unchanged by PC61 treatment (Fig. 5.6 C/D).

Although there were more lymphocytes capable of producing cytokine immediately prior to challenge in PC61 treated/BCG vaccinated mice, the quality of these cells may have been compromised during their development. To explore this possibility, the mean fluorescence intensity (MFI) of $\mathrm{CD} 4^{+}$and $\mathrm{CD} 8^{+}$lymphocytes staining positive for IFN- $\gamma$ or IL-2 in the lung and the spleen was assessed. There were no significant differences in MFI between BCG vaccinated groups for either cytokine by $\mathrm{CD}^{+}$or $\mathrm{CD}^{+}$lymphocytes in the spleen of the lung (data not shown).

Therefore, inactivation of Tregs during BCG vaccination led to a larger pool of antigen-responsive $\mathrm{CD}^{+}$and $\mathrm{CD} 8^{+}$lymphocytes in the spleen and lung 6 weeks after vaccination and immediately prior to infectious challenge. Furthermore, it appears that the increase in the number of antigen-responsive lymphocytes did not correlate with a decrease in their quality, as assessed by comparison of cytokine stain MFI. 


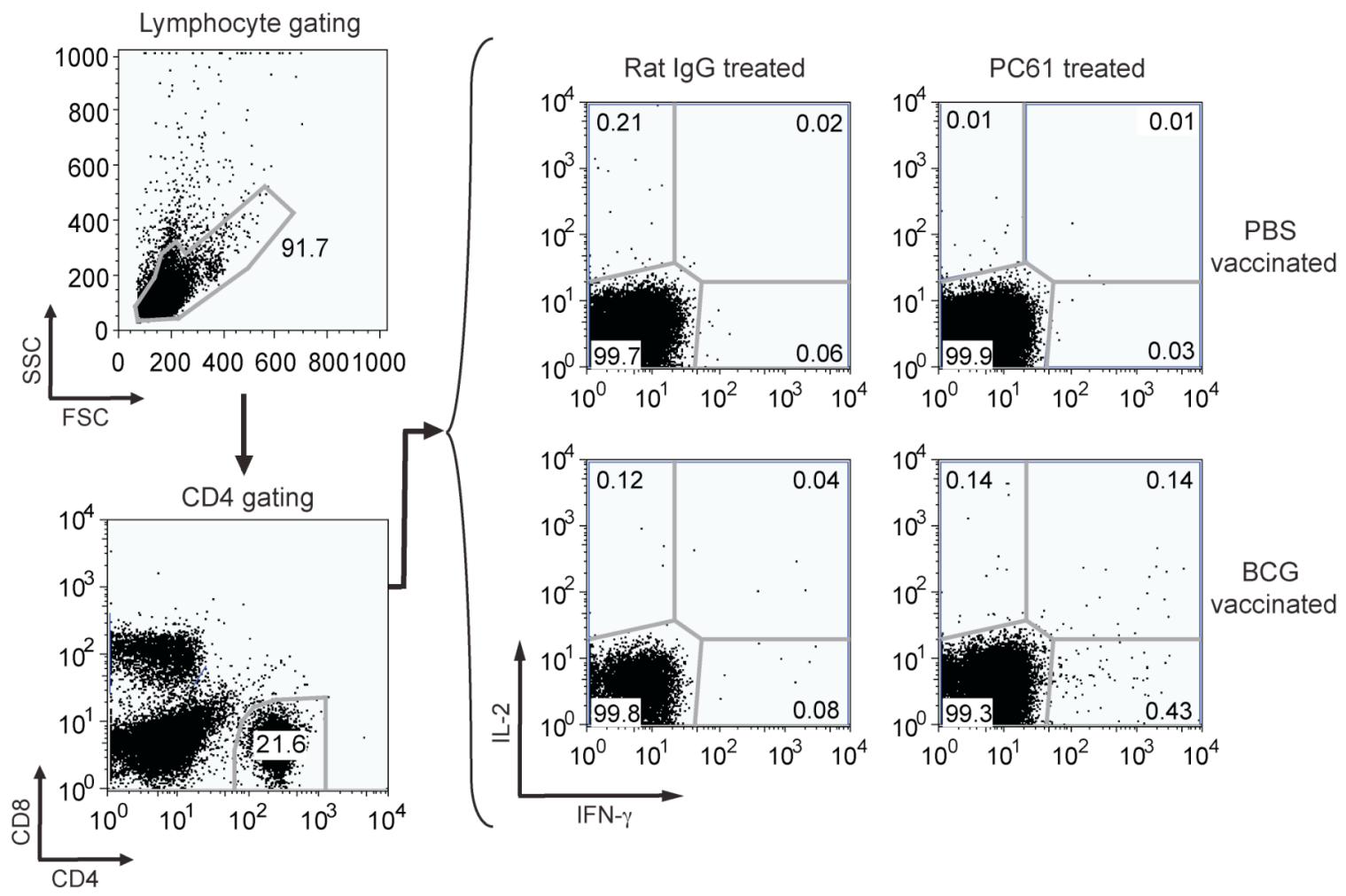

Figure 5.5: IFN- $\gamma$ and IL-2 production by $\mathrm{CD4}^{+}$lymphocytes 6 weeks after BCG vaccination

Mice were administered either rat IgG or PC61 antibody i.p and vaccinated 3 days later with BCG or mock vaccinated with PBS At six weeks after vaccination, spleens were harvested and isolated splenocytes were stimulated with CFP overnight before being assessed for cytokine production. Displayed are representative flow cytometry plots of lymphocyte gating, CD4 gating and IFN- $\gamma / \mathrm{IL}-2$ staining for restimulated splenocytes from rat IgG treated and PC61 treated mice treated with PBS or vaccinated with BCG. Numbers in the gates on flow cytometry plots represent the percent of lymphocytes within that gate from the total parent population. Five animals were analysed per treatment group. Results represent one experiment. 
Spleen

A.

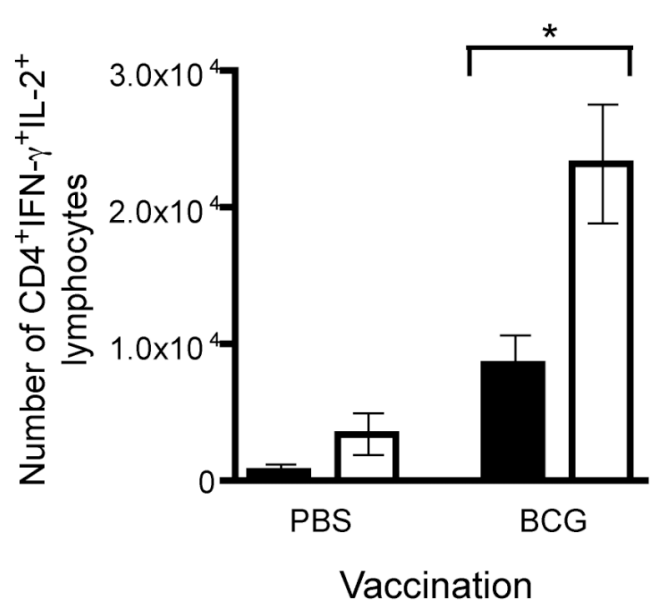

C.

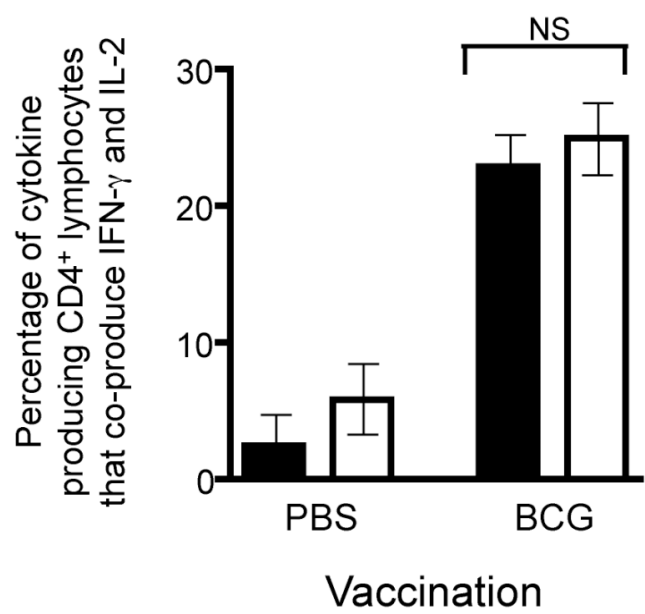

Lung

B.

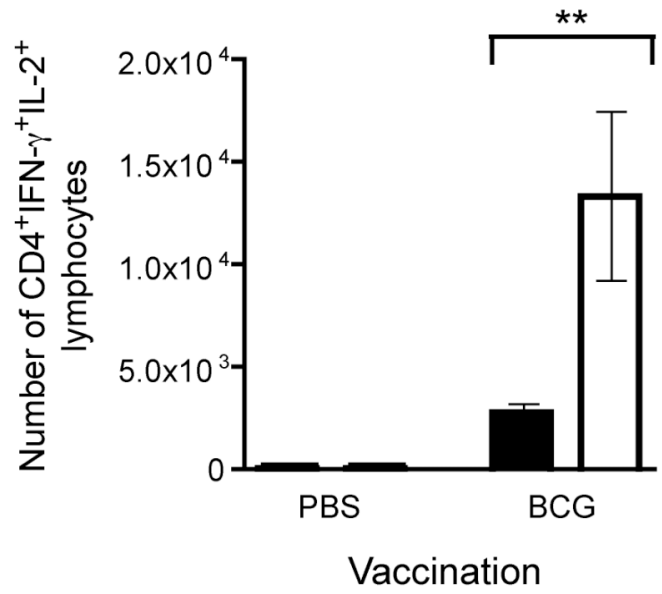

D.
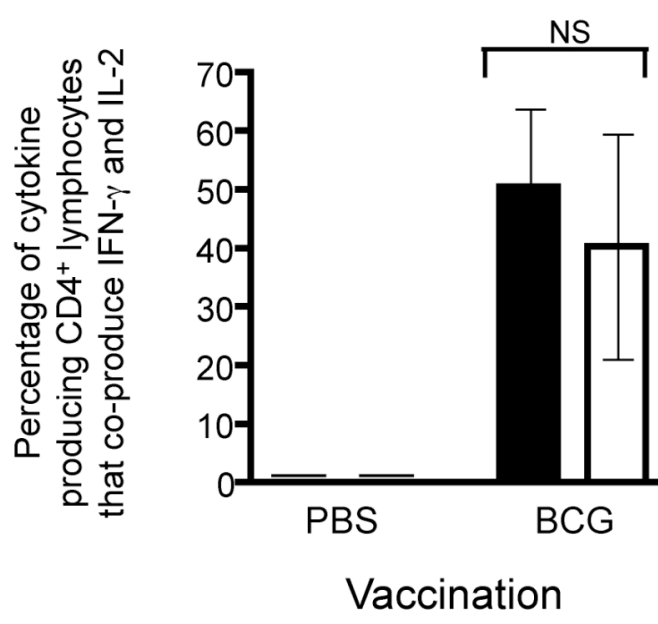

Key:

Rat IgG treated PC61 treated

Figure 5.6: IFN- $\gamma$ and IL-2 co-production by $\mathrm{CD}^{+}$lymphocytes 6 weeks after BCG vaccination

Mice were administered either rat IgG or PC61 antibody i.p and vaccinated 3 days later with BCG or mock vaccinated with PBS. At six weeks after vaccination, lymphocytes were isolated from spleens and lungs and restimulated with CFP overnight before being assessed for cytokine production by flow cytometry. A) Average number of $\mathrm{CD} 4^{+} \mathrm{IFN}-\gamma^{+} \mathrm{IL}-2^{+}$lymphocytes in the spleen. B) Average number of CD4 $4^{+} \mathrm{IFN}-\gamma^{+} \mathrm{IL}-2^{+}$lymphocytes in the lung. C) Percentage of cytokine producing $\mathrm{CD} 4^{+}$lymphocytes that co-produce IFN- $\gamma$ and IL-2 in the spleen. D) Percentage of cytokine producing $\mathrm{CD}^{+}{ }^{+}$lymphocytes that co-produce IFN- $\gamma$ and IL- 2 in the lung. Bars represent mean values \pm SEM. Five animals were analysed per treatment group. Treatment groups were compared using the two-tailed Mann Whitney test; NS indicates $p$ > $0.05, *$ indicates $\mathrm{p} \leq 0.05, * *$ indicates $\mathrm{p} \leq 0.01$. Results represent one experiment. 


\subsubsection{The secondary immune response for BCG vaccination}

Increased numbers of antigen-responsive cells prior to infectious challenge have been observed in other models of Treg inactivation prior to vaccination and this has correlated with an amplified immune response to infectious challenge [338, 339]. However, increased numbers of antigen-responsive cells present in the host prior to secondary antigen exposure do not guarantee that the cells will respond efficiently to the infectious challenge. To assess the response of lymphocytes to an infectious challenge, numbers of cytokine producing lymphocytes were quantified over the course of a BCG infection and responses were compared between treatment groups.

Vaccinated mice were challenged with BCG i.n. and lymphocytes were isolated immediately prior to challenge and at days 3,8 and 15 after challenge (Fig 5.1 C). Lymphocytes were isolated from the mediastinal lymph nodes draining the site of infection and from the lung. These lymphocytes were restimulated with CFP, processed for intracellular cytokine staining and analysed by flow cytometry for the production of IFN- $\gamma$ and IL-2. Some lymph nodes isolated from BCG vaccinated mice at day 15 after BCG infection were fibrotic and therefore the day 15 time point for mediastinal lymph nodes was not included.

In the mediastinal lymph node, numbers of both IFN $-\gamma^{+} \mathrm{CD} 4^{+}$and IFN- $\gamma^{+} \mathrm{CD} 8^{+}$ lymphocytes increased markedly in BCG vaccinated mice over the early course of the infection (Fig. 5.7 A/B). However, in PC61 treated/BCG vaccinated mice the increase in cytokine producing lymphocytes was accelerated compared to rat IgG treated/BCG vaccinated mice, where there were more IFN- $\gamma$ producing lymphocytes at day 3 after challenge in these mice (Fig. 5.7 A/B). At the times analysed, the maximum numbers of antigen-responsive lymphocytes for both PC61 treated/BCG vaccinated mice and rat IgG treated/BCG vaccinated mice were similar (Fig. 5.7 A/B). However, statistical significance for differences observed between treatments groups could not be assessed for mediastinal lymph nodes as these had been pooled for each treatment group to provide enough cells for restimulation and flow cytometric analysis.

In the lung immediately prior to challenge, the average number of IFN $-\gamma^{+} \mathrm{CD} 4^{+}$ and IFN $-\gamma^{+} \mathrm{CD} 8^{+}$lymphocytes was already elevated in PC61 treated/BCG vaccinated mice compared to rat IgG treated/BCG vaccinated mice (Figs. 5.4 B/D and 5.7 C/D). By day 3 after infection, there were still significantly more IFN- $\gamma^{+} \mathrm{CD} 4^{+}$lymphocytes in 
PC61 treated/BCG vaccinated mice compared to rat IgG treated/BCG vaccinated mice (Fig. 5.7 C). A similar trend at day 3 was also observed for IFN- $\gamma^{+} \mathrm{CD} 8^{+}$lymphocytes (Fig. 5.7 D), although differences were not statistically significant. Furthermore, at the times analysed, the maximum numbers of IFN $-\gamma^{+} \mathrm{CD}^{+}$lymphocytes in the lung were similar across PC61 treated and rat IgG treated groups for BCG vaccinated mice (Fig. $5.7 \mathrm{~A} / \mathrm{B} / \mathrm{C})$.

Therefore, when Tregs were inactivated during BCG vaccination, the secondary response to a mycobacterial challenge was accelerated compared to BCG control vaccinated animals in both the draining lymph node and the lung but, at the times assessed in this study, the response magnitude was not significantly amplified. 

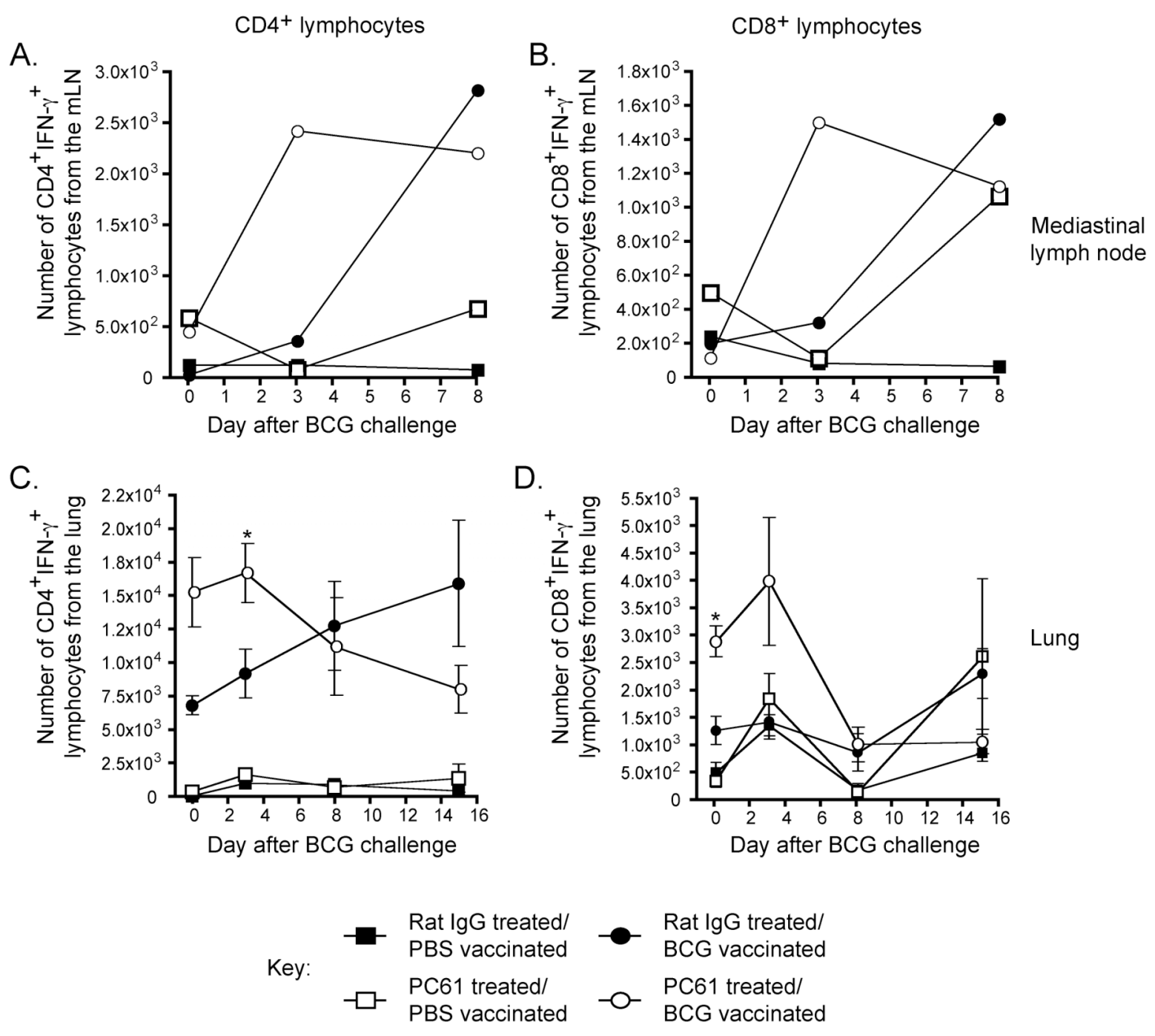

Figure 5.7: The secondary immune response after i.n. BCG challenge

Mice were administered either rat IgG (filled symbols) or PC61 antibody (empty symbols) i.p., 3 days before vaccination with BCG (circles) or mock vaccination with PBS (squares). At six weeks after vaccination mice were infected i.n. with BCG. Lymphocytes were harvested immediately prior to infection and at days 3, 8 and 15 after infection from the mediastinal lymph node and the lung. Lymphocytes were restimulated overnight with CFP before being assessed for cytokine production by flow cytometry. A) Number of CD4 ${ }^{+} \mathrm{IFN}-\gamma^{+}$lymphocytes in the mediastinal lymph node. B) Number of $\mathrm{CD} 8^{+} \mathrm{IFN}-\gamma^{+}$lymphocytes in the mediastinal lymph node. C) Average number of $\mathrm{CD}^{+} \mathrm{IFN}-\gamma^{+}$lymphocytes in the lung. D) Average number of $\mathrm{CD} 8^{+}$IFN- $\gamma^{+}$lymphocytes in the lung. Symbols for the lung represent mean values $\pm \mathrm{SEM}$. Four to five animals were analysed per treatment group at each time point. Treatment groups were compared using the two-tailed Mann Whitney test; * indicates $\mathrm{p} \leq 0.05$. The day three time point was repeated twice with similar results. 


\subsubsection{Short-term protection for BCG vaccination}

An accelerated but not amplified secondary immune response after Treg inactivation prior to vaccination has not been observed in other vaccination models. However, accelerating the influx of cytokine-producing cells to the infected lung tissue could conceivably result in earlier control of pulmonary mycobacterial infection and reduce dissemination from the lung to other tissues $[84,171]$. Therefore, bacterial burden in the lung and spleen were assessed after a virulent mycobacterial aerosol challenge.

Vaccinated animals were challenged by aerosol with M. tuberculosis or M. bovis 6 weeks after vaccination and bacterial numbers in the tissues were assessed 5 weeks after infection (Fig. 5.1 D). BCG vaccination, compared to PBS control vaccination, afforded on average a $0.79-0.87 \log _{10}$ reduction in bacterial numbers in the lung after $M$. tuberculosis infection (Fig. $5.8 \mathrm{~A}$ ), a 1.32-1.54 $\log _{10}$ reduction in bacterial numbers in the spleen after M. tuberculosis infection (Fig. $5.8 \mathrm{~B}$ ) and a $1.33-1.39 \log _{10}$ reduction in bacterial numbers in the lung after M. bovis infection (Fig. 5.8 C). However, despite significant differences in both the primary immune response and the kinetics of the secondary immune response, there was no difference in bacterial numbers in the lung after M. tuberculosis or M. bovis infection between PC61 treated and control treated BCG vaccinated mice (Fig. $5.8 \mathrm{~A} / \mathrm{C}$ ). This demonstrates that control of mycobacterial infection in the lung by BCG vaccination was not affected by antibody-mediated Treg inactivation. Furthermore, there was no difference in bacterial numbers in the spleen after M. tuberculosis infection between PC61 treated and control treated BCG vaccinated mice (Fig. 5.8 B), suggesting that dissemination of mycobacterial infection to the spleen was not affected by Treg inactivation prior to BCG vaccination.

Therefore, after an infectious challenge early (6 weeks) after vaccination, protection afforded by the BCG vaccine was not affected by PC61 treatment prior to vaccination. 
A.

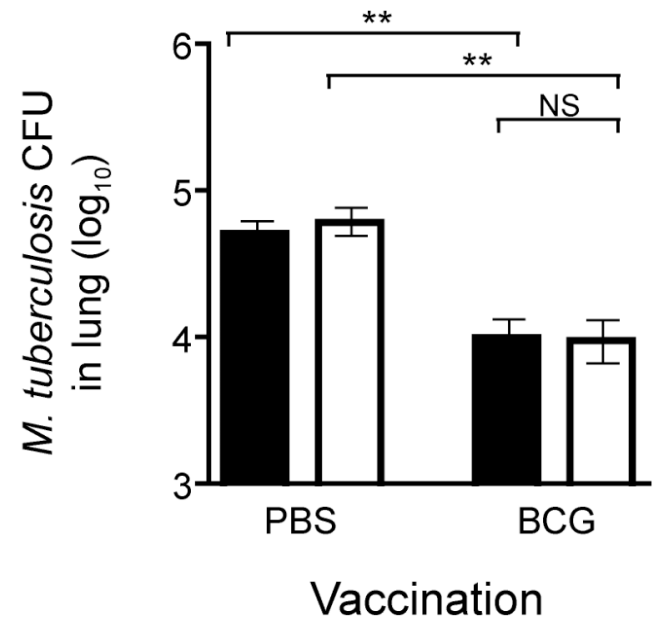

B.

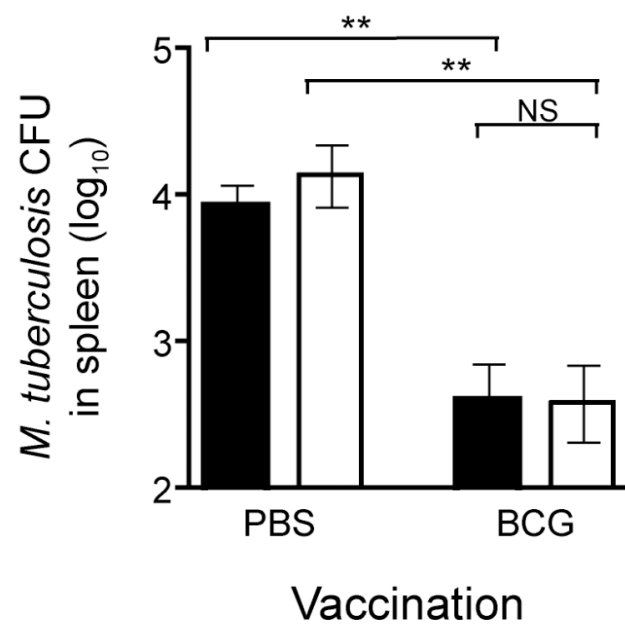

C.

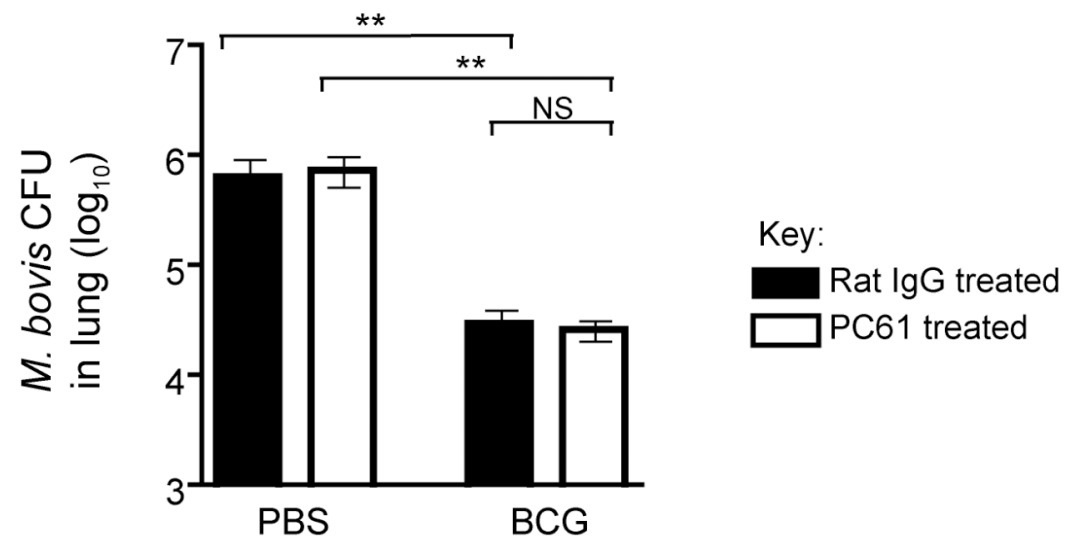

Vaccination

\section{Figure 5.8: Tissue bacterial numbers after early virulent mycobacterial challenge}

Mice were treated with rat IgG (filled bars) or PC61 (empty bars) 3 days before vaccination with BCG or mock vaccination with PBS. At 6 weeks after vaccination mice were infected by aerosol with $M$. tuberculosis or M. bovis. Bacterial numbers in the lung were determined 5 weeks after infection. A) Bacterial numbers in the lung after M. tuberculosis infection. B) Bacterial numbers in the spleen after M. tuberculosis infection. C) Bacterial numbers in the lung after $M$. bovis infection. Symbols represent geometric means \pm GEM. Five animals were analysed per treatment group. Treatment groups were compared using the two-tailed Mann Whitney test; NS indicates $\mathrm{p}>0.05,{ }^{*}$ indicates $\mathrm{p} \leq 0.01$.Results in A represent two replicate experiments, while $\mathrm{B}$ or $\mathrm{C}$ represent single experiments. 


\subsubsection{Long-term protection for BCG vaccination}

Treg inactivation significantly increased the number of lymphocytes producing cytokines, including IFN- $\chi$, during and after the primary immune response to BCG vaccination (Figs. 5.2/5.3). Currently, it is thought that the BCG vaccine protects against $\mathrm{Tb}$ infection through induction of Th1 immunity [170]. The current model of Th1 memory development proposes that increased numbers of IFN- $\gamma$-producing cells during and after vaccination do not correlate with enhanced protective memory, as cells that produce IFN- $\gamma$ do not survive long-term [221]. If the model described above applies to BCG-mediated immunity, then using Treg inactivation prior to vaccination to generate more IFN- $\gamma$ producing lymphocytes during the primary immune response could be detrimental to the longevity of protection afforded by BCG. To determine if this was indeed the case, long-term protection afforded by BCG vaccination after Treg inactivation was evaluated.

Vaccinated animals were challenged by aerosol with $M$. tuberculosis or M. bovis 15 weeks after vaccination and bacterial numbers in the tissues were assessed 5 weeks after infection (Fig. 5.1 D). Similar to results shown in Fig. 5.7, BCG vaccination afforded on average a $0.52-0.82 \log _{10}$ reduction in bacterial numbers in the lung after $M$. tuberculosis infection (Fig. $5.9 \mathrm{~A}$ ) and a 1.51-1.63 $\log _{10}$ reduction in bacterial numbers in the lung after M. bovis infection (Fig. 5.9 B) compared to PBS control vaccinated animals. However, there was again no significant difference in bacterial numbers in the lung caused by PC61 treatment with either M. tuberculosis or M. bovis infection (Fig. $5.9 \mathrm{~A} / \mathrm{B})$.

This illustrated that the inactivation of Tregs prior to BCG vaccination and the expansion of IFN- $\gamma$-producing lymphocytes during the primary immune response did not decrease protection after BCG vaccination against a late (15 week) infectious challenge. However, it also reinforced that control of M. tuberculosis or M. bovis infection in the lung by BCG vaccination is not affected by Treg inactivation. 
A.

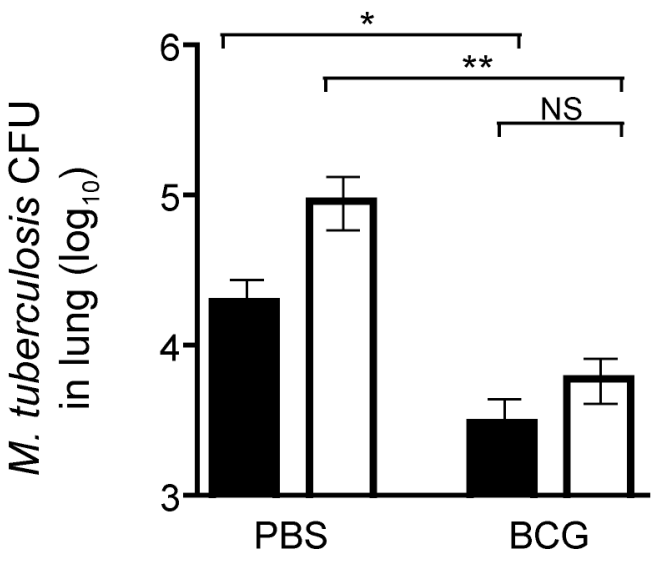

Vaccination

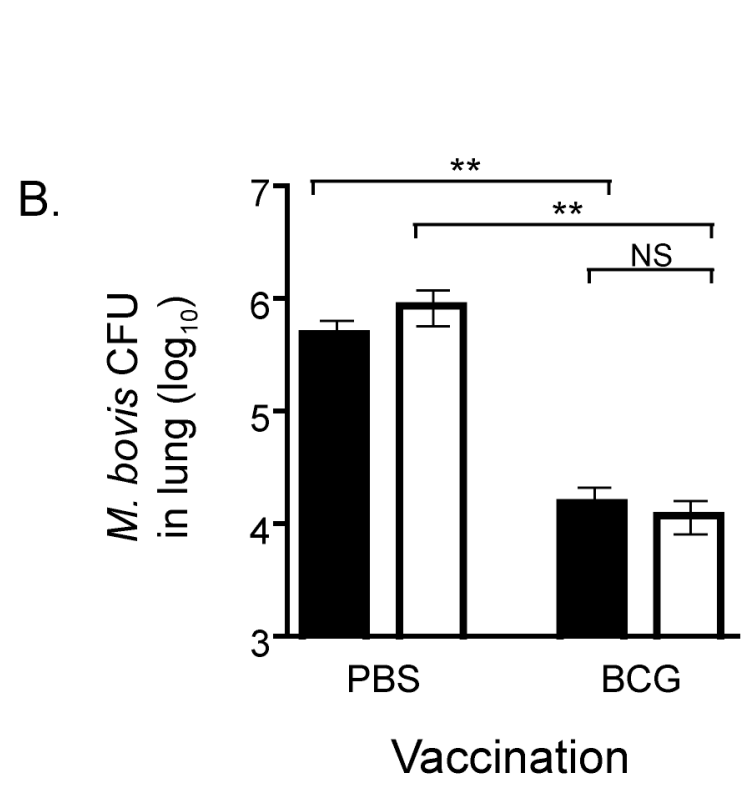

Key:

Rat IgG treated

PC61 treated

Figure 5.9: Lung bacterial numbers after late $M$. tuberculosis or $M$. bovis challenge

Mice were treated with rat IgG (filled bars) or PC61 (empty bars) 3 days before vaccination with BCG or mock vaccination with PBS. At 15 weeks after vaccination mice were infected by aerosol with M. tuberculosis or M. bovis. Bacterial numbers in the lung were determined 5 weeks after infection. A) Bacterial numbers in the lung after M. tuberculosis infection. B) Bacterial numbers in the lung after M. bovis infection. Symbols represent geometric mean values \pm GEM. Five animals were analysed per treatment group. Treatment groups were compared using the two-tailed Mann Whitney test; NS indicates $\mathrm{p}>0.05$, * indicates $\mathrm{p} \leq 0.05$, ** indicates $\mathrm{p} \leq 0.01$. Results represent single experiments. 


\subsubsection{Lung pathology for BCG vaccination}

The pulmonary inflammation induced in the lung following M. tuberculosis or M. bovis infection was assessed after early and late challenges. Lung tissue was collected concurrently with bacterial burden studies (Figs. 5.8 and 5.9), prepared for histological analysis and scored for the degree of inflammation. When M. tuberculosis infected samples were harvested, the degree of inflammation was low and differences between treatment groups were insignificant (data not shown). However, M. bovis infection causes more extensive inflammation in mice than M. tuberculosis infection [382], and the degree of pulmonary inflammation in $M$. bovis infected mice yielded significant differences between groups.

Mild, localised granulomatous inflammation, with epithelioid histiocytes and lymphocytes present within the pulmonary interstitium and alveolar spaces, was observed in PBS treated groups after either early (Fig. 5.10 A) or late (data not shown) $M$. bovis challenge. This inflammation was reduced in BCG vaccinated mice after either early (Fig. 5.10 A) or late (data not shown) M. bovis challenge. BCG vaccinated mice exhibited a trend for lower lung pathology composite scores (Fig. 5.10 B) and had significantly lower numbers of lung lesions (Fig. 5.10 C) than PBS treated groups after early $M$. bovis challenge. These patterns were also observed after late $M$. bovis challenge (data not shown). However, the degree and composition of pulmonary inflammation between PC61 and control treated mice that were BCG vaccinated was similar (Fig 5.10 A/B/C).

These results show that inactivation of Tregs during vaccination did not affect the ability of BCG to mitigate the extent of pathological damage in the lung associated with mycobacterial infection. 


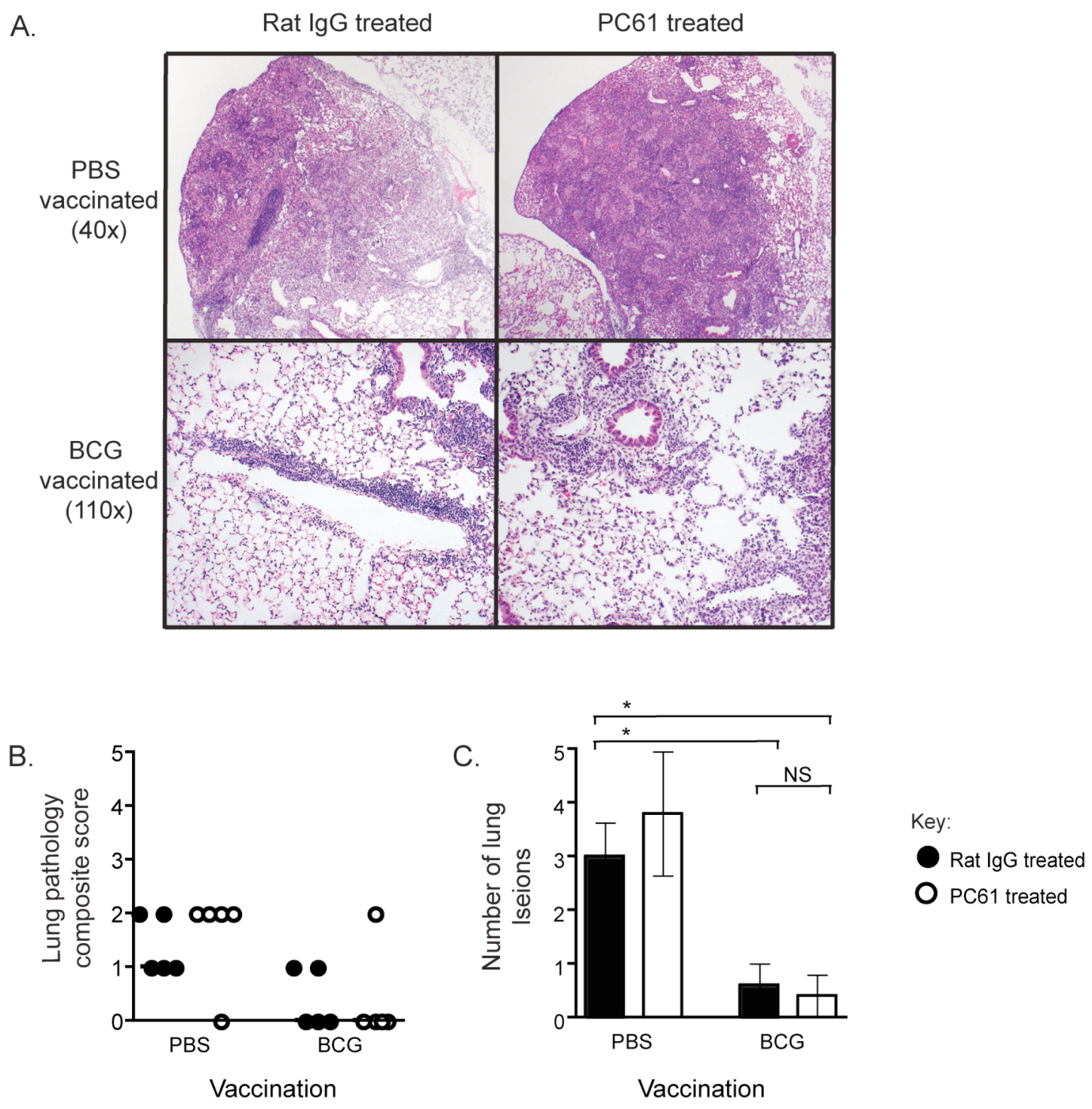

\section{Figure 5.10: Pulmonary inflammation after $M$. bovis infection}

Mice were treated with rat IgG (filled bars) or PC61 (empty bars) 3 days before vaccination with BCG or mock vaccination with PBS. At 6 weeks after vaccination mice were infected by aerosol with M. bovis. Lung tissue samples were fixed, sectioned and stained with haematoxylin and eosin before being graded for pulmonary inflammation. A) Sections from lungs taken week 5 after M. bovis infection. One micrograph, representative of sections from five animals, is shown for each treatment group. Micrographs for PBS vaccinated mice were taken at 40x magnification, whereas micrographs for BCG vaccinated mice were taken at 110x magnification. B) Composite pathology score (ranging from 0 (least severe) to 5 (most severe)) for lungs after M. bovis infection. Symbols represent individual values and median values are indicated by the horizontal black line (although some symbols obscure this line). C) Numbers of lesions per lung sample. Bars represent mean values \pm SEM. Five animals were analysed per treatment. Statistical significance was determined by the two-tailed Mann Whitney test; NS indicates $\mathrm{p}>0.05, *$ indicates $\mathrm{p} \leq 0.05$. Results represent one experiment and similar results were found for mice infected with $M$. bovis 15 weeks after vaccination. 


\subsubsection{Vaccine-induced immune responses to Tb DNA vaccines}

My studies have shown that inactivation of Tregs during BCG vaccination did not affect protective outcomes of BCG vaccination, such as bacterial numbers in tissues or pulmonary inflammation. These findings contrast with studies where inactivation of Tregs has been shown to enhance the protective efficacy of vaccines against other pathogens, such as $P$. berghei and HSV-1 [338, 339]. The BCG vaccine is the only currently available vaccine for $\mathrm{Tb}$, but other vaccines against $\mathrm{Tb}$, including DNA vaccines, have been developed and methods for enhancing DNA vaccine immunogenicity are of significant interest. If inactivation of Tregs prior to Tb DNA vaccination resulted in enhanced vaccine efficacy, then more effective Tb vaccine strategies could be designed that minimise Treg activation. Therefore, I hypothesised that Treg inactivation prior to $\mathrm{Tb}$ DNA vaccination would enhance primary and secondary immune responses, as well as protection afforded against virulent $M$. tuberculosis infection. Two Tb DNA vaccines were used to assess this; pcDNA3-85A [433] and pKUN85A (chapter 4).

To examine this hypothesis, groups of mice were treated with either PC61 or control rat IgG followed by homologous prime-boost vaccination with one of the vaccines (Fig. 5.1 A). DNA vaccines were administered i.m., with 2 weeks rest between the prime and boost vaccinations. For pcDNA3-85A, a standard dose of $100 \mu \mathrm{g}$ was administered [433]. For pKUN85A, a $25 \mu \mathrm{g}$ dose was chosen as this dose induced maximal cytokine production and lymphocyte proliferation at two weeks after vaccination during assessment of vaccine immunogenicity (Figs. 4.10/4.11). As a positive control, other groups of PC61 or rat IgG treated mice were vaccinated with BCG at the same time as the DNA vaccine boosts (Fig. 5.1 A). Two weeks after the DNA vaccine boost, lymphocytes were harvested from the spleen and restimulated with Ag85A, the mycobacterial antigen encoded by both of the DNA vaccines (Fig. 5.1 B). Restimulated lymphocytes were either assessed for IFN- $\gamma$ or IL-2 production by flow cytometry or for proliferation by $\left[{ }^{3} \mathrm{H}\right]$ thymidine incorporation.

PC61 treatment alone induced a small but significant increases in basal IFN- $\gamma$ production by $\mathrm{CD} 8^{+}$lymphocytes (Fig. $5.11 \mathrm{~A}$ ) and proliferation (Fig. 5.11 E) in PBS vaccinated mice. PC61 treatment did not significantly alter the number of CD8 ${ }^{+} \mathrm{IFN}-\gamma^{+}$ or $\mathrm{CD}^{+} \mathrm{IFN}^{-} \gamma^{+} \mathrm{T}$ lymphocytes in BCG, pKUN85A or pcDNA3-85A vaccinated mice (Fig. $5.11 \mathrm{~A} / \mathrm{C}$ ). PC61 treatment also did not affect the number of CD8 ${ }^{+} \mathrm{IL}_{-} 2^{+}$or 
$\mathrm{CD}^{+} \mathrm{IL}^{-}{ }^{+} \mathrm{T}$ lymphocytes in pKUN85A or pcDNA3-85A vaccinated mice (Fig. 5.11 B/D), although both cell populations were significantly increased in PC61 treated compared to control treated BCG vaccinated mice (Fig. 5.11 B/D). Finally, lymphocyte proliferation was significantly increased in PC61 treated compared to control treated pKUN85A vaccinated mice, but PC61 treatment did not alter proliferation in either BCG or pcDNA3-85A vaccinated mice (Fig. 5.11 E).

Therefore, inactivation of Tregs prior to pKUN85A vaccination led to modest increase in the proliferative response to Ag85A at two weeks after vaccination. In contrast, inactivation of Tregs prior to pcDNA3-85A vaccination did not affect lymphocyte proliferation or the numbers of cytokine producing lymphocytes two weeks after vaccination. 
A.

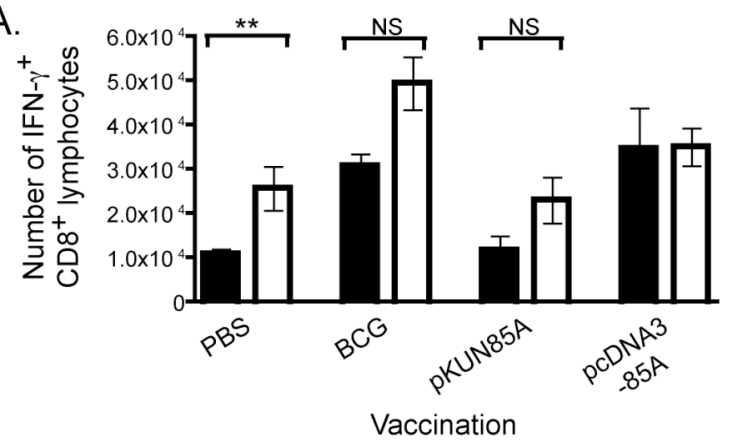

C.

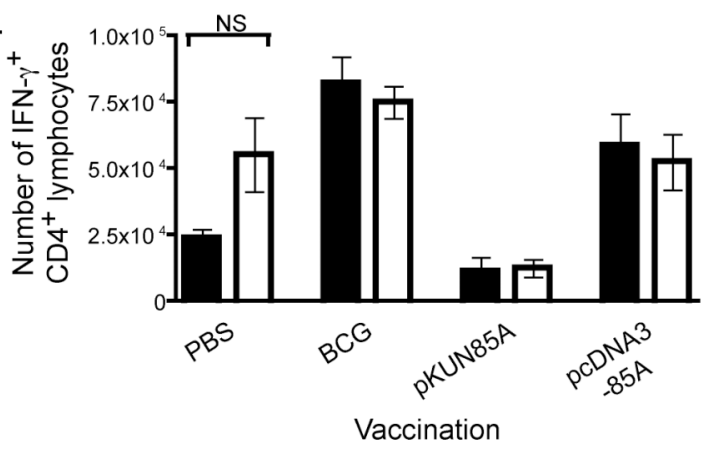

B.

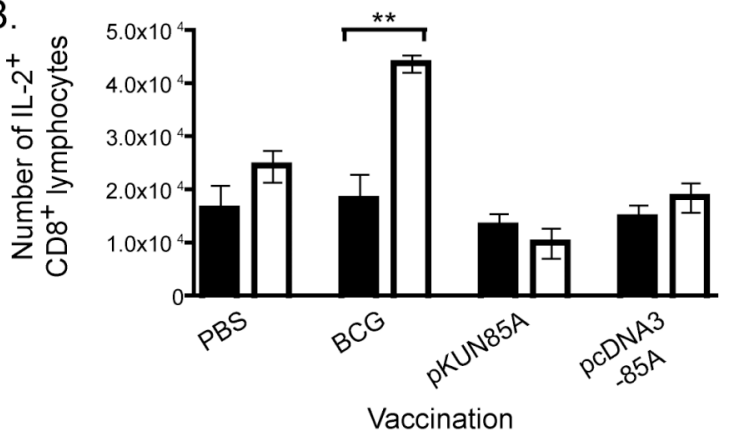

D.

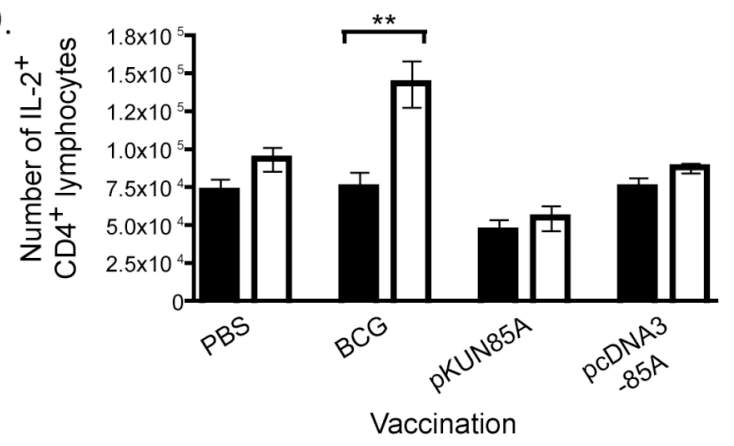

\section{E.}

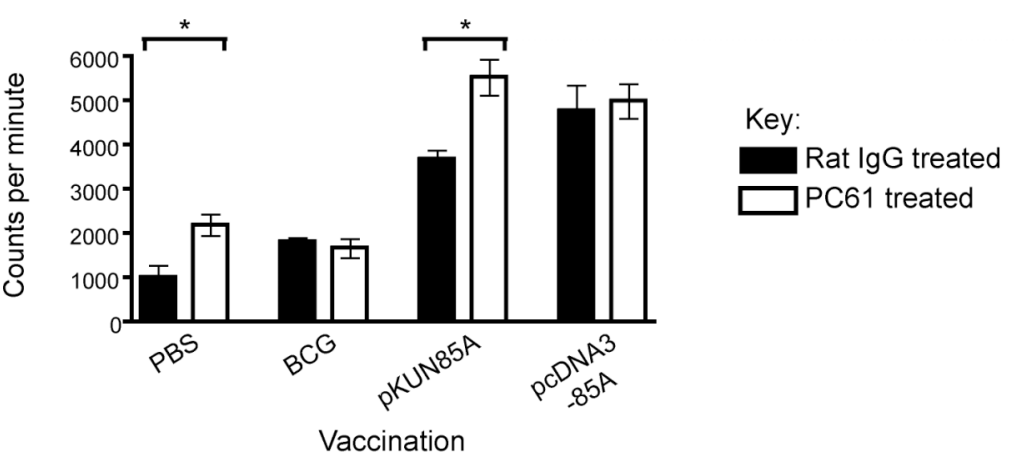

Figure 5.11: Vaccine-induced immune responses after DNA vaccination

Mice were treated with rat IgG or PC61 then vaccinated with BCG, pKUN85A or pcDNA3-85A 3 days later. Mice vaccinated with pKUN85A or pcDNA3-85A received a boost vaccination two weeks later. Two weeks after BCG vaccination and two weeks after the boost pKUN85A or pcDNA3-85A vaccinations, splenocytes were isolated and restimulated with Ag85A before being assessed for cytokine production by flow cytometry and for lymphocyte proliferation by $\left[{ }^{3} \mathrm{H}\right]$ thymidine incorporation assay. A) Average number of CD8 ${ }^{+} \mathrm{IFN}-\gamma^{+}$lymphocytes. B) Average number of $\mathrm{CD} 8^{+} \mathrm{IL}-2^{+}$lymphocytes. C) Average number of $\mathrm{CD} 4^{+} \mathrm{IFN}-\gamma^{+}$lymphocytes. D) Average number of CD4 ${ }^{+} \mathrm{IL}-2^{+}$lymphocytes. E) Lymphocyte proliferation as measured by $\left[{ }^{3} \mathrm{H}\right]$ thymidine incorporation. Bars represent mean values per spleen \pm SEM. Five animals were analysed per treatment group. Treatment groups were compared using the two-tailed Mann Whitney test; NS indicates $\mathrm{p}>0.05, *$ indicates $\mathrm{p} \leq 0.05, * *$ indicates $\mathrm{p} \leq 0.01$. Results represent one experiment. 


\subsubsection{Secondary immune responses for Tb DNA vaccines}

Inactivation of Tregs caused a modest enhancement of the primary immune response after pKUN85A vaccination but the effect of Treg inactivation prior to DNA vaccination on the secondary immune response remained to be tested.

To evaluate the secondary immune responses of DNA vaccinated mice to infectious challenge, vaccinated mice were infected i.n. with BCG 6 weeks after boosting and lymphocytes were isolated at day 3 after infection (Fig. 5.1 C). Lymphocytes were isolated from the spleen and lung and restimulated with Ag85A. Restimulated lymphocytes were processed for intracellular cytokine staining and analysed by flow cytometry for the production of IFN- $\gamma$ and IL-2. Cytokine production was assessed at day 3 after infection, as the most striking differences between PC61 and control treated BCG vaccinated mice were observed at day 3 after infection (Fig. 5.7).

At day 3 after challenge in the spleen, for BCG vaccination, numbers of $\mathrm{CD} 4{ }^{+}$IFN $-\gamma^{+}$lymphocytes were significantly increased in PC61 treated compared to rat IgG treated mice (Fig. 5.12 C). $\mathrm{CD} 8^{+}$IFN- $\gamma^{+}$lymphocytes were also increased but the difference was not statistically significant (Fig. 5.12 A). For pcDNA3-85A vaccination, the same trends were observed for $\mathrm{CD} 4^{+} \mathrm{IFN}-\gamma^{+}$and $\mathrm{CD} 8^{+} \mathrm{IFN}-\gamma^{+}$lymphocytes (Fig. 5.12 $\mathrm{A} / \mathrm{C})$ and, in addition, numbers of $\mathrm{CD} 8^{+} \mathrm{IL}_{-} 2^{+}$and $\mathrm{CD} 4^{+} \mathrm{IL}-2^{+}$lymphocytes were significantly increased in PC61 treated compared to rat IgG treated mice (Fig. 5.12 $\mathrm{B} / \mathrm{D})$. For pKUN85A vaccination, numbers of cytokine producing lymphocytes in the spleen in PC61 treated mice did not differ significantly from rat IgG treated mice and were largely comparable to PBS vaccinated control mice (Fig. 5.12 A-D). 

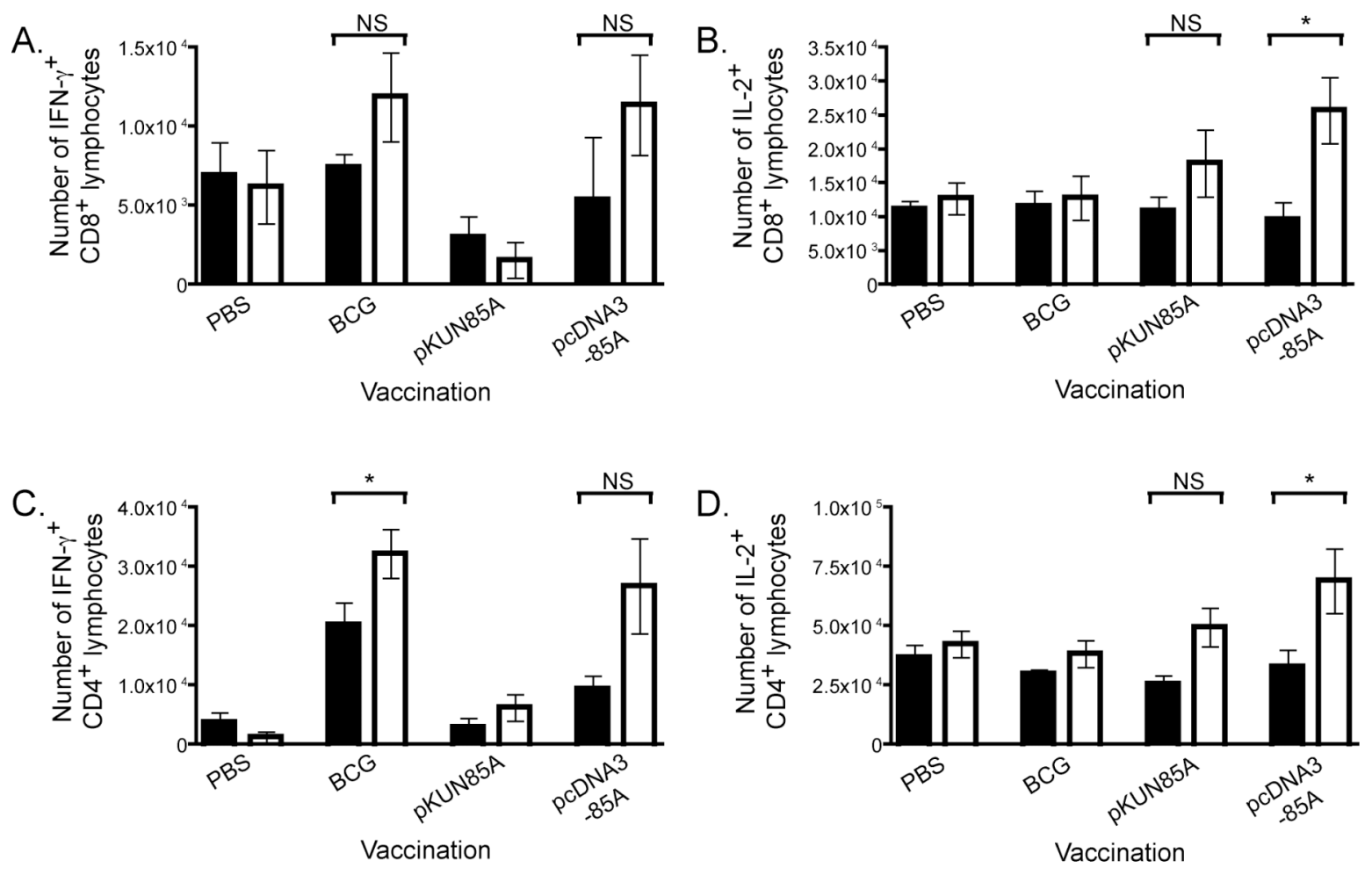

Key:

Rat IgG treated

PC61 treated

\section{Figure 5.12: Secondary immune responses in the spleen after DNA vaccination}

Mice were treated with rat IgG or PC61 then vaccinated with BCG, pKUN85A or pcDNA3-85A 3 days later. Mice vaccinated with pKUN85A or pcDNA3-85A received a boost vaccination two weeks later. Six weeks after BCG vaccination and six weeks after the boost pKUN85A or pcDNA3-85A vaccinations, mice were challenged i.n. with BCG. Three days after challenge, splenocytes were isolated and restimulated with Ag85A before being assessed for cytokine production by flow cytometry. A) Average number of CD8 ${ }^{+}$IFN $-\gamma^{+}$lymphocytes. B) Average number of CD8 ${ }^{+} \mathrm{IL}^{-} 2^{+}$lymphocytes. C) Average number of CD4 ${ }^{+} \mathrm{IFN}-\gamma^{+}$lymphocytes. D) Average number of $\mathrm{CD}^{+}{ }^{+} \mathrm{IL}-2^{+}$lymphocytes. Bars represent mean values per spleen $\pm \mathrm{SEM}$.

Four to five animals were analysed per treatment group. Treatment groups were compared using the two-tailed Mann Whitney test; NS indicates $p>0.05, *$ indicates $p \leq 0.05$. Results represent one experiment. 
At day 3 after challenge in the lung, there were no significant differences between PC61 treated and rat IgG treated mice in the numbers of IFN $-\gamma^{+} \mathrm{CD} 8^{+}, \mathrm{IL}-2^{+} \mathrm{CD} 8^{+}$, IFN$\gamma^{+} \mathrm{CD} 4^{+}$or IL-2 ${ }^{+} \mathrm{CD} 4^{+}$lymphocytes for any of the DNA vaccinated groups (Fig. 5.13 $\mathrm{A} / \mathrm{B} / \mathrm{C} / \mathrm{D})$. A small but significant increase in numbers of IFN $-\gamma^{+} \mathrm{CD} 8^{+}$lymphocytes was observed with PC61 treatment in the PBS treated control groups, which may indicate an increase in basal cytokine production (Fig. $5.13 \mathrm{~A}$ ).

The numbers and proportions of IFN $-\gamma^{+} \mathrm{IL}-2^{+} \mathrm{CD} 4^{+}$lymphocytes were also assessed in both the spleen and lung for each treatment group. In the spleens, pcDNA3$85 \mathrm{~A}$ vaccinated mice exhibited a trend for increased numbers of IFN- $\gamma^{+} \mathrm{IL}-2^{+} \mathrm{CD} 4^{+}$ lymphocytes in PC61 treated compared to rat IgG treated mice, although the difference was not statistically significant (Fig. 5.14 A). In the lung, there were very low numbers of IFN- $\gamma^{+}$IL-2 ${ }^{+} \mathrm{CD} 4^{+}$lymphocytes in pKUN85A or pcDNA3-85A vaccinated mice and these numbers did not differ significantly between PC61 treated and rat IgG treated groups (Fig. 5.14 B). When proportions of IFN $-\gamma^{+} \mathrm{IL}-2^{+} \mathrm{CD} 4^{+}$lymphocytes within the total IFN- $\gamma$ or IL-2 producing CD4 ${ }^{+}$lymphocyte population were assessed, the proportions were unchanged by PC61 treatment for all vaccination groups in both the spleen and lung (Fig. 5.14 C/D), although there was large variation in the proportions of IFN $-\gamma^{+} \mathrm{IL}-2^{+} \mathrm{CD} 4^{+}$lymphocytes in the lung due to low total numbers of cytokineproducing cells.

In summary, at 3 days after infectious challenge, PC61 treated/pcDNA3-85A vaccinated mice had increased numbers of cytokine producing $\mathrm{CD}^{+}$and $\mathrm{CD} 4^{+}$ lymphocytes in the spleen compared to rat $\mathrm{IgG}$ treated/pcDNA3-85A vaccinated mice. Significant differences between PC61 treated and rat IgG treated groups for each DNA vaccine were not observed in the lung. Numbers of cytokine producing $\mathrm{CD} 8^{+}$and $\mathrm{CD} 4^{+}$ lymphocytes in pKUN85A vaccinated mice were low in both the spleen and lung and comparable in many cases to PBS vaccinated control mice, and did not exhibit significant differences between PC61 treated and rat IgG treated groups. 
A.

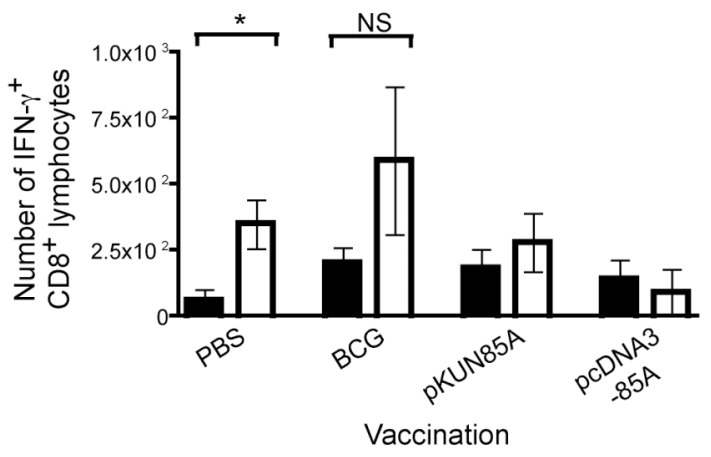

C.

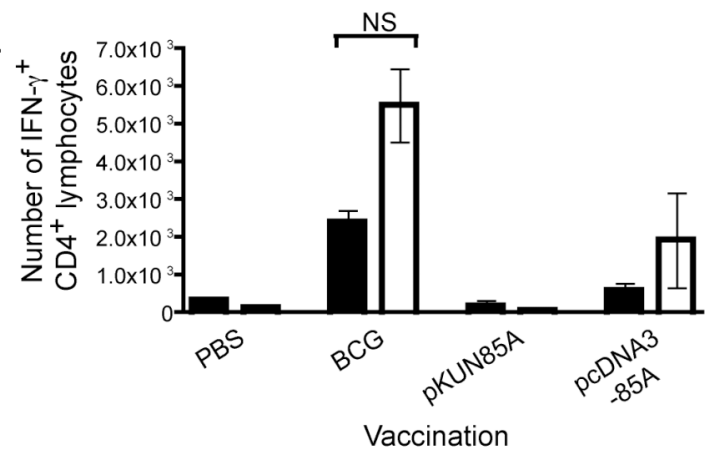

B.

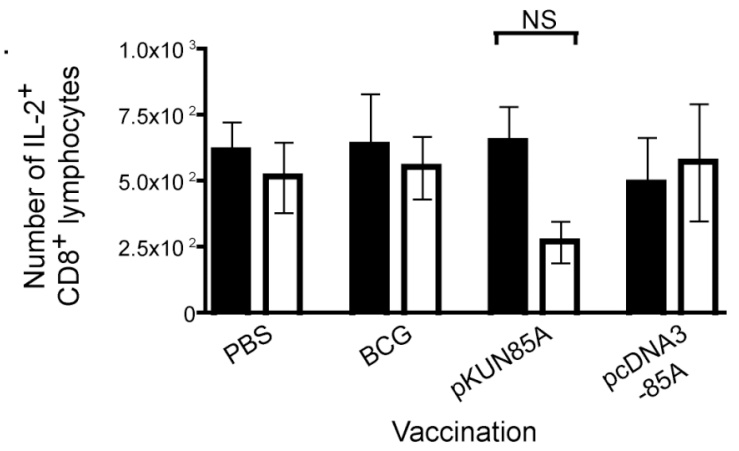

D.

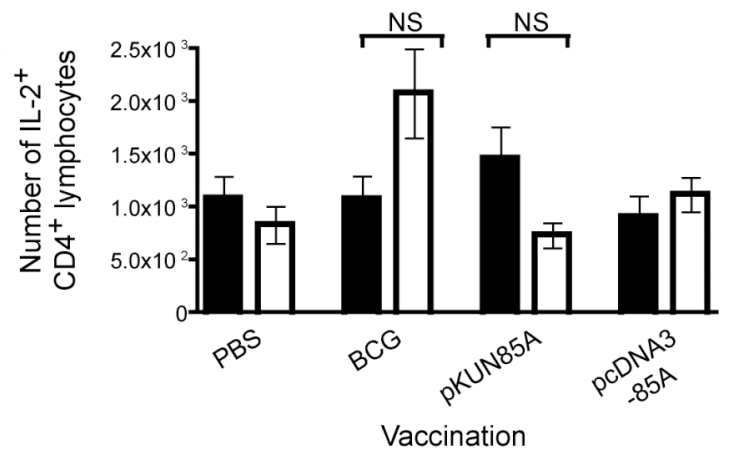

Key:

Rat IgG treated

PC61 treated

\section{Figure 5.13: Secondary immune response in the lung after DNA vaccination}

Mice were treated with rat IgG or PC61 then vaccinated with BCG, pKUN85A or pcDNA3-85A 3 days later. Mice vaccinated with pKUN85A or pcDNA3-85A received a boost vaccination two weeks later. Six weeks after BCG vaccination and six weeks after the boost pKUN85A or pcDNA3-85A vaccinations, mice were challenged i.n. with BCG. Three days after challenge, lungs were harvested and isolated lymphocytes were restimulated with Ag85A before being assessed for cytokine production by flow cytometry. A) Average number of $\mathrm{CD} 8^{+} \mathrm{IFN}-\gamma^{+}$ lymphocytes. B) Average number of $\mathrm{CD} 8^{+} \mathrm{IL}-2^{+}$lymphocytes. C) Average number of $\mathrm{CD} 4^{+} \mathrm{IFN}-\gamma^{+}$lymphocytes. D) Average number of CD4 ${ }^{+} \mathrm{IL}-2^{+}$lymphocytes. Bars represent mean values per lung \pm SEM. Four to five animals were analysed per treatment group. Treatment groups were compared using the two-tailed Mann Whitney test; NS indicates $\mathrm{p}>0.05$, * indicates $\mathrm{p} \leq 0.05$. Results represent one experiment. 
Spleen
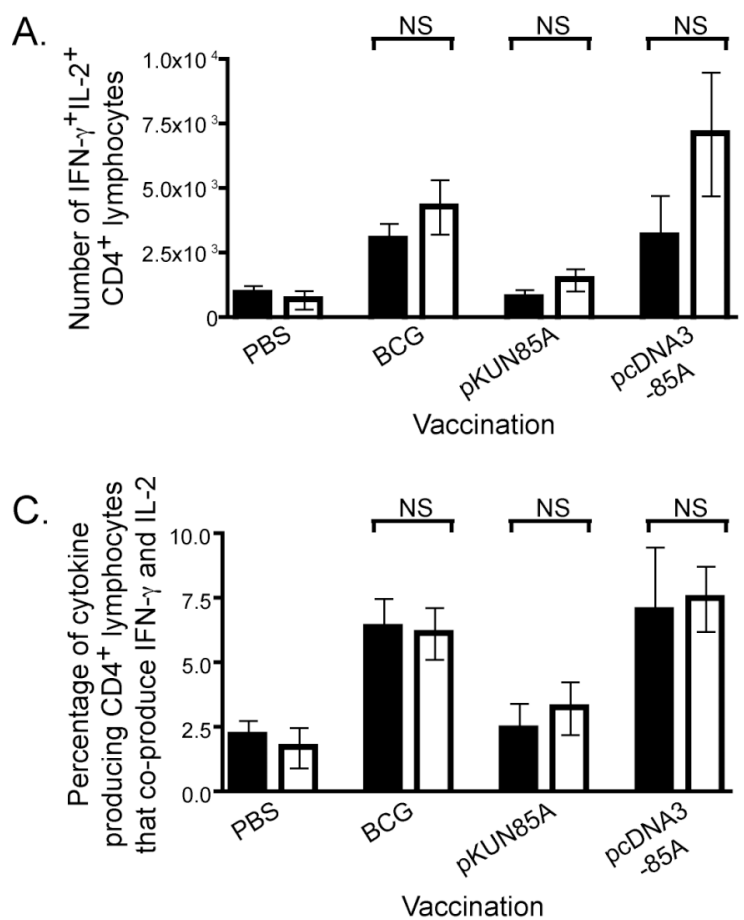

Lung

B. +

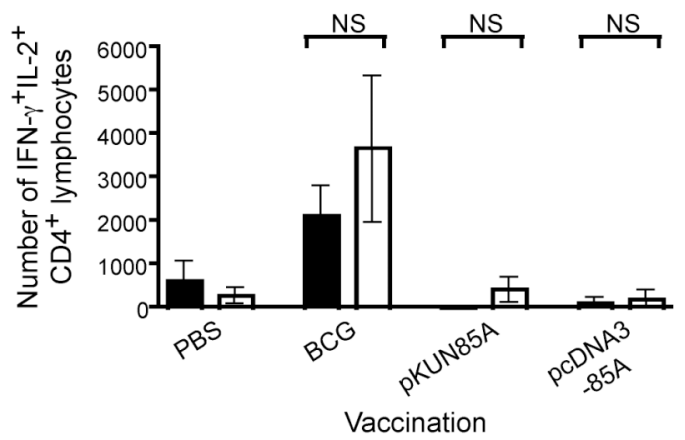

D.

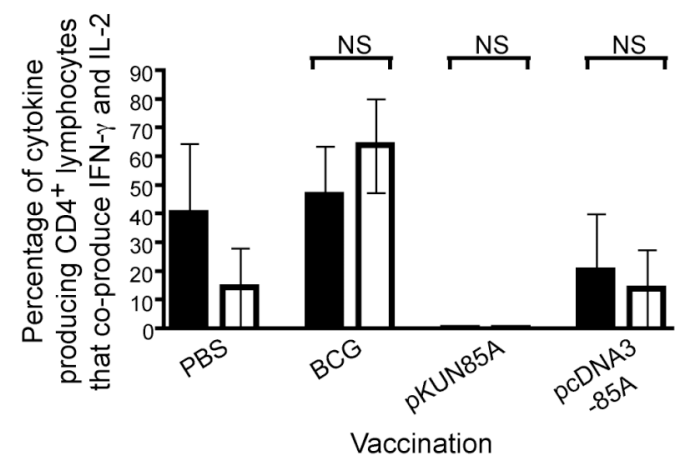

Figure 5.14: IFN- $\gamma$ and IL-2 co-production by $\mathrm{CD4}^{+}$lymphocytes after DNA vaccination

Mice were treated with rat IgG or PC61 then vaccinated with BCG, pKUN85A or pcDNA3-85A 3 days later. Mice vaccinated with pKUN85A or pcDNA3-85A received a boost vaccination two weeks later. Six weeks after BCG vaccination and six weeks after the boost pKUN85A or pcDNA3-85A vaccinations, mice were challenged i.n. with BCG. Three days after challenge, spleens and lungs were harvested and isolated lymphocytes were restimulated with $\mathrm{Ag} 85 \mathrm{~A}$ before being assessed for cytokine production by flow cytometry. A) Average number of $\mathrm{CD} 4^{+} \mathrm{IFN}-\gamma^{+} \mathrm{IL}-2^{+}$lymphocytes in the spleen. B) Average number of CD4 $4^{+} \mathrm{IFN}-\gamma^{+} \mathrm{IL}-2^{+}$ lymphocytes in the lung. C) Percentage of cytokine producing CD4 ${ }^{+}$lymphocytes that coproduce IFN- $\gamma$ and IL-2 in the spleen. D) Percentage of cytokine producing $\mathrm{CD} 4^{+}$lymphocytes that co-produce IFN- $\gamma$ and IL-2 in the lung. Bars represent mean values per tissue \pm SEM. Four to five animals were analysed per treatment group. Treatment groups were compared using the two-tailed Mann Whitney test; NS indicates $p>0.05$. Results represent one experiment. 


\subsubsection{Protection from infectious challenge for Tb DNA vaccines}

PC61 treatment prior to vaccination significantly increased numbers of cytokine producing lymphocytes in the spleen but not the lung after infectious challenge in pcDNA3-85A vaccinated mice. Although the effects of Treg inactivation on vaccineinduced and secondary immune responses after DNA vaccination were relatively minor compared to those seen following BCG vaccination, the effect of Treg inactivation on the protective efficacy of pKUN85A and pcDNA3-85A remained to be assessed.

Vaccinated animals were challenged by aerosol with M. tuberculosis 6 weeks after vaccination (Fig. 5.1 D). Bacterial numbers in the lung and spleen were assessed 5 weeks after infection. Lung samples were collected concurrently and prepared for histological analysis but the degree of inflammation in the lung at the time was too low for differences between groups to be significant (data not shown).

When bacterial numbers in the lung were assessed, BCG vaccination afforded a significant $\sim 1 \log _{10}$ reduction in the lung and spleen after $M$. tuberculosis infection compared to control treated PBS vaccinated animals and PC61 treatment did not affect bacterial numbers either tissue (Fig. 5.15 A/B). Control treated mice vaccinated with pKUN85A, using a $25 \mu \mathrm{g}$ dose, did not exhibit reduced bacterial numbers in either the lung or the spleen compared to control treated PBS vaccinated mice and PC61 treatment prior to pKUN85A vaccination did not alter bacterial numbers (Fig. $5.15 \mathrm{~A} / \mathrm{B}$ ). Control treated mice vaccinated with pcDNA3-85A exhibited a trend for reduced bacterial numbers in the lung and spleen compared to control treated PBS vaccinated mice, but the differences were not statistically significant $(p=0.1508$ for lung, $p=0.0714$ for spleen) (Fig. 5.15 A/B). However, PC61 treatment prior to pcDNA3-85A vaccination led to a significant increase in bacterial numbers in the spleen (Fig. $5.15 \mathrm{~A} / \mathrm{B}$ ), suggesting protection from dissemination to the spleen afforded by pcDNA3-85A vaccination may be abrogated in this group.

These results suggest that, while Treg inactivation prior to vaccination did not affect the BCG vaccine, it may diminish protection against dissemination afforded by the pcDNA3-85A DNA vaccine. In addition, the $25 \mu \mathrm{g}$ dose chosen for pKUN85A vaccination did not confer measurable protection in terms of reduced bacterial numbers in the lung or spleen, and Treg inactivation prior to vaccination does not affect this. 


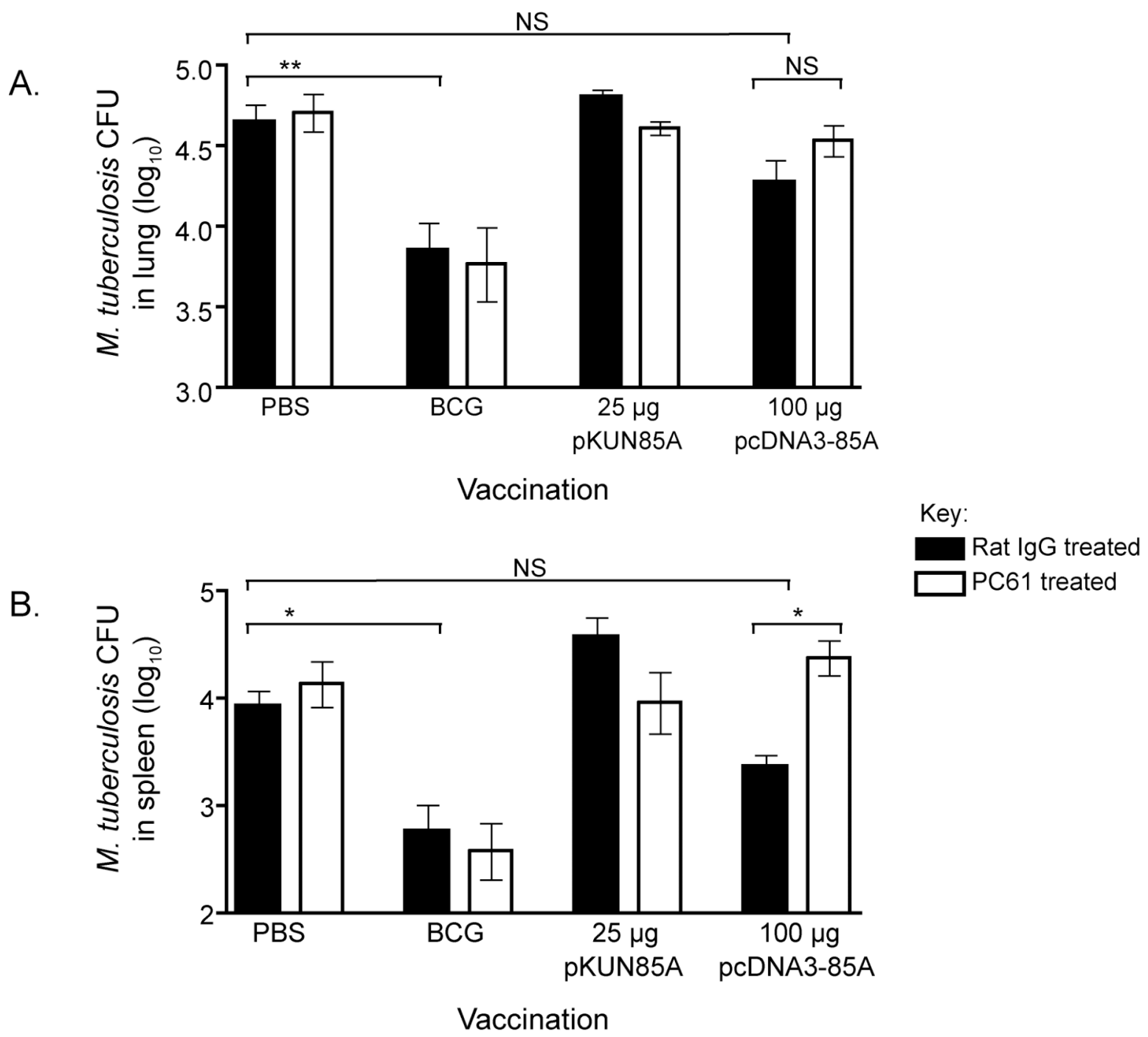

Figure 5.15: Protection afforded by DNA vaccination

Mice were treated with rat IgG or PC61 then vaccinated with BCG, pKUN85A or pcDNA3-85A 3 days later. Mice vaccinated with pKUN85A or pcDNA3-85A received a boost vaccination two weeks later. Six weeks after BCG vaccination and six weeks after the boost pKUN85A or pcDNA3-85A vaccinations, mice were challenged by aerosol with $M$. tuberculosis. Bacterial numbers in the lung were determined 5 weeks after infection. A) Bacterial numbers in the lung and B) bacterial numbers in the spleen after M. tuberculosis infection. Symbols represent geometric mean values \pm GEM. Five animals were analysed per treatment group. Treatment groups were compared using the two-tailed Mann Whitney test; NS indicates $\mathrm{p}>0.05$, ** indicates $\mathrm{p} \leq 0.01$. Results represent one experiment. 


\subsection{Discussion}

I hypothesised that Treg inactivation through PC61 treatment prior to BCG or plasmid DNA vaccination would enhance the primary and secondary immune responses and would also enhance the protective efficacy of the vaccines against $\mathrm{Tb}$.

In keeping with my hypothesis, for BCG vaccination, an increase in the number of cytokine producing antigen-specific $\mathrm{CD}^{+}$lymphocytes in the spleen and lymph nodes was observed during the primary immune response (Fig. 5.3) and the secondary immune response in the lung upon infectious challenge was accelerated (Fig. 5.7). Contrary to my hypothesis, the enhancement or acceleration of primary and secondary immune responses by Treg inactivation did not enhance protection afforded by BCG vaccination (Figs. 5.8-5.10).

The outcomes from Treg inactivation prior to Tb DNA vaccination also differed from my original hypothesis. For pcDNA3-85A vaccination, inactivation of Tregs prior to priming did not significantly affect the primary immune response to vaccination (Fig. 5.11) but did increase numbers of cytokine producing $\mathrm{CD} 8^{+}$and $\mathrm{CD} 4^{+}$lymphocytes in the spleen 3 days after infectious challenge (Fig. 5.12). Interestingly, Treg inactivation prior to pcDNA3-85A vaccination resulted in a significant increase in bacterial numbers in the spleen after M. tuberculosis infection (Fig. 5.15). The study evaluating the effect of Treg inactivation on pcDNA3-85A vaccination requires confirmation and will be investigated further.

Inactivation of Tregs prior to pKUN85A vaccination modestly increased lymphocyte proliferation two weeks after boosting. However, vaccination with the 25 $\mu \mathrm{g}$ dose of pKUN85A did not reduce bacterial numbers in the lung or spleen after $M$. tuberculosis challenge. The $25 \mu \mathrm{g}$ dose was chosen on the basis that it resulted in maximal mean cytokine production and proliferation after in vitro restimulation of lymphocytes isolated from vaccinated mice (Figs. 4.10/4.11), rather protection data (Figs. 4.12/4.13). Protection data was not available at the time that these experiments were initiated, as protection studies for $\mathrm{Tb}$ vaccines can take 4-6 months to allow for vaccination, infection and processing of tissues for assessment of bacterial numbers. Given the decrease in protection that has been observed with high doses of plasmid DNA replicon vaccines [433], it is possible that the $25 \mu \mathrm{g}$ dose of pKUN85A was too high to mediate protection against mycobacterial challenge. In future studies, a dose of pKUN85A titrated to confer significant protection against $M$. tuberculosis challenge 
will be used to assess whether Treg inactivation prior to pKUN85A vaccination enhances vaccine efficacy.

Overall, the protective efficacy of the BCG vaccine is not affected, whereas the protective efficacy of the pcDNA3-85A vaccine may be diminished by Treg inactivation prior to vaccination. Both of these results contrast with results obtained in other studies assessing the impact of Treg inactivation on vaccines against other infectious diseases.

\subsubsection{Comparison to other studies}

Inactivation of Tregs prior to vaccination against HSV-1 [338], hepatitis B virus [336] or concurrently with malarial vaccination [339], has been shown to result in increased primary immune responses and secondary immune responses, similar to the enhanced immune responses observed in this chapter. However, in contrast to our findings, inactivation of Tregs prior to or concurrently with vaccination against HSV-1 or malaria resulted in enhanced protection from challenge [338, 339].

When comparing these studies to my own, there are several points of difference between the vaccines that may account for the distinct outcomes observed after Treg inactivation. The malarial and HSV-1 vaccines utilised a single protein antigen and induced protection predominantly mediated by $\mathrm{CD} 8^{+} \mathrm{T}$ cells $[338,339]$. $\mathrm{BCG}$ is a live vaccine with a large number of different antigenic targets and $\mathrm{CD} 4^{+}$more so than $\mathrm{CD} 8^{+}$ $\mathrm{T}$ cells are thought to mediate protection against $\mathrm{Tb}[61,468]$. The different outcomes caused by Treg inactivation prior to vaccination for each vaccine could be due to Tregs affecting the development of the discrete lymphocyte populations, which mediate protection for the different vaccines in distinct ways.

Tregs can inhibit production of $\gamma$ c family cytokines, such as IL-2, by responder $\mathrm{T}$ cells $[289,291]$ and signalling from these cytokines is vital for optimal memory development (section 1.3.4.3). For example, IL-2 signalling during $\mathrm{CD}^{+} \mathrm{T}$ cell activation has been shown to promote expansion of the effector population and $\mathrm{CD} 8^{+}$ memory $\mathrm{T}$ cells that receive IL-2 signalling during priming accumulate in vastly larger numbers after secondary stimulation [202]. In addition, IL-2 signalling during $\mathrm{CD}^{+} \mathrm{T}$ cell activation has been shown to promote re-expression of IL-7R $\alpha$ on the resulting 
population [201]. Signalling from IL-7 is important for maintenance of CD4 ${ }^{+}$memory T cells [201] and therefore early IL-2 signalling facilitates the later survival of CD4 ${ }^{+}$ memory T cells.

Studies of the effect of IL-2 signalling on $\mathrm{CD} 4^{+}$memory T cell development have not differentiated between memory subsets within the $\mathrm{CD} 4^{+} \mathrm{T}$ cell population. Although $\mathrm{CD}^{+}$and $\mathrm{CD}^{+} \mathrm{T}$ cells have subtly different requirements for cytokine signalling for memory induction and maintenance, it is possible that different subsets of $\mathrm{CD}^{+} \mathrm{T}$ cells, such as Th1, Th2, Th17, Treg and other yet to be identified subsets, also have different cytokine requirements. Therefore, if the development of protective memory were reliant on signalling from cytokines such as IL-2, Treg inactivation prior to vaccination would be anticipated to enhance the protective efficacy of vaccination. On the contrary, if development of protective memory was not reliant on such signalling, Treg inactivation prior to vaccination might not affect the protective efficacy.

Both $\mathrm{CD} 4^{+} \mathrm{T}$ cells and $\mathrm{CD} 8^{+} \mathrm{T}$ cells potentially contribute to protection mediated by vaccination with the live BCG vaccine, although $\mathrm{CD} 4^{+} \mathrm{T}$ cells are regarded as the major protective population [170]. The protective $\mathrm{CD}^{+} \mathrm{T}$ cell subset for the BCG vaccine is not definitively known, but results presented in this thesis have suggested some refinements. Inactivation of Tregs prior to BCG vaccination resulted in increased numbers of IFN $-\gamma^{+}$and IL-2 ${ }^{+} \mathrm{CD} 4^{+} \mathrm{T}$ cells in the draining lymph node and spleen two weeks after vaccination (Fig. 5.3), and increased numbers of IFN- $\gamma^{+}$and IL-2 $2^{+} \mathrm{IFN}-\gamma^{+}$ $\mathrm{CD} 4^{+} \mathrm{T}$ cells in the spleen and lung six weeks after vaccination (Figs. 5.4/5.6). Treg inactivation prior to BCG vaccination did not affect the protective efficacy of the BCG vaccine (Figs. 5.8/5.9). This suggests that IFN- $\gamma^{+}, \mathrm{IL}_{-} 2^{+}$or IL-2 ${ }^{+} \mathrm{IFN}-\gamma^{+} \mathrm{CD} 4^{+} \mathrm{T}$ cell subsets do not define the protective subset arising from BCG vaccination. As a result, one might speculate that the quality and/or quantity of the memory $\mathrm{T}$ cell subset responsible for protection after BCG vaccination is unaffected by Treg inactivation prior to vaccination (Fig. 5.16) 


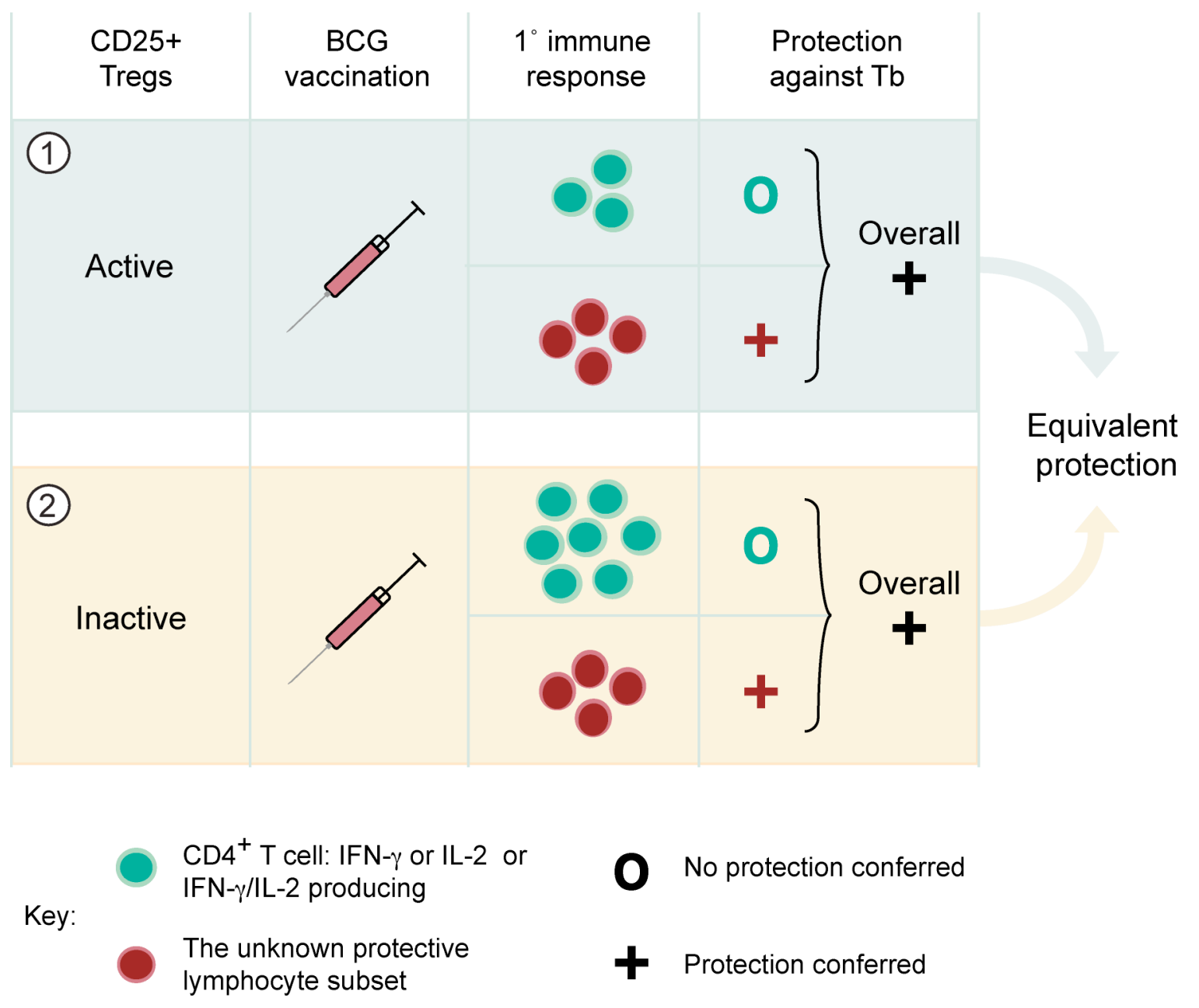

Figure 5.16: Schematic for hypothesised effect of Treg inactivation on lymphocyte subsets and protection after BCG vaccination

For the live BCG vaccine, 1) if Tregs are active, a robust cell-mediated response develops and a protective lymphocyte subset develops and confers protection against Tb infection. 2) If Tregs are inactive, enhanced activation signals, such as increased IL-2 signalling, could enhance the development of IFN- $\gamma$, IL-2 or IFN- $\gamma /$ IL-2 producing $C D 4^{+} \mathrm{T}$ cell subsets. I hypothesise that these cellular subsets do not define the protective subset for the BCG vaccine. I also hypothesise that enhanced activation signals when Tregs have been inactivated do not affect the development of the lymphocyte subset that mediates protection. Therefore, Treg inactivation does not alter protection from infection for the BCG vaccine. 
By contrast, preliminary data suggests that inactivation of Tregs may diminish protection afforded by pcDNA3-85A (Fig. 5.15). The only published experimental model where protective memory is lost after Treg inhibition is during L. major murine infection [305]. It was hypothesised that protection was abrogated as the immune response is increased to the point that low-grade infection was resolved and the source of persistent antigen for maintenance of memory was removed. It has been reported that $\mathrm{CD} 4{ }^{+} \mathrm{CD} 25^{+}$Tregs can expand after DNA vaccination and suppress immune responses [469], although the degree of Treg expansion is enhanced with certain routes of vaccination, such as mucosal vaccination. The effect of Tregs on the persistence of cells transfected with a DNA vaccine construct has not been assessed and if inactivation of Tregs prior to DNA vaccination results in increased clearance of transfected cells, it could negatively impact on vaccine protective efficacy.

Therefore, the differing effects of Treg inactivation on the various vaccines tested could reflect the diversity of protective mechanisms elicited by the vaccines.

\subsection{2 $\mathrm{CD}^{+}{ }^{+} \mathrm{CD} 25^{-}{ }^{-}$Oxp $^{+}$and induced Tregs}

It is important to note that similar to the discussion presented in chapter 3 , there are other subsets of functional Tregs that may suppress immune responses to BCG and DNA vaccination, as the anti-CD25 mAb PC61 inactivated only CD4 ${ }^{+} \mathrm{CD} 25^{+}$natural Tregs.

$\mathrm{CD} 4^{+} \mathrm{CD} 25^{-} \mathrm{T}$ cells that express Foxp3 are unaffected by PC61 treatment [374]. Since expression of Foxp3 correlates highly with suppressive Treg activity [282], these lymphocytes represent a major Treg population that persists after PC61 treatment and may affect memory development and protective efficacy of the vaccines.

Furthermore, vaccination may induce the development of Tregs that affect the primary and secondary immune responses and the resulting protective efficacy. There is evidence that BCG vaccination can induce Treg development from naïve $\mathrm{CD} 4^{+} \mathrm{CD} 25^{-} \mathrm{T}$ cells. DCs stimulated with BCG produce IL-10 and, when incubated with naïve T cells, can induce a subset of cells that produce IL-10, which is consistent with the Tr1 subset of induced Tregs [470]. In addition, BCG vaccinated infants have increased expression of Foxp3 mRNA in mycobacteria-stimulated peripheral blood samples, which may be 
indicative of either Foxp3 $3^{+}$Treg expansion or induction of Tregs [471]. While it is interesting to note that Foxp3 expression isw induced in BCG vaccinated infants despite this group being effectively protected from $\mathrm{Tb}$ infection, it would be informative to compare this to induction of Foxp3 expression in BCG vaccinated adults in whom the vaccine is less effective. Increased Foxp3 expression in adults compared to infants after vaccination could indicate that Foxp $3^{+}$Treg expansion or induction of Tregs contribute to the diminshed efficacy of BCG in adults compared to children.

Induction of Tregs or expansion of natural Tregs may also occur after DNA vaccination. Increased numbers of $\mathrm{T}$ cells with a suppressive phenotype are found after vaccination but this may be more pronounced with certain routes of vaccination, such as mucosal vaccination. For example, intranasal vaccination with DNA encoding proinsulin was able to inhibit the development of autoimmune diabetes in non-obese diabetic mice [461]. Through adoptive transfer experiments, it was determined that both $\mathrm{CD} 25^{+}$and $\mathrm{CD} 25^{-} \mathrm{CD} 4^{+} \mathrm{T}$ cells could mediate this inhibition [461]. However, this inhibition was completely abrogated by pre-incubation of transferred $\mathrm{T}$ cells with the anti-GITR mAb DTA-1, which is thought to inhibit suppression by Tregs through modulation of GITR/GITRL signaling [461]. Whether Tregs are induced with other routes of DNA vaccination and whether Tregs induced by DNA vaccination migrate to the vaccination site have not been assessed.

\subsubsection{Implications for models of Th1 immunity}

A key aspect of this chapter is that the results allow us to further define the appropriate secondary response that enables protection from $\mathrm{Tb}$ infection. Current dogma suggests an effective $\mathrm{Tb}$ vaccine should induce a strong Th1 primary immune response characterised by $\mathrm{CD}^{+} \mathrm{T}$ cells producing IFN- $\gamma$ (reviewed in [472-474]). Following a linear model of memory development [475], such a vaccine would thereby result in a larger pool of Th1 memory lymphocytes and a secondary immune response with an increased magnitude or speed of onset. The results presented in this chapter showed that inactivation of Tregs during BCG vaccination induced a larger vaccine-induced immune response (Fig. 5.3) and larger subsequent pool of $\mathrm{CD} 4^{+}$and $\mathrm{CD} 8^{+}$lymphocytes that could express IFN- $\gamma$ in response to mycobacterial antigen (Fig. 5.4), as well as an accelerated secondary immune response (Fig. 5.7). However, these factors were not 
sufficient to reduce bacterial burden (Figs. 5.8/5.9). Therefore, this study lends weight to mounting evidence against the current dogma of $\mathrm{Tb}$ protective immunity in two areas.

Firstly, it illustrates that after vaccination, increased numbers of IFN- $\gamma$ producing lymphocytes locally in the lung and systemically in the spleen after vaccination and before infection does not correlate with increased protection from infectious challenge. This finding is consistent with other studies [167, 476].

Secondly, it suggests that accelerating the existing BCG-induced secondary immune response in the lung is insufficient to enhance protection against $\mathrm{Tb}$ infection. It has been suggested that BCG vaccination results in lower bacterial burden in the lung because it accelerates the arrival of the IFN- $\gamma$ expressing $\mathrm{CD} 4^{+}$lymphocytes in the lung $[47,84,171]$. The earlier exposure of infected alveolar macrophages to IFN- $\gamma$ could arrest the growth of mycobacteria residing in macrophages earlier after infection, resulting in the $\sim 1 \log _{10}$ reduction in lung bacterial numbers in the lung and spleen that is typically observed in BCG vaccinated compared to naïve animals. However, inactivation of Tregs prior to BCG vaccination accelerated the secondary immune response compared to BCG vaccination alone (Fig. 5.7), with no corresponding decrease in bacterial numbers in the lung or spleen (Figs. 5.8/5.9).

Recently, the protective efficacy of vaccines targeting Th1 immunity has been shown to correlate with their ability to induce an increased proportion of multifunctional $\mathrm{CD}^{+} \mathrm{Th} 1$ cells that are capable of producing multiple cytokines rather then just IFN- $\gamma$ [477]. This study used a L. major model of Th1 immunity and the multifunctional $\mathrm{CD}^{+}$Th1 cells produced a combination of IL-2, TNF- $\alpha$ and IFN- $\gamma$ [477]. It has been proposed that $\mathrm{T}$ cells that produce solely IFN- $\gamma$ represent a terminal differentiation state [221] and they do not contribute to long-term Th1 immunity. However, multifunctional $\mathrm{CD} 4^{+} \mathrm{Th} 1$ cells are long-lived and capable of producing high amounts of all three cytokines upon in vitro restimulation [477]. As a result, it has been proposed that multi-cytokine producing $\mathrm{CD}^{+}{ }^{\mathrm{T}}$ cells are the mediators of Th1 immunity.

TNF- $\alpha$ production was not assessed in these studies, as the number of cellular markers that could be simultaneously assessed was limited by 4-colour flow cytometry. However, co-production of IL-2 and IFN- $\gamma$ was evaluated. Co-production of IL-2 and 
IFN- $\gamma$ was not observed 2 weeks after BCG vaccination (Fig. 5.2) but by week 6 after vaccination, a small population of IL- $2^{+} \mathrm{IFN}-\gamma^{+} \mathrm{CD} 4^{+}$lymphocytes was observed (Fig. 5.5/5.6). Co-production of IL- 2 and IFN- $\gamma$ by CD4 $4^{+}$lymphocytes was also observed 3 days after infectious challenge for BCG, pcDNA3-85A and pKUN85A vaccinated groups (Fig. 5.14). The numbers of IFN $-\gamma^{+} \mathrm{IL}-2^{+} \mathrm{CD} 4^{+}$lymphocytes were significantly increased in PC61 treated compared to control treated BCG vaccinated mice in both the spleen and lung immediately prior to infectious challenge (Fig. 5.6). This significant increase did not correlate with a decrease in bacterial numbers in the lung or spleen after virulent mycobacterial challenge (Figs. 5.8/5.9).

When IFN $-\gamma^{+} \mathrm{IL}-2^{+} \mathrm{CD} 4^{+}$lymphocytes were evaluated as a proportion of the total IFN- $\gamma$ or IL-2 producing CD4 ${ }^{+}$lymphocyte population, PC61 treatment did not alter the proportion of these cells 6 weeks after BCG vaccination (Fig. 5.6 C/D) or 3 days after infectious challenge in BCG vaccinated animals (Fig. 5.14 C/D). This correlates with the fact that PC61 treatment did not cause a difference in the protective efficacy of the BCG vaccine (Figs. 5.8/5.9). Therefore, the proportion rather than absolute numbers of $\mathrm{CD}^{+}$lymphocytes producing multiple cytokines may prove to be a useful correlate of protective efficacy for the BCG vaccine.

Interestingly, preliminary results for pcDNA3-85A vaccination suggest that proportions of IFN- $\gamma^{+} \mathrm{IL}-2^{+} \mathrm{CD} 4^{+}$lymphocytes might not consistently correlate with the protective efficacy of all Tb vaccines. Proportions of IFN- $\gamma^{+} \mathrm{IL}-2^{+} \mathrm{CD} 4^{+}$lymphocytes were comparable between PC61 treated and control treated pcDNA3-85A vaccinated mice in the spleen 3 days after infectious challenge (Fig. 5.14 C/D); however, bacterial numbers in the spleen were significantly higher in PC61 treated compared to control treated pcDNA3-85A vaccinated mice after M. tuberculosis challenge (Fig. 5.15). It might not be appropriate to look for an immune correlate of protection against $\mathrm{Tb}$ shared between BCG and pcDNA3-85A vaccination and this issue will be discussed in greater depth in section 6.3.4.

Overall, these results illustrate that several proposed immune correlates of protection for $\mathrm{Tb}$ do not consistently correlate with vaccine protective efficacy after Treg inactivation. In future studies, it would be interesting to assess IL-2, IFN- $\gamma$ and TNF- $\alpha$ co-production, to more accurately assess the proportions of multi-functional $\mathrm{CD}^{+}$Th1 cells after vaccination and test this as an immune correlate of protection for Tb. 


\subsection{Summary}

Two key outcomes emerge from this study. Firstly, inactivation of natural Tregs may diminish the protective efficacy of pcDNA385A, resulting in slight increases in the secondary immune response but diminished protection from infectious challenge in the spleen. Secondly and in contrast, natural $\mathrm{CD} 4^{+} \mathrm{CD} 25^{+}$Tregs did not have a significant effect during vaccination on protective outcome of the BCG vaccine.

These outcomes also had implications for the validity of several proposed immune correlates of protection for $\mathrm{Tb}$. They lend weight to mounting evidence that increased numbers of IFN- $\gamma$ producing $\mathrm{CD}^{+}$lymphocytes [476] do not correlate with enhanced protection. The data also suggests that accelerating the appearance of IFN- $\gamma$ producing $\mathrm{T}$ cells in the lung following infection $[47,84,171]$ does not correlate with enhanced protection. Finally, the assessment of whether the proportion of multifunctional $\mathrm{CD}^{+} \mathrm{Th} 1$ cells [477] is a correlate of $\mathrm{Tb}$ vaccine efficacy requires evaluation of TNF- $\alpha$ as well as IFN- $\gamma$ and IL-2 production by $\mathrm{CD}^{+} \mathrm{T}$ cells. This data illustrated that, while the number of IFN $-\gamma^{+} \mathrm{IL}-2^{+} \mathrm{CD} 4^{+} \mathrm{T}$ cells did not consistently correlate with protection against $\mathrm{Tb}$ infection, the proportion of IFN $-\gamma^{+} \mathrm{IL}-2^{+} \mathrm{CD} 4^{+} \mathrm{T}$ cells may be a correlate of BCG vaccine efficacy. Thus, while Treg inactivation during vaccination does not enhance the protective efficacy of the BCG vaccine, it has provided insights into aspects of the immune response that do not limit the efficacy of BCG and assists in narrowing the search for more effective immune correlates of protection against $\mathrm{Tb}$. 
Chapter 6:

\section{General Discussion}


At the time this study was initiated, Treg immunology was a relatively new field and natural Tregs had been shown to suppress immune responses and protection in some infection and vaccination models. However, the influence of Tregs during primary and memory responses to mycobacterial infection had not been assessed using an in vivo model. Given the effects of natural Tregs on other models, there was much speculation that natural Tregs would similarly suppress immune responses and protection during $\mathrm{Tb}$ infection and vaccination. It is interesting that, in this study, inactivation of natural Tregs enhanced primary and secondary immune responses but did not enhance control of a primary $\mathrm{Tb}$ infection or protection from a secondary infectious challenge.

This discussion firstly summarises the results presented in this thesis. Secondly, it outlines ways of refining and optimising the DNA vaccine, pKUN85A, which was developed during this thesis. Thirdly, it explores other avenues through which Treg subsets may affect mycobacterial infection or vaccination and which were not addressed in this thesis. Finally, the broader implications of this study are examined for proposed immunological correlates of protection from $\mathrm{Tb}$ infection.

\subsection{Summary of experiments}

The main question addressed in this thesis was whether natural $\mathrm{CD} 4{ }^{+} \mathrm{CD} 25^{+}$Tregs suppressed the protective primary and memory immune responses against mycobacterial infection.

I had initially hypothesised that Tregs would suppress both the immune response to mycobacterial infection and the immune response elicited by vaccination. To evaluate this, Tregs were inactivated in vivo using antibody-mediated inactivation prior to infection or vaccination, including pKUN85A vaccination, and resulting immune responses were followed. By inactivating Tregs, I hypothesised that immunosuppression mediated by Tregs would be relieved and thereby control of infection or vaccinemediated protection respectively would be enhanced. Several different mycobacterial infections were used in this study, including i.n. BCG infection and aerosol M. bovis or M. tuberculosis infection. The effect of Treg inactivation on three different $\mathrm{Tb}$ vaccines was also assessed: BCG, pcDNA3-85A and the pKUN85A plasmid DNA replicon vaccine, which was developed during the course of this thesis (chapter 4). 
Initial studies revealed that natural Tregs suppressed the acute immune response to mycobacterial infections (Figs. 3.8-3.10). However, inactivation of Tregs did not affect bacterial numbers in the tissues during early stages of disease (Figs. 3.11-3.13) and only transiently reduced lung pathology in M. bovis infected mice (Fig. 3.14). Natural Tregs also suppressed immune responses to BCG vaccination, since inactivation of Tregs prior to BCG vaccination led to an enhanced vaccine-induced immune response (Fig. 5.3) and accelerated the immune response to secondary challenge (Fig. 5.7). However, this accelerated secondary immune response did not affect the protective efficacy of the BCG (Figs. 5.8/5.9). Preliminary studies with the pcDNA3-85A plasmid DNA vaccine suggest that inactivation of Tregs prior to vaccination may diminish vaccine-mediated protection from infectious challenge (Fig. 5.15).

Overall, this body of work suggests that natural Tregs suppress immune responses after mycobacterial infection or vaccination against $\mathrm{Tb}$ but this does not significantly inhibit control of a primary mycobacterial infection or protection from infection after $\mathrm{Tb}$ vaccination. This work also has implications for the validity of different immunological correlates of protection for $\mathrm{Tb}$ and for models of protective memory development after vaccination, which will be discussed in sections 6.3.

\subsection{Optimisation of the pKUN85A DNA replicon vaccine}

\subsubsection{Techniques for optimising pKUN85A efficacy}

The novel pKUN85A Tb vaccine was developed, as outlined in chapter 4, with the aim of creating the first non-cytopathic plasmid DNA replicon vaccine against $\mathrm{Tb}$. Vaccination with a non-cytopathic plasmid DNA replicon was anticipated to result in prolonged antigen expression in vivo and efficient activation of $\mathrm{CD} 8^{+} \mathrm{T}$ cells.

The pKUN85A construct was created by cloning the $A g 85 A$ gene from the pcDNA3-85A plasmid DNA vaccine into the pKUNrep6 vector (Fig. 4.5). Orientation PCRs (Fig. 4.7) and digests (Fig. 4.8) followed by sequencing around the cloning site of this construct (Appendix 8.2) revealed that the $\mathrm{Ag} 85 \mathrm{~A}$ gene inserted in the correct orientation and in the correct frame with the surrounding ORF. Transfection of pKUN85A into the NIH-3T3 murine fibroblast cell-line demonstrated that NS-1 protein was produced from the ORF that also contained the $A g 85 A$ gene (Fig. 4.9). When 
pKUN85A was administered i.m. to mice, it induced an Ag85A-directed immune response (Figs. 4.10/4.11). These data suggest that pKUN85A facilitates production of Ag85A protein in vivo and initiates an Ag85A-specific immune response. The dose titration of pKUN85A with respect to protection was complicated by high variation within treatment groups (Fig. 4.12) and further dose titrations of pKUN85A will be performed to determine the dose that confers maximal protection from $\mathrm{Tb}$.

Once an appropriate dose of pKUN85A has been determined, it would be informative to characterise the type of immune response induced by the vaccine. $\mathrm{Tb}$ vaccines are often designed to activate $\mathrm{CD}^{+}{ }^{\mathrm{T}}$ cells and induce $\mathrm{Th} 1$ immunity but plasmid DNA replicon vaccines are known to induce cell-mediated immunity and robust $\mathrm{CD}^{+} \mathrm{T}$ cell activation. Restimulation of lymphocytes from pKUN85A vaccinated mice with whole Ag85A protein resulted in cytokine production by $\mathrm{CD}^{+} \mathrm{T}$ cells (Fig. $4.11 \mathrm{~B} / \mathrm{D})$, but this is likely to be a result of bystander activation of $\mathrm{CD}^{+} \mathrm{T}$ cells mediated by CD28 signalling rather than true antigen-driven cytokine production [445]. To assess antigen-driven cytokine production by $\mathrm{CD}^{+} \mathrm{T}$ cells, lymphocytes should be restimulated with APCs loaded with an MHC I restricted peptide from Ag85A. Currently, there have not been any MHC I restricted peptides from Ag85A identified for C57BL/6 mice, but an MHC I restricted peptide from Ag85A has been identified for BALB/c mice $[430,446,447,478]$. Therefore, induction of CD8 ${ }^{+} \mathrm{T}$ cell responses will be assessed after vaccination of BALB/c mice with pKUN85A and, thereafter, the contribution of $\mathrm{CD}^{+} \mathrm{T}$ cells to protection from mycobacterial infection afforded by pKUN85A vaccination will be assessed.

Additionally, vaccination with pKUN85A could be optimised in a number of ways, including choice of antigen, optimisation of antigen expression, optimisation of vaccine delivery or enhancement of vaccine adjuvancy (section 4.4.4). However, the most promising way to enhance the efficacy of the pKUN85A vaccine is to incorporate it into a heterologous prime-boost vaccination approach. DNA vaccines have been used in human clinical trials as part of a heterologous prime-boost approach to enhance vaccine efficacy (reviewed in $[232,479,480]$ ). It is possible that pKUN85A would be more effective as a component of a heterologous prime-boost vaccine in combination with adjuvanted protein subunit $\mathrm{Tb}$ vaccines, BCG or viral vectors containing Ag85A. 


\subsubsection{Progressing towards clinical trials}

There are several major hurdles for efficacy testing and study design before a DNA vaccine, such as pKUN85A, could be considered for Tb vaccine clinical trials.

Firstly, the safety and efficacy of a vaccine needs to be validated in a number of animal models. For $\mathrm{Tb}$ vaccines, pre-clinical studies are usually performed in murine models followed by the guinea pig model. The guinea pig model exhibits lung pathology that that more closely models the human granuloma, with central necrosis, and displays progressive disease not only in the lung but also the spleen, which only occurs in highly susceptible humans $[22,186]$. Due to their high susceptibility, guinea pigs are often used as a final pre-clinical model to test novel Tb vaccine efficacy. During testing in animal models, vaccines are compared to the BCG vaccine, which is used as the gold standard for $\mathrm{Tb}$ vaccines. Vaccine efficacy for a $\mathrm{Tb}$ vaccine would need to be at least equivalent if not better than BCG to progress into clinical trials.

Preclinical safety testing for DNA vaccines also carries a particular set of considerations. DNA vaccine plasmids could theoretically insert into the genome of the transfected host cell or DNA vaccination could result in the development of anti-DNA antibodies and autoimmunity, but so far neither of these outcomes has been observed in practice (reviewed in [481]). In fact, plasmid DNA vaccines have been used in numerous preclinical safety trials and phase I human clinical trials and they have a consistently high safety record. However, evaluating vaccine efficacy in humans for $\mathrm{Tb}$ vaccines can be a complicated and prolonged process.

Clinical trials to assess $\mathrm{Tb}$ vaccine efficacy are complicated by two issues.

Firstly, $\mathrm{Tb}$ does not often progress rapidly to symptomatic disease and may persist in its latent form. Secondly, the latent form of $\mathrm{Tb}$ infection can be difficult to diagnose. Therefore, vaccinated individuals must be followed for a long period, using relatively labour-intensive methods to diagnose disease, to accurately assess vaccine efficacy. The prolonged and involved nature of these studies could be minimised for $\mathrm{Tb}$ if an accurate immune correlate of protection could be identified and efficacy could be assessed earlier after vaccination.

Despite potential issues associated with human clinical trials of $\mathrm{Tb}$ vaccines, there have been a number of recent trials [254]. One recent study recruited individuals already vaccinated with BCG and boosted them with a viral vector expressing Ag85A 
[480]. This viral vector boost resulted in strong induction of IFN- $\gamma$ production by $\mathrm{T}$ cells assessed by ELISpot after boosting and these responses were assessed as an indicator of vaccine immunogenicity [480].

Unfortunately, while parameters such as IFN- $\gamma$ production and antigen-specific proliferation are used to assess $\mathrm{Tb}$ vaccine immunogenicity, these parameters do not consistently correlate with maximal protection from infection [167]. Additionally, small changes in the dose of plasmid DNA replicons can have a major effect on the efficacy of the vaccine in a homologous prime-boost setting [433]. Given the sensitivity of plasmid DNA replicons to dose and the lack of an immune correlate of protection, an efficacy trial for pKUN85A would need to test different doses of pKUN85A and relate this to protection from $\mathrm{Tb}$ infection rather than immunogenicity. Therefore, a clinical trial to assess efficacy of a plasmid DNA replicon vaccine, such as pKUN85A, against $\mathrm{Tb}$ would be relatively large, prolonged and difficult to perform compared to a clinical trial for vaccines against other infections.

Overall, vaccination with pKUN85A requires a number of refinements to enhance vaccine efficacy (discussed in section 4.4.4) and these refinements need to be tested in small animal models, such as the $\mathrm{C} 57 \mathrm{BL} / 6$ and $\mathrm{BALB} / \mathrm{c}$ murine models of $\mathrm{Tb}$. If the vaccine is able to improve upon the efficacy seen with BCG, pre-clinical efficacy trials could be considered using other animal models, such as the highly susceptible guinea pig model. Depending on the success of pre-clinical trials, human clinical trials could be considered. However, human clinical trials of vaccine efficacy would be complex to undertake, considering the lack of an immune correlate of protection for $\mathrm{Tb}$ and the need for plasmid DNA replicon vaccine dose to be carefully titrated with respect to protection.

\subsection{Tregs and anti-mycobacterial immunity}

The experiments in this thesis were driven by the hypothesis that inactivating natural $\mathrm{CD} 4^{+} \mathrm{CD} 25^{+}$Tregs prior to mycobacterial infection or vaccination would enhance acute immune responses and thereby enhance control of primary infection or enhance development of protective memory. 
This thesis focussed on natural Tregs and the acute immune response to mycobacterial infection, primarily because of tools available to manipulate Tregs in vivo. At the outset of these studies, these tools were limited to anti-CD25 antibodies, such as PC61, and antibodies blocking or signalling through the GITR/GITRL pathway, such as DTA-1 and 5F-1. In vivo administration of PC61 was used to inactivate Tregs but results contained in this thesis outlined several limitations of this method:

- PC61 treatment inactivated $\mathrm{CD} 4^{+} \mathrm{CD} 25^{+}$Foxp $3^{+}$lymphocytes efficiently but did not target $\mathrm{CD}^{+} \mathrm{CD}^{-} 5^{-}$Foxp $^{+}$lymphocytes (Fig. 3.2). Foxp3 is a better correlate of Treg suppressive phenotype than CD25 and CD $4^{+} \mathrm{CD} 25^{-}$Foxp $3^{+}$lymphocytes possess significant suppressive function [282]. As a result, PC61 treatment inactivated natural $\mathrm{CD} 4^{+} \mathrm{CD} 25^{+} \mathrm{Foxp}^{+}$lymphocytes effectively but suppressive $\mathrm{CD}^{+} \mathrm{CD} 25^{-} \mathrm{Foxp}^{+}$lymphocytes were retained and comprised up to $50 \%$ of the total Foxp $3^{+}$Treg population in tissues like the lung and spleen of naïve mice [282].

- After a single treatment with PC61, CD25 ${ }^{+}$Tregs were inactivated for up to 23 days but eventually repopulated the periphery (Fig. 3.3). The onset of repopulation may coincide with the clearance of the PC61 antibody from the periphery (Fig. 3.5). Therefore, PC61 treatment prior to infection or vaccination inactivated Tregs during the acute immune response but inactivation did not persist during later stages of infection or vaccination.

- While PC61 treatment inactivated natural CD25 $5^{+}$Treg subsets in a naïve mouse, induced subsets of Tregs may arise upon infection or vaccination. This study did not address the role of induced Treg subsets during mycobacterial infection and vaccination.

Therefore, my thesis illustrates that $\mathrm{CD} 25^{+}$Tregs do not suppress protection in mycobacterial infection and vaccination models to the degree that has been observed for other infection and vaccination models, but this does not rule out a role for Tregs altogether. Induced Tregs or memory Tregs may affect anti-mycobacterial immunity and Treg activity may be induced in certain immunological settings, such as coinfection, chronic mycobacterial infection or virulent mycobacterial infection, as will be discussed below. Recently, several tools have been developed that will enable deeper exploration of Treg involvement for anti-mycobacterial immunity and potential applications of these tools will be outlined. 


\subsubsection{Induced Tregs and anti-mycobacterial immunity}

Induced Tregs can arise under a number of different conditions, including low-dose antigen presentation and antigen presentation during conditions in which TGF- $\beta$ or IL10 is present (section 1.4.3). Currently, there have not been any studies directly assessing the role of induced Tregs during mycobacterial infection or vaccination. However, it is possible that during mycobacterial infection or vaccination against $\mathrm{Tb}$, antigen presentation occurs in conditions of low-dose antigen or elevated TGF- $\beta$ or IL10 , as described below.

Although substantial amounts of antigen may be available during mycobacterial infection and vaccination, it is possible that mycobacterial antigen presentation is not optimal, resulting in the induction of Tregs. MHC II presentation is down-regulated in mycobacteria infected macrophages and DCs by suppression of H-2DM gene expression [166] and inhibition of release of MHC II molecules into physiologically relevant cellular compartments [165]. Therefore, although antigen is phagocytosed during mycobacterial infection and vaccination, the amount of antigen presented by APCs containing mycobacteria may be low, resulting in the differentiation of induced Tregs.

IL-10 or TGF- $\beta$ may also be produced during presentation of mycobacterial antigen. APCs can be stimulated to produce IL-10 upon exposure to mycobacterial products. For example, an LPS-free preparation of M. tuberculosis heat shock protein 70 inhibited maturation of murine DC precursors in vitro and in vivo [482]. DCs cultured in these conditions produced IL-10 and inhibited T cell proliferation in vitro [482]. These results illustrate that mycobacterial proteins can suppress APC maturation and activation, and can induce IL-10 production in a context that would promote induction of Tregs, resulting in immunosuppression.

Furthermore, IL-10 and TGF- $\beta$ producing $\mathrm{CD} 4^{+} \mathrm{CD} 25^{+} \mathrm{T}$ cells have been detected in the lungs of M. tuberculosis infected mice. [483]. Co-expression of IL-10 and TGF- $\beta$ by $C D 4{ }^{+} \mathrm{CD} 25^{+}$T cells was not assessed but neutralisation of either IL-10 or TGF- $\beta$ enhanced in vitro IFN- $\gamma$ production by lung lymphocytes [483]. Therefore, $\mathrm{CD} 4{ }^{+} \mathrm{CD} 25^{+}$Tregs producing IL-10 or TGF- $\beta$ can access the lung and suppress local 
responses. These Tregs could have been natural CD $25^{+}$Tregs or induced Tregs, as induced Tregs can upregulate CD25 upon TCR stimulation.

Production of IL-10 by APCs not only occurs after exposure of APCs to $M$. tuberculosis proteins but also after exposure of APCs to BCG. DCs matured in the presence of BCG decreased the amount of IL-12 they produced but increased IL-10 production upon stimulation with CD40L compared to LPS-matured DCs [484]. When BCG-matured DCs were used to prime naïve T cells, the T cells produced IL-10 but had no bias for IFN- $\gamma$ or IL-4 production compared to LPS-matured DCs [484]. Therefore, APCs stimulated with BCG in vitro induce conditions conducive for Treg induction during antigen presentation to naïve $T$ cells, and these naïve $T$ cells acquire an induced Treg-like phenotype.

Finally, the ability of DNA vaccination to induce Tregs or even expand natural Tregs has not been widely explored. It is known that delivery of DNA vaccines to mucosal surfaces can result in tolerogenic responses against antigens encoded in the construct. Vaccination with DNA encoding proinsulin by an i.n. route resulted in suppression of autoimmune diabetes in non-obese diabetic mice [461]. When mechanisms responsible for suppression were explored, it was found to be mediated by both $\mathrm{CD} 25^{+}$and $\mathrm{CD} 25^{-} \mathrm{CD} 4^{+} \mathrm{T}$ cells in a GITR-dependent mechanism [461]. These results support the hypothesis that DNA vaccination via mucosal surfaces can result in natural Treg activation or induction of Tregs. Natural Treg activation or induction of Tregs following i.m. DNA vaccination has not been directly assessed but inactivation of Tregs prior to pcDNA3-85A did not significantly enhance the primary immune response (Fig. 5.11), although it did enhance the secondary response (Fig. 5.12). These results illustrate that $\mathrm{CD} 4{ }^{+} \mathrm{CD} 25^{+}$Tregs can suppress the development of secondary immune responses after i.m. DNA vaccination and suggest that Tregs are activated or induced upon vaccination.

Considering the conditions, such as sub-optimal antigen presentation or antigen presentation in the presence of IL-10 and TGF- $\beta$, which occur during mycobacterial infection or vaccination, Treg populations may be induced. As a result, I hypothesise that an induced Treg population could mediate immunosuppression during primary mycobacterial infection and during $\mathrm{Tb}$ vaccination. This would introduce a degree of redundancy into the system. Immunosuppressive responses detected during mycobacterial infection and vaccination could be as a result of suppression mediated by 
natural Tregs or by induced Tregs. During inactivation of $\mathrm{CD} 4{ }^{+} \mathrm{CD} 25^{+}$Tregs, the suppressive activity of induced Tregs unaffected by PC61 treatment could mask any effect of inactivation of natural Tregs. Therefore, significant questions remain, including whether induced Tregs suppress bacterial clearance after mycobacterial infection or suppress the protective efficacy of Tb vaccines, and whether there is redundancy between the immunosuppressive mechanisms of natural and induced Tregs.

\subsubsection{Tregs during chronic mycobacterial infection}

Tregs suppress immune responses predominantly during chronic infections [305, 330, $333,349,375]$, and mycobacterial infections are well known for their chronicity. Therefore, Tregs could be more important for immunosuppression in the granuloma during the chronic phase of mycobacterial infection, a point also briefly explored in this thesis.

Tregs have been shown to accumulate in mycobacterial lesions. Results presented in this thesis demonstrated that $\mathrm{CD} 4^{+} \mathrm{CD} 25^{+}$Foxp $3^{+}$Tregs accumulate in the lung during the acute phase of mycobacterial infection (Figs. 3.5/3.6). Another recent study by Scott-Browne, et al. demonstrated that Foxp3 $3^{+}$Tregs accumulate along with other lymphocytic infiltrate in the lung in granulomatous lesions [384] and thus are likely an integral part of the granuloma. It remains to be seen whether these Tregs suppress immune responses in the granuloma and, to date, no studies evaluating colocalisation of Foxp3 and Treg mediator molecules such as IL-10 and TGF- $\beta$ have been published. While it is possible that the Tregs themselves secrete these molecules, it is also possible that Tregs direct other effector cells within the granuloma to secrete suppressive cytokines, such as is the case with L. major infection in both Th1- and Th2biased murine models [360,361]. It is also not known which Treg subsets, whether natural or induced Tregs, make up the majority of infiltrate to the lung.

Tregs contribute to the cellular composition of the granuloma but whether Tregs suppress bacterial clearance during chronic mycobacterial infection has not been extensively examined. In this thesis, Treg activity was modulated during the chronic phase of mycobacterial infection to address this point. Neither enhancing Treg activity through administration of 5F-1 (anti-GITRL mAb) nor inhibiting Treg activity through administration of PC61 (anti-CD25 mAb) and/or DTA-1 (anti-GITR mAb) altered the 
bacterial numbers present in the lung (Fig. 3.15). Inhibition of Treg activity through administration of PC61 and DTA-1 during chronic infection with L. sigmodontis has been shown to reduce pathogen load [330] and it is interesting that a similar effect was not apparent for M. tuberculosis infection.

There are potential limitations associated with using these mAbs to assess the effect of Tregs during chronic M. tuberculosis infection. Administration of PC61 during an active immune response has been controversial, since recently activated effector $\mathrm{T}$ cells upregulate CD25 and, thereby, PC61 may inhibit the activity of both Tregs and T effector cells. While this could occur theoretically, enhanced effector responses were observed in mice that had PC61 persisting in their circulation during the first week of mycobacterial infection (Figs. 3.5/3.8-3.10), suggesting that the presence of PC61 does not inhibit the development of effector immune responses.

Anti-GITR or anti-GITRL mAbs can be used to modulate the activity of Tregs through the GITR/GITRL signalling pathway. The use of these antibodies in vivo presupposes that the activity of Tregs present during chronic mycobacterial infection is affected by GITR/GITRL signalling. During L. sigmodontis infection, there is substantial expression of GITR on lymphocytes associated with lesions [330] and some GITR expression has been observed by lymphocytes in PBMCs from M. tuberculosis infected patients [370, 371]. However, the exact mechanism of GITR/GITRL signalling is not well characterised and whether it interferes with mechanisms of suppression utilised by Tregs present during chronic mycobacterial infection is uncertain. Finally, as noted in the previous section, induced Tregs may develop over time and play a role during chronic stages of mycobacterial infection. The effect of PC61, DTA-1 and 5F-1 mAbs on the activity of induced Tregs has not been characterised.

Thus, it remains to be fully explored whether Tregs suppress clearance of bacteria during the chronic stages of mycobacterial infection. Specifically, it remains to be seen whether targeted inhibition of natural and/or induced Treg function during the chronic phase of mycobacterial infection will enhance tissue pathology or decrease tissue bacterial numbers. It is apparent from results presented in this thesis (Figs. 3.5/3.6) and other recently published studies [384] that Tregs infiltrate the lung and granuloma during mycobacterial infection, which supports the hypothesis that Treg activity can modulate the granuloma environment during chronic infection. 


\subsubsection{Tregs during virulent mycobacterial disease}

Treg inactivation did not affect bacterial numbers in the lung after pulmonary BCG, $M$. tuberculosis or M. bovis infection (Fig. 3.11/3.13), but may result in transiently diminished pulmonary inflammation after M. bovis infection (Fig. 3.14). The preliminary finding that Treg inactivation could diminish pulmonary pathology after $M$. bovis infection requires further testing; however, if this proves to be the case, there are interesting implications for the role of Tregs in the pathology of virulent mycobacterial infection. In chapter 3, it was speculated that, since $M$. bovis infection is more virulent in mice compared to BCG or M. tuberculosis infection [382], Tregs might somehow contribute to this virulence.

It has recently been found that Tregs can have altered kinetics in more virulent mycobacterial infections. After infection of mice with a hypervirulent strain of $M$. tuberculosis, bacterial numbers in the tissue increased rapidly and pathology rapidly progressed [485]. This correlated with a very rapid Th1 immune response but also with a rapid emergence of $\mathrm{CD} 4{ }^{+} \mathrm{CD} 25^{+} \mathrm{Foxp} 3^{+}$Tregs [485]. This paper suggested that the rapid emergence of Tregs suppressed the Th1 immune response before it was able to control the infection, resulting in hypervirulence. However, it would be interesting to inactivate Tregs prior to infection with the more virulent $M$. tuberculosis infection. If Treg inactivation resulted in a reduction of inflammation similar to the reduction observed with Treg inactivation prior to $M$. bovis infection, it would suggest that Tregs contributed to a mechanism of virulence.

\subsubsection{Cross-reactivity or bystander Treg suppression}

The methodology in this thesis was used to assess whether natural Tregs suppress protective immune responses to mycobacterial infection in animals that were not known to have encountered any pathogens previously. However, human subjects are not naïve prior to mycobacterial infection or BCG vaccination. They may be exposed to environmental mycobacteria that possess cross-reactive antigens or may be infected with other pathogens at the time of infection or vaccination. Co-infections or crossreactivity with tolergenic antigens could result in scenarios in which Tregs can modulate the outcome of infection or vaccination. These scenarios have not been explored in these studies but there is evidence in the literature that Tregs induced during 
co-infection or induced to cross-reactive antigen could suppress immune responses or protection to mycobacterial infection and vaccination, as described below.

Tregs are activated or induced in an antigen-specific manner, as they require TCR stimulation with their cognate antigen to initiate their suppressive capabilities. Once activated or induced, Tregs can suppress other responses in a non-antigen specific manner [290], particularly through soluble mediators such as cytokines. Thus, Tregs activated during co-infection or during a previous infection that possesses shared antigens could confer generalised bystander suppression on responses to mycobacterial infection or vaccination (reviewed in [486]).

Environmental mycobacteria may induce or activate Tregs specific for antigens cross-reactive with BCG or M. tuberculosis antigens. For example, the environmental mycobacterium $M$. vaccae can promote Treg-mediated suppression for co-administered antigens. When a suspension of heat-killed M. vaccae was administered with a sensitising antigen for airway hypersensitivity assays, the eosinophilic response during challenge was greatly suppressed [487]. The cellular population responsible for suppression was $C D 4^{+} \mathrm{CD} 45 \mathrm{RB}^{\mathrm{lo}}$, which is a classic Treg phenotype [488], and the suppression was dependent on the presence of IL-10 and TGF- $\beta$ [487]. Therefore, after exposure to environmental mycobacteria such as $M$. vaccae, Tregs specific for codelivered and cross-reactive mycobacterial antigens may be induced.

In addition, co-infection with helminths or other pathogens that effectively induce Tregs could result in bystander suppression of immune responses to mycobacterial infection or vaccination. Helminth co-infections correlate with diminished responses to both mycobacterial infection and BCG vaccination in human studies. Human Tb patients that had concomitant helminth infections had decreased IFN- $\gamma$ production but increased IL-10 production by PBMCs in response to mycobacterial antigen compared to helminth-free Tb patients [489]. For BCG vaccination it has been found that de-worming treatment prior to vaccination leads to increased proliferation and IFN- $\gamma$ production by PBMCs after in vitro restimulation with mycobacterial antigen [490]. These results suggest that helminth co-infection results in immunosuppression of mycobacterial immune responses and Treg activity could contribute to this suppression. 
Therefore, Tregs may be activated during co-infection or during a previous cross-reactive infection. The effects of Treg cross-reactivity or bystander suppression on anti-mycobacterial immunity remain intriguing possibilities for future study.

\subsubsection{Treg memory}

For the experiments described in thesis, Tregs were inactivated prior to vaccination and primary and secondary immune responses were then assessed. It was hypothesised that the enhanced effector responses observed after secondary challenge were due to alterations in Th1 memory cell populations caused by the lack of suppression by Tregs during priming. However, an alternative explanation is that inactivation of Tregs during priming resulted in failure to induce a Treg memory population. This hypothetical previously activated Treg memory population may be essential for suppression of the secondary immune response.

Treg memory is difficult to define. A Treg memory subset could arise from natural or induced Treg populations, and Treg memory has not been widely studied. The conventional definition of a memory cell is a long-lived cell that has experienced antigen and responds quantitatively or qualitatively better than naïve cells to secondary stimulation. It is widely thought that natural Tregs are selected in the thymus based on intermediate affinity TCR interactions with self-peptide leading to a semi-activated cell surface marker phenotype [491]. Due to their phenotype, natural Tregs are never truly naïve, even upon egress from the thymus. However, Tregs that then encounter antigen in the lymphoid tissues reach a heightened state of activation and can exhibit additional markers of activation, such as loss of CCR7 and CD62L expression and increased CD103 expression [492, 493]. By virtue of this altered expression of chemokine receptors and cell surface adhesion molecules, these activated Tregs migrate into tissues. Presumably both natural and induced Tregs migrate into tissues and tissue resident Tregs are referred to as having an effector/memory phenotype [492, 493]. Lymphoid tissue resident Tregs with a more naïve phenotype are efficient at inhibiting proliferation of naïve $\mathrm{T}$ cells and tissue resident Tregs with an effector/memory phenotype are efficient at inhibiting ongoing inflammatory responses [492]. However, it has not been assessed whether naïve or effector/memory Tregs are long-lived or whether the response of effector/memory Tregs to secondary stimulation is enhanced 
compared to a primary response. As these aspects are central tenets of the definition of a memory cell, it is premature to call tissue resident Tregs true memory cells.

Thus, it is not known to what degree antigen-experienced Tregs from a primary immune response affect the secondary immune response. In mice vaccinated against $L$. monocytogenes, antibody-mediated Treg inactivation immediately prior to secondary challenge releases suppression on $\mathrm{CD}^{+} \mathrm{T}$ cell effector mechanisms [337]. Whether Treg suppression during secondary challenge was a result of de novo Treg activation or activation of antigen-experienced, tissue-resident Tregs residual after the initial vaccination was not assessed. To ascertain whether suppression of secondary immune responses is due to long-lived memory Tregs or de novo activation of Tregs, the kinetics of accumulation and persistence of Tregs in the tissues would need to be observed during primary and secondary immune responses.

\subsubsection{Recent progress and remaining questions}

As alluded to above, a number of avenues remain to be explored to test whether Tregs suppress anti-mycobacterial immunity, and the recent development of several experimental tools will assist these studies. Mice that express GFP as a fusion protein with Foxp3 [282] enable better tracking of Tregs in general as Foxp3 is a tighter correlate of the Treg suppressive phenotype than markers like CD25, GITR and CD154. Furthermore, mice that express GFP and a diphtheria toxin receptor under the control of the Foxp3 promoter [494] will permit targeted depletion of Foxp3 expressing cells at discrete stages during an active immune response. These tools could be used to resolve a number of outstanding issues for the role of Tregs in anti-mycobacterial immunity, including:

- Are Tregs induced during mycobacterial infection or vaccination? To address this issue, a $\mathrm{CD}^{+} \mathrm{CD}^{-} 5^{-} \mathrm{Foxp}^{-} \mathrm{GFP}^{-}$population could be sorted from naïve GFP-Foxp3 transgenic mice and adoptively transferred into congenic mice before infection or vaccination. The transferred $\mathrm{CD} 4^{+} \mathrm{CD} 25^{-} \mathrm{Foxp}^{-} \mathrm{GFP}^{-}$ population should contain naïve $\mathrm{T}$ cells but no Tregs. If a significant $\mathrm{CD} 4^{+} \mathrm{Foxp}^{+} \mathrm{GFP}^{+}$population develops after infection or vaccination, then that would suggest that Tregs are induced after exposure to mycobacterial antigen. 
- Are the Tregs that migrate to the granuloma during mycobacterial infection natural or induced Tregs? To address this issue, $\mathrm{CD} 4^{+} \mathrm{CD} 25^{+} \mathrm{Foxp}^{+} \mathrm{GFP}^{+}$Tregs and $\mathrm{CD}^{+} \mathrm{CD}^{+} 5^{-} \mathrm{Foxp}^{-} \mathrm{GFP}^{-}$naïve T cells could be sorted from naïve GFPFoxp3 transgenic mice. $\mathrm{CD} 4^{+} \mathrm{CD} 25^{-} \mathrm{Foxp}^{-} \mathrm{GFP}^{-}$naïve T cells could be stimulated in vitro with mycobacterial antigen under conditions that result in Treg induction. These populations could be adoptively transferred into separate congenic mice early or late during mycobacterial infection. Localisation of the transferred populations in non-lymphoid and lymphoid tissues could be observed. The cellular composition of the granuloma could be examined to determine which cell type predominates in the granuloma.

- Do natural or induced Foxp3 ${ }^{+}$Tregs facilitate pathogen persistence and prevent pathology during chronic mycobacterial infection? To examine this, mice expressing the diphtheria toxin receptor under the control of the Foxp3 promoter could be infected with mycobacteria. Once the infection reaches a chronic phase, treatment of the mice with diphtheria toxin would deplete all Foxp $3^{+}$Tregs, including both natural and induced Tregs. The integrity of the granuloma and bacterial numbers in tissues could be assessed. Increased pathology after Treg depletion would indicate that Tregs act during chronic mycobacterial infection to suppress tissue pathology, as has been observed in other infections. However, decreased bacterial numbers in tissues would indicate that Tregs act at later stages of mycobacterial infection to facilitate pathogen persistence.

- Do long-lived Treg cells develop during mycobacterial infection or vaccination? Are these Treg cells derived from natural or induced populations? $\mathrm{CD}^{+} \mathrm{CD} 25^{+} \mathrm{Foxp}^{+} \mathrm{GFP}^{+}$Tregs and $\mathrm{CD} 4^{+} \mathrm{CD} 25^{-} \mathrm{Foxp}^{-} \mathrm{GFP}^{-}$naïve $\mathrm{T}$ cells could be sorted from naïve GFP-Foxp3 transgenic mice. These populations could be adoptively transferred into congenic mice prior to mycobacterial infection or vaccination. The expansion and persistence of congenic Foxp $3^{+} \mathrm{GFP}^{+}$Tregs after priming could be followed. The tissue distribution of effector/memory phenotype Tregs could also be assessed as other studies have shown that effector/memory Tregs localise in peripheral tissue due to altered chemokine receptor and adhesion molecule expression [492].

- If long-lived Treg cells develop during mycobacterial infection or vaccination, it raises the question of whether these are memory cells in the sense that their suppressive function is enhanced upon secondary challenge. To assess this, 
Foxp $3^{+}$Tregs could be sorted from previously infected or vaccinated mice, and the cells adoptively transferred into naïve congenic mice. As a control, Foxp $3^{+}$ Tregs could be sorted from naïve mice and transferred into naïve congenic mice, and all recipient mice would then be subsequently infected with mycobacteria. If expansion of Foxp $3^{+} \mathrm{GFP}^{+}$Tregs and suppression of the immune response to infectious challenge was enhanced in mice that receive Foxp $3^{+} \mathrm{GFP}^{+}$Tregs from infected or vaccinated donors compared to naïve donors, that would suggest that long-lived Treg cells possess an enhanced ability to respond to secondary antigen stimulus. This would fit the definition of a memory cell as a cell that responds quantitatively or qualitatively better than naïve cells to secondary stimulation.

\subsubsection{Summary: Tregs and anti-mycobacterial immunity}

Results presented in this thesis demonstrated that inactivating natural Tregs prior to mycobacterial infection or vaccination enhanced the acute primary immune response to infection and enhanced primary and memory immune responses to BCG vaccination. Despite these enhanced immune responses, and in contrast to other infection and vaccination models, inactivating natural Tregs did not have any effect on protection from primary mycobacterial infection or the protective efficacy of the BCG vaccine.

Overall, this study illustrates that under naïve WT conditions, $\mathrm{CD} 4^{+} \mathrm{CD} 25^{+}$ Tregs modestly suppress immune responses to mycobacterial infection and vaccination in the murine model but do not affect protection from infection. However, it is possible that Tregs can be induced, expanded or their activity enhanced under certain conditions as outlined above. In a heterogeneous outbred human population, exposed to a diverse array of environmental conditions, there may be more variation in Treg numbers or activity upon mycobacterial infection or vaccination compared to an inbred murine population, such as was used in this thesis. A study by Kursar, et al. indicated that extremely high proportions of natural Tregs could suppress bacterial clearance after $M$. tuberculosis infection [383]. Therefore, in scenarios in which Tregs represent a high proportion of $\mathrm{CD} 4^{+} \mathrm{T}$ cells, then inhibition of Treg-mediated suppression or avoidance of Treg activation could be employed as strategies to enhance immunotherapy or vaccination against $\mathrm{Tb}$. 


\subsection{Lessons for the immunological basis of mycobacterial protection}

Contrary to the broad hypothesis of this thesis, inactivation of natural Tregs prior to mycobacterial infection and vaccination did not enhance control of infection or protective efficacy of the vaccines. Although this suggests that modulation of natural Treg activity would not be useful as a general immunotherapeutic approach to enhance bacterial clearance after $\mathrm{Tb}$ infection, the effects of Treg inactivation prior to vaccination against $\mathrm{Tb}$ on primary and secondary immune responses can contribute to the search for more effective correlates of protection for mycobacteria.

\subsubsection{IFN $-\gamma^{+} \mathrm{CD} 4^{+} \mathrm{T}$ lymphocytes}

The production of IFN- $\gamma$ by $\mathrm{CD}^{+}$lymphocytes is widely regarded as vital for antimycobacterial immunity (section 1.2.3.3). Humans with mutations in the IFN- $\gamma$ receptor gene are highly vulnerable to mycobacterial infection $[67,68]$ and mice deficient in IFN- $\gamma$ or the IFN- $\gamma$ receptor are exquisitely sensitive to $M$. tuberculosis infection, as the infection rapidly progresses until death with very high bacterial numbers in the lung $[49,64-66]$. Control of bacterial growth in the lung after mycobacterial infection in murine models coincides with the influx of IFN- $\gamma$ producing $\mathrm{CD}^{+} \mathrm{T}$ cells [171]. Furthermore, treatment of infected macrophages with IFN- $\gamma$ can enhance functions of those macrophages that can eliminate mycobacteria, or at least inhibit their growth, such as autophagy [97] and production of ROI [99] or RNI [100, 102, 105, 106]. Therefore, the current model of the protective primary immune response against mycobacteria is that IFN- $\gamma$ produced by activated $\mathrm{CD} 4^{+} \mathrm{T}$ cells activates mycobacteriocidal activities of infected macrophages, which degrade or inhibit the growth of the mycobacteria that they have phagocytosed.

Control of bacterial growth in the lung after mycobacterial infection in BCGvaccinated mice also coincides with the earlier influx of IFN- $\gamma$ producing $\mathrm{CD}^{+}{ }^{+} \mathrm{T}$ cells compared to naïve mice [171]. Given the importance of IFN- $\gamma$ during the primary immune response to mycobacteria, it has been presumed that IFN- $\gamma$ production by $\mathrm{CD}^{+}$ $\mathrm{T}$ cells is also the key mediator of protective immunity after vaccination (section 1.2.6). To that end, IFN- $\gamma$ production by $\mathrm{CD}^{+}$lymphocytes was assessed in this thesis, and this measure was used to test the hypothesis that inactivating Tregs prior to 
mycobacterial infection or vaccination against $\mathrm{Tb}$ would enhance anti-mycobacterial immune responses.

The presumption that IFN- $\gamma$ is the key mediator of both primary and secondary immunity has been held for many years. However, it is becoming increasingly obvious that, while production of IFN- $\gamma$ mediates protection against a primary $\mathrm{Tb}$ infection, the amount of IFN- $\gamma$ induced during mycobacterial infection does not correlate with the degree of protection from infection. For example, IL-4 -/- mice infected with $M$. tuberculosis displayed increased IFN- $\gamma$ production by splenocytes and increased IFN- $\gamma$ mRNA expression in the lung up to 4 weeks after infection compared to WT mice [85]. Despite enhanced IFN- $\gamma$ production, IL-4 -/- mice did not exhibit any difference in tissue bacterial numbers compared to WT mice [85]. Similarly the number of IFN$\gamma^{+} \mathrm{CD}^{+} \mathrm{T}$ cells induced during vaccination against $\mathrm{Tb}$ does not correlate with the protective efficacy of the vaccine. For example, in a comparison of mucosal and subcutaneous BCG vaccination, numbers of IFN $-\gamma^{+} \mathrm{CD} 4^{+} \mathrm{T}$ cells induced by mucosal BCG were much lower in the draining lymph node, spleen and lung tissue compared to subcutaneous BCG vaccination, when assessed two weeks after vaccination [167]. Despite profound differences in numbers of IFN $-\gamma^{+} \mathrm{CD} 4^{+} \mathrm{T}$ cells two weeks after vaccination, both mucosal and subcutaneous BCG vaccination conferred a similar degree of protection from challenge with M. tuberculosis [167].

Results presented in this thesis corroborate the hypothesis that numbers of IFN$\gamma^{+} \mathrm{CD} 4^{+} \mathrm{T}$ cells do not correlate with the degree of protection either for primary or secondary immune responses. Inactivation of Tregs prior to mycobacterial infection led to increased numbers of IFN $-\gamma^{+} \mathrm{CD}^{+} \mathrm{T}$ cells in the spleen (Figs. 3.9/3.10) but did not affect bacterial numbers in the lung (Figs. 3.11/3.13). Inactivation of Tregs prior to BCG vaccination led to increased numbers of IFN $-\gamma^{+} \mathrm{CD}^{+} \mathrm{T}$ cells in the lung and spleen after vaccination (Fig. 5.4) but did not affect bacterial numbers in the lung or spleen after infectious challenge (Figs. 5.8/5.9).

Since numbers of IFN $-\gamma^{+} \mathrm{CD} 4^{+} \mathrm{T}$ cells after vaccination do not correlate with protection from infectious challenge, vaccinologists desperately need an immunological correlate of protection from $\mathrm{Tb}$. Several alternatives have been suggested, including the timing of $\mathrm{CD}^{+} \mathrm{T}$ cell influx to the lung in vaccinated mice after challenge $[84,171$, 495] and the presence of high proportions of multifunctional Th1 CD4 ${ }^{+} \mathrm{T}$ cells [477], which are each discussed in detail below. 


\subsubsection{Timing of IFN $-\gamma^{+} \mathrm{CD} 4^{+} \mathrm{T}$ cell influx to the lung}

In $\mathrm{BCG}$ vaccinated mice, the influx of IFN $-\gamma^{+} \mathrm{CD}^{+} \mathrm{T}$ cells to the lung is earlier and correlates with bacterial numbers stabilising earlier in the lung and lower bacterial numbers compared to naïve mice [171]. Earlier expression of type I cytokines after mycobacterial challenge, including IFN- $\gamma$, was shown to occur in mice vaccinated with either BCG or a DNA vaccine cocktail encoding several mycobacterial antigens, when compared to naïve mice [495].

Recently, this concept has been extended by a study in which mice were vaccinated with mycobacterial peptide in adjuvant then challenged with $M$. tuberculosis. The influx of IFN- $\gamma^{+} \mathrm{CD}^{+}$lymphocytes to the lung was earlier and correlated with lower bacterial numbers in peptide vaccinated mice compared to naïve mice [84]. Importantly, the influx of IFN $-\gamma^{+} \mathrm{CD} 4^{+}$lymphocytes to the lung was preceded by an influx of IL-17 ${ }^{+} \mathrm{CD} 4^{+}$lymphocytes. Blocking IL-17 production diminished the influx of IFN $-\gamma^{+} \mathrm{CD}^{+}$lymphocytes. Therefore, this model of mycobacterial protective immunity after vaccination suggests that vaccination induces IL- $17^{+} \mathrm{CD} 4^{+} \mathrm{T}$ cells that infiltrate the lung earlier after challenge than in naïve animals. The IL- $17^{+} \mathrm{CD} 4^{+} \mathrm{T}$ cells recruit IFN $-\gamma^{+} \mathrm{CD} 4^{+} \mathrm{T}$ cells, which mediate control of bacterial numbers.

In this thesis, inactivation of Tregs prior to BCG vaccination accelerated the influx of IFN- $\gamma^{+} \mathrm{CD} 4^{+}$lymphocytes to the lung after challenge compared to control treated BCG vaccinated mice (Fig. 5.7). Despite acceleration of IFN- $\gamma^{+} \mathrm{CD} 4^{+}$ lymphocyte influx, Treg inactivation prior to BCG vaccination did not alter bacterial numbers in the lung compared to control treated BCG vaccinated mice (Figs. 5.8/5.9). IL-17 production was not assessed in this thesis but it would be interesting to see whether the influx of $\mathrm{IL}-17^{+} \mathrm{CD} 4^{+}$lymphocytes to the lung was also accelerated when Tregs were inactivated prior to BCG vaccination compared to control treated BCG vaccinated mice. A similar acceleration would be consistent with the proposed role of IL-17 producers mediating the influx of IFN- $\gamma^{+} \mathrm{CD} 4^{+}$lymphocytes.

Two issues arise concerning the results presented in this thesis. The first and most obvious issue is that these results call into question whether the timing of IFN$\gamma^{+} \mathrm{CD} 4^{+}$lymphocyte influx to the lung is an appropriate correlate of protection for $\mathrm{Tb}$. Since accelerating IFN $-\gamma^{+} \mathrm{CD} 4^{+}$lymphocyte influx by inactivating Tregs prior to vaccination did not correlate with changes in bacterial numbers, the timing of IFN- 
$\gamma^{+} \mathrm{CD} 4^{+}$lymphocyte influx does not appear to be a consistent correlate of protection for $\mathrm{Tb}$. It also suggests for therapeutic settings that ensuring an accelerated influx of IFN$\gamma^{+} \mathrm{CD} 4^{+}$lymphocytes to the lung upon secondary challenge will not guarantee that the resulting $\mathrm{Tb}$ vaccine is more effective.

The second issue that arises is whether IFN $-\gamma^{+} \mathrm{CD} 4^{+}$lymphocytes are actually the lymphocytes that mediate the reduction in bacterial numbers in the lung in vaccinated animals. Since the accelerated arrival of IFN- $\gamma^{+} \mathrm{CD} 4^{+}$lymphocytes did not affect bacterial numbers, it suggests that IFN $-\gamma^{+} \mathrm{CD} 4^{+}$lymphocytes are not responsible for control of bacterial numbers after infectious challenge and the kinetics or quality of another cell population that is responsible for control of bacterial numbers is unaffected by Treg inactivation. While IFN- $\gamma$ production by $\mathrm{CD}^{+} \mathrm{T}$ cells undoubtedly contributes to control of primary mycobacterial infection, cellular subsets producing other related cytokines may be responsible for protection in a vaccination setting. Early expression of type I cytokines and chemokines at the level of mRNA and protein has been demonstrated to correlate with the protective efficacies of BCG vaccination and a DNA Tb vaccine cocktail [495]. The type I cytokines and chemokines that were expressed earlier in vaccinated mice included IFN- $\gamma$ but also included IL-12 and the chemokine RANTES. In addition, earlier expression of IFN- $\gamma$ occurred not only in $\mathrm{CD} 4^{+} \mathrm{T}$ cells but also $\mathrm{CD}^{+} \mathrm{T}$ cells, as assessed by flow cytometry. This thesis focussed on the kinetics of IFN- $\gamma^{+} \mathrm{CD} 4^{+}$lymphocytes in the lung and draining lymph node upon infectious challenge and changes in the numbers or kinetics of this population did not always correlate with changes in protection. A fuller assessment of the kinetics of CD4 ${ }^{+} \mathrm{T}$ cells and $\mathrm{CD} 8^{+} \mathrm{T}$ cells producing chemokines or cytokines, such as IFN- $\gamma$, IL-2, TNF- $\alpha$, and IL-17, upon challenge in vaccinated mice, could be compared to protective efficacies of the vaccines. Correlations between the appearance of cellular subsets and protection may give important insights as to which cell type is responsible for protection from $\mathrm{Tb}$.

\subsubsection{Multifunctional Th1 lymphocytes}

In a $L$. major vaccination model, an increased proportion of $\mathrm{CD}^{+} \mathrm{T}$ cells producing IFN- $\gamma$, IL-2 and TNF- $\alpha$ amongst the total cytokine (IFN- $\gamma$, IL-2 or TNF- $\alpha$ ) producing $\mathrm{CD}^{+} \mathrm{T}$ population has been shown to correlate with protection [477]. These IFN- $\gamma$, IL2 and TNF- $\alpha$ producing $\mathrm{CD}^{+}{ }^{+} \mathrm{T}$ cells have been termed multi-functional Th1 
lymphocytes. L. major is an intracellular protozoal pathogen, which is reliant on a robust Th1 immune response for control of the infection, and this provides a model for Th1 immunity. Based on results gained in the L. major model, it has been proposed that successful vaccines relying on Th1 immunity will induce a higher proportion of multifunctional Th1 lymphocytes [477].

Multi-functional $\mathrm{CD}^{+}$Th1 lymphocytes produce increased amounts of each cytokine upon restimulation, as determined by flow cytometric analysis [477]. It has also been proposed that $\mathrm{CD}^{+} \mathrm{T}$ cells that do not exclusively produce IFN- $\gamma$ are longerlived than $\mathrm{CD}^{+} \mathrm{T}$ cells committed to exclusive IFN- $\gamma$ production [221]. Therefore, it is reasoned that multi-functional $\mathrm{CD}^{+} \mathrm{Th} 1$ lymphocytes are more effective at mediating protection than $\mathrm{CD}^{+} \mathrm{T}$ cells committed to only IFN- $\gamma$ production as they persist longer after vaccination and respond with increased amounts of these cytokines upon secondary challenge. Furthermore, it has been shown that BCG-vaccinated human subjects possess multifunctional cells that can produce all three cytokines after in vitro restimulation with mycobacterial proteins [477]. Consequently, it is proposed that successful $\mathrm{Tb}$ vaccines should promote the development of increased proportions of multi-functional Th1 lymphocytes to promote long-lived, robust Th1 immunity.

In this thesis, IFN- $\gamma$, IL-2 and TNF- $\alpha$ production by CD4 ${ }^{+} \mathrm{T}$ cells were not assessed simultaneously, but IL-2/IFN- $\gamma$ co-production by $\mathrm{CD} 4^{+} \mathrm{T}$ cells was assessed. At two weeks after either BCG i.n. infection or BCG s.c. vaccination, IL-2 and IFN- $\gamma$ production resolved to distinct populations within the $\mathrm{CD} 4{ }^{+}$lymphocyte compartment and IL-2/IFN- $\gamma$ co-producers were not observed (Figs. 3.8/3.9 and 5.2). By six weeks after BCG vaccination, a small population of IL-2/IFN- $\gamma$ co-producing CD4 ${ }^{+}$ lymphocytes was observed in both the spleen and the lung (Figs. 5.5/5.6). After DNA vaccination, an IL-2/IFN- $\gamma$ co-producing population of $\mathrm{CD}^{+}$lymphocytes was also observed when secondary immune responses were observed three days after infectious challenge (Fig. 5.14). Therefore, in the murine model, a population of IL-2/IFN- $\gamma$ coproducing $\mathrm{CD}^{+}$lymphocytes could be observed in BCG or DNA vaccinated mice after vaccination or after secondary challenge.

Numbers of IL-2/IFN- $\gamma$ co-producing $\mathrm{CD}^{+}$lymphocytes were assessed for the different vaccination groups and compared to protection data. Treg inactivation prior to BCG vaccination significantly increased the number of IL-2/IFN- $\gamma$ co-producing CD4 ${ }^{+}$ lymphocytes at six weeks after vaccination in both the lungs and spleen (Fig. 5.6). This 
did not correlate with any change in bacterial numbers in the lung or spleen after mycobacterial challenge (Figs. 5.8/5.9).

However, the proportions of IFN- $\gamma / \mathrm{IL}-2$ co-producing $\mathrm{CD} 4{ }^{+}$lymphocytes within the total IFN- $\gamma$ or IL-2 producing $\mathrm{CD}^{+}$lymphocyte population were also assessed. At 6 weeks after BCG vaccination, the proportion of IFN- $\gamma / \mathrm{IL}-2$ co-producing CD4 ${ }^{+}$ lymphocytes was unchanged by PC61 treatment (Fig. 5.6.). This data correlated with protection, since bacterial numbers in the lung and spleen after virulent infectious challenge were unchanged in BCG vaccinated mice, regardless of PC61 treatment (Figs. $5.8 / 5.9)$.

Preliminary data suggests that the proportions of IFN- $\gamma / \mathrm{IL}-2$ co-producing CD4 ${ }^{+}$ lymphocytes after $\mathrm{Tb}$ vaccination might not always correlate with differences in protection against virulent challenge. Three days after infectious challenge, the proportions of IFN- $\gamma / \mathrm{IL}-2$ co-producing $\mathrm{CD} 4^{+}$lymphocytes in the spleens and lungs of pcDNA3-85A vaccinated mice appeared unchanged, regardless of PC61 treatment (Fig. 5.14). When bacterial numbers in tissues were assessed after virulent infectious, bacterial numbers in the spleen were significantly increased in PC61 treated compared to rat IgG treated pcDNA3-85A vaccinated mice (Fig. 5.15). However, it may not be appropriate to compare the BCG vaccine and the pcDNA3-85A vaccine in the search for a broad immune correlate of protection for $\mathrm{Tb}$, as will be discussed in section 6.4.4.

Taken as a whole, these data indicate that, although numbers of IL-2/IFN- $\gamma$ coproducing $\mathrm{CD}^{+}$lymphocytes do not consistently correlate with protection, proportions of IL-2/IFN- $\gamma$ co-producing $\mathrm{CD}^{+}$lymphocytes may correlate with the protective efficacy of the BCG vaccine. The study that identified multifunctional Th1 cells assessed TNF- $\alpha$ production, as well as IL- 2 and IFN- $\gamma$ production, and the frequency of multifunctional Th1 cells amongst total cytokine producing $\mathrm{CD} 4^{+} \mathrm{T}$ cells correlated with the protective efficacy of the L. major vaccines [477]. As a result, it would be interesting to assess TNF- $\alpha$ production, as well as IL- 2 and IFN- $\gamma$ production, for the BCG, pcDNA3-85A and pKUN85A vaccines.

It would also be informative to assess the kinetics of the development of the TNF- $\alpha /$ IL-2/IFN- $\gamma$ co-producing CD4 ${ }^{+}$lymphocyte population for the BCG, pcDNA3-

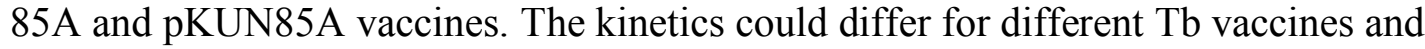
the relative numbers and proportions of this population could differ depending on the 
time after vaccination that these parameters are assessed. The proportion of TNF- $\alpha /$ IL$2 / \mathrm{IFN}-\gamma$ producing $\mathrm{CD} 4^{+} \mathrm{T}$ cells amongst total cytokine producing $\mathrm{CD} 4^{+} \mathrm{T}$ cells may prove to be a valuable immune correlate of protection for $\mathrm{Tb}$ vaccines.

\subsubsection{Summary: Immune correlates of protection for $\mathrm{Tb}$}

Overall, the findings in this thesis were used to assess the applicability of several proposed correlates of protection for $\mathrm{Tb}$ vaccination strategies. The results illustrated that the number of IFN $-\gamma^{+} \mathrm{CD} 4^{+}$lymphocytes, the timing of IFN- $\gamma^{+} \mathrm{CD} 4^{+}$lymphocyte influx to the lung and the number of IFN- $\gamma / \mathrm{IL}-2$ co-producing $\mathrm{CD} 4^{+}$lymphocytes did not consistently correlate with the protective efficacy of $\mathrm{Tb}$ vaccines. However, the proportion of IFN- $\gamma / \mathrm{IL}-2$ co-producing $\mathrm{CD}^{+}$lymphocytes may correlate with the protective efficacy of the BCG vaccine. This study did not assess TNF- $\alpha$, IFN- $\gamma$ and IL2 co-production, and therefore the applicability of the proportion of multifunctional Th1 cells as a correlate of protection against $\mathrm{Tb}$ remains an intriguing possibility. The results presented in this thesis have illustrated scenarios in which several proposed immune correlates of protection for $\mathrm{Tb}$ do not apply and hence narrowed the search for a broad immune correlate for protection against $\mathrm{Tb}$.

The search for a broad immune correlate of protection for all $\mathrm{Tb}$ vaccines assumes that there is only one protective mechanism and one protective cell subset that all $\mathrm{Tb}$ vaccines induce. An ideal correlate of protection is a parameter that is measurable early after vaccination that goes on to contribute to the mechanism of protection during infectious challenge. If there are multiple different memory cell subsets that can mediate protection and different $\mathrm{Tb}$ vaccines can induce different protective subsets, then a broad immune correlate for all $\mathrm{Tb}$ vaccines might not exist. For example, it might not be appropriate to look for a correlate shared by a live vaccine such as BCG, which is thought to rely on Th1 immunity, with a DNA vaccine, where $\mathrm{CD} 8^{+} \mathrm{T}$ cell-dependent mechanisms might contribute more to protection from infection. This highlights the importance of understanding protective mechanisms induced by vaccines before using them to search for broad immune correlates of protection encompassing all vaccines targeting a single pathogen. 


\subsection{Conclusions}

Using a murine C57BL/6 model of mycobacterial infection and vaccination and in vivo administration of anti-CD25 (clone PC61) mAb to inactivate CD25 ${ }^{+}$natural Tregs, this thesis has offered several conclusions relating to anti-mycobacterial immunity and the role of Tregs:

- The plasmid DNA replicon vaccine, pKUN85A, was created and i.m. vaccination with pKUN85A induced an Ag85A-driven immune response, as assessed by IFN- $\gamma$ and IL-2 production from $\mathrm{CD}^{+} \mathrm{T}$ cells and bystander cytokine production from $\mathrm{CD}^{+} \mathrm{T}$ cells. Preliminary data suggests homologous prime-boost vaccination with $\mathrm{pKUN85A}$ is not as protective as BCG vaccination against virulent mycobacterial challenge, although further refinements will be made to enhance pKUN85A vaccine efficacy.

- Enhanced immune responses were observed when natural Tregs were inactivated prior to mycobacterial infection.

- Inactivation of natural Tregs prior to mycobacterial infection did not affect bacterial numbers in the lung during acute mycobacterial infections, although inactivation of natural Tregs prior to $M$. bovis infection may cause transient reductions in lung pathology.

- Enhanced primary immune responses, increased numbers of antigen-responsive cells in the spleen and lung prior to secondary challenge and an accelerated secondary immune response upon secondary challenge were observed when natural Tregs were inactivated prior to BCG vaccination.

- Inactivation of Tregs prior to BCG vaccination did not affect the protective efficacy of the BCG vaccine.

- Preliminary data suggests that inactivation of Tregs prior to pcDNA3-85A vaccination modestly enhanced secondary immune responses but may have led to reduced protection in the spleen.

In summary, this thesis illustrates that BCG, M. tuberculosis and M. bovis contrast with other infection and vaccination models, as inactivation of natural CD25 $5^{+}$Tregs by in vivo treatment with PC61 does not affect protection in terms of pathogen load.

However, this thesis also highlights a general concern for $\mathrm{Tb}$ vaccine design. Using the current dogma for protective immunity against $\mathrm{Tb}$, several hypotheses were proposed 
during the course of this thesis that were later found to be incorrect. These assumptions based on the current dogma were logical but the outcomes reflect the fact that there are fundamental flaws in the current model and therefore $\mathrm{Tb}$ vaccine research lacks a robust, consistent model of protection against $\mathrm{Tb}$. As a result, researchers also currently lack an accurate immune correlate of protection for the development of a more effective $\mathrm{Tb}$ vaccine. Identification of the cellular subset responsible for protection against mycobacterial infection would facilitate rational vaccine design for $\mathrm{Tb}$, but due to this gap in our understanding, truly rational vaccine design for $\mathrm{Tb}$ is at an impasse at present. However, through empirical experimentation supported by rational hypotheses, vaccine design can progress. Lessons learned from failed and successful vaccine designs can be used as clues to build knowledge about protective cellular subsets and correlates of protection. Eventually, this knowledge will narrow the gap between immunology and vaccinology, permitting truly targeted design of $\mathrm{Tb}$ vaccines. 


\section{References}

Konomi, N., Lebwohl, E., Mowbray, K., Tattersall, I. and Zhang, D., Detection of mycobacterial DNA in Andean mummies. J Clin Microbiol 2002. 40: 4738-4740.

2 Nerlich, A. G., Haas, C. J., Zink, A., Szeimies, U. and Hagedorn, H. G., Molecular evidence for tuberculosis in an ancient Egyptian mummy. Lancet 1997. 350: 1404.

3 Salo, W. L., Aufderheide, A. C., Buikstra, J. and Holcomb, T. A., Identification of Mycobacterium tuberculosis DNA in a pre-Columbian Peruvian mummy. Proc Natl Acad Sci U S A 1994. 91: 2091-2094.

4 Zimmerman, M. R., Pulmonary and osseous tuberculosis in an Egyptian mummy. Bull N Y Acad Med 1979. 55: 604-608.

5 Global tuberculosis control: surveillance, planning, financing. WHO report 2007. Geneva, World Health Organization (WHO/HTM/TB/2007.376).

6 Shinnick, T. M. and Good, R. C., Mycobacterial taxonomy. Eur J Clin Microbiol Infect Dis 1994. 13: 884-901.

7 Fu, L. M. and Fu-Liu, C. S., Is Mycobacterium tuberculosis a closer relative to Gram-positive or Gram-negative bacterial pathogens? Tuberculosis (Edinb) 2002. 82: 85-90.

Shoub, H. L., A Comparison of the Ziehl-Neelsen and Schulte-Tigges Methods of Staining Tubercle Bacilli. J Bacteriol 1923. 8: 121-126.

9 James, B. W., Williams, A. and Marsh, P. D., The physiology and pathogenicity of Mycobacterium tuberculosis grown under controlled conditions in a defined medium. $J$ Appl Microbiol 2000. 88: 669-677.

10 Wayne, L. G., Synchronized replication of Mycobacterium tuberculosis. Infect Immun 1977. 17: 528-530.

11 North, R. J. and Izzo, A. A., Mycobacterial virulence. Virulent strains of Mycobacteria tuberculosis have faster in vivo doubling times and are better equipped to resist growth-inhibiting functions of macrophages in the presence and absence of specific immunity. J Exp Med 1993. 177: 1723-1733.

12 Tsai, M. C., Chakravarty, S., Zhu, G., Xu, J., Tanaka, K., Koch, C., Tufariello, J., et al., Characterization of the tuberculous granuloma in murine and human lungs: cellular composition and relative tissue oxygen tension. Cell Microbiol 2006. 8: 218-232.

13 Cole, S. T., Brosch, R., Parkhill, J., Garnier, T., Churcher, C., Harris, D., Gordon, S. V., et al., Deciphering the biology of Mycobacterium tuberculosis from the complete genome sequence. Nature 1998. 393: 537-544.

14 Fleischmann, R. D., Alland, D., Eisen, J. A., Carpenter, L., White, O., Peterson, J., DeBoy, R., et al., Whole-genome comparison of Mycobacterium tuberculosis clinical and laboratory strains. J Bacteriol 2002. 184: 5479-5490.

15 Garnier, T., Eiglmeier, K., Camus, J. C., Medina, N., Mansoor, H., Pryor, M., Duthoy, S., et al., The complete genome sequence of Mycobacterium bovis. Proc Natl Acad Sci U S A 2003. 100: 7877-7882.

16 Brosch, R., Gordon, S. V., Garnier, T., Eiglmeier, K., Frigui, W., Valenti, P., Dos Santos, S., et al., Genome plasticity of BCG and impact on vaccine efficacy. Proc Natl Acad Sci U S A 2007. 104: 5596-5601. 
17 Brosch, R., Pym, A. S., Gordon, S. V. and Cole, S. T., The evolution of mycobacterial pathogenicity: clues from comparative genomics. Trends Microbiol 2001. 9: 452-458.

18 Matzinger, P., Tolerance, danger, and the extended family. Annu Rev Immunol 1994. 12: 991-1045.

19 Guermonprez, P., Valladeau, J., Zitvogel, L., Thery, C. and Amigorena, S., Antigen presentation and T cell stimulation by dendritic cells. Annu Rev Immunol 2002. 20: 621-667.

20 Sugita, M., Barral, D. C. and Brenner, M. B., Pathways of CD1 and lipid antigen delivery, trafficking, processing, loading, and presentation. Curr Top Microbiol Immunol 2007. 314: 143-164.

21 Mosmann, T. R., Cherwinski, H., Bond, M. W., Giedlin, M. A. and Coffman, R. L., Two types of murine helper T cell clone. I. Definition according to profiles of lymphokine activities and secreted proteins. J Immunol 1986. 136: 2348-2357.

22 North, R. J. and Jung, Y. J., Immunity to tuberculosis. Annu Rev Immunol 2004. 22: 599-623.

23 Vynnycky, E. and Fine, P. E., Lifetime risks, incubation period, and serial interval of tuberculosis. Am J Epidemiol 2000. 152: 247-263.

24 Gatfield, J. and Pieters, J., Essential role for cholesterol in entry of mycobacteria into macrophages. Science 2000. 288: 1647-1650.

25 Bonar, A., Chmiela, M., Rudnicka, W. and Rozalska, B., Mannose-binding lectin enhances the attachment and phagocytosis of mycobacteria in vitro. Arch Immunol Ther Exp (Warsz) 2005. 53: 437-441.

26 Hickman, S. P., Chan, J. and Salgame, P., Mycobacterium tuberculosis induces differential cytokine production from dendritic cells and macrophages with divergent effects on naive $T$ cell polarization. J Immunol 2002. 168: 46364642.

27 Bodnar, K. A., Serbina, N. V. and Flynn, J. L., Fate of Mycobacterium tuberculosis within murine dendritic cells. Infect Immun 2001. 69: 800-809.

28 Scanga, C. A., Bafica, A., Feng, C. G., Cheever, A. W., Hieny, S. and Sher, A., MyD88-deficient mice display a profound loss in resistance to Mycobacterium tuberculosis associated with partially impaired Th1 cytokine and nitric oxide synthase 2 expression. Infect Immun 2004. 72: 2400-2404.

29 Fremond, C. M., Yeremeev, V., Nicolle, D. M., Jacobs, M., Quesniaux, V. F. and Ryffel, B., Fatal Mycobacterium tuberculosis infection despite adaptive immune response in the absence of MyD88. J Clin Invest 2004. 114: 1790-1799.

30 Bafica, A., Scanga, C. A., Feng, C. G., Leifer, C., Cheever, A. and Sher, A., TLR9 regulates Th1 responses and cooperates with TLR2 in mediating optimal resistance to Mycobacterium tuberculosis. J Exp Med 2005. 202: 1715-1724.

31 Ladel, C. H., Szalay, G., Riedel, D. and Kaufmann, S. H., Interleukin-12 secretion by Mycobacterium tuberculosis-infected macrophages. Infect Immun 1997. 65: 1936-1938.

32 Henderson, R. A., Watkins, S. C. and Flynn, J. L., Activation of human dendritic cells following infection with Mycobacterium tuberculosis. J Immunol 1997. 159: 635-643.

33 Cooper, A. M., Kipnis, A., Turner, J., Magram, J., Ferrante, J. and Orme, I. M., Mice lacking bioactive IL-12 can generate protective, antigen-specific cellular responses to mycobacterial infection only if the IL-12 p40 subunit is present. J Immunol 2002. 168: 1322-1327.

34 Wakeham, J., Wang, J., Magram, J., Croitoru, K., Harkness, R., Dunn, P., Zganiacz, A. and Xing, Z., Lack of both types 1 and 2 cytokines, tissue 
inflammatory responses, and immune protection during pulmonary infection by Mycobacterium bovis bacille Calmette-Guerin in IL-12-deficient mice. $J$ Immunol 1998. 160: 6101-6111.

35 Altare, F., Durandy, A., Lammas, D., Emile, J. F., Lamhamedi, S., Le Deist, F., Drysdale, P., et al., Impairment of mycobacterial immunity in human interleukin-12 receptor deficiency. Science 1998. 280: 1432-1435.

36 Feng, C. G., Kaviratne, M., Rothfuchs, A. G., Cheever, A., Hieny, S., Young, H. A., Wynn, T. A. and Sher, A., NK cell-derived IFN-gamma differentially regulates innate resistance and neutrophil response in T celldeficient hosts infected with Mycobacterium tuberculosis. J Immunol 2006. 177: 7086-7093.

37 Wang, J., Wakeham, J., Harkness, R. and Xing, Z., Macrophages are a significant source of type 1 cytokines during mycobacterial infection. $J$ Clin Invest 1999. 103: 1023-1029.

38 Fenton, M. J., Vermeulen, M. W., Kim, S., Burdick, M., Strieter, R. M. and Kornfeld, H., Induction of gamma interferon production in human alveolar macrophages by Mycobacterium tuberculosis. Infect Immun 1997. 65: 51495156.

39 Schaible, U. E., Winau, F., Sieling, P. A., Fischer, K., Collins, H. L., Hagens, K., Modlin, R. L., et al., Apoptosis facilitates antigen presentation to T lymphocytes through MHC-I and CD1 in tuberculosis. Nat Med 2003. 9: 10391046.

40 Winau, F., Weber, S., Sad, S., de Diego, J., Hoops, S. L., Breiden, B., Sandhoff, K., et al., Apoptotic vesicles crossprime CD8 T cells and protect against tuberculosis. Immunity 2006. 24: 105-117.

41 Winau, F., Kaufmann, S. H. and Schaible, U. E., Apoptosis paves the detour path for CD8 T cell activation against intracellular bacteria. Cell Microbiol 2004. 6: 599-607.

42 Guermonprez, P. and Amigorena, S., Pathways for antigen cross presentation. Springer Semin Immunopathol 2005. 26: 257-271.

43 Mazzaccaro, R. J., Gedde, M., Jensen, E. R., van Santen, H. M., Ploegh, H. L., Rock, K. L. and Bloom, B. R., Major histocompatibility class I presentation of soluble antigen facilitated by Mycobacterium tuberculosis infection. Proc Natl Acad Sci U S A 1996. 93: 11786-11791.

44 van der Wel, N., Hava, D., Houben, D., Fluitsma, D., van Zon, M., Pierson, J., Brenner, M. and Peters, P. J., M. tuberculosis and M. leprae translocate from the phagolysosome to the cytosol in myeloid cells. Cell 2007. 129: 12871298.

45 Leveton, C., Barnass, S., Champion, B., Lucas, S., De Souza, B., Nicol, M., Banerjee, D. and Rook, G., T-cell-mediated protection of mice against virulent Mycobacterium tuberculosis. Infect Immun 1989. 57: 390-395.

46 Muller, I., Cobbold, S. P., Waldmann, H. and Kaufmann, S. H., Impaired resistance to Mycobacterium tuberculosis infection after selective in vivo depletion of L3T4+ and Lyt-2+ T cells. Infect Immun 1987. 55: 2037-2041.

47 Chackerian, A. A., Perera, T. V. and Behar, S. M., Gamma interferonproducing CD4+ T lymphocytes in the lung correlate with resistance to infection with Mycobacterium tuberculosis. Infect Immun 2001. 69: 2666-2674.

Ehlers, S., Benini, J., Held, H. D., Roeck, C., Alber, G. and Uhlig, S.,

Alphabeta $\mathrm{T}$ cell receptor-positive cells and interferon-gamma, but not inducible nitric oxide synthase, are critical for granuloma necrosis in a mouse model of mycobacteria-induced pulmonary immunopathology. J Exp Med 2001. 194: 1847-1859. 
Mogues, T., Goodrich, M. E., Ryan, L., LaCourse, R. and North, R. J., The relative importance of $\mathrm{T}$ cell subsets in immunity and immunopathology of airborne Mycobacterium tuberculosis infection in mice. J Exp Med 2001. 193: 271-280.

50 Caruso, A. M., Serbina, N., Klein, E., Triebold, K., Bloom, B. R. and Flynn, J. L., Mice deficient in CD4 T cells have only transiently diminished levels of IFN-gamma, yet succumb to tuberculosis. J Immunol 1999. 162: 5407-5416.

51 Behar, S. M., Dascher, C. C., Grusby, M. J., Wang, C. R. and Brenner, M. B., Susceptibility of mice deficient in CD1D or TAP1 to infection with Mycobacterium tuberculosis. J Exp Med 1999. 189: 1973-1980.

Rolph, M. S., Raupach, B., Kobernick, H. H., Collins, H. L., Perarnau, B., Lemonnier, F. A. and Kaufmann, S. H., MHC class Ia-restricted T cells partially account for beta2-microglobulin-dependent resistance to Mycobacterium tuberculosis. Eur J Immunol 2001. 31: 1944-1949.

53 Sousa, A. O., Mazzaccaro, R. J., Russell, R. G., Lee, F. K., Turner, O. C., Hong, S., Van Kaer, L. and Bloom, B. R., Relative contributions of distinct MHC class I-dependent cell populations in protection to tuberculosis infection in mice. Proc Natl Acad Sci U S A 2000. 97: 4204-4208.

54 Urdahl, K. B., Liggitt, D. and Bevan, M. J., CD8+ T cells accumulate in the lungs of Mycobacterium tuberculosis-infected Kb-/-Db-/- mice, but provide minimal protection. J Immunol 2003. 170: 1987-1994.

55 Schaible, U. E., Collins, H. L., Priem, F. and Kaufmann, S. H., Correction of the iron overload defect in beta-2-microglobulin knockout mice by lactoferrin abolishes their increased susceptibility to tuberculosis. $J$ Exp Med 2002. 196: 1507-1513.

56 Fischer, K., Scotet, E., Niemeyer, M., Koebernick, H., Zerrahn, J., Maillet, S., Hurwitz, R., et al., Mycobacterial phosphatidylinositol mannoside is a natural antigen for CD1d-restricted T cells. Proc Natl Acad Sci U S A 2004. 101: 10685-10690.

57 Sieling, P. A., Chatterjee, D., Porcelli, S. A., Prigozy, T. I., Mazzaccaro, R. J., Soriano, T., Bloom, B. R., et al., CD1-restricted T cell recognition of microbial lipoglycan antigens. Science 1995. 269: 227-230.

58 Skold, M. and Behar, S. M., Role of CD1d-restricted NKT cells in microbial immunity. Infect Immun 2003. 71: 5447-5455.

59 Tanaka, Y., Morita, C. T., Tanaka, Y., Nieves, E., Brenner, M. B. and Bloom, B. R., Natural and synthetic non-peptide antigens recognized by human gamma delta T cells. Nature 1995. 375: 155-158.

60 D'Souza, C. D., Cooper, A. M., Frank, A. A., Mazzaccaro, R. J., Bloom, B. R. and Orme, I. M., An anti-inflammatory role for gamma delta T lymphocytes in acquired immunity to Mycobacterium tuberculosis. J Immunol 1997. 158: 1217-1221.

61 Ladel, C. H., Hess, J., Daugelat, S., Mombaerts, P., Tonegawa, S. and Kaufmann, S. H., Contribution of alpha/beta and gamma/delta T lymphocytes to immunity against Mycobacterium bovis bacillus Calmette Guerin: studies with T cell receptor-deficient mutant mice. Eur J Immunol 1995. 25: 838-846. Itohara, S., Nakanishi, N., Kanagawa, O., Kubo, R. and Tonegawa, S., Monoclonal antibodies specific to native murine T-cell receptor gamma delta: analysis of gamma delta $\mathrm{T}$ cells during thymic ontogeny and in peripheral lymphoid organs. Proc Natl Acad Sci U S A 1989. 86: 5094-5098. Wherry, E. J., Teichgraber, V., Becker, T. C., Masopust, D., Kaech, S. M., Antia, R., von Andrian, U. H. and Ahmed, R., Lineage relationship and 
protective immunity of memory CD8 T cell subsets. Nat Immunol 2003. 4: 225234.

64 Cooper, A. M., Dalton, D. K., Stewart, T. A., Griffin, J. P., Russell, D. G. and Orme, I. M., Disseminated tuberculosis in interferon gamma genedisrupted mice. J Exp Med 1993. 178: 2243-2247.

65 Flynn, J. L., Chan, J., Triebold, K. J., Dalton, D. K., Stewart, T. A. and Bloom, B. R., An essential role for interferon gamma in resistance to Mycobacterium tuberculosis infection. J Exp Med 1993. 178: 2249-2254.

66 Dalton, D. K., Pitts-Meek, S., Keshav, S., Figari, I. S., Bradley, A. and Stewart, T. A., Multiple defects of immune cell function in mice with disrupted interferon-gamma genes. Science 1993. 259: 1739-1742.

67 Jouanguy, E., Altare, F., Lamhamedi, S., Revy, P., Emile, J. F., Newport, M., Levin, M., et al., Interferon-gamma-receptor deficiency in an infant with fatal bacille Calmette-Guerin infection. N Engl J Med 1996. 335: 1956-1961.

68 Newport, M. J., Huxley, C. M., Huston, S., Hawrylowicz, C. M., Oostra, B. A., Williamson, R. and Levin, M., A mutation in the interferon-gammareceptor gene and susceptibility to mycobacterial infection. N Engl J Med 1996. 335: 1941-1949.

69 Junqueira-Kipnis, A. P., Kipnis, A., Jamieson, A., Juarrero, M. G., Diefenbach, A., Raulet, D. H., Turner, J. and Orme, I. M., NK cells respond to pulmonary infection with Mycobacterium tuberculosis, but play a minimal role in protection. $J$ Immunol 2003. 171: 6039-6045.

70 Emoto, M., Emoto, Y., Buchwalow, I. B. and Kaufmann, S. H., Induction of IFN-gamma-producing CD4+ natural killer T cells by Mycobacterium bovis bacillus Calmette Guerin. Eur J Immunol 1999. 29: 650-659.

71 Feng, C. G., Bean, A. G., Hooi, H., Briscoe, H. and Britton, W. J., Increase in gamma interferon-secreting $\mathrm{CD} 8(+)$, as well as CD4(+), T cells in lungs following aerosol infection with Mycobacterium tuberculosis. Infect Immun 1999. 67: 3242-3247.

72 Orme, I. M., Roberts, A. D., Griffin, J. P. and Abrams, J. S., Cytokine secretion by CD4 T lymphocytes acquired in response to Mycobacterium tuberculosis infection. J Immunol 1993. 151: 518-525.

73 Flynn, J. L., Goldstein, M. M., Chan, J., Triebold, K. J., Pfeffer, K., Lowenstein, C. J., Schreiber, R., et al., Tumor necrosis factor-alpha is required in the protective immune response against Mycobacterium tuberculosis in mice. Immunity 1995. 2: 561-572.

74 Mohan, V. P., Scanga, C. A., Yu, K., Scott, H. M., Tanaka, K. E., Tsang, E., Tsai, M. M., et al., Effects of tumor necrosis factor alpha on host immune response in chronic persistent tuberculosis: possible role for limiting pathology. Infect Immun 2001. 69: 1847-1855.

75 Keane, J., Gershon, S., Wise, R. P., Mirabile-Levens, E., Kasznica, J., Schwieterman, W. D., Siegel, J. N. and Braun, M. M., Tuberculosis associated with infliximab, a tumor necrosis factor alpha-neutralizing agent. $N$ Engl J Med 2001. 345: 1098-1104.

76 Jovanovic, D. V., Di Battista, J. A., Martel-Pelletier, J., Jolicoeur, F. C., He, Y., Zhang, M., Mineau, F. and Pelletier, J. P., IL-17 stimulates the production and expression of proinflammatory cytokines, IL-beta and TNF-alpha, by human macrophages. J Immunol 1998. 160: 3513-3521.

77 Laan, M., Cui, Z. H., Hoshino, H., Lotvall, J., Sjostrand, M., Gruenert, D. C., Skoogh, B. E. and Linden, A., Neutrophil recruitment by human IL-17 via C-X-C chemokine release in the airways. J Immunol 1999. 162: 2347-2352. 
78 Lockhart, E., Green, A. M. and Flynn, J. L., IL-17 production is dominated by gammadelta T cells rather than CD4 T cells during Mycobacterium tuberculosis infection. J Immunol 2006. 177: 4662-4669.

Bettelli, E., Carrier, Y., Gao, W., Korn, T., Strom, T. B., Oukka, M., Weiner, H. L. and Kuchroo, V. K., Reciprocal developmental pathways for the generation of pathogenic effector TH17 and regulatory T cells. Nature 2006. 441: 235-238.

80 Veldhoen, M., Hocking, R. J., Atkins, C. J., Locksley, R. M. and Stockinger, B., TGFbeta in the context of an inflammatory cytokine milieu supports de novo differentiation of IL-17-producing T cells. Immunity 2006. 24: 179-189.

81 Mangan, P. R., Harrington, L. E., O'Quinn, D. B., Helms, W. S., Bullard, D. C., Elson, C. O., Hatton, R. D., et al., Transforming growth factor-beta induces development of the $\mathrm{T}(\mathrm{H}) 17$ lineage. Nature 2006. 441: 231-234.

82 Happel, K. I., Lockhart, E. A., Mason, C. M., Porretta, E., Keoshkerian, E., Odden, A. R., Nelson, S. and Ramsay, A. J., Pulmonary interleukin-23 gene delivery increases local T-cell immunity and controls growth of Mycobacterium tuberculosis in the lungs. Infect Immun 2005. 73: 5782-5788.

83 Wozniak, T. M., Ryan, A. A., Triccas, J. A. and Britton, W. J., Plasmid interleukin-23 (IL-23), but not plasmid IL-27, enhances the protective efficacy of a DNA vaccine against Mycobacterium tuberculosis infection. Infect Immun 2006. 74: 557-565.

84 Khader, S. A., Bell, G. K., Pearl, J. E., Fountain, J. J., Rangel-Moreno, J., Cilley, G. E., Shen, F., et al., IL-23 and IL-17 in the establishment of protective pulmonary CD4+ T cell responses after vaccination and during Mycobacterium tuberculosis challenge. Nat Immunol 2007. 8: 369-377.

85 Saunders, B. M., Frank, A. A., Orme, I. M. and Cooper, A. M., Interleukin-6 induces early gamma interferon production in the infected lung but is not required for generation of specific immunity to Mycobacterium tuberculosis infection. Infect Immun 2000. 68: 3322-3326.

86 Neufert, C., Becker, C., Wirtz, S., Fantini, M. C., Weigmann, B., Galle, P. R. and Neurath, M. F., IL-27 controls the development of inducible regulatory T cells and Th17 cells via differential effects on STAT1. Eur J Immunol 2007. 37: 1809-1816.

87 Pearl, J. E., Khader, S. A., Solache, A., Gilmartin, L., Ghilardi, N., deSauvage, F. and Cooper, A. M., IL-27 signaling compromises control of bacterial growth in mycobacteria-infected mice. J Immunol 2004. 173: 74907496.

88 Refaeli, Y., Van Parijs, L., Alexander, S. I. and Abbas, A. K., Interferon gamma is required for activation-induced death of T lymphocytes. $J$ Exp Med 2002. 196: 999-1005.

89 Li, X., McKinstry, K. K., Swain, S. L. and Dalton, D. K., IFN-gamma acts directly on activated CD4+ T cells during mycobacterial infection to promote apoptosis by inducing components of the intracellular apoptosis machinery and by inducing extracellular proapoptotic signals. J Immunol 2007. 179: 939-949.

90 Dalton, D. K., Haynes, L., Chu, C. Q., Swain, S. L. and Wittmer, S., Interferon gamma eliminates responding CD4 T cells during mycobacterial infection by inducing apoptosis of activated CD4 T cells. J Exp Med 2000. 192: 117-122.

91 North, R. J., Mice incapable of making IL-4 or IL-10 display normal resistance to infection with Mycobacterium tuberculosis. Clin Exp Immunol 1998. 113: 5558 . 
92 Desjardins, M., Biogenesis of phagolysosomes: the 'kiss and run' hypothesis. Trends Cell Biol 1995. 5: 183-186.

93 Desjardins, M., Huber, L. A., Parton, R. G. and Griffiths, G., Biogenesis of phagolysosomes proceeds through a sequential series of interactions with the endocytic apparatus. J Cell Biol 1994. 124: 677-688.

94 Mellman, I., Fuchs, R. and Helenius, A., Acidification of the endocytic and exocytic pathways. Annu Rev Biochem 1986. 55: 663-700.

95 Ohkuma, S. and Poole, B., Fluorescence probe measurement of the intralysosomal $\mathrm{pH}$ in living cells and the perturbation of $\mathrm{pH}$ by various agents. Proc Natl Acad Sci U S A 1978. 75: 3327-3331.

96 Cohn, Z. A., The fate of bacteria within phagocytic cells. I. The degradation of isotopically labeled bacteria by polymorphonuclear leucocytes and macrophages. J Exp Med 1963. 117: 27-42.

97 Gutierrez, M. G., Master, S. S., Singh, S. B., Taylor, G. A., Colombo, M. I. and Deretic, V., Autophagy is a defense mechanism inhibiting BCG and Mycobacterium tuberculosis survival in infected macrophages. Cell 2004. 119: 753-766.

98 Singh, S. B., Davis, A. S., Taylor, G. A. and Deretic, V., Human IRGM induces autophagy to eliminate intracellular mycobacteria. Science 2006. 313: 1438-1441.

99 Adams, L. B., Dinauer, M. C., Morgenstern, D. E. and Krahenbuhl, J. L., Comparison of the roles of reactive oxygen and nitrogen intermediates in the host response to Mycobacterium tuberculosis using transgenic mice. Tuber Lung Dis 1997. 78: 237-246.

100 Chan, J., Xing, Y., Magliozzo, R. S. and Bloom, B. R., Killing of virulent Mycobacterium tuberculosis by reactive nitrogen intermediates produced by activated murine macrophages. J Exp Med 1992. 175: 1111-1122.

101 Flesch, I. E. and Kaufmann, S. H., Attempts to characterize the mechanisms involved in mycobacterial growth inhibition by gamma-interferon-activated bone marrow macrophages. Infect Immun 1988. 56: 1464-1469.

102 Flesch, I. E. and Kaufmann, S. H., Mechanisms involved in mycobacterial growth inhibition by gamma interferon-activated bone marrow macrophages: role of reactive nitrogen intermediates. Infect Immun 1991. 59: 3213-3218.

103 Walker, L. and Lowrie, D. B., Killing of Mycobacterium microti by immunologically activated macrophages. Nature 1981. 293: 69-71.

104 Cooper, A. M., Segal, B. H., Frank, A. A., Holland, S. M. and Orme, I. M., Transient loss of resistance to pulmonary tuberculosis in $\mathrm{p} 47$ (phox-/-) mice. Infect Immun 2000. 68: 1231-1234.

105 Flesch, I. and Kaufmann, S. H., Mycobacterial growth inhibition by interferon-gamma-activated bone marrow macrophages and differential susceptibility among strains of Mycobacterium tuberculosis. J Immunol 1987. 138: 4408-4413.

106 Denis, M., Interferon-gamma-treated murine macrophages inhibit growth of tubercle bacilli via the generation of reactive nitrogen intermediates. Cell Immunol 1991. 132: 150-157.

107 Flesch, I. E., Hess, J. H., Oswald, I. P. and Kaufmann, S. H., Growth inhibition of Mycobacterium bovis by IFN-gamma stimulated macrophages: regulation by endogenous tumor necrosis factor-alpha and by IL-10. Int Immunol 1994. 6: 693-700.

108 Flynn, J. L., Scanga, C. A., Tanaka, K. E. and Chan, J., Effects of aminoguanidine on latent murine tuberculosis. J Immunol 1998. 160: 17961803. 
109 Nicholson, S., Bonecini-Almeida Mda, G., Lapa e Silva, J. R., Nathan, C., Xie, Q. W., Mumford, R., Weidner, J. R., et al., Inducible nitric oxide synthase in pulmonary alveolar macrophages from patients with tuberculosis. $J$ Exp Med 1996. 183: 2293-2302.

110 Wang, C. H., Liu, C. Y., Lin, H. C., Yu, C. T., Chung, K. F. and Kuo, H. P., Increased exhaled nitric oxide in active pulmonary tuberculosis due to inducible NO synthase upregulation in alveolar macrophages. Eur Respir J 1998. 11: 809815.

111 De Libero, G., Flesch, I. and Kaufmann, S. H., Mycobacteria-reactive Lyt-2+ T cell lines. Eur J Immunol 1988. 18: 59-66.

112 Martineau, A. R., Newton, S. M., Wilkinson, K. A., Kampmann, B., Hall, B. M., Nawroly, N., Packe, G. E., et al., Neutrophil-mediated innate immune resistance to mycobacteria. J Clin Invest 2007. 117: 1988-1994.

113 Esin, S., Batoni, G., Pardini, M., Favilli, F., Bottai, D., Maisetta, G., Florio, W., et al., Functional characterization of human natural killer cells responding to Mycobacterium bovis bacille Calmette-Guerin. Immunology 2004. 112: 143152.

114 Gansert, J. L., Kiessler, V., Engele, M., Wittke, F., Rollinghoff, M., Krensky, A. M., Porcelli, S. A., et al., Human NKT cells express granulysin and exhibit antimycobacterial activity. J Immunol 2003. 170: 3154-3161.

115 Dieli, F., Ivanyi, J., Marsh, P., Williams, A., Naylor, I., Sireci, G., Caccamo, N., et al., Characterization of lung gamma delta T cells following intranasal infection with Mycobacterium bovis bacillus Calmette-Guerin. J Immunol 2003. 170: $463-469$.

116 Silva, C. L. and Lowrie, D. B., Identification and characterization of murine cytotoxic T cells that kill Mycobacterium tuberculosis. Infect Immun 2000. 68: 3269-3274.

117 Serbina, N. V. and Flynn, J. L., Early emergence of CD8(+) T cells primed for production of type 1 cytokines in the lungs of Mycobacterium tuberculosisinfected mice. Infect Immun 1999. 67: 3980-3988.

118 Canaday, D. H., Wilkinson, R. J., Li, Q., Harding, C. V., Silver, R. F. and Boom, W. H., CD4(+) and CD8(+) T cells kill intracellular Mycobacterium tuberculosis by a perforin and Fas/Fas ligand-independent mechanism. $J$ Immunol 2001. 167: 2734-2742.

119 Stenger, S., Mazzaccaro, R. J., Uyemura, K., Cho, S., Barnes, P. F., Rosat, J. P., Sette, A., et al., Differential effects of cytolytic T cell subsets on intracellular infection. Science 1997. 276: 1684-1687.

120 Cooper, A. M., D'Souza, C., Frank, A. A. and Orme, I. M., The course of Mycobacterium tuberculosis infection in the lungs of mice lacking expression of either perforin- or granzyme-mediated cytolytic mechanisms. Infect Immun 1997. 65: 1317-1320.

121 Laochumroonvorapong, P., Wang, J., Liu, C. C., Ye, W., Moreira, A. L., Elkon, K. B., Freedman, V. H. and Kaplan, G., Perforin, a cytotoxic molecule which mediates cell necrosis, is not required for the early control of mycobacterial infection in mice. Infect Immun 1997. 65: 127-132.

122 Stenger, S., Hanson, D. A., Teitelbaum, R., Dewan, P., Niazi, K. R., Froelich, C. J., Ganz, T., et al., An antimicrobial activity of cytolytic T cells mediated by granulysin. Science 1998. 282: 121-125.

123 Ernst, W. A., Thoma-Uszynski, S., Teitelbaum, R., Ko, C., Hanson, D. A., Clayberger, C., Krensky, A. M., et al., Granulysin, a T cell product, kills bacteria by altering membrane permeability. J Immunol 2000. 165: 7102-7108. 
124 Clayberger, C. and Krensky, A. M., Granulysin. Curr Opin Immunol 2003. 15: 560-565.

125 Saunders, B. M., Frank, A. A. and Orme, I. M., Granuloma formation is required to contain bacillus growth and delay mortality in mice chronically infected with Mycobacterium tuberculosis. Immunology 1999. 98: 324-328.

126 Ulrichs, T., Kosmiadi, G. A., Trusov, V., Jorg, S., Pradl, L., Titukhina, M., Mishenko, V., et al., Human tuberculous granulomas induce peripheral lymphoid follicle-like structures to orchestrate local host defence in the lung. $J$ Pathol 2004. 204: 217-228.

127 Cosma, C. L., Humbert, O. and Ramakrishnan, L., Superinfecting mycobacteria home to established tuberculous granulomas. Nat Immunol 2004. 5: $828-835$.

128 Algood, H. M., Lin, P. L., Yankura, D., Jones, A., Chan, J. and Flynn, J. L., TNF influences chemokine expression of macrophages in vitro and that of $\mathrm{CD} 11 \mathrm{~b}+$ cells in vivo during Mycobacterium tuberculosis infection. J Immunol 2004. 172: 6846-6857.

129 Scanga, C. A., Mohan, V. P., Yu, K., Joseph, H., Tanaka, K., Chan, J. and Flynn, J. L., Depletion of CD4(+) T cells causes reactivation of murine persistent tuberculosis despite continued expression of interferon gamma and nitric oxide synthase 2. J Exp Med 2000. 192: 347-358.

130 Gonzalez-Juarrero, M., Turner, O. C., Turner, J., Marietta, P., Brooks, J. V. and Orme, I. M., Temporal and spatial arrangement of lymphocytes within lung granulomas induced by aerosol infection with Mycobacterium tuberculosis. Infect Immun 2001. 69: 1722-1728.

131 Turner, J., D'Souza, C. D., Pearl, J. E., Marietta, P., Noel, M., Frank, A. A., Appelberg, R., et al., CD8- and CD95/95L-dependent mechanisms of resistance in mice with chronic pulmonary tuberculosis. Am J Respir Cell Mol Biol 2001. 24: 203-209.

132 Lazarevic, V., Nolt, D. and Flynn, J. L., Long-term control of Mycobacterium tuberculosis infection is mediated by dynamic immune responses. J Immunol 2005. 175: 1107-1117.

133 Corbett, E. L., Watt, C. J., Walker, N., Maher, D., Williams, B. G., Raviglione, M. C. and Dye, C., The growing burden of tuberculosis: global trends and interactions with the HIV epidemic. Arch Intern Med 2003. 163: 1009-1021.

134 Turner, J., Gonzalez-Juarrero, M., Ellis, D. L., Basaraba, R. J., Kipnis, A., Orme, I. M. and Cooper, A. M., In vivo IL-10 production reactivates chronic pulmonary tuberculosis in C57BL/6 mice. J Immunol 2002. 169: 6343-6351.

135 Verbon, A., Juffermans, N., Van Deventer, S. J., Speelman, P., Van Deutekom, H. and Van Der Poll, T., Serum concentrations of cytokines in patients with active tuberculosis (TB) and after treatment. Clin Exp Immunol 1999. 115: 110-113.

136 Toossi, Z., Gogate, P., Shiratsuchi, H., Young, T. and Ellner, J. J., Enhanced production of TGF-beta by blood monocytes from patients with active tuberculosis and presence of TGF-beta in tuberculous granulomatous lung lesions. J Immunol 1995. 154: 465-473.

137 Dahl, K. E., Shiratsuchi, H., Hamilton, B. D., Ellner, J. J. and Toossi, Z., Selective induction of transforming growth factor beta in human monocytes by lipoarabinomannan of Mycobacterium tuberculosis. Infect Immun 1996. 64: 399405.

138 Hirsch, C. S., Ellner, J. J., Blinkhorn, R. and Toossi, Z., In vitro restoration of $\mathrm{T}$ cell responses in tuberculosis and augmentation of monocyte effector 
function against Mycobacterium tuberculosis by natural inhibitors of transforming growth factor beta. Proc Natl Acad Sci U S A 1997. 94: 3926-3931.

139 Voskuil, M. I., Schnappinger, D., Visconti, K. C., Harrell, M. I., Dolganov, G. M., Sherman, D. R. and Schoolnik, G. K., Inhibition of respiration by nitric oxide induces a Mycobacterium tuberculosis dormancy program. J Exp Med 2003. 198: 705-713.

140 Schnappinger, D., Ehrt, S., Voskuil, M. I., Liu, Y., Mangan, J. A., Monahan, I. M., Dolganov, G., et al., Transcriptional Adaptation of Mycobacterium tuberculosis within Macrophages: Insights into the Phagosomal Environment. J Exp Med 2003. 198: 693-704.

141 Shi, L., Jung, Y. J., Tyagi, S., Gennaro, M. L. and North, R. J., Expression of Th1-mediated immunity in mouse lungs induces a Mycobacterium tuberculosis transcription pattern characteristic of nonreplicating persistence. Proc Natl Acad Sci U S A 2003. 100: 241-246.

142 Yuan, Y., Crane, D. D., Simpson, R. M., Zhu, Y. Q., Hickey, M. J., Sherman, D. R. and Barry, C. E., 3rd, The 16-kDa alpha-crystallin (Acr) protein of Mycobacterium tuberculosis is required for growth in macrophages. Proc Natl Acad Sci U S A 1998. 95: 9578-9583.

143 Wayne, L. G. and Sramek, H. A., Antigenic differences between extracts of actively replicating and synchronized resting cells of Mycobacterium tuberculosis. Infect Immun 1979. 24: 363-370.

144 Sorensen, A. L., Nagai, S., Houen, G., Andersen, P. and Andersen, A. B., Purification and characterization of a low-molecular-mass T-cell antigen secreted by Mycobacterium tuberculosis. Infect Immun 1995. 63: 1710-1717.

145 Demissie, A., Leyten, E. M., Abebe, M., Wassie, L., Aseffa, A., Abate, G., Fletcher, H., et al., Recognition of stage-specific mycobacterial antigens differentiates between acute and latent infections with Mycobacterium tuberculosis. Clin Vaccine Immunol 2006. 13: 179-186.

146 Roupie, V., Romano, M., Zhang, L., Korf, H., Lin, M. Y., Franken, K. L., Ottenhoff, T. H., et al., Immunogenicity of eight dormancy regulon-encoded proteins of Mycobacterium tuberculosis in DNA-vaccinated and tuberculosisinfected mice. Infect Immun 2007. 75: 941-949.

147 Armstrong, J. A. and Hart, P. D., Response of cultured macrophages to Mycobacterium tuberculosis, with observations on fusion of lysosomes with phagosomes. J Exp Med 1971. 134: 713-740.

148 Hart, P. D., Armstrong, J. A., Brown, C. A. and Draper, P., Ultrastructural study of the behavior of macrophages toward parasitic mycobacteria. Infect Immun 1972. 5: 803-807.

149 Mwandumba, H. C., Russell, D. G., Nyirenda, M. H., Anderson, J., White, S. A., Molyneux, M. E. and Squire, S. B., Mycobacterium tuberculosis resides in nonacidified vacuoles in endocytically competent alveolar macrophages from patients with tuberculosis and HIV infection. J Immunol 2004. 172: 4592-4598.

150 Russell, D. G., Dant, J. and Sturgill-Koszycki, S., Mycobacterium avium- and Mycobacterium tuberculosis-containing vacuoles are dynamic, fusion-competent vesicles that are accessible to glycosphingolipids from the host cell plasmalemma. J Immunol 1996. 156: 4764-4773.

151 Ferrari, G., Langen, H., Naito, M. and Pieters, J., A coat protein on phagosomes involved in the intracellular survival of mycobacteria. Cell 1999. 97: 435-447.

152 Suzuki, K., Takeshita, F., Nakata, N., Ishii, N. and Makino, M., Localization of CORO1A in the Macrophages Containing Mycobacterium leprae. Acta Histochem Cytochem 2006. 39: 107-112. 
153 Crowle, A. J., Dahl, R., Ross, E. and May, M. H., Evidence that vesicles containing living, virulent Mycobacterium tuberculosis or Mycobacterium avium in cultured human macrophages are not acidic. Infect Immun 1991. 59: 18231831.

154 Sturgill-Koszycki, S., Schlesinger, P. H., Chakraborty, P., Haddix, P. L., Collins, H. L., Fok, A. K., Allen, R. D., et al., Lack of acidification in Mycobacterium phagosomes produced by exclusion of the vesicular protonATPase. Science 1994. 263: 678-681.

155 Xu, S., Cooper, A., Sturgill-Koszycki, S., van Heyningen, T., Chatterjee, D., Orme, I., Allen, P. and Russell, D. G., Intracellular trafficking in Mycobacterium tuberculosis and Mycobacterium avium-infected macrophages. $J$ Immunol 1994. 153: 2568-2578.

156 Gordon, A. H., Hart, P. D. and Young, M. R., Ammonia inhibits phagosomelysosome fusion in macrophages. Nature 1980. 286: 79-80.

157 Chan, J., Fan, X. D., Hunter, S. W., Brennan, P. J. and Bloom, B. R., Lipoarabinomannan, a possible virulence factor involved in persistence of Mycobacterium tuberculosis within macrophages. Infect Immun 1991. 59: 17551761.

158 Chan, J., Fujiwara, T., Brennan, P., McNeil, M., Turco, S. J., Sibille, J. C., Snapper, M., et al., Microbial glycolipids: possible virulence factors that scavenge oxygen radicals. Proc Natl Acad Sci U S A 1989. 86: 2453-2457.

159 Ehrt, S., Shiloh, M. U., Ruan, J., Choi, M., Gunzburg, S., Nathan, C., Xie, Q. and Riley, L. W., A novel antioxidant gene from Mycobacterium tuberculosis. J Exp Med 1997. 186: 1885-1896.

160 Ruan, J., St John, G., Ehrt, S., Riley, L. and Nathan, C., noxR3, a novel gene from Mycobacterium tuberculosis, protects Salmonella typhimurium from nitrosative and oxidative stress. Infect Immun 1999. 67: 3276-3283.

161 Bryk, R., Lima, C. D., Erdjument-Bromage, H., Tempst, P. and Nathan, C., Metabolic enzymes of mycobacteria linked to antioxidant defense by a thioredoxin-like protein. Science 2002. 295: 1073-1077.

162 St John, G., Brot, N., Ruan, J., Erdjument-Bromage, H., Tempst, P., Weissbach, H. and Nathan, C., Peptide methionine sulfoxide reductase from Escherichia coli and Mycobacterium tuberculosis protects bacteria against oxidative damage from reactive nitrogen intermediates. Proc Natl Acad Sci US A 2001. 98: 9901-9906.

163 Ting, L. M., Kim, A. C., Cattamanchi, A. and Ernst, J. D., Mycobacterium tuberculosis inhibits IFN-gamma transcriptional responses without inhibiting activation of STAT1. J Immunol 1999. 163: 3898-3906.

164 Pai, R. K., Convery, M., Hamilton, T. A., Boom, W. H. and Harding, C. V., Inhibition of IFN-gamma-induced class II transactivator expression by a $19-\mathrm{kDa}$ lipoprotein from Mycobacterium tuberculosis: a potential mechanism for immune evasion. J Immunol 2003. 171: 175-184.

165 Hmama, Z., Gabathuler, R., Jefferies, W. A., de Jong, G. and Reiner, N. E., Attenuation of HLA-DR expression by mononuclear phagocytes infected with Mycobacterium tuberculosis is related to intracellular sequestration of immature class II heterodimers. J Immunol 1998. 161: 4882-4893.

166 Noss, E. H., Harding, C. V. and Boom, W. H., Mycobacterium tuberculosis inhibits MHC class II antigen processing in murine bone marrow macrophages. Cell Immunol 2000. 201: 63-74.

167 Mittrucker, H. W., Steinhoff, U., Kohler, A., Krause, M., Lazar, D., Mex, P., Miekley, D. and Kaufmann, S. H., Poor correlation between BCG 
vaccination-induced $\mathrm{T}$ cell responses and protection against tuberculosis. Proc Natl Acad Sci U S A 2007. 104: 12434-12439.

168 Lefford, M. J., Transfer of adoptive immunity to tuberculosis in mice. Infect Immun 1975. 11: 1174-1181.

169 Pedrazzini, T., Hug, K. and Louis, J. A., Importance of L3T4+ and Lyt-2+ cells in the immunologic control of infection with Mycobacterium bovis strain bacillus Calmette-Guerin in mice. Assessment by elimination of T cell subsets in vivo. J Immunol 1987. 139: 2032-2037.

170 Feng, C. G. and Britton, W. J., CD4+ and CD8+ T cells mediate adoptive immunity to aerosol infection of Mycobacterium bovis bacillus Calmette-Guerin. J Infect Dis 2000. 181: 1846-1849.

171 Jung, Y. J., Ryan, L., LaCourse, R. and North, R. J., Properties and protective value of the secondary versus primary $\mathrm{T}$ helper type 1 response to airborne Mycobacterium tuberculosis infection in mice. J Exp Med 2005. 201: 1915-1924.

172 Cowley, S. C. and Elkins, K. L., CD4+ T cells mediate IFN-gammaindependent control of Mycobacterium tuberculosis infection both in vitro and in vivo. J Immunol 2003. 171: 4689-4699.

173 Goldsack, L. and Kirman, J. R., Half-truths and selective memory: Interferon gamma, CD4(+) T cells and protective memory against tuberculosis.

Tuberculosis (Edinb) 2007.

174 Flynn, J. L., Lessons from experimental Mycobacterium tuberculosis infections. Microbes Infect 2006. 8: 1179-1188.

175 Medina, E. and North, R. J., Resistance ranking of some common inbred mouse strains to Mycobacterium tuberculosis and relationship to major histocompatibility complex haplotype and Nramp1 genotype. Immunology 1998. 93: $270-274$.

176 Chackerian, A. A., Alt, J. M., Perera, T. V., Dascher, C. C. and Behar, S. M., Dissemination of Mycobacterium tuberculosis is influenced by host factors and precedes the initiation of T-cell immunity. Infect Immun 2002. 70: 45014509.

177 Dheda, K., Booth, H., Huggett, J. F., Johnson, M. A., Zumla, A. and Rook, G. A., Lung remodeling in pulmonary tuberculosis. J Infect Dis 2005. 192: 1201-1209.

178 Gehr, P., Mwangi, D. K., Ammann, A., Maloiy, G. M., Taylor, C. R. and Weibel, E. R., Design of the mammalian respiratory system. V. Scaling morphometric pulmonary diffusing capacity to body mass: wild and domestic mammals. Respir Physiol 1981. 44: 61-86.

179 Co, D. O., Hogan, L. H., Kim, S. I. and Sandor, M., Mycobacterial granulomas: keys to a long-lasting host-pathogen relationship. Clin Immunol 2004. 113: 130-136.

180 Grode, L., Seiler, P., Baumann, S., Hess, J., Brinkmann, V., Nasser Eddine, A., Mann, P., et al., Increased vaccine efficacy against tuberculosis of recombinant Mycobacterium bovis bacille Calmette-Guerin mutants that secrete listeriolysin. J Clin Invest 2005. 115: 2472-2479.

181 Hinchey, J., Lee, S., Jeon, B. Y., Basaraba, R. J., Venkataswamy, M. M., Chen, B., Chan, J., et al., Enhanced priming of adaptive immunity by a proapoptotic mutant of Mycobacterium tuberculosis. J Clin Invest 2007. 117: 2279-2288.

182 Saxena, R. K., Weissman, D., Simpson, J. and Lewis, D. M., Murine model of BCG lung infection: dynamics of lymphocyte subpopulations in lung interstitium and tracheal lymph nodes. J Biosci 2002. 27: 143-153. 
183 Fulton, S. A., Martin, T. D., Redline, R. W. and Henry Boom, W., Pulmonary immune responses during primary mycobacterium bovis- CalmetteGuerin bacillus infection in C57Bl/6 mice. Am J Respir Cell Mol Biol 2000. 22: 333-343.

184 Alsaadi, A. I. and Smith, D. W., The fate of virulent and attenuated Mycobacteria in guinea pigs infected by the respiratory route. Am Rev Respir Dis 1973. 107: 1041-1046.

185 Lurie, M. B., Zappasodi, P. and Tickner, C., On the nature of genetic resistance to tuberculosis in the light of the host-parasite relationships in natively resistant and susceptible rabbits. Am Rev Tuberc 1955. 72: 297-329.

186 Smith, D. W. and Harding, G. E., Animal model of human disease. Pulmonary tuberculosis. Animal model: Experimental airborne tuberculosis in the guinea pig. Am J Pathol 1977. 89: 273-276.

187 Capuano, S. V., 3rd, Croix, D. A., Pawar, S., Zinovik, A., Myers, A., Lin, P. L., Bissel, S., et al., Experimental Mycobacterium tuberculosis infection of cynomolgus macaques closely resembles the various manifestations of human M. tuberculosis infection. Infect Immun 2003. 71: 5831-5844.

188 Plotkin, S. A., Vaccines: past, present and future. Nat Med 2005. 11: S5-11.

189 Graham, B. S., Pathogenesis of respiratory syncytial virus vaccine-augmented pathology. Am J Respir Crit Care Med 1995. 152: S63-66.

190 Kaufmann, S. H., Baumann, S. and Nasser Eddine, A., Exploiting immunology and molecular genetics for rational vaccine design against tuberculosis. Int J Tuberc Lung Dis 2006. 10: 1068-1079.

191 McHeyzer-Williams, L. J. and McHeyzer-Williams, M. G., Antigen-specific memory B cell development. Annu Rev Immunol 2005. 23: 487-513.

192 Manz, R. A., Hauser, A. E., Hiepe, F. and Radbruch, A., Maintenance of serum antibody levels. Annu Rev Immunol 2005. 23: 367-386.

193 Tough, D. F. and Sprent, J., Turnover of naive- and memory-phenotype T cells. J Exp Med 1994. 179: 1127-1135.

194 Lalvani, A., Brookes, R., Hambleton, S., Britton, W. J., Hill, A. V. and McMichael, A. J., Rapid effector function in CD8+ memory T cells. J Exp Med 1997. 186: 859-865.

195 Sallusto, F., Lenig, D., Forster, R., Lipp, M. and Lanzavecchia, A., Two subsets of memory $\mathrm{T}$ lymphocytes with distinct homing potentials and effector functions. Nature 1999. 401: 708-712.

196 Champagne, P., Ogg, G. S., King, A. S., Knabenhans, C., Ellefsen, K., Nobile, M., Appay, V., et al., Skewed maturation of memory HIV-specific CD8 T lymphocytes. Nature 2001. 410: 106-111.

197 Debes, G. F., Hopken, U. E. and Hamann, A., In vivo differentiated cytokineproducing CD4(+) T cells express functional CCR7. J Immunol 2002. 168: 5441-5447.

198 Unsoeld, H., Krautwald, S., Voehringer, D., Kunzendorf, U. and Pircher, H., Cutting edge: CCR7+ and CCR7- memory T cells do not differ in immediate effector cell function. J Immunol 2002. 169: 638-641.

199 Ahmadzadeh, M., Hussain, S. F. and Farber, D. L., Heterogeneity of the memory CD4 T cell response: persisting effectors and resting memory T cells. $J$ Immunol 2001. 166: 926-935.

200 Dooms, H., Kahn, E., Knoechel, B. and Abbas, A. K., IL-2 induces a competitive survival advantage in T lymphocytes. J Immunol 2004. 172: 59735979. 
201 Dooms, H., Wolslegel, K., Lin, P. and Abbas, A. K., Interleukin-2 enhances CD4+ T cell memory by promoting the generation of IL-7R alpha-expressing cells. J Exp Med 2007. 204: 547-557.

202 Williams, M. A., Tyznik, A. J. and Bevan, M. J., Interleukin-2 signals during priming are required for secondary expansion of CD8+ memory T cells. Nature 2006. 441: 890-893.

203 Sun, J. C. and Bevan, M. J., Defective CD8 T cell memory following acute infection without CD4 T cell help. Science 2003. 300: 339-342.

204 Sun, J. C., Williams, M. A. and Bevan, M. J., CD4+ T cells are required for the maintenance, not programming, of memory CD8+ T cells after acute infection. Nat Immunol 2004. 5: 927-933.

205 Seddon, B., Tomlinson, P. and Zamoyska, R., Interleukin 7 and T cell receptor signals regulate homeostasis of CD4 memory cells. Nat Immunol 2003. 4: 680-686.

206 Murali-Krishna, K., Lau, L. L., Sambhara, S., Lemonnier, F., Altman, J. and Ahmed, R., Persistence of memory CD8 T cells in MHC class I-deficient mice. Science 1999. 286: 1377-1381.

207 Tan, J. T., Ernst, B., Kieper, W. C., LeRoy, E., Sprent, J. and Surh, C. D., Interleukin (IL)-15 and IL-7 jointly regulate homeostatic proliferation of memory phenotype CD8+ cells but are not required for memory phenotype CD4+ cells. J Exp Med 2002. 195: 1523-1532.

208 Zhang, X., Sun, S., Hwang, I., Tough, D. F. and Sprent, J., Potent and selective stimulation of memory-phenotype CD8+ T cells in vivo by IL-15. Immunity 1998. 8: 591-599.

209 Kennedy, M. K., Glaccum, M., Brown, S. N., Butz, E. A., Viney, J. L., Embers, M., Matsuki, N., et al., Reversible defects in natural killer and memory CD8 T cell lineages in interleukin 15-deficient mice. J Exp Med 2000. 191: 771-780.

210 Ahmed, R. and Gray, D., Immunological memory and protective immunity: understanding their relation. Science 1996. 272: 54-60.

211 Hu, H., Huston, G., Duso, D., Lepak, N., Roman, E. and Swain, S. L., CD4(+) T cell effectors can become memory cells with high efficiency and without further division. Nat Immunol 2001. 2: 705-710.

212 Kaech, S. M. and Ahmed, R., Memory CD8+ T cell differentiation: initial antigen encounter triggers a developmental program in naive cells. Nat Immunol 2001. 2: 415-422.

213 Kaech, S. M., Hemby, S., Kersh, E. and Ahmed, R., Molecular and functional profiling of memory CD8 T cell differentiation. Cell 2002. 111: 837-851.

214 Opferman, J. T., Ober, B. T. and Ashton-Rickardt, P. G., Linear differentiation of cytotoxic effectors into memory T lymphocytes. Science 1999. 283: 1745-1748.

215 Manjunath, N., Shankar, P., Wan, J., Weninger, W., Crowley, M. A., Hieshima, K., Springer, T. A., et al., Effector differentiation is not prerequisite for generation of memory cytotoxic T lymphocytes. J Clin Invest 2001. 108: 871-878.

216 Lauvau, G., Vijh, S., Kong, P., Horng, T., Kerksiek, K., Serbina, N., Tuma, R. A. and Pamer, E. G., Priming of memory but not effector CD8 T cells by a killed bacterial vaccine. Science 2001. 294: 1735-1739.

217 Farber, D. L., Remembrance of antigens past: new insights into memory T cells. Scand J Immunol 2003. 58: 145-154.

218 Murali-Krishna, K., Altman, J. D., Suresh, M., Sourdive, D. J., Zajac, A. J., Miller, J. D., Slansky, J. and Ahmed, R., Counting antigen-specific CD8 T 
cells: a reevaluation of bystander activation during viral infection. Immunity 1998. 8: 177-187.

219 Wu, C. Y., Kirman, J. R., Rotte, M. J., Davey, D. F., Perfetto, S. P., Rhee, E. G., Freidag, B. L., et al., Distinct lineages of T(H)1 cells have differential capacities for memory cell generation in vivo. Nat Immunol 2002. 3: 852-858.

220 Badovinac, V. P., Tvinnereim, A. R. and Harty, J. T., Regulation of antigenspecific CD8+ T cell homeostasis by perforin and interferon-gamma. Science 2000. 290: 1354-1358.

221 Foulds, K. E., Wu, C. Y. and Seder, R. A., Th1 memory: implications for vaccine development. Immunol Rev 2006. 211: 58-66.

222 Goraya, J. S. and Virdi, V. S., Bacille Calmette-Guerin lymphadenitis. Postgrad Med J 2002. 78: 327-329.

223 Berman, S., Lowenthal, J. P., Grogan, E. W., Altieri, P. L. and Benenson, A. S., Bacterial Vaccine In Multidose Tablet Form For Parenteral Use. Appl Microbiol 1963. 11: 423-426.

224 Kantele, A., Arvilommi, H., Kantele, J. M., Rintala, L. and Makela, P. H., Comparison of the human immune response to live oral, killed oral or killed parenteral Salmonella typhi TY21A vaccines. Microb Pathog 1991. 10: 117126.

225 Offit, P. A., The Cutter incident, 50 years later. $N$ Engl J Med 2005. 352: 14111412.

226 Vaananen, P. and Vaheri, A., Large-scale purification of rubella virus and preparation of an experimental split rubella virus vaccine. Appl Microbiol 1971. 22: $255-259$.

227 Caldwell, S. R., Varghese, J. and Puri, N. K., Large scale purification process for recombinant NS1-OspA as a candidate vaccine for Lyme disease. Bioseparation 1996. 6: 115-123.

228 Peters, J., Fowler, E., Gatton, M., Chen, N., Saul, A. and Cheng, Q., High diversity and rapid changeover of expressed var genes during the acute phase of Plasmodium falciparum infections in human volunteers. Proc Natl Acad Sci U S A 2002. 99: 10689-10694.

229 Wolff, J. A., Malone, R. W., Williams, P., Chong, W., Acsadi, G., Jani, A. and Felgner, P. L., Direct gene transfer into mouse muscle in vivo. Science 1990. 247: 1465-1468.

230 Tang, D. C., DeVit, M. and Johnston, S. A., Genetic immunization is a simple method for eliciting an immune response. Nature 1992. 356: 152-154.

231 Ulmer, J. B., Donnelly, J. J., Parker, S. E., Rhodes, G. H., Felgner, P. L., Dwarki, V. J., Gromkowski, S. H., et al., Heterologous protection against influenza by injection of DNA encoding a viral protein. Science 1993. 259: 1745-1749.

232 Donnelly, J. J., Wahren, B. and Liu, M. A., DNA vaccines: progress and challenges. J Immunol 2005. 175: 633-639.

233 Huygen, K., On the use of DNA vaccines for the prophylaxis of mycobacterial diseases. Infect Immun 2003. 71: 1613-1621.

234 Akbari, O., Panjwani, N., Garcia, S., Tascon, R., Lowrie, D. and Stockinger, B., DNA vaccination: transfection and activation of dendritic cells as key events for immunity. J Exp Med 1999. 189: 169-178.

235 Chattergoon, M. A., Robinson, T. M., Boyer, J. D. and Weiner, D. B., Specific immune induction following DNA-based immunization through in vivo transfection and activation of macrophages/antigen-presenting cells. J Immunol 1998. 160: $5707-5718$. 
236 Donnelly, J. J., Liu, M. A. and Ulmer, J. B., Antigen presentation and DNA vaccines. Am J Respir Crit Care Med 2000. 162: S190-193.

237 Dupuis, M., Denis-Mize, K., Woo, C., Goldbeck, C., Selby, M. J., Chen, M., Otten, G. R., et al., Distribution of DNA vaccines determines their immunogenicity after intramuscular injection in mice. J Immunol 2000. 165: 2850-2858.

238 Iwasaki, A., Torres, C. A., Ohashi, P. S., Robinson, H. L. and Barber, B. H., The dominant role of bone marrow-derived cells in CTL induction following plasmid DNA immunization at different sites. J Immunol 1997. 159: 11-14.

239 Shirota, H., Petrenko, L., Hong, C. and Klinman, D. M., Potential of transfected muscle cells to contribute to DNA vaccine immunogenicity. $J$ Immunol 2007. 179: 329-336.

240 Torres, C. A., Iwasaki, A., Barber, B. H. and Robinson, H. L., Differential dependence on target site tissue for gene gun and intramuscular DNA immunizations. J Immunol 1997. 158: 4529-4532.

241 Fu, T. M., Ulmer, J. B., Caulfield, M. J., Deck, R. R., Friedman, A., Wang, S., Liu, X., et al., Priming of cytotoxic T lymphocytes by DNA vaccines: requirement for professional antigen presenting cells and evidence for antigen transfer from myocytes. Mol Med 1997. 3: 362-371.

242 Porgador, A., Irvine, K. R., Iwasaki, A., Barber, B. H., Restifo, N. P. and Germain, R. N., Predominant role for directly transfected dendritic cells in antigen presentation to $\mathrm{CD} 8+\mathrm{T}$ cells after gene gun immunization. $J$ Exp Med 1998. 188: 1075-1082.

243 Oettinger, T., Jorgensen, M., Ladefoged, A., Haslov, K. and Andersen, P., Development of the Mycobacterium bovis BCG vaccine: review of the historical and biochemical evidence for a genealogical tree. Tuber Lung Dis 1999. 79: 243-250.

244 Rodrigues, L. C., Diwan, V. K. and Wheeler, J. G., Protective effect of BCG against tuberculous meningitis and miliary tuberculosis: a meta-analysis. Int $J$ Epidemiol 1993. 22: 1154-1158.

245 Wiegeshaus, E., Balasubramanian, V. and Smith, D. W., Immunity to tuberculosis from the perspective of pathogenesis. Infect Immun 1989. 57: 36713676.

246 Colditz, G. A., Brewer, T. F., Berkey, C. S., Wilson, M. E., Burdick, E., Fineberg, H. V. and Mosteller, F., Efficacy of BCG vaccine in the prevention of tuberculosis. Meta-analysis of the published literature. Jama 1994. 271: 698702.

247 Sterne, J. A., Rodrigues, L. C. and Guedes, I. N., Does the efficacy of BCG decline with time since vaccination? Int J Tuberc Lung Dis 1998. 2: 200-207.

248 Aronson, N. E., Santosham, M., Comstock, G. W., Howard, R. S., Moulton, L. H., Rhoades, E. R. and Harrison, L. H., Long-term efficacy of BCG vaccine in American Indians and Alaska Natives: A 60-year follow-up study. Jama 2004. 291: 2086-2091.

249 Gheorghiu, M. and Lagrange, P. H., Viability, heat stability and immunogenicity of four BCG vaccines prepared from four different BCG strains. Ann Immunol (Paris) 1983. 134C: 125-147.

250 Elias, D., Akuffo, H. and Britton, S., Helminthes could influence the outcome of vaccines against TB in the tropics. Parasite Immunol 2006. 28: 507-513.

251 Rook, G. A., Dheda, K. and Zumla, A., Immune responses to tuberculosis in developing countries: implications for new vaccines. Nat Rev Immunol 2005. 5: 661-667. 
252 Young, S. L., Slobbe, L., Wilson, R., Buddle, B. M., de Lisle, G. W. and Buchan, G. S., Environmental strains of Mycobacterium avium interfere with immune responses associated with Mycobacterium bovis BCG vaccination. Infect Immun 2007. 75: 2833-2840.

253 Brandt, L., Feino Cunha, J., Weinreich Olsen, A., Chilima, B., Hirsch, P., Appelberg, R. and Andersen, P., Failure of the Mycobacterium bovis BCG vaccine: some species of environmental mycobacteria block multiplication of BCG and induction of protective immunity to tuberculosis. Infect Immun 2002. 70: $672-678$.

254 Marris, E., A dozen vaccine candidates bring shot of hope to TB epidemic. Nat Med 2007. 13: 274.

255 Goonetilleke, N. P., McShane, H., Hannan, C. M., Anderson, R. J., Brookes, R. H. and Hill, A. V., Enhanced immunogenicity and protective efficacy against Mycobacterium tuberculosis of bacille Calmette-Guerin vaccine using mucosal administration and boosting with a recombinant modified vaccinia virus Ankara. J Immunol 2003. 171: 1602-1609.

256 McShane, H., Pathan, A. A., Sander, C. R., Keating, S. M., Gilbert, S. C., Huygen, K., Fletcher, H. A. and Hill, A. V., Recombinant modified vaccinia virus Ankara expressing antigen 85A boosts BCG-primed and naturally acquired antimycobacterial immunity in humans. Nat Med 2004. 10: 1240-1244.

257 Mollenkopf, H. J., Kursar, M. and Kaufmann, S. H., Immune response to postprimary tuberculosis in mice: Mycobacterium tuberculosis and Miycobacterium bovis bacille Calmette-Guerin induce equal protection. J Infect Dis 2004. 190: 588-597.

258 Gershon, R. K. and Kondo, K., Infectious immunological tolerance. Immunology 1971. 21: 903-914.

259 Gershon, R. K., Cohen, P., Hencin, R. and Liebhaber, S. A., Suppressor T cells. J Immunol 1972. 108: 586-590.

260 Moller, G., Do suppressor T cells exist? Scand J Immunol 1988. 27: 247-250.

261 Sakaguchi, S., Sakaguchi, N., Asano, M., Itoh, M. and Toda, M., Immunologic self-tolerance maintained by activated T cells expressing IL-2 receptor alpha-chains (CD25). Breakdown of a single mechanism of selftolerance causes various autoimmune diseases. J Immunol 1995. 155: 11511164.

262 Taguchi, O. and Nishizuka, Y., Autoimmune oophoritis in thymectomized mice: T cell requirement in adoptive cell transfer. Clin Exp Immunol 1980. 42: 324-331.

263 Taguchi, O., Nishizuka, Y., Sakakura, T. and Kojima, A., Autoimmune oophoritis in thymectomized mice: detection of circulating antibodies against oocytes. Clin Exp Immunol 1980. 40: 540-553.

264 Takeuchi, T., Rudd, C. E., Schlossman, S. F. and Morimoto, C., Induction of suppression following autologous mixed lymphocyte reaction; role of a novel 2H4 antigen. Eur J Immunol 1987. 17: 97-103.

265 Starr, T. K., Jameson, S. C. and Hogquist, K. A., Positive and negative selection of T cells. Annu Rev Immunol 2003. 21: 139-176.

266 Itoh, M., Takahashi, T., Sakaguchi, N., Kuniyasu, Y., Shimizu, J., Otsuka, F. and Sakaguchi, S., Thymus and autoimmunity: production of CD25+CD4+ naturally anergic and suppressive $T$ cells as a key function of the thymus in maintaining immunologic self-tolerance. J Immunol 1999. 162: 5317-5326.

267 Shevach, E. M., McHugh, R. S., Thornton, A. M., Piccirillo, C., Natarajan, K. and Margulies, D. H., Control of autoimmunity by regulatory T cells. $A d v$ Exp Med Biol 2001. 490: 21-32. 
268 van Santen, H. M., Benoist, C. and Mathis, D., Number of T reg cells that differentiate does not increase upon encounter of agonist ligand on thymic epithelial cells. J Exp Med 2004. 200: 1221-1230.

269 Jordan, M. S., Boesteanu, A., Reed, A. J., Petrone, A. L., Holenbeck, A. E., Lerman, M. A., Naji, A. and Caton, A. J., Thymic selection of CD4+CD25+ regulatory $\mathrm{T}$ cells induced by an agonist self-peptide. Nat Immunol 2001. 2: 301306.

270 Read, S., Malmstrom, V. and Powrie, F., Cytotoxic T lymphocyte-associated antigen 4 plays an essential role in the function of CD25(+)CD4(+) regulatory cells that control intestinal inflammation. J Exp Med 2000. 192: 295-302.

271 McHugh, R. S., Whitters, M. J., Piccirillo, C. A., Young, D. A., Shevach, E. M., Collins, M. and Byrne, M. C., CD4(+)CD25(+) immunoregulatory T cells: gene expression analysis reveals a functional role for the glucocorticoid-induced TNF receptor. Immunity 2002. 16: 311-323.

272 Hartigan-O'Connor, D. J., Poon, C., Sinclair, E. and McCune, J. M., Human CD4+ regulatory T cells express lower levels of the IL-7 receptor alpha chain (CD127), allowing consistent identification and sorting of live cells. J Immunol Methods 2007. 319: 41-52.

273 Liu, W., Putnam, A. L., Xu-Yu, Z., Szot, G. L., Lee, M. R., Zhu, S., Gottlieb, P. A., et al., CD127 expression inversely correlates with FoxP3 and suppressive function of human CD4+ T reg cells. J Exp Med 2006. 203: 1701-1711.

274 Papiernik, M., de Moraes, M. L., Pontoux, C., Vasseur, F. and Penit, C., Regulatory CD4 T cells: expression of IL-2R alpha chain, resistance to clonal deletion and IL-2 dependency. Int Immunol 1998. 10: 371-378.

275 Schneider, M. A., Meingassner, J. G., Lipp, M., Moore, H. D. and Rot, A., CCR7 is required for the in vivo function of CD4+ CD25+ regulatory T cells. $J$ Exp Med 2007. 204: 735-745.

276 Huehn, J., Siegmund, K., Lehmann, J. C., Siewert, C., Haubold, U., Feuerer, M., Debes, G. F., et al., Developmental stage, phenotype, and migration distinguish naive- and effector/memory-like CD4+ regulatory T cells. $J$ Exp Med 2004. 199: 303-313.

277 Fontenot, J. D., Gavin, M. A. and Rudensky, A. Y., Foxp3 programs the development and function of CD4+CD25+ regulatory T cells. Nat Immunol 2003. 4: 330-336.

278 Khattri, R., Cox, T., Yasayko, S. A. and Ramsdell, F., An essential role for Scurfin in CD4+CD25+ T regulatory cells. Nat Immunol 2003. 4: 337-342.

279 Bennett, C. L., Christie, J., Ramsdell, F., Brunkow, M. E., Ferguson, P. J., Whitesell, L., Kelly, T. E., et al., The immune dysregulation, polyendocrinopathy, enteropathy, X-linked syndrome (IPEX) is caused by mutations of FOXP3. Nat Genet 2001. 27: 20-21.

280 Brunkow, M. E., Jeffery, E. W., Hjerrild, K. A., Paeper, B., Clark, L. B., Yasayko, S. A., Wilkinson, J. E., et al., Disruption of a new forkhead/wingedhelix protein, scurfin, results in the fatal lymphoproliferative disorder of the scurfy mouse. Nat Genet 2001. 27: 68-73.

281 Fontenot, J. D. and Rudensky, A. Y., A well adapted regulatory contrivance: regulatory $\mathrm{T}$ cell development and the forkhead family transcription factor Foxp3. Nat Immunol 2005. 6: 331-337.

282 Fontenot, J. D., Rasmussen, J. P., Williams, L. M., Dooley, J. L., Farr, A. G. and Rudensky, A. Y., Regulatory T cell lineage specification by the forkhead transcription factor foxp3. Immunity 2005. 22: 329-341. 
283 Tommasini, A., Ferrari, S., Moratto, D., Badolato, R., Boniotto, M., Pirulli, D., Notarangelo, L. D. and Andolina, M., X-chromosome inactivation analysis in a female carrier of FOXP3 mutation. Clin Exp Immunol 2002. 130: 127-130.

284 Zheng, Y., Josefowicz, S. Z., Kas, A., Chu, T. T., Gavin, M. A. and Rudensky, A. Y., Genome-wide analysis of Foxp3 target genes in developing and mature regulatory T cells. Nature 2007. 445: 936-940.

285 Sugimoto, N., Oida, T., Hirota, K., Nakamura, K., Nomura, T., Uchiyama, T. and Sakaguchi, S., Foxp3-dependent and -independent molecules specific for CD25+CD4+ natural regulatory T cells revealed by DNA microarray analysis. Int Immunol 2006. 18: 1197-1209.

286 Chen, C., Rowell, E. A., Thomas, R. M., Hancock, W. W. and Wells, A. D., Transcriptional regulation by Foxp3 is associated with direct promoter occupancy and modulation of histone acetylation. J Biol Chem 2006. 281: 36828-36834.

287 Bettelli, E., Dastrange, M. and Oukka, M., Foxp3 interacts with nuclear factor of activated T cells and NF-kappa B to repress cytokine gene expression and effector functions of T helper cells. Proc Natl Acad Sci U S A 2005. 102: 51385143.

288 Ivanov, II, McKenzie, B. S., Zhou, L., Tadokoro, C. E., Lepelley, A., Lafaille, J. J., Cua, D. J. and Littman, D. R., The orphan nuclear receptor RORgammat directs the differentiation program of proinflammatory IL-17+ T helper cells. Cell 2006. 126: 1121-1133.

289 Thornton, A. M. and Shevach, E. M., CD4+CD25+ immunoregulatory T cells suppress polyclonal $\mathrm{T}$ cell activation in vitro by inhibiting interleukin 2 production. J Exp Med 1998. 188: 287-296.

290 Thornton, A. M. and Shevach, E. M., Suppressor effector function of CD4+CD25+ immunoregulatory T cells is antigen nonspecific. J Immunol 2000. 164: 183-190.

291 Thornton, A. M., Donovan, E. E., Piccirillo, C. A. and Shevach, E. M., Cutting edge: IL-2 is critically required for the in vitro activation of CD4+CD25+ T cell suppressor function. J Immunol 2004. 172: 6519-6523.

292 Piccirillo, C. A. and Shevach, E. M., Cutting edge: control of CD8+ T cell activation by CD4+CD25+ immunoregulatory cells. J Immunol 2001. 167: 1137-1140.

293 Salomon, B., Lenschow, D. J., Rhee, L., Ashourian, N., Singh, B., Sharpe, A. and Bluestone, J. A., B7/CD28 costimulation is essential for the homeostasis of the CD4+CD25+ immunoregulatory $\mathrm{T}$ cells that control autoimmune diabetes. Immunity 2000. 12: 431-440.

294 Seo, S. J., Fields, M. L., Buckler, J. L., Reed, A. J., Mandik-Nayak, L., Nish, S. A., Noelle, R. J., et al., The impact of T helper and T regulatory cells on the regulation of anti-double-stranded DNA B cells. Immunity 2002. 16: 535-546.

295 Azuma, T., Takahashi, T., Kunisato, A., Kitamura, T. and Hirai, H., Human CD4+ CD25+ regulatory T cells suppress NKT cell functions. Cancer Res 2003. 63: 4516-4520.

296 Taams, L. S., van Amelsfort, J. M., Tiemessen, M. M., Jacobs, K. M., de Jong, E. C., Akbar, A. N., Bijlsma, J. W. and Lafeber, F. P., Modulation of monocyte/macrophage function by human CD4+CD25+ regulatory T cells. Hum Immunol 2005. 66: 222-230.

297 Cederbom, L., Hall, H. and Ivars, F., CD4+CD25+ regulatory T cells downregulate co-stimulatory molecules on antigen-presenting cells. Eur J Immunol 2000. 30: 1538-1543. 
298 D'Cruz, L. M. and Klein, L., Development and function of agonist-induced CD25+Foxp3+ regulatory T cells in the absence of interleukin 2 signaling. Nat Immunol 2005. 6: 1152-1159.

299 Fontenot, J. D., Rasmussen, J. P., Gavin, M. A. and Rudensky, A. Y., A function for interleukin 2 in Foxp3-expressing regulatory T cells. Nat Immunol 2005. 6: 1142-1151.

300 Pandiyan, P., Zheng, L., Ishihara, S., Reed, J. and Lenardo, M. J., $\mathrm{CD} 4(+) \mathrm{CD} 25(+)$ Foxp3(+) regulatory T cells induce cytokine deprivationmediated apoptosis of effector CD4(+) T cells. Nat Immunol 2007. 8: 13531362.

301 Chambers, C. A. and Allison, J. P., Costimulatory regulation of T cell function. Curr Opin Cell Biol 1999. 11: 203-210.

302 Takahashi, T., Tagami, T., Yamazaki, S., Uede, T., Shimizu, J., Sakaguchi, N., Mak, T. W. and Sakaguchi, S., Immunologic self-tolerance maintained by $\mathrm{CD} 25(+) \mathrm{CD} 4(+)$ regulatory $\mathrm{T}$ cells constitutively expressing cytotoxic $\mathrm{T}$ lymphocyte-associated antigen 4. J Exp Med 2000. 192: 303-310.

303 Shevach, E. M., CD4+ CD25+ suppressor T cells: more questions than answers. Nat Rev Immunol 2002. 2: 389-400.

304 Nakamura, K., Kitani, A. and Strober, W., Cell contact-dependent immunosuppression by $\mathrm{CD} 4(+) \mathrm{CD} 25(+)$ regulatory $\mathrm{T}$ cells is mediated by cell surface-bound transforming growth factor beta. J Exp Med 2001. 194: 629-644.

305 Belkaid, Y., Piccirillo, C. A., Mendez, S., Shevach, E. M. and Sacks, D. L., CD4+CD25+ regulatory T cells control Leishmania major persistence and immunity. Nature 2002. 420: 502-507.

306 Du, W., Wong, F. S., Li, M. O., Peng, J., Qi, H., Flavell, R. A., Sherwin, R. and Wen, L., TGF-beta signaling is required for the function of insulin-reactive T regulatory cells. J Clin Invest 2006. 116: 1360-1370.

307 Grossman, W. J., Verbsky, J. W., Barchet, W., Colonna, M., Atkinson, J. P. and Ley, T. J., Human T regulatory cells can use the perforin pathway to cause autologous target cell death. Immunity 2004. 21: 589-601.

308 Gondek, D. C., Lu, L. F., Quezada, S. A., Sakaguchi, S. and Noelle, R. J., Cutting edge: contact-mediated suppression by CD4+CD25+ regulatory cells involves a granzyme B-dependent, perforin-independent mechanism. J Immunol 2005. 174: 1783-1786.

309 Shevach, E. M., DiPaolo, R. A., Andersson, J., Zhao, D. M., Stephens, G. L. and Thornton, A. M., The lifestyle of naturally occurring CD4+ CD25+ Foxp3+ regulatory T cells. Immunol Rev 2006. 212: 60-73.

310 Kretschmer, K., Apostolou, I., Hawiger, D., Khazaie, K., Nussenzweig, M. C. and von Boehmer, H., Inducing and expanding regulatory $\mathrm{T}$ cell populations by foreign antigen. Nat Immunol 2005. 6: 1219-1227.

311 Kang, H. K., Michaels, M. A., Berner, B. R. and Datta, S. K., Very low-dose tolerance with nucleosomal peptides controls lupus and induces potent regulatory T cell subsets. J Immunol 2005. 174: 3247-3255.

312 Barrat, F. J., Cua, D. J., Boonstra, A., Richards, D. F., Crain, C., Savelkoul, H. F., de Waal-Malefyt, R., et al., In vitro generation of interleukin 10producing regulatory $\mathrm{CD} 4(+) \mathrm{T}$ cells is induced by immunosuppressive drugs and inhibited by T helper type 1 (Th1)- and Th2-inducing cytokines. $J$ Exp Med 2002. 195: 603-616.

313 O'Garra, A. and Vieira, P., Regulatory T cells and mechanisms of immune system control. Nat Med 2004. 10: 801-805.

314 Vieira, P. L., Christensen, J. R., Minaee, S., O'Neill, E. J., Barrat, F. J., Boonstra, A., Barthlott, T., et al., IL-10-secreting regulatory T cells do not 
express Foxp3 but have comparable regulatory function to naturally occurring CD4+CD25+ regulatory T cells. J Immunol 2004. 172: 5986-5993.

315 McGuirk, P., McCann, C. and Mills, K. H., Pathogen-specific T regulatory 1 cells induced in the respiratory tract by a bacterial molecule that stimulates interleukin 10 production by dendritic cells: a novel strategy for evasion of protective T helper type 1 responses by Bordetella pertussis. J Exp Med 2002. 195: 221-231.

316 Fantini, M. C., Becker, C., Monteleone, G., Pallone, F., Galle, P. R. and Neurath, M. F., Cutting edge: TGF-beta induces a regulatory phenotype in CD4+CD25- T cells through Foxp3 induction and down-regulation of Smad7. $J$ Immunol 2004. 172: 5149-5153.

317 Yamagiwa, S., Gray, J. D., Hashimoto, S. and Horwitz, D. A., A role for TGF-beta in the generation and expansion of CD4+CD25+ regulatory T cells from human peripheral blood. J Immunol 2001. 166: 7282-7289.

318 Davidson, T. S., DiPaolo, R. J., Andersson, J. and Shevach, E. M., Cutting Edge: IL-2 is essential for TGF-beta-mediated induction of Foxp3+ T regulatory cells. J Immunol 2007. 178: 4022-4026.

319 Laurence, A., Tato, C. M., Davidson, T. S., Kanno, Y., Chen, Z., Yao, Z., Blank, R. B., et al., Interleukin-2 signaling via STAT5 constrains T helper 17 cell generation. Immunity 2007. 26: 371-381.

320 Jiang, H. and Chess, L., The specific regulation of immune responses by CD8+ $\mathrm{T}$ cells restricted by the MHC class Ib molecule, Qa-1. Annu Rev Immunol 2000. 18: $185-216$.

321 Gilliet, M. and Liu, Y. J., Generation of human CD8 T regulatory cells by CD40 ligand-activated plasmacytoid dendritic cells. J Exp Med 2002. 195: 695704.

322 Fischer, K., Voelkl, S., Heymann, J., Przybylski, G. K., Mondal, K., Laumer, M., Kunz-Schughart, L., et al., Isolation and characterization of human antigen-specific TCR alpha beta+ CD4(-)CD8- double-negative regulatory T cells. Blood 2005. 105: 2828-2835.

323 Zhang, D., Yang, W., Degauque, N., Tian, Y., Mikita, A. and Zheng, X. X., New differentiation pathway for double-negative regulatory $T$ cells that regulates the magnitude of immune responses. Blood 2007. 109: 4071-4079.

324 Pasare, C. and Medzhitov, R., Toll pathway-dependent blockade of CD4+CD25+ T cell-mediated suppression by dendritic cells. Science 2003. 299: 1033-1036.

325 King, I. L. and Segal, B. M., Cutting edge: IL-12 induces CD4+CD25- T cell activation in the presence of T regulatory cells. J Immunol 2005. 175: 641-645.

326 Sutmuller, R. P., den Brok, M. H., Kramer, M., Bennink, E. J., Toonen, L. W., Kullberg, B. J., Joosten, L. A., et al., Toll-like receptor 2 controls expansion and function of regulatory T cells. J Clin Invest 2006. 116: 485-494.

327 Peng, G., Guo, Z., Kiniwa, Y., Voo, K. S., Peng, W., Fu, T., Wang, D. Y., et al., Toll-like receptor 8-mediated reversal of $\mathrm{CD} 4+$ regulatory $\mathrm{T}$ cell function. Science 2005. 309: 1380-1384.

328 Caramalho, I., Lopes-Carvalho, T., Ostler, D., Zelenay, S., Haury, M. and Demengeot, J., Regulatory T cells selectively express toll-like receptors and are activated by lipopolysaccharide. J Exp Med 2003. 197: 403-411.

329 Shevach, E. M., From vanilla to 28 flavors: multiple varieties of T regulatory cells. Immunity 2006. 25: 195-201.

330 Taylor, M. D., LeGoff, L., Harris, A., Malone, E., Allen, J. E. and Maizels, R. M., Removal of regulatory $\mathrm{T}$ cell activity reverses hyporesponsiveness and leads to filarial parasite clearance in vivo. J Immunol 2005. 174: 4924-4933. 
331 Suvas, S., Azkur, A. K., Kim, B. S., Kumaraguru, U. and Rouse, B. T., CD4+CD25+ regulatory T cells control the severity of viral immunoinflammatory lesions. J Immunol 2004. 172: 4123-4132.

332 Raghavan, S., Fredriksson, M., Svennerholm, A. M., Holmgren, J. and Suri-Payer, E., Absence of CD4+CD25+ regulatory T cells is associated with a loss of regulation leading to increased pathology in Helicobacter pylori-infected mice. Clin Exp Immunol 2003. 132: 393-400.

333 Long, T. T., Nakazawa, S., Onizuka, S., Huaman, M. C. and Kanbara, H., Influence of CD4+CD25+ T cells on Plasmodium berghei NK65 infection in BALB/c mice. Int J Parasitol 2003. 33: 175-183.

334 Hisaeda, H., Maekawa, Y., Iwakawa, D., Okada, H., Himeno, K., Kishihara, K., Tsukumo, S. and Yasutomo, K., Escape of malaria parasites from host immunity requires CD4+CD25+ regulatory T cells. Nat Med 2004. 10: 29-30.

335 Heuer, J. G., Zhang, T., Zhao, J., Ding, C., Cramer, M., Justen, K. L., Vonderfecht, S. L. and Na, S., Adoptive transfer of in vitro-stimulated CD4+CD25+ regulatory $\mathrm{T}$ cells increases bacterial clearance and improves survival in polymicrobial sepsis. J Immunol 2005. 174: 7141-7146.

336 Furuichi, Y., Tokuyama, H., Ueha, S., Kurachi, M., Moriyasu, F. and Kakimi, K., Depletion of CD25+CD4+T cells (Tregs) enhances the HBVspecific CD8+ T cell response primed by DNA immunization. World $J$ Gastroenterol 2005. 11: 3772-3777.

337 Kursar, M., Kohler, A., Kaufmann, S. H. and Mittrucker, H. W., Depletion of CD4+ T cells during immunization with nonviable Listeria monocytogenes causes enhanced CD8+ T cell-mediated protection against listeriosis. J Immunol 2004. 172: 3167-3172.

338 Toka, F. N., Suvas, S. and Rouse, B. T., CD4+ CD25+ T cells regulate vaccine-generated primary and memory $\mathrm{CD} 8+\mathrm{T}$-cell responses against herpes simplex virus type 1. J Virol 2004. 78: 13082-13089.

339 Moore, A. C., Gallimore, A., Draper, S. J., Watkins, K. R., Gilbert, S. C. and Hill, A. V., Anti-CD25 antibody enhancement of vaccine-induced immunogenicity: increased durable cellular immunity with reduced immunodominance. J Immunol 2005. 175: 7264-7273.

340 Ausubel, F. M. (Ed.) Current Protocols in Molecular Biology. John Wiley \& Sons, Inc., Hoboken 2001.

341 Wilson, T. M., de Lisle, G. W. and Collins, D. M., Effect of inhA and katG on isoniazid resistance and virulence of Mycobacterium bovis. Mol Microbiol 1995. 15: 1009-1015.

342 Kent, P., Kubica, GP, Public Health Mycobacteriology. A Guide for the Level III Laboratory. U.S. Department of Health and Human Services, Public Health Service., Atlanta: 1985.

343 Ortega, G., Robb, R. J., Shevach, E. M. and Malek, T. R., The murine IL 2 receptor. I. Monoclonal antibodies that define distinct functional epitopes on activated T cells and react with activated B cells. J Immunol 1984. 133: 19701975.

344 Lowenthal, J. W., Corthesy, P., Tougne, C., Lees, R., MacDonald, H. R. and Nabholz, M., High and low affinity IL 2 receptors: analysis by IL 2 dissociation rate and reactivity with monoclonal anti-receptor antibody PC61. J Immunol 1985. 135: 3988-3994.

345 Loughry, A., Fairchild, S., Athanasou, N., Edwards, J. and Hall, F. C., Inflammatory arthritis and dermatitis in thymectomized, CD25+ cell-depleted adult mice. Rheumatology (Oxford) 2005. 44: 299-308. 
346 Cao, Q., Wang, L., Du, F., Sheng, H., Zhang, Y., Wu, J., Shen, B., et al., Downregulation of CD4+CD25+ regulatory T cells may underlie enhanced Th1 immunity caused by immunization with activated autologous T cells. Cell Res 2007. 17: 627-637.

347 Poole, T. (Ed.) The UFAW Handbook on the Care and Management of Laboratory Animals. Blackwell Science Ltd., Oxford, 1999, $7^{\text {th }}$ Edition.

348 Jainchill, J. L., Aaronson, S. A. and Todaro, G. J., Murine sarcoma and leukemia viruses: assay using clonal lines of contact-inhibited mouse cells. $J$ Virol 1969. 4: 549-553.

349 Boettler, T., Spangenberg, H. C., Neumann-Haefelin, C., Panther, E., Urbani, S., Ferrari, C., Blum, H. E., et al., T cells with a CD4+CD25+ regulatory phenotype suppress in vitro proliferation of virus-specific CD8+ $\mathrm{T}$ cells during chronic hepatitis C virus infection. J Virol 2005. 79: 7860-7867.

350 Taguchi, O. and Takahashi, T., Administration of anti-interleukin-2 receptor alpha antibody in vivo induces localized autoimmune disease. Eur J Immunol 1996. 26: 1608-1612.

351 McHugh, R. S. and Shevach, E. M., Cutting edge: depletion of CD4+CD25+ regulatory $\mathrm{T}$ cells is necessary, but not sufficient, for induction of organ-specific autoimmune disease. J Immunol 2002. 168: 5979-5983.

352 McNeill, A., Spittle, E. and Backstrom, B. T., Partial depletion of CD69lowexpressing natural regulatory $\mathrm{T}$ cells with the anti-CD25 monoclonal antibody PC61. Scand J Immunol 2007. 65: 63-69.

353 Kohm, A. P., McMahon, J. S., Podojil, J. R., Begolka, W. S., DeGutes, M., Kasprowicz, D. J., Ziegler, S. F. and Miller, S. D., Cutting Edge: Anti-CD25 monoclonal antibody injection results in the functional inactivation, not depletion, of CD4+CD25+ T regulatory cells. J Immunol 2006. 176: 3301-3305.

354 Bismuth, G., Moreau, J. L., Somme, G., Duphot, M., Dautry-Varsat, A., Robb, R. J. and Theze, J., Regulation of interleukin 2 (IL2) receptor expression: IL2 as an inducing signal for the expression of its own receptor on a murine T helper cell line. Eur J Immunol 1985. 15: 723-727.

355 Shevach, E. M. and Stephens, G. L., The GITR-GITRL interaction: costimulation or contrasuppression of regulatory activity? Nat Rev Immunol 2006. 6: 613-618.

356 Stephens, G. L., McHugh, R. S., Whitters, M. J., Young, D. A., Luxenberg, D., Carreno, B. M., Collins, M. and Shevach, E. M., Engagement of glucocorticoid-induced TNFR family-related receptor on effector T cells by its ligand mediates resistance to suppression by CD4+CD25+ T cells. $J$ Immunol 2004. 173: 5008-5020.

357 Shimizu, J., Yamazaki, S., Takahashi, T., Ishida, Y. and Sakaguchi, S., Stimulation of CD25(+)CD4(+) regulatory T cells through GITR breaks immunological self-tolerance. Nat Immunol 2002. 3: 135-142.

358 Belkaid, Y., Hoffmann, K. F., Mendez, S., Kamhawi, S., Udey, M. C., Wynn, T. A. and Sacks, D. L., The role of interleukin (IL)-10 in the persistence of Leishmania major in the skin after healing and the therapeutic potential of anti-IL-10 receptor antibody for sterile cure. J Exp Med 2001. 194: 1497-1506.

359 Suffia, I., Reckling, S. K., Salay, G. and Belkaid, Y., A role for CD103 in the retention of CD4+CD25+ Treg and control of Leishmania major infection. $J$ Immunol 2005. 174: 5444-5455.

360 Nagase, H., Jones, K. M., Anderson, C. F. and Noben-Trauth, N., Despite increased CD4+Foxp3+ cells within the infection site, BALB/c IL-4 receptordeficient mice reveal CD4+Foxp3-negative T cells as a source of IL-10 in Leishmania major susceptibility. J Immunol 2007. 179: 2435-2444. 
361 Anderson, C. F., Oukka, M., Kuchroo, V. J. and Sacks, D., CD4(+)CD25()Foxp3(-) Th1 cells are the source of IL-10-mediated immune suppression in chronic cutaneous leishmaniasis. J Exp Med 2007. 204: 285-297.

362 Aseffa, A., Gumy, A., Launois, P., MacDonald, H. R., Louis, J. A. and Tacchini-Cottier, F., The early IL-4 response to Leishmania major and the resulting Th2 cell maturation steering progressive disease in BALB/c mice are subject to the control of regulatory CD4+CD25+ T cells. J Immunol 2002. 169: 3232-3241.

363 Mustafa, A. S. and Godal, T., In vitro induction of human suppressor T cells by mycobacterial antigens. BCG activated OKT4+ cells mediate suppression of antigen induced T cell proliferation. Clin Exp Immunol 1983. 52: 29-37.

364 Mustafa, A. S. and Godal, T., BCG-induced suppressor T cells optimal conditions for in vitro induction and mode of action. Clin Exp Immunol 1985. 62: 474-481.

365 Delgado, J. C., Tsai, E. Y., Thim, S., Baena, A., Boussiotis, V. A., Reynes, J. M., Sath, S., et al., Antigen-specific and persistent tuberculin anergy in a cohort of pulmonary tuberculosis patients from rural Cambodia. Proc Natl Acad Sci U $S$ A 2002. 99: 7576-7581.

366 Guyot-Revol, V., Innes, J. A., Hackforth, S., Hinks, T. and Lalvani, A., Regulatory $\mathrm{T}$ cells are expanded in blood and disease sites in patients with tuberculosis. Am J Respir Crit Care Med 2006. 173: 803-810.

367 Ribeiro-Rodrigues, R., Resende Co, T., Rojas, R., Toossi, Z., Dietze, R., Boom, W. H., Maciel, E. and Hirsch, C. S., A role for CD4CD25 T cells in regulation of the immune response during human tuberculosis. Clin Exp Immunol 2006. 144: 25-34.

368 Chen, X., Zhou, B., Li, M., Deng, Q., Wu, X., Le, X., Wu, C., et al., $\mathrm{CD} 4(+) \mathrm{CD} 25(+) \mathrm{FoxP} 3(+)$ regulatory T cells suppress Mycobacterium tuberculosis immunity in patients with active disease. Clin Immunol 2007. 123: 50-59.

369 Gazzola, L., Tincati, C., Gori, A., Saresella, M., Marventano, I. and Zanini, F., FoxP3 mRNA expression in regulatory $\mathrm{T}$ cells from patients with tuberculosis. Am J Respir Crit Care Med 2006. 174: 356; author reply 357.

370 Hougardy, J. M., Verscheure, V., Locht, C. and Mascart, F., In vitro expansion of $\mathrm{CD} 4(+) \mathrm{CD} 25($ high)FOXP3(+)CD127(low/-) regulatory T cells from peripheral blood lymphocytes of healthy Mycobacterium tuberculosisinfected humans. Microbes Infect 2007. 9: 1325-1332.

371 Li, L., Lao, S. H. and Wu, C. Y., Increased frequency of CD4(+)CD25(high) Treg cells inhibit BCG-specific induction of IFN-gamma by CD4(+) T cells from TB patients. Tuberculosis (Edinb) 2007. 87: 526-534.

372 Hougardy, J. M., Place, S., Hildebrand, M., Drowart, A., Debrie, A. S., Locht, C. and Mascart, F., Regulatory T cells depress immune responses to protective antigens in active tuberculosis. Am J Respir Crit Care Med 2007. 176: 409-416.

373 Baumann, S., Nasser Eddine, A. and Kaufmann, S. H., Progress in tuberculosis vaccine development. Curr Opin Immunol 2006. 18: 438-448.

374 Quinn, K. M., McHugh, R. S., Rich, F. J., Goldsack, L. M., de Lisle, G. W., Buddle, B. M., Delahunt, B. and Kirman, J. R., Inactivation of CD4+ CD25+ regulatory $\mathrm{T}$ cells during early mycobacterial infection increases cytokine production but does not affect pathogen load. Immunol Cell Biol 2006. 84: 467474. 
375 Suvas, S., Kumaraguru, U., Pack, C. D., Lee, S. and Rouse, B. T., CD4+CD25+ T cells regulate virus-specific primary and memory CD8+ T cell responses. J Exp Med 2003. 198: 889-901.

376 Hogan, L. H., Markofski, W., Bock, A., Barger, B., Morrissey, J. D. and Sandor, M., Mycobacterium bovis BCG-induced granuloma formation depends on gamma interferon and CD40 ligand but does not require CD28. Infect Immun 2001. 69: 2596-2603.

377 Wedlock, D. N., Vesosky, B., Skinner, M. A., de Lisle, G. W., Orme, I. M. and Buddle, B. M., Vaccination of cattle with Mycobacterium bovis culture filtrate proteins and interleukin-2 for protection against bovine tuberculosis. Infect Immun 2000. 68: 5809-5815.

378 Coleman, J. D. and Cooke, M. M., Mycobacterium bovis infection in wildlife in New Zealand. Tuberculosis (Edinb) 2001. 81: 191-202.

379 Couper, K. N., Blount, D. G., de Souza, J. B., Suffia, I., Belkaid, Y. and Riley, E. M., Incomplete depletion and rapid regeneration of Foxp3+ regulatory $\mathrm{T}$ cells following anti-CD25 treatment in malaria-infected mice. J Immunol 2007. 178: 4136-4146.

380 Jung, Y. J., Ryan, L., LaCourse, R. and North, R. J., Increased interleukin-10 expression is not responsible for failure of $\mathrm{T}$ helper 1 immunity to resolve airborne Mycobacterium tuberculosis infection in mice. Immunology 2003. 109: 295-299.

381 Erb, K. J., Kirman, J., Delahunt, B., Chen, W. and Le Gros, G., IL-4, IL-5 and IL-10 are not required for the control of $M$. bovis-BCG infection in mice. Immunol Cell Biol 1998. 76: 41-46.

382 Medina, E., Ryan, L., LaCourse, R. and North, R. J., Superior virulence of Mycobacterium bovis over Mycobacterium tuberculosis (Mtb) for Mtb-resistant and Mtb-susceptible mice is manifest as an ability to cause extrapulmonary disease. Tuberculosis (Edinb) 2006. 86: 20-27.

383 Kursar, M., Koch, M., Mittrucker, H. W., Nouailles, G., Bonhagen, K., Kamradt, T. and Kaufmann, S. H., Cutting Edge: Regulatory T cells prevent efficient clearance of Mycobacterium tuberculosis. J Immunol 2007. 178: 26612665.

384 Scott-Browne, J. P., Shafiani, S., Tucker-Heard, G., Ishida-Tsubota, K., Fontenot, J. D., Rudensky, A. Y., Bevan, M. J. and Urdahl, K. B., Expansion and function of Foxp3-expressing T regulatory cells during tuberculosis. $J$ Exp Med 2007. 204: 2159-2169.

385 Andersen, P., Askgaard, D., Gottschau, A., Bennedsen, J., Nagai, S. and Heron, I., Identification of immunodominant antigens during infection with Mycobacterium tuberculosis. Scand J Immunol 1992. 36: 823-831.

386 Pacholczyk, R., Ignatowicz, H., Kraj, P. and Ignatowicz, L., Origin and T cell receptor diversity of Foxp3+CD4+CD25+ T cells. Immunity 2006. 25: 249259.

387 Huygen, K., Content, J., Denis, O., Montgomery, D. L., Yawman, A. M., Deck, R. R., DeWitt, C. M., et al., Immunogenicity and protective efficacy of a tuberculosis DNA vaccine. Nat Med 1996. 2: 893-898.

388 Tascon, R. E., Colston, M. J., Ragno, S., Stavropoulos, E., Gregory, D. and Lowrie, D. B., Vaccination against tuberculosis by DNA injection. Nat Med 1996. 2: 888-892.

389 Tanghe, A., Denis, O., Lambrecht, B., Motte, V., van den Berg, T. and Huygen, K., Tuberculosis DNA vaccine encoding Ag85A is immunogenic and protective when administered by intramuscular needle injection but not by epidermal gene gun bombardment. Infect Immun 2000. 68: 3854-3860. 
390 Denis, O., Tanghe, A., Palfliet, K., Jurion, F., van den Berg, T. P., Vanonckelen, A., Ooms, J., et al., Vaccination with plasmid DNA encoding mycobacterial antigen 85A stimulates a CD4+ and CD8+ T-cell epitopic repertoire broader than that stimulated by Mycobacterium tuberculosis $\mathrm{H} 37 \mathrm{Rv}$ infection. Infect Immun 1998. 66: 1527-1533.

391 Przybylowski, M., Bartido, S., Borquez-Ojeda, O., Sadelain, M. and Riviere, I., Production of clinical-grade plasmid DNA for human Phase I clinical trials and large animal clinical studies. Vaccine 2007. 25: 5013-5024.

392 Leitner, W. W., Ying, H., Driver, D. A., Dubensky, T. W. and Restifo, N. P., Enhancement of tumor-specific immune response with plasmid DNA replicon vectors. Cancer Res 2000. 60: 51-55.

393 DiCiommo, D. P. and Bremner, R., Rapid, high level protein production using DNA-based Semliki Forest virus vectors. J Biol Chem 1998. 273: 18060-18066.

394 Dubensky, T. W., Jr., Driver, D. A., Polo, J. M., Belli, B. A., Latham, E. M., Ibanez, C. E., Chada, S., et al., Sindbis virus DNA-based expression vectors: utility for in vitro and in vivo gene transfer. $J$ Virol 1996. 70: 508-519.

395 Jones, C. T., Patkar, C. G. and Kuhn, R. J., Construction and applications of yellow fever virus replicons. Virology 2005. 331: 247-259.

396 Khromykh, A. A. and Westaway, E. G., Subgenomic replicons of the flavivirus Kunjin: construction and applications. J Virol 1997. 71: 1497-1505.

397 Leitner, W. W., Hwang, L. N., deVeer, M. J., Zhou, A., Silverman, R. H., Williams, B. R., Dubensky, T. W., et al., Alphavirus-based DNA vaccine breaks immunological tolerance by activating innate antiviral pathways. Nat Med 2003. 9: 33-39.

398 Derrick, S. C., Yang, A. L. and Morris, S. L., Vaccination with a Sindbis virus-based DNA vaccine expressing antigen $85 \mathrm{~B}$ induces protective immunity against Mycobacterium tuberculosis. Infect Immun 2005. 73: 7727-7735.

399 Leitner, W. W., Bergmann-Leitner, E. S., Hwang, L. N. and Restifo, N. P., Type I Interferons are essential for the efficacy of replicase-based DNA vaccines. Vaccine 2006. 24: 5110-5118.

400 Leitner, W. W., Ying, H. and Restifo, N. P., DNA and RNA-based vaccines: principles, progress and prospects. Vaccine 1999. 18: 765-777.

401 Alexopoulou, L., Holt, A. C., Medzhitov, R. and Flavell, R. A., Recognition of double-stranded RNA and activation of NF-kappaB by Toll-like receptor 3 . Nature 2001. 413: 732-738.

402 Yoneyama, M., Kikuchi, M., Natsukawa, T., Shinobu, N., Imaizumi, T., Miyagishi, M., Taira, K., et al., The RNA helicase RIG-I has an essential function in double-stranded RNA-induced innate antiviral responses. Nat Immunol 2004. 5: 730-737.

403 Galabru, J. and Hovanessian, A., Autophosphorylation of the protein kinase dependent on double-stranded RNA. J Biol Chem 1987. 262: 15538-15544.

404 Hovanessian, A. G., The double stranded RNA-activated protein kinase induced by interferon: dsRNA-PK. J Interferon Res 1989. 9: 641-647.

405 Rhoads, R. E., Regulation of eukaryotic protein synthesis by initiation factors. $J$ Biol Chem 1993. 268: 3017-3020.

406 Kim, T. W., Hung, C. F., Ling, M., Juang, J., He, L., Hardwick, J. M., Kumar, S. and Wu, T. C., Enhancing DNA vaccine potency by coadministration of DNA encoding antiapoptotic proteins. J Clin Invest 2003. 112: 109-117.

407 Leitner, W. W., Hwang, L. N., Bergmann-Leitner, E. S., Finkelstein, S. E., Frank, S. and Restifo, N. P., Apoptosis is essential for the increased efficacy of alphaviral replicase-based DNA vaccines. Vaccine 2004. 22: 1537-1544. 
408 Leitner, W. W. and Restifo, N. P., DNA vaccines and apoptosis: to kill or not to kill? J Clin Invest 2003. 112: 22-24.

409 Varnavski, A. N., Young, P. R. and Khromykh, A. A., Stable high-level expression of heterologous genes in vitro and in vivo by noncytopathic DNAbased Kunjin virus replicon vectors. J Virol 2000. 74: 4394-4403.

410 Liu, W. J., Wang, X. J., Mokhonov, V. V., Shi, P. Y., Randall, R. and Khromykh, A. A., Inhibition of interferon signaling by the New York 99 strain and Kunjin subtype of West Nile virus involves blockage of STAT1 and STAT2 activation by nonstructural proteins. J Virol 2005. 79: 1934-1942.

411 Anraku, I., Harvey, T. J., Linedale, R., Gardner, J., Harrich, D., Suhrbier, A. and Khromykh, A. A., Kunjin virus replicon vaccine vectors induce protective CD8+ T-cell immunity. J Virol 2002. 76: 3791-3799.

412 Harvey, T. J., Anraku, I., Linedale, R., Harrich, D., Mackenzie, J., Suhrbier, A. and Khromykh, A. A., Kunjin virus replicon vectors for human immunodeficiency virus vaccine development. J Virol 2003. 77: 7796-7803.

413 Herd, K. A., Harvey, T., Khromykh, A. A. and Tindle, R. W., Recombinant Kunjin virus replicon vaccines induce protective T-cell immunity against human papillomavirus 16 E7-expressing tumour. Virology 2004. 319: 237-248.

414 Ward, S. M., Tindle, R. W., Khromykh, A. A. and Gowans, E. J., Generation of CTL responses using Kunjin replicon RNA. Immunol Cell Biol 2003. 81: 7378.

415 Closs, O., Harboe, M., Axelsen, N. H., Bunch-Christensen, K. and Magnusson, M., The antigens of Mycobacterium bovis, strain BCG, studied by crossed immunoelectrophoresis: a reference system. Scand J Immunol 1980. 12: 249-263.

416 Wiker, H. G., Harboe, M., Nagai, S. and Bennedsen, J., Quantitative and qualitative studies on the major extracellular antigen of Mycobacterium tuberculosis H37Rv and Mycobacterium bovis BCG. Am Rev Respir Dis 1990. 141: $830-838$.

417 Borremans, M., de Wit, L., Volckaert, G., Ooms, J., de Bruyn, J., Huygen, K., van Vooren, J. P., et al., Cloning, sequence determination, and expression of a 32-kilodalton-protein gene of Mycobacterium tuberculosis. Infect Immun 1989. 57: 3123-3130.

418 Content, J., de la Cuvellerie, A., De Wit, L., Vincent-Levy-Frebault, V., Ooms, J. and De Bruyn, J., The genes coding for the antigen 85 complexes of Mycobacterium tuberculosis and Mycobacterium bovis BCG are members of a gene family: cloning, sequence determination, and genomic organization of the gene coding for antigen 85-C of M. tuberculosis. Infect Immun 1991. 59: 32053212 .

419 Harth, G., Lee, B. Y., Wang, J., Clemens, D. L. and Horwitz, M. A., Novel insights into the genetics, biochemistry, and immunocytochemistry of the 30kilodalton major extracellular protein of Mycobacterium tuberculosis. Infect Immun 1996. 64: 3038-3047.

420 Rinke de Wit, T. F., Bekelie, S., Osland, A., Wieles, B., Janson, A. A. and Thole, J. E., The Mycobacterium leprae antigen 85 complex gene family: identification of the genes for the 85A, 85C, and related MPT51 proteins. Infect Immun 1993. 61: 3642-3647.

421 Abou-Zeid, C., Ratliff, T. L., Wiker, H. G., Harboe, M., Bennedsen, J. and Rook, G. A., Characterization of fibronectin-binding antigens released by Mycobacterium tuberculosis and Mycobacterium bovis BCG. Infect Immun 1988. 56: 3046-3051. 
422 Ratliff, T. L., McGarr, J. A., Abou-Zeid, C., Rook, G. A., Stanford, J. L., Aslanzadeh, J. and Brown, E. J., Attachment of mycobacteria to fibronectincoated surfaces. J Gen Microbiol 1988. 134: 1307-1313.

423 Belisle, J. T., Vissa, V. D., Sievert, T., Takayama, K., Brennan, P. J. and Besra, G. S., Role of the major antigen of Mycobacterium tuberculosis in cell wall biogenesis. Science 1997. 276: 1420-1422.

424 McNeil, M. R. and Brennan, P. J., Structure, function and biogenesis of the cell envelope of mycobacteria in relation to bacterial physiology, pathogenesis and drug resistance; some thoughts and possibilities arising from recent structural information. Res Microbiol 1991. 142: 451-463.

425 Chatterjee, D., The mycobacterial cell wall: structure, biosynthesis and sites of drug action. Curr Opin Chem Biol 1997. 1: 579-588.

426 Huygen, K., Abramowicz, D., Vandenbussche, P., Jacobs, F., De Bruyn, J., Kentos, A., Drowart, A., et al., Spleen cell cytokine secretion in Mycobacterium bovis BCG-infected mice. Infect Immun 1992. 60: 2880-2886.

427 Silver, R. F., Wallis, R. S. and Ellner, J. J., Mapping of T cell epitopes of the 30-kDa alpha antigen of Mycobacterium bovis strain bacillus Calmette-Guerin in purified protein derivative (PPD)-positive individuals. J Immunol 1995. 154: 4665-4674.

428 Huygen, K., Van Vooren, J. P., Turneer, M., Bosmans, R., Dierckx, P. and De Bruyn, J., Specific lymphoproliferation, gamma interferon production, and serum immunoglobulin $\mathrm{G}$ directed against a purified $32 \mathrm{kDa}$ mycobacterial protein antigen (P32) in patients with active tuberculosis. Scand J Immunol 1988. 27: 187-194.

429 Huygen, K., Lozes, E., Gilles, B., Drowart, A., Palfliet, K., Jurion, F., Roland, I., et al., Mapping of TH1 helper T-cell epitopes on major secreted mycobacterial antigen 85A in mice infected with live Mycobacterium bovis BCG. Infect Immun 1994. 62: 363-370.

430 D'Souza, S., Rosseels, V., Romano, M., Tanghe, A., Denis, O., Jurion, F., Castiglione, N., et al., Mapping of murine Th1 helper T-Cell epitopes of mycolyl transferases Ag85A, Ag85B, and Ag85C from Mycobacterium tuberculosis. Infect Immun 2003. 71: 483-493.

431 Launois, P., DeLeys, R., Niang, M. N., Drowart, A., Andrien, M., Dierckx, P., Cartel, J. L., et al., T-cell-epitope mapping of the major secreted mycobacterial antigen Ag85A in tuberculosis and leprosy. Infect Immun 1994. 62: $3679-3687$.

432 Lozes, E., Huygen, K., Content, J., Denis, O., Montgomery, D. L., Yawman, A. M., et al., Immunogenicity and efficacy of a tuberculosis DNA vaccine encoding the components of the secreted antigen 85 complex. Vaccine 1997. 15: 830-833.

433 Kirman, J. R., Turon, T., Su, H., Li, A., Kraus, C., Polo, J. M., Belisle, J., Morris, S. and Seder, R. A., Enhanced immunogenicity to Mycobacterium tuberculosis by vaccination with an alphavirus plasmid replicon expressing antigen 85A. Infect Immun 2003. 71: 575-579.

434 Khromykh, A. A., Harvey, T. J., Abedinia, M. and Westaway, E. G., Expression and purification of the seven nonstructural proteins of the flavivirus Kunjin in the E. coli and the baculovirus expression systems. J Virol Methods 1996. 61: 47-58.

435 Khromykh, A. A. and Westaway, E. G., Completion of Kunjin virus RNA sequence and recovery of an infectious RNA transcribed from stably cloned fulllength cDNA. J Virol 1994. 68: 4580-4588. 
436 Hogarth, P. J., Logan, K. E., Ferraz, J. C., Hewinson, R. G. and Chambers, M. A., Protective efficacy induced by Mycobacterium bovis bacille CalmetteGuerin can be augmented in an antigen independent manner by use of noncoding plasmid DNA. Vaccine 2006. 24: 95-101.

437 Loots, K., Vleugels, B., Ons, E., Vanrompay, D. and Goddeeris, B. M., Evaluation of the persistence and gene expression of an anti-Chlamydophila psittaci DNA vaccine in turkey muscle. BMC Vet Res 2006. 2: 18.

438 Swain, S. L., Generation and in vivo persistence of polarized Th1 and Th2 memory cells. Immunity 1994. 1: 543-552.

439 Swain, S. L., Hu, H. and Huston, G., Class II-independent generation of CD4 memory T cells from effectors. Science 1999. 286: 1381-1383.

440 Kassiotis, G., Garcia, S., Simpson, E. and Stockinger, B., Impairment of immunological memory in the absence of MHC despite survival of memory $\mathrm{T}$ cells. Nat Immunol 2002. 3: 244-250.

441 Tanchot, C., Lemonnier, F. A., Perarnau, B., Freitas, A. A. and Rocha, B., Differential requirements for survival and proliferation of CD8 naive or memory T cells. Science 1997. 276: 2057-2062.

442 Gray, D. and Matzinger, P., T cell memory is short-lived in the absence of antigen. J Exp Med 1991. 174: 969-974.

443 Olsen, A. W., Brandt, L., Agger, E. M., van Pinxteren, L. A. and Andersen, P., The influence of remaining live BCG organisms in vaccinated mice on the maintenance of immunity to tuberculosis. Scand J Immunol 2004. 60: 273-277.

444 Tannis, L. L., Gauthier, A., Evelegh, C., Parsons, R., Nyholt, D., Khromykh, A. and Bramson, J. L., Semliki forest virus and Kunjin virus RNA replicons elicit comparable cellular immunity but distinct humoral immunity. Vaccine 2005. 23: 4189-4194.

445 Gilbertson, B., Germano, S., Steele, P., Turner, S., Fazekas de St Groth, B. and Cheers, C., Bystander activation of CD8+ T lymphocytes during experimental mycobacterial infection. Infect Immun 2004. 72: 6884-6891.

446 D'Souza, S., Denis, O., Scorza, T., Nzabintwali, F., Verschueren, H. and Huygen, K., CD4+ T cells contain Mycobacterium tuberculosis infection in the absence of CD8+ T cells in mice vaccinated with DNA encoding Ag85A. Eur J Immunol 2000. 30: 2455-2459.

447 Denis, O. and Huygen, K., Characterization of the culture filtrate-specific cytotoxic T lymphocyte response induced by Bacillus Calmette-Guerin vaccination in H-2b mice. Int Immunol 1999. 11: 209-216.

448 Denis, O., Stroobant, V., Colau, D., D'Souza, S. and Huygen, K., Culture filtrate specific $\mathrm{H}-2$ (b) restricted $\mathrm{CD} 8+\mathrm{T}$ cells activated in vivo by Mycobacterium tuberculosis or bovis BCG recognize a restricted number of immunodominant peptides. Immunol Lett 2002. 81: 115-124.

449 Romano, M., Roupie, V., Wang, X. M., Denis, O., Jurion, F., Adnet, P. Y., Laali, R. and Huygen, K., Immunogenicity and protective efficacy of tuberculosis DNA vaccines combining mycolyl-transferase Ag85A and phosphate transport receptor PstS-3. Immunology 2006. 118: 321-332.

450 Lew, D., Parker, S. E., Latimer, T., Abai, A. M., Kuwahara-Rundell, A., Doh, S. G., Yang, Z. Y., et al., Cancer gene therapy using plasmid DNA: pharmacokinetic study of DNA following injection in mice. Hum Gene Ther 1995. 6: 553-564.

451 Beyer, T., Herrmann, M., Reiser, C., Bertling, W. and Hess, J., Bacterial carriers and virus-like-particles as antigen delivery devices: role of dendritic cells in antigen presentation. Curr Drug Targets Infect Disord 2001. 1: 287-302. 
452 D'Souza, S., Rosseels, V., Denis, O., Tanghe, A., De Smet, N., Jurion, F., Palfliet, K., et al., Improved tuberculosis DNA vaccines by formulation in cationic lipids. Infect Immun 2002. 70: 3681-3688.

453 Evans, R. K., Zhu, D. M., Casimiro, D. R., Nawrocki, D. K., Mach, H., Troutman, R. D., Tang, A., et al., Characterization and biological evaluation of a microparticle adjuvant formulation for plasmid DNA vaccines. J Pharm Sci 2004. 93: 1924-1939.

454 Ulmer, J. B., DeWitt, C. M., Chastain, M., Friedman, A., Donnelly, J. J., McClements, W. L., Caulfield, M. J., et al., Enhancement of DNA vaccine potency using conventional aluminum adjuvants. Vaccine 1999. 18: 18-28. Selby, M., Goldbeck, C., Pertile, T., Walsh, R. and Ulmer, J., Enhancement of DNA vaccine potency by electroporation in vivo. J Biotechnol 2000. 83: 147152.

456 Widera, G., Austin, M., Rabussay, D., Goldbeck, C., Barnett, S. W., Chen, M., Leung, L., et al., Increased DNA vaccine delivery and immunogenicity by electroporation in vivo. J Immunol 2000. 164: 4635-4640.

457 Chow, Y. H., Huang, W. L., Chi, W. K., Chu, Y. D. and Tao, M. H., Improvement of hepatitis B virus DNA vaccines by plasmids coexpressing hepatitis B surface antigen and interleukin-2. J Virol 1997. 71: 169-178.

458 Eo, S. K., Lee, S., Kumaraguru, U. and Rouse, B. T., Immunopotentiation of DNA vaccine against herpes simplex virus via co-delivery of plasmid DNA expressing CCR7 ligands. Vaccine 2001. 19: 4685-4693.

459 Gurunathan, S., Irvine, K. R., Wu, C. Y., Cohen, J. I., Thomas, E., Prussin, C., Restifo, N. P. and Seder, R. A., CD40 ligand/trimer DNA enhances both humoral and cellular immune responses and induces protective immunity to infectious and tumor challenge. J Immunol 1998. 161: 4563-4571.

460 Yamamoto, S., Yamamoto, T., Shimada, S., Kuramoto, E., Yano, O., Kataoka, T. and Tokunaga, T., DNA from bacteria, but not from vertebrates, induces interferons, activates natural killer cells and inhibits tumor growth. Microbiol Immunol 1992. 36: 983-997.

461 Every, A. L., Kramer, D. R., Mannering, S. I., Lew, A. M. and Harrison, L. C., Intranasal vaccination with proinsulin DNA induces regulatory CD4+ T cells that prevent experimental autoimmune diabetes. J Immunol 2006. 176: 46084615.

462 Stoitzner, P., Tripp, C. H., Eberhart, A., Price, K. M., Jung, J. Y., Bursch, L., Ronchese, F. and Romani, N., Langerhans cells cross-present antigen derived from skin. Proc Natl Acad Sci U S A 2006. 103: 7783-7788.

463 Mendez, S., Belkaid, Y., Seder, R. A. and Sacks, D., Optimization of DNA vaccination against cutaneous leishmaniasis. Vaccine 2002. 20: 3702-3708.

464 Woodland, D. L., Jump-starting the immune system: prime-boosting comes of age. Trends Immunol 2004. 25: 98-104.

465 Estcourt, M. J., Ramsay, A. J., Brooks, A., Thomson, S. A., Medveckzy, C. J. and Ramshaw, I. A., Prime-boost immunization generates a high frequency, high-avidity CD8(+) cytotoxic T lymphocyte population. Int Immunol 2002. 14: 31-37.

466 McConkey, S. J., Reece, W. H., Moorthy, V. S., Webster, D., Dunachie, S., Butcher, G., Vuola, J. M., et al., Enhanced T-cell immunogenicity of plasmid DNA vaccines boosted by recombinant modified vaccinia virus Ankara in humans. Nat Med 2003. 9: 729-735.

467 Kamath, A. T., Groat, N. L., Bean, A. G. and Britton, W. J., Protective effect of DNA immunization against mycobacterial infection is associated with the 
early emergence of interferon-gamma (IFN-gamma)-secreting lymphocytes. Clin Exp Immunol 2000. 120: 476-482.

468 Kipnis, A., Irwin, S., Izzo, A. A., Basaraba, R. J. and Orme, I. M., Memory T lymphocytes generated by Mycobacterium bovis BCG vaccination reside within a CD4 CD44lo CD62 Ligandhi population. Infect Immun 2005. 73: 77597764.

469 Silver, P. B., Agarwal, R. K., Su, S. B., Suffia, I., Grajewski, R. S., Luger, D., Chan, C. C., et al., Hydrodynamic Vaccination with DNA Encoding an Immunologically Privileged Retinal Antigen Protects from Autoimmunity through Induction of Regulatory T Cells. J Immunol 2007. 179: 5146-5158.

470 Madura Larsen, J., Stabell Benn, C., Fillie, Y., van der Kleij, D., Aaby, P. and Yazdanbakhsh, M., BCG stimulated dendritic cells induce an interleukin10 producing T-cell population with no T helper 1 or T helper 2 bias in vitro. Immunology 2007. 121: 276-282.

471 Hanekom, W. A., The immune response to BCG vaccination of newborns. Ann NY Acad Sci 2005. 1062: 69-78.

472 Doherty, T. M. and Andersen, P., Vaccines for tuberculosis: novel concepts and recent progress. Clin Microbiol Rev 2005. 18: 687-702.

473 Flynn, J. L., Immunology of tuberculosis and implications in vaccine development. Tuberculosis (Edinb) 2004. 84: 93-101.

474 Reed, S. G., Alderson, M. R., Dalemans, W., Lobet, Y. and Skeiky, Y. A., Prospects for a better vaccine against tuberculosis. Tuberculosis (Edinb) 2003. 83: 213-219.

475 Seder, R. A. and Ahmed, R., Similarities and differences in CD4+ and CD8+ effector and memory T cell generation. Nat Immunol 2003. 4: 835-842.

476 Majlessi, L., Simsova, M., Jarvis, Z., Brodin, P., Rojas, M. J., Bauche, C., Nouze, C., et al., An increase in antimycobacterial Th1-cell responses by primeboost protocols of immunization does not enhance protection against tuberculosis. Infect Immun 2006. 74: 2128-2137.

477 Darrah, P. A., Patel, D. T., De Luca, P. M., Lindsay, R. W., Davey, D. F., Flynn, B. J., Hoff, S. T., et al., Multifunctional TH1 cells define a correlate of vaccine-mediated protection against Leishmania major. Nat Med 2007. 13: 843850.

478 Denis, O., Lozes, E. and Huygen, K., Induction of cytotoxic T-cell responses against culture filtrate antigens in Mycobacterium bovis bacillus CalmetteGuerin-infected mice. Infect Immun 1997. 65: 676-684.

479 Moore, A. C. and Hill, A. V., Progress in DNA-based heterologous primeboost immunization strategies for malaria. Immunol Rev 2004. 199: 126-143.

480 Gilbert, S. C., Moorthy, V. S., Andrews, L., Pathan, A. A., McConkey, S. J., Vuola, J. M., Keating, S. M., et al., Synergistic DNA-MVA prime-boost vaccination regimes for malaria and tuberculosis. Vaccine 2006. 24: 4554-4561.

481 Schalk, J. A., Mooi, F. R., Berbers, G. A., van Aerts, L. A., Ovelgonne, H. and Kimman, T. G., Preclinical and clinical safety studies on DNA vaccines. Hum Vaccin 2006. 2: 45-53.

482 Motta, A., Schmitz, C., Rodrigues, L., Ribeiro, F., Teixeira, C., Detanico, T., Bonan, C., et al., Mycobacterium tuberculosis heat-shock protein 70 impairs maturation of dendritic cells from bone marrow precursors, induces interleukin10 production and inhibits T-cell proliferation in vitro. Immunology 2007. 121: 462-472.

483 Mason, C. M., Porretta, E., Zhang, P. and Nelson, S., CD4+ CD25+ transforming growth factor-beta-producing $\mathrm{T}$ cells are present in the lung in 
murine tuberculosis and may regulate the host inflammatory response. Clin Exp Immunol 2007. 148: 537-545.

484 Madura Larsen, J., Benn, C. S., Fillie, Y., van der Kleij, D., Aaby, P. and Yazdanbakhsh, M., BCG stimulated dendritic cells induce an interleukin-10 producing T-cell population with no T helper 1 or T helper 2 bias in vitro. Immunology 2007. 121: 276-282.

485 Ordway, D., Henao-Tamayo, M., Harton, M., Palanisamy, G., Troudt, J., Shanley, C., Basaraba, R. J. and Orme, I. M., The hypervirulent Mycobacterium tuberculosis strain HN878 induces a potent TH1 response followed by rapid down-regulation. J Immunol 2007. 179: 522-531.

486 van Riet, E., Hartgers, F. C. and Yazdanbakhsh, M., Chronic helminth infections induce immunomodulation: consequences and mechanisms. Immunobiology 2007. 212: 475-490.

487 Zuany-Amorim, C., Sawicka, E., Manlius, C., Le Moine, A., Brunet, L. R., Kemeny, D. M., Bowen, G., Rook, G. and Walker, C., Suppression of airway eosinophilia by killed Mycobacterium vaccae-induced allergen-specific regulatory T-cells. Nat Med 2002. 8: 625-629.

488 Powrie, F., Carlino, J., Leach, M. W., Mauze, S. and Coffman, R. L., A critical role for transforming growth factor-beta but not interleukin 4 in the suppression of T helper type 1-mediated colitis by CD45RB(low) CD4+ T cells. $J$ Exp Med 1996. 183: 2669-2674.

489 Resende Co, T., Hirsch, C. S., Toossi, Z., Dietze, R. and Ribeiro-Rodrigues, R., Intestinal helminth co-infection has a negative impact on both antiMycobacterium tuberculosis immunity and clinical response to tuberculosis therapy. Clin Exp Immunol 2007. 147: 45-52.

490 Elias, D., Wolday, D., Akuffo, H., Petros, B., Bronner, U. and Britton, S., Effect of deworming on human $\mathrm{T}$ cell responses to mycobacterial antigens in helminth-exposed individuals before and after bacille Calmette-Guerin (BCG) vaccination. Clin Exp Immunol 2001. 123: 219-225.

491 Schwartz, R. H., Natural regulatory T cells and self-tolerance. Nat Immunol 2005. 6: 327-330.

492 Menning, A., Hopken, U. E., Siegmund, K., Lipp, M., Hamann, A. and Huehn, J., Distinctive role of CCR7 in migration and functional activity of naive- and effector/memory-like Treg subsets. Eur J Immunol 2007. 37: 15751583.

493 Stephens, G. L., Andersson, J. and Shevach, E. M., Distinct subsets of FoxP3+ regulatory T cells participate in the control of immune responses. $J$ Immunol 2007. 178: 6901-6911.

494 Lahl, K., Loddenkemper, C., Drouin, C., Freyer, J., Arnason, J., Eberl, G., Hamann, A., et al., Selective depletion of Foxp3+ regulatory T cells induces a scurfy-like disease. J Exp Med 2007. 204: 57-63.

495 Goter-Robinson, C., Derrick, S. C., Yang, A. L., Jeon, B. Y. and Morris, S. L., Protection against an aerogenic Mycobacterium tuberculosis infection in BCG-immunized and DNA-vaccinated mice is associated with early type I cytokine responses. Vaccine 2006. 24: 3522-3529.

496 Morel, C., Badell, E., Abadie, V., Robledo, M., Setterblad, N., Gluckman, J. C., Gicquel, B., et al., Mycobacterium bovis BCG-infected neutrophils and dendritic cells cooperate to induce specific $\mathrm{T}$ cell responses in humans and mice. Eur J Immunol 2008. 38: 437-447.

497 Agnello, D., Lankford, C. S., Bream, J., Morinobu, A., Gadina, M., O'Shea, J. J. and Frucht, D. M., Cytokines and transcription factors that regulate T 
helper cell differentiation: new players and new insights. J Clin Immunol 2003. 23: $147-161$. 


\section{Appendix}

\section{1: Primer designs and properties}

Molecular weight, projected melting temperature, secondary structure and primer dimer projections were calculated using the Sigma Genosys primer design website, http://www.sigma-genosys.com/calc/DNACalc.asp. Projected annealing temperatures were calculated using $\mathrm{T}_{\mathrm{A}}=\mathrm{T}_{\mathrm{M}}-5$ to $10{ }^{\circ} \mathrm{C}$.

Table 8.1: Primers for amplification of the $A g 85 A$ insert

\begin{tabular}{|l|l|}
\hline 5' Ag85A primer & GCGACGCGTTGCAGCTTGTTGACAGGGTTCG \\
\hline Number of bases & 31 \\
\hline Molecular weight (Da) & 9598.97 \\
\hline $\mathrm{T}_{\mathrm{M}}:$ projected melting temperature $\left({ }^{\circ} \mathrm{C}\right)$ & 85.22 \\
\hline $\mathrm{T}_{\mathrm{A}}:$ projected annealing temperature $\left({ }^{\circ} \mathrm{C}\right)$ & 80.22 to 75.22 \\
\hline GC content $(\%)$ & 61.29 \\
\hline Projected secondary structure & Weak \\
\hline Projected primer dimer & None \\
\hline
\end{tabular}

\begin{tabular}{|l|l|}
\hline 3' Ag85A primer & GATACGCGTGACCCAGTGCCCGTTGCAGG \\
\hline Number of bases & 29 \\
\hline Molecular weight (Da) & 8919.6 \\
\hline $\mathrm{T}_{\mathrm{M}}:$ projected melting temperature $\left({ }^{\circ} \mathrm{C}\right)$ & 83.89 \\
\hline $\mathrm{T}_{\mathrm{A}}:$ projected annealing temperature $\left({ }^{\circ} \mathrm{C}\right)$ & 78.89 to 73.89 \\
\hline GC content $(\%)$ & 65.52 \\
\hline Projected secondary structure & Weak \\
\hline Projected primer dimer & None \\
\hline
\end{tabular}


Table 8.2: Primers for orientation PCR and sequencing of the $A g 85 A$ insert

\begin{tabular}{|l|l|}
\hline 5' CS primer & CAGTGCGAGCTGTTTCTTAGC \\
\hline Number of bases & 21 \\
\hline Molecular weight $(\mathrm{Da})$ & 6428.0 \\
\hline $\mathrm{T}_{\mathrm{M}}:$ projected melting temperature $\left({ }^{\circ} \mathrm{C}\right)$ & 64 \\
\hline $\mathrm{T}_{\mathrm{A}}:$ projected annealing temperature $\left({ }^{\circ} \mathrm{C}\right)$ & 59 to 54 \\
\hline GC content $(\%)$ & 52.38 \\
\hline Projected secondary structure & Weak \\
\hline Projected primer dimer & None \\
\hline
\end{tabular}

\begin{tabular}{|l|l|}
\hline 3' CS primer & CAGTATCAGCATGCACGTTC \\
\hline Number of bases & 20 \\
\hline Molecular weight $(\mathrm{Da})$ & 6076.9 \\
\hline $\mathrm{T}_{\mathrm{M}}:$ projected melting temperature $\left({ }^{\circ} \mathrm{C}\right)$ & 61.9 \\
\hline $\mathrm{T}_{\mathrm{A}}:$ projected annealing temperature $\left({ }^{\circ} \mathrm{C}\right)$ & 56.9 to 51.9 \\
\hline GC content $(\%)$ & 50 \\
\hline Projected secondary structure & Weak \\
\hline Projected primer dimer & None \\
\hline
\end{tabular}

\begin{tabular}{|l|l|}
\hline 5' Mid primer & CGGTCTTTCGATGGCTGCTTC \\
\hline Number of bases & 21 \\
\hline Molecular weight (Da) & 6395.0 \\
\hline $\mathrm{T}_{\mathrm{M}}:$ projected melting temperature $\left({ }^{\circ} \mathrm{C}\right)$ & 70 \\
\hline $\mathrm{T}_{\mathrm{A}}:$ projected annealing temperature $\left({ }^{\circ} \mathrm{C}\right)$ & 65 to 60 \\
\hline GC content $(\%)$ & 57.14 \\
\hline Projected secondary structure & None \\
\hline Projected primer dimer & None \\
\hline
\end{tabular}




\begin{tabular}{|l|l|}
\hline 3' Mid primer & CATGTCGGAGGCCTTGTAGC \\
\hline Number of bases & 20 \\
\hline Molecular weight $(\mathrm{Da})$ & 6148.9 \\
\hline $\mathrm{T}_{\mathrm{M}}:$ projected melting temperature $\left({ }^{\circ} \mathrm{C}\right)$ & 66.8 \\
\hline $\mathrm{T}_{\mathrm{A}}:$ projected annealing temperature $\left({ }^{\circ} \mathrm{C}\right)$ & 61.8 to 56.8 \\
\hline GC content $(\%)$ & 60 \\
\hline Projected secondary structure & None \\
\hline Projected primer dimer & None \\
\hline
\end{tabular}




\section{2: Sequencing of pKUN85A}

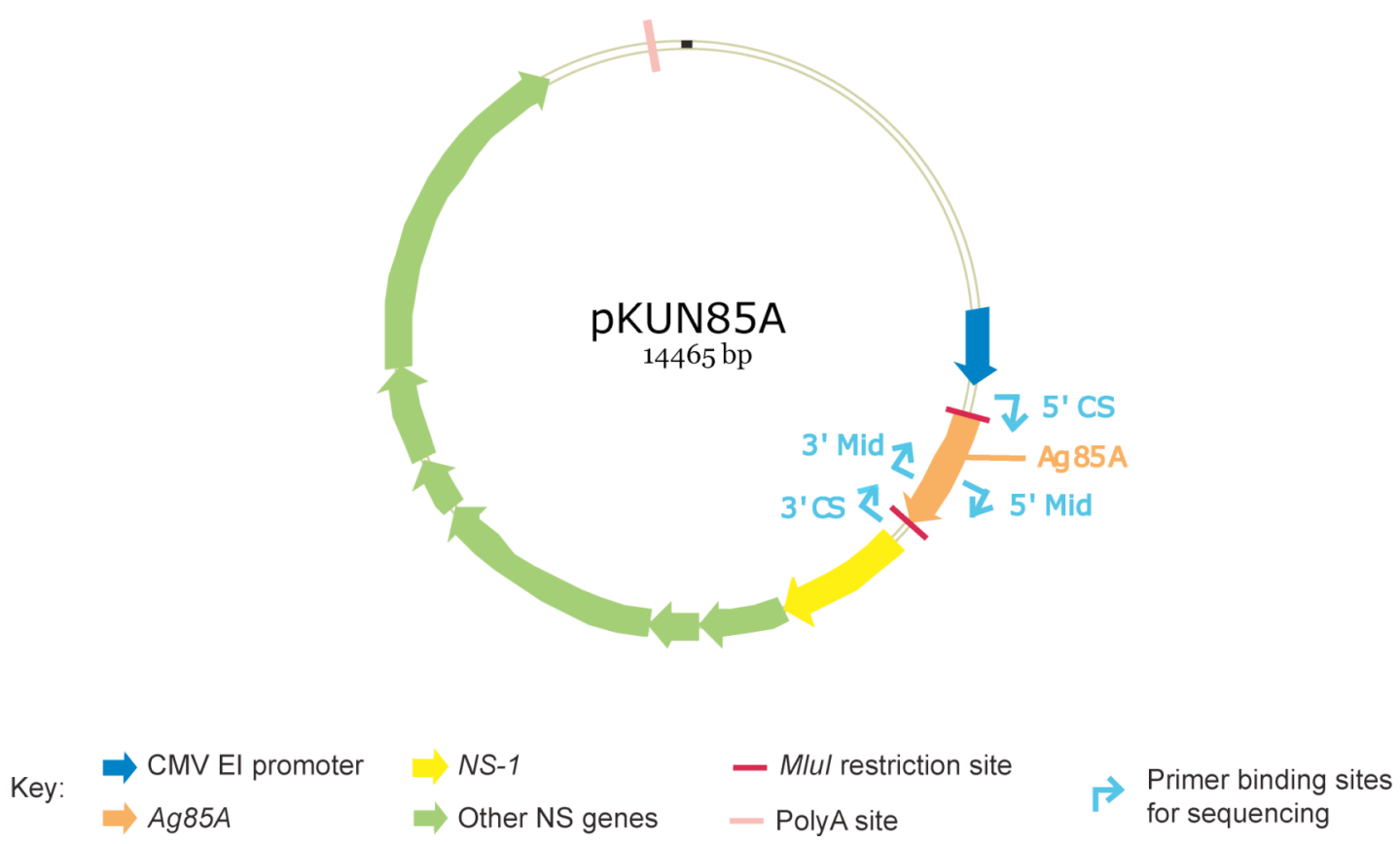

\section{Figure 8.1: Primer design for sequencing of transformant plasmids}

Primers were designed to sequence the length of the $\mathrm{Ag} 85 \mathrm{~A}$ insert and into vector DNA at the 5, and 3' ends of the insert. Total bp size of the plasmids is indicated and the first $b p$ is designated by the black notch at the top of the plasmid. MluI restriction sites either end of the $A g 85 \mathrm{~A}$ insert are indicated at $4239 \mathrm{bp}$ and $5223 \mathrm{bp}$. Primer binding sites for sequencing are indicated. 5' CS binds at 4057-4077 bp and 5' Mid binds at 4740-4760 bp and both result in sequence running from 5' to 3'. 3' CS binds at 5376-5395 bp and 3' Mid binds at 4889-4908 bp and both result in sequence running from 3 ' to 5 '.

For the following page:

\section{Figure 8.2: Sequencing alignment for transformant 37}

The sequences acquired for transformant 37, using the 3' Mid, 5' Mid, 3' CS and 5' CS primers described above, were compiled to provide the subject sequence. This subject sequence was aligned with the query sequence, which was the projected sequence of pKUN85A after insertion of the $A g 85 A$ insert. The MluI restriction sites either side of the $A g 85 A$ insert are underlined. For some bases highlighted in red, sequencing analysis software did not define the nucleotide.

However, these bases were verified as being the correct projected nucleotide after analysis of the corresponding electropherogram (data not shown). $\mathrm{M}_{511}=\mathrm{A}, \mathrm{Y}_{836}=\mathrm{C}$. 


\begin{tabular}{|c|c|c|c|}
\hline Query & 4223 & 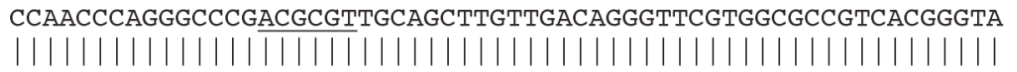 & 4282 \\
\hline Sbjct & 1 & CCAACCCAGGGCCCGACGCGTTGCAGCTTGTTGACAGGGTTCGTGGCGCCGTCACGGGTA & 60 \\
\hline Query & 4283 & $\begin{array}{l}\text { TGTCGCGTCGACTCGTGGTCGGGGCCGTCGGCGCGGCCCTAGTGTCGGGTCTGGTCGGCG } \\
||||||||||||||||||||||||||||||||||||||||||||||||||||||||||||\end{array}$ & 4342 \\
\hline Sbjct & 61 & TGTCGCGTCGACTCGTGGTCGGGGCCGTCGGCGCGGCCCTAGTGTCGGGTCTGGTCGGCG & 120 \\
\hline Query & 4343 & $\begin{array}{l}\text { CCGTCGGTGGCACGGCGACCGCGGGGGCATTTTCCCGGCCGGGCTTGCCGGTGGAGTACC } \\
||||||||||||||||||||||||||||||||||||||||||||||||||||||||||\end{array}$ & 4402 \\
\hline Sbjet & 121 & CCGTCGGTGGCACGGCGACCGCGGGGGCATTTTCCCGGCCGGGCTTGCCGGTGGAGTACC & 180 \\
\hline Query & 4403 & 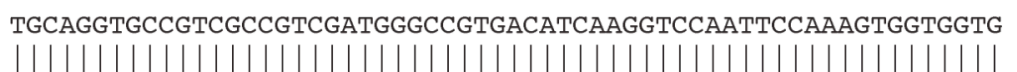 & 4462 \\
\hline Sbjet & 181 & TGCAGGTGCCGTCGCCGTCGATGGGCCGTGACATCAAGGTCCAATTCCAAAGTGGTGGTG & 240 \\
\hline Query & 4463 & $\begin{array}{l}\text { CCAACTCGCCCGCCTGTACCTGCTCGACGGCTGCGCGCGCAGGACGACTTCAGCGGCT } \\
||||||||||||||||||||||||||||||||||||||||||||||||||||||||||||\end{array}$ & 4522 \\
\hline Sbjet & 241 & CCAACTCGCCCGCCCTGTACCTGCTCGACGGCCTGCGCGCGCAGGACGACTTCAGCGGCT & 300 \\
\hline Query & 4523 & 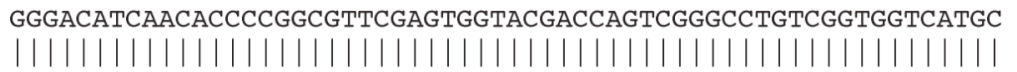 & 4582 \\
\hline Sbjet & 301 & GGGACATCAACACCCCGGCGTTCGAGTGGTACGACCAGTCGGGCCTGTCGGTGGTCATGC & 360 \\
\hline Query & 4583 & $\begin{array}{l}\text { CGGTGGGTGGCCAGTCAAGCTTCTACTCCGACTGGTACCAGCCCGCCTGCGGCAAGGCCG } \\
|||||||||||||||||||||||||||||||||||||||||||||||||||||||| \mid\end{array}$ & 4642 \\
\hline Sbjet & 361 & CGGTGGGTGGCCAGTCAAGCTTCTACTCCGACTGGTACCAGCCCGCCTGCGGCAAGGCCG & 420 \\
\hline Query & 4643 & 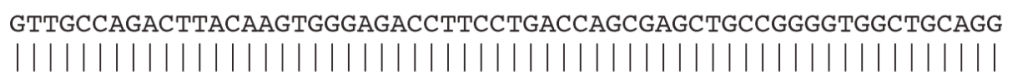 & 4702 \\
\hline Sbjet & 421 & GTTGCCAGACTTACAAGTGGGAGACCTTCCTGACCAGCGAGCTGCCGGGGTGGCTGCAGG & 480 \\
\hline Query & 4703 & $\begin{array}{l}\text { CCAACAGGCACGTCAAGCCCACCGGAAGCGCCGTCGTCGGTCTTTCGATGGCTGCTTCTT } \\
||||||||||||||||||||||||||||||||||||||||||||||||||||||||\end{array}$ & 4762 \\
\hline Sbjet & 481 & CCAACAGGCACGTCAAGCCCMCCGGAAGCGCCGTCGTCGGTCTTTCGATGGCTGCTTCTT & 540 \\
\hline Query & 4763 & $\begin{array}{l}\text { CGGCGCTGACGCTGGCGATCTATCACCCCCAGCAGTTCGTCTACGCGGGAGCGATGTCGG } \\
|||||||||||||||||||||||||||||||||||||||||||||||||||||||||| \mid\end{array}$ & 4822 \\
\hline Sbjet & 541 & CGGCGCTGACGCTGGCGATCTATCACCCCCAGCAGTTCGTCTACGCGGGAGCGATGTCGG & 600 \\
\hline Query & 4823 & 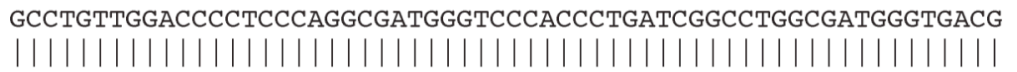 & 4882 \\
\hline Sbjet & 601 & GCCTGTTGGACCCCTCCCAGGCGATGGGTCCCACCCTGATCGGCCTGGCGATGGGTGACG & 660 \\
\hline Query & 4883 & $\begin{array}{l}\text { CTGGCGGCTACAAGGCCTCCGACATGTGGGGCCGAAGGAGGACCCGGGTGGCAGCGCA } \\
|||||||||||||||||||||||||||||||||||||||||||||||||||||| \mid\end{array}$ & 4942 \\
\hline Sbjet & 661 & CTGGCGGCTACAAGGCCTCCGACATGTGGGGCCCGAAGGAGGACCCGGCGTGGCAGCGCA & 720 \\
\hline Query & 4943 & 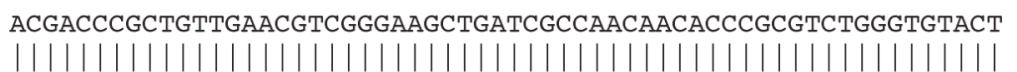 & 5002 \\
\hline Sbjet & 721 & ACGACCCGCTGTTGAACGTCGGGAAGCTGATCGCCAACAACACCCGCGTCTGGGTGTACT & 780 \\
\hline Query & 5003 & 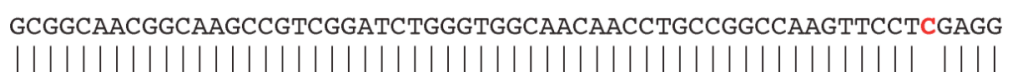 & 5062 \\
\hline Sbjet & 781 & GCGGCAACGGCAAGCCGTCGGATCTGGGTGGCAACAACCTGCCGGCCAAGTTCCTYGAGG & 840 \\
\hline Query & 5063 & $\begin{array}{l}\text { GCTTCGTGCGGACCAGCAACATCAAGTTCCAAGACGCCTACAACGCCGGTGGCGGCCACA } \\
|||||||||||||||||||||||||||||||||||||||||||||||||||||||| \mid\end{array}$ & 5122 \\
\hline Sbjet & 841 & GCTTCGTGCGGACCAGCAACATCAAGTTCCAAGACGCCTACAACGCCGGTGGCGGCCACA & 900 \\
\hline Query & 5123 & 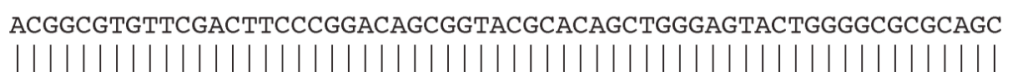 & 5182 \\
\hline Sbjet & 901 & ACGGCGTGTTCGACTTCCCGGACAGCGGTACGCACAGCTGGGAGTACTGGGGCGCGCAGC & 960 \\
\hline Query & 5183 & $\begin{array}{l}\text { TCAACGCTATGAAGCCCGACCTGCAACGGGCACTGGGTCACGCGTATG } 5230 \\
|||||||||||||||||||||||||||||||||||||||||||||| \mid\end{array}$ & \\
\hline Sbjet & 961 & TCAACGCTATGAAGCCCGACCTGCAACGGGCACTGGGTCACGCGTATG & \\
\hline
\end{tabular}




\section{3: Solutions and media}

\section{Freezing Media}

- 9 mL Fetal Bovine Serum (FBS) (PAA Laboratories GmbH, Pasching, Austria)

- $1 \mathrm{~mL}$ Dimethyl Sulfoxide (Sigma)

Mix together under sterile conditions and resuspend cells to be frozen in this at $2 \times 10^{6}$ cells $/ \mathrm{mL}$, Freeze aliquots immediately but slowly in ethanol at $-80{ }^{\circ} \mathrm{C}$.

\section{Incomplete Iscove's Modified Dulbecco's Medium}

- $\quad 3.024 \mathrm{~g} \mathrm{NaHCO}_{3}(\mathrm{BDH})$

- 1 sachet of powdered IMDM (Gibco BRL)

- $1000 \mathrm{~mL}$ of MilliQ $\mathrm{H}_{2} \mathrm{O}$

Add water to powders. Filter sterilise using a VacuCap60 filter (Pall Life Sciences). Store at $4{ }^{\circ} \mathrm{C}$ until use.

\section{Complete Iscove's Modified Dulbecco's Medium- 20, 10, 5, 1 or 0 \%}

- $10 \mathrm{~mL}$ of Gibco Penicillin-Streptomycin liquid (Invitrogen)

- $10 \mathrm{~mL}$ of Gibco GlutaMAX ${ }^{\mathrm{TM}}-1$ (Invitrogen)

- $1 \mathrm{~mL}$ of Gibco 2-Mercaptoethanol liquid (1000x) (Invitrogen)

- $200 \mathrm{~mL}(20 \%), 100 \mathrm{~mL}(10 \%), 50 \mathrm{~mL}(5 \%), 10 \mathrm{~mL}(1 \%)$ or $0 \mathrm{~mL}(0 \%)$ of FBS (PAA Laboratories GmbH)

- Top up to $1000 \mathrm{~mL}$ with iIMDM (as above)

Store at $4^{\circ} \mathrm{C}$ for up to 4 weeks. 


\section{Purification Wash Buffer}

- $2.84 \mathrm{~g} \mathrm{Na}_{2} \mathrm{HPO}_{4}(\mathrm{BDH})$

- $1000 \mathrm{~mL}$ of $\mathrm{dH}_{2} \mathrm{O}$

Adjust $\mathrm{pH}$ to 8.0 . Store at $4{ }^{\circ} \mathrm{C}$ but warm to room temperature and filter to degas and remove particulates prior to use.

\section{Purification Elution Buffer}

- $\quad 3.75 \mathrm{~g}$ of Glycine (BDH)

- $\quad 500 \mathrm{~mL}$ of $\mathrm{dH}_{2} \mathrm{O}$

Use $\mathrm{HCl}$ to adjust $\mathrm{pH}$ to 2.7 . Store at $4{ }^{\circ} \mathrm{C}$ but warm to room temperature and filter to degas and remove particulates prior to use.

\section{Neutralisation/Stabilisation Buffer}

- $6.06 \mathrm{~g}$ of Tris $(\mathrm{BDH})$

- $50 \mathrm{~mL}$ of $\mathrm{dH}_{2} \mathrm{O}$

Use $\mathrm{HCl}$ to adjust $\mathrm{pH}$ to 9.0. Filter to remove particulates prior to use.

\section{Phosphate Buffered Saline}

- $8 \mathrm{~g} \mathrm{NaCl}$

- $0.2 \mathrm{~g} \mathrm{KH}_{2} \mathrm{PO}_{4}$

- $1.15 \mathrm{~g} \mathrm{Na}_{2} \mathrm{HPO}_{4}$ (anhydrous)

- $0.2 \mathrm{~g} \mathrm{KCl}$

- $1000 \mathrm{~mL}$ MilliQ water

All components are contained in 1 sachet of Dulbecco's Phosphate Buffered Saline mix (Gibco). Add water to components and adjust $\mathrm{pH}$ to 7.4. Filter sterilise using a VacuCap60 filter (Pall Life Sciences). Store at $4{ }^{\circ} \mathrm{C}$ until use. 


\section{Tween Albumin Broth}

- 1.3 g of Dubos Broth Base (Difco, BD Diagnostic Systems, Sparks, MD, USA)

- $180 \mathrm{~mL}$ of $\mathrm{dH}_{2} \mathrm{O}$

Autoclave at $121{ }^{\circ} \mathrm{C}$ for 15 minutes, cool to $45^{\circ} \mathrm{C}$ and add $20 \mathrm{~mL}$ of sterile oleic acidalbumin-dextrose (OADC) supplement (Difco). Filter if desired.

\section{PBS/Tween $80-0.5$ or $0.05 \%$}

- $1000 \mathrm{~mL}$ of PBS (as described above)

- $5 \mathrm{~mL}(0.5 \%)$ or $0.5 \mathrm{~mL}(0.05 \%)$ of Tween 80 (Sigma)

Dissolve Tween80 into PBS, some heating may be required. Filter sterilise using a VacuCap60 filter (Pall Life Sciences). Store at $4{ }^{\circ} \mathrm{C}$ until use.

\section{Middlebrooks 7H11 agar plates}

- 10.5 g of 7 H11 agar (Difco)

- $450 \mathrm{~mL}$ of $\mathrm{dH}_{2} \mathrm{O}$

- $5 \mathrm{ml}$ of Glycerol BP 100\% (for growing M. tuberculosis or M. bovis BCG) or $2.01 \mathrm{~g}$ of Sodium Pyruvate (for growing M. bovis) (both from Sigma)

Boil to dissolve the agar and then autoclave the solution at $121^{\circ} \mathrm{C}$ for 10 minutes. Cool to $56^{\circ} \mathrm{C}$ in a waterbath then add $0.5 \mathrm{~mL}$ of polymyxin $\mathrm{B}(200 \mathrm{~K} \mathrm{U} / \mathrm{mL})(\mathrm{Gibco} \mathrm{BRL}), 0.5$ $\mathrm{mL}$ of carbenicillin $(50 \mathrm{mg} / \mathrm{mL})$ (Gibco BRL) and $50 \mathrm{ml}$ of sterile OADC supplement (Difco) and mix thoroughly. Pour $\sim 20 \mathrm{~mL}$ of agar into sterile plates (Biolab, Auckland, New Zealand) and cool and dry plates in a Class II cabinet overnight. Store at $4^{\circ} \mathrm{C}$ for up to 2 months and keep plates protected from light. 


\section{0x Anaesthetic}

- $10 \mathrm{~mL}$ of Ketamine at $100 \mathrm{mg} / \mathrm{mL}$ (Phoenix, Auckland, New Zealand)

- $1.5 \mathrm{~mL}$ of Xylazine at $20 \mathrm{mg} / \mathrm{mL}$ (Phoenix)

Store 10x anaesthetic stock at room temperature. Dilute 1:10 immediately prior to use. Use at $100 \mu \mathrm{L}$ ( $26 \mu \mathrm{g}$ of xylazine and $870 \mu \mathrm{g}$ of ketamine) per $10 \mathrm{~g}$ body weight for anaesthesia or $400 \mu \mathrm{L}$ (104 $\mu \mathrm{g}$ of xylazine and $3480 \mu \mathrm{g}$ of ketamine) per $10 \mathrm{~g}$ body weight for lethal anaesthesia.

\section{Alsevers Solution}

- $20.5 \mathrm{~g}$ of Dextrose (BDH)

- $4.2 \mathrm{~g}$ of $\mathrm{NaCl}(\mathrm{BDH})$

- $8.0 \mathrm{~g}$ of $\mathrm{Na}$ citrate $(\mathrm{BDH})$

- Top up to $1000 \mathrm{~mL}$ of $\mathrm{dH}_{2} \mathrm{O}$

Autoclave at $121{ }^{\circ} \mathrm{C}$ for 15 minutes, cool and aliquot into sterile containers. Store at room temperature until use.

\section{ACT Lysis Buffer}

- Solution 1:

○ $8.3 \mathrm{~g}$ of $\mathrm{NH}_{4} \mathrm{Cl}(\mathrm{BDH})$

○ $1000 \mathrm{~mL}$ of MilliQ $\mathrm{H}_{2} \mathrm{O}$

- Solution 2:

○ $20.6 \mathrm{~g}$ Tris $(\mathrm{BDH})$

○ $900 \mathrm{~mL}$ of MilliQ $\mathrm{H}_{2} \mathrm{O}$

Adjust the $\mathrm{pH}$ of solution 2 to 7.65 and top it up to a total volume of $1000 \mathrm{~mL}$. Take $900 \mathrm{~mL}$ of solution 1 and mix with $100 \mathrm{~mL}$ of solution 2. Adjust the $\mathrm{pH}$ of the mixed solutions to 7.2. Filter sterilise using a VacuCap60 filter (Pall Life Sciences). Store at 4 ${ }^{\circ} \mathrm{C}$ until use but allow solution to warm to room temperature before use. 


\section{Flow Cytometry Buffer}

- $1000 \mathrm{~mL}$ of PBS (as described above)

- $10 \mathrm{~mL}$ FBS (PAA Laboratories GmbH) or 10g Bovine Serum Albumin (Gibco)

Prepare under sterile conditions. Store at $4^{\circ} \mathrm{C}$ for up to 4 weeks

\section{Lung Digestion Media (per lung)}

- $5 \mathrm{~mL}$ of $0 \%$ cIMDM (as described above)

- $12 \mathrm{mg}$ of Collagenase Type I (Gibco)

- $5 \mathrm{mg}$ of DNAase Type I (Roche)

Mix components immediately before use under sterile conditions. Use $5 \mathrm{~mL}$ of lung digestion media per lung.

\section{Percoll Diluent}

- $45 \mathrm{~mL}$ of $10 \times$ PBS

- $3 \mathrm{~mL}$ of $0.6 \mathrm{M} \mathrm{HCl}$

- $132 \mathrm{~mL}$ of $\mathrm{dH}_{2} \mathrm{O}$

Filter sterilise using a VacuCap60 filter (Pall Life Sciences). Store at $4{ }^{\circ} \mathrm{C}$ until use.

\section{$70 \%$ Percoll}

- $63 \mathrm{~mL}$ of Percoll Diluent (as described above)

- $37 \mathrm{~mL}$ of Percoll (GE Healthcare)

Mix liquids and adjust $\mathrm{pH}$ to 7.2. Filter sterilise using a VacuCap60 filter (Pall Life Sciences). Store at $4{ }^{\circ} \mathrm{C}$ until use. 


\section{4\% Paraformaldehyde Solution}

- $6 \mathrm{~g}$ of anhydrous $\mathrm{Na}_{2} \mathrm{H}_{2} \mathrm{PO}_{4}(\mathrm{BDH})$

- $17.9 \mathrm{~g}$ of $\mathrm{Na}_{2} \mathrm{HPO}_{4} .12 \mathrm{H}_{2} \mathrm{O}(\mathrm{BDH})$

- $900 \mathrm{~mL}$ of MilliQ $\mathrm{H}_{2} \mathrm{O}$

Mix together and warm on a hot plate. Carefully add $40 \mathrm{~g}$ of paraformaldehyde (Sigma) so as to not disperse into the air. Stir with heating on the hot plate for 30-45 min until dissolved. Add small amounts of $\mathrm{NaOH}$ if needed to help dissolving. Cool the solution and top up with MilliQ $\mathrm{H}_{2} \mathrm{O}$ to a volume of $950 \mathrm{~mL}$. Adjust the $\mathrm{pH}$ to 7.0 and bring the final volume to $1000 \mathrm{~mL}$ with more MilliQ $\mathrm{H}_{2} \mathrm{O}$. Aliquot and store immediately at $20^{\circ} \mathrm{C}$ in $50 \mathrm{ml}$ tubes for up to 1 year. Minimise exposure to light and defrost several hours prior to use. If cloudy it may require warming to re-dissolve. Store thawed aliquots at $4{ }^{\circ} \mathrm{C}$ and use within 3 days.

\section{Saponin Buffer}

- $1000 \mathrm{~mL}$ of PBS (as described above)

- $5 \mathrm{~g}$ of Bovine Serum Albumin (Gibco)

- $1 \mathrm{~g}$ of Saponin (Sigma)

Dissolve BSA in PBS, then add saponin and mix gently for 15 min. Filter sterilise, aliquot and keep at $4{ }^{\circ} \mathrm{C}$ for short-term storage or $-20^{\circ} \mathrm{C}$ for long-term storage.

\section{ELISA Coating Buffer}

- $3.56 \mathrm{~g}$ of $\mathrm{Na}_{2} \mathrm{CO}_{3}$ (or $4.23 \mathrm{~g}$ of $\left.\mathrm{Na}_{2} \mathrm{CO}_{3} .10 \mathrm{H}_{2} 0\right)(\mathrm{BDH})$

- $8.40 \mathrm{~g}$ of $\mathrm{NaHCO}_{3}(\mathrm{BDH})$

- $1000 \mathrm{~mL}$ of $\mathrm{dH}_{2} \mathrm{O}$

Store at $4{ }^{\circ} \mathrm{C}$ for no longer than 3 weeks 


\section{0x ELISA Wash Buffer}

- $1000 \mathrm{~mL}$ of $10 \mathrm{x}$ PBS

- $5 \mathrm{~mL}$ of Tween20 (Sigma)

Dissolve Tween20 in 10x PBS and adjust $\mathrm{pH}$ to 7.4 . Store at $4^{\circ} \mathrm{C}$ for several months. Dilute to make 1x ELISA Wash Buffer immediately prior to use.

\section{ELISA Assay Diluent}

- $500 \mathrm{~mL}$ of PBS (as described above)

- $50 \mathrm{~mL}$ FBS (PAA Laboratories GmbH)

Freshly prepare under sterile conditions and use within 3 days of preparation with storage at $4{ }^{\circ} \mathrm{C}$.

\section{ELISA Stop Solution}

- $2 \mathrm{~N} \mathrm{H}_{2} \mathrm{SO}_{4}$

Store at $4^{\circ} \mathrm{C}$ for several months.

\section{LB Amp ${ }^{+}$agar plates}

- $500 \mathrm{~mL}$ of $\mathrm{dH}_{2} 0$

- $6.25 \mathrm{~g}$ of Bacto-Agar (BD Biosciences)

- $12.5 \mathrm{~g}$ of LB Broth Base (Invitrogen)

Autoclave at $121{ }^{\circ} \mathrm{C}$ for 15 minutes, cool to $45^{\circ} \mathrm{C}$ and add $0.5 \mathrm{~mL}$ ampicillin (100 $\mu \mathrm{g} / \mathrm{mL}$ ) (Sigma). Pour $\sim 20 \mathrm{~mL}$ of agar into sterile agar plates (Biolab) and cool for 20 minutes store at $4^{\circ} \mathrm{C}$ for up to 4 weeks. 


\section{LB Amp ${ }^{+}$liquid broth}

- $\quad 500 \mathrm{~mL}$ of $\mathrm{dH}_{2} \mathrm{O}$

- $\quad 12.5 \mathrm{~g}$ of LB Broth Base (Invitrogen)

Autoclave at $121{ }^{\circ} \mathrm{C}$ for 15 minutes to sterilise and store at room temperature until needed. Just before use, add a $0.5 \mathrm{~mL}$ freshly thawed aliquot of ampicillin $(100 \mu \mathrm{g} / \mathrm{mL})$ (Sigma). Store at $4^{\circ} \mathrm{C}$ for up to 7 days.

\section{Glycerol Stocks}

- $850 \mu \mathrm{L}$ of $E$. coli grown in $\mathrm{LB} \mathrm{Amp}{ }^{+}$liquid broth

- $150 \mu \mathrm{L}$ of Glycerol BP (Sigma)

Vortex thoroughly and freeze immediately at $-80{ }^{\circ} \mathrm{C}$.

\section{Lysis Buffer}

- $\quad 6.71 \mathrm{~mL}$ MilliQ $\mathrm{H}_{2} \mathrm{O}$

- $0.5 \mathrm{~mL}$ of Tris- $\mathrm{HCl}(1 \mathrm{M}, \mathrm{pH} 8.0)(\mathrm{BDH})$

- $1.5 \mathrm{~mL}$ of $\mathrm{NaCl}(1 \mathrm{M})(\mathrm{BDH})$

- $\quad 0.1 \mathrm{~mL}$ of Sodium Dodecyl Sulphate (10\%) (Sigma)

- $1 \mathrm{~mL}$ of NP-40 (10\%) (Sigma)

- $\quad 0.09 \mathrm{~mL}$ of Triton $\mathrm{X}-100(100 \%)($ Sigma $)$

- $0.1 \mathrm{~mL}$ of Phenylmethanesulfonyl Fluoride (PMSF) (100mM) (Sigma)

Mix it all of the above and freeze in $1 \mathrm{~mL}$ aliquots immediately. Thaw aliquots just before use. PMSF is a protease inhibitor that only has a half-life of $30 \mathrm{~min}$ at room temp, so keep lysis buffer and cell lysates at $4{ }^{\circ} \mathrm{C}$. 


\section{0x TAE buffer}

- $121 \mathrm{~g}$ of Tris $(\mathrm{BDH})$

- $28.55 \mathrm{~mL}$ of Glacial Acetic Acid (BDH)

- $2 \mathrm{~mL}$ of $0.5 \mathrm{M}$ EDTA (ph 8.0)

- $500 \mathrm{~mL}$ of MilliQ $\mathrm{H}_{2} \mathrm{O}$

Autoclave in a $1 \mathrm{~L} \mathrm{Schott} \mathrm{bottle} \mathrm{at} 121{ }^{\circ} \mathrm{C}$ for 15 minutes to sterilise and store at room temperature until needed. Before use dilute 50x.

\section{Agarose gel- 2, 1.5 or $0.8 \%$}

- $100 \mathrm{~mL}$ TAE buffer

- $2 \mathrm{~g}(2 \%), 1.5 \mathrm{~g}(1.5 \%)$ or $0.8 \mathrm{~g}(0.8 \%)$ of UltraPure ${ }^{\mathrm{TM}}$ Agarose (Invitrogen)

Melt the agarose by putting on for initially $3 \mathrm{~min}$ at high in the microwave, swirling and continuing to melt $1 \mathrm{~min}$ at a time until the solution boils and is clear. Store at $40{ }^{\circ} \mathrm{C}$ for up to 6 weeks. Once poured, leave for $30 \mathrm{~min}$ to cool.

\section{PCR Loading Buffer}

- $19.5 \mathrm{~g}$ of Sucrose (BDH)

- $3 \mathrm{~mL}$ of $0.1 \mathrm{M}$ EDTA (Sigma)

- $3 \mathrm{~mL}$ of $0.1 \mathrm{M}$ Tris- $\mathrm{HCl}(\mathrm{pH} 7.5)(\mathrm{BDH})$

- $90 \mathrm{mg}$ of Bromophenol Blue (Sigma)

- Up to $30 \mathrm{~mL}$ with MilliQ $\mathrm{H}_{2} \mathrm{O}$

Initially dissolve sucrose in a minimum volume of water as it has substantial mass itself. Heat to dissolve. Mix components, aliquot into $1 \mathrm{~mL}$ batches and freeze at $-20{ }^{\circ} \mathrm{C}$ until needed. Store thawed aliquots at $4{ }^{\circ} \mathrm{C}$. 


\section{0x Western Transfer buffer}

- $\quad 3.03 \mathrm{~g}$ of Tris (BDH)

- $144 \mathrm{~g}$ of Glycine (BDH)

- $1000 \mathrm{~mL}$ of MilliQ $\mathrm{H}_{2} \mathrm{O}$

Store at $4{ }^{\circ} \mathrm{C}$ for several months. Before use dilute $10 \mathrm{x}$ and add methanol to $20 \% \mathrm{v} / \mathrm{v}$ final.

\section{0x Tris Buffered Saline}

- $48.5 \mathrm{~g}$ of Tris $(\mathrm{BDH})$

- $88.7 \mathrm{~g}$ of $\mathrm{NaCl}(\mathrm{BDH})$

- $5 \mathrm{~mL}$ of Tween20 (Sigma)

- $950 \mathrm{~mL}$ of MilliQ $\mathrm{H}_{2} \mathrm{O}$

Adjust $\mathrm{pH}$ to 8.6 with $\mathrm{HCl}$ and top up to a final volume of $1000 \mathrm{~mL}$. Store at $4{ }^{\circ} \mathrm{C}$ for several months. Before use dilute 10x.

\section{Western Blocking Solution}

- $100 \mathrm{~mL} 1 \mathrm{x}$ TBS (as described above)

- $4 \mathrm{~g}$ of Anchor Low-Fat Milk Powder (Fonterra Brands NZ Ltd., Auckland New Zealand)

Mix well to ensure that the milk powder is resuspended. Store at $4^{\circ} \mathrm{C}$ for up to 3 days then discard. 


\section{4: Publications}

Publications arising from work presented in this thesis include:

2006

Quinn, K. M., McHugh, R. S., Rich, F. J., Goldsack, L. M., de Lisle, G. W., Buddle, B. M., Delahunt, B. and Kirman, J. R., Inactivation of $\mathrm{CD} 4{ }^{+} \mathrm{CD} 25^{+}$regulatory T cells during early mycobacterial infection increases cytokine production but does not affect pathogen load. Immunol Cell Biol 2006. 84: 467-474.

2007

Quinn, K. M., Rich, F. J., Goldsack, L. M., de Lisle, G. W., Buddle, B. M., Delahunt, B. and Kirman, J. R., Accelerating the secondary immune response by inactivating $\mathrm{CD} 4{ }^{+} \mathrm{CD} 25^{+} \mathrm{T}$ regulatory cells prior to $\mathrm{BCG}$ vaccination does not enhance protection against Tuberculosis. Revised manuscript submitted, European Journal of Immunology 
